
\title{
Food and Agriculture United Nations
}

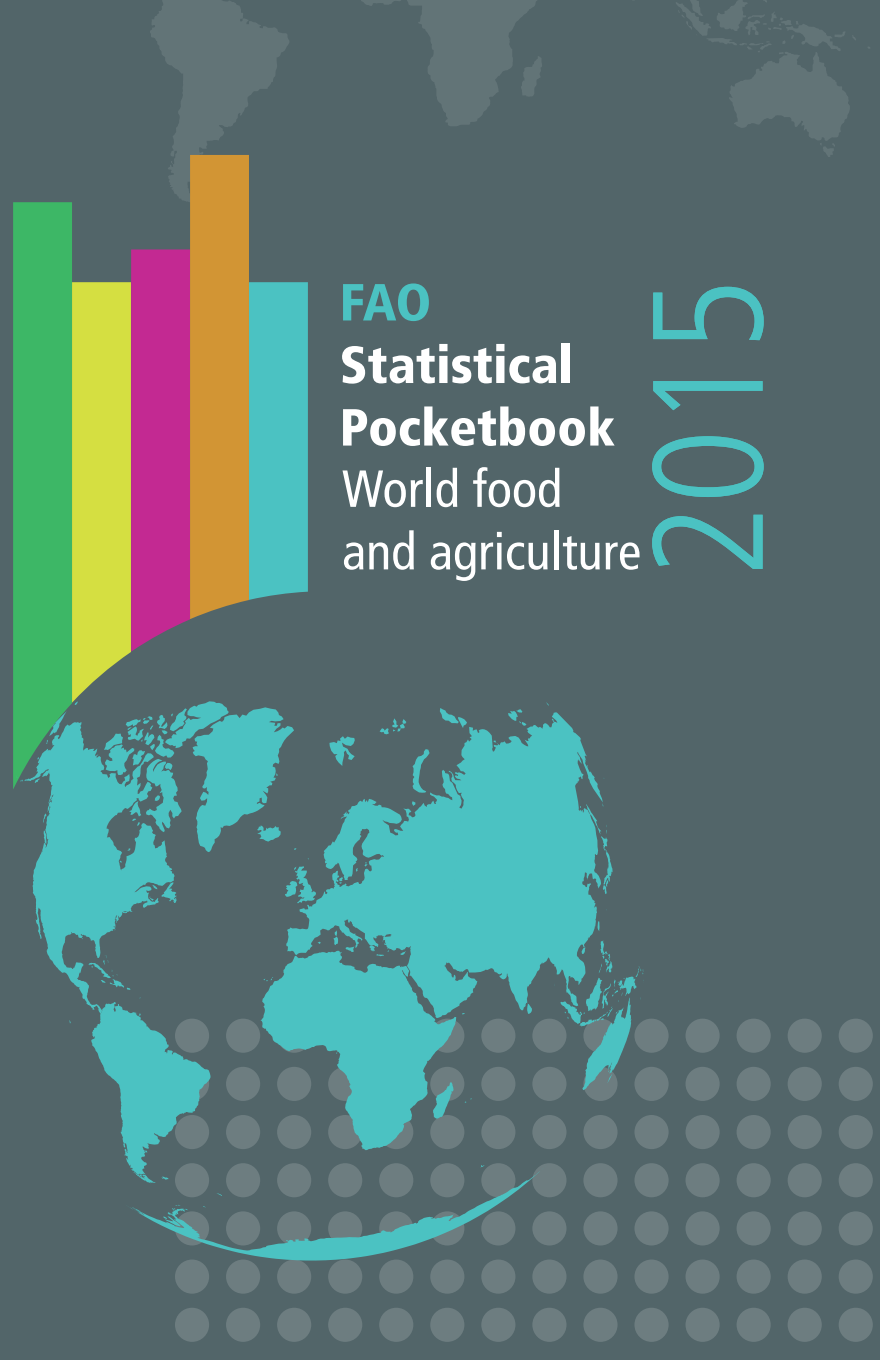




\section{FAO Statistical Pocketbook}

\section{5}




\begin{abstract}
The designations employed and the presentation of material in this information product do not imply the expression of any opinion whatsoever on the part of the Food and Agriculture Organization of the United Nations (FAO) concerning the legal or development status of any country, territory, city or area or of its authorities, or concerning the delimitation of its frontiers or boundaries. The mention of specific companies or products of manufacturers, whether or not these have been patented, does not imply that these have been endorsed or recommended by FAO in preference to others of a similar nature that are not mentioned.

The views expressed in this information product are those of the author(s) and do not necessarily reflect the views or policies of FAO.

ISBN 978-92-5-108802-9

(c) FAO, 2015

FAO encourages the use, reproduction and dissemination of material in this information product. Except where otherwise indicated, material may be copied, downloaded and product. Except where otherwise indicated, material may be copied, downloaded and
printed for private study, research and teaching purposes, or for use in non-commercial printed for private study, research and teaching purposes, or for use in non-commercial
products or services, provided that appropriate acknowledgement of FAO as the source products or services, provided that appropriate acknowledgement of FAO as the source
and copyright holder is given and that FAO's endorsement of users' views, products or and copyright holder is given and
services is not implied in any way.

All requests for translation and adaptation rights, and for resale and other commercial All requests for translation and adaptation rights, and for resale and other commercia use rights should be mat copyright@fao.org.

FAO information products are available on the FAO website (www.fao.org/publications) and can be purchased through publications-sales@fao.org.
\end{abstract}




\section{Foreword}

2015 is a pivotal year as we look back on the progress we have made on the development agenda, and forward on what we must still accomplish.

This is the end of a 15-year cycle where the international community partnered with developing countries to tackle the Millennium Development Goals. We are now taking stock of what we can learn from that effort. For example we know that the commitment to halve the percentage of hungry people, that is, to reach the MDG 1c target, has been almost met at the global level. Indeed, 72 of the 129 countries monitored for progress have reached that MDG target, while 29 of them also reached the more ambitious WFS goal by at least halving the number of undernourished people in their populations.

This year also marks the beginning of the new post-2015 Sustainable Development Agenda. Again the international community will unite around new priorities for the future.

Clear indicators to measure progress towards these international goals is of paramount importance. Timely and robust statistics are the fundamental tool in monitoring the myriad efforts being made, both with an eye to early detection of problems and the recognition of success. The better the data, the better the policies that can be designed. And the better the data, the easier it is to measure the impact of policies or to hold governments accountable for the pledges they make.

This publication presents selected key indicators related to agriculture and food security that the international community, governments, the private sector and civil society can use to assess current trends and prioritize their actions. It is divided into two main sections, one thematic and one country-specific. It presents a variety of dimensions of agriculture and food security along four main focus areas:

The setting measures the state of the agricultural resource base by assessing the supply of land, labour, capital and inputs, and examining the pressure on the world food system stemming from demographic and macroeconomic change.

Hunger dimensions gauges the state of food insecurity and malnutrition, and highlights the four dimensions - availability, access, stability and utilization that determine the scale of hunger and the shape of undernourishment.

Food supply evaluates the past and present productive capacity of world agriculture, together with the role of trade, in meeting the world's demand for food, feed and other products.

Environment examines the sustainability of agriculture in the context of the pressure it exerts on its ecological surroundings, including the interaction of agriculture with climate change.

This Pocketbook is part of the FAO Statistical Yearbook suite of products and is just one of the tools that can be used as building blocks for monitoring progress and formulating policy. It includes data from FAOSTAT, other FAO databases and international organizations.

FAO is deeply committed to helping countries strengthen their statistical systems to improve the timeliness and quality of their data. And it will continue to do so through the SDG process.

Pietro Gennari

Chief Statistician and Director, FAO Statistics Division 


\section{Contents}

Demographics

Economy

Labour

Inputs

Investment

Undernourishment

Food availability

Food access

Economic and political stability

Food utilization

Dietary energy supply

Crop production

Crops

Livestock

Fisheries

Agricultural trade

Land

Water

Energy

Forestry

Climate change

Country Profiles 


\section{Demographics}

A combination of declining mortality rates, prolonged life expectancy and younger populations in regions with high fertility contributes to population growth in the world. While growth rates have been slowing since the late 1960s, the world's population has nevertheless doubled since then, to over 7 billion people. Population growth is generally highest where income levels are low. This is especially true in cities. Since 2008, there have been more people living in cities than in rural areas.

CHART 2: Population, average annual growth (2004-2014)

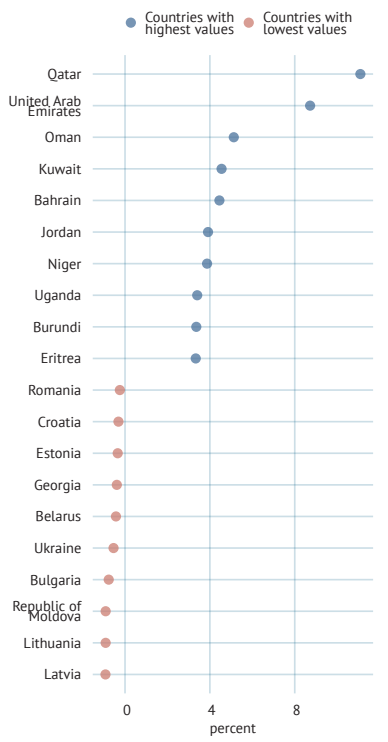

CHART 1: World rural and urban population (1961 to 2030)

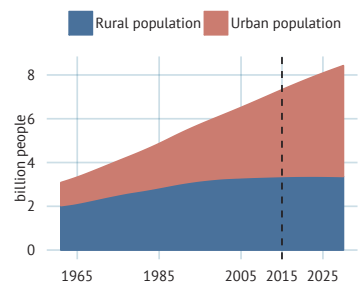

Data after 2010 are projections.

CHART 3: Life expectancy at birth, countries with the highest and lowest values (2013)

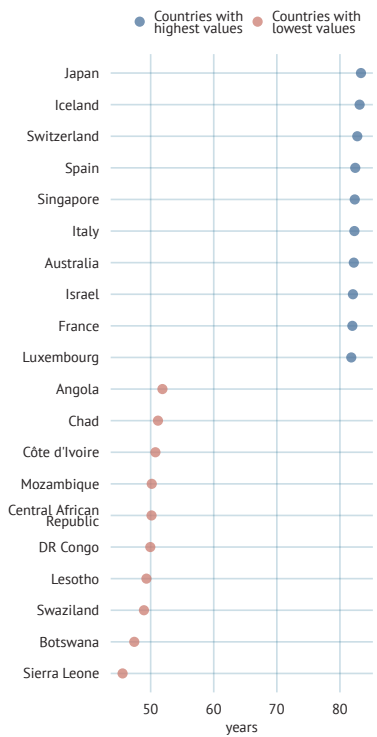

CHART 4: Total economically active population in Asia (2000 to 2014)

$\rightarrow$ Central Asia $\rightarrow$ Eastern Asia $\rightarrow$ South-Eastern Asia $\rightarrow$ Southern Asia $\rightarrow$ Western Asia

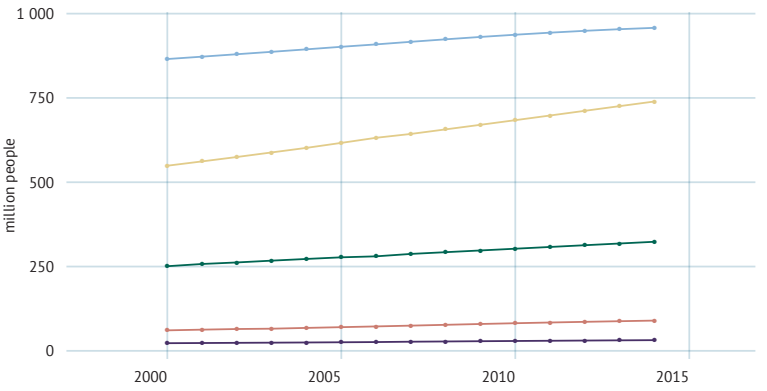


FIGURE 1: Rural population, share of total population (percent, 2014)

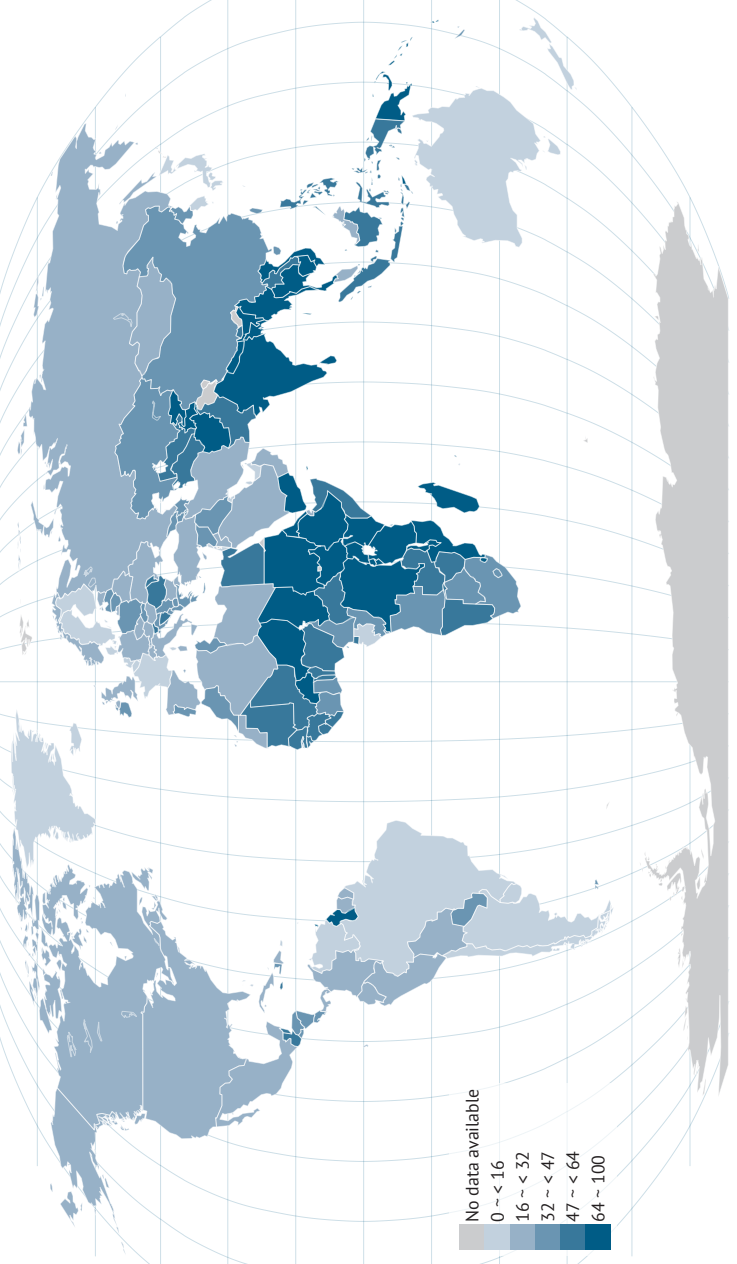




\section{Economy}

While some sectors have been hard hit, agriculture has demonstrated resilience during the recent economic downturn. Changes in the wider economy, including growing global integration, affect the performance of the agriculture sector. Higher overall economic growth also raises consumers' incomes and hence food demand. Changing interest rates influence capital investments, land values and storage levels, while inflation affects input prices, revenues and credit costs. Fluctuations in exchange rates have an important bearing on international competitiveness and trade flows.

\section{CHART 6: Agriculture value added per worker,} countries with the highest values (2013)

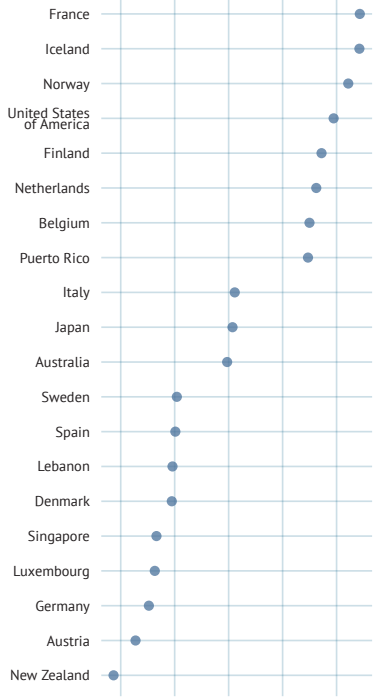

$\begin{array}{lllll}30 & 40 & 50 & 60 & 70\end{array}$ constant 2000 thousand US\$
CHART 5: Value added in agriculture, industry and services, share of GDP (2013)

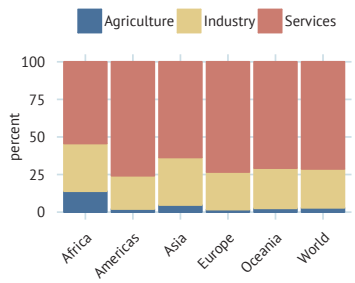

CHART 7: Value added in agriculture, average annual growth (2003-2013)

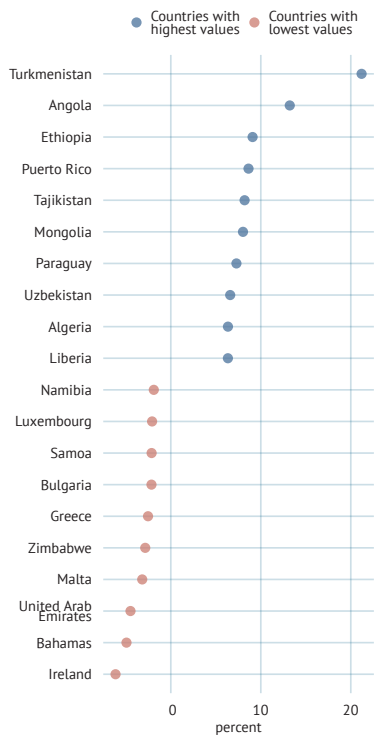

CHART 8: Value added in agriculture as share of GDP

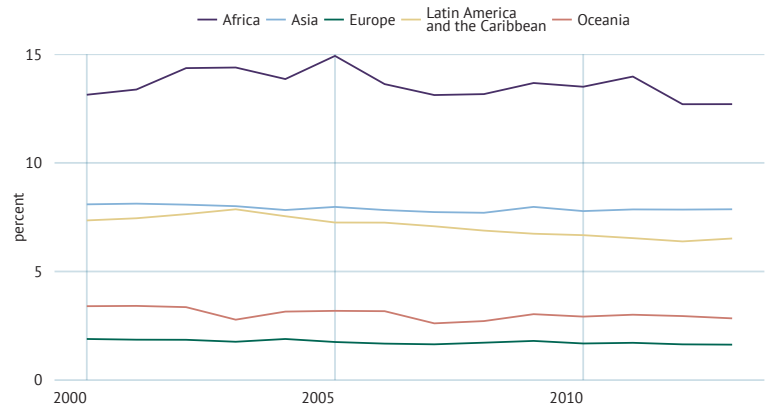


FIGURE 2: Value added in agriculture, share of GDP (percent, 2010 to 2013*)

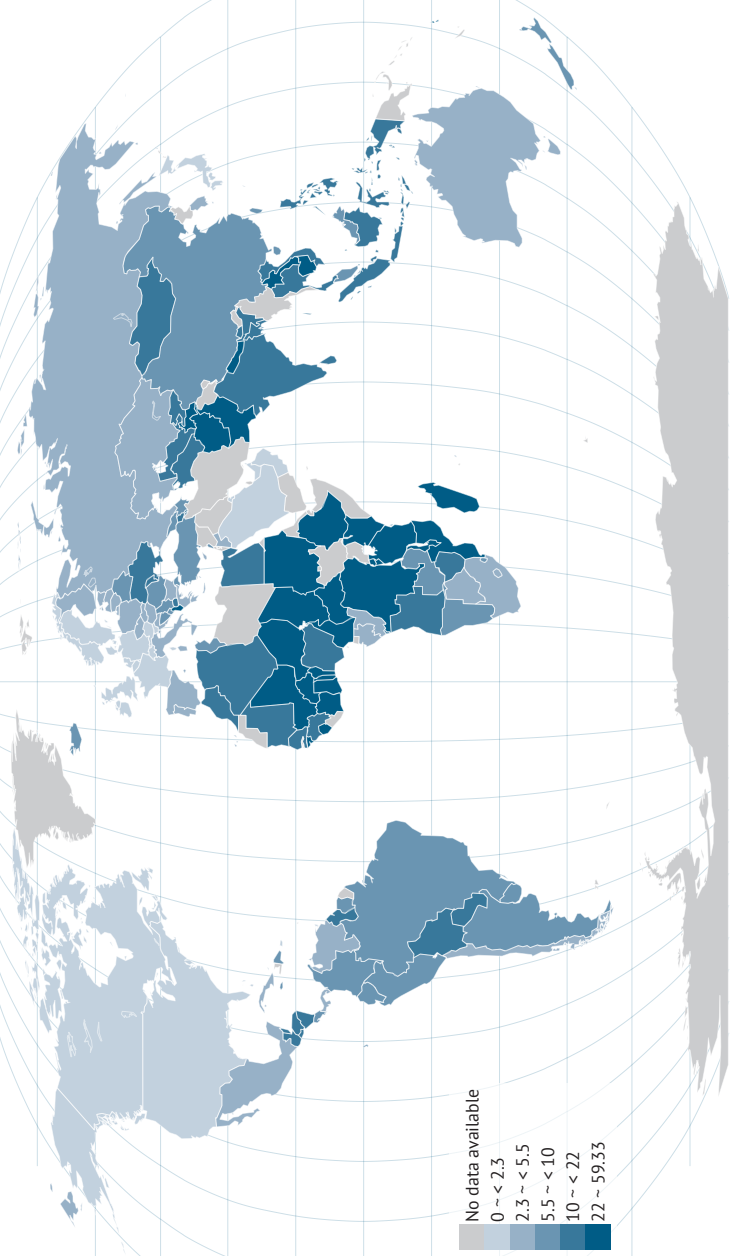




\section{Labour}

A strong labour market is the foundation of sustained well-being and economic growth, inclusion and social cohesion. Therefore access to safe, productive and remunerated work is essential. Yet many workers, especially the most vulnerable, do not enter into formal wage employment but are instead self-employed or participate in unpaid family work, such as in agriculture. This is especially the case with subsistence farming. As a large share of the working poor are involved in agriculture, developments in this sector have a major impact on welfare.

CHART 10: Female employment in agriculture, share of female employment (percent, 2012)

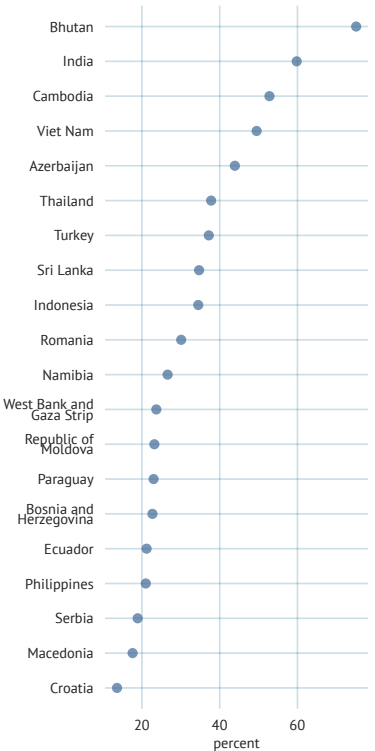

\section{CHART 11: Male employment in agriculture,} share of male employment (percent, 2012)

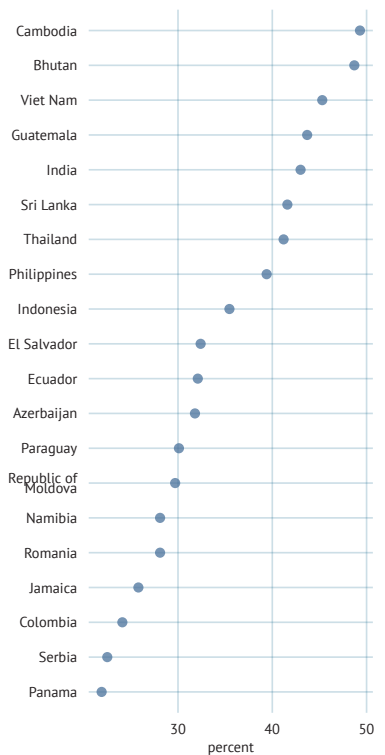

CHART 12: Female employment in agriculture, share of female employment (2000-2014)

$\rightarrow$ Africa $\rightarrow$ Americas $\rightarrow$ Asia $\rightarrow$ Europe $\rightarrow$ Oceania $\rightarrow$ World

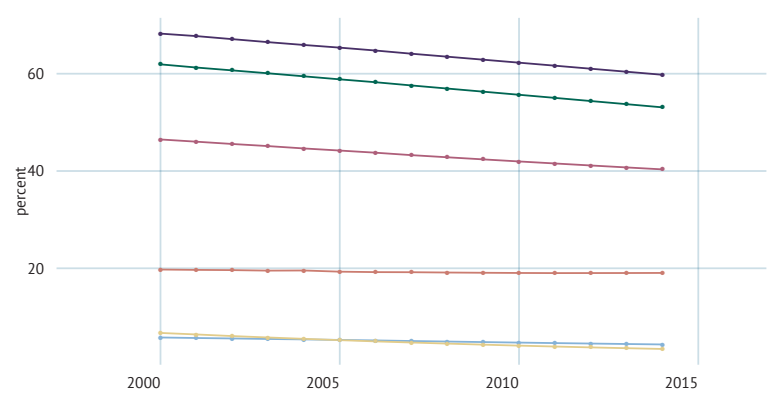


FIGURE 3: Employment in agriculture, share of total employment (percent, 2007 to $2012^{*}$ )

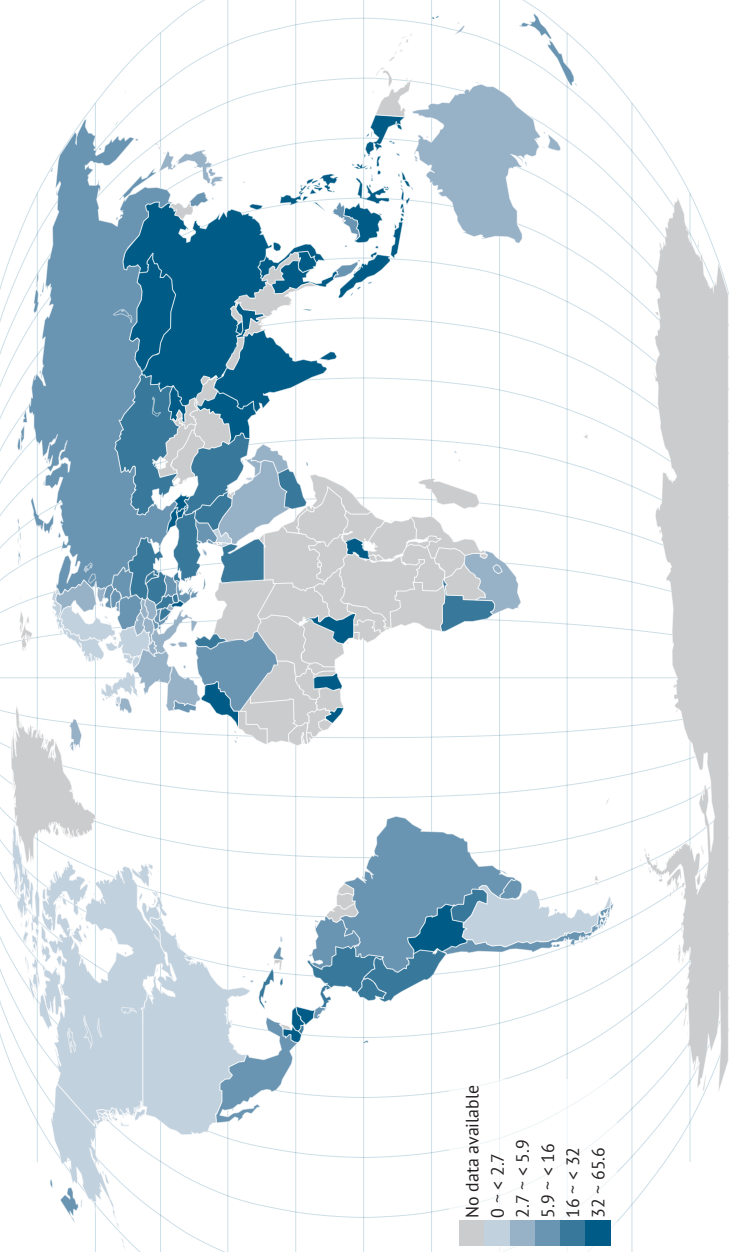




\section{Inputs}

Adequate access to inputs, including land, pesticides and fertilizers, is vital for agricultural production and growth. Throughout Asia and in parts of Latin America, expanding seed and fertilizer use has been accompanied by investments in irrigation, rural roads, marketing infrastructure and financial services, paving the way for dynamic commercial input markets. In other regions, such as sub-Saharan Africa, the uptake of agricultural inputs is relatively low because it is often cheaper to expand cropland to have higher production.

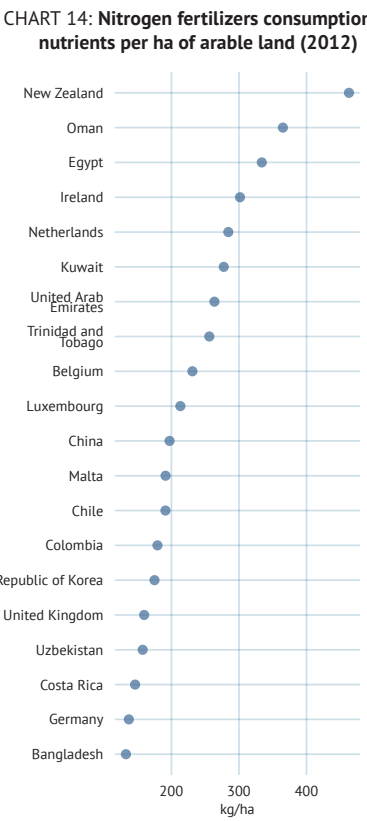

CHART 13: Fertilizer consumption in nutrients per ha of arable land (2002 to 2012)

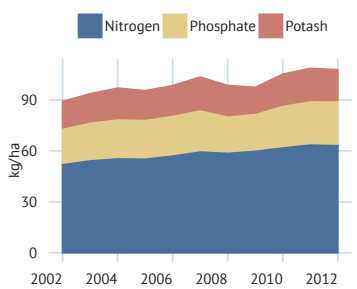

CHART 15: Phosphate fertilizers consumption in nutrients per ha of arable land (2012)

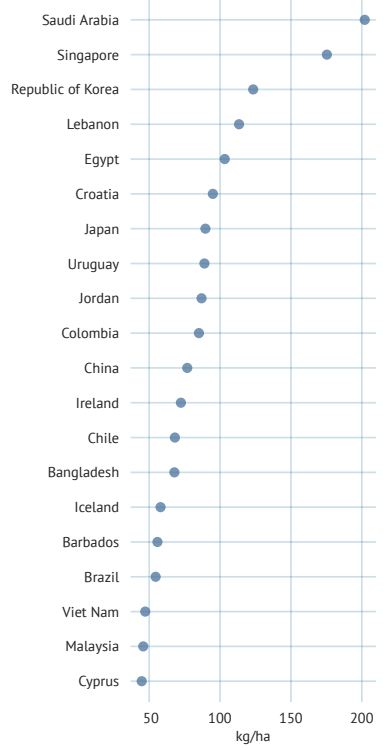

CHART 16: Fertilizer consumption in nutrients per ha of arable land (2012)

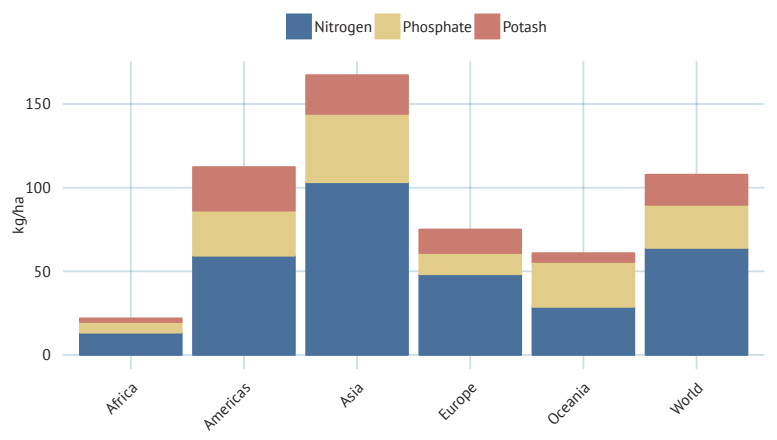


FIGURE 4: Pesticides per ha of arable land (kg/ha, 2007 to 2012*)

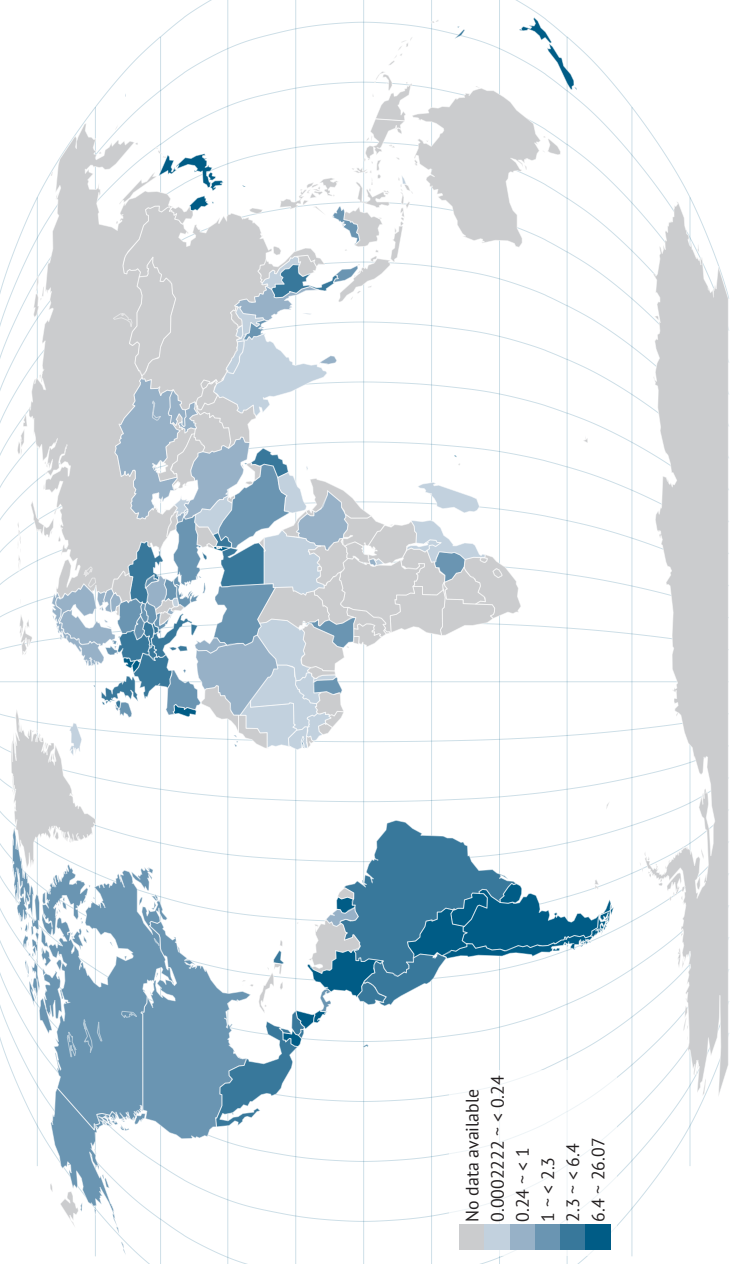




\section{Investment}

Investing in agriculture is one of the most effective strategies for reducing poverty and hunger, and promoting sustainability. The regions of the world where hunger and extreme poverty are most widespread today - South Asia and sub-Saharan Africa - have seen flat or declining rates of investment per worker in agriculture over the past thirty years. Farmers tend to be the largest investors in developing country agriculture, and therefore their investment decisions are paramount for any strategy aimed at improving agricultural investment.

CHART 18: Total credit to agriculture, top 20 countries in 2014 (2000 and 2012)

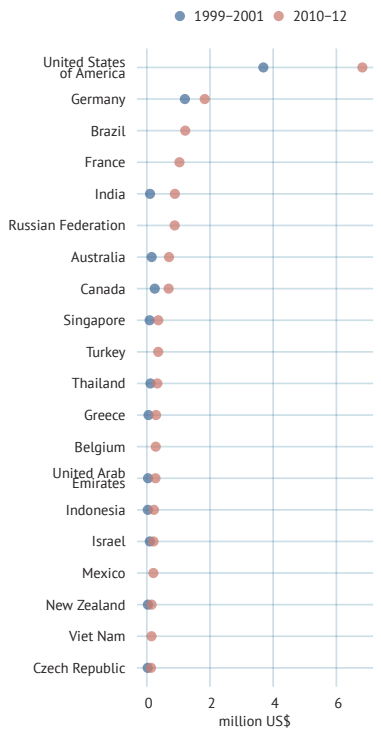

CHART 17: Aid flows to agriculture, share of total aid (1995-2013)

$\rightarrow$ Agriculture, narrow $\rightarrow$ Agriculture, broad

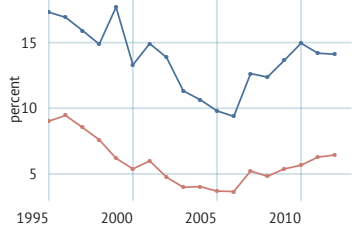

CHART 19: Agri-Orientation Index, highest and lowest values (average 2008-2012)

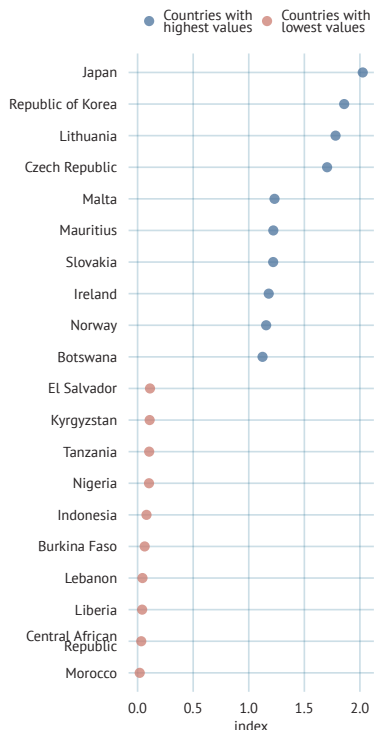

CHART 20: Aid flows to agriculture, broad (1995-2013)

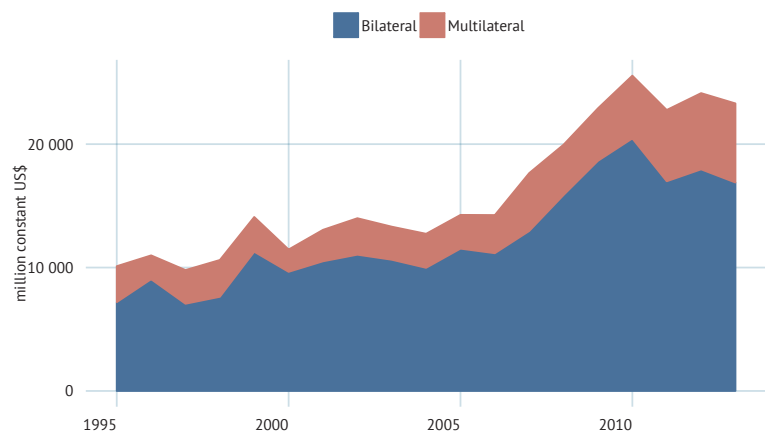


FIGURE 5: Share of government expenditure on agriculture, share of total outlays (percent, 2008 to $2012^{*}$ )

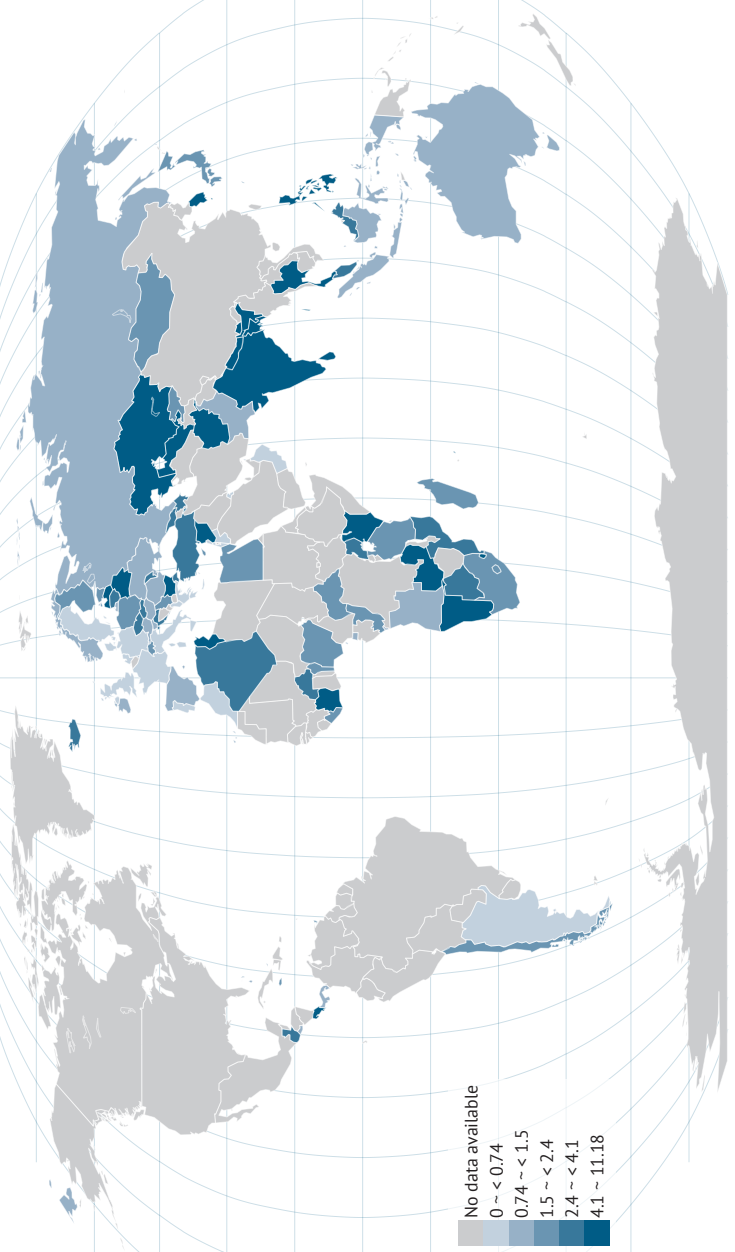




\section{Undernourishment}

Undernourishment is a state, lasting for at least one year, of inability to acquire enough food, defined as a level of food intake insufficient to meet dietary energy requirements. About 795 million people - just over one in every nine people - in the world still lack sufficient food for conducting an active and healthy life. Yet progress has been made, even in the presence of significant population growth. Two hundred and sixteen million million fewer people suffer from undernourishment than 25 years ago and 167 million fewer than a decade ago.

\section{CHART 21: Asian countries with the highest number of undernourished in 2014-16}

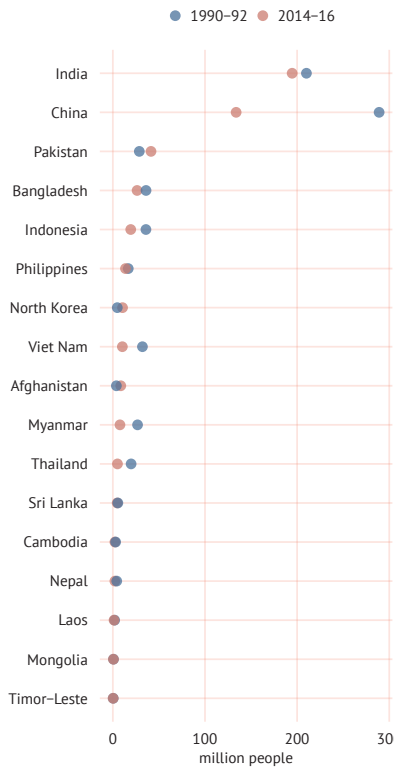

TABLE 1: Prevalence of undernourishment (percent)

\begin{tabular}{lrr}
\hline & $1990-92$ & $2014-16$ \\
\hline World & 18.6 & 10.9 \\
Developed countries & $<5.0$ & $<5.0$ \\
Developing countries & 23.3 & 12.9 \\
Africa & 27.6 & 20.0 \\
Asia & 23.6 & 12.1 \\
Latin America and the & 14.7 & 5.5 \\
Caribbean & & \\
Oceania & 15.7 & 14.2 \\
\hline
\end{tabular}

CHART 22: African countries with the highest number of undernourished in 2014-16

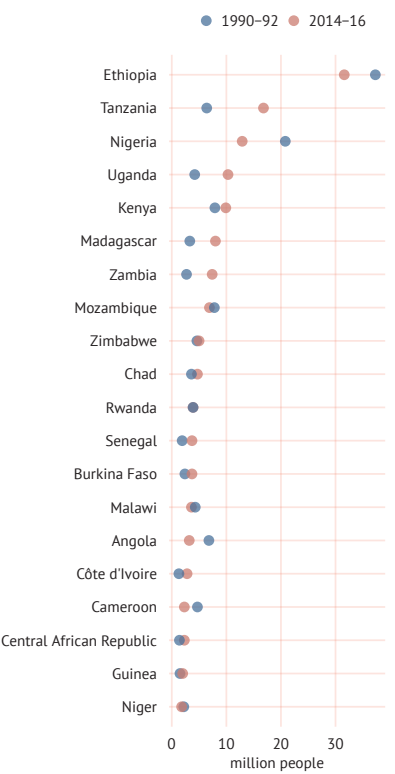

CHART 23: Number of people undernourished

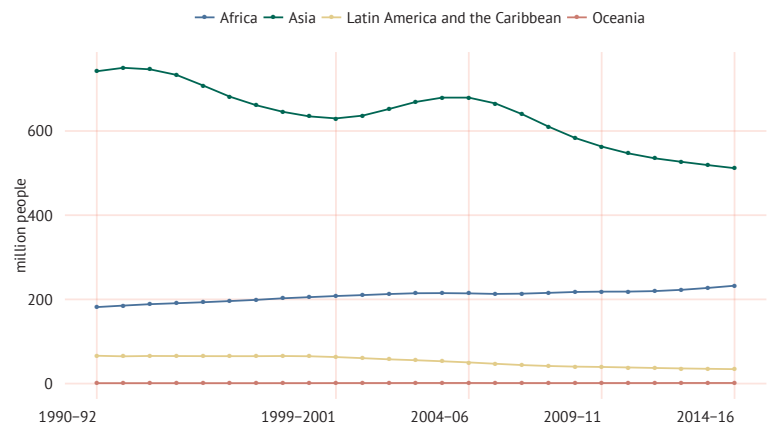


FIGURE 6: Prevalence of undernourishment (percent, 2014-16)

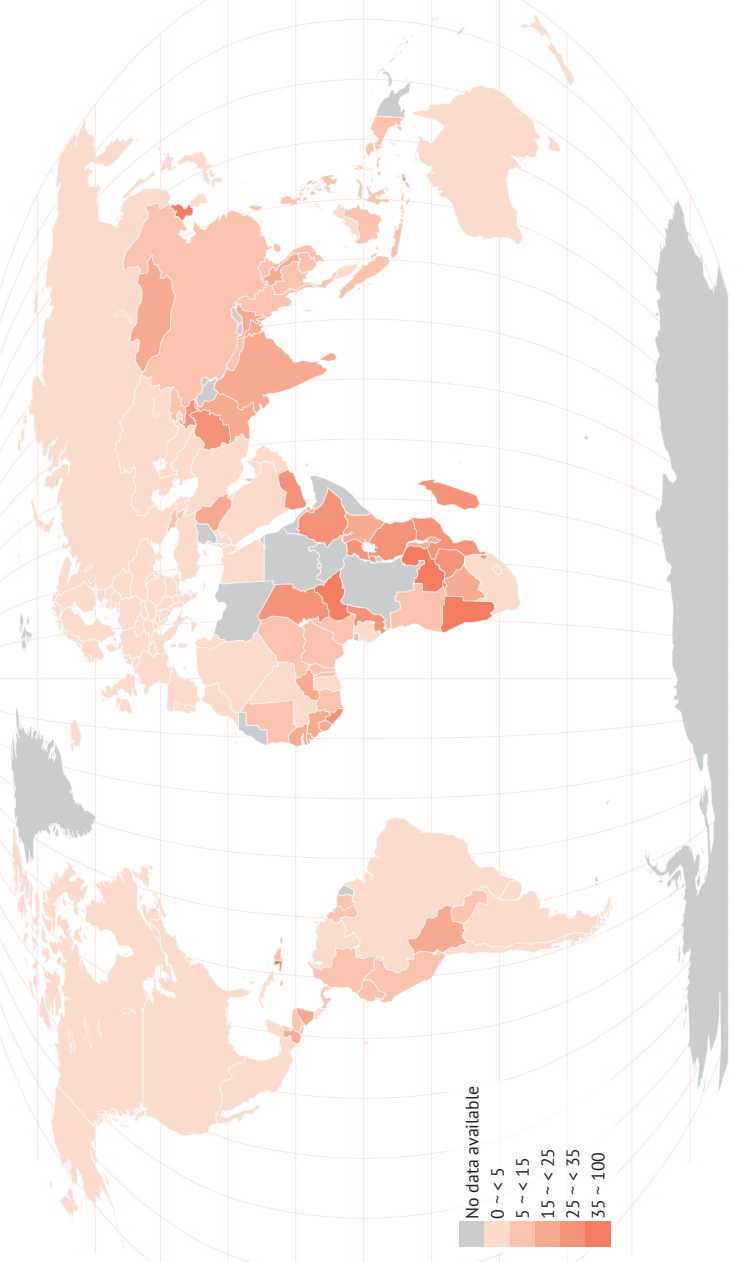




\section{Food availability}

Availability is an important dimension of food security. Supplying enough food to the reference population is a necessary, but insufficient, condition for ensuring adequate access for individuals. Over recent decades, trends in food production per capita have been generally positive across most regions. However, growth rates in Africa have been lower for the last 20 years, despite notable exceptions. In most countries and regions, high food availability is associated with relatively low prevalence of undernourishment. However, outcome indicators show that high food availability does not always guarantee high food security.

CHART 25: Energy supply derived from cereals, roots and tubers, top 20 countries in 2009-2011

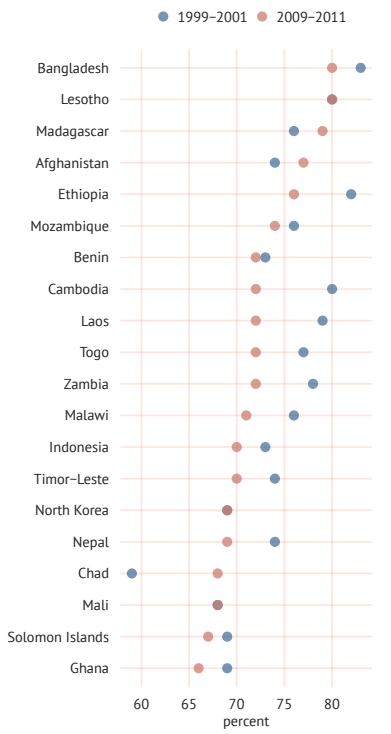

CHART 24: Average dietary energy supply adequacy, 3 year averages (1990 to 2015)

$$
\begin{aligned}
& \text { - Africa } \quad-\text { Oceania } \\
& \text { - Asia } \quad \text { - World }
\end{aligned}
$$

$\rightarrow$ Latin Am. and the Carib.

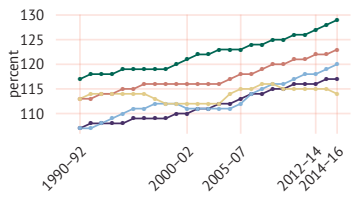

CHART 26: Average protein supply, top 20 countries in 2009-2011

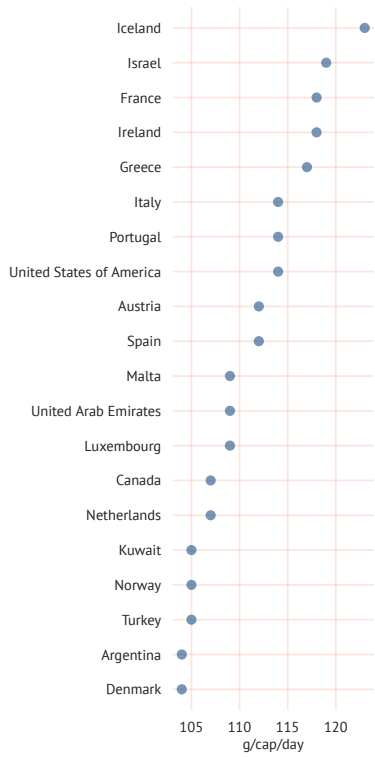

CHART 27: Average supply of protein of animal origin

$\rightarrow$ Africa $\rightarrow$ Asia $\rightarrow$ Latin Am. and the Carib. $\rightarrow$ Oceania $\rightarrow$ World

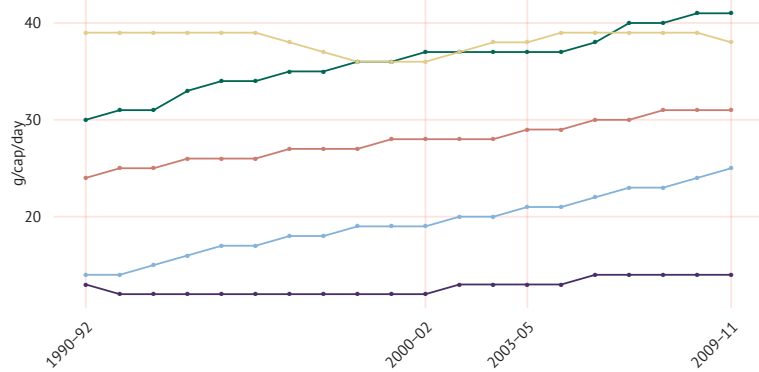


FIGURE 7: Average value of food production, constant 2004-2006 I\$ per person (3 year average, 2011-13)

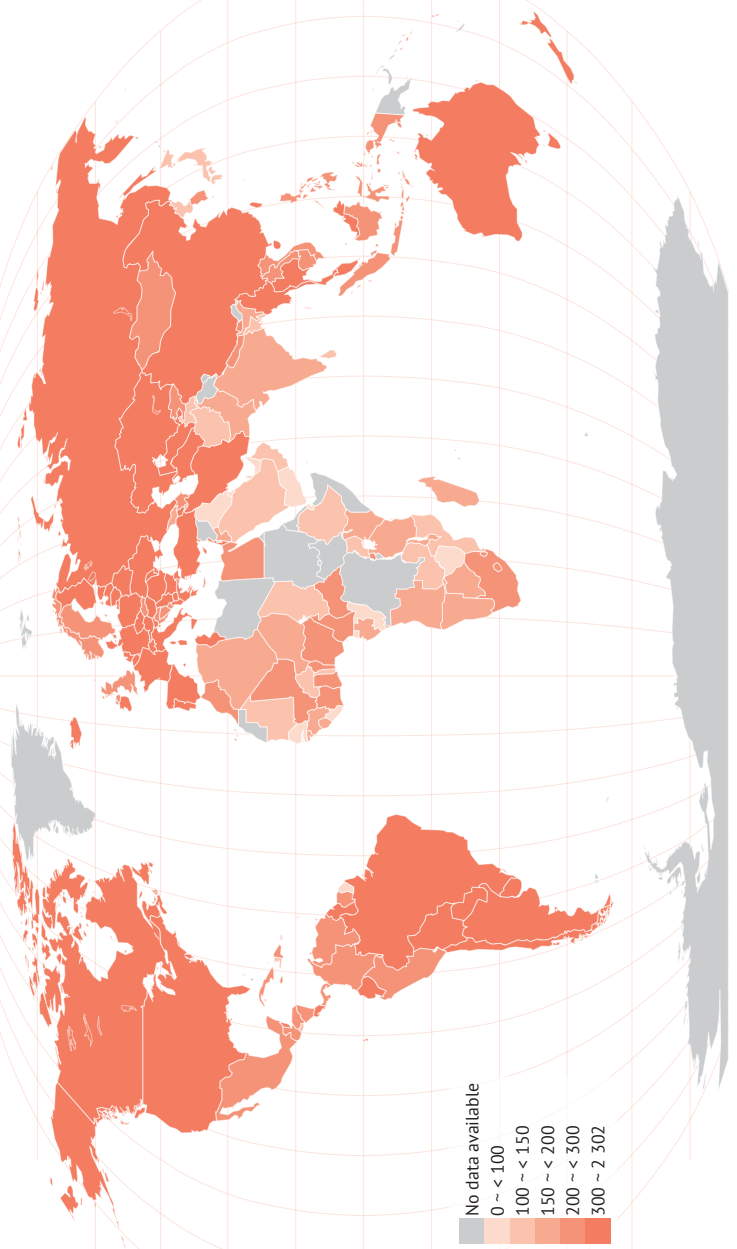




\section{Food access}

An adequate supply of food does not in itself guarantee household level food security. Access to food is primarily determined by incomes, food prices and the ability of households and individuals to obtain access to social support. Individuals' access to food is also heavily influenced by social variables, including gender positioning and power hierarchies within households. In addition to economic affordability, physical access to food is also facilitated by adequate infrastructure, such as railway lines and paved roads.

CHART 29: Domestic food price level index, top 20 countries in 2014 (2000 to $2014^{*}$ )

$2000 \cdot 2014$

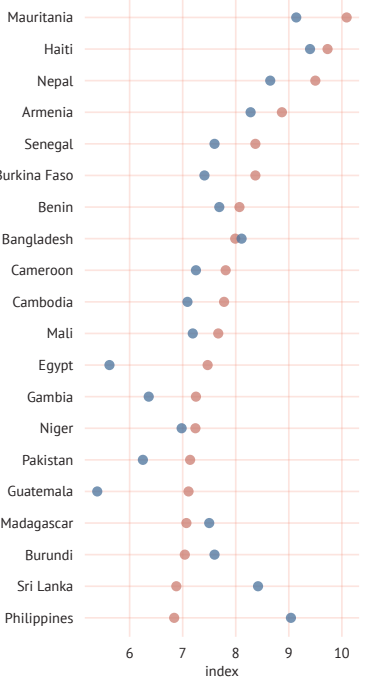

CHART 28: Depth of food decifit, kcal/capita/day (3 year averages)

$$
\begin{aligned}
& \rightarrow \text { Africa } \\
& \rightarrow \text { Asia } \\
& \rightarrow \text { Latin Am. and the Carib. }
\end{aligned}
$$

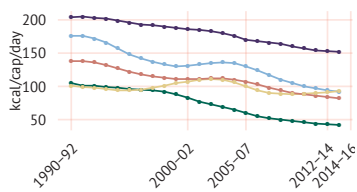

CHART 30: Prevalence of undernourishment, highest 20 countries in 2014-16 ( 3 year averages)

- 1999-2001 2014-16

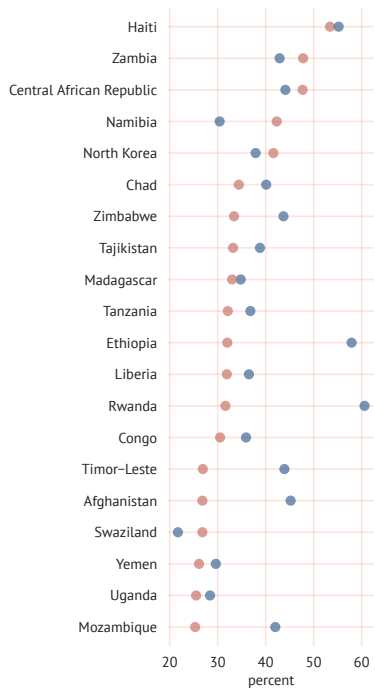

CHART 31: GDP per capita, PPP, constant 2011 international \$

$\rightarrow$ Africa $\rightarrow$ Asia $\rightarrow$ Latin Am. and the Carib. $\rightarrow$ Oceania $\rightarrow$ World

10000

药

5000

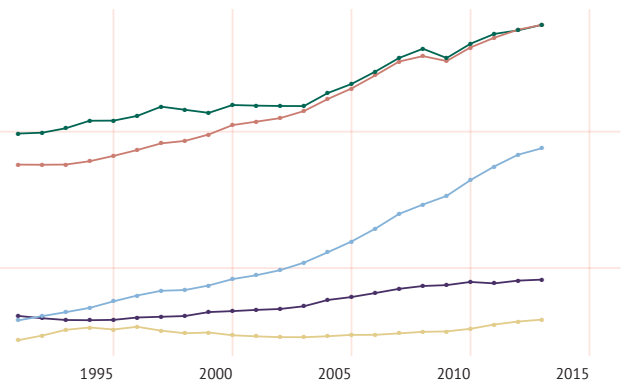


FIGURE 8: Road density, per 100 square km of land area (2007 to 2011*)

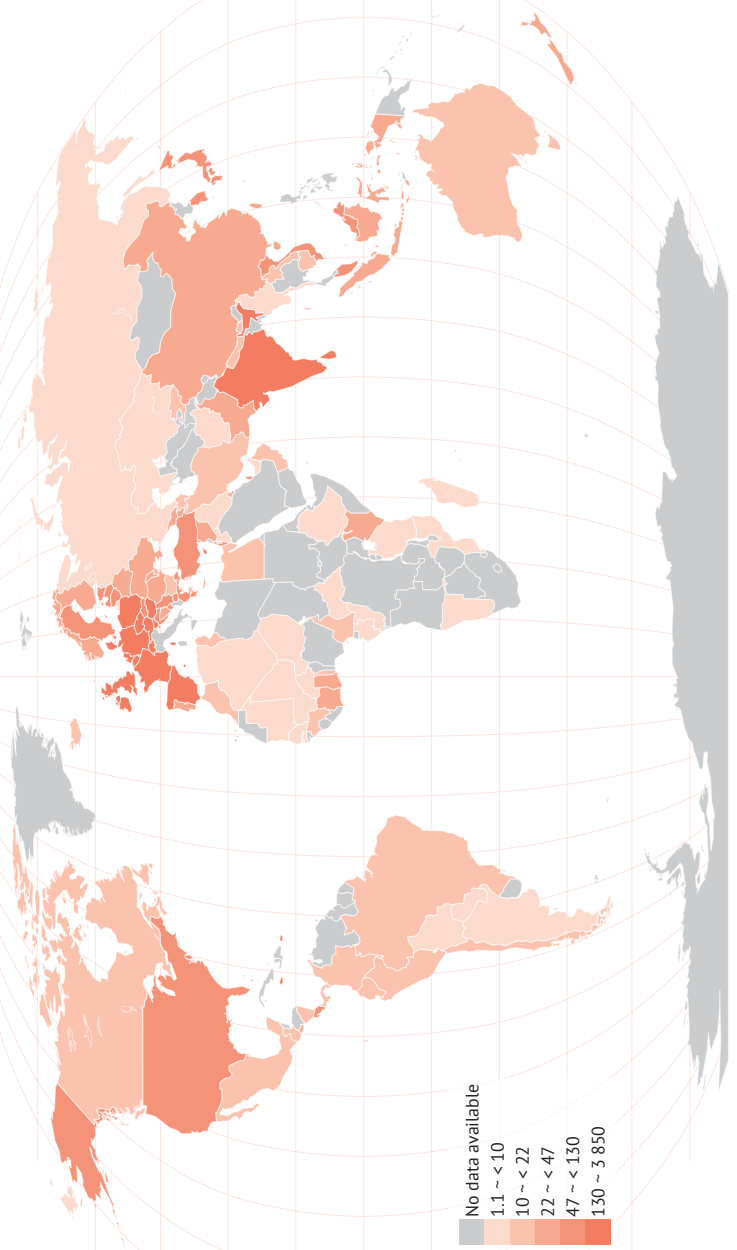




\section{Economic and political stability}

Over the last ten years, food and agricultural markets have entered an unexpectedly turbulent phase, characterized by large supply shortfalls, price swings. Political and economic uncertainties, coupled with extreme weather conditions, can have direct and adverse impacts on food security. The poorer the household, the stronger the impact of external shocks, as poor households spend a proportionally higher share of their incomes on food.
CHART 32: Per capita food production variability, constant 2004-2006 thousand international \$

$$
\begin{array}{ll}
\rightarrow \text { Africa } & \rightarrow \text { Oceania } \\
- \text { Asia } & - \text { World }
\end{array}
$$

$\rightarrow$ Latin Am. and the Carib.

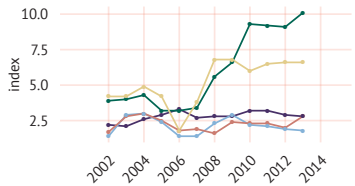

CHART 33: Per capita food supply variability, top CHART 34: Domestic food price volatility index, 20 countries in 2011, kcal/capita/day top 20 countries in 2014

$2000 \cdot 2011$

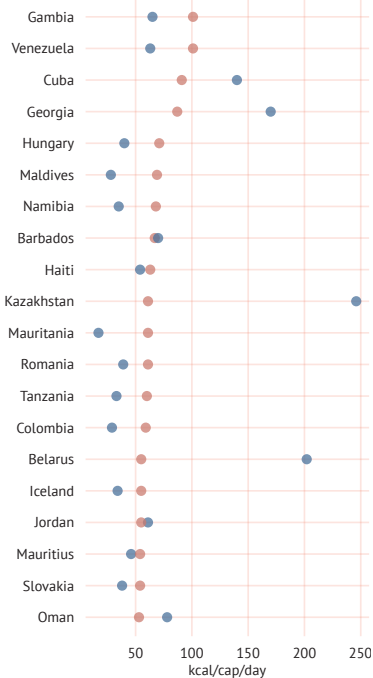

$2000 \cdot 2014$

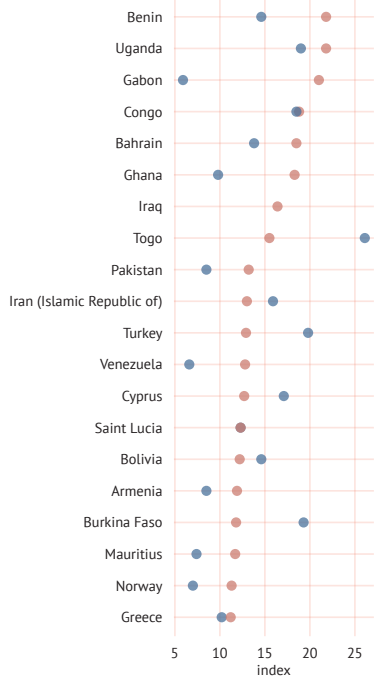

CHART 35: Value of food imports as a share of total merchandise exports (3 year averages)

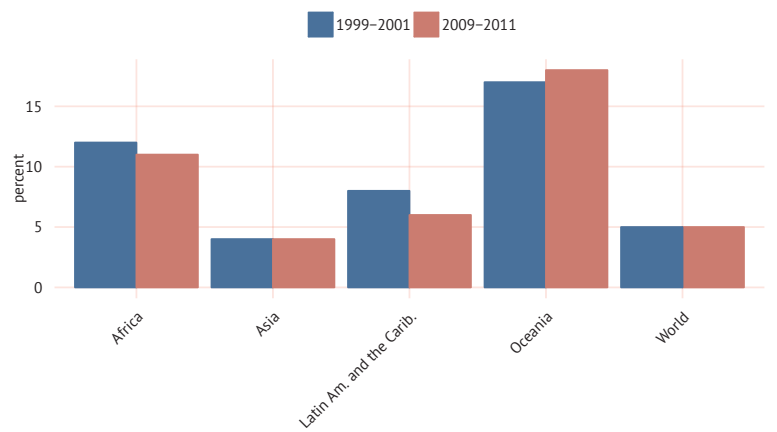


FIGURE 9: Political stability and absence of violence/terrorism, index (2013)

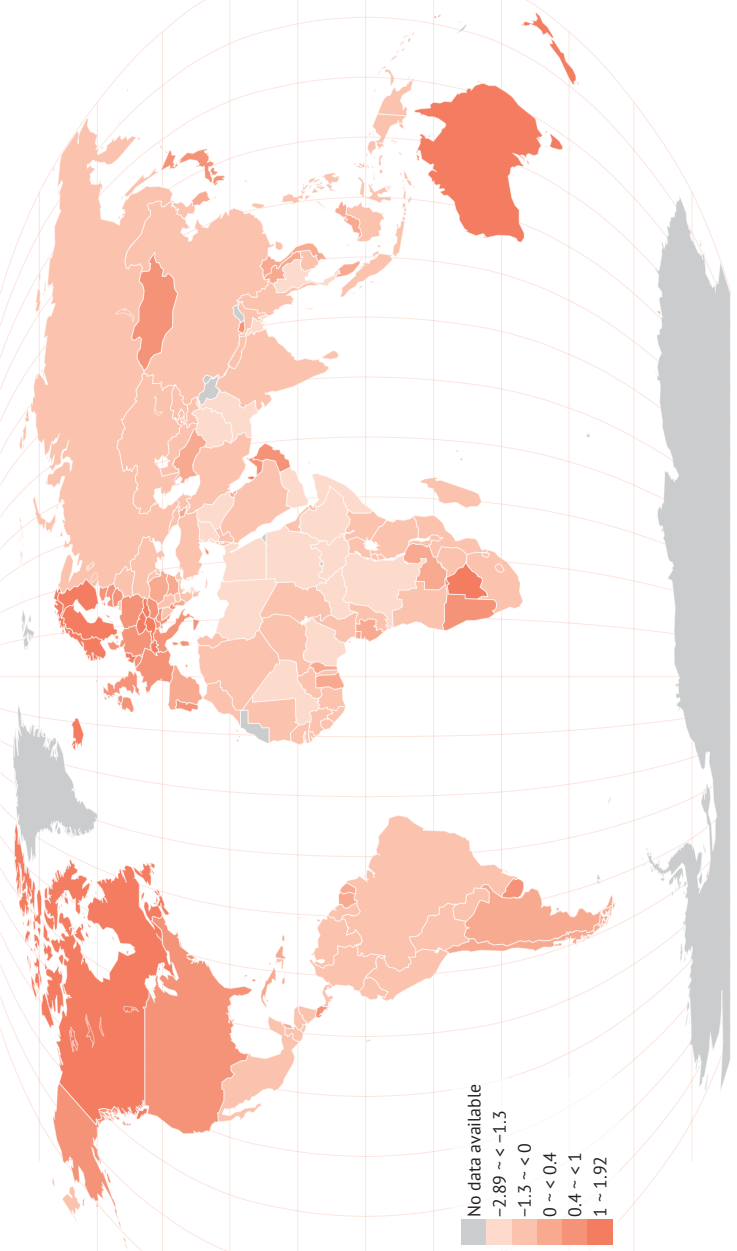




\section{Food utilization}

Utilization emphasizes the nutritional aspects of food security. It is commonly understood as the way the body makes the most of nutrients from food. Sufficient energy and nutrient intake includes nutritious and safe diets, a clean environment, access to health care, diversity of a diet and intra-household distribution of food. Poor utilization within a population can impose economic and social costs in countries at all economic levels.

\section{CHART 36: Percentage of children under 5 who} are stunted, highest 20 countries

$\left(2006-2014^{*}\right)$
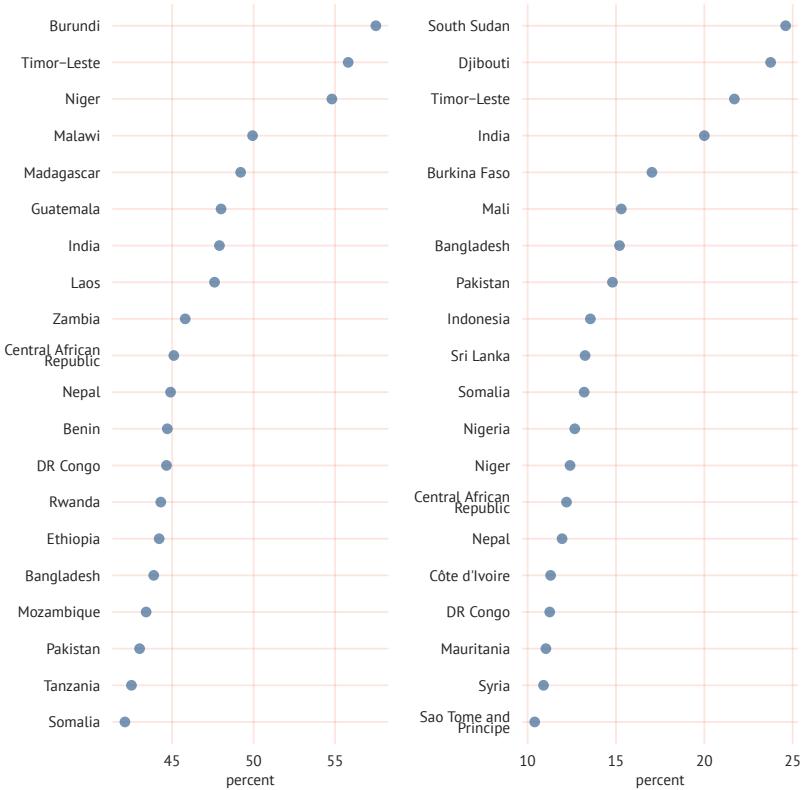

CHART 38: Access to improved water source and sanitation facilities
TABLE 2: Countries with highest share of children under 5 who are underweight, percent

\begin{tabular}{lrr}
\hline & Year & $\%$ \\
\hline Timor-Leste & 2009 & 45.3 \\
Eritrea & 2010 & 38.8 \\
Niger & 2012 & 37.9 \\
Yemen & 2011 & 35.5 \\
\hline Bangladesh & 2013 & 31.9 \\
\hline
\end{tabular}

CHART 37: Percentage of children under 5 affected by wasting, highest 20 countries (2006 - 2014*)

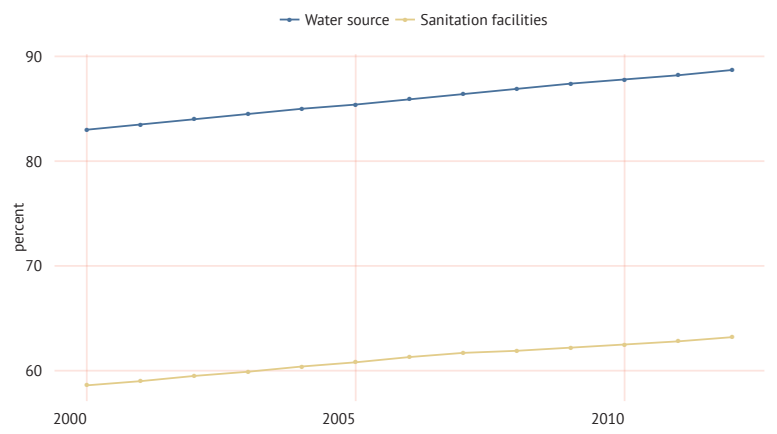


FIGURE 10: Percentage of anaemia among children under 5, percent (2011)

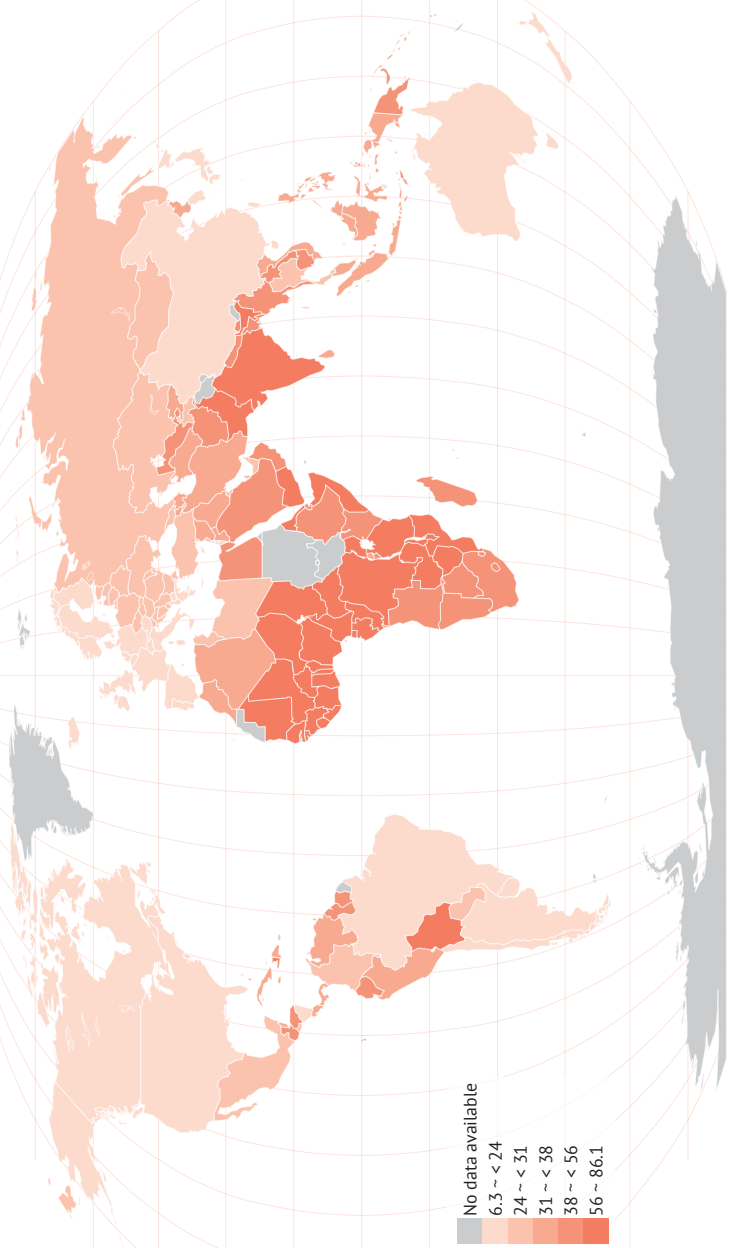




\section{Dietary energy supply}

The dietary energy supply (DES) is the food available for human consumption, expressed in kilocalories per person per day. At the country level, it is calculated as a measure of food available for human use after taking out all non-food utilization, including exports, industrial use, animal feed, seed, wastage and changes in stocks. In 1961 the average global calorie availability was as low as 2196 $\mathrm{kcal} / \mathrm{cap} /$ day; by 2011, it had reached 2870 $\mathrm{kcal} / \mathrm{cap} / \mathrm{day}$, and was centered more around a narrow base of staple grains as well as meat and dairy products.

\section{CHART 40: Dietary energy supply, top 20} countries in 2015

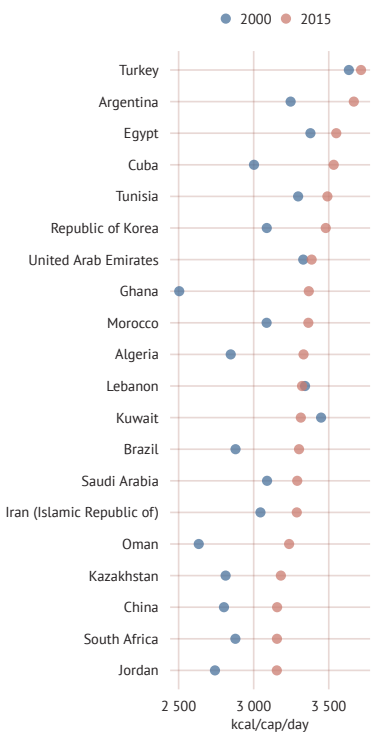

CHART 39: Share of dietary energy supply, kcal/capita/day (2009-2011)

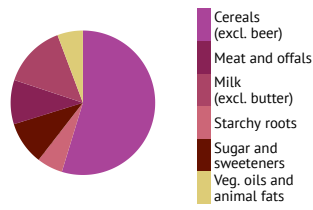

CHART 41: Dietary energy supply, bottom 20 countries in 2015

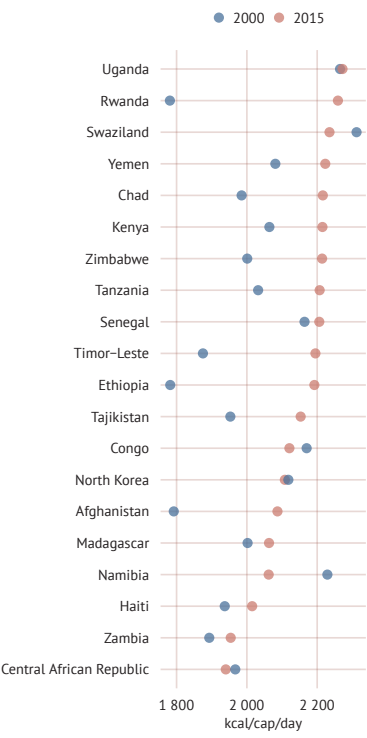

CHART 42: Dietary energy supply, kcal/cap/day

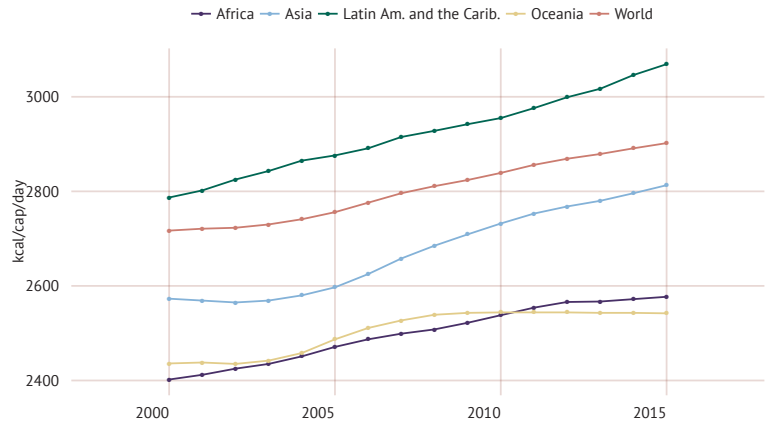


FIGURE 11: Average dietary energy supply adequacy, percent (2014-2016)

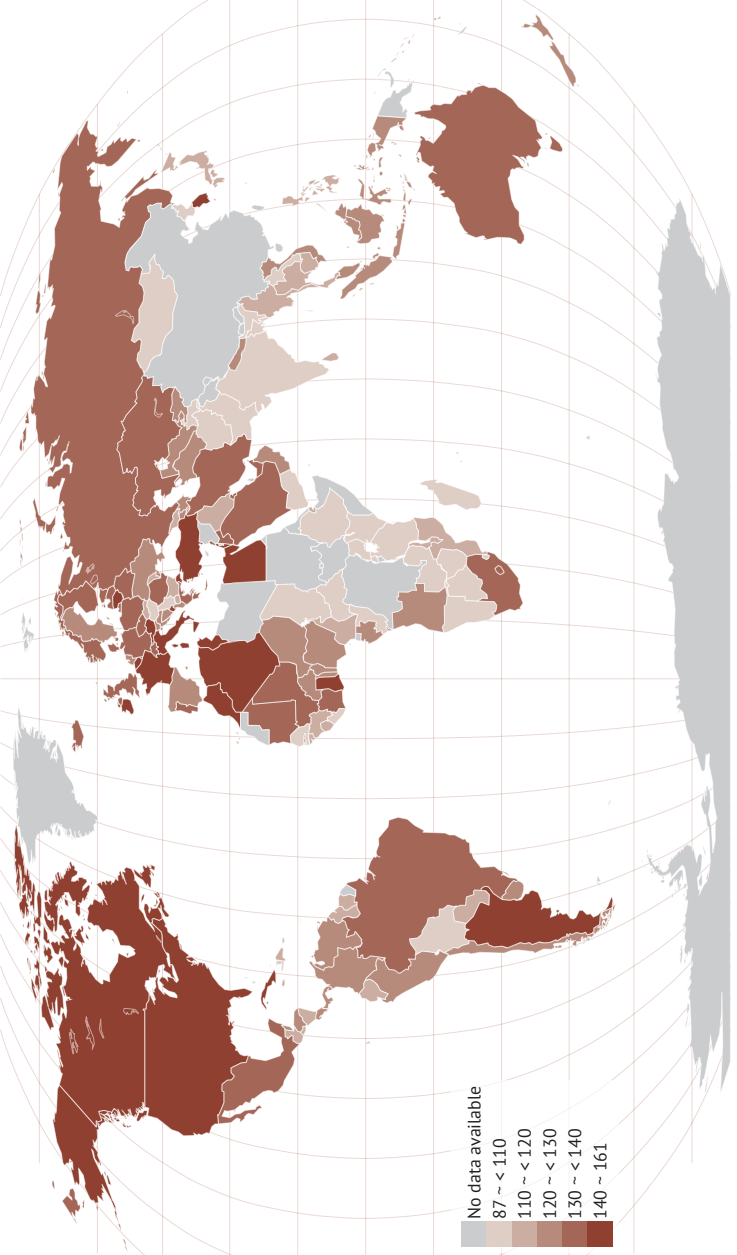




\section{Crop production}

The majority of people in developing countries live in rural areas, and most of them depend on agriculture for their livelihoods. Over the past 50 years, growth in crop production has been driven largely by higher yields per unit of land, and crop intensification. Trends are not uniform across regions, however. Most of the growth in wheat and rice production in Asia and Northern Africa has been from gains in yield, while expansion of harvested land has led to production growth of maize in Latin America and in sub-Saharan Africa.

CHART 43: Top 20 crop producing countries in 2012 based on net per capita crop production value

$2000 \cdot 2012$

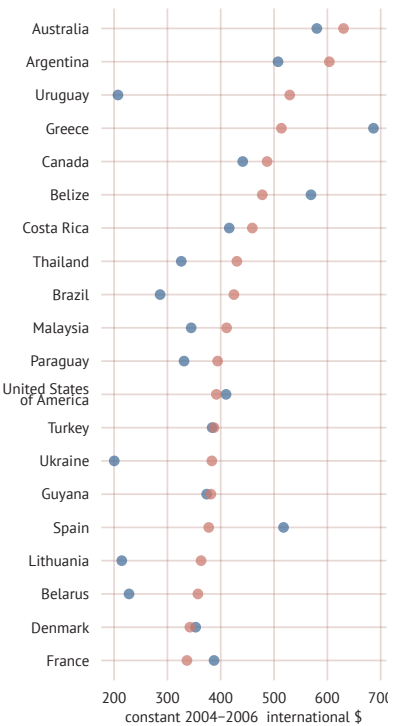

TABLE 3: Fastest growing products based on quantities (average anual growth rate, 2000 to 2013)

\begin{tabular}{lr}
\hline & $\%$ \\
\hline Fruit, pome nes & 9.8 \\
\hline Bambara beans & 9.2 \\
\hline Walnuts, with shell & 9.1 \\
\hline Pistachios & 8.5 \\
\hline Vanilla & 8.0 \\
\hline
\end{tabular}

CHART 44: Top 20 food producing countries in 2012 based on net food per capita production value

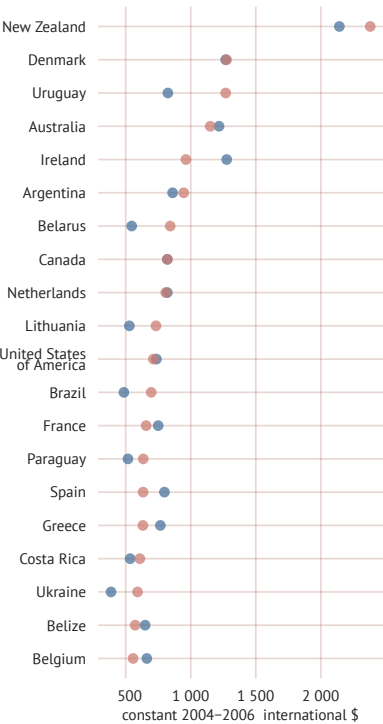

CHART 45: Average annual growth in cereals production (2000-13)

Production quantity Harvested area Yield

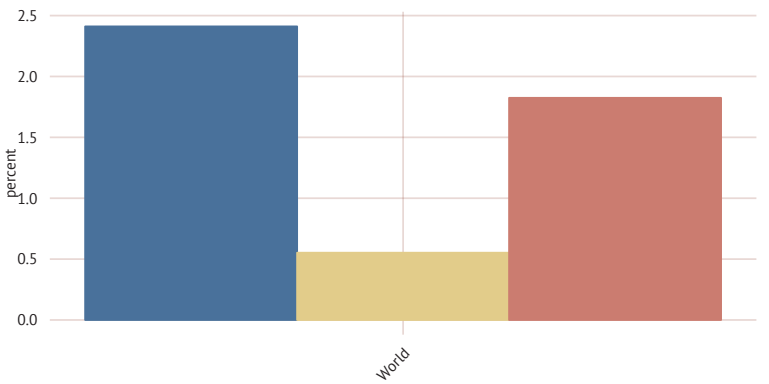


FIGURE 12: Crops, gross per capita production index $(2004-06=100,2013)$

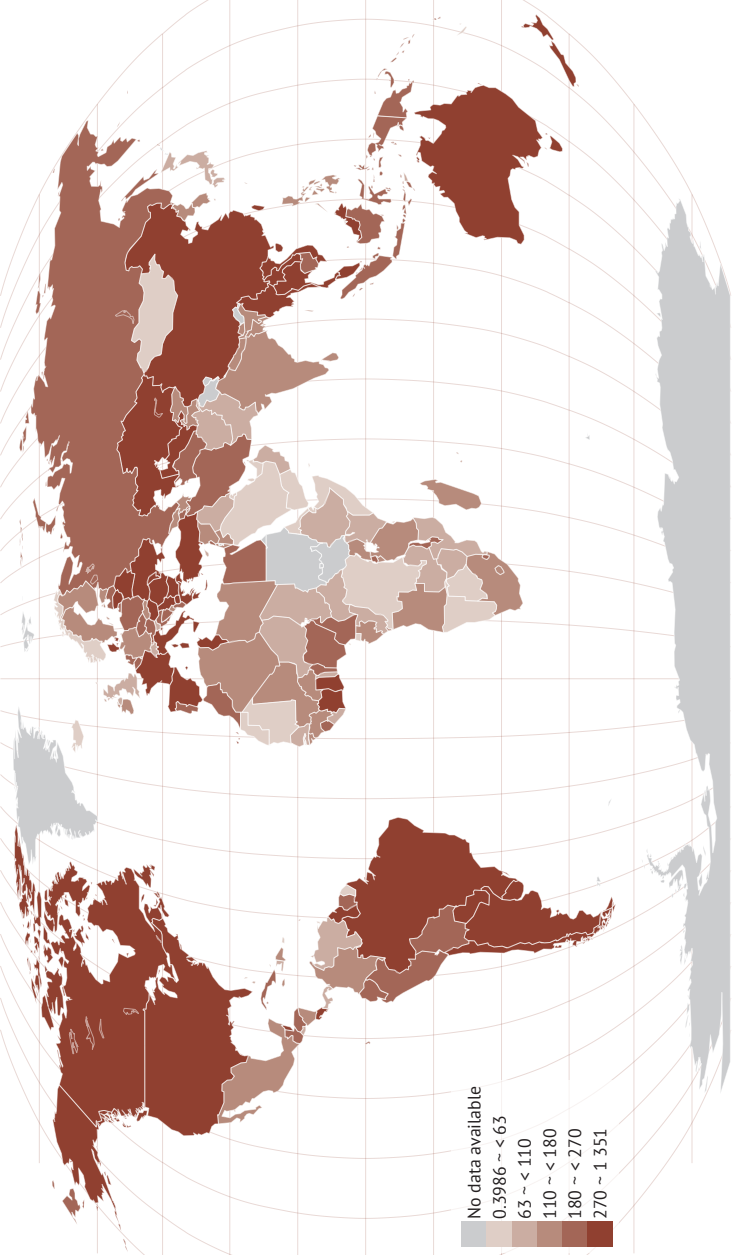




\section{Crops}

Cereals, which include wheat, rice, barley, maize, rye, oats and millet, make up the majority of the production of the crop sector. They continue to be the most important food source for human consumption. Yet external factors, such as rising incomes and urbanization, are causing diets to shift towards diets that are higher in protein, fats and sugar. In addition, livestock and biofuel production have and will most likely grow at a faster rate than crop production. This is causing a shift away from crops, like wheat and rice, towards coarse grains and oilseeds to meet demands for food, feed and biofuel.

\section{CHART 46: Top 20 rice producing countries, pe} capita

2000 - 2012

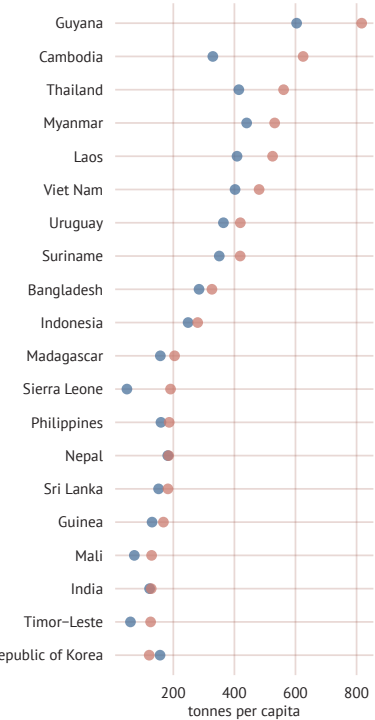

CHART 48: Cereals, yield
TABLE 4: Top five items produced in $\mathbf{2 0 1 3 ,}$ thousand tonnes

\begin{tabular}{lrr}
\hline & 2000 & 2013 \\
\hline Sugar cane & 1256380 & 1877110 \\
Maize & 592479 & 1016740 \\
Rice, paddy & 599355 & 745710 \\
Wheat & 585691 & 713183 \\
\hline Potatoes & 327600 & 368096 \\
\hline
\end{tabular}

\section{CHART 47: Top 20 wheat producing countries,} per capita

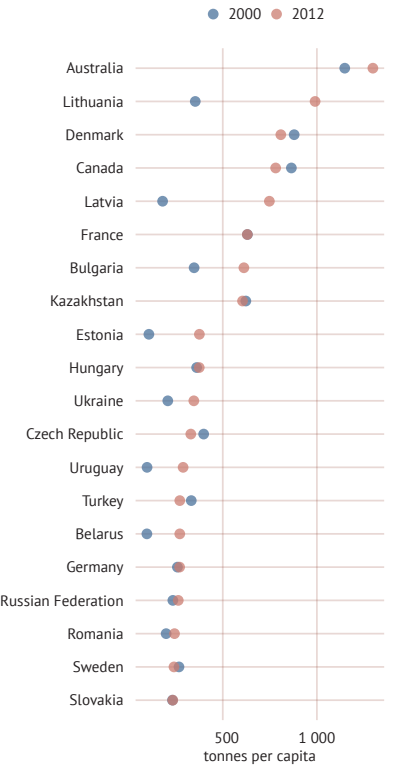

tonnes per capita

- Africa - Americas - Asia - Europe - Oceania - World

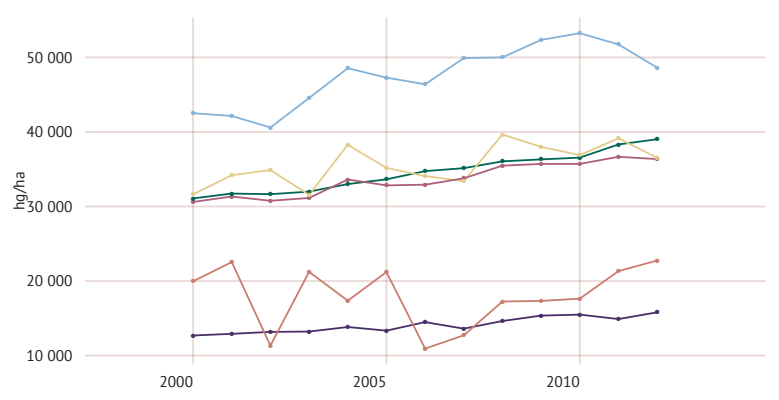


FIGURE 13: Cereal production, tonnes/cap (2013)

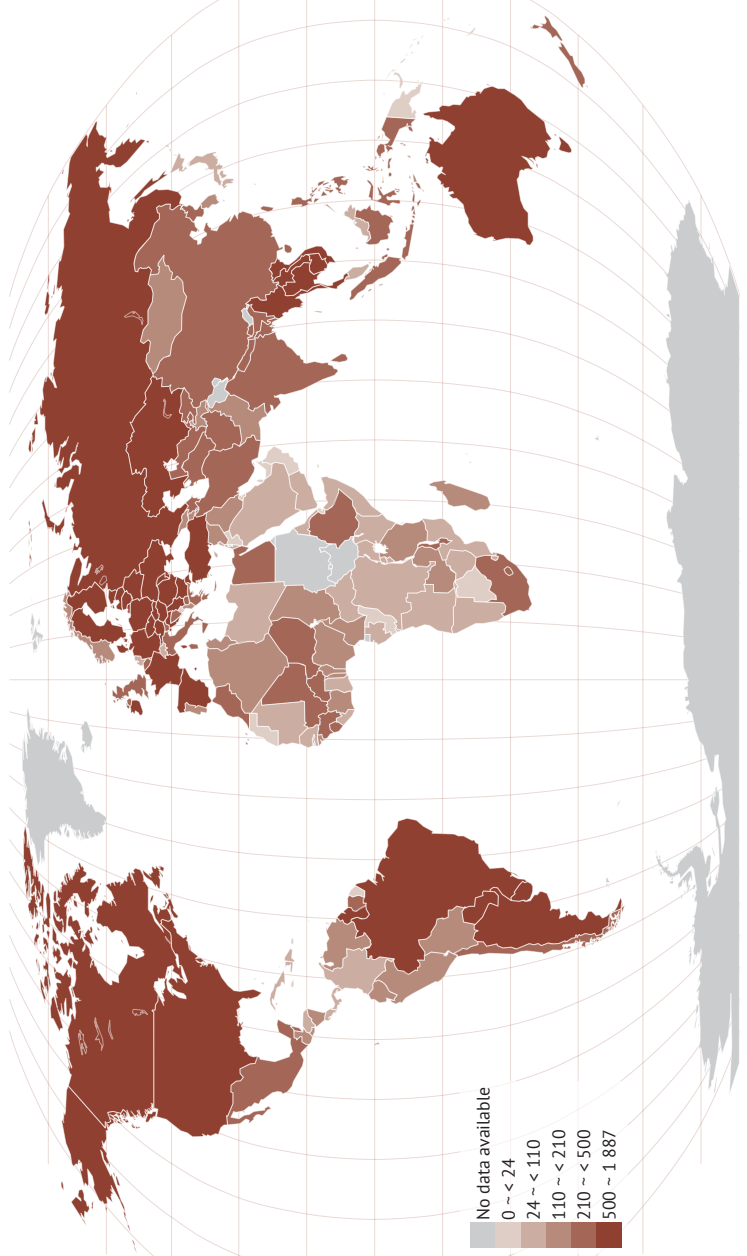




\section{Livestock}

The world food economy is being increasingly driven by the shift of diets towards animal-based products such as meat, milk and dairy. As a result, agriculture is being affected, not only through growth of livestock production, but also through linkages to other sectors that supply feeding stuffs, such as crops and fisheries. Globally livestock production is the largest user of agricultural land and therefore also leaves a significant imprint on the environment.

CHART 49: Total milk production, top and bottom 10 countries (2012)
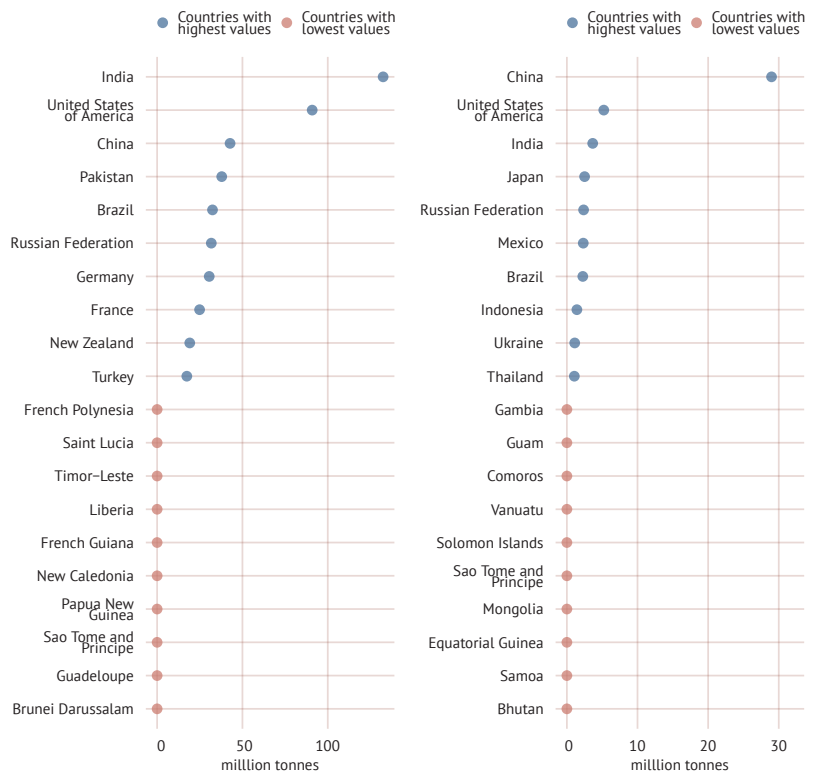

CHART 51: Pig production (heads)

Africa Americas Asia Europe Oceania

2000

2013
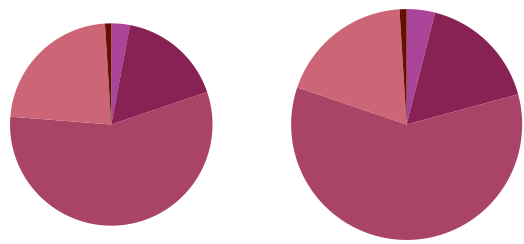
FIGURE 14: Cattle and buffaloes per ha of agricultural area, heads per ha (2012)

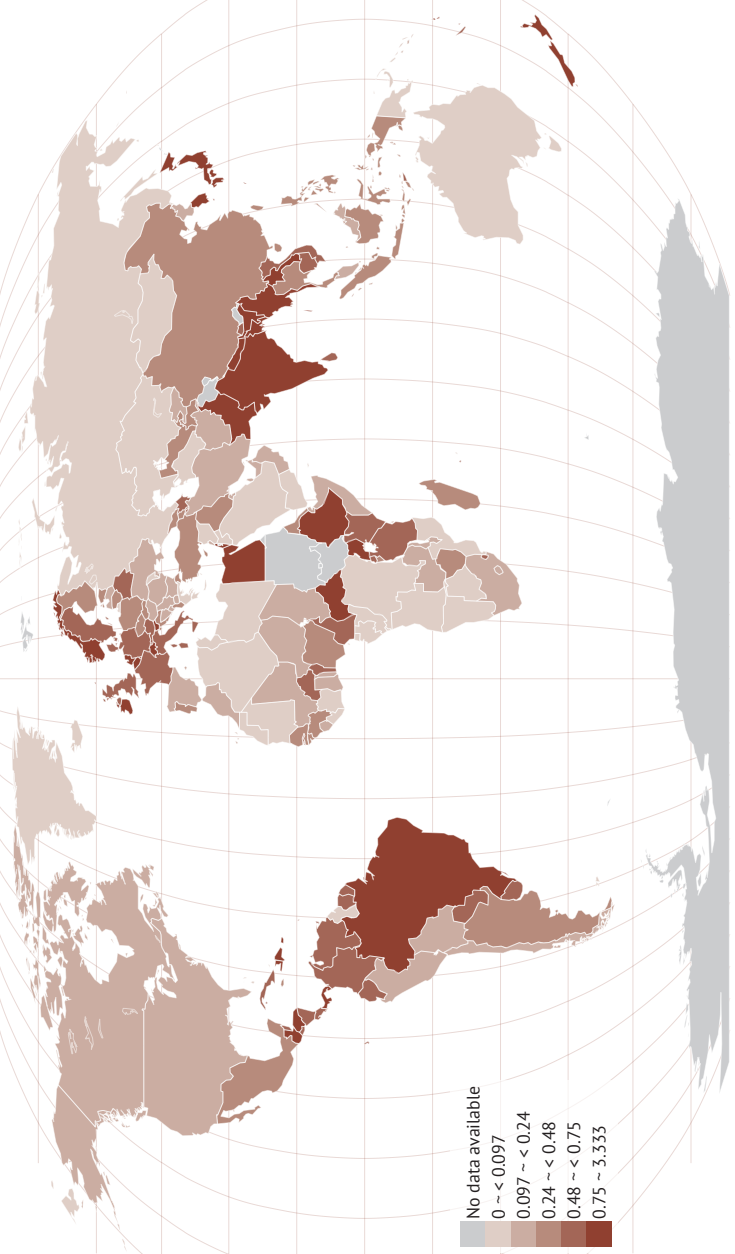




\section{Fisheries}

Fish is an important component in people's diets, providing about 3.1 billion people with almost 20 percent of their average intake of animal protein. Capture fisheries continue to dominate world output, but aquaculture accounts for a growing percentage of total fish supply. Fishery sectors are particularly important in developing countries, providing both food and livelihoods.

\section{CHART 53: 20 countries with highest value of} capture production (2013)

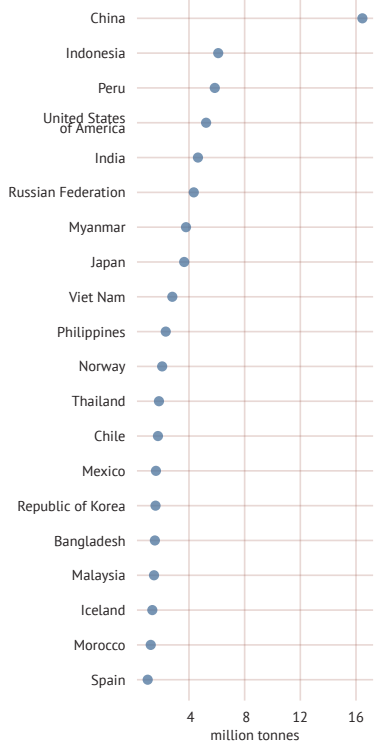

\section{CHART 52: Per capita fish food supply}

From capture fisheries

From aquaculture

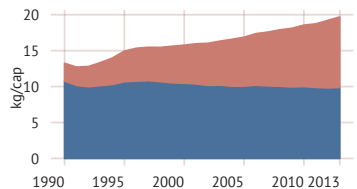

CHART 54: 20 countries with highest value of aquaculture production (2013)

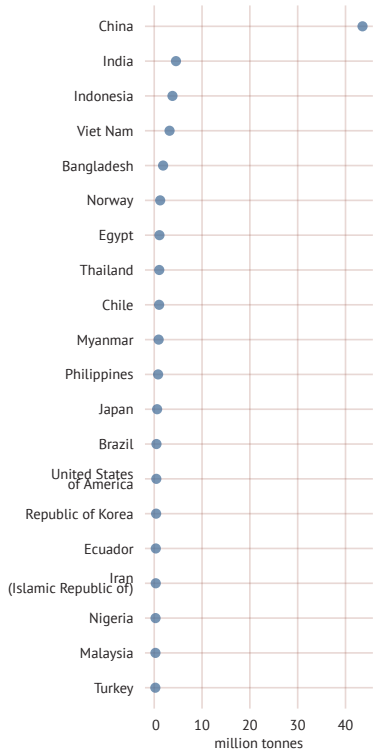

CHART 55: State of the world's fishery stocks (1974 - 2011)

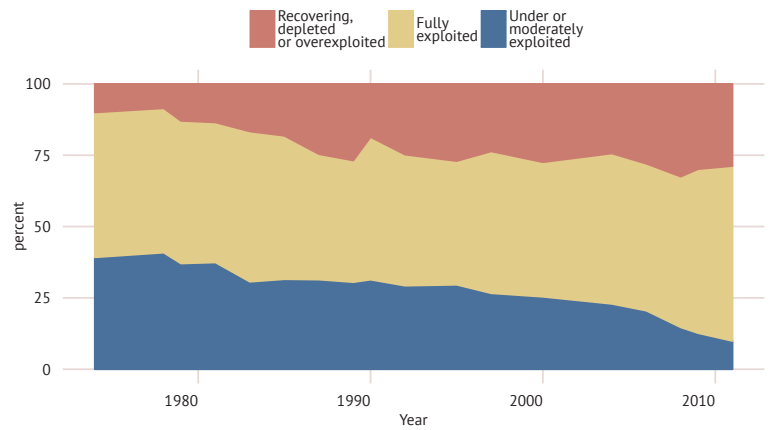


FIGURE 15: Fish production index $(2004-06=100,2013)$

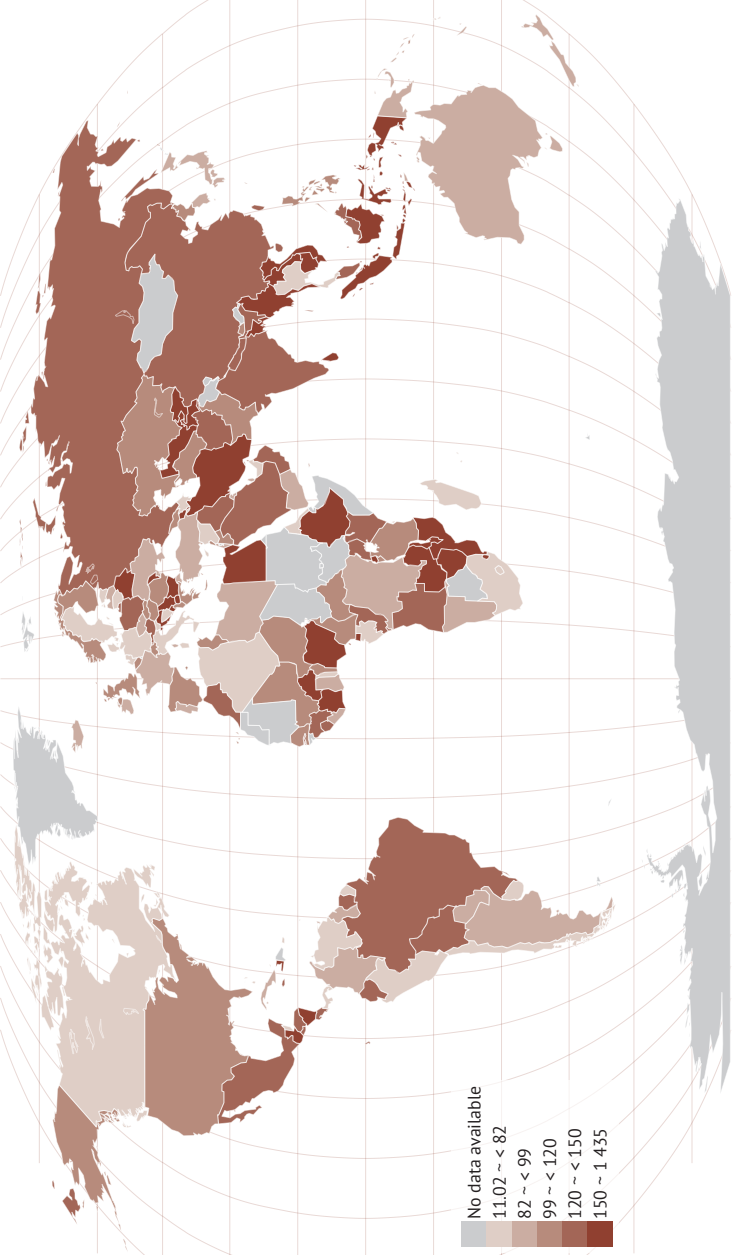




\section{Agricultural trade}

Most of the food consumed worldwide is grown locally. Where there is not enough local production to meet demand, trade has been instrumental in filling the gap. The scale of food and agricultural trade today is unprecedented. In real terms, the value of international flows has increased around fivefold over the past 50 years, reflecting global trends in the overall volume of trade. However, this expansion has been unevenly distributed across regions. High-income countries have generally outpaced developing regions, although several of the latter have comparative advantages in food and agricultural production.

CHART 56: Top food importing countries in 2012

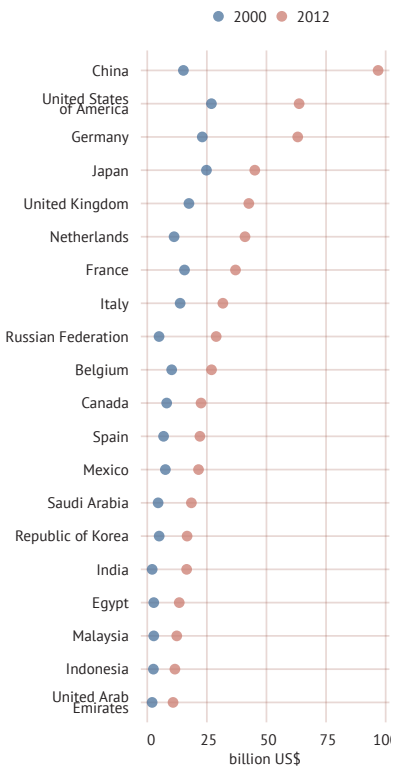

TABLE 6: Exports and Imports of food, million US\$ (2012)

\begin{tabular}{lrr}
\hline & Export value & Import Value \\
\hline Europe & 403 & 418 \\
Asia & 160 & 264 \\
\hline Americas & 266 & 159 \\
Africa & 29 & 64 \\
\hline Oceania & 45 & 14 \\
\hline
\end{tabular}

CHART 57: Top food exporting countries in $\mathbf{2 0 1 2}$

- 2000 e 2012

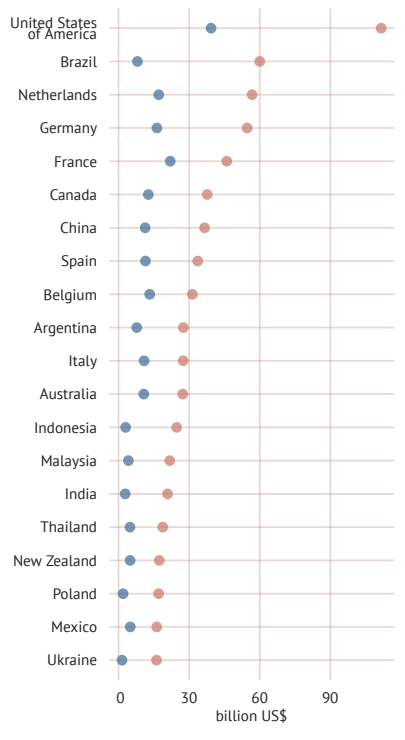

CHART 58: Cereal exports

$\rightarrow$ Africa $\rightarrow$ Americas $\rightarrow$ Asia $\rightarrow$ Europe $\rightarrow$ Oceania

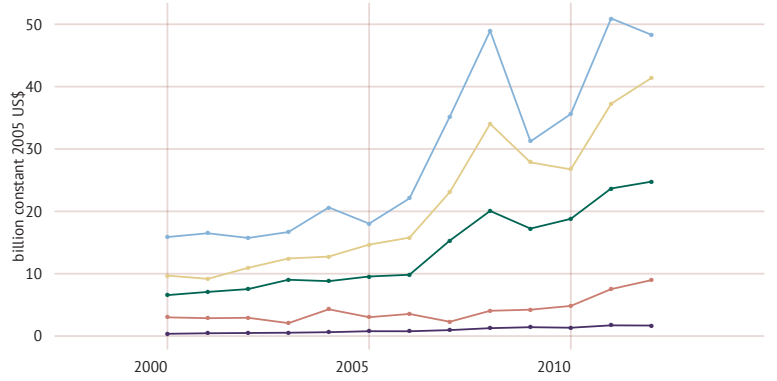


FIGURE 16: Import value index $(2004-2006=100,2011)$

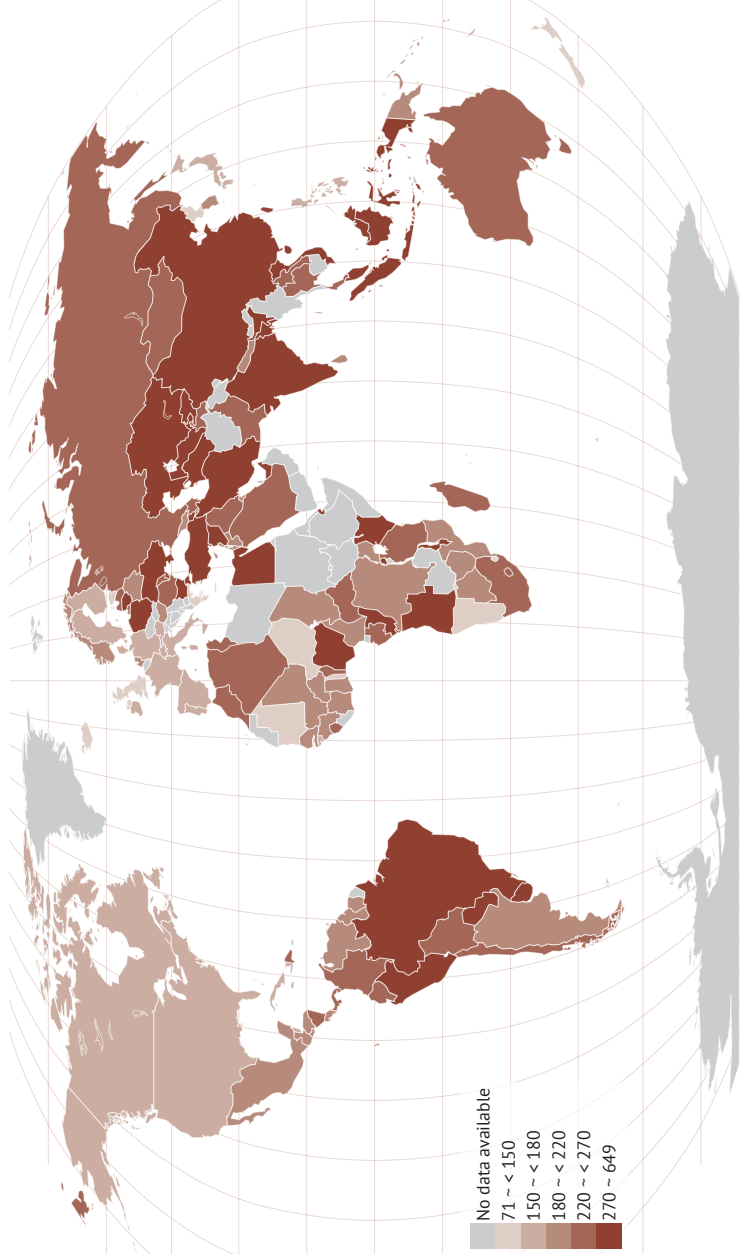




\section{Land}

Land is necessary for sustainable agricultural development, essential ecosystem functions and food security. More than 1.5 billion hectares - about 12 percent of the world's land area - are used for crop production. Although large amounts of land are potentially suitable for agriculture, much of it is covered by forests, protected for environmental reasons or are part of urban areas. Some 90 percent of agricultural land is in Latin America and sub-Saharan Africa. At the other extreme, there is almost none available for agricultural expansion in Southern Asia, the Western Asia and Northern Africa.

CHART 60: Arable land per capita, top 20 countries (2012)

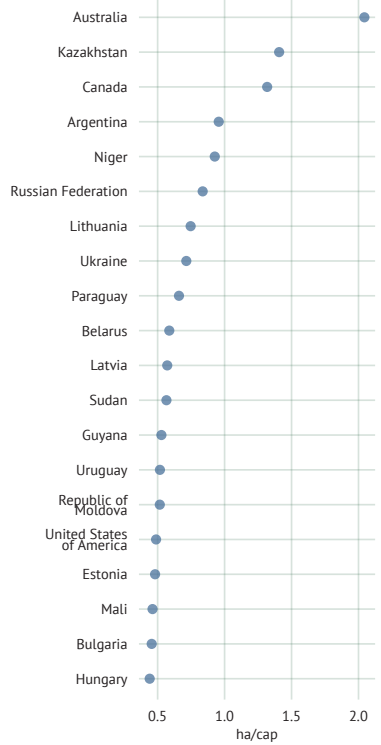

\section{CHART 59: Land area}

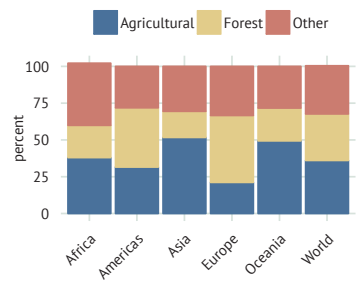

CHART 61: Arable land per capita, bottom 20 countries (2012)

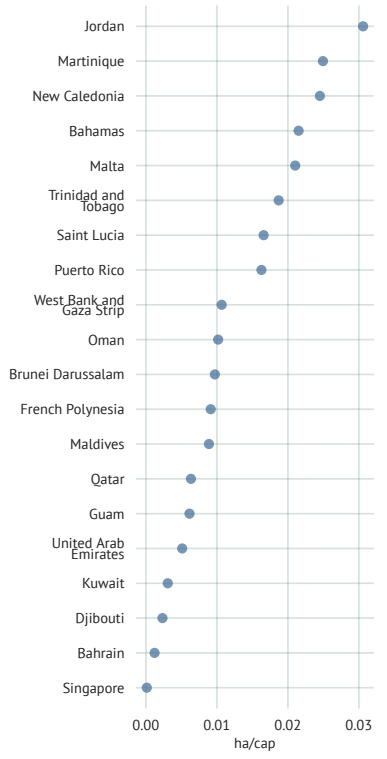

CHART 62: Agricultural area

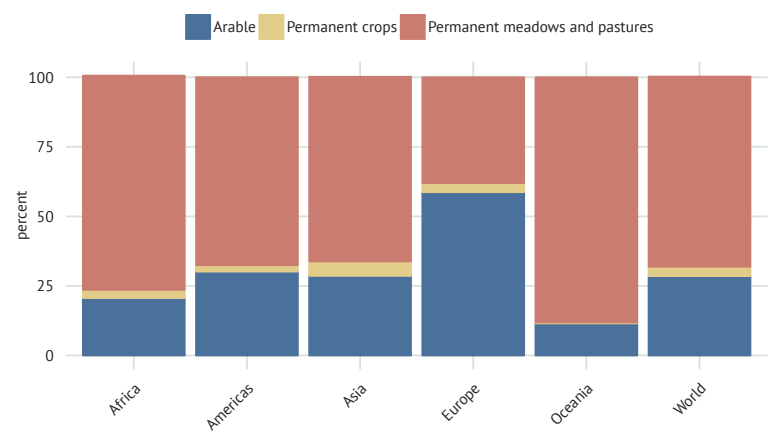


FIGURE 17: Cropland per capita, ha per cap (2012)

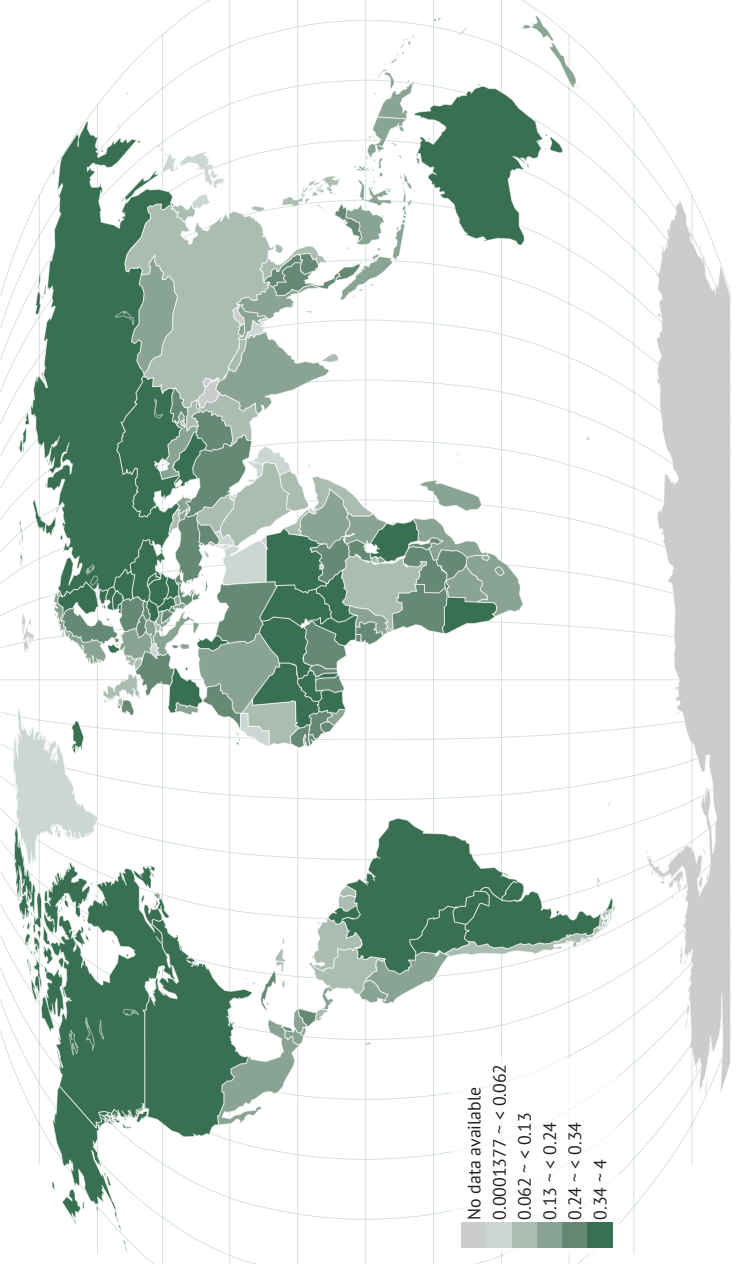




\section{Water}

Global demand for water has risen sharply over the last century. Total annual water withdrawal from agriculture, municipalities and industries rose from less than $580 \mathrm{~km}^{3}$ in 1900 to more than $3900 \mathrm{~km}^{3}$ in 2010 . Agriculture accounts for approximately 70 percent of total freshwater withdrawal in the world, mostly through irrigation. This has been crucial for gains in food production since irrigation reduces drought risk and encourages crop diversification, thus also enhancing rural incomes. While irrigated agriculture represents about 20 percent of the cultivated land, it contributes to 40 percent of global food production.

CHART 64: Freshwater withdrawal by industrial sector, share of total, highest 20 (1999 to 2013)

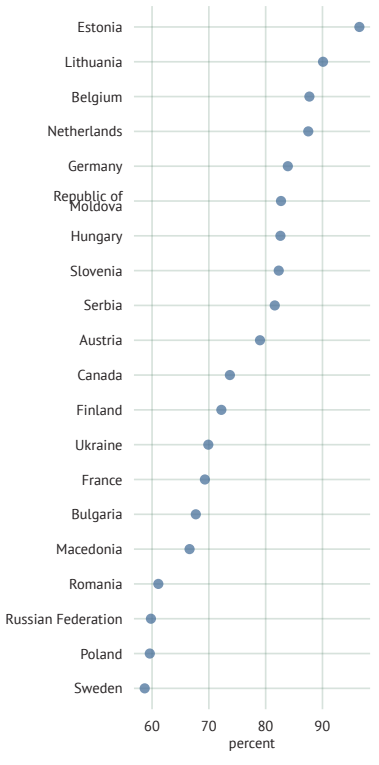

\section{CHART 63: Countries with the lowest renewable} water resources per capita

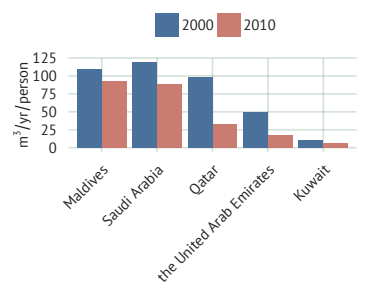

CHART 65: Freshwater withdrawal by agricultural sector, share of total, highest 20 (1999 to 2013)

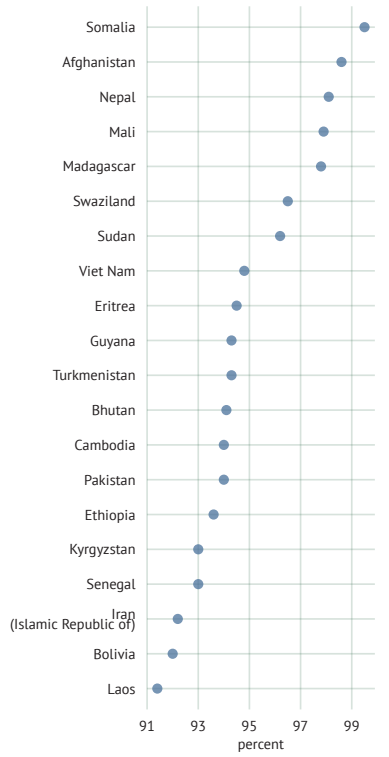

CHART 66: Countries with the highest renewable water resources per capita

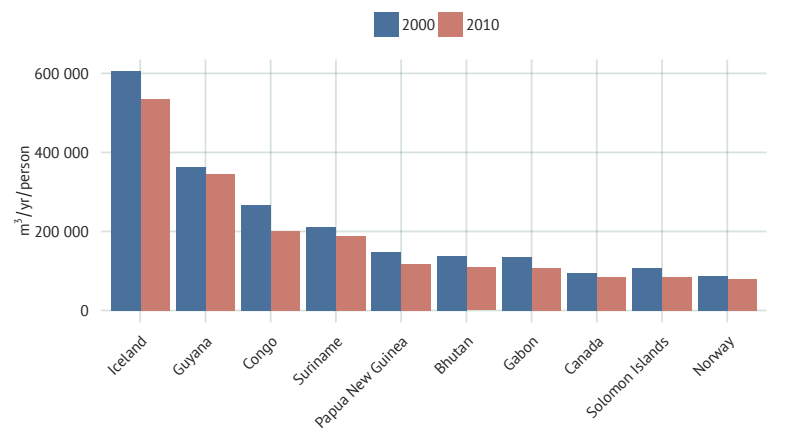


FIGURE 18: Freshwater resources withdrawn by agriculture (percent, 1999-2013*)

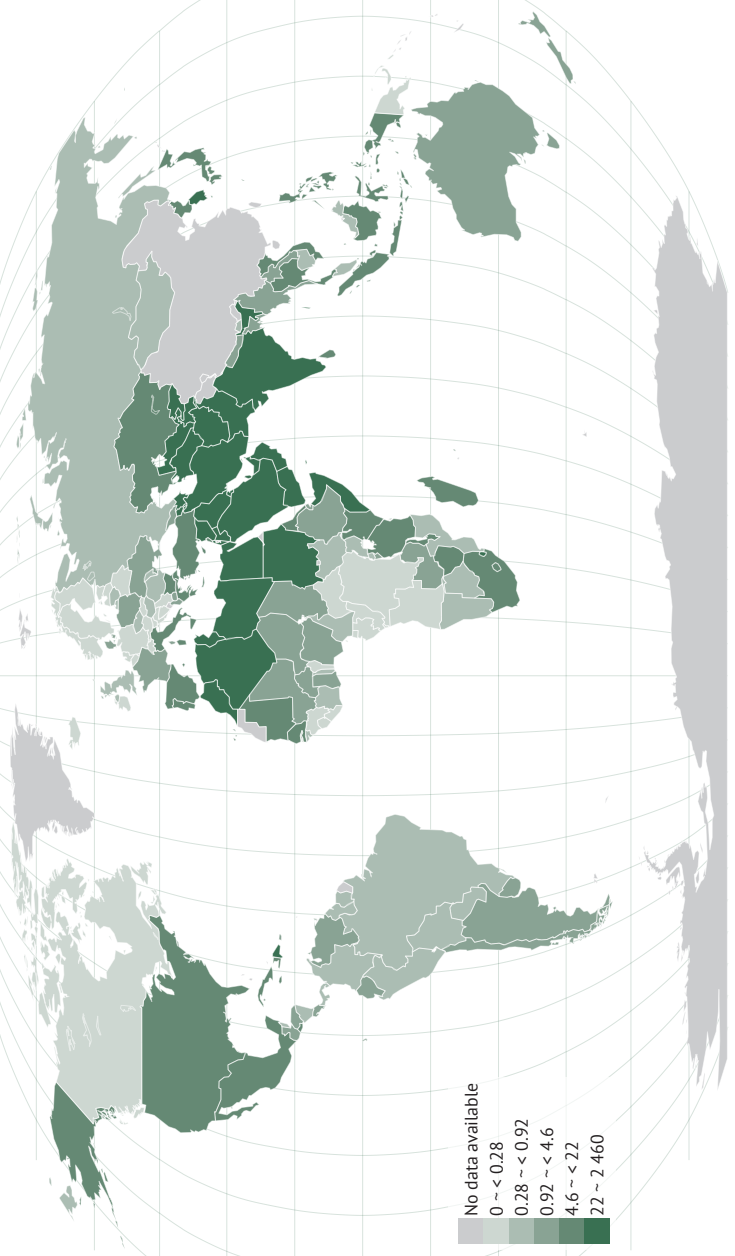




\section{Energy}

Energy is an important input for the agri-food chain and is used to power agricultural machinery, heat greenhouses, power irrigation systems, but also to manufacture equipment, fertilizers, pesticides and other agro-chemicals.

The amount of energy consumed by agriculture is increasing worldwide as mechanization, especially in developing countries, increases. At the same time agriculture produces energy in the form of bioenergy. Bioenergy production increased sharply over the last years to meet the new demand for liquid biofuels for transport (e.g., ethanol and biodiesel) and solid biomass for power such as pellets or wood chips.

\section{CHART 68: Total energy consumption in} agriculture, top 20 countries (2012)

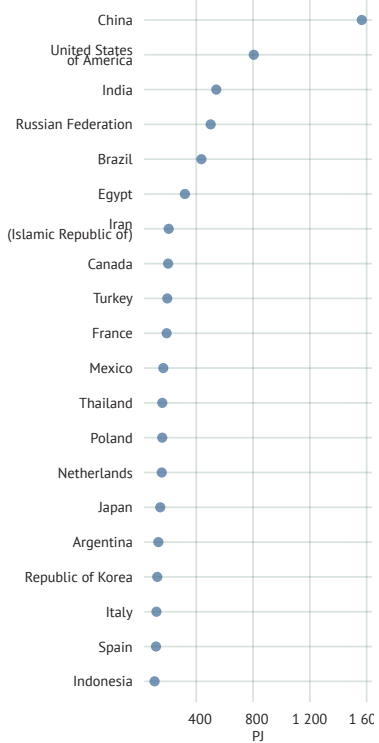

\section{CHART 67: Global wood pellet production}

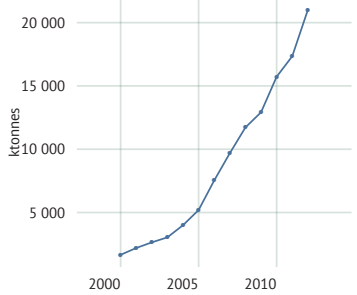

CHART 69: Bioenergy as a \% of total renewable energy, selected countries (2012)

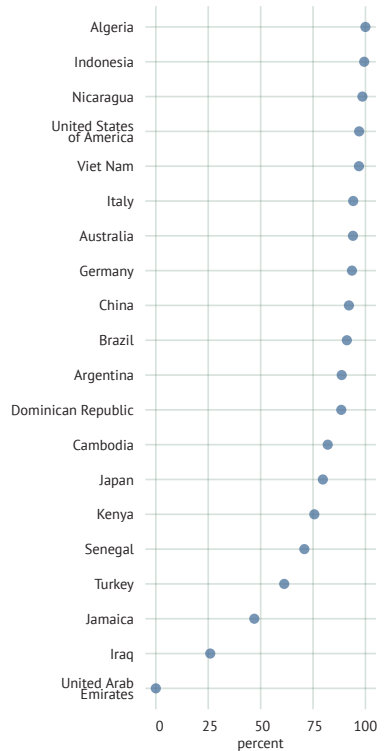

CHART 70: Biodiesel, biogas, biogasoline and other bio-oil consumption

$\rightarrow$ Biodiesel $\rightarrow$ Biogases $\rightarrow$ Biogasoline $\rightarrow$ Other liquid biofuels

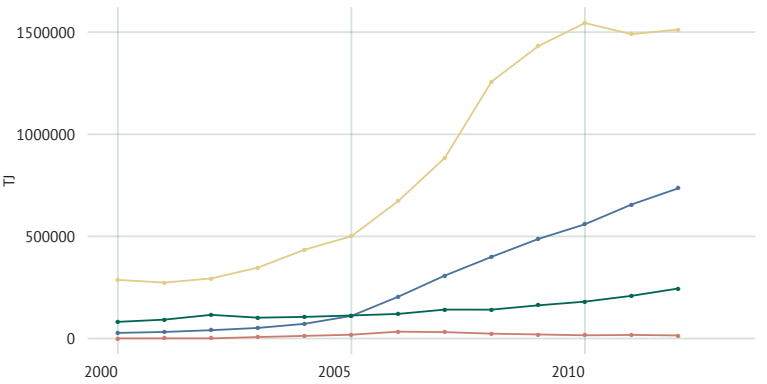


FIGURE 19: Energy consumption for power irrigation, million kWh (2011)

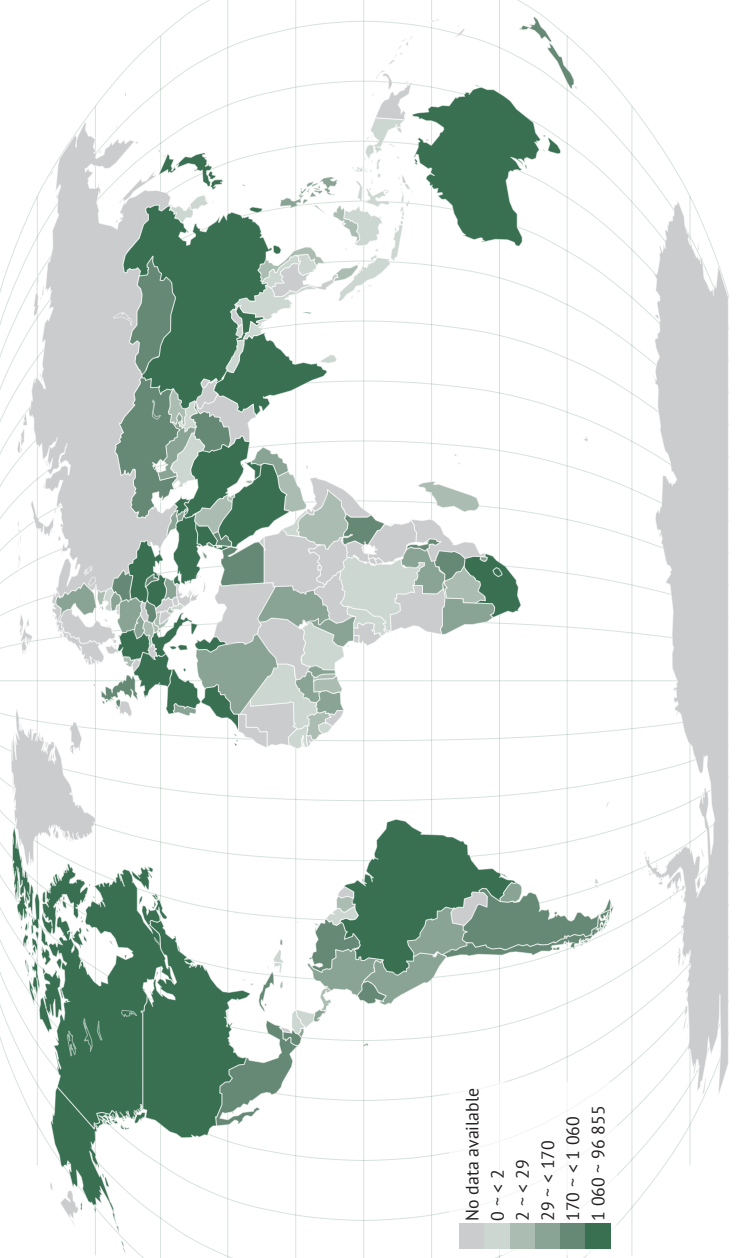




\section{Forestry}

Forests make vital contributions to biodiversity. They also sustain a range of economic activities and act as a source of food, medicine and fuel for more than a billion people. The latest estimate of the world's total forest area is more than 4 billion hectares, corresponding to about 30 percent of total land area. But today forests face unprecedented pressures. Changes in land cover have caused the most pressing environmental issue in recent decades. The impact of deforestation and land use intensification, especially on soil degradation, have been significant.

\section{CHART 72: Top 20 exporters of forest products} (2012)

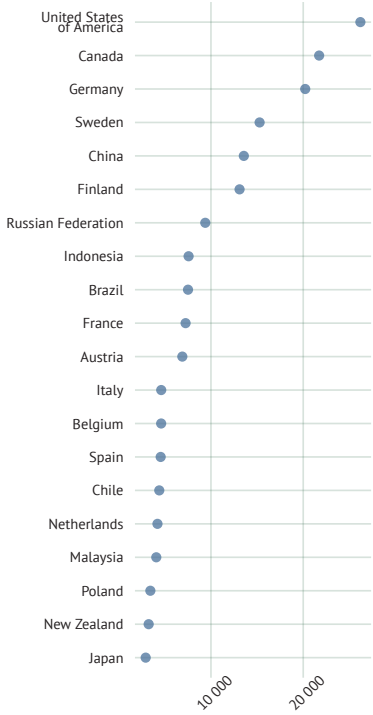

billion US\$
CHART 71: Production of selected forest products

$$
\begin{aligned}
& \rightarrow \text { Recovered paper } \\
& \rightarrow \text { Wood pulp } \\
& \rightarrow \text { Paper and paperboard }
\end{aligned}
$$

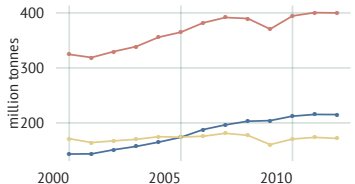

CHART 73: Top 20 importers of forest products (2012)

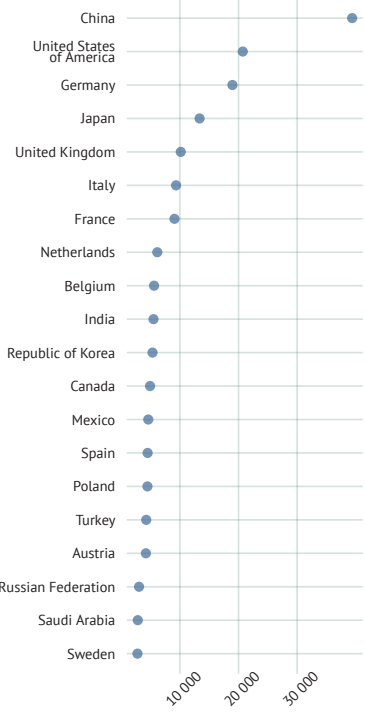

billion US\$

CHART 74: Forest characteristics (planted forest, primary forest, and other naturally regenerated forest) (2010)

primary forest planted forest other naturally regenerated forest

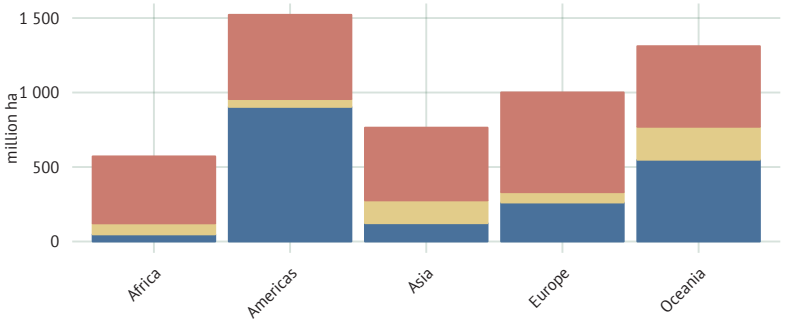


FIGURE 20: Forest area as share of total land area, percent (2012)

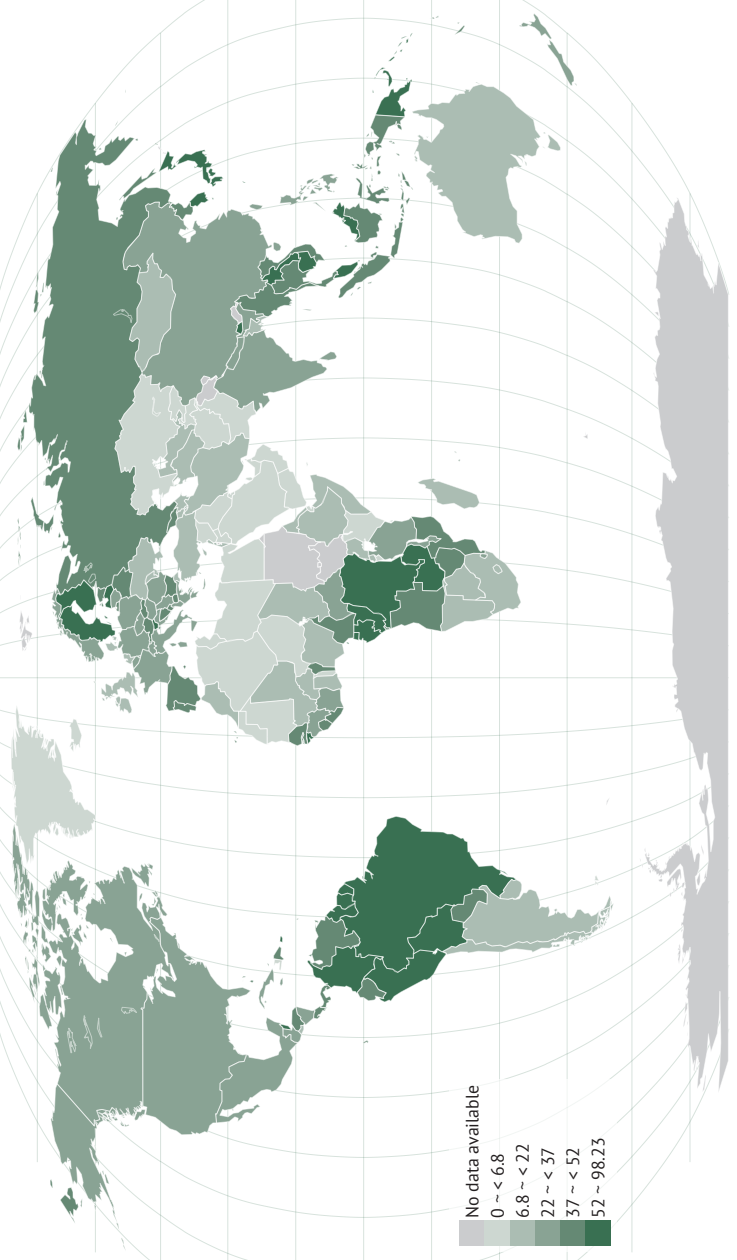




\section{Climate change}

The severity and speed of climate change is presenting an unprecedented challenge. Current global surface temperatures are now about 0.6 degrees Celsius higher than the average for the last century. This increase is consistent with model predictions of the effects of rising atmospheric concentrations of carbon dioxide $\left(\mathrm{CO}_{2}\right)$ and other $\mathrm{GHGs}$, which are a result of human activity. The poorest and most food-insecure regions around the globe are the most vulnerable. Already scarce land and water resources will likely become even more scarce, and insufficient technical and financial means will make adaptation to a changing climate very difficult.

CHART 76: Greehouse gas emissions in agriculture, highest 20 countries in 2012

$2000 \cdot 2012$

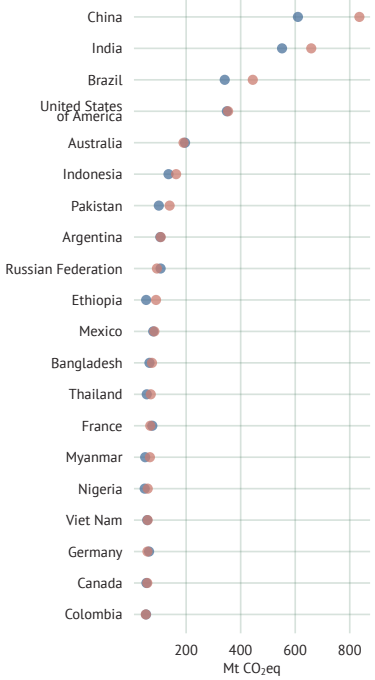

CHART 75: Greenhouse gas emissions in agriculture

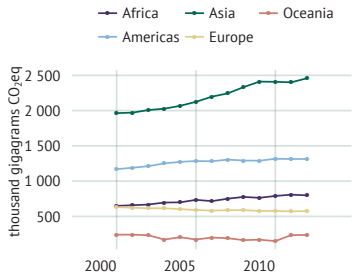

CHART 77: Land use total emissions, highest 20 countries in 2012

- 2000 - 2012

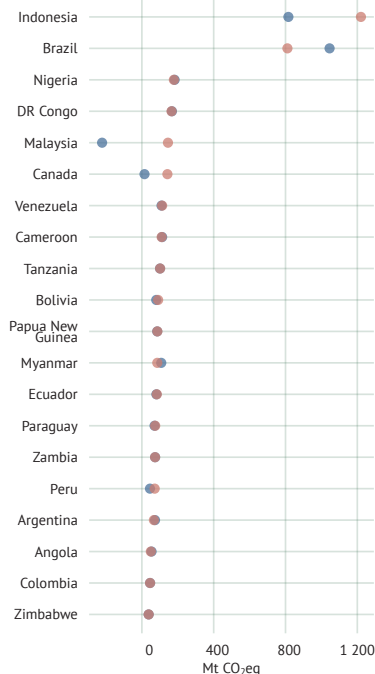

CHART 78: Emissions by subsectors in 2012

All GHG agricultural sectors Net forest conversion

Cultivation histoils and peat fires Forest Burning savanna

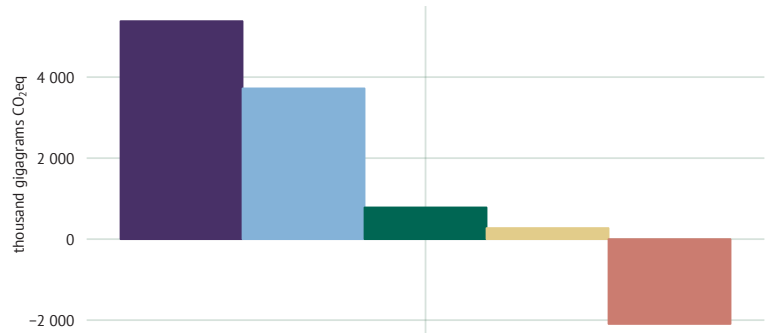


FIGURE 21: Total greenhouse gas emissions from agriculture, forestry and other land use, gigagrams $\mathrm{CO}_{2}$ eq (2012)

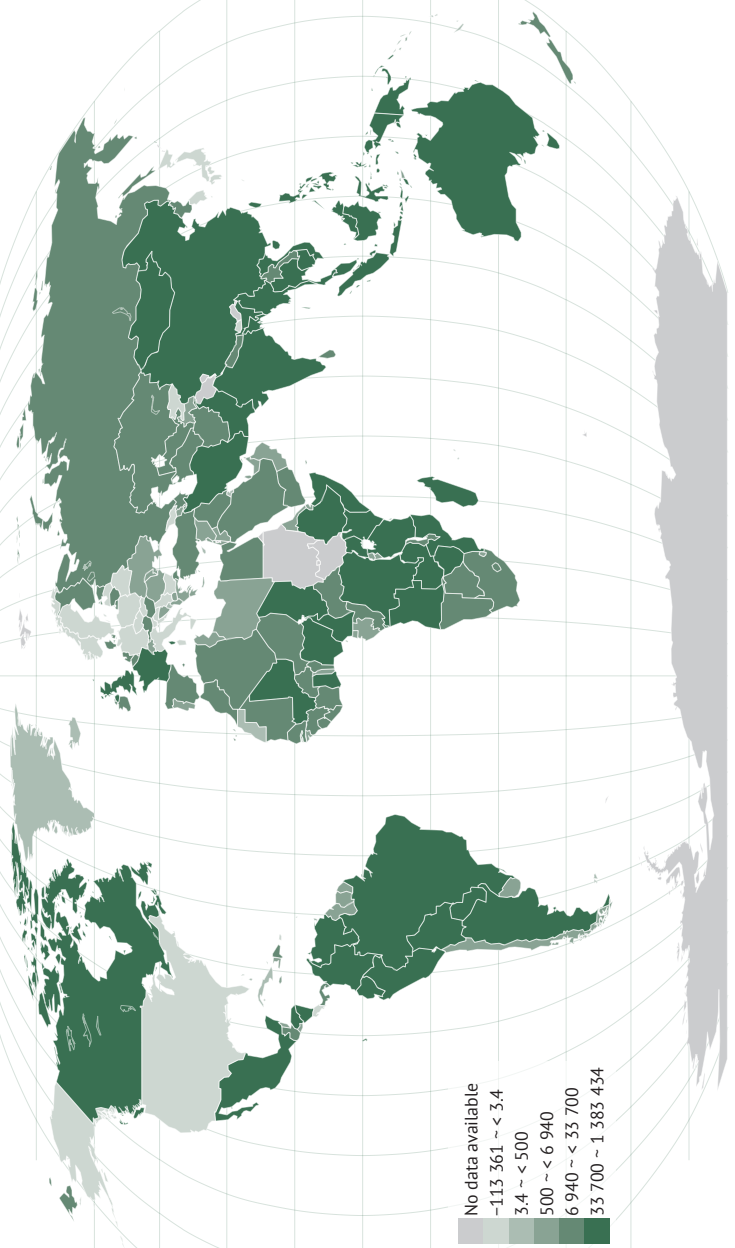






\begin{tabular}{|c|c|c|c|}
\hline & 1990 & 2000 & 2014 \\
\hline \multicolumn{4}{|l|}{ The setting } \\
\hline Population, total (mln) & 5320.8 & 6127.7 & 7243.8 \\
\hline Population, rural (mln) & 3033 & 3263.4 & 3362.5 \\
\hline \multicolumn{4}{|l|}{ Govt expenditure on ag (\% total outlays) } \\
\hline Area harvested (mln ha) & 1952 & 2061 & 2781 \\
\hline Cropping intensity ratio & 0.4 & 0.4 & \\
\hline \multicolumn{4}{|l|}{ Water resources (1 $000 \mathrm{~m}^{3} /$ person/year) } \\
\hline \multicolumn{4}{|l|}{ Area equipped for irrigation (1 $000 \mathrm{ha})$} \\
\hline \multicolumn{4}{|l|}{ Area irrigated (\% area equipped for irrigation) } \\
\hline Employment in agriculture (\%) & 35.3 & 38 & 30.7 \\
\hline Employment in agriculture, female (\%) & 9.2 & 20.3 & 25.2 \\
\hline Fertilizers, Nitrogen (kg of nutrients per ha) & & 64.9 & 85.8 \\
\hline Fertilizers, Phosphate (kg of nutrients per ha) & & 25.9 & 33.2 \\
\hline Fertilizers, Potash (kg nutrients per ha) & & 18.2 & 20.4 \\
\hline Energy consump, power irrigation (mln kWh) & 35981 & 130786 & 325448 \\
\hline \multicolumn{4}{|l|}{ Agr value added per worker (constant US\$) } \\
\hline \multicolumn{4}{|l|}{ Hunger dimensions } \\
\hline Dietary energy supply (kcal/pc/day) & 2621 & 2717 & 2891 \\
\hline Average dietary energy supply adequacy (\%) & 114 & 116 & 122 \\
\hline Dietary en supp, cereals/roots/tubers (\%) & 58 & 55 & 52 \\
\hline Prevalence of undernourishment (\%) & 18.2 & 15 & 11 \\
\hline GDP per capita (US\$, PPP) & 8832 & 10241 & 13915 \\
\hline Domestic food price volatility (index) & & 3.6 & 6.4 \\
\hline Cereal import dependency ratio (\%) & -0.4 & -0.2 & 50.7 \\
\hline \multicolumn{4}{|l|}{ Underweight, children under-5 (\%) } \\
\hline Improved water source (\% pop) & 78.5 & 83 & 88.7 \\
\hline \multicolumn{4}{|l|}{ Food supply } \\
\hline Food production value, (2004-2006 mln I\$) & 1294508 & 1618814 & 2246912 \\
\hline Agriculture, value added (\% GDP) & & 4 & 4 \\
\hline Food exports (mln US\$) & 215425 & 276704 & 945572 \\
\hline Food imports (mln US\$) & 237329 & 294271 & 966964 \\
\hline \multicolumn{4}{|l|}{ Production indices $(2004-06=100)$} \\
\hline Net food & 73 & 90 & 121 \\
\hline Net crops & 72 & 89 & 123 \\
\hline Cereals & 82 & 92 & 123 \\
\hline Vegetable oils & 51 & 77 & 141 \\
\hline Roots and tubers & 74 & 94 & 119 \\
\hline Fruit and vegetables & 58 & 86 & 127 \\
\hline Sugar & 86 & 93 & 132 \\
\hline Livestock & 76 & 92 & 115 \\
\hline Milk & 83 & 89 & 114 \\
\hline Meat & 74 & 91 & 118 \\
\hline Fish & 72 & 92 & 119 \\
\hline \multicolumn{4}{|l|}{ Net trade (mln US\$) } \\
\hline Cereals & -2447 & -4525 & -6979 \\
\hline Fruit and vegetables & -9430 & -7461 & -5811 \\
\hline Meat & -2574 & -682 & 5056 \\
\hline Dairy products & -663 & 165 & 1169 \\
\hline Fish & -3882 & -4295 & 1257 \\
\hline \multicolumn{4}{|l|}{ Environment } \\
\hline Forest area (\%) & 33 & 32 & 32 \\
\hline \multicolumn{4}{|l|}{ Renewable water res withdrawn (\% of total) } \\
\hline Terrestrial protect areas (\% total land area) & 9 & 12 & 14 \\
\hline Organic area (\% total agricultural area) & & & 1 \\
\hline \multicolumn{4}{|l|}{ Water withdrawal by agriculture (\% of total) } \\
\hline Biofuel production (thousand kt of oil eq.) & 3987 & 18110 & 381064 \\
\hline Wood pellet prod. (1 000 tonnes) & & & 26154 \\
\hline Net GHG emissions from AFOLU ( $\mathrm{CO}_{2}$ eq, $\mathrm{Mt}$ ) & 8075 & 7449 & 8165 \\
\hline
\end{tabular}




\begin{tabular}{|c|c|c|c|}
\hline & 1990 & 2000 & 2014 \\
\hline \multicolumn{4}{|l|}{ The setting } \\
\hline Population, total (mln) & 630 & 808.3 & 1138.2 \\
\hline Population, rural (mln) & 428 & 520.8 & 675.5 \\
\hline \multicolumn{4}{|l|}{ Govt expenditure on ag (\% total outlays) } \\
\hline Area harvested (mln ha) & 111 & 169 & 280 \\
\hline Cropping intensity ratio & 0.1 & 0.2 & \\
\hline \multicolumn{4}{|l|}{ Water resources (1 $000 \mathrm{~m}^{3} /$ person/year) } \\
\hline \multicolumn{4}{|l|}{ Area equipped for irrigation (1 $000 \mathrm{ha})$} \\
\hline \multicolumn{4}{|l|}{ Area irrigated (\% area equipped for irrigation) } \\
\hline Employment in agriculture (\%) & 30.9 & 24.5 & 27.6 \\
\hline Employment in agriculture, female (\%) & 40.9 & 29.4 & 31.1 \\
\hline Fertilizers, Nitrogen (kg of nutrients per ha) & & 14.1 & 13.8 \\
\hline Fertilizers, Phosphate (kg of nutrients per ha) & & 4.7 & 5.9 \\
\hline Fertilizers, Potash (kg nutrients per ha) & & 2.3 & 2.2 \\
\hline Energy consump, power irrigation (mln kWh) & 3163 & 14718 & 20667 \\
\hline \multicolumn{4}{|l|}{ Agr value added per worker (constant US\$) } \\
\hline \multicolumn{4}{|l|}{ Hunger dimensions } \\
\hline Dietary energy supply (kcal/pc/day) & 2338 & 2402 & 2572 \\
\hline Average dietary energy supply adequacy (\%) & 108 & 110 & 117 \\
\hline Dietary en supp, cereals/roots/tubers (\%) & 66 & 65 & 63 \\
\hline Prevalence of undernourishment (\%) & 27.2 & 25.7 & 20 \\
\hline GDP per capita (US\$, PPP) & 3315 & 3421 & 4575 \\
\hline Domestic food price volatility (index) & & 5.7 & 9.2 \\
\hline Cereal import dependency ratio (\%) & 25.9 & 28.1 & 42 \\
\hline \multicolumn{4}{|l|}{ Underweight, children under-5 (\%) } \\
\hline Improved water source (\% pop) & 55.5 & 61.1 & 68.7 \\
\hline \multicolumn{4}{|l|}{ Food supply } \\
\hline Food production value, (2004-2006 mln I\$) & 93685 & 128481 & 202196 \\
\hline Agriculture, value added (\% GDP) & & 16 & 14 \\
\hline Food exports (mln US\$) & 6744 & 7815 & 28015 \\
\hline Food imports (mln US\$) & 12451 & 16135 & 70074 \\
\hline \multicolumn{4}{|l|}{ Production indices $(2004-06=100)$} \\
\hline Net food & 60 & 82 & 129 \\
\hline Net crops & 59 & 82 & 127 \\
\hline Cereals & 66 & 79 & 131 \\
\hline Vegetable oils & 58 & 79 & 128 \\
\hline Roots and tubers & 50 & 80 & 133 \\
\hline Fruit and vegetables & 58 & 82 & 124 \\
\hline Sugar & 76 & 94 & 114 \\
\hline Livestock & 65 & 84 & 126 \\
\hline Milk & 59 & 77 & 125 \\
\hline Meat & 67 & 87 & 127 \\
\hline Fish & 65 & 90 & 119 \\
\hline \multicolumn{4}{|l|}{ Net trade (mln US\$) } \\
\hline Cereals & -4973 & -7262 & -28752 \\
\hline Fruit and vegetables & 1058 & 1192 & 4334 \\
\hline Meat & -454 & -626 & -4517 \\
\hline Dairy products & -1365 & -1269 & -4067 \\
\hline Fish & 590 & 1752 & 225 \\
\hline \multicolumn{4}{|l|}{ Environment } \\
\hline Forest area (\%) & 25 & 24 & 22 \\
\hline \multicolumn{4}{|l|}{ Renewable water res withdrawn (\% of total) } \\
\hline Terrestrial protect areas (\% total land area) & 9 & 10 & 14 \\
\hline Organic area (\% total agricultural area) & & & 0 \\
\hline \multicolumn{4}{|l|}{ Water withdrawal by agriculture (\% of total) } \\
\hline Biofuel production (thousand kt of oil eq.) & 246 & 425 & 512 \\
\hline Wood pellet prod. (1 000 tonnes) & & & 95 \\
\hline Net GHG emissions from AFOLU ( $\mathrm{CO}_{2}$ eq, $\mathrm{Mt}$ ) & 1751 & 1817 & 1865 \\
\hline
\end{tabular}




\begin{tabular}{|c|c|c|c|}
\hline & 1990 & 2000 & 2014 \\
\hline \multicolumn{4}{|l|}{ The setting } \\
\hline Population, total (mln) & 3146.8 & 3717.4 & 4342.3 \\
\hline Population, rural (mln) & 2142.5 & 2325.5 & 2293.3 \\
\hline \multicolumn{4}{|l|}{ Govt expenditure on ag (\% total outlays) } \\
\hline Area harvested (mln ha) & 873 & 996 & 1348 \\
\hline Cropping intensity ratio & 0.7 & 0.6 & \\
\hline \multicolumn{4}{|l|}{ Water resources (1 $000 \mathrm{~m}^{3} /$ person/year) } \\
\hline \multicolumn{4}{|l|}{ Area equipped for irrigation (1 $000 \mathrm{ha})$} \\
\hline \multicolumn{4}{|l|}{ Area irrigated (\% area equipped for irrigation) } \\
\hline Employment in agriculture (\%) & 50.1 & 49.5 & 38.6 \\
\hline Employment in agriculture, female (\%) & 9.7 & 26.1 & 44.2 \\
\hline Fertilizers, Nitrogen (kg of nutrients per ha) & & 110.6 & 165.6 \\
\hline Fertilizers, Phosphate (kg of nutrients per ha) & & 39.8 & 62.5 \\
\hline Fertilizers, Potash (kg nutrients per ha) & & 20.2 & 28.4 \\
\hline Energy consump, power irrigation (mln kWh) & 7614 & 38316 & 82411 \\
\hline \multicolumn{4}{|l|}{ Agr value added per worker (constant US\$) } \\
\hline \multicolumn{4}{|l|}{ Hunger dimensions } \\
\hline Dietary energy supply (kcal/pc/day) & 2435 & 2573 & 2796 \\
\hline Average dietary energy supply adequacy (\%) & 108 & 112 & 119 \\
\hline Dietary en supp, cereals/roots/tubers (\%) & 66 & 63 & 57 \\
\hline Prevalence of undernourishment (\%) & 23 & 17.6 & 12.4 \\
\hline GDP per capita (US\$, PPP) & 3017 & 4595 & 9392 \\
\hline Domestic food price volatility (index) & & 5.3 & 9.3 \\
\hline Cereal import dependency ratio (\%) & 5.3 & 5.8 & 93.3 \\
\hline \multicolumn{4}{|l|}{ Underweight, children under-5 (\%) } \\
\hline Improved water source (\% pop) & & & 90.8 \\
\hline \multicolumn{4}{|l|}{ Food supply } \\
\hline Food production value, (2004-2006 mln I\$) & 508899 & 757934 & 1134641 \\
\hline Agriculture, value added (\% GDP) & & 7 & 10 \\
\hline Food exports (mln US\$) & 26095 & 40997 & 181141 \\
\hline Food imports (mln US\$) & 55240 & 84465 & 325015 \\
\hline \multicolumn{4}{|l|}{ Production indices $(2004-06=100)$} \\
\hline Net food & 68 & 88 & 128 \\
\hline Net crops & 69 & 88 & 129 \\
\hline Cereals & 83 & 93 & 123 \\
\hline Vegetable oils & 46 & 75 & 143 \\
\hline Roots and tubers & 68 & 97 & 129 \\
\hline Fruit and vegetables & 42 & 81 & 140 \\
\hline Sugar & 82 & 102 & 134 \\
\hline Livestock & 64 & 85 & 126 \\
\hline Milk & 51 & 78 & 122 \\
\hline Meat & 51 & 87 & 128 \\
\hline Fish & 54 & 85 & 136 \\
\hline \multicolumn{4}{|l|}{ Net trade (mln US\$) } \\
\hline Cereals & -13330 & -12967 & -35498 \\
\hline Fruit and vegetables & 979 & -2128 & 669 \\
\hline Meat & -5691 & -11087 & -27384 \\
\hline Dairy products & -3090 & -4559 & -14876 \\
\hline Fish & -4395 & -5573 & 6725 \\
\hline \multicolumn{4}{|l|}{ Environment } \\
\hline Forest area (\%) & 23 & 18 & 18 \\
\hline \multicolumn{4}{|l|}{ Renewable water res withdrawn (\% of total) } \\
\hline Terrestrial protect areas (\% total land area) & 9 & 11 & 12 \\
\hline Organic area (\% total agricultural area) & & & 0 \\
\hline \multicolumn{4}{|l|}{ Water withdrawal by agriculture (\% of total) } \\
\hline Biofuel production (thousand kt of oil eq.) & 928 & 1520 & 30089 \\
\hline Wood pellet prod. (1 000 tonnes) & & & 1585 \\
\hline Net GHG emissions from AFOLU ( $\mathrm{CO}_{2}$ eq, $\mathrm{Mt}$ ) & 2246 & 2311 & 3409 \\
\hline
\end{tabular}




\begin{tabular}{|c|c|c|c|}
\hline & 1990 & 2000 & 2014 \\
\hline \multicolumn{4}{|l|}{ The setting } \\
\hline Population, total (mln) & 445.2 & 526.3 & 623.4 \\
\hline Population, rural (mln) & 132.1 & 129.1 & 125 \\
\hline \multicolumn{4}{|l|}{ Govt expenditure on ag (\% total outlays) } \\
\hline \multicolumn{4}{|l|}{ Area harvested (mln ha) } \\
\hline \multicolumn{4}{|l|}{ Cropping intensity ratio } \\
\hline \multicolumn{4}{|l|}{ Water resources (1 $000 \mathrm{~m}^{3} /$ person/year) } \\
\hline \multicolumn{4}{|l|}{ Area equipped for irrigation ( $1000 \mathrm{ha}$ ) } \\
\hline \multicolumn{4}{|l|}{ Area irrigated (\% area equipped for irrigation) } \\
\hline Employment in agriculture (\%) & 19.5 & 14.2 & 15.8 \\
\hline Employment in agriculture, female (\%) & 13.7 & 6.2 & 7.6 \\
\hline \multicolumn{4}{|l|}{ Fertilizers, Nitrogen (kg of nutrients per ha) } \\
\hline \multicolumn{4}{|l|}{ Fertilizers, Phosphate (kg of nutrients per ha) } \\
\hline \multicolumn{4}{|l|}{ Fertilizers, Potash (kg nutrients per ha) } \\
\hline \multicolumn{4}{|l|}{ Energy consump, power irrigation (mln kWh) } \\
\hline \multicolumn{4}{|l|}{ Agr value added per worker (constant US\$) } \\
\hline \multicolumn{4}{|l|}{ Hunger dimensions } \\
\hline Dietary energy supply (kcal/pc/day) & 2702 & 2787 & 3046 \\
\hline Average dietary energy supply adequacy (\%) & 118 & 120 & 128 \\
\hline Dietary en supp, cereals/roots/tubers (\%) & 42 & 41 & 40 \\
\hline Prevalence of undernourishment (\%) & 14.1 & 12.1 & 5.7 \\
\hline GDP per capita (US\$, PPP) & 9837 & 10976 & 13915 \\
\hline Domestic food price volatility (index) & & 3.5 & 5.9 \\
\hline Cereal import dependency ratio (\%) & 13 & 12 & 49.7 \\
\hline \multicolumn{4}{|l|}{ Underweight, children under-5 (\%) } \\
\hline Improved water source (\% pop) & 85.1 & 89.8 & 94 \\
\hline \multicolumn{4}{|l|}{ Food supply } \\
\hline Food production value, (2004-2006 mln I\$) & 138170 & 192134 & 297533 \\
\hline Agriculture, value added (\% GDP) & & 5 & 6 \\
\hline Food exports (mln US\$) & 23052 & 31727 & 142567 \\
\hline Food imports (mln US\$) & 11115 & 22055 & 67602 \\
\hline \multicolumn{4}{|l|}{ Production indices (2004-06=100) } \\
\hline Net food & 60 & 83 & 129 \\
\hline Net crops & 64 & 83 & 131 \\
\hline Cereals & 62 & 88 & 139 \\
\hline \multicolumn{4}{|l|}{ Vegetable oils } \\
\hline \multicolumn{4}{|l|}{ Roots and tubers } \\
\hline Fruit and vegetables & & & \\
\hline Sugar & & & \\
\hline Livestock & 58 & 84 & 123 \\
\hline Milk & & & \\
\hline Meat & & & \\
\hline Fish & 82 & 107 & 71 \\
\hline Net trade (mln US\$) & & & \\
\hline Cereals & -1766 & -3293 & 1077 \\
\hline Fruit and vegetables & 6023 & 8082 & 23534 \\
\hline Meat & 1119 & 899 & 14206 \\
\hline Dairy products & -1154 & -1303 & -2237 \\
\hline Fish & 2311 & 4644 & 9889 \\
\hline Environment & & & \\
\hline Forest area (\%) & 52 & 50 & 47 \\
\hline Renewable water res withdrawn (\% of total) & & & \\
\hline Terrestrial protect areas (\% total land area) & 9 & 14 & 21 \\
\hline Organic area (\% total agricultural area) & & & 1 \\
\hline Water withdrawal by agriculture (\% of total) & & & \\
\hline Biofuel production (thousand kt of oil eq.) & 1619 & 1730 & 61706 \\
\hline Wood pellet prod. (1 000 tonnes) & & & 122 \\
\hline Net GHG emissions from AFOLU ( $\mathrm{CO}_{2}$ eq, $\mathrm{Mt}$ ) & 2462 & 2415 & 2335 \\
\hline
\end{tabular}




\begin{tabular}{|c|c|c|c|}
\hline & 1990 & 2000 & 2014 \\
\hline \multicolumn{4}{|l|}{ The setting } \\
\hline Population, total (mln) & 27 & 31.2 & 38.8 \\
\hline Population, rural (mln) & 7.9 & 9.2 & 11.3 \\
\hline \multicolumn{4}{|l|}{ Govt expenditure on ag (\% total outlays) } \\
\hline Area harvested (mln ha) & 29 & 42 & 37 \\
\hline Cropping intensity ratio & 0.1 & 0.1 & \\
\hline \multicolumn{4}{|l|}{ Water resources (1 $000 \mathrm{~m}^{3} /$ person/year) } \\
\hline \multicolumn{4}{|l|}{ Area equipped for irrigation (1 $000 \mathrm{ha})$} \\
\hline \multicolumn{4}{|l|}{ Area irrigated (\% area equipped for irrigation) } \\
\hline Employment in agriculture (\%) & 6.2 & 17.1 & 3.8 \\
\hline Employment in agriculture, female (\%) & 4.5 & 3.9 & 4.4 \\
\hline Fertilizers, Nitrogen (kg of nutrients per ha) & & 27 & 29.5 \\
\hline Fertilizers, Phosphate (kg of nutrients per ha) & & 32.2 & 27.6 \\
\hline Fertilizers, Potash (kg nutrients per ha) & & 7.9 & 5.4 \\
\hline Energy consump, power irrigation (mln kWh) & 415 & 1028 & 8667 \\
\hline \multicolumn{4}{|l|}{ Agr value added per worker (constant US\$) } \\
\hline \multicolumn{4}{|l|}{ Hunger dimensions } \\
\hline Dietary energy supply (kcal/pc/day) & 2477 & 2436 & 2543 \\
\hline Average dietary energy supply adequacy (\%) & 114 & 112 & 115 \\
\hline Dietary en supp, cereals/roots/tubers (\%) & 49 & 50 & 48 \\
\hline Prevalence of undernourishment (\%) & 15.2 & 16.1 & 14 \\
\hline GDP per capita (US\$, PPP) & 2269 & 2536 & 3110 \\
\hline Domestic food price volatility (index) & & 13.6 & 8.3 \\
\hline Cereal import dependency ratio (\%) & 95.2 & 95.9 & 95.4 \\
\hline \multicolumn{4}{|l|}{ Underweight, children under-5 (\%) } \\
\hline Improved water source (\% pop) & 49.7 & 53.2 & 55.5 \\
\hline \multicolumn{4}{|l|}{ Food supply } \\
\hline Food production value, (2004-2006 mln I\$) & 23873 & 32768 & 38664 \\
\hline Agriculture, value added (\% GDP) & & 4 & 3 \\
\hline Food exports (mln US\$) & 10836 & 16050 & 45536 \\
\hline Food imports (mln US\$) & 2128 & 3343 & 12669 \\
\hline \multicolumn{4}{|l|}{ Production indices $(2004-06=100)$} \\
\hline Net food & 72 & 99 & 116 \\
\hline Net crops & 69 & 105 & 126 \\
\hline Cereals & 77 & 113 & 117 \\
\hline Vegetable oils & 41 & 122 & 215 \\
\hline Roots and tubers & 80 & 90 & 110 \\
\hline Fruit and vegetables & 66 & 88 & 104 \\
\hline Sugar & 70 & 104 & 71 \\
\hline Livestock & 79 & 97 & 107 \\
\hline Milk & 56 & 92 & 113 \\
\hline Meat & 79 & 96 & 105 \\
\hline Fish & 51 & 78 & 85 \\
\hline \multicolumn{4}{|l|}{ Net trade (mln US\$) } \\
\hline Cereals & 2451 & 2809 & 8606 \\
\hline Fruit and vegetables & 514 & 861 & 1257 \\
\hline Meat & 3419 & 4366 & 10465 \\
\hline Dairy products & 1642 & 3458 & 10513 \\
\hline Fish & 642 & 1280 & 1095 \\
\hline \multicolumn{4}{|l|}{ Environment } \\
\hline Forest area (\%) & 23 & 23 & 22 \\
\hline \multicolumn{4}{|l|}{ Renewable water res withdrawn (\% of total) } \\
\hline Terrestrial protect areas (\% total land area) & 7 & 9 & 13 \\
\hline Organic area (\% total agricultural area) & & & 3 \\
\hline \multicolumn{4}{|l|}{ Water withdrawal by agriculture (\% of total) } \\
\hline Biofuel production (thousand kt of oil eq.) & 210 & 282 & 1990 \\
\hline Wood pellet prod. (1 000 tonnes) & & & 32 \\
\hline Net GHG emissions from AFOLU ( $\mathrm{CO}_{2}$ eq, $\mathrm{Mt}$ ) & 334 & 321 & 340 \\
\hline
\end{tabular}




\begin{tabular}{|c|c|c|c|}
\hline & 1990 & 2000 & 2014 \\
\hline \multicolumn{4}{|l|}{ The setting } \\
\hline Population, total (mln) & 11.7 & 20.6 & 31.3 \\
\hline Population, rural (mln) & 9.6 & 16.4 & 23.6 \\
\hline Govt expenditure on ag (\% total outlays) & & 5.3 & 4.3 \\
\hline Area harvested (mln ha) & 3 & 2 & 7 \\
\hline Cropping intensity ratio & 0.1 & 0.1 & \\
\hline Water resources (1 $000 \mathrm{~m}^{3} /$ person/year) & 6 & 3 & 2 \\
\hline Area equipped for irrigation (1 $000 \mathrm{ha})$ & & & 3208 \\
\hline Area irrigated (\% area equipped for irrigation) & & & 59.1 \\
\hline \multicolumn{4}{|l|}{ Employment in agriculture (\%) } \\
\hline \multicolumn{4}{|l|}{ Employment in agriculture, female (\%) } \\
\hline Fertilizers, Nitrogen (kg of nutrients per ha) & & 2.6 & 4.6 \\
\hline Fertilizers, Phosphate (kg of nutrients per ha) & & 0.7 & 0 \\
\hline Fertilizers, Potash (kg nutrients per ha) & & 0 & 0 \\
\hline Energy consump, power irrigation (mln kWh) & 275 & 275 & 275 \\
\hline Agr value added per worker (constant US\$) & & 424 & 396 \\
\hline \multicolumn{4}{|l|}{ Hunger dimensions } \\
\hline Dietary energy supply (kcal/pc/day) & 1872 & 1792 & 2089 \\
\hline Average dietary energy supply adequacy (\%) & 92 & 89 & 100 \\
\hline Dietary en supp, cereals/roots/tubers (\%) & 75 & 76 & 77 \\
\hline Prevalence of undernourishment (\%) & 39.2 & 45.2 & 26 \\
\hline GDP per capita (US\$, PPP) & & 1097 & 1884 \\
\hline \multicolumn{4}{|l|}{ Domestic food price volatility (index) } \\
\hline Cereal import dependency ratio (\%) & 5.7 & 29.6 & 22.1 \\
\hline Underweight, children under-5 (\%) & & 44.9 & \\
\hline Improved water source (\% pop) & 4.9 & 22.1 & 64.2 \\
\hline \multicolumn{4}{|l|}{ Food supply } \\
\hline Food production value, (2004-2006 mln I\$) & 1959 & 2423 & 3393 \\
\hline Agriculture, value added (\% GDP) & & 38 & 24 \\
\hline Food exports (mln US\$) & 95 & 37 & 161 \\
\hline Food imports (mln US\$) & 170 & 158 & 1081 \\
\hline \multicolumn{4}{|l|}{ Production indices (2004-06=100) } \\
\hline Net food & 69 & 86 & 120 \\
\hline Net crops & 68 & 69 & 134 \\
\hline Cereals & 60 & 43 & 142 \\
\hline Vegetable oils & 87 & 87 & 95 \\
\hline Roots and tubers & 75 & 79 & 100 \\
\hline Fruit and vegetables & 81 & 93 & 120 \\
\hline Sugar & 94 & 65 & 180 \\
\hline Livestock & 71 & 105 & 104 \\
\hline Milk & 50 & 98 & 108 \\
\hline Meat & 84 & 109 & 101 \\
\hline Fish & 97 & 90 & 141 \\
\hline \multicolumn{4}{|l|}{ Net trade (mln US\$) } \\
\hline Cereals & & & -372 \\
\hline Fruit and vegetables & 89 & 31 & -42 \\
\hline \multicolumn{4}{|l|}{ Meat } \\
\hline Dairy products & & & -63 \\
\hline Fish & & & 0 \\
\hline \multicolumn{4}{|l|}{ Environment } \\
\hline Forest area (\%) & 2 & 2 & 2 \\
\hline Renewable water res withdrawn (\% of total) & & 99 & 99 \\
\hline Terrestrial protect areas (\% total land area) & 0 & 0 & 0 \\
\hline Organic area (\% total agricultural area) & & & 0 \\
\hline Water withdrawal by agriculture (\% of total) & & 99 & 99 \\
\hline \multicolumn{4}{|l|}{ Biofuel production (thousand kt of oil eq.) } \\
\hline \multicolumn{4}{|l|}{ Wood pellet prod. (1 000 tonnes) } \\
\hline Net GHG emissions from AFOLU ( $\mathrm{CO}_{2}$ eq, $\mathrm{Mt}$ ) & 8 & 10 & 14 \\
\hline
\end{tabular}




\section{Albania}

\begin{tabular}{|c|c|c|c|}
\hline & 1990 & 2000 & 2014 \\
\hline \multicolumn{4}{|l|}{ The setting } \\
\hline Population, total (mln) & 3.4 & 3.3 & 3.2 \\
\hline Population, rural (mln) & 2.2 & 1.9 & 1.4 \\
\hline \multicolumn{4}{|l|}{ Govt expenditure on ag (\% total outlays) } \\
\hline Area harvested (mln ha) & 1 & 1 & 1 \\
\hline Cropping intensity ratio & 0.8 & 0.5 & \\
\hline Water resources (1 $000 \mathrm{~m}^{3} /$ person/year) & 9 & 9 & 10 \\
\hline Area equipped for irrigation (1 $000 \mathrm{ha}$ ) & & & 331 \\
\hline Area irrigated (\% area equipped for irrigation) & & & 60.8 \\
\hline Employment in agriculture (\%) & & 71.8 & 41.5 \\
\hline Employment in agriculture, female (\%) & & & 52.6 \\
\hline Fertilizers, Nitrogen (kg of nutrients per ha) & & 64.3 & 55.9 \\
\hline Fertilizers, Phosphate (kg of nutrients per ha) & & 34.7 & 35 \\
\hline Fertilizers, Potash (kg nutrients per ha) & & 0 & 0 \\
\hline Energy consump, power irrigation (mln kWh) & 0 & 0 & 0 \\
\hline Agr value added per worker (constant US\$) & 1234 & 2486 & 3915 \\
\hline \multicolumn{4}{|l|}{ Hunger dimensions } \\
\hline \multicolumn{4}{|l|}{ Dietary energy supply (kcal/pc/day) } \\
\hline Average dietary energy supply adequacy (\%) & 118 & 117 & 120 \\
\hline Dietary en supp, cereals/roots/tubers (\%) & 58 & 51 & 41 \\
\hline Prevalence of undernourishment (\%) & $<5.0$ & $<5.0$ & $<5.0$ \\
\hline GDP per capita (US\$, PPP) & 4350 & 5305 & 9961 \\
\hline Domestic food price volatility (index) & & 10.8 & 10.3 \\
\hline Cereal import dependency ratio (\%) & 45.5 & 45.6 & 40.6 \\
\hline Underweight, children under-5 (\%) & & 17 & 6.3 \\
\hline Improved water source (\% pop) & & 96.5 & 95.7 \\
\hline \multicolumn{4}{|l|}{ Food supply } \\
\hline Food production value, (2004-2006 mln I\$) & 640 & 826 & 1222 \\
\hline Agriculture, value added (\% GDP) & 36 & 29 & 23 \\
\hline Food exports (mln US\$) & 54 & 4 & 40 \\
\hline Food imports (mln US\$) & 89 & 185 & 589 \\
\hline \multicolumn{4}{|l|}{ Production indices (2004-06=100) } \\
\hline Net food & 67 & 86 & 128 \\
\hline Net crops & 84 & 86 & 149 \\
\hline Cereals & 182 & 112 & 131 \\
\hline Vegetable oils & 55 & 86 & 196 \\
\hline Roots and tubers & 34 & 94 & 119 \\
\hline Fruit and vegetables & 62 & 76 & 152 \\
\hline Sugar & 390 & 97 & 92 \\
\hline Livestock & 55 & 89 & 110 \\
\hline Milk & 48 & 88 & 107 \\
\hline Meat & 70 & 94 & 113 \\
\hline Fish & 222 & 54 & 110 \\
\hline \multicolumn{4}{|l|}{ Net trade (mln US\$) } \\
\hline Cereals & -30 & -68 & -203 \\
\hline Fruit and vegetables & 50 & -41 & -65 \\
\hline Meat & -6 & -20 & -69 \\
\hline Dairy products & & -4 & -21 \\
\hline Fish & 6 & 1 & 6 \\
\hline \multicolumn{4}{|l|}{ Environment } \\
\hline Forest area (\%) & 29 & 28 & 28 \\
\hline Renewable water res withdrawn (\% of total) & & & 40 \\
\hline Terrestrial protect areas (\% total land area) & 3 & 7 & 11 \\
\hline Organic area (\% total agricultural area) & & & 0 \\
\hline Water withdrawal by agriculture (\% of total) & & & 40 \\
\hline \multicolumn{4}{|l|}{ Biofuel production (thousand kt of oil eq.) } \\
\hline Wood pellet prod. (1 000 tonnes) & & & 5 \\
\hline Net GHG emissions from AFOLU ( $\mathrm{CO}_{2}$ eq, $\mathrm{Mt}$ ) & 3 & 3 & 2 \\
\hline
\end{tabular}




\begin{tabular}{|c|c|c|c|}
\hline & 1990 & 2000 & 2014 \\
\hline \multicolumn{4}{|l|}{ The setting } \\
\hline Population, total (mln) & 26.2 & 31.7 & 39.9 \\
\hline Population, rural (mln) & 12.6 & 12.4 & 9.8 \\
\hline \multicolumn{4}{|l|}{ Govt expenditure on ag (\% total outlays) } \\
\hline Area harvested (mln ha) & 2 & 3 & 5 \\
\hline Cropping intensity ratio & 0.1 & 0.1 & \\
\hline Water resources (1 $000 \mathrm{~m}^{3} /$ person/year) & 0 & 0 & 0 \\
\hline Area equipped for irrigation (1 $000 \mathrm{ha}$ ) & & & 570 \\
\hline Area irrigated (\% area equipped for irrigation) & & 79.6 & \\
\hline Employment in agriculture (\%) & & 21.1 & 10.8 \\
\hline Employment in agriculture, female (\%) & & 11 & 3 \\
\hline Fertilizers, Nitrogen (kg of nutrients per ha) & & 2.3 & 9.9 \\
\hline Fertilizers, Phosphate (kg of nutrients per ha) & & 0.8 & 7.2 \\
\hline Fertilizers, Potash (kg nutrients per ha) & & 2.9 & 4.6 \\
\hline Energy consump, power irrigation (mln kWh) & 96 & 96 & 96 \\
\hline Agr value added per worker (constant US\$) & 2036 & 2025 & 3719 \\
\hline \multicolumn{4}{|l|}{ Hunger dimensions } \\
\hline Dietary energy supply (kcal/pc/day) & 2830 & 2847 & 3310 \\
\hline Average dietary energy supply adequacy (\%) & 129 & 124 & 143 \\
\hline Dietary en supp, cereals/roots/tubers (\%) & 60 & 60 & 57 \\
\hline Prevalence of undernourishment (\%) & 7.1 & 8.7 & $<5.0$ \\
\hline GDP per capita (US\$, PPP) & 10113 & 9886 & 12893 \\
\hline Domestic food price volatility (index) & & 13.9 & 5.5 \\
\hline Cereal import dependency ratio (\%) & 76.1 & 79.7 & 67.6 \\
\hline Underweight, children under-5 (\%) & 9.2 & 5.4 & 3.7 \\
\hline Improved water source (\% pop) & 93.9 & 89.4 & 83.9 \\
\hline \multicolumn{4}{|l|}{ Food supply } \\
\hline Food production value, (2004-2006 mln I\$) & 2646 & 3640 & 8218 \\
\hline Agriculture, value added (\% GDP) & 11 & 9 & 10 \\
\hline Food exports (mln US\$) & 25 & 19 & 277 \\
\hline Food imports (mln US\$) & 2101 & 2264 & 8478 \\
\hline \multicolumn{4}{|l|}{ Production indices $(2004-06=100)$} \\
\hline Net food & 51 & 70 & 158 \\
\hline Net crops & 41 & 57 & 167 \\
\hline Cereals & 35 & 20 & 128 \\
\hline Vegetable oils & 60 & 64 & 165 \\
\hline Roots and tubers & 34 & 57 & 240 \\
\hline Fruit and vegetables & 42 & 65 & 171 \\
\hline \multicolumn{4}{|l|}{ Sugar } \\
\hline Livestock & 70 & 90 & 145 \\
\hline Milk & 55 & 82 & 170 \\
\hline Meat & 74 & 98 & 127 \\
\hline Fish & 70 & 88 & 79 \\
\hline \multicolumn{4}{|l|}{ Net trade (mln US\$) } \\
\hline Cereals & -834 & -1086 & -3494 \\
\hline Fruit and vegetables & -154 & -178 & -897 \\
\hline Meat & -24 & -35 & -260 \\
\hline Dairy products & -495 & -424 & -1263 \\
\hline Fish & -5 & -8 & -60 \\
\hline \multicolumn{4}{|l|}{ Environment } \\
\hline Forest area (\%) & 1 & 1 & 1 \\
\hline Renewable water res withdrawn (\% of total) & & 61 & 61 \\
\hline Terrestrial protect areas (\% total land area) & 6 & 6 & 7 \\
\hline Organic area (\% total agricultural area) & & & 0 \\
\hline Water withdrawal by agriculture (\% of total) & & 61 & 61 \\
\hline \multicolumn{4}{|l|}{ Biofuel production (thousand kt of oil eq.) } \\
\hline \multicolumn{4}{|l|}{ Wood pellet prod. (1 000 tonnes) } \\
\hline Net GHG emissions from AFOLU ( $\mathrm{CO}_{2}$ eq, $\mathrm{Mt}$ ) & 9 & 10 & 13 \\
\hline
\end{tabular}




\section{Angola}

\begin{tabular}{|c|c|c|c|}
\hline & 1990 & 2000 & 2014 \\
\hline \multicolumn{4}{|l|}{ The setting } \\
\hline Population, total (mln) & 10.3 & 13.9 & 22.1 \\
\hline Population, rural (mln) & 6.5 & 7.1 & 8.5 \\
\hline Govt expenditure on ag (\% total outlays) & & 0.8 & 1.4 \\
\hline Area harvested (mln ha) & 2 & 5 & 18 \\
\hline Cropping intensity ratio & 0 & 0.1 & \\
\hline Water resources (1 $000 \mathrm{~m}^{3} /$ person/year) & 14 & 11 & 7 \\
\hline Area equipped for irrigation (1 $000 \mathrm{ha}$ ) & & & 86 \\
\hline Area irrigated (\% area equipped for irrigation) & & 29 & \\
\hline Employment in agriculture (\%) & 5.1 & & \\
\hline \multicolumn{4}{|l|}{ Employment in agriculture, female (\%) } \\
\hline Fertilizers, Nitrogen (kg of nutrients per ha) & & 0.5 & 5 \\
\hline Fertilizers, Phosphate (kg of nutrients per ha) & & 0.4 & 2.3 \\
\hline Fertilizers, Potash (kg nutrients per ha) & & 0.8 & 2.4 \\
\hline \multicolumn{4}{|l|}{ Energy consump, power irrigation (mln kWh) } \\
\hline Agr value added per worker (constant US\$) & 483 & 279 & 867 \\
\hline \multicolumn{4}{|l|}{ Hunger dimensions } \\
\hline Dietary energy supply (kcal/pc/day) & 1611 & 1810 & 2502 \\
\hline Average dietary energy supply adequacy (\%) & 78 & 88 & 120 \\
\hline Dietary en supp, cereals/roots/tubers (\%) & 64 & 67 & 60 \\
\hline Prevalence of undernourishment (\%) & 64.6 & 51.1 & 15.3 \\
\hline GDP per capita (US\$, PPP) & 4232 & 3387 & 7488 \\
\hline Domestic food price volatility (index) & & 89.8 & 13.7 \\
\hline Cereal import dependency ratio (\%) & 53.5 & 53.7 & 56.7 \\
\hline Underweight, children under-5 (\%) & & 27.5 & 15.6 \\
\hline Improved water source (\% pop) & 42.4 & 45.7 & 54.3 \\
\hline \multicolumn{4}{|l|}{ Food supply } \\
\hline Food production value, (2004-2006 mln I\$) & 790 & 1301 & 4440 \\
\hline Agriculture, value added (\% GDP) & 33 & 6 & 9 \\
\hline Food exports (mln US\$) & 0 & 0 & 19 \\
\hline Food imports (mln US\$) & 353 & 334 & 2707 \\
\hline \multicolumn{4}{|l|}{ Production indices $(2004-06=100)$} \\
\hline Net food & 38 & 63 & 213 \\
\hline Net crops & 29 & 53 & 236 \\
\hline Cereals & 31 & 66 & 204 \\
\hline Vegetable oils & 67 & 78 & 144 \\
\hline Roots and tubers & 19 & 49 & 191 \\
\hline Fruit and vegetables & 45 & 49 & 356 \\
\hline Sugar & 71 & 92 & 134 \\
\hline Livestock & 69 & 97 & 136 \\
\hline Milk & 69 & 86 & 83 \\
\hline Meat & 65 & 100 & 154 \\
\hline Fish & 60 & 107 & 124 \\
\hline \multicolumn{4}{|l|}{ Net trade (mln US\$) } \\
\hline Cereals & -87 & -119 & -746 \\
\hline Fruit and vegetables & -26 & -26 & -209 \\
\hline Meat & -80 & -79 & -809 \\
\hline Dairy products & -67 & -8 & -82 \\
\hline Fish & -50 & -5 & -228 \\
\hline \multicolumn{4}{|l|}{ Environment } \\
\hline Forest area (\%) & 49 & 48 & 47 \\
\hline Renewable water res withdrawn (\% of total) & & & 21 \\
\hline Terrestrial protect areas (\% total land area) & 12 & 12 & 12 \\
\hline Organic area (\% total agricultural area) & & & 0 \\
\hline Water withdrawal by agriculture (\% of total) & & & 21 \\
\hline Biofuel production (thousand kt of oil eq.) & 1 & 0 & \\
\hline \multicolumn{4}{|l|}{ Wood pellet prod. (1 000 tonnes) } \\
\hline Net GHG emissions from AFOLU ( $\mathrm{CO}_{2}$ eq, $\left.\mathrm{Mt}\right)$ & 79 & 80 & 79 \\
\hline
\end{tabular}




\begin{tabular}{|c|c|c|c|}
\hline & 1990 & 2000 & 2014 \\
\hline \multicolumn{4}{|l|}{ The setting } \\
\hline Population, total (mln) & 32.6 & 36.9 & 41.8 \\
\hline Population, rural (mln) & 4.2 & 3.6 & 2.9 \\
\hline \multicolumn{4}{|l|}{ Govt expenditure on ag (\% total outlays) } \\
\hline Area harvested (mln ha) & 20 & 39 & 51 \\
\hline Cropping intensity ratio & 0.2 & 0.3 & \\
\hline Water resources (1 $000 \mathrm{~m}^{3} /$ person/year) & 27 & 24 & 21 \\
\hline Area equipped for irrigation (1 $000 \mathrm{ha})$ & & & 2360 \\
\hline Area irrigated (\% area equipped for irrigation) & & & 91.7 \\
\hline Employment in agriculture (\%) & 0.4 & 0.7 & 0.6 \\
\hline Employment in agriculture, female (\%) & 0.2 & 0.2 & 0.3 \\
\hline Fertilizers, Nitrogen (kg of nutrients per ha) & & 25.8 & 24.8 \\
\hline Fertilizers, Phosphate (kg of nutrients per ha) & & 14.7 & 13.1 \\
\hline Fertilizers, Potash (kg nutrients per ha) & & 1.4 & 1 \\
\hline Energy consump, power irrigation (mln kWh) & 142 & 142 & 885 \\
\hline Agr value added per worker (constant US\$) & 7175 & 9794 & 13176 \\
\hline \multicolumn{4}{|l|}{ Hunger dimensions } \\
\hline Dietary energy supply (kcal/pc/day) & 3116 & 3246 & 3606 \\
\hline Average dietary energy supply adequacy (\%) & 132 & 136 & 149 \\
\hline Dietary en supp, cereals/roots/tubers (\%) & 34 & 34 & 36 \\
\hline Prevalence of undernourishment (\%) & $<5.0$ & $<5.0$ & $<5.0$ \\
\hline \multicolumn{4}{|l|}{ GDP per capita (US\$, PPP) } \\
\hline \multicolumn{4}{|l|}{ Domestic food price volatility (index) } \\
\hline Cereal import dependency ratio (\%) & -96.7 & -158.7 & -168.5 \\
\hline Underweight, children under-5 (\%) & & 4.7 & 2.3 \\
\hline Improved water source (\% pop) & 93.8 & 96.5 & 98.7 \\
\hline \multicolumn{4}{|l|}{ Food supply } \\
\hline Food production value, (2004-2006 mln I\$) & 22555 & 30583 & 42146 \\
\hline Agriculture, value added (\% GDP) & 8 & 5 & 8 \\
\hline Food exports (mln US\$) & 5141 & 7746 & 27534 \\
\hline Food imports (mln US\$) & 102 & 982 & 1084 \\
\hline \multicolumn{4}{|l|}{ Production indices $(2004-06=100)$} \\
\hline Net food & 63 & 86 & 118 \\
\hline Net crops & 54 & 82 & 126 \\
\hline Cereals & 56 & 107 & 142 \\
\hline Vegetable oils & 48 & 77 & 129 \\
\hline Roots and tubers & 82 & 111 & 105 \\
\hline Fruit and vegetables & 74 & 86 & 102 \\
\hline Sugar & 66 & 77 & 99 \\
\hline Livestock & 84 & 94 & 111 \\
\hline Milk & 66 & 107 & 124 \\
\hline Meat & 89 & 91 & 107 \\
\hline Fish & 55 & 91 & 86 \\
\hline \multicolumn{4}{|l|}{ Net trade (mln US\$) } \\
\hline Cereals & 1450 & 2548 & 10586 \\
\hline Fruit and vegetables & 579 & 645 & 2897 \\
\hline Meat & 867 & 604 & 1830 \\
\hline Dairy products & 121 & 291 & 1260 \\
\hline Fish & 310 & 722 & 1164 \\
\hline \multicolumn{4}{|l|}{ Environment } \\
\hline Forest area (\%) & 13 & 12 & 11 \\
\hline Renewable water res withdrawn (\% of total) & & & 74 \\
\hline Terrestrial protect areas (\% total land area) & 5 & 6 & 7 \\
\hline Organic area (\% total agricultural area) & & & 2 \\
\hline Water withdrawal by agriculture (\% of total) & & & 74 \\
\hline Biofuel production (thousand kt of oil eq.) & 74 & 40 & 49086 \\
\hline Wood pellet prod. (1 000 tonnes) & & & 9 \\
\hline Net GHG emissions from AFOLU ( $\mathrm{CO}_{2}$ eq, $\mathrm{Mt}$ ) & 186 & 178 & 174 \\
\hline
\end{tabular}




\begin{tabular}{|c|c|c|c|}
\hline & 1990 & 2000 & 2014 \\
\hline \multicolumn{4}{|l|}{ The setting } \\
\hline Population, total (mln) & 3.4 & 3.1 & 3 \\
\hline Population, rural (mln) & 1.1 & 1.1 & 1.1 \\
\hline Govt expenditure on ag (\% total outlays) & & 6.3 & 4.3 \\
\hline Area harvested (mln ha) & & 0 & 1 \\
\hline Cropping intensity ratio & & 0.3 & \\
\hline Water resources (1 $000 \mathrm{~m}^{3} /$ person/year) & 2 & 3 & 3 \\
\hline Area equipped for irrigation (1 $000 \mathrm{ha}$ ) & & & 274 \\
\hline Area irrigated (\% area equipped for irrigation) & & & 64.4 \\
\hline Employment in agriculture (\%) & & 46 & 38.9 \\
\hline Employment in agriculture, female (\%) & & 43.4 & 45.5 \\
\hline Fertilizers, Nitrogen (kg of nutrients per ha) & & 23 & 32 \\
\hline Fertilizers, Phosphate (kg of nutrients per ha) & & 0 & 0.9 \\
\hline Fertilizers, Potash (kg nutrients per ha) & & 0 & 0.8 \\
\hline Energy consump, power irrigation (mln kWh) & 0 & & 63 \\
\hline Agr value added per worker (constant US\$) & 2234 & 3476 & 10006 \\
\hline \multicolumn{4}{|l|}{ Hunger dimensions } \\
\hline Dietary energy supply (kcal/pc/day) & 2179 & 2253 & 2862 \\
\hline Average dietary energy supply adequacy (\%) & 95 & 95 & 118 \\
\hline Dietary en supp, cereals/roots/tubers (\%) & 65 & 62 & 43 \\
\hline Prevalence of undernourishment (\%) & 27.3 & 21.4 & 6.3 \\
\hline GDP per capita (US\$, PPP) & 3736 & 2919 & 7527 \\
\hline Domestic food price volatility (index) & & 8.5 & 11.9 \\
\hline Cereal import dependency ratio (\%) & 62.1 & 56.7 & 55.7 \\
\hline Underweight, children under-5 (\%) & & 2.6 & 5.3 \\
\hline Improved water source (\% pop) & 90.6 & 92.6 & 99.8 \\
\hline \multicolumn{4}{|l|}{ Food supply } \\
\hline Food production value, $(2004-2006 \mathrm{mln}$ I\$) & 546 & 568 & 1087 \\
\hline Agriculture, value added (\% GDP) & 17 & 26 & 22 \\
\hline Food exports (mln US\$) & 0 & 4 & 59 \\
\hline Food imports (mln US\$) & 118 & 161 & 589 \\
\hline \multicolumn{4}{|l|}{ Production indices $(2004-06=100)$} \\
\hline Net food & 65 & 68 & 129 \\
\hline Net crops & 58 & 58 & 132 \\
\hline Cereals & 81 & 63 & 161 \\
\hline \multicolumn{4}{|l|}{ Vegetable oils } \\
\hline Roots and tubers & 63 & 43 & 117 \\
\hline Fruit and vegetables & 54 & 59 & 129 \\
\hline Sugar & 6 & 6 & 488 \\
\hline Livestock & 72 & 80 & 124 \\
\hline Milk & 67 & 76 & 112 \\
\hline Meat & 84 & 86 & 142 \\
\hline Fish & 679 & 177 & 1435 \\
\hline \multicolumn{4}{|l|}{ Net trade (mln US\$) } \\
\hline Cereals & & -82 & -182 \\
\hline Fruit and vegetables & -1 & -3 & -27 \\
\hline Meat & & -23 & -89 \\
\hline Dairy products & & -10 & -34 \\
\hline Fish & & -4 & 14 \\
\hline \multicolumn{4}{|l|}{ Environment } \\
\hline Forest area (\%) & 12 & 11 & 9 \\
\hline Renewable water res withdrawn (\% of total) & & & 66 \\
\hline Terrestrial protect areas (\% total land area) & 7 & 7 & 8 \\
\hline Organic area (\% total agricultural area) & & & 0 \\
\hline Water withdrawal by agriculture (\% of total) & & & 66 \\
\hline \multicolumn{4}{|l|}{ Biofuel production (thousand kt of oil eq.) } \\
\hline Wood pellet prod. (1 000 tonnes) & & & 0 \\
\hline Net GHG emissions from AFOLU ( $\mathrm{CO}_{2}$ eq, $\mathrm{Mt}$ ) & 2 & 2 & 2 \\
\hline
\end{tabular}




\begin{tabular}{|c|c|c|c|}
\hline & 1990 & 2000 & 2014 \\
\hline \multicolumn{4}{|l|}{ The setting } \\
\hline Population, total (mln) & 17.1 & 19.3 & 23.6 \\
\hline Population, rural (mln) & 2.5 & 2.5 & 2.4 \\
\hline \multicolumn{4}{|l|}{ Govt expenditure on ag (\% total outlays) } \\
\hline Area harvested (mln ha) & 24 & 38 & 36 \\
\hline Cropping intensity ratio & 0.1 & 0.1 & \\
\hline Water resources (1 $000 \mathrm{~m}^{3} /$ person/year) & 29 & 26 & 21 \\
\hline Area equipped for irrigation (1 $000 \mathrm{ha})$ & & & 2550 \\
\hline \multicolumn{4}{|l|}{ Area irrigated (\% area equipped for irrigation) } \\
\hline Employment in agriculture (\%) & 5.3 & 5 & 3.3 \\
\hline Employment in agriculture, female (\%) & 3.8 & 3.5 & 2.2 \\
\hline Fertilizers, Nitrogen (kg of nutrients per ha) & & 19.8 & 23.3 \\
\hline Fertilizers, Phosphate (kg of nutrients per ha) & & 21.7 & 17.2 \\
\hline Fertilizers, Potash (kg nutrients per ha) & & 4.7 & 4.2 \\
\hline Energy consump, power irrigation (mln kWh) & 113 & 284 & 1379 \\
\hline Agr value added per worker (constant US\$) & 26995 & 41209 & 52701 \\
\hline \multicolumn{4}{|l|}{ Hunger dimensions } \\
\hline \multicolumn{4}{|l|}{ Dietary energy supply (kcal/pc/day) } \\
\hline Average dietary energy supply adequacy (\%) & 123 & 122 & 132 \\
\hline Dietary en supp, cereals/roots/tubers (\%) & 25 & 25 & 25 \\
\hline Prevalence of undernourishment (\%) & $<5.0$ & $<5.0$ & $<5.0$ \\
\hline GDP per capita (US\$, PPP) & 28604 & 35253 & 42834 \\
\hline \multicolumn{4}{|l|}{ Domestic food price volatility (index) } \\
\hline Cereal import dependency ratio (\%) & -179.3 & -151.4 & -144.9 \\
\hline Underweight, children under-5 (\%) & & 0 & 0.2 \\
\hline Improved water source (\% pop) & 100 & 100 & 100 \\
\hline \multicolumn{4}{|l|}{ Food supply } \\
\hline Food production value, (2004-2006 mln I\$) & 15583 & 21996 & 25035 \\
\hline Agriculture, value added (\% GDP) & 5 & 3 & 3 \\
\hline Food exports (mln US\$) & 7094 & 10726 & 27285 \\
\hline Food imports (mln US\$) & 1039 & 1959 & 8036 \\
\hline \multicolumn{4}{|l|}{ Production indices $(2004-06=100)$} \\
\hline Net food & 74 & 105 & 120 \\
\hline Net crops & 67 & 108 & 129 \\
\hline Cereals & 76 & 113 & 117 \\
\hline Vegetable oils & 28 & 146 & 293 \\
\hline Roots and tubers & 91 & 92 & 102 \\
\hline Fruit and vegetables & 61 & 83 & 95 \\
\hline Sugar & 65 & 102 & 73 \\
\hline Livestock & 86 & 104 & 106 \\
\hline Milk & 64 & 107 & 94 \\
\hline Meat & 79 & 100 & 112 \\
\hline Fish & 83 & 83 & 87 \\
\hline \multicolumn{4}{|l|}{ Net trade (mln US\$) } \\
\hline Cereals & 2662 & 3056 & 9024 \\
\hline Fruit and vegetables & 98 & 384 & 162 \\
\hline Meat & 2228 & 2834 & 6751 \\
\hline Dairy products & 478 & 1407 & 1584 \\
\hline Fish & 188 & 485 & -615 \\
\hline \multicolumn{4}{|l|}{ Environment } \\
\hline Forest area (\%) & 20 & 20 & 19 \\
\hline Renewable water res withdrawn (\% of total) & & & 66 \\
\hline Terrestrial protect areas (\% total land area) & 7 & 9 & 13 \\
\hline Organic area (\% total agricultural area) & & & 3 \\
\hline Water withdrawal by agriculture (\% of total) & & & 66 \\
\hline Biofuel production (thousand kt of oil eq.) & 169 & 225 & 1908 \\
\hline Wood pellet prod. (1 000 tonnes) & & & 2 \\
\hline Net GHG emissions from AFOLU ( $\mathrm{CO}_{2}$ eq, $\mathrm{Mt}$ ) & 186 & 210 & 227 \\
\hline
\end{tabular}




\section{Austria}

\begin{tabular}{|c|c|c|c|}
\hline & 1990 & 2000 & 2014 \\
\hline \multicolumn{4}{|l|}{ The setting } \\
\hline Population, total (mln) & 7.7 & 8 & 8.5 \\
\hline Population, rural (mln) & 2.6 & 2.7 & 2.7 \\
\hline \multicolumn{4}{|l|}{ Govt expenditure on ag (\% total outlays) } \\
\hline Area harvested (mln ha) & 5 & 5 & 5 \\
\hline Cropping intensity ratio & 1.5 & 1.4 & \\
\hline Water resources (1 $000 \mathrm{~m}^{3} /$ person/year) & 10 & 10 & 9 \\
\hline Area equipped for irrigation (1 $000 \mathrm{ha})$ & & & 117 \\
\hline Area irrigated (\% area equipped for irrigation) & & & 37.1 \\
\hline Employment in agriculture (\%) & 7.9 & 5.8 & 4.9 \\
\hline Employment in agriculture, female (\%) & 9.4 & 6.1 & 4.5 \\
\hline Fertilizers, Nitrogen (kg of nutrients per ha) & & 215.5 & 63.5 \\
\hline Fertilizers, Phosphate (kg of nutrients per ha) & & 25.6 & 17 \\
\hline Fertilizers, Potash (kg nutrients per ha) & & 54.6 & 23.2 \\
\hline Energy consump, power irrigation (mln kWh) & 0 & 4 & 4 \\
\hline Agr value added per worker (constant US\$) & 13450 & 19849 & 37805 \\
\hline \multicolumn{4}{|l|}{ Hunger dimensions } \\
\hline \multicolumn{4}{|l|}{ Dietary energy supply (kcal/pc/day) } \\
\hline Average dietary energy supply adequacy (\%) & 141 & 150 & 150 \\
\hline Dietary en supp, cereals/roots/tubers (\%) & 23 & 27 & 26 \\
\hline Prevalence of undernourishment (\%) & $<5.0$ & $<5.0$ & $<5.0$ \\
\hline GDP per capita (US\$, PPP) & 31053 & 38548 & 44056 \\
\hline Domestic food price volatility (index) & & 3.6 & 5.9 \\
\hline Cereal import dependency ratio (\%) & -14.4 & -8.9 & 6.3 \\
\hline \multicolumn{4}{|l|}{ Underweight, children under-5 (\%) } \\
\hline Improved water source (\% pop) & 100 & 100 & 100 \\
\hline \multicolumn{4}{|l|}{ Food supply } \\
\hline Food production value, (2004-2006 mln I\$) & 3911 & 4140 & 4225 \\
\hline Agriculture, value added (\% GDP) & 4 & 2 & 1 \\
\hline Food exports (mln US\$) & 1043 & 2343 & 8396 \\
\hline Food imports (mln US\$) & 1779 & 2995 & 9461 \\
\hline \multicolumn{4}{|l|}{ Production indices $(2004-06=100)$} \\
\hline Net food & 94 & 100 & 102 \\
\hline Net crops & 90 & 94 & 96 \\
\hline Cereals & 95 & 92 & 96 \\
\hline Vegetable oils & 68 & 84 & 119 \\
\hline Roots and tubers & 108 & 98 & 85 \\
\hline Fruit and vegetables & 84 & 98 & 94 \\
\hline Sugar & 88 & 91 & 123 \\
\hline Livestock & 97 & 101 & 104 \\
\hline Milk & 107 & 107 & 109 \\
\hline Meat & 92 & 97 & 100 \\
\hline Fish & 132 & 118 & 129 \\
\hline \multicolumn{4}{|l|}{ Net trade (mln US\$) } \\
\hline Cereals & 54 & -45 & 46 \\
\hline Fruit and vegetables & -717 & -516 & -1349 \\
\hline Meat & 89 & 38 & 506 \\
\hline Dairy products & 81 & 167 & 511 \\
\hline Fish & -146 & -151 & -435 \\
\hline \multicolumn{4}{|l|}{ Environment } \\
\hline Forest area (\%) & 46 & 47 & 47 \\
\hline Renewable water res withdrawn (\% of total) & & 3 & 3 \\
\hline Terrestrial protect areas (\% total land area) & 21 & 24 & 24 \\
\hline Organic area (\% total agricultural area) & & & 17 \\
\hline Water withdrawal by agriculture (\% of total) & & 3 & 3 \\
\hline Biofuel production (thousand kt of oil eq.) & & 30 & 2476 \\
\hline Wood pellet prod. (1 000 tonnes) & & & 945 \\
\hline Net GHG emissions from AFOLU ( $\mathrm{CO}_{2}$ eq, $\left.\mathrm{Mt}\right)$ & -2 & -6 & 11 \\
\hline
\end{tabular}




\begin{tabular}{|c|c|c|c|}
\hline & 1990 & 2000 & 2014 \\
\hline \multicolumn{4}{|l|}{ The setting } \\
\hline Population, total (mln) & 7.6 & 8.1 & 9.5 \\
\hline Population, rural (mln) & 3.6 & 3.9 & 4.3 \\
\hline Govt expenditure on ag (\% total outlays) & & 4.3 & 2.9 \\
\hline Area harvested (mln ha) & & 1 & 3 \\
\hline Cropping intensity ratio & & 0.3 & \\
\hline Water resources (1 $000 \mathrm{~m}^{3} /$ person/year) & 5 & 4 & 4 \\
\hline Area equipped for irrigation (1 $000 \mathrm{ha})$ & & & 1428 \\
\hline Area irrigated (\% area equipped for irrigation) & & & 95.3 \\
\hline Employment in agriculture (\%) & 30.9 & 41 & 37.7 \\
\hline Employment in agriculture, female (\%) & & 46.1 & 43.9 \\
\hline Fertilizers, Nitrogen (kg of nutrients per ha) & & 6.8 & 14.2 \\
\hline Fertilizers, Phosphate (kg of nutrients per ha) & & 0.1 & 2.2 \\
\hline Fertilizers, Potash (kg nutrients per ha) & & 0.7 & 1.6 \\
\hline Energy consump, power irrigation (mln kWh) & 0 & 366 & 1464 \\
\hline Agr value added per worker (constant US\$) & 850 & 885 & 1434 \\
\hline \multicolumn{4}{|l|}{ Hunger dimensions } \\
\hline Dietary energy supply (kcal/pc/day) & 2221 & 2380 & 3030 \\
\hline Average dietary energy supply adequacy (\%) & 98 & 102 & 126 \\
\hline Dietary en supp, cereals/roots/tubers (\%) & 69 & 69 & 63 \\
\hline Prevalence of undernourishment (\%) & 23.6 & 22.5 & $<5.0$ \\
\hline GDP per capita (US\$, PPP) & 8513 & 4459 & 16593 \\
\hline \multicolumn{4}{|l|}{ Domestic food price volatility (index) } \\
\hline Cereal import dependency ratio (\%) & 42 & 33.1 & 37.7 \\
\hline Underweight, children under-5 (\%) & & 14 & 8.4 \\
\hline Improved water source (\% pop) & 69.9 & 74 & 80.2 \\
\hline \multicolumn{4}{|l|}{ Food supply } \\
\hline Food production value, (2004-2006 mln I\$) & 1211 & 1395 & 2578 \\
\hline Agriculture, value added (\% GDP) & 29 & 17 & 6 \\
\hline Food exports (mln US\$) & 21 & 31 & 679 \\
\hline Food imports (mln US\$) & 187 & 200 & 1018 \\
\hline \multicolumn{4}{|l|}{ Production indices $(2004-06=100)$} \\
\hline Net food & 66 & 76 & 140 \\
\hline Net crops & 78 & 72 & 120 \\
\hline Cereals & 55 & 73 & 138 \\
\hline Vegetable oils & 102 & 50 & 68 \\
\hline Roots and tubers & 16 & 47 & 97 \\
\hline Fruit and vegetables & 78 & 75 & 132 \\
\hline Sugar & 17 & 54 & 216 \\
\hline Livestock & 63 & 79 & 166 \\
\hline Milk & 63 & 82 & 145 \\
\hline Meat & 57 & 76 & 189 \\
\hline Fish & 526 & 239 & 14 \\
\hline \multicolumn{4}{|l|}{ Net trade (mln US\$) } \\
\hline Cereals & & -116 & -432 \\
\hline Fruit and vegetables & 13 & 14 & 206 \\
\hline Meat & -30 & -24 & -10 \\
\hline Dairy products & & -8 & -53 \\
\hline Fish & & 2 & -13 \\
\hline \multicolumn{4}{|l|}{ Environment } \\
\hline Forest area $(\%)$ & 11 & 11 & 11 \\
\hline Renewable water res withdrawn (\% of total) & & & 76 \\
\hline Terrestrial protect areas (\% total land area) & 6 & 7 & 7 \\
\hline Organic area (\% total agricultural area) & & & 0 \\
\hline Water withdrawal by agriculture (\% of total) & & & 76 \\
\hline \multicolumn{4}{|l|}{ Biofuel production (thousand kt of oil eq.) } \\
\hline Wood pellet prod. (1 000 tonnes) & & & 0 \\
\hline Net GHG emissions from AFOLU ( $\mathrm{CO}_{2}$ eq, $\mathrm{Mt}$ ) & 5 & 5 & 7 \\
\hline
\end{tabular}




\begin{tabular}{|c|c|c|c|}
\hline & 1990 & 2000 & 2014 \\
\hline \multicolumn{4}{|l|}{ The setting } \\
\hline Population, total (mln) & 0.5 & 0.7 & 1.3 \\
\hline Population, rural (mln) & 0.1 & 0.1 & 0.1 \\
\hline \multicolumn{4}{|l|}{ Govt expenditure on ag (\% total outlays) } \\
\hline Area harvested (mln ha) & 0 & 1 & 0 \\
\hline Cropping intensity ratio & 62.5 & 69 & \\
\hline Water resources (1 $000 \mathrm{~m}^{3} /$ person/year) & 0 & 0 & 0 \\
\hline Area equipped for irrigation (1 $000 \mathrm{ha})$ & & & 4 \\
\hline Area irrigated (\% area equipped for irrigation) & & 100 & \\
\hline Employment in agriculture (\%) & 2.4 & 1.5 & 1.1 \\
\hline Employment in agriculture, female (\%) & 0 & 0.2 & 0 \\
\hline Fertilizers, Nitrogen (kg of nutrients per ha) & & 0 & 403.1 \\
\hline Fertilizers, Phosphate (kg of nutrients per ha) & & 50.6 & 371.9 \\
\hline Fertilizers, Potash (kg nutrients per ha) & & 52.9 & 46.9 \\
\hline Energy consump, power irrigation (mln kWh) & 0 & 2 & 2 \\
\hline \multicolumn{4}{|l|}{ Agr value added per worker (constant US\$) } \\
\hline \multicolumn{4}{|l|}{ Hunger dimensions } \\
\hline \multicolumn{4}{|l|}{ Dietary energy supply (kcal/pc/day) } \\
\hline \multicolumn{4}{|l|}{ Average dietary energy supply adequacy (\%) } \\
\hline \multicolumn{4}{|l|}{ Dietary en supp, cereals/roots/tubers (\%) } \\
\hline \multicolumn{4}{|l|}{ Prevalence of undernourishment (\%) } \\
\hline GDP per capita (US\$, PPP) & 35398 & 45063 & 42444 \\
\hline Domestic food price volatility (index) & & 13.8 & 18.5 \\
\hline \multicolumn{4}{|l|}{ Cereal import dependency ratio (\%) } \\
\hline Underweight, children under-5 (\%) & & 7.6 & \\
\hline Improved water source (\% pop) & 94.9 & 98.9 & 100 \\
\hline \multicolumn{4}{|l|}{ Food supply } \\
\hline Food production value, (2004-2006 mln I\$) & 19 & 26 & 44 \\
\hline Agriculture, value added (\% GDP) & 1 & 1 & \\
\hline Food exports (mln US\$) & 1 & 28 & 147 \\
\hline Food imports (mln US\$) & 226 & 334 & 877 \\
\hline \multicolumn{4}{|l|}{ Production indices $(2004-06=100)$} \\
\hline Net food & 91 & 122 & 207 \\
\hline Net crops & 61 & 111 & 123 \\
\hline \multicolumn{4}{|l|}{ Cereals } \\
\hline \multicolumn{4}{|l|}{ Vegetable oils } \\
\hline Roots and tubers & 485 & 172 & 1000 \\
\hline Fruit and vegetables & 61 & 110 & 125 \\
\hline \multicolumn{4}{|l|}{ Sugar } \\
\hline Livestock & 137 & 141 & 338 \\
\hline Milk & 144 & 148 & 83 \\
\hline Meat & 148 & 157 & 1007 \\
\hline Fish & 58 & 84 & 107 \\
\hline \multicolumn{4}{|l|}{ Net trade (mln US\$) } \\
\hline Cereals & -32 & -49 & -133 \\
\hline Fruit and vegetables & -63 & -104 & -174 \\
\hline Meat & -43 & -44 & -178 \\
\hline Dairy products & -27 & -48 & -145 \\
\hline Fish & -1 & 5 & 6 \\
\hline \multicolumn{4}{|l|}{ Environment } \\
\hline Forest area (\%) & 0 & 1 & 1 \\
\hline Renewable water res withdrawn (\% of total) & & 44 & 44 \\
\hline Terrestrial protect areas (\% total land area) & 1 & 3 & 3 \\
\hline \multicolumn{4}{|l|}{ Organic area (\% total agricultural area) } \\
\hline Water withdrawal by agriculture (\% of total) & & 44 & 44 \\
\hline \multicolumn{4}{|l|}{ Biofuel production (thousand kt of oil eq.) } \\
\hline Wood pellet prod. (1 000 tonnes) & & & \\
\hline Net GHG emissions from AFOLU ( $\mathrm{CO}_{2}$ eq, $\left.\mathrm{Mt}\right)$ & 0 & 0 & 0 \\
\hline
\end{tabular}




\begin{tabular}{|c|c|c|c|}
\hline & 1990 & 2000 & 2014 \\
\hline \multicolumn{4}{|l|}{ The setting } \\
\hline Population, total (mln) & 107.4 & 132.4 & 158.5 \\
\hline Population, rural (mln) & 86.1 & 101.2 & 111.2 \\
\hline Govt expenditure on ag (\% total outlays) & & 2.9 & 15.2 \\
\hline Area harvested (mln ha) & 28 & 40 & 55 \\
\hline Cropping intensity ratio & 2.7 & 4.2 & \\
\hline Water resources (1 $000 \mathrm{~m}^{3} /$ person/year) & 11 & 9 & 8 \\
\hline Area equipped for irrigation (1 $000 \mathrm{ha})$ & & & 5300 \\
\hline Area irrigated (\% area equipped for irrigation) & 88.3 & & \\
\hline Employment in agriculture (\%) & 66.4 & 62.1 & 48.1 \\
\hline Employment in agriculture, female (\%) & 84.9 & 76.9 & 68.1 \\
\hline Fertilizers, Nitrogen (kg of nutrients per ha) & & 115.6 & 147.4 \\
\hline Fertilizers, Phosphate (kg of nutrients per ha) & & 24.3 & 75.3 \\
\hline Fertilizers, Potash (kg nutrients per ha) & & 20.3 & 56 \\
\hline Energy consump, power irrigation (mln kWh) & 0 & 0 & 0 \\
\hline Agr value added per worker (constant US\$) & 267 & 347 & 602 \\
\hline \multicolumn{4}{|l|}{ Hunger dimensions } \\
\hline Dietary energy supply (kcal/pc/day) & 2041 & 2285 & 2470 \\
\hline Average dietary energy supply adequacy (\%) & 95 & 104 & 108 \\
\hline Dietary en supp, cereals/roots/tubers (\%) & 84 & 84 & 80 \\
\hline Prevalence of undernourishment (\%) & 34.7 & 23.1 & 16.9 \\
\hline GDP per capita (US\$, PPP) & 1239 & 1606 & 2853 \\
\hline Domestic food price volatility (index) & & 3.3 & 4.5 \\
\hline Cereal import dependency ratio (\%) & 5.6 & 11.6 & 10.8 \\
\hline Underweight, children under-5 (\%) & 61.5 & 42.3 & 31.9 \\
\hline Improved water source (\% pop) & 68 & 76 & 84.8 \\
\hline \multicolumn{4}{|l|}{ Food supply } \\
\hline Food production value, (2004-2006 mln I\$) & 10276 & 14307 & 21639 \\
\hline Agriculture, value added (\% GDP) & 33 & 24 & 16 \\
\hline Food exports (mln US\$) & 15 & 13 & 71 \\
\hline Food imports (mln US\$) & 627 & 1320 & 4712 \\
\hline \multicolumn{4}{|l|}{ Production indices $(2004-06=100)$} \\
\hline Net food & 64 & 89 & 135 \\
\hline Net crops & 66 & 91 & 138 \\
\hline Cereals & 68 & 97 & 134 \\
\hline Vegetable oils & 122 & 97 & 133 \\
\hline Roots and tubers & 26 & 63 & 184 \\
\hline Fruit and vegetables & 49 & 56 & 157 \\
\hline Sugar & 119 & 111 & 73 \\
\hline Livestock & 57 & 82 & 131 \\
\hline Milk & 60 & 81 & 136 \\
\hline Meat & 62 & 86 & 120 \\
\hline Fish & 38 & 75 & 154 \\
\hline \multicolumn{4}{|l|}{ Net trade (mln US\$) } \\
\hline Cereals & -224 & -375 & -800 \\
\hline Fruit and vegetables & -88 & -141 & -619 \\
\hline Meat & 4 & 0 & -3 \\
\hline Dairy products & -90 & -85 & -249 \\
\hline Fish & 167 & 339 & 442 \\
\hline \multicolumn{4}{|l|}{ Environment } \\
\hline Forest area $(\%)$ & 11 & 11 & 11 \\
\hline Renewable water res withdrawn (\% of total) & & & 88 \\
\hline Terrestrial protect areas (\% total land area) & 1 & 5 & 5 \\
\hline Organic area (\% total agricultural area) & & & 0 \\
\hline Water withdrawal by agriculture (\% of total) & & & 88 \\
\hline Biofuel production (thousand kt of oil eq.) & 172 & 206 & 235 \\
\hline \multicolumn{4}{|l|}{ Wood pellet prod. (1 000 tonnes) } \\
\hline Net GHG emissions from AFOLU ( $\mathrm{CO}_{2}$ eq, $\mathrm{Mt}$ ) & 91 & 97 & 106 \\
\hline
\end{tabular}




\begin{tabular}{|c|c|c|c|}
\hline & 1990 & 2000 & 2014 \\
\hline \multicolumn{4}{|l|}{ The setting } \\
\hline Population, total (mln) & 0.3 & 0.3 & 0.3 \\
\hline Population, rural (mln) & 0.2 & 0.2 & 0.2 \\
\hline Govt expenditure on ag (\% total outlays) & & 2.4 & 1.7 \\
\hline Area harvested (mln ha) & 1 & 1 & 0 \\
\hline Cropping intensity ratio & 31.9 & 34.9 & \\
\hline Water resources (1 $000 \mathrm{~m}^{3} /$ person/year) & 0 & 0 & 0 \\
\hline Area equipped for irrigation (1 $000 \mathrm{ha}$ ) & & & 5 \\
\hline \multicolumn{4}{|l|}{ Area irrigated (\% area equipped for irrigation) } \\
\hline Employment in agriculture (\%) & 6.3 & 3.7 & 2.8 \\
\hline Employment in agriculture, female (\%) & 5.5 & 3.3 & 2.2 \\
\hline Fertilizers, Nitrogen (kg of nutrients per ha) & & 48.1 & 103.5 \\
\hline Fertilizers, Phosphate (kg of nutrients per ha) & & 29.8 & 60.9 \\
\hline Fertilizers, Potash (kg nutrients per ha) & & 1.9 & 9.2 \\
\hline \multicolumn{4}{|l|}{ Energy consump, power irrigation (mln kWh) } \\
\hline Agr value added per worker (constant US\$) & 8473 & 10368 & 13668 \\
\hline \multicolumn{4}{|l|}{ Hunger dimensions } \\
\hline Dietary energy supply (kcal/pc/day) & 2933 & 2887 & 3071 \\
\hline Average dietary energy supply adequacy (\%) & 120 & 117 & 123 \\
\hline Dietary en supp, cereals/roots/tubers (\%) & 34 & 33 & 32 \\
\hline Prevalence of undernourishment (\%) & $<5.0$ & $<5.0$ & $<5.0$ \\
\hline GDP per capita (US\$, PPP) & 13025 & 14714 & 15299 \\
\hline Domestic food price volatility (index) & & 8.2 & 5.4 \\
\hline Cereal import dependency ratio (\%) & 97.8 & 100 & 100 \\
\hline Underweight, children under-5 (\%) & & & 3.5 \\
\hline Improved water source (\% pop) & 95.4 & 99.1 & 99.8 \\
\hline \multicolumn{4}{|l|}{ Food supply } \\
\hline Food production value, $(2004-2006 \mathrm{mln}$ I\$) & 54 & 50 & 47 \\
\hline Agriculture, value added (\% GDP) & 4 & 2 & 2 \\
\hline Food exports (mln US\$) & 43 & 43 & 47 \\
\hline Food imports (mln US\$) & 88 & 95 & 242 \\
\hline \multicolumn{4}{|l|}{ Production indices $(2004-06=100)$} \\
\hline Net food & 110 & 102 & 97 \\
\hline Net crops & 131 & 129 & 84 \\
\hline Cereals & 886 & 116 & 131 \\
\hline Vegetable oils & 108 & 97 & 110 \\
\hline Roots and tubers & 254 & 142 & 38 \\
\hline Fruit and vegetables & 61 & 101 & 102 \\
\hline Sugar & 158 & 142 & 73 \\
\hline Livestock & 97 & 85 & 106 \\
\hline Milk & 231 & 129 & 101 \\
\hline Meat & 88 & 83 & 105 \\
\hline Fish & 143 & 151 & 142 \\
\hline \multicolumn{4}{|l|}{ Net trade (mln US\$) } \\
\hline Cereals & -18 & -21 & -42 \\
\hline Fruit and vegetables & -14 & -22 & -43 \\
\hline Meat & -18 & -13 & -26 \\
\hline Dairy products & -9 & -10 & -22 \\
\hline Fish & -6 & -10 & -25 \\
\hline \multicolumn{4}{|l|}{ Environment } \\
\hline Forest area (\%) & 19 & 19 & 19 \\
\hline Renewable water res withdrawn (\% of total) & & & 54 \\
\hline Terrestrial protect areas (\% total land area) & 0 & 0 & 0 \\
\hline \multicolumn{4}{|l|}{ Organic area (\% total agricultural area) } \\
\hline Water withdrawal by agriculture (\% of total) & & & 54 \\
\hline Biofuel production (thousand kt of oil eq.) & 2 & 1 & 1 \\
\hline \multicolumn{4}{|l|}{ Wood pellet prod. (1 000 tonnes) } \\
\hline Net GHG emissions from AFOLU ( $\mathrm{CO}_{2}$ eq, $\mathrm{Mt}$ ) & 0 & 0 & 0 \\
\hline
\end{tabular}




\begin{tabular}{|c|c|c|c|}
\hline & 1990 & 2000 & 2014 \\
\hline \multicolumn{4}{|l|}{ The setting } \\
\hline Population, total (mln) & 10.2 & 10 & 9.3 \\
\hline Population, rural (mln) & 3.4 & 3 & 2.2 \\
\hline Govt expenditure on ag (\% total outlays) & & 10.2 & 9.8 \\
\hline Area harvested (mln ha) & & 9 & 7 \\
\hline Cropping intensity ratio & & 0.9 & \\
\hline Water resources (1 $000 \mathrm{~m}^{3} /$ person/year) & 6 & 6 & 6 \\
\hline Area equipped for irrigation (1 $000 \mathrm{ha}$ ) & & & 114 \\
\hline Area irrigated (\% area equipped for irrigation) & & & 26.8 \\
\hline Employment in agriculture (\%) & 21.6 & 21.2 & 10.5 \\
\hline Employment in agriculture, female (\%) & & & 7.8 \\
\hline Fertilizers, Nitrogen (kg of nutrients per ha) & & 55.7 & 100.9 \\
\hline Fertilizers, Phosphate (kg of nutrients per ha) & & 15.6 & 40.1 \\
\hline Fertilizers, Potash (kg nutrients per ha) & & 64.3 & 130.4 \\
\hline Energy consump, power irrigation (mln kWh) & 253 & 253 & 253 \\
\hline Agr value added per worker (constant US\$) & 2795 & 3107 & 9835 \\
\hline \multicolumn{4}{|l|}{ Hunger dimensions } \\
\hline \multicolumn{4}{|l|}{ Dietary energy supply (kcal/pc/day) } \\
\hline Average dietary energy supply adequacy (\%) & 130 & 123 & 133 \\
\hline Dietary en supp, cereals/roots/tubers (\%) & 48 & 46 & 38 \\
\hline Prevalence of undernourishment (\%) & $<5.0$ & $<5.0$ & $<5.0$ \\
\hline GDP per capita (US\$, PPP) & 8084 & 7300 & 17055 \\
\hline Domestic food price volatility (index) & & 9.8 & 6 \\
\hline Cereal import dependency ratio (\%) & 23.2 & 26 & 1.4 \\
\hline Underweight, children under-5 (\%) & & & 1.3 \\
\hline Improved water source (\% pop) & 99.5 & 99.6 & 99.6 \\
\hline \multicolumn{4}{|l|}{ Food supply } \\
\hline Food production value, (2004-2006 mln I\$) & 5162 & 3641 & 5746 \\
\hline Agriculture, value added (\% GDP) & 24 & 14 & 9 \\
\hline Food exports (mln US\$) & 153 & 413 & 4120 \\
\hline Food imports (mln US\$) & 516 & 701 & 2070 \\
\hline \multicolumn{4}{|l|}{ Production indices $(2004-06=100)$} \\
\hline Net food & 113 & 80 & 126 \\
\hline Net crops & 102 & 76 & 96 \\
\hline Cereals & 110 & 68 & 130 \\
\hline Vegetable oils & 24 & 63 & 407 \\
\hline Roots and tubers & 124 & 90 & 64 \\
\hline Fruit and vegetables & 80 & 60 & 85 \\
\hline Sugar & 46 & 44 & 129 \\
\hline Livestock & 115 & 85 & 136 \\
\hline Milk & 100 & 81 & 119 \\
\hline Meat & 134 & 88 & 161 \\
\hline Fish & 294 & 109 & 213 \\
\hline \multicolumn{4}{|l|}{ Net trade (mln US\$) } \\
\hline Cereals & -257 & -272 & -136 \\
\hline Fruit and vegetables & -60 & -42 & -274 \\
\hline Meat & 46 & 2 & 957 \\
\hline Dairy products & 30 & 91 & 1749 \\
\hline Fish & -4 & -62 & -172 \\
\hline \multicolumn{4}{|l|}{ Environment } \\
\hline Forest area (\%) & 39 & 41 & 43 \\
\hline Renewable water res withdrawn (\% of total) & & 19 & 19 \\
\hline Terrestrial protect areas (\% total land area) & 7 & 7 & 8 \\
\hline \multicolumn{4}{|l|}{ Organic area (\% total agricultural area) } \\
\hline Water withdrawal by agriculture (\% of total) & & 19 & 19 \\
\hline Biofuel production (thousand kt of oil eq.) & & 6 & 1042 \\
\hline Wood pellet prod. (1 000 tonnes) & & & 1 \\
\hline Net GHG emissions from AFOLU ( $\mathrm{CO}_{2}$ eq, $\mathrm{Mt}$ ) & 14 & 8 & -8 \\
\hline
\end{tabular}




\begin{tabular}{|c|c|c|c|}
\hline & 1990 & 2000 & 2014 \\
\hline \multicolumn{4}{|l|}{ The setting } \\
\hline Population, total (mln) & & 10.3 & 11.1 \\
\hline Population, rural (mln) & & 0.3 & 0.3 \\
\hline \multicolumn{4}{|l|}{ Govt expenditure on ag (\% total outlays) } \\
\hline Area harvested (mln ha) & & 6 & 4 \\
\hline Cropping intensity ratio & & 4.4 & \\
\hline Water resources (1 $000 \mathrm{~m}^{3} /$ person/year) & 2 & 2 & 2 \\
\hline Area equipped for irrigation (1 $000 \mathrm{ha}$ ) & & & 23 \\
\hline Area irrigated (\% area equipped for irrigation) & & & 24.3 \\
\hline Employment in agriculture (\%) & 3.1 & 1.9 & 1.2 \\
\hline Employment in agriculture, female (\%) & 1.9 & 1.2 & 0.7 \\
\hline Fertilizers, Nitrogen (kg of nutrients per ha) & & 211.3 & 238 \\
\hline Fertilizers, Phosphate (kg of nutrients per ha) & & 58.2 & 28.4 \\
\hline Fertilizers, Potash (kg nutrients per ha) & & 44.3 & 28 \\
\hline \multicolumn{4}{|l|}{ Energy consump, power irrigation (mln kWh) } \\
\hline Agr value added per worker (constant US\$) & & 40464 & 68736 \\
\hline \multicolumn{4}{|l|}{ Hunger dimensions } \\
\hline \multicolumn{4}{|l|}{ Dietary energy supply (kcal/pc/day) } \\
\hline Average dietary energy supply adequacy (\%) & & 149 & 151 \\
\hline Dietary en supp, cereals/roots/tubers (\%) & & 26 & 30 \\
\hline Prevalence of undernourishment (\%) & $<5.0$ & $<5.0$ & $<5.0$ \\
\hline GDP per capita (US\$, PPP) & 30798 & 37404 & 40609 \\
\hline Domestic food price volatility (index) & & 6.6 & 6 \\
\hline Cereal import dependency ratio (\%) & & 56.1 & 57 \\
\hline \multicolumn{4}{|l|}{ Underweight, children under-5 (\%) } \\
\hline Improved water source (\% pop) & 100 & 100 & 100 \\
\hline \multicolumn{4}{|l|}{ Food supply } \\
\hline Food production value, (2004-2006 mln I\$) & & 5994 & 5683 \\
\hline Agriculture, value added (\% GDP) & & 1 & 1 \\
\hline Food exports (mln US\$) & & 13240 & 31389 \\
\hline Food imports (mln US\$) & & 10245 & 26957 \\
\hline \multicolumn{4}{|l|}{ Production indices $(2004-06=100)$} \\
\hline Net food & & 107 & 102 \\
\hline Net crops & & 102 & 95 \\
\hline Cereals & & 90 & 110 \\
\hline Vegetable oils & & 76 & 189 \\
\hline Roots and tubers & & 102 & 122 \\
\hline Fruit and vegetables & & 99 & 102 \\
\hline Sugar & & 103 & 74 \\
\hline Livestock & & 110 & 106 \\
\hline Milk & & 124 & 117 \\
\hline Meat & & 106 & 103 \\
\hline Fish & 167 & 126 & 103 \\
\hline \multicolumn{4}{|l|}{ Net trade (mln US\$) } \\
\hline Cereals & & 272 & -1 \\
\hline Fruit and vegetables & & 565 & 1859 \\
\hline Meat & & 1337 & 2586 \\
\hline Dairy products & & -131 & -46 \\
\hline Fish & -525 & -559 & -976 \\
\hline \multicolumn{4}{|l|}{ Environment } \\
\hline Forest area $(\%)$ & & 22 & 22 \\
\hline Renewable water res withdrawn (\% of total) & & & 1 \\
\hline Terrestrial protect areas (\% total land area) & 12 & 15 & 23 \\
\hline Organic area (\% total agricultural area) & & & 4 \\
\hline Water withdrawal by agriculture (\% of total) & & & 1 \\
\hline Biofuel production (thousand kt of oil eq.) & & 4 & 8506 \\
\hline Wood pellet prod. (1 000 tonnes) & & & 390 \\
\hline Net GHG emissions from AFOLU ( $\mathrm{CO}_{2}$ eq, $\left.\mathrm{Mt}\right)$ & & 9 & 8 \\
\hline
\end{tabular}




\begin{tabular}{|c|c|c|c|}
\hline & 1990 & 2000 & 2014 \\
\hline \multicolumn{4}{|l|}{ The setting } \\
\hline Population, total (mln) & 0.2 & 0.2 & 0.3 \\
\hline Population, rural (mln) & 0.1 & 0.1 & 0.2 \\
\hline \multicolumn{4}{|l|}{ Govt expenditure on ag (\% total outlays) } \\
\hline Area harvested (mln ha) & 1 & 1 & 1 \\
\hline Cropping intensity ratio & 8.6 & 7.4 & \\
\hline Water resources (1 $000 \mathrm{~m}^{3} /$ person/year) & 116 & 91 & 65 \\
\hline Area equipped for irrigation (1 $000 \mathrm{ha})$ & & & 4 \\
\hline Area irrigated (\% area equipped for irrigation) & & & 100 \\
\hline Employment in agriculture (\%) & 24.2 & 27.5 & 19.5 \\
\hline Employment in agriculture, female (\%) & 4.7 & 6.4 & 3.3 \\
\hline Fertilizers, Nitrogen (kg of nutrients per ha) & & 295.6 & 49.2 \\
\hline Fertilizers, Phosphate (kg of nutrients per ha) & & 66.3 & 55.2 \\
\hline Fertilizers, Potash (kg nutrients per ha) & & 20.5 & 16.5 \\
\hline \multicolumn{4}{|l|}{ Energy consump, power irrigation (mln kWh) } \\
\hline Agr value added per worker (constant US\$) & 2933 & 3780 & 4331 \\
\hline \multicolumn{4}{|l|}{ Hunger dimensions } \\
\hline Dietary energy supply (kcal/pc/day) & 2682 & 2696 & 2773 \\
\hline Average dietary energy supply adequacy (\%) & 126 & 125 & 123 \\
\hline Dietary en supp, cereals/roots/tubers (\%) & 38 & 40 & 40 \\
\hline Prevalence of undernourishment (\%) & 7.3 & 6.5 & 6.3 \\
\hline GDP per capita (US\$, PPP) & 5166 & 7215 & 8215 \\
\hline Domestic food price volatility (index) & & & 27.9 \\
\hline Cereal import dependency ratio (\%) & 35.5 & 29.7 & 11.1 \\
\hline Underweight, children under-5 (\%) & 5.4 & & 6.2 \\
\hline Improved water source (\% pop) & 72.9 & 85.1 & 99.3 \\
\hline \multicolumn{4}{|l|}{ Food supply } \\
\hline Food production value, (2004-2006 mln I\$) & 88 & 149 & 178 \\
\hline Agriculture, value added (\% GDP) & 20 & 17 & 15 \\
\hline Food exports (mln US\$) & 94 & 158 & 205 \\
\hline Food imports (mln US\$) & 38 & 60 & 87 \\
\hline \multicolumn{4}{|l|}{ Production indices $(2004-06=100)$} \\
\hline Net food & 51 & 85 & 102 \\
\hline Net crops & 51 & 91 & 103 \\
\hline Cereals & 53 & 89 & 208 \\
\hline Vegetable oils & 220 & 109 & 466 \\
\hline Roots and tubers & 84 & 197 & 102 \\
\hline Fruit and vegetables & 33 & 86 & 93 \\
\hline Sugar & 99 & 101 & 98 \\
\hline Livestock & 52 & 64 & 114 \\
\hline Milk & 36 & 40 & 144 \\
\hline Meat & 51 & 63 & 112 \\
\hline Fish & 2 & 26 & 31 \\
\hline \multicolumn{4}{|l|}{ Net trade (mln US\$) } \\
\hline Cereals & -7 & -13 & -18 \\
\hline Fruit and vegetables & 34 & 112 & 132 \\
\hline Meat & -4 & -6 & -7 \\
\hline Dairy products & -6 & -14 & -17 \\
\hline Fish & 5 & 29 & 28 \\
\hline \multicolumn{4}{|l|}{ Environment } \\
\hline Forest area (\%) & 70 & 65 & 60 \\
\hline Renewable water res withdrawn (\% of total) & & 68 & 68 \\
\hline Terrestrial protect areas (\% total land area) & 26 & 35 & 37 \\
\hline Organic area (\% total agricultural area) & & & 1 \\
\hline Water withdrawal by agriculture (\% of total) & & 68 & 68 \\
\hline Biofuel production (thousand kt of oil eq.) & 3 & 3 & 2 \\
\hline \multicolumn{4}{|l|}{ Wood pellet prod. (1 000 tonnes) } \\
\hline Net GHG emissions from AFOLU ( $\mathrm{CO}_{2}$ eq, $\mathrm{Mt}$ ) & 5 & 5 & 5 \\
\hline
\end{tabular}




\begin{tabular}{|c|c|c|c|}
\hline & 1990 & 2000 & 2014 \\
\hline \multicolumn{4}{|l|}{ The setting } \\
\hline Population, total (mln) & 5 & 6.9 & 10.6 \\
\hline Population, rural (mln) & 3.3 & 4.3 & 5.6 \\
\hline \multicolumn{4}{|l|}{ Govt expenditure on ag (\% total outlays) } \\
\hline Area harvested (mln ha) & 2 & 4 & 7 \\
\hline Cropping intensity ratio & 0.9 & 1.3 & \\
\hline Water resources (1 $000 \mathrm{~m}^{3} /$ person/year) & 5 & 4 & 3 \\
\hline Area equipped for irrigation (1 $000 \mathrm{ha})$ & & & 23 \\
\hline Area irrigated (\% area equipped for irrigation) & & & 74.7 \\
\hline Employment in agriculture (\%) & & 42.7 & \\
\hline Employment in agriculture, female (\%) & & 32.7 & \\
\hline Fertilizers, Nitrogen (kg of nutrients per ha) & & 0.5 & 10.7 \\
\hline Fertilizers, Phosphate (kg of nutrients per ha) & & 0.2 & 5.3 \\
\hline Fertilizers, Potash (kg nutrients per ha) & & 0.2 & 3.5 \\
\hline Energy consump, power irrigation (mln kWh) & 0 & 14 & 14 \\
\hline Agr value added per worker (constant US\$) & 640 & 865 & 1278 \\
\hline \multicolumn{4}{|l|}{ Hunger dimensions } \\
\hline Dietary energy supply (kcal/pc/day) & 2159 & 2262 & 2792 \\
\hline Average dietary energy supply adequacy (\%) & 102 & 106 & 127 \\
\hline Dietary en supp, cereals/roots/tubers (\%) & 74 & 73 & 72 \\
\hline Prevalence of undernourishment (\%) & 28.5 & 23.9 & 8.1 \\
\hline GDP per capita (US\$, PPP) & 1362 & 1525 & 1733 \\
\hline Domestic food price volatility (index) & & 14.6 & 21.8 \\
\hline Cereal import dependency ratio (\%) & 36.7 & 11.5 & 22.2 \\
\hline Underweight, children under-5 (\%) & & 21.5 & 20.2 \\
\hline Improved water source (\% pop) & 57.1 & 66.1 & 76.1 \\
\hline \multicolumn{4}{|l|}{ Food supply } \\
\hline Food production value, (2004-2006 mln I\$) & 695 & 1218 & 2185 \\
\hline Agriculture, value added (\% GDP) & 35 & 35 & 36 \\
\hline Food exports (mln US\$) & 17 & 40 & 373 \\
\hline Food imports (mln US\$) & 92 & 106 & 1612 \\
\hline \multicolumn{4}{|l|}{ Production indices $(2004-06=100)$} \\
\hline Net food & 48 & 85 & 152 \\
\hline Net crops & 46 & 89 & 149 \\
\hline Cereals & 50 & 92 & 165 \\
\hline Vegetable oils & 51 & 95 & 123 \\
\hline Roots and tubers & 40 & 80 & 145 \\
\hline Fruit and vegetables & 60 & 83 & 168 \\
\hline Sugar & 48 & 92 & 91 \\
\hline Livestock & 70 & 83 & 129 \\
\hline Milk & 60 & 86 & 120 \\
\hline Meat & 70 & 82 & 128 \\
\hline Fish & 101 & 85 & 114 \\
\hline \multicolumn{4}{|l|}{ Net trade (mln US\$) } \\
\hline Cereals & -57 & -23 & -625 \\
\hline Fruit and vegetables & -4 & 5 & 94 \\
\hline Meat & -8 & -35 & -371 \\
\hline Dairy products & -6 & -13 & -30 \\
\hline Fish & -2 & -7 & -35 \\
\hline \multicolumn{4}{|l|}{ Environment } \\
\hline Forest area (\%) & 51 & 45 & 40 \\
\hline Renewable water res withdrawn (\% of total) & & 45 & 45 \\
\hline Terrestrial protect areas (\% total land area) & 24 & 25 & 26 \\
\hline Organic area (\% total agricultural area) & & & 0 \\
\hline Water withdrawal by agriculture (\% of total) & & 45 & 45 \\
\hline Biofuel production (thousand kt of oil eq.) & 0 & 0 & 0 \\
\hline \multicolumn{4}{|l|}{ Wood pellet prod. (1 000 tonnes) } \\
\hline Net GHG emissions from AFOLU ( $\mathrm{CO}_{2}$ eq, $\mathrm{Mt}$ ) & 19 & 20 & 15 \\
\hline
\end{tabular}




\begin{tabular}{|c|c|c|c|}
\hline & 1990 & 2000 & 2014 \\
\hline \multicolumn{4}{|l|}{ The setting } \\
\hline Population, total (mln) & 0.5 & 0.6 & 0.8 \\
\hline Population, rural (mln) & 0.4 & 0.4 & 0.5 \\
\hline Govt expenditure on ag (\% total outlays) & & 11.3 & 11.6 \\
\hline Area harvested (mln ha) & 0 & 0 & 0 \\
\hline Cropping intensity ratio & 0.7 & 0.6 & \\
\hline Water resources (1 $000 \mathrm{~m}^{3} /$ person/year) & 146 & 138 & 103 \\
\hline Area equipped for irrigation (1 $000 \mathrm{ha})$ & & & 32 \\
\hline Area irrigated (\% area equipped for irrigation) & & & 100 \\
\hline Employment in agriculture (\%) & & 79.8 & 62.2 \\
\hline Employment in agriculture, female (\%) & & 88.7 & 75.1 \\
\hline Fertilizers, Nitrogen (kg of nutrients per ha) & & 7.1 & 9.2 \\
\hline Fertilizers, Phosphate (kg of nutrients per ha) & & 1.6 & 3.6 \\
\hline Fertilizers, Potash (kg nutrients per ha) & & 1.2 & 2.6 \\
\hline Energy consump, power irrigation (mln kWh) & 0 & 0 & 0 \\
\hline Agr value added per worker (constant US\$) & 817 & 947 & 629 \\
\hline \multicolumn{4}{|l|}{ Hunger dimensions } \\
\hline \multicolumn{4}{|l|}{ Dietary energy supply (kcal/pc/day) } \\
\hline \multicolumn{4}{|l|}{ Average dietary energy supply adequacy (\%) } \\
\hline \multicolumn{4}{|l|}{ Dietary en supp, cereals/roots/tubers (\%) } \\
\hline \multicolumn{4}{|l|}{ Prevalence of undernourishment (\%) } \\
\hline GDP per capita (US\$, PPP) & 2332 & 3596 & 7167 \\
\hline Domestic food price volatility (index) & & & 6.4 \\
\hline \multicolumn{4}{|l|}{ Cereal import dependency ratio (\%) } \\
\hline Underweight, children under-5 (\%) & & 14.1 & 12.8 \\
\hline Improved water source (\% pop) & & 86.1 & 98.1 \\
\hline \multicolumn{4}{|l|}{ Food supply } \\
\hline Food production value, $(2004-2006 \mathrm{mln}$ I\$) & 101 & 101 & 142 \\
\hline Agriculture, value added (\% GDP) & 35 & 27 & 17 \\
\hline Food exports (mln US\$) & 10 & 10 & 33 \\
\hline Food imports (mln US\$) & 14 & 20 & 124 \\
\hline \multicolumn{4}{|l|}{ Production indices $(2004-06=100)$} \\
\hline Net food & 69 & 69 & 97 \\
\hline Net crops & 62 & 61 & 97 \\
\hline Cereals & 73 & 62 & 99 \\
\hline Vegetable oils & 45 & 50 & 16 \\
\hline Roots and tubers & 65 & 55 & 83 \\
\hline Fruit and vegetables & 52 & 66 & 92 \\
\hline Sugar & 96 & 99 & 117 \\
\hline Livestock & 98 & 99 & 96 \\
\hline Milk & 85 & 96 & 89 \\
\hline Meat & 108 & 100 & 102 \\
\hline Fish & 259 & 157 & 104 \\
\hline \multicolumn{4}{|l|}{ Net trade (mln US\$) } \\
\hline Cereals & -8 & -9 & -39 \\
\hline Fruit and vegetables & 7 & 5 & 5 \\
\hline Meat & & 0 & -14 \\
\hline Dairy products & 0 & -2 & -18 \\
\hline Fish & & & -5 \\
\hline \multicolumn{4}{|l|}{ Environment } \\
\hline Forest area (\%) & 65 & 79 & 86 \\
\hline Renewable water res withdrawn (\% of total) & & & 94 \\
\hline Terrestrial protect areas (\% total land area) & 14 & 28 & 28 \\
\hline Organic area (\% total agricultural area) & & & 1 \\
\hline Water withdrawal by agriculture (\% of total) & & & 94 \\
\hline \multicolumn{4}{|l|}{ Biofuel production (thousand kt of oil eq.) } \\
\hline \multicolumn{4}{|l|}{ Wood pellet prod. (1 000 tonnes) } \\
\hline Net GHG emissions from AFOLU ( $\mathrm{CO}_{2}$ eq, $\mathrm{Mt}$ ) & -2 & -6 & -8 \\
\hline
\end{tabular}




\begin{tabular}{|c|c|c|c|}
\hline & 1990 & 2000 & 2014 \\
\hline \multicolumn{4}{|l|}{ The setting } \\
\hline Population, total (mln) & 6.8 & 8.5 & 10.8 \\
\hline Population, rural (mln) & 3 & 3.2 & 3.5 \\
\hline \multicolumn{4}{|l|}{ Govt expenditure on ag (\% total outlays) } \\
\hline Area harvested (mln ha) & 4 & 4 & 8 \\
\hline Cropping intensity ratio & 0.1 & 0.1 & \\
\hline Water resources (1 $000 \mathrm{~m}^{3} /$ person/year) & 84 & 68 & 54 \\
\hline Area equipped for irrigation (1 $000 \mathrm{ha})$ & & & 300 \\
\hline Area irrigated (\% area equipped for irrigation) & & & 100 \\
\hline Employment in agriculture (\%) & 1.2 & 36.8 & 32.1 \\
\hline Employment in agriculture, female (\%) & 0.2 & 35.3 & 32.9 \\
\hline Fertilizers, Nitrogen (kg of nutrients per ha) & & 1.8 & 6 \\
\hline Fertilizers, Phosphate (kg of nutrients per ha) & & 1.5 & 2.7 \\
\hline Fertilizers, Potash (kg nutrients per ha) & & 0.5 & 1.1 \\
\hline Energy consump, power irrigation (mln kWh) & 0 & & 46 \\
\hline Agr value added per worker (constant US\$) & 594 & 607 & 658 \\
\hline \multicolumn{4}{|l|}{ Hunger dimensions } \\
\hline Dietary energy supply (kcal/pc/day) & 1996 & 2066 & 2277 \\
\hline Average dietary energy supply adequacy (\%) & 94 & 96 & 103 \\
\hline Dietary en supp, cereals/roots/tubers (\%) & 52 & 51 & 52 \\
\hline Prevalence of undernourishment (\%) & 36.5 & 34.6 & 16.6 \\
\hline GDP per capita (US\$, PPP) & 3740 & 4330 & 5934 \\
\hline Domestic food price volatility (index) & & 14.6 & 12.2 \\
\hline Cereal import dependency ratio (\%) & 21.6 & 27.8 & 18.7 \\
\hline Underweight, children under-5 (\%) & 9.7 & 5.9 & 4.5 \\
\hline Improved water source (\% pop) & 68.6 & 78.9 & 88.1 \\
\hline \multicolumn{4}{|l|}{ Food supply } \\
\hline Food production value, (2004-2006 mln I\$) & 1536 & 2144 & 3547 \\
\hline Agriculture, value added (\% GDP) & 17 & 15 & 13 \\
\hline Food exports (mln US\$) & 143 & 254 & 1004 \\
\hline Food imports (mln US\$) & 74 & 236 & 494 \\
\hline \multicolumn{4}{|l|}{ Production indices $(2004-06=100)$} \\
\hline Net food & 59 & 82 & 136 \\
\hline Net crops & 54 & 85 & 133 \\
\hline Cereals & 50 & 77 & 136 \\
\hline Vegetable oils & 16 & 78 & 156 \\
\hline Roots and tubers & 94 & 96 & 127 \\
\hline Fruit and vegetables & 89 & 117 & 108 \\
\hline Sugar & 69 & 64 & 144 \\
\hline Livestock & 65 & 80 & 134 \\
\hline Milk & 48 & 82 & 177 \\
\hline Meat & 65 & 80 & 131 \\
\hline Fish & 110 & 97 & 122 \\
\hline \multicolumn{4}{|l|}{ Net trade (mln US\$) } \\
\hline Cereals & -33 & -87 & -116 \\
\hline Fruit and vegetables & 15 & 29 & 194 \\
\hline Meat & -1 & -3 & 2 \\
\hline Dairy products & -5 & -12 & 0 \\
\hline Fish & 0 & -9 & -16 \\
\hline \multicolumn{4}{|l|}{ Environment } \\
\hline Forest area (\%) & 58 & 55 & 52 \\
\hline Renewable water res withdrawn (\% of total) & & & 92 \\
\hline Terrestrial protect areas (\% total land area) & 9 & 19 & 21 \\
\hline Organic area (\% total agricultural area) & & & 0 \\
\hline Water withdrawal by agriculture (\% of total) & & & 92 \\
\hline Biofuel production (thousand kt of oil eq.) & 13 & 21 & 19 \\
\hline \multicolumn{4}{|l|}{ Wood pellet prod. (1 000 tonnes) } \\
\hline Net GHG emissions from AFOLU ( $\mathrm{CO}_{2}$ eq, $\mathrm{Mt}$ ) & 99 & 99 & 115 \\
\hline
\end{tabular}




\begin{tabular}{|c|c|c|c|}
\hline & 1990 & 2000 & 2014 \\
\hline \multicolumn{4}{|l|}{ The setting } \\
\hline Population, total (mln) & 3.9 & 3.8 & 3.8 \\
\hline Population, rural (mln) & 2.3 & 2.2 & 1.9 \\
\hline \multicolumn{4}{|l|}{ Govt expenditure on ag (\% total outlays) } \\
\hline Area harvested (mln ha) & & 1 & 1 \\
\hline Cropping intensity ratio & & 0.4 & \\
\hline Water resources (1 $000 \mathrm{~m}^{3} /$ person/year) & 10 & 10 & 10 \\
\hline Area equipped for irrigation (1 $000 \mathrm{ha})$ & & & 3 \\
\hline \multicolumn{4}{|l|}{ Area irrigated (\% area equipped for irrigation) } \\
\hline Employment in agriculture (\%) & & & 20.5 \\
\hline Employment in agriculture, female (\%) & & & 22.7 \\
\hline Fertilizers, Nitrogen (kg of nutrients per ha) & & 10.6 & 79.8 \\
\hline Fertilizers, Phosphate (kg of nutrients per ha) & & 3.5 & 9.7 \\
\hline Fertilizers, Potash (kg nutrients per ha) & & 3.6 & 9.7 \\
\hline \multicolumn{4}{|l|}{ Energy consump, power irrigation (mln kWh) } \\
\hline Agr value added per worker (constant US\$) & & & 13840 \\
\hline \multicolumn{4}{|l|}{ Hunger dimensions } \\
\hline \multicolumn{4}{|l|}{ Dietary energy supply (kcal/pc/day) } \\
\hline Average dietary energy supply adequacy (\%) & 100 & 116 & 125 \\
\hline Dietary en supp, cereals/roots/tubers (\%) & 66 & 60 & 50 \\
\hline Prevalence of undernourishment (\%) & $<5.0$ & $<5.0$ & $<5.0$ \\
\hline GDP per capita (US\$, PPP) & & 6158 & 9387 \\
\hline Domestic food price volatility (index) & & & 6.3 \\
\hline Cereal import dependency ratio (\%) & 8.7 & 29.3 & 34.8 \\
\hline Underweight, children under-5 (\%) & & 4.2 & 1.5 \\
\hline Improved water source (\% pop) & 97.1 & 97.6 & 99.6 \\
\hline \multicolumn{4}{|l|}{ Food supply } \\
\hline Food production value, (2004-2006 mln I\$) & 687 & 524 & 954 \\
\hline Agriculture, value added (\% GDP) & & 11 & 8 \\
\hline Food exports (mln US\$) & 14 & 35 & 318 \\
\hline Food imports (mln US\$) & 105 & 364 & 1275 \\
\hline \multicolumn{4}{|l|}{ Production indices $(2004-06=100)$} \\
\hline Net food & 84 & 64 & 117 \\
\hline Net crops & 71 & 60 & 111 \\
\hline Cereals & 70 & 67 & 89 \\
\hline Vegetable oils & 107 & 61 & 90 \\
\hline Roots and tubers & 74 & 64 & 85 \\
\hline Fruit and vegetables & 59 & 49 & 134 \\
\hline Sugar & 1142857 & 1714 & 103 \\
\hline Livestock & 89 & 74 & 119 \\
\hline Milk & 67 & 84 & 106 \\
\hline Meat & 179 & 35 & 163 \\
\hline Fish & 0 & 3 & 44 \\
\hline \multicolumn{4}{|l|}{ Net trade (mln US\$) } \\
\hline Cereals & -14 & -80 & -261 \\
\hline Fruit and vegetables & -18 & -24 & -104 \\
\hline Meat & -20 & -40 & -113 \\
\hline Dairy products & -7 & -34 & -57 \\
\hline Fish & -5 & -11 & -26 \\
\hline \multicolumn{4}{|l|}{ Environment } \\
\hline Forest area $(\%)$ & 43 & 43 & 43 \\
\hline Renewable water res withdrawn (\% of total) & & & 0 \\
\hline Terrestrial protect areas (\% total land area) & 1 & 1 & 1 \\
\hline Organic area (\% total agricultural area) & & & 0 \\
\hline Water withdrawal by agriculture (\% of total) & & & 0 \\
\hline \multicolumn{4}{|l|}{ Biofuel production (thousand kt of oil eq.) } \\
\hline Wood pellet prod. (1 000 tonnes) & & & 200 \\
\hline Net GHG emissions from AFOLU ( $\mathrm{CO}_{2}$ eq, $\mathrm{Mt}$ ) & -9 & -9 & 3 \\
\hline
\end{tabular}




\begin{tabular}{|c|c|c|c|}
\hline & 1990 & 2000 & 2014 \\
\hline \multicolumn{4}{|l|}{ The setting } \\
\hline Population, total (mln) & 1.4 & 1.8 & 2 \\
\hline Population, rural (mln) & 0.8 & 0.8 & 0.7 \\
\hline Govt expenditure on ag (\% total outlays) & & 3.9 & 2.7 \\
\hline Area harvested (mln ha) & 0 & 0 & 0 \\
\hline Cropping intensity ratio & 0 & 0 & \\
\hline Water resources (1 $000 \mathrm{~m}^{3} /$ person/year) & 9 & 7 & 6 \\
\hline Area equipped for irrigation (1 $000 \mathrm{ha}$ ) & & & 2 \\
\hline Area irrigated (\% area equipped for irrigation) & 100 & & \\
\hline Employment in agriculture (\%) & & 19.7 & 29.9 \\
\hline Employment in agriculture, female (\%) & & 17 & 24.3 \\
\hline Fertilizers, Nitrogen (kg of nutrients per ha) & & & 53 \\
\hline Fertilizers, Phosphate (kg of nutrients per ha) & & & 0.8 \\
\hline Fertilizers, Potash (kg nutrients per ha) & & & 0.5 \\
\hline Energy consump, power irrigation (mln kWh) & 3 & 3 & 3 \\
\hline Agr value added per worker (constant US\$) & 888 & 621 & 734 \\
\hline \multicolumn{4}{|l|}{ Hunger dimensions } \\
\hline Dietary energy supply (kcal/pc/day) & 2153 & 2117 & 2326 \\
\hline Average dietary energy supply adequacy (\%) & 97 & 92 & 99 \\
\hline Dietary en supp, cereals/roots/tubers (\%) & 51 & 51 & 49 \\
\hline Prevalence of undernourishment (\%) & 28.6 & 35.6 & 24.8 \\
\hline GDP per capita (US\$, PPP) & 8094 & 10274 & 15247 \\
\hline Domestic food price volatility (index) & & 5.5 & 3.6 \\
\hline Cereal import dependency ratio (\%) & 79.3 & 89.8 & 80.8 \\
\hline Underweight, children under-5 (\%) & & 10.7 & 11.2 \\
\hline Improved water source (\% pop) & 91.9 & 94.8 & 96.8 \\
\hline \multicolumn{4}{|l|}{ Food supply } \\
\hline Food production value, (2004-2006 mln I\$) & 218 & 201 & 293 \\
\hline Agriculture, value added (\% GDP) & 5 & 3 & 2 \\
\hline Food exports (mln US\$) & 71 & 99 & 106 \\
\hline Food imports (mln US\$) & 167 & 308 & 564 \\
\hline \multicolumn{4}{|l|}{ Production indices (2004-06=100) } \\
\hline Net food & 97 & 89 & 130 \\
\hline Net crops & 92 & 92 & 96 \\
\hline Cereals & 154 & 74 & 124 \\
\hline Vegetable oils & 23 & 88 & 170 \\
\hline Roots and tubers & 67 & 87 & 101 \\
\hline Fruit and vegetables & 94 & 89 & 131 \\
\hline \multicolumn{4}{|l|}{ Sugar } \\
\hline Livestock & 98 & 89 & 137 \\
\hline Milk & 108 & 116 & 113 \\
\hline Meat & 97 & 83 & 143 \\
\hline \multicolumn{4}{|l|}{ Fish } \\
\hline \multicolumn{4}{|l|}{ Net trade (mln US\$) } \\
\hline Cereals & -41 & -88 & -156 \\
\hline Fruit and vegetables & -31 & -67 & -115 \\
\hline Meat & 56 & 66 & 47 \\
\hline Dairy products & -26 & -36 & -60 \\
\hline Fish & -5 & -11 & -12 \\
\hline \multicolumn{4}{|l|}{ Environment } \\
\hline Forest area (\%) & 24 & 22 & 20 \\
\hline Renewable water res withdrawn (\% of total) & & 41 & 41 \\
\hline Terrestrial protect areas (\% total land area) & 30 & 37 & 37 \\
\hline \multicolumn{4}{|l|}{ Organic area (\% total agricultural area) } \\
\hline Water withdrawal by agriculture (\% of total) & & 41 & 41 \\
\hline \multicolumn{4}{|l|}{ Biofuel production (thousand kt of oil eq.) } \\
\hline \multicolumn{4}{|l|}{ Wood pellet prod. (1 000 tonnes) } \\
\hline Net GHG emissions from AFOLU ( $\mathrm{CO}_{2}$ eq, $\mathrm{Mt}$ ) & 18 & 21 & 18 \\
\hline
\end{tabular}




\begin{tabular}{|c|c|c|c|}
\hline & 1990 & 2000 & 2014 \\
\hline \multicolumn{4}{|l|}{ The setting } \\
\hline Population, total (mln) & 149.6 & 174.5 & 202 \\
\hline Population, rural (mln) & 39 & 32.8 & 29.4 \\
\hline \multicolumn{4}{|l|}{ Govt expenditure on ag (\% total outlays) } \\
\hline Area harvested (mln ha) & 263 & 328 & 739 \\
\hline Cropping intensity ratio & 1.1 & 1.3 & \\
\hline Water resources (1 $000 \mathrm{~m}^{3} /$ person/year) & 58 & 50 & 43 \\
\hline Area equipped for irrigation (1 $000 \mathrm{ha})$ & & & 5400 \\
\hline Area irrigated (\% area equipped for irrigation) & & & 96.8 \\
\hline Employment in agriculture (\%) & 22.8 & 20.7 & 15.3 \\
\hline Employment in agriculture, female (\%) & 13.3 & 16.1 & 11 \\
\hline Fertilizers, Nitrogen (kg of nutrients per ha) & & 37.2 & 58.6 \\
\hline Fertilizers, Phosphate (kg of nutrients per ha) & & 55.4 & 59.8 \\
\hline Fertilizers, Potash (kg nutrients per ha) & & 59.7 & 63.4 \\
\hline Energy consump, power irrigation (mln kWh) & 44 & 2565 & 6034 \\
\hline Agr value added per worker (constant US\$) & 1712 & 2464 & 5470 \\
\hline \multicolumn{4}{|l|}{ Hunger dimensions } \\
\hline Dietary energy supply (kcal/pc/day) & 2788 & 2879 & 3276 \\
\hline Average dietary energy supply adequacy (\%) & 119 & 122 & 134 \\
\hline Dietary en supp, cereals/roots/tubers (\%) & 38 & 35 & 34 \\
\hline Prevalence of undernourishment (\%) & 14.3 & 12.3 & $<5.0$ \\
\hline GDP per capita (US\$, PPP) & 9997 & 11015 & 14555 \\
\hline Domestic food price volatility (index) & & 6 & 4.4 \\
\hline Cereal import dependency ratio (\%) & 16.4 & 15.3 & -3 \\
\hline Underweight, children under-5 (\%) & & 3.7 & 2.2 \\
\hline Improved water source (\% pop) & 88.5 & 93.5 & 97.5 \\
\hline \multicolumn{4}{|l|}{ Food supply } \\
\hline Food production value, (2004-2006 mln I\$) & 52671 & 78722 & 140046 \\
\hline Agriculture, value added (\% GDP) & 8 & 6 & 6 \\
\hline Food exports (mln US\$) & 4690 & 8031 & 59994 \\
\hline Food imports (mln US\$) & 1797 & 3373 & 8276 \\
\hline \multicolumn{4}{|l|}{ Production indices $(2004-06=100)$} \\
\hline Net food & 51 & 77 & 137 \\
\hline Net crops & 59 & 77 & 140 \\
\hline Cereals & 54 & 78 & 157 \\
\hline Vegetable oils & 41 & 65 & 157 \\
\hline Roots and tubers & 92 & 90 & 90 \\
\hline Fruit and vegetables & 74 & 92 & 108 \\
\hline Sugar & 60 & 75 & 175 \\
\hline Livestock & 45 & 78 & 128 \\
\hline Milk & 59 & 81 & 135 \\
\hline Meat & 41 & 76 & 125 \\
\hline Fish & 62 & 82 & 121 \\
\hline \multicolumn{4}{|l|}{ Net trade (mln US\$) } \\
\hline Cereals & -749 & -1586 & 3439 \\
\hline Fruit and vegetables & 1328 & 985 & 1491 \\
\hline Meat & 316 & 1795 & 14937 \\
\hline Dairy products & -168 & -374 & -536 \\
\hline Fish & -55 & -85 & -1036 \\
\hline \multicolumn{4}{|l|}{ Environment } \\
\hline Forest area (\%) & 69 & 65 & 62 \\
\hline Renewable water res withdrawn (\% of total) & & & 60 \\
\hline Terrestrial protect areas (\% total land area) & 7 & 14 & 26 \\
\hline Organic area (\% total agricultural area) & & & 0 \\
\hline Water withdrawal by agriculture (\% of total) & & & 60 \\
\hline Biofuel production (thousand kt of oil eq.) & 1033 & 1148 & 2238 \\
\hline Wood pellet prod. (1 000 tonnes) & & & 62 \\
\hline Net GHG emissions from AFOLU ( $\mathrm{CO}_{2}$ eq, $\left.\mathrm{Mt}\right)$ & 1345 & 1387 & 1255 \\
\hline
\end{tabular}




\begin{tabular}{|c|c|c|c|}
\hline & 1990 & 2000 & 2014 \\
\hline \multicolumn{4}{|l|}{ The setting } \\
\hline Population, total (mln) & 0.3 & 0.3 & 0.4 \\
\hline Population, rural (mln) & 0.1 & 0.1 & 0.1 \\
\hline Govt expenditure on ag (\% total outlays) & & 0.6 & 0.6 \\
\hline Area harvested (mln ha) & 0 & 0 & 0 \\
\hline Cropping intensity ratio & 18.8 & 19.8 & \\
\hline Water resources (1 $000 \mathrm{~m}^{3} /$ person/year) & 33 & 26 & 20 \\
\hline Area equipped for irrigation (1 $000 \mathrm{ha}$ ) & & & 1 \\
\hline Area irrigated (\% area equipped for irrigation) & & 63 & \\
\hline Employment in agriculture (\%) & 2 & 1.4 & \\
\hline Employment in agriculture, female (\%) & 1.6 & 0.3 & \\
\hline Fertilizers, Nitrogen (kg of nutrients per ha) & & 39 & 73.2 \\
\hline Fertilizers, Phosphate (kg of nutrients per ha) & & 27.5 & 20 \\
\hline Fertilizers, Potash (kg nutrients per ha) & & 35.5 & 20.2 \\
\hline Energy consump, power irrigation (mln kWh) & 0 & 0 & 0 \\
\hline Agr value added per worker (constant US\$) & 21093 & 64178 & 83868 \\
\hline \multicolumn{4}{|l|}{ Hunger dimensions } \\
\hline Dietary energy supply (kcal/pc/day) & 2841 & 2828 & 3067 \\
\hline Average dietary energy supply adequacy (\%) & 124 & 122 & 129 \\
\hline Dietary en supp, cereals/roots/tubers (\%) & 49 & 51 & 46 \\
\hline Prevalence of undernourishment (\%) & $<5.0$ & $<5.0$ & $<5.0$ \\
\hline GDP per capita (US\$, PPP) & 77076 & 74475 & 69474 \\
\hline Domestic food price volatility (index) & & 5.6 & 4.7 \\
\hline Cereal import dependency ratio (\%) & 98.7 & 100 & 98.3 \\
\hline \multicolumn{4}{|l|}{ Underweight, children under-5 (\%) } \\
\hline \multicolumn{4}{|l|}{ Improved water source (\% pop) } \\
\hline \multicolumn{4}{|l|}{ Food supply } \\
\hline Food production value, (2004-2006 mln I\$) & 8 & 25 & 50 \\
\hline Agriculture, value added (\% GDP) & 1 & 1 & 1 \\
\hline Food exports (mln US\$) & 7 & 0 & 2 \\
\hline Food imports (mln US\$) & 113 & 136 & 327 \\
\hline \multicolumn{4}{|l|}{ Production indices $(2004-06=100)$} \\
\hline Net food & 27 & 85 & 167 \\
\hline Net crops & 58 & 92 & 104 \\
\hline Cereals & 121 & 37 & 238 \\
\hline Vegetable oils & 40 & 57 & 87 \\
\hline Roots and tubers & 54 & 80 & 135 \\
\hline Fruit and vegetables & 54 & 95 & 97 \\
\hline \multicolumn{4}{|l|}{ Sugar } \\
\hline Livestock & 20 & 83 & 183 \\
\hline Milk & 41 & 123 & 69 \\
\hline Meat & 13 & 84 & 201 \\
\hline Fish & 83 & 91 & 169 \\
\hline \multicolumn{4}{|l|}{ Net trade (mln US\$) } \\
\hline Cereals & -29 & -40 & -127 \\
\hline Fruit and vegetables & -27 & -23 & -47 \\
\hline Meat & -12 & -8 & -33 \\
\hline Dairy products & -9 & -14 & -19 \\
\hline Fish & -7 & -15 & -40 \\
\hline \multicolumn{4}{|l|}{ Environment } \\
\hline Forest area (\%) & 78 & 75 & 71 \\
\hline \multicolumn{4}{|l|}{ Renewable water res withdrawn (\% of total) } \\
\hline Terrestrial protect areas (\% total land area) & 37 & 44 & 44 \\
\hline \multicolumn{4}{|l|}{ Organic area (\% total agricultural area) } \\
\hline \multicolumn{4}{|l|}{ Water withdrawal by agriculture (\% of total) } \\
\hline \multicolumn{4}{|l|}{ Biofuel production (thousand kt of oil eq.) } \\
\hline \multicolumn{4}{|l|}{ Wood pellet prod. (1 000 tonnes) } \\
\hline Net GHG emissions from AFOLU ( $\mathrm{CO}_{2}$ eq, $\left.\mathrm{Mt}\right)$ & 2 & 2 & 2 \\
\hline
\end{tabular}




\section{Bulgaria}

\begin{tabular}{|c|c|c|c|}
\hline & 1990 & 2000 & 2014 \\
\hline \multicolumn{4}{|l|}{ The setting } \\
\hline Population, total (mln) & 8.8 & 8 & 7.2 \\
\hline Population, rural (mln) & 3 & 2.5 & 1.8 \\
\hline Govt expenditure on ag (\% total outlays) & & 3.6 & 0.6 \\
\hline Area harvested (mln ha) & 8 & 4 & 8 \\
\hline Cropping intensity ratio & 1.3 & 0.8 & \\
\hline Water resources (1 $000 \mathrm{~m}^{3} /$ person/year) & 2 & 3 & 3 \\
\hline Area equipped for irrigation (1 $000 \mathrm{ha}$ ) & & & 102 \\
\hline Area irrigated (\% area equipped for irrigation) & & & 69.5 \\
\hline Employment in agriculture (\%) & 18.5 & 13.1 & 6.4 \\
\hline Employment in agriculture, female (\%) & & 10.6 & 4.3 \\
\hline Fertilizers, Nitrogen (kg of nutrients per ha) & & 95 & 95.8 \\
\hline Fertilizers, Phosphate (kg of nutrients per ha) & & 51.4 & 20.9 \\
\hline Fertilizers, Potash (kg nutrients per ha) & & 0.9 & 5.1 \\
\hline Energy consump, power irrigation (mln kWh) & 0 & & 46 \\
\hline Agr value added per worker (constant US\$) & 5694 & 9643 & 18789 \\
\hline \multicolumn{4}{|l|}{ Hunger dimensions } \\
\hline \multicolumn{4}{|l|}{ Dietary energy supply (kcal/pc/day) } \\
\hline Average dietary energy supply adequacy (\%) & 119 & 113 & 116 \\
\hline Dietary en supp, cereals/roots/tubers (\%) & 42 & 42 & 43 \\
\hline Prevalence of undernourishment (\%) & $<5.0$ & $<5.0$ & $<5.0$ \\
\hline GDP per capita (US\$, PPP) & 9333 & 9075 & 15695 \\
\hline Domestic food price volatility (index) & & 12.6 & 5.9 \\
\hline Cereal import dependency ratio (\%) & -3.2 & -17.6 & -92.2 \\
\hline \multicolumn{4}{|l|}{ Underweight, children under-5 (\%) } \\
\hline Improved water source (\% pop) & 99.9 & 99.7 & 99.5 \\
\hline \multicolumn{4}{|l|}{ Food supply } \\
\hline Food production value, (2004-2006 mln I\$) & 4587 & 2997 & 3143 \\
\hline Agriculture, value added (\% GDP) & 17 & 12 & 5 \\
\hline Food exports (mln US\$) & 738 & 284 & 3224 \\
\hline Food imports (mln US\$) & 334 & 277 & 2108 \\
\hline \multicolumn{4}{|l|}{ Production indices $(2004-06=100)$} \\
\hline Net food & 176 & 115 & 120 \\
\hline Net crops & 150 & 87 & 129 \\
\hline Cereals & 131 & 68 & 136 \\
\hline Vegetable oils & 38 & 41 & 205 \\
\hline Roots and tubers & 90 & 79 & 35 \\
\hline Fruit and vegetables & 299 & 169 & 99 \\
\hline Sugar & 2248 & 89 & 63 \\
\hline Livestock & 238 & 153 & 91 \\
\hline Milk & 161 & 111 & 85 \\
\hline Meat & 357 & 226 & 100 \\
\hline Fish & 569 & 123 & 217 \\
\hline \multicolumn{4}{|l|}{ Net trade (mln US\$) } \\
\hline Cereals & 39 & 16 & 1095 \\
\hline Fruit and vegetables & 334 & 14 & -87 \\
\hline Meat & 124 & 2 & -296 \\
\hline Dairy products & 24 & 10 & -79 \\
\hline Fish & 13 & -4 & -43 \\
\hline \multicolumn{4}{|l|}{ Environment } \\
\hline Forest area (\%) & 30 & 31 & 37 \\
\hline Renewable water res withdrawn (\% of total) & & & 16 \\
\hline Terrestrial protect areas (\% total land area) & 2 & 4 & 37 \\
\hline Organic area (\% total agricultural area) & & & 1 \\
\hline Water withdrawal by agriculture (\% of total) & & & 16 \\
\hline Biofuel production (thousand kt of oil eq.) & & 1 & 516 \\
\hline Wood pellet prod. (1 000 tonnes) & & & 120 \\
\hline Net GHG emissions from AFOLU ( $\mathrm{CO}_{2}$ eq, $\left.\mathrm{Mt}\right)$ & 2 & -6 & -9 \\
\hline
\end{tabular}




\section{Burkina Faso}

\begin{tabular}{|c|c|c|c|}
\hline & 1990 & 2000 & 2014 \\
\hline \multicolumn{4}{|l|}{ The setting } \\
\hline Population, total (mln) & 8.8 & 11.6 & 17.4 \\
\hline Population, rural (mln) & 7.6 & 9.5 & 12.4 \\
\hline \multicolumn{4}{|l|}{ Govt expenditure on ag (\% total outlays) } \\
\hline Area harvested (mln ha) & 3 & 3 & 5 \\
\hline Cropping intensity ratio & 0.3 & 0.3 & \\
\hline Water resources (1 $000 \mathrm{~m}^{3} /$ person/year) & 2 & 1 & 1 \\
\hline Area equipped for irrigation (1 $000 \mathrm{ha})$ & & & 55 \\
\hline Area irrigated (\% area equipped for irrigation) & & & 85 \\
\hline Employment in agriculture (\%) & & 85.1 & 84.8 \\
\hline Employment in agriculture, female (\%) & & 87.4 & 87.2 \\
\hline Fertilizers, Nitrogen (kg of nutrients per ha) & & 5.2 & 4.7 \\
\hline Fertilizers, Phosphate (kg of nutrients per ha) & & 2.6 & 3.1 \\
\hline Fertilizers, Potash (kg nutrients per ha) & & 2.5 & 3.2 \\
\hline Energy consump, power irrigation (mln kWh) & 9 & 9 & 10 \\
\hline Agr value added per worker (constant US\$) & 243 & 189 & 190 \\
\hline \multicolumn{4}{|l|}{ Hunger dimensions } \\
\hline Dietary energy supply (kcal/pc/day) & 2317 & 2331 & 2707 \\
\hline Average dietary energy supply adequacy (\%) & 107 & 108 & 123 \\
\hline Dietary en supp, cereals/roots/tubers (\%) & 65 & 66 & 65 \\
\hline Prevalence of undernourishment (\%) & 23.2 & 26.6 & 20.7 \\
\hline GDP per capita (US\$, PPP) & 848 & 1080 & 1630 \\
\hline Domestic food price volatility (index) & & 19.3 & 11.8 \\
\hline Cereal import dependency ratio (\%) & 7.5 & 10.1 & 9.8 \\
\hline Underweight, children under-5 (\%) & 29.6 & 35.2 & 26.2 \\
\hline Improved water source (\% pop) & 43.6 & 59.9 & 81.7 \\
\hline \multicolumn{4}{|l|}{ Food supply } \\
\hline Food production value, (2004-2006 mln I\$) & 922 & 1253 & 2246 \\
\hline Agriculture, value added (\% GDP) & 29 & 19 & 22 \\
\hline Food exports (mln US\$) & 20 & 35 & 176 \\
\hline Food imports (mln US\$) & 75 & 128 & 367 \\
\hline \multicolumn{4}{|l|}{ Production indices $(2004-06=100)$} \\
\hline Net food & 51 & 69 & 124 \\
\hline Net crops & 45 & 60 & 142 \\
\hline Cereals & 44 & 68 & 143 \\
\hline Vegetable oils & 51 & 54 & 199 \\
\hline Roots and tubers & 73 & 102 & 223 \\
\hline Fruit and vegetables & 64 & 97 & 80 \\
\hline Sugar & 84 & 89 & 107 \\
\hline Livestock & 55 & 76 & 85 \\
\hline Milk & 58 & 77 & 123 \\
\hline Meat & 53 & 75 & 75 \\
\hline Fish & 76 & 92 & 224 \\
\hline \multicolumn{4}{|l|}{ Net trade (mln US\$) } \\
\hline Cereals & -43 & -68 & -207 \\
\hline Fruit and vegetables & -8 & -12 & 34 \\
\hline Meat & -1 & 0 & -1 \\
\hline Dairy products & -17 & -13 & -19 \\
\hline Fish & -6 & -1 & -10 \\
\hline \multicolumn{4}{|l|}{ Environment } \\
\hline Forest area (\%) & 25 & 23 & 20 \\
\hline Renewable water res withdrawn (\% of total) & & & 51 \\
\hline Terrestrial protect areas (\% total land area) & 14 & 14 & 15 \\
\hline Organic area (\% total agricultural area) & & & 0 \\
\hline Water withdrawal by agriculture (\% of total) & & & 51 \\
\hline Biofuel production (thousand kt of oil eq.) & 1 & 1 & 1 \\
\hline \multicolumn{4}{|l|}{ Wood pellet prod. (1 000 tonnes) } \\
\hline Net GHG emissions from AFOLU ( $\mathrm{CO}_{2}$ eq, $\left.\mathrm{Mt}\right)$ & 24 & 26 & 31 \\
\hline
\end{tabular}




\section{Burundi}

\begin{tabular}{|c|c|c|c|}
\hline & 1990 & 2000 & 2014 \\
\hline \multicolumn{4}{|l|}{ The setting } \\
\hline Population, total (mln) & 5.6 & 6.7 & 10.5 \\
\hline Population, rural (mln) & 5.3 & 6.1 & 9.2 \\
\hline \multicolumn{4}{|l|}{ Govt expenditure on ag (\% total outlays) } \\
\hline Area harvested (mln ha) & 2 & 2 & 3 \\
\hline Cropping intensity ratio & 0.8 & 0.9 & \\
\hline Water resources (1 $000 \mathrm{~m}^{3} /$ person/year) & 2 & 2 & 1 \\
\hline Area equipped for irrigation (1 $000 \mathrm{ha})$ & & & 23 \\
\hline \multicolumn{4}{|l|}{ Area irrigated (\% area equipped for irrigation) } \\
\hline Employment in agriculture (\%) & & 92.2 & \\
\hline Employment in agriculture, female (\%) & & 96.6 & \\
\hline Fertilizers, Nitrogen (kg of nutrients per ha) & & 0.3 & 2.7 \\
\hline Fertilizers, Phosphate (kg of nutrients per ha) & & 0 & 2.4 \\
\hline Fertilizers, Potash (kg nutrients per ha) & & 0 & 0.6 \\
\hline \multicolumn{4}{|l|}{ Energy consump, power irrigation (mln kWh) } \\
\hline Agr value added per worker (constant US\$) & 233 & 183 & 132 \\
\hline \multicolumn{4}{|l|}{ Hunger dimensions } \\
\hline \multicolumn{4}{|l|}{ Dietary energy supply (kcal/pc/day) } \\
\hline \multicolumn{4}{|l|}{ Average dietary energy supply adequacy (\%) } \\
\hline \multicolumn{4}{|l|}{ Dietary en supp, cereals/roots/tubers (\%) } \\
\hline \multicolumn{4}{|l|}{ Prevalence of undernourishment (\%) } \\
\hline GDP per capita (US\$, PPP) & 1050 & 723 & 747 \\
\hline Domestic food price volatility (index) & & 51.9 & 8.3 \\
\hline Cereal import dependency ratio (\%) & 18.9 & 14.3 & 30.3 \\
\hline Underweight, children under-5 (\%) & & 38.9 & 29.1 \\
\hline Improved water source (\% pop) & 68.8 & 71.8 & 75.3 \\
\hline \multicolumn{4}{|l|}{ Food supply } \\
\hline Food production value, $(2004-2006 \mathrm{mln}$ I\$) & 967 & 858 & 1368 \\
\hline Agriculture, value added (\% GDP) & 56 & 48 & 39 \\
\hline Food exports (mln US\$) & 0 & 2 & 0 \\
\hline Food imports (mln US\$) & 21 & 23 & 173 \\
\hline \multicolumn{4}{|l|}{ Production indices $(2004-06=100)$} \\
\hline Net food & 102 & 91 & 145 \\
\hline Net crops & 89 & 86 & 129 \\
\hline Cereals & 90 & 84 & 80 \\
\hline Vegetable oils & 73 & 52 & 234 \\
\hline Roots and tubers & 96 & 95 & 230 \\
\hline Fruit and vegetables & 93 & 92 & 139 \\
\hline Sugar & 71 & 97 & 183 \\
\hline Livestock & 111 & 90 & 125 \\
\hline Milk & 168 & 106 & 229 \\
\hline Meat & 102 & 88 & 106 \\
\hline Fish & 129 & 129 & 100 \\
\hline \multicolumn{4}{|l|}{ Net trade (mln US\$) } \\
\hline Cereals & -11 & -14 & -84 \\
\hline Fruit and vegetables & 0 & -2 & -5 \\
\hline Meat & & 0 & 0 \\
\hline Dairy products & & -1 & -3 \\
\hline Fish & 0 & 0 & -2 \\
\hline \multicolumn{4}{|l|}{ Environment } \\
\hline Forest area (\%) & 11 & 8 & 7 \\
\hline Renewable water res withdrawn (\% of total) & & 77 & 77 \\
\hline Terrestrial protect areas (\% total land area) & 4 & 5 & 5 \\
\hline Organic area (\% total agricultural area) & & & 0 \\
\hline Water withdrawal by agriculture (\% of total) & & 77 & 77 \\
\hline Biofuel production (thousand kt of oil eq.) & 0 & 1 & 0 \\
\hline \multicolumn{4}{|l|}{ Wood pellet prod. (1 000 tonnes) } \\
\hline Net GHG emissions from AFOLU ( $\mathrm{CO}_{2}$ eq, $\left.\mathrm{Mt}\right)$ & 10 & 7 & 6 \\
\hline
\end{tabular}




\begin{tabular}{|c|c|c|c|}
\hline & 1990 & 2000 & 2014 \\
\hline \multicolumn{4}{|l|}{ The setting } \\
\hline Population, total (mln) & 0.4 & 0.4 & 0.5 \\
\hline Population, rural (mln) & 0.2 & 0.2 & 0.2 \\
\hline Govt expenditure on ag (\% total outlays) & & & 3.3 \\
\hline Area harvested (mln ha) & 0 & 0 & 0 \\
\hline Cropping intensity ratio & 7 & 4.2 & \\
\hline Water resources (1 $000 \mathrm{~m}^{3} /$ person/year) & 1 & 1 & 1 \\
\hline Area equipped for irrigation (1 $000 \mathrm{ha}$ ) & & & 4 \\
\hline Area irrigated (\% area equipped for irrigation) & & 65.5 & \\
\hline \multicolumn{4}{|l|}{ Employment in agriculture (\%) } \\
\hline \multicolumn{4}{|l|}{ Employment in agriculture, female (\%) } \\
\hline \multicolumn{4}{|l|}{ Fertilizers, Nitrogen (kg of nutrients per ha) } \\
\hline \multicolumn{4}{|l|}{ Fertilizers, Phosphate (kg of nutrients per ha) } \\
\hline \multicolumn{4}{|l|}{ Fertilizers, Potash (kg nutrients per ha) } \\
\hline Energy consump, power irrigation (mln kWh) & 0 & 0 & 0 \\
\hline Agr value added per worker (constant US\$) & 1025 & 2708 & 4410 \\
\hline \multicolumn{4}{|l|}{ Hunger dimensions } \\
\hline Dietary energy supply (kcal/pc/day) & 2463 & 2344 & 2821 \\
\hline Average dietary energy supply adequacy (\%) & 114 & 105 & 118 \\
\hline Dietary en supp, cereals/roots/tubers (\%) & 53 & 50 & 46 \\
\hline Prevalence of undernourishment (\%) & 13.4 & 18.7 & 10 \\
\hline GDP per capita (US\$, PPP) & 1610 & 3772 & 6210 \\
\hline Domestic food price volatility (index) & & & 5.4 \\
\hline Cereal import dependency ratio (\%) & 90.3 & 72.9 & 94 \\
\hline Underweight, children under-5 (\%) & & 11.8 & \\
\hline Improved water source (\% pop) & 80.4 & 82.7 & 89.3 \\
\hline \multicolumn{4}{|l|}{ Food supply } \\
\hline Food production value, (2004-2006 mln I\$) & 24 & 41 & 39 \\
\hline Agriculture, value added (\% GDP) & 14 & 13 & 8 \\
\hline Food exports (mln US\$) & 2 & 0 & 0 \\
\hline Food imports (mln US\$) & 35 & 56 & 184 \\
\hline \multicolumn{4}{|l|}{ Production indices $(2004-06=100)$} \\
\hline Net food & 55 & 92 & 89 \\
\hline Net crops & 64 & 105 & 100 \\
\hline Cereals & 193 & 411 & 98 \\
\hline Vegetable oils & 156 & 97 & 129 \\
\hline Roots and tubers & 111 & 91 & 147 \\
\hline Fruit and vegetables & 43 & 93 & 94 \\
\hline Sugar & 68 & 94 & 104 \\
\hline Livestock & 51 & 84 & 77 \\
\hline Milk & 41 & 66 & 76 \\
\hline Meat & 56 & 87 & 72 \\
\hline \multicolumn{4}{|l|}{ Fish } \\
\hline \multicolumn{4}{|l|}{ Net trade (mln US\$) } \\
\hline Cereals & -11 & -15 & -50 \\
\hline Fruit and vegetables & -3 & -9 & -29 \\
\hline Meat & -1 & -4 & -26 \\
\hline Dairy products & -5 & -8 & -32 \\
\hline Fish & 2 & 1 & 40 \\
\hline \multicolumn{4}{|l|}{ Environment } \\
\hline Forest area (\%) & 14 & 20 & 21 \\
\hline Renewable water res withdrawn (\% of total) & & 91 & 91 \\
\hline Terrestrial protect areas (\% total land area) & 2 & 2 & 2 \\
\hline \multicolumn{4}{|l|}{ Organic area (\% total agricultural area) } \\
\hline Water withdrawal by agriculture (\% of total) & & 91 & 91 \\
\hline \multicolumn{4}{|l|}{ Biofuel production (thousand kt of oil eq.) } \\
\hline \multicolumn{4}{|l|}{ Wood pellet prod. (1 000 tonnes) } \\
\hline Net GHG emissions from AFOLU ( $\mathrm{CO}_{2}$ eq, $\mathrm{Mt}$ ) & 0 & 0 & 0 \\
\hline
\end{tabular}




\begin{tabular}{|c|c|c|c|}
\hline & 1990 & 2000 & 2014 \\
\hline \multicolumn{4}{|l|}{ The setting } \\
\hline Population, total (mln) & 9.1 & 12.2 & 15.4 \\
\hline Population, rural (mln) & 7.6 & 10 & 12.2 \\
\hline Govt expenditure on ag (\% total outlays) & & 1.3 & 0.8 \\
\hline Area harvested (mln ha) & 3 & 4 & 10 \\
\hline Cropping intensity ratio & 0.6 & 0.9 & \\
\hline Water resources (1 $000 \mathrm{~m}^{3} /$ person/year) & 53 & 39 & 31 \\
\hline Area equipped for irrigation (1 $000 \mathrm{ha})$ & & & 354 \\
\hline Area irrigated (\% area equipped for irrigation) & & & 89.7 \\
\hline Employment in agriculture (\%) & & 73.7 & 51 \\
\hline Employment in agriculture, female (\%) & & 74.9 & 52.8 \\
\hline Fertilizers, Nitrogen (kg of nutrients per ha) & & 1.4 & 10.7 \\
\hline Fertilizers, Phosphate (kg of nutrients per ha) & & 2.2 & 5 \\
\hline Fertilizers, Potash (kg nutrients per ha) & & 0.2 & 0.9 \\
\hline Energy consump, power irrigation (mln kWh) & 0 & 0 & 0 \\
\hline Agr value added per worker (constant US\$) & 349 & 375 & 514 \\
\hline \multicolumn{4}{|l|}{ Hunger dimensions } \\
\hline Dietary energy supply (kcal/pc/day) & 2034 & 2114 & 2488 \\
\hline Average dietary energy supply adequacy (\%) & 101 & 99 & 112 \\
\hline Dietary en supp, cereals/roots/tubers (\%) & 83 & 78 & 72 \\
\hline Prevalence of undernourishment (\%) & 29 & 32 & 15 \\
\hline GDP per capita (US\$, PPP) & 1004 & 1368 & 2944 \\
\hline Domestic food price volatility (index) & & 17.5 & 4.7 \\
\hline Cereal import dependency ratio (\%) & 5.2 & 3.3 & -1.4 \\
\hline Underweight, children under-5 (\%) & & 39.5 & 29 \\
\hline Improved water source (\% pop) & 21.5 & 41.5 & 71.3 \\
\hline \multicolumn{4}{|l|}{ Food supply } \\
\hline Food production value, (2004-2006 mln I\$) & 1124 & 1767 & 4292 \\
\hline Agriculture, value added (\% GDP) & 47 & 38 & 30 \\
\hline Food exports (mln US\$) & 7 & 5 & 177 \\
\hline Food imports (mln US\$) & 12 & 73 & 399 \\
\hline \multicolumn{4}{|l|}{ Production indices $(2004-06=100)$} \\
\hline Net food & 47 & 74 & 181 \\
\hline Net crops & 47 & 72 & 200 \\
\hline Cereals & 45 & 73 & 179 \\
\hline Vegetable oils & 15 & 22 & 66 \\
\hline Roots and tubers & 10 & 19 & 734 \\
\hline Fruit and vegetables & 82 & 98 & 123 \\
\hline Sugar & 209 & 129 & 476 \\
\hline Livestock & 54 & 91 & 90 \\
\hline Milk & 80 & 94 & 108 \\
\hline Meat & 53 & 91 & 88 \\
\hline Fish & 27 & 72 & 175 \\
\hline \multicolumn{4}{|l|}{ Net trade (mln US\$) } \\
\hline Cereals & -7 & -30 & 55 \\
\hline Fruit and vegetables & 0 & -5 & -2 \\
\hline Meat & 0 & 0 & -2 \\
\hline Dairy products & 0 & -6 & -9 \\
\hline Fish & 0 & 32 & 38 \\
\hline \multicolumn{4}{|l|}{ Environment } \\
\hline Forest area (\%) & 73 & 65 & 56 \\
\hline Renewable water res withdrawn (\% of total) & & & 94 \\
\hline Terrestrial protect areas (\% total land area) & 0 & 23 & 26 \\
\hline Organic area (\% total agricultural area) & & & 0 \\
\hline Water withdrawal by agriculture (\% of total) & & & 94 \\
\hline \multicolumn{4}{|l|}{ Biofuel production (thousand kt of oil eq.) } \\
\hline \multicolumn{4}{|l|}{ Wood pellet prod. (1 000 tonnes) } \\
\hline Net GHG emissions from AFOLU ( $\mathrm{CO}_{2}$ eq, $\mathrm{Mt}$ ) & 41 & 43 & 42 \\
\hline
\end{tabular}




\begin{tabular}{|c|c|c|c|}
\hline & 1990 & 2000 & 2014 \\
\hline \multicolumn{4}{|l|}{ The setting } \\
\hline Population, total (mln) & 12.1 & 15.9 & 22.8 \\
\hline Population, rural (mln) & 7.3 & 8.7 & 10.5 \\
\hline \multicolumn{4}{|l|}{ Govt expenditure on ag (\% total outlays) } \\
\hline Area harvested (mln ha) & 3 & 4 & 7 \\
\hline Cropping intensity ratio & 0.3 & 0.4 & \\
\hline Water resources (1 $000 \mathrm{~m}^{3} /$ person/year) & 23 & 18 & 13 \\
\hline Area equipped for irrigation (1 $000 \mathrm{ha}$ ) & & & 29 \\
\hline \multicolumn{4}{|l|}{ Area irrigated (\% area equipped for irrigation) } \\
\hline Employment in agriculture (\%) & & 61.3 & 53.3 \\
\hline Employment in agriculture, female (\%) & & 64.7 & 58.1 \\
\hline Fertilizers, Nitrogen (kg of nutrients per ha) & & 3.2 & 4.5 \\
\hline Fertilizers, Phosphate (kg of nutrients per ha) & & 2 & 1.8 \\
\hline Fertilizers, Potash (kg nutrients per ha) & & 2.9 & 3.8 \\
\hline Energy consump, power irrigation (mln kWh) & 0 & 13 & 13 \\
\hline Agr value added per worker (constant US\$) & 540 & 760 & 1264 \\
\hline \multicolumn{4}{|l|}{ Hunger dimensions } \\
\hline Dietary energy supply (kcal/pc/day) & 2055 & 2133 & 2596 \\
\hline Average dietary energy supply adequacy (\%) & 95 & 97 & 117 \\
\hline Dietary en supp, cereals/roots/tubers (\%) & 58 & 57 & 54 \\
\hline Prevalence of undernourishment (\%) & 36.7 & 32.3 & 10.2 \\
\hline GDP per capita (US\$, PPP) & 2768 & 2404 & 2739 \\
\hline Domestic food price volatility (index) & & 8.3 & 10 \\
\hline Cereal import dependency ratio (\%) & 31.2 & 30.2 & 25.8 \\
\hline Underweight, children under-5 (\%) & 18 & 17.8 & 15.1 \\
\hline Improved water source (\% pop) & 51.3 & 61.7 & 74.1 \\
\hline \multicolumn{4}{|l|}{ Food supply } \\
\hline Food production value, (2004-2006 mln I\$) & 1874 & 2548 & 5279 \\
\hline Agriculture, value added (\% GDP) & 25 & 22 & 23 \\
\hline Food exports (mln US\$) & 259 & 172 & 602 \\
\hline Food imports (mln US\$) & 212 & 215 & 934 \\
\hline \multicolumn{4}{|l|}{ Production indices (2004-06=100) } \\
\hline Net food & 57 & 77 & 160 \\
\hline Net crops & 55 & 77 & 161 \\
\hline Cereals & 43 & 66 & 163 \\
\hline Vegetable oils & 61 & 69 & 141 \\
\hline Roots and tubers & 58 & 81 & 157 \\
\hline Fruit and vegetables & 50 & 73 & 182 \\
\hline Sugar & 108 & 100 & 89 \\
\hline Livestock & 73 & 86 & 124 \\
\hline Milk & 79 & 86 & 114 \\
\hline Meat & 72 & 86 & 126 \\
\hline Fish & 52 & 82 & 113 \\
\hline \multicolumn{4}{|l|}{ Net trade (mln US\$) } \\
\hline Cereals & -101 & -106 & -642 \\
\hline Fruit and vegetables & 25 & 46 & 65 \\
\hline Meat & -5 & -14 & -14 \\
\hline Dairy products & -17 & -14 & -54 \\
\hline Fish & -45 & -27 & -247 \\
\hline \multicolumn{4}{|l|}{ Environment } \\
\hline Forest area (\%) & 51 & 47 & 41 \\
\hline Renewable water res withdrawn (\% of total) & & 76 & 76 \\
\hline Terrestrial protect areas (\% total land area) & 6 & 8 & 11 \\
\hline Organic area (\% total agricultural area) & & & 0 \\
\hline Water withdrawal by agriculture (\% of total) & & 76 & 76 \\
\hline Biofuel production (thousand kt of oil eq.) & 2 & 3 & 7 \\
\hline \multicolumn{4}{|l|}{ Wood pellet prod. (1 000 tonnes) } \\
\hline Net GHG emissions from AFOLU ( $\mathrm{CO}_{2}$ eq, $\mathrm{Mt}$ ) & 123 & 126 & 121 \\
\hline
\end{tabular}




\begin{tabular}{|c|c|c|c|}
\hline & 1990 & 2000 & 2014 \\
\hline \multicolumn{4}{|l|}{ The setting } \\
\hline Population, total (mln) & 27.7 & 30.7 & 35.5 \\
\hline Population, rural (mln) & 6.5 & 6.3 & 6.8 \\
\hline Govt expenditure on ag (\% total outlays) & & 3 & 0.6 \\
\hline Area harvested (mln ha) & 57 & 51 & 66 \\
\hline Cropping intensity ratio & 0.8 & 0.8 & \\
\hline Water resources (1 $000 \mathrm{~m}^{3} /$ person/year) & 105 & 95 & 82 \\
\hline Area equipped for irrigation (1 $000 \mathrm{ha}$ ) & & & 870 \\
\hline Area irrigated (\% area equipped for irrigation) & & & 69.5 \\
\hline Employment in agriculture (\%) & 4.1 & 3.3 & 2.4 \\
\hline Employment in agriculture, female (\%) & 2.4 & 1.9 & 1.3 \\
\hline Fertilizers, Nitrogen (kg of nutrients per ha) & & 36.2 & 51 \\
\hline Fertilizers, Phosphate (kg of nutrients per ha) & & 15 & 15.8 \\
\hline Fertilizers, Potash (kg nutrients per ha) & & 7.7 & 7.6 \\
\hline Energy consump, power irrigation (mln kWh) & 18 & 18 & 1329 \\
\hline \multicolumn{4}{|l|}{ Agr value added per worker (constant US\$) } \\
\hline \multicolumn{4}{|l|}{ Hunger dimensions } \\
\hline \multicolumn{4}{|l|}{ Dietary energy supply (kcal/pc/day) } \\
\hline Average dietary energy supply adequacy (\%) & 127 & 140 & 145 \\
\hline Dietary en supp, cereals/roots/tubers (\%) & 26 & 28 & 28 \\
\hline Prevalence of undernourishment (\%) & $<5.0$ & $<5.0$ & $<5.0$ \\
\hline GDP per capita (US\$, PPP) & 31118 & 37259 & 41899 \\
\hline Domestic food price volatility (index) & & 5.3 & 7.1 \\
\hline Cereal import dependency ratio (\%) & -104.3 & -66.8 & -81 \\
\hline \multicolumn{4}{|l|}{ Underweight, children under-5 (\%) } \\
\hline Improved water source (\% pop) & 99.8 & 99.8 & 99.8 \\
\hline \multicolumn{4}{|l|}{ Food supply } \\
\hline Food production value, (2004-2006 mln I\$) & 17406 & 21564 & 27181 \\
\hline Agriculture, value added (\% GDP) & & & 2 \\
\hline Food exports (mln US\$) & 7300 & 12663 & 37701 \\
\hline Food imports (mln US\$) & 5173 & 8147 & 22561 \\
\hline \multicolumn{4}{|l|}{ Production indices $(2004-06=100)$} \\
\hline Net food & 74 & 92 & 115 \\
\hline Net crops & 82 & 96 & 142 \\
\hline Cereals & 114 & 101 & 136 \\
\hline Vegetable oils & 46 & 84 & 188 \\
\hline Roots and tubers & 60 & 92 & 96 \\
\hline Fruit and vegetables & 84 & 95 & 99 \\
\hline Sugar & 127 & 111 & 81 \\
\hline Livestock & 68 & 89 & 85 \\
\hline Milk & 101 & 103 & 106 \\
\hline Meat & 60 & 86 & 79 \\
\hline Fish & 132 & 88 & 80 \\
\hline \multicolumn{4}{|l|}{ Net trade (mln US\$) } \\
\hline Cereals & 3318 & 2977 & 7473 \\
\hline Fruit and vegetables & -1920 & -1405 & -3751 \\
\hline Meat & 184 & 1617 & 1565 \\
\hline Dairy products & 44 & -66 & -220 \\
\hline Fish & 1649 & 1430 & 1541 \\
\hline \multicolumn{4}{|l|}{ Environment } \\
\hline Forest area (\%) & 34 & 34 & 34 \\
\hline Renewable water res withdrawn (\% of total) & & & 5 \\
\hline Terrestrial protect areas (\% total land area) & 5 & 7 & 9 \\
\hline Organic area (\% total agricultural area) & & & 1 \\
\hline Water withdrawal by agriculture (\% of total) & & & 5 \\
\hline Biofuel production (thousand kt of oil eq.) & & 12 & 225 \\
\hline Wood pellet prod. (1 000 tonnes) & & & 1900 \\
\hline Net GHG emissions from AFOLU ( $\mathrm{CO}_{2}$ eq, $\mathrm{Mt}$ ) & 162 & 69 & 200 \\
\hline
\end{tabular}


Central African Republic

\begin{tabular}{|c|c|c|c|}
\hline & 1990 & 2000 & 2014 \\
\hline \multicolumn{4}{|l|}{ The setting } \\
\hline Population, total (mln) & 2.9 & 3.6 & 4.7 \\
\hline Population, rural (mln) & 1.8 & 2.3 & 2.8 \\
\hline \multicolumn{4}{|l|}{ Govt expenditure on ag (\% total outlays) } \\
\hline Area harvested (mln ha) & 1 & 1 & 1 \\
\hline Cropping intensity ratio & 0.2 & 0.2 & \\
\hline Water resources (1 $000 \mathrm{~m}^{3} /$ person/year) & 48 & 39 & 31 \\
\hline Area equipped for irrigation (1 $000 \mathrm{ha})$ & & & 1 \\
\hline \multicolumn{4}{|l|}{ Area irrigated (\% area equipped for irrigation) } \\
\hline \multicolumn{4}{|l|}{ Employment in agriculture (\%) } \\
\hline \multicolumn{4}{|l|}{ Employment in agriculture, female (\%) } \\
\hline \multicolumn{4}{|l|}{ Fertilizers, Nitrogen (kg of nutrients per ha) } \\
\hline \multicolumn{4}{|l|}{ Fertilizers, Phosphate (kg of nutrients per ha) } \\
\hline \multicolumn{4}{|l|}{ Fertilizers, Potash (kg nutrients per ha) } \\
\hline \multicolumn{4}{|l|}{ Energy consump, power irrigation (mln kWh) } \\
\hline Agr value added per worker (constant US\$) & 430 & 510 & 456 \\
\hline \multicolumn{4}{|l|}{ Hunger dimensions } \\
\hline Dietary energy supply (kcal/pc/day) & 1887 & 1967 & 1926 \\
\hline Average dietary energy supply adequacy (\%) & 87 & 90 & 87 \\
\hline Dietary en supp, cereals/roots/tubers (\%) & 57 & 56 & 56 \\
\hline Prevalence of undernourishment (\%) & 48.4 & 44.1 & 44.5 \\
\hline GDP per capita (US\$, PPP) & 780 & 701 & 584 \\
\hline \multicolumn{4}{|l|}{ Domestic food price volatility (index) } \\
\hline Cereal import dependency ratio (\%) & 29.3 & 20.8 & 21.4 \\
\hline Underweight, children under-5 (\%) & & 21.8 & 23.5 \\
\hline Improved water source (\% pop) & 58.8 & 62.5 & 68.2 \\
\hline \multicolumn{4}{|l|}{ Food supply } \\
\hline Food production value, (2004-2006 mln I\$) & 489 & 727 & 966 \\
\hline Agriculture, value added (\% GDP) & 49 & 52 & 58 \\
\hline Food exports (mln US\$) & 17 & 12 & 16 \\
\hline Food imports (mln US\$) & 35 & 18 & 59 \\
\hline \multicolumn{4}{|l|}{ Production indices (2004-06=100) } \\
\hline Net food & 62 & 91 & 121 \\
\hline Net crops & 77 & 100 & 122 \\
\hline Cereals & 38 & 72 & 119 \\
\hline Vegetable oils & 68 & 84 & 101 \\
\hline Roots and tubers & 74 & 103 & 127 \\
\hline Fruit and vegetables & 77 & 100 & 112 \\
\hline Sugar & 67 & 99 & 121 \\
\hline Livestock & 54 & 88 & 124 \\
\hline Milk & 70 & 95 & 123 \\
\hline Meat & 52 & 88 & 126 \\
\hline Fish & 52 & 60 & 120 \\
\hline \multicolumn{4}{|l|}{ Net trade (mln US\$) } \\
\hline Cereals & -12 & -8 & -23 \\
\hline Fruit and vegetables & -1 & 0 & -1 \\
\hline Meat & -1 & 0 & -1 \\
\hline Dairy products & -3 & -1 & -3 \\
\hline Fish & -2 & 0 & -3 \\
\hline \multicolumn{4}{|l|}{ Environment } \\
\hline Forest area (\%) & 37 & 37 & 36 \\
\hline Renewable water res withdrawn (\% of total) & & & 1 \\
\hline Terrestrial protect areas (\% total land area) & 18 & 18 & 18 \\
\hline \multicolumn{4}{|l|}{ Organic area (\% total agricultural area) } \\
\hline Water withdrawal by agriculture (\% of total) & & & 1 \\
\hline \multicolumn{4}{|l|}{ Biofuel production (thousand kt of oil eq.) } \\
\hline \multicolumn{4}{|l|}{ Wood pellet prod. (1 000 tonnes) } \\
\hline Net GHG emissions from AFOLU ( $\mathrm{CO}_{2}$ eq, $\left.\mathrm{Mt}\right)$ & 33 & 39 & 30 \\
\hline
\end{tabular}




\begin{tabular}{|c|c|c|c|}
\hline & 1990 & 2000 & 2014 \\
\hline \multicolumn{4}{|l|}{ The setting } \\
\hline Population, total (mln) & 6 & 8.3 & 13.2 \\
\hline Population, rural (mln) & 4.7 & 6.5 & 10.3 \\
\hline \multicolumn{4}{|l|}{ Govt expenditure on ag (\% total outlays) } \\
\hline Area harvested (mln ha) & 1 & 2 & 3 \\
\hline Cropping intensity ratio & 0 & 0 & \\
\hline Water resources (1 $000 \mathrm{~m}^{3} /$ person/year) & 8 & 6 & 4 \\
\hline Area equipped for irrigation (1 $000 \mathrm{ha})$ & & & 30 \\
\hline Area irrigated (\% area equipped for irrigation) & & 86.6 & \\
\hline Employment in agriculture (\%) & 83 & 83 & \\
\hline Employment in agriculture, female (\%) & 85.9 & 85.9 & \\
\hline \multicolumn{4}{|l|}{ Fertilizers, Nitrogen (kg of nutrients per ha) } \\
\hline \multicolumn{4}{|l|}{ Fertilizers, Phosphate (kg of nutrients per ha) } \\
\hline \multicolumn{4}{|l|}{ Fertilizers, Potash (kg nutrients per ha) } \\
\hline Energy consump, power irrigation (mln kWh) & 8 & 8 & 9 \\
\hline Agr value added per worker (constant US\$) & & & 1287 \\
\hline \multicolumn{4}{|l|}{ Hunger dimensions } \\
\hline Dietary energy supply (kcal/pc/day) & 1818 & 1985 & 2171 \\
\hline Average dietary energy supply adequacy (\%) & 85 & 93 & 101 \\
\hline Dietary en supp, cereals/roots/tubers (\%) & 66 & 58 & 68 \\
\hline Prevalence of undernourishment (\%) & 55.7 & 40.1 & 36.1 \\
\hline GDP per capita (US\$, PPP) & 1111 & 998 & 2022 \\
\hline Domestic food price volatility (index) & & 15.4 & 11.7 \\
\hline Cereal import dependency ratio (\%) & 7.1 & 4.9 & 7.7 \\
\hline Underweight, children under-5 (\%) & & 29.4 & 30.3 \\
\hline Improved water source (\% pop) & 39.8 & 44.7 & 50.7 \\
\hline \multicolumn{4}{|l|}{ Food supply } \\
\hline Food production value, $(2004-2006 \mathrm{mln}$ I\$) & 719 & 984 & 1531 \\
\hline Agriculture, value added (\% GDP) & 29 & 42 & 53 \\
\hline Food exports (mln US\$) & 33 & 52 & 39 \\
\hline Food imports (mln US\$) & 27 & 32 & 154 \\
\hline \multicolumn{4}{|l|}{ Production indices $(2004-06=100)$} \\
\hline Net food & 56 & 77 & 120 \\
\hline Net crops & 51 & 72 & 116 \\
\hline Cereals & 35 & 54 & 159 \\
\hline Vegetable oils & 29 & 80 & 90 \\
\hline Roots and tubers & 94 & 77 & 134 \\
\hline Fruit and vegetables & 88 & 88 & 105 \\
\hline Sugar & 81 & 89 & 105 \\
\hline Livestock & 74 & 90 & 115 \\
\hline Milk & 64 & 90 & 114 \\
\hline Meat & 76 & 90 & 116 \\
\hline \multicolumn{4}{|l|}{ Fish } \\
\hline \multicolumn{4}{|l|}{ Net trade (mln US\$) } \\
\hline Cereals & -7 & -8 & -63 \\
\hline Fruit and vegetables & 0 & 0 & -4 \\
\hline Meat & -2 & 0 & -1 \\
\hline Dairy products & -2 & -4 & -5 \\
\hline Fish & & 0 & -1 \\
\hline \multicolumn{4}{|l|}{ Environment } \\
\hline Forest area (\%) & 10 & 10 & 9 \\
\hline Renewable water res withdrawn (\% of total) & & & 76 \\
\hline Terrestrial protect areas (\% total land area) & 10 & 10 & 17 \\
\hline \multicolumn{4}{|l|}{ Organic area (\% total agricultural area) } \\
\hline Water withdrawal by agriculture (\% of total) & & & 76 \\
\hline Biofuel production (thousand kt of oil eq.) & 1 & 1 & 1 \\
\hline \multicolumn{4}{|l|}{ Wood pellet prod. (1 000 tonnes) } \\
\hline Net GHG emissions from AFOLU ( $\mathrm{CO}_{2}$ eq, $\mathrm{Mt}$ ) & 33 & 39 & 36 \\
\hline
\end{tabular}




\begin{tabular}{|c|c|c|c|}
\hline & 1990 & 2000 & 2014 \\
\hline \multicolumn{4}{|l|}{ The setting } \\
\hline Population, total (mln) & 13.2 & 15.5 & 17.8 \\
\hline Population, rural (mln) & 2.2 & 2.2 & 1.8 \\
\hline \multicolumn{4}{|l|}{ Govt expenditure on ag (\% total outlays) } \\
\hline Area harvested (mln ha) & 3 & 4 & 4 \\
\hline Cropping intensity ratio & 0.2 & 0.3 & \\
\hline Water resources (1 $000 \mathrm{~m}^{3} /$ person/year) & 70 & 60 & 52 \\
\hline Area equipped for irrigation (1 $000 \mathrm{ha})$ & & & 1110 \\
\hline Area irrigated (\% area equipped for irrigation) & & & 98.6 \\
\hline Employment in agriculture (\%) & 19.3 & 14.4 & 10.3 \\
\hline Employment in agriculture, female (\%) & 5.9 & 4.6 & 5.1 \\
\hline Fertilizers, Nitrogen (kg of nutrients per ha) & & 147.4 & 256.6 \\
\hline Fertilizers, Phosphate (kg of nutrients per ha) & & 124.3 & 91.4 \\
\hline Fertilizers, Potash (kg nutrients per ha) & & 58.5 & 10.4 \\
\hline Energy consump, power irrigation (mln kWh) & 0 & & 667 \\
\hline Agr value added per worker (constant US\$) & 3224 & 4429 & 6638 \\
\hline \multicolumn{4}{|l|}{ Hunger dimensions } \\
\hline Dietary energy supply (kcal/pc/day) & 2722 & 2834 & 3047 \\
\hline Average dietary energy supply adequacy (\%) & 116 & 119 & 125 \\
\hline Dietary en supp, cereals/roots/tubers (\%) & 46 & 45 & 44 \\
\hline Prevalence of undernourishment (\%) & 7.1 & $<5.0$ & $<5.0$ \\
\hline GDP per capita (US\$, PPP) & 9199 & 14623 & 21714 \\
\hline Domestic food price volatility (index) & & 4 & 7.4 \\
\hline Cereal import dependency ratio (\%) & 26.7 & 39.8 & 38.8 \\
\hline Underweight, children under-5 (\%) & & 0.6 & 0.5 \\
\hline Improved water source (\% pop) & 90.4 & 94.8 & 98.8 \\
\hline \multicolumn{4}{|l|}{ Food supply } \\
\hline Food production value, $(2004-2006 \mathrm{mln}$ I\$) & 4179 & 5980 & 8424 \\
\hline Agriculture, value added (\% GDP) & 9 & 6 & 3 \\
\hline Food exports (mln US\$) & 1014 & 1996 & 8004 \\
\hline Food imports (mln US\$) & 232 & 946 & 4022 \\
\hline \multicolumn{4}{|l|}{ Production indices $(2004-06=100)$} \\
\hline Net food & 58 & 83 & 117 \\
\hline Net crops & 61 & 82 & 117 \\
\hline Cereals & 79 & 69 & 102 \\
\hline Vegetable oils & 108 & 89 & 335 \\
\hline Roots and tubers & 65 & 79 & 96 \\
\hline Fruit and vegetables & 55 & 83 & 119 \\
\hline Sugar & 99 & 131 & 80 \\
\hline Livestock & 56 & 83 & 117 \\
\hline Milk & 60 & 86 & 115 \\
\hline Meat & 52 & 81 & 115 \\
\hline Fish & 100 & 90 & 54 \\
\hline \multicolumn{4}{|l|}{ Net trade (mln US\$) } \\
\hline Cereals & 6 & -170 & -533 \\
\hline Fruit and vegetables & 875 & 1441 & 5326 \\
\hline Meat & 15 & -87 & -95 \\
\hline Dairy products & -18 & -27 & 26 \\
\hline Fish & 858 & 1746 & 3911 \\
\hline \multicolumn{4}{|l|}{ Environment } \\
\hline Forest area (\%) & 21 & 21 & 22 \\
\hline Renewable water res withdrawn (\% of total) & & & 83 \\
\hline Terrestrial protect areas (\% total land area) & 16 & 17 & 19 \\
\hline Organic area (\% total agricultural area) & & & 0 \\
\hline Water withdrawal by agriculture (\% of total) & & & 83 \\
\hline Biofuel production (thousand kt of oil eq.) & 1 & 0 & 0 \\
\hline Wood pellet prod. (1 000 tonnes) & & & 10 \\
\hline Net GHG emissions from AFOLU ( $\mathrm{CO}_{2}$ eq, $\mathrm{Mt}$ ) & 14 & -1 & 5 \\
\hline
\end{tabular}




\begin{tabular}{|c|c|c|c|}
\hline & 1990 & 2000 & 2014 \\
\hline \multicolumn{4}{|l|}{ The setting } \\
\hline Population, total (mln) & 1191.8 & 1309.6 & 1425 \\
\hline Population, rural (mln) & 864.1 & 827.7 & 641.6 \\
\hline Govt expenditure on ag (\% total outlays) & & 1.3 & 1.9 \\
\hline Area harvested (mln ha) & 405 & 407 & 553 \\
\hline Cropping intensity ratio & 0.8 & 0.8 & \\
\hline Water resources (1 $000 \mathrm{~m}^{3} /$ person/year) & 2 & 2 & 2 \\
\hline Area equipped for irrigation (1 $000 \mathrm{ha}$ ) & & & 69390 \\
\hline Area irrigated (\% area equipped for irrigation) & & & 86.1 \\
\hline Employment in agriculture (\%) & 56.1 & 49.8 & 36.7 \\
\hline Employment in agriculture, female (\%) & 0.4 & 0.2 & 0.2 \\
\hline Fertilizers, Nitrogen (kg of nutrients per ha) & & 251.6 & 422.2 \\
\hline Fertilizers, Phosphate (kg of nutrients per ha) & & 85.8 & 155.7 \\
\hline Fertilizers, Potash (kg nutrients per ha) & & 43 & 66 \\
\hline Energy consump, power irrigation (mln kWh) & 1178 & 1337 & 7813 \\
\hline \multicolumn{4}{|l|}{ Agr value added per worker (constant US\$) } \\
\hline \multicolumn{4}{|l|}{ Hunger dimensions } \\
\hline Dietary energy supply (kcal/pc/day) & 2545 & 2802 & 3132 \\
\hline Average dietary energy supply adequacy (\%) & 109 & 116 & 128 \\
\hline Dietary en supp, cereals/roots/tubers (\%) & 67 & 61 & 52 \\
\hline Prevalence of undernourishment (\%) & 23.4 & 16.2 & 9.8 \\
\hline GDP per capita (US\$, PPP) & 1623 & 3780 & 11778 \\
\hline Domestic food price volatility (index) & & 10.8 & 8.1 \\
\hline Cereal import dependency ratio (\%) & 1.4 & 0.1 & 100 \\
\hline Underweight, children under-5 (\%) & 12.6 & 7.4 & 3.4 \\
\hline \multicolumn{4}{|l|}{ Improved water source (\% pop) } \\
\hline \multicolumn{4}{|l|}{ Food supply } \\
\hline Food production value, (2004-2006 mln I\$) & 196044 & 336354 & 518851 \\
\hline Agriculture, value added (\% GDP) & & 13 & 9 \\
\hline Food exports (mln US\$) & 7371 & 11300 & 36567 \\
\hline Food imports (mln US\$) & 10104 & 15163 & 96838 \\
\hline \multicolumn{4}{|l|}{ Production indices $(2004-06=100)$} \\
\hline \multicolumn{4}{|l|}{ Net food } \\
\hline \multicolumn{4}{|l|}{ Net crops } \\
\hline Cereals & 97 & 97 & 125 \\
\hline Vegetable oils & 59 & 96 & 111 \\
\hline Roots and tubers & 72 & 109 & 117 \\
\hline Fruit and vegetables & 26 & 75 & 145 \\
\hline Sugar & 83 & 80 & 141 \\
\hline \multicolumn{4}{|l|}{ Livestock } \\
\hline Milk & 23 & 40 & 126 \\
\hline Meat & 44 & 87 & 128 \\
\hline Fish & 31 & 85 & 140 \\
\hline \multicolumn{4}{|l|}{ Net trade (mln US\$) } \\
\hline Cereals & -3013 & -42 & -8335 \\
\hline Fruit and vegetables & 1198 & 1566 & 8588 \\
\hline Meat & 777 & -577 & -5731 \\
\hline Dairy products & -415 & -587 & -4748 \\
\hline Fish & 643 & 365 & 7824 \\
\hline \multicolumn{4}{|l|}{ Environment } \\
\hline \multicolumn{4}{|l|}{ Forest area (\%) } \\
\hline Renewable water res withdrawn (\% of total) & & & 65 \\
\hline Terrestrial protect areas (\% total land area) & 14 & 15 & 17 \\
\hline Organic area (\% total agricultural area) & & & 0 \\
\hline Water withdrawal by agriculture (\% of total) & & & 65 \\
\hline Biofuel production (thousand kt of oil eq.) & 118 & 174 & 266 \\
\hline Wood pellet prod. (1 000 tonnes) & & & 400 \\
\hline Net GHG emissions from AFOLU ( $\mathrm{CO}_{2}$ eq, $\mathrm{Mt}$ ) & 446 & 279 & 544 \\
\hline
\end{tabular}




\begin{tabular}{|c|c|c|c|}
\hline & 1990 & 2000 & 2014 \\
\hline \multicolumn{4}{|l|}{ The setting } \\
\hline Population, total (mln) & 33.3 & 39.9 & 48.9 \\
\hline Population, rural (mln) & 10.6 & 11.1 & 11.7 \\
\hline \multicolumn{4}{|l|}{ Govt expenditure on ag (\% total outlays) } \\
\hline Area harvested (mln ha) & 28 & 34 & 35 \\
\hline Cropping intensity ratio & 0.6 & 0.8 & \\
\hline Water resources (1 $000 \mathrm{~m}^{3} /$ person/year) & 71 & 59 & 49 \\
\hline Area equipped for irrigation (1 $000 \mathrm{ha})$ & & & 1090 \\
\hline Area irrigated (\% area equipped for irrigation) & & & 36.2 \\
\hline Employment in agriculture (\%) & 1.4 & 1.1 & 16.9 \\
\hline Employment in agriculture, female (\%) & 0.6 & 0.5 & 6.9 \\
\hline Fertilizers, Nitrogen (kg of nutrients per ha) & & 163.3 & 390.9 \\
\hline Fertilizers, Phosphate (kg of nutrients per ha) & & 54.6 & 185.5 \\
\hline Fertilizers, Potash (kg nutrients per ha) & & 106.9 & 167.8 \\
\hline Energy consump, power irrigation (mln kWh) & 94 & 94 & 94 \\
\hline Agr value added per worker (constant US\$) & 3654 & 2721 & 3982 \\
\hline \multicolumn{4}{|l|}{ Hunger dimensions } \\
\hline Dietary energy supply (kcal/pc/day) & 2678 & 2786 & 2829 \\
\hline Average dietary energy supply adequacy (\%) & 119 & 122 & 121 \\
\hline Dietary en supp, cereals/roots/tubers (\%) & 38 & 37 & 35 \\
\hline Prevalence of undernourishment (\%) & 12.8 & 9.9 & 9.5 \\
\hline GDP per capita (US\$, PPP) & 7752 & 8414 & 12025 \\
\hline Domestic food price volatility (index) & & 8.8 & 4.5 \\
\hline Cereal import dependency ratio (\%) & 38 & 53.9 & 63.3 \\
\hline Underweight, children under-5 (\%) & & 4.9 & 3.4 \\
\hline Improved water source (\% pop) & 88.4 & 89.9 & 91.2 \\
\hline \multicolumn{4}{|l|}{ Food supply } \\
\hline Food production value, (2004-2006 mln I\$) & 8177 & 10216 & 13623 \\
\hline Agriculture, value added (\% GDP) & 17 & 9 & 7 \\
\hline Food exports (mln US\$) & 598 & 1063 & 2717 \\
\hline Food imports (mln US\$) & 261 & 944 & 4360 \\
\hline \multicolumn{4}{|l|}{ Production indices $(2004-06=100)$} \\
\hline Net food & 70 & 87 & 117 \\
\hline Net crops & 79 & 88 & 108 \\
\hline Cereals & 103 & 92 & 93 \\
\hline Vegetable oils & 44 & 78 & 139 \\
\hline Roots and tubers & 106 & 83 & 118 \\
\hline Fruit and vegetables & 54 & 89 & 121 \\
\hline Sugar & 71 & 87 & 89 \\
\hline Livestock & 69 & 86 & 120 \\
\hline Milk & 62 & 94 & 99 \\
\hline Meat & 75 & 82 & 130 \\
\hline Fish & 74 & 113 & 85 \\
\hline \multicolumn{4}{|l|}{ Net trade (mln US\$) } \\
\hline Cereals & -126 & -358 & -1966 \\
\hline Fruit and vegetables & 315 & 383 & 395 \\
\hline Meat & 16 & -29 & -108 \\
\hline Dairy products & -3 & 0 & -112 \\
\hline Fish & 74 & 116 & -170 \\
\hline \multicolumn{4}{|l|}{ Environment } \\
\hline Forest area (\%) & 56 & 55 & 54 \\
\hline Renewable water res withdrawn (\% of total) & & & 54 \\
\hline Terrestrial protect areas (\% total land area) & 19 & 20 & 21 \\
\hline Organic area (\% total agricultural area) & & & 0 \\
\hline Water withdrawal by agriculture (\% of total) & & & 54 \\
\hline Biofuel production (thousand kt of oil eq.) & 67 & 60 & 9285 \\
\hline \multicolumn{4}{|l|}{ Wood pellet prod. (1 000 tonnes) } \\
\hline Net GHG emissions from AFOLU ( $\mathrm{CO}_{2}$ eq, $\mathrm{Mt}$ ) & 102 & 99 & 98 \\
\hline
\end{tabular}




\begin{tabular}{|c|c|c|c|}
\hline & 1990 & 2000 & 2014 \\
\hline \multicolumn{4}{|l|}{ The setting } \\
\hline Population, total (mln) & 0.4 & 0.5 & 0.8 \\
\hline Population, rural (mln) & 0.3 & 0.4 & 0.5 \\
\hline \multicolumn{4}{|l|}{ Govt expenditure on ag (\% total outlays) } \\
\hline Area harvested (mln ha) & 0 & 0 & 0 \\
\hline Cropping intensity ratio & 0.9 & 1.1 & \\
\hline Water resources (1 $000 \mathrm{~m}^{3} /$ person/year) & 3 & 2 & 2 \\
\hline Area equipped for irrigation (1 $000 \mathrm{ha}$ ) & & & 0 \\
\hline \multicolumn{4}{|l|}{ Area irrigated (\% area equipped for irrigation) } \\
\hline \multicolumn{4}{|l|}{ Employment in agriculture (\%) } \\
\hline \multicolumn{4}{|l|}{ Employment in agriculture, female (\%) } \\
\hline \multicolumn{4}{|l|}{ Fertilizers, Nitrogen (kg of nutrients per ha) } \\
\hline \multicolumn{4}{|l|}{ Fertilizers, Phosphate (kg of nutrients per ha) } \\
\hline \multicolumn{4}{|l|}{ Fertilizers, Potash (kg nutrients per ha) } \\
\hline \multicolumn{4}{|l|}{ Energy consump, power irrigation (mln kWh) } \\
\hline Agr value added per worker (constant US\$) & 747 & 771 & 817 \\
\hline \multicolumn{4}{|l|}{ Hunger dimensions } \\
\hline \multicolumn{4}{|l|}{ Dietary energy supply (kcal/pc/day) } \\
\hline \multicolumn{4}{|l|}{ Average dietary energy supply adequacy (\%) } \\
\hline \multicolumn{4}{|l|}{ Dietary en supp, cereals/roots/tubers (\%) } \\
\hline \multicolumn{4}{|l|}{ Prevalence of undernourishment (\%) } \\
\hline GDP per capita (US\$, PPP) & 1540 & 1343 & 1400 \\
\hline \multicolumn{4}{|l|}{ Domestic food price volatility (index) } \\
\hline Cereal import dependency ratio (\%) & 70.2 & 69 & 71.2 \\
\hline Underweight, children under-5 (\%) & 16.2 & 25 & 16.9 \\
\hline Improved water source (\% pop) & 87 & 92 & 95.1 \\
\hline \multicolumn{4}{|l|}{ Food supply } \\
\hline Food production value, (2004-2006 mln I\$) & 51 & 61 & 72 \\
\hline Agriculture, value added (\% GDP) & 41 & 49 & 35 \\
\hline Food exports (mln US\$) & 11 & 6 & 27 \\
\hline Food imports (mln US\$) & 16 & 13 & 81 \\
\hline \multicolumn{4}{|l|}{ Production indices $(2004-06=100)$} \\
\hline Net food & 80 & 95 & 113 \\
\hline Net crops & 78 & 95 & 113 \\
\hline Cereals & 75 & 87 & 149 \\
\hline Vegetable oils & 73 & 84 & 110 \\
\hline Roots and tubers & 72 & 93 & 124 \\
\hline Fruit and vegetables & 75 & 93 & 101 \\
\hline \multicolumn{4}{|l|}{ Sugar } \\
\hline Livestock & 92 & 99 & 113 \\
\hline Milk & 89 & 101 & 117 \\
\hline Meat & 95 & 99 & 112 \\
\hline Fish & & & \\
\hline Net trade (mln US\$) & & & \\
\hline Cereals & & -7 & -38 \\
\hline Fruit and vegetables & -1 & -1 & -2 \\
\hline Meat & & -8 & -28 \\
\hline Dairy products & & -2 & -3 \\
\hline Fish & -1 & 0 & -3 \\
\hline Environment & & & \\
\hline Forest area (\%) & 6 & 4 & 1 \\
\hline Renewable water res withdrawn (\% of total) & & 47 & 47 \\
\hline Terrestrial protect areas (\% total land area) & & 1 & 10 \\
\hline Organic area (\% total agricultural area) & & & 2 \\
\hline Water withdrawal by agriculture (\% of total) & & 47 & 47 \\
\hline Biofuel production (thousand kt of oil eq.) & & & \\
\hline Wood pellet prod. (1 000 tonnes) & & & \\
\hline Net GHG emissions from AFOLU ( $\mathrm{CO}_{2}$ eq, $\mathrm{Mt}$ ) & 0 & 0 & 0 \\
\hline
\end{tabular}




\begin{tabular}{|c|c|c|c|}
\hline & 1990 & 2000 & 2014 \\
\hline \multicolumn{4}{|l|}{ The setting } \\
\hline Population, total (mln) & 2.4 & 3.1 & 4.6 \\
\hline Population, rural (mln) & 1.1 & 1.3 & 1.6 \\
\hline \multicolumn{4}{|l|}{ Govt expenditure on ag (\% total outlays) } \\
\hline Area harvested (mln ha) & 1 & 1 & 1 \\
\hline Cropping intensity ratio & 0.1 & 0.1 & \\
\hline Water resources (1 $000 \mathrm{~m}^{3} /$ person/year) & 349 & 266 & 187 \\
\hline Area equipped for irrigation (1 $000 \mathrm{ha}$ ) & & & 2 \\
\hline \multicolumn{4}{|l|}{ Area irrigated (\% area equipped for irrigation) } \\
\hline Employment in agriculture (\%) & & & 35.4 \\
\hline Employment in agriculture, female (\%) & & & 39.3 \\
\hline Fertilizers, Nitrogen (kg of nutrients per ha) & & 0 & 6.1 \\
\hline Fertilizers, Phosphate (kg of nutrients per ha) & & 0 & 0.7 \\
\hline Fertilizers, Potash (kg nutrients per ha) & & 0 & 2.5 \\
\hline Energy consump, power irrigation (mln kWh) & 0 & 0 & 0 \\
\hline Agr value added per worker (constant US\$) & 502 & 405 & 838 \\
\hline \multicolumn{4}{|l|}{ Hunger dimensions } \\
\hline Dietary energy supply (kcal/pc/day) & 1995 & 2170 & 2132 \\
\hline Average dietary energy supply adequacy (\%) & 91 & 99 & 97 \\
\hline Dietary en supp, cereals/roots/tubers (\%) & 60 & 61 & 61 \\
\hline Prevalence of undernourishment (\%) & 43.6 & 35.9 & 29 \\
\hline GDP per capita (US\$, PPP) & 5264 & 4623 & 5680 \\
\hline Domestic food price volatility (index) & & 18.5 & 18.8 \\
\hline Cereal import dependency ratio (\%) & 94.8 & 95.2 & 92.9 \\
\hline Underweight, children under-5 (\%) & & & 11.8 \\
\hline Improved water source (\% pop) & & 69.2 & 75.3 \\
\hline \multicolumn{4}{|l|}{ Food supply } \\
\hline Food production value, (2004-2006 mln I\$) & 203 & 268 & 441 \\
\hline Agriculture, value added (\% GDP) & 13 & 5 & 5 \\
\hline Food exports (mln US\$) & 14 & 16 & 17 \\
\hline Food imports (mln US\$) & 67 & 136 & 623 \\
\hline \multicolumn{4}{|l|}{ Production indices $(2004-06=100)$} \\
\hline Net food & 63 & 83 & 136 \\
\hline Net crops & 66 & 86 & 127 \\
\hline Cereals & 25 & 45 & 137 \\
\hline Vegetable oils & 93 & 88 & 134 \\
\hline Roots and tubers & 71 & 83 & 126 \\
\hline Fruit and vegetables & 54 & 94 & 127 \\
\hline Sugar & 45 & 76 & 109 \\
\hline Livestock & 51 & 67 & 164 \\
\hline Milk & 83 & 87 & 112 \\
\hline Meat & 51 & 67 & 165 \\
\hline Fish & 85 & 81 & 129 \\
\hline \multicolumn{4}{|l|}{ Net trade (mln US\$) } \\
\hline Cereals & -35 & -45 & -248 \\
\hline Fruit and vegetables & -2 & -14 & -30 \\
\hline Meat & -13 & -17 & -204 \\
\hline Dairy products & -4 & -17 & -33 \\
\hline Fish & -19 & -18 & -52 \\
\hline \multicolumn{4}{|l|}{ Environment } \\
\hline Forest area (\%) & 67 & 66 & 66 \\
\hline Renewable water res withdrawn (\% of total) & & 9 & 9 \\
\hline Terrestrial protect areas (\% total land area) & 5 & 9 & 30 \\
\hline \multicolumn{4}{|l|}{ Organic area (\% total agricultural area) } \\
\hline Water withdrawal by agriculture (\% of total) & & 9 & 9 \\
\hline Biofuel production (thousand kt of oil eq.) & 1 & 1 & 2 \\
\hline \multicolumn{4}{|l|}{ Wood pellet prod. (1 000 tonnes) } \\
\hline Net GHG emissions from AFOLU ( $\mathrm{CO}_{2}$ eq, $\left.\mathrm{Mt}\right)$ & 12 & 12 & 12 \\
\hline
\end{tabular}




\begin{tabular}{|c|c|c|c|}
\hline & 1990 & 2000 & 2014 \\
\hline \multicolumn{4}{|l|}{ The setting } \\
\hline Population, total (mln) & 3.1 & 3.9 & 4.9 \\
\hline Population, rural (mln) & 1.5 & 1.6 & 1.7 \\
\hline \multicolumn{4}{|l|}{ Govt expenditure on ag (\% total outlays) } \\
\hline Area harvested (mln ha) & 3 & 4 & 4 \\
\hline Cropping intensity ratio & 1.1 & 2.1 & \\
\hline Water resources (1 $000 \mathrm{~m}^{3} /$ person/year) & 37 & 29 & 23 \\
\hline Area equipped for irrigation (1 $000 \mathrm{ha})$ & & & 102 \\
\hline Area irrigated (\% area equipped for irrigation) & & & 100 \\
\hline Employment in agriculture (\%) & 25.9 & 20.4 & 13.4 \\
\hline Employment in agriculture, female (\%) & 6.4 & 5.4 & 3.9 \\
\hline Fertilizers, Nitrogen (kg of nutrients per ha) & & 310.4 & 349.2 \\
\hline Fertilizers, Phosphate (kg of nutrients per ha) & & 150.6 & 90.4 \\
\hline Fertilizers, Potash (kg nutrients per ha) & & 386.3 & 265.4 \\
\hline Energy consump, power irrigation (mln kWh) & 0 & 38 & 38 \\
\hline Agr value added per worker (constant US\$) & 3199 & 4499 & 6813 \\
\hline \multicolumn{4}{|l|}{ Hunger dimensions } \\
\hline Dietary energy supply (kcal/pc/day) & 2735 & 2801 & 2897 \\
\hline Average dietary energy supply adequacy (\%) & 118 & 119 & 119 \\
\hline Dietary en supp, cereals/roots/tubers (\%) & 34 & 35 & 34 \\
\hline Prevalence of undernourishment (\%) & 5.4 & 5.2 & 5.5 \\
\hline GDP per capita (US\$, PPP) & 7333 & 9565 & 13431 \\
\hline Domestic food price volatility (index) & & 5.6 & 7.6 \\
\hline Cereal import dependency ratio (\%) & 72.3 & 79.7 & 82.4 \\
\hline Underweight, children under-5 (\%) & 2.5 & 4.5 & 1.1 \\
\hline Improved water source (\% pop) & 93.1 & 95 & 96.6 \\
\hline \multicolumn{4}{|l|}{ Food supply } \\
\hline Food production value, (2004-2006 mln I\$) & 1406 & 2077 & 2941 \\
\hline Agriculture, value added (\% GDP) & 12 & 9 & 6 \\
\hline Food exports (mln US\$) & 527 & 1223 & 3080 \\
\hline Food imports (mln US\$) & 139 & 382 & 1306 \\
\hline \multicolumn{4}{|l|}{ Production indices $(2004-06=100)$} \\
\hline Net food & 59 & 87 & 124 \\
\hline Net crops & 62 & 89 & 122 \\
\hline Cereals & 128 & 140 & 117 \\
\hline Vegetable oils & 42 & 77 & 169 \\
\hline Roots and tubers & 47 & 80 & 109 \\
\hline Fruit and vegetables & 53 & 84 & 126 \\
\hline Sugar & 61 & 89 & 114 \\
\hline Livestock & 71 & 91 & 123 \\
\hline Milk & 59 & 92 & 136 \\
\hline Meat & 78 & 92 & 116 \\
\hline Fish & 43 & 103 & 125 \\
\hline \multicolumn{4}{|l|}{ Net trade (mln US\$) } \\
\hline Cereals & -55 & -116 & -376 \\
\hline Fruit and vegetables & 398 & 874 & 1776 \\
\hline Meat & 45 & 30 & 22 \\
\hline Dairy products & -5 & 5 & 74 \\
\hline Fish & 46 & 98 & 93 \\
\hline \multicolumn{4}{|l|}{ Environment } \\
\hline Forest area (\%) & 50 & 47 & 52 \\
\hline Renewable water res withdrawn (\% of total) & & & 57 \\
\hline Terrestrial protect areas (\% total land area) & 19 & 24 & 27 \\
\hline Organic area (\% total agricultural area) & & & 0 \\
\hline Water withdrawal by agriculture (\% of total) & & & 57 \\
\hline Biofuel production (thousand kt of oil eq.) & 6 & 10 & 16 \\
\hline Wood pellet prod. (1 000 tonnes) & & & 30 \\
\hline Net GHG emissions from AFOLU ( $\mathrm{CO}_{2}$ eq, $\mathrm{Mt}$ ) & 11 & 9 & -4 \\
\hline
\end{tabular}




\begin{tabular}{|c|c|c|c|}
\hline & 1990 & 2000 & 2014 \\
\hline \multicolumn{4}{|l|}{ The setting } \\
\hline Population, total (mln) & 4.8 & 4.5 & 4.3 \\
\hline Population, rural (mln) & 2.2 & 2 & 1.8 \\
\hline Govt expenditure on ag (\% total outlays) & & 2.9 & 3.9 \\
\hline Area harvested (mln ha) & & 3 & 3 \\
\hline Cropping intensity ratio & & 2.4 & \\
\hline Water resources (1 $000 \mathrm{~m}^{3} /$ person/year) & 22 & 24 & 25 \\
\hline Area equipped for irrigation (1 $000 \mathrm{ha}$ ) & & & 24 \\
\hline \multicolumn{4}{|l|}{ Area irrigated (\% area equipped for irrigation) } \\
\hline Employment in agriculture (\%) & & 14.5 & 13.7 \\
\hline Employment in agriculture, female (\%) & & 15.3 & 13.6 \\
\hline Fertilizers, Nitrogen (kg of nutrients per ha) & & 143.1 & 139 \\
\hline Fertilizers, Phosphate (kg of nutrients per ha) & & 73.9 & 108.1 \\
\hline Fertilizers, Potash (kg nutrients per ha) & & 75.9 & 53.8 \\
\hline Energy consump, power irrigation (mln kWh) & 0 & 1 & 3 \\
\hline Agr value added per worker (constant US\$) & & 10494 & 23815 \\
\hline \multicolumn{4}{|l|}{ Hunger dimensions } \\
\hline \multicolumn{4}{|l|}{ Dietary energy supply (kcal/pc/day) } \\
\hline Average dietary energy supply adequacy (\%) & 93 & 105 & 121 \\
\hline Dietary en supp, cereals/roots/tubers (\%) & 37 & 39 & 32 \\
\hline Prevalence of undernourishment (\%) & $<5.0$ & $<5.0$ & $<5.0$ \\
\hline GDP per capita (US\$, PPP) & & 15644 & 20049 \\
\hline Domestic food price volatility (index) & & 12.4 & 2.7 \\
\hline Cereal import dependency ratio (\%) & -5.3 & -0.7 & -12.4 \\
\hline Underweight, children under-5 (\%) & & 0.5 & \\
\hline Improved water source (\% pop) & 98.4 & 98.5 & 98.6 \\
\hline \multicolumn{4}{|l|}{ Food supply } \\
\hline Food production value, (2004-2006 mln I\$) & 1157 & 1060 & 1082 \\
\hline Agriculture, value added (\% GDP) & & 6 & 4 \\
\hline Food exports (mln US\$) & 296 & 224 & 1104 \\
\hline Food imports (mln US\$) & 306 & 456 & 1819 \\
\hline \multicolumn{4}{|l|}{ Production indices $(2004-06=100)$} \\
\hline Net food & 105 & 96 & 98 \\
\hline Net crops & 100 & 104 & 98 \\
\hline Cereals & 87 & 89 & 100 \\
\hline Vegetable oils & 49 & 63 & 137 \\
\hline Roots and tubers & 171 & 191 & 62 \\
\hline Fruit and vegetables & 140 & 143 & 99 \\
\hline Sugar & 39 & 35 & 77 \\
\hline Livestock & 97 & 77 & 92 \\
\hline Milk & 78 & 77 & 90 \\
\hline Meat & 123 & 74 & 96 \\
\hline Fish & 0 & 59 & 186 \\
\hline \multicolumn{4}{|l|}{ Net trade (mln US\$) } \\
\hline Cereals & 50 & 7 & 19 \\
\hline Fruit and vegetables & -52 & -107 & -291 \\
\hline Meat & -12 & -32 & -189 \\
\hline Dairy products & -23 & -30 & -75 \\
\hline Fish & 26 & 5 & 42 \\
\hline \multicolumn{4}{|l|}{ Environment } \\
\hline Forest area (\%) & 33 & 34 & 34 \\
\hline Renewable water res withdrawn (\% of total) & & & 1 \\
\hline Terrestrial protect areas (\% total land area) & 8 & 10 & 14 \\
\hline Organic area (\% total agricultural area) & & & 2 \\
\hline Water withdrawal by agriculture (\% of total) & & & 1 \\
\hline Biofuel production (thousand kt of oil eq.) & & 0 & 2 \\
\hline Wood pellet prod. (1 000 tonnes) & & & 190 \\
\hline Net GHG emissions from AFOLU ( $\mathrm{CO}_{2}$ eq, $\mathrm{Mt}$ ) & -9 & -9 & -9 \\
\hline
\end{tabular}




\begin{tabular}{|c|c|c|c|}
\hline & 1990 & 2000 & 2014 \\
\hline \multicolumn{4}{|l|}{ The setting } \\
\hline Population, total (mln) & 10.6 & 11.1 & 11.3 \\
\hline Population, rural (mln) & 2.8 & 2.7 & 2.8 \\
\hline \multicolumn{4}{|l|}{ Govt expenditure on ag (\% total outlays) } \\
\hline Area harvested (mln ha) & 82 & 36 & 14 \\
\hline Cropping intensity ratio & 12.1 & 5.6 & \\
\hline Water resources (1 $000 \mathrm{~m}^{3} /$ person/year) & 4 & 3 & 3 \\
\hline Area equipped for irrigation (1 $000 \mathrm{ha}$ ) & & & 870 \\
\hline Area irrigated (\% area equipped for irrigation) & & 84.8 & \\
\hline Employment in agriculture (\%) & 25.9 & 27.1 & 19.7 \\
\hline Employment in agriculture, female (\%) & & 14.2 & 8.8 \\
\hline Fertilizers, Nitrogen (kg of nutrients per ha) & & 6.6 & 24 \\
\hline Fertilizers, Phosphate (kg of nutrients per ha) & & 3.2 & 10.6 \\
\hline Fertilizers, Potash (kg nutrients per ha) & & 6.6 & 15.6 \\
\hline Energy consump, power irrigation (mln kWh) & 0 & 916 & 916 \\
\hline Agr value added per worker (constant US\$) & 4223 & 3032 & 4634 \\
\hline \multicolumn{4}{|l|}{ Hunger dimensions } \\
\hline Dietary energy supply (kcal/pc/day) & 2395 & 3002 & 3459 \\
\hline Average dietary energy supply adequacy (\%) & 100 & 124 & 140 \\
\hline Dietary en supp, cereals/roots/tubers (\%) & 38 & 43 & 44 \\
\hline Prevalence of undernourishment (\%) & 13.1 & 5.6 & $<5.0$ \\
\hline GDP per capita (US\$, PPP) & 13532 & 11154 & 18796 \\
\hline \multicolumn{4}{|l|}{ Domestic food price volatility (index) } \\
\hline Cereal import dependency ratio (\%) & 82.7 & 71.7 & 75.5 \\
\hline Underweight, children under-5 (\%) & & 3.4 & \\
\hline Improved water source (\% pop) & & 90.7 & 94 \\
\hline \multicolumn{4}{|l|}{ Food supply } \\
\hline Food production value, $(2004-2006 \mathrm{mln} I \$)$ & 4320 & 3375 & 2821 \\
\hline Agriculture, value added (\% GDP) & 14 & 8 & 5 \\
\hline Food exports (mln US\$) & 4561 & 551 & 499 \\
\hline Food imports (mln US\$) & 803 & 631 & 1845 \\
\hline \multicolumn{4}{|l|}{ Production indices $(2004-06=100)$} \\
\hline Net food & 158 & 123 & 103 \\
\hline Net crops & 134 & 112 & 90 \\
\hline Cereals & 82 & 113 & 145 \\
\hline Vegetable oils & 38 & 61 & 33 \\
\hline Roots and tubers & 28 & 65 & 101 \\
\hline Fruit and vegetables & 35 & 81 & 77 \\
\hline Sugar & 528 & 235 & 104 \\
\hline Livestock & 186 & 125 & 144 \\
\hline Milk & 241 & 144 & 138 \\
\hline Meat & 178 & 126 & 150 \\
\hline Fish & 341 & 184 & 94 \\
\hline \multicolumn{4}{|l|}{ Net trade (mln US\$) } \\
\hline Cereals & & -295 & -828 \\
\hline Fruit and vegetables & 94 & 15 & -68 \\
\hline Meat & -111 & -80 & -379 \\
\hline Dairy products & -105 & -87 & -182 \\
\hline Fish & 65 & 44 & 34 \\
\hline \multicolumn{4}{|l|}{ Environment } \\
\hline Forest area (\%) & 19 & 23 & 28 \\
\hline Renewable water res withdrawn (\% of total) & & & 65 \\
\hline Terrestrial protect areas (\% total land area) & 4 & 4 & 12 \\
\hline Organic area (\% total agricultural area) & & & 0 \\
\hline Water withdrawal by agriculture (\% of total) & & & 65 \\
\hline Biofuel production (thousand kt of oil eq.) & 150 & 87 & 29 \\
\hline \multicolumn{4}{|l|}{ Wood pellet prod. (1 000 tonnes) } \\
\hline Net GHG emissions from AFOLU ( $\mathrm{CO}_{2}$ eq, $\mathrm{Mt}$ ) & -2 & -16 & 0 \\
\hline
\end{tabular}




\begin{tabular}{|c|c|c|c|}
\hline & 1990 & 2000 & 2014 \\
\hline \multicolumn{4}{|l|}{ The setting } \\
\hline Population, total (mln) & 0.8 & 0.9 & 1.2 \\
\hline Population, rural (mln) & 0.3 & 0.3 & 0.3 \\
\hline \multicolumn{4}{|l|}{ Govt expenditure on ag (\% total outlays) } \\
\hline Area harvested (mln ha) & 1 & 1 & 0 \\
\hline Cropping intensity ratio & 4.2 & 5.6 & \\
\hline Water resources (1 $000 \mathrm{~m}^{3} /$ person/year) & 1 & 1 & 1 \\
\hline Area equipped for irrigation (1 $000 \mathrm{ha})$ & & & 46 \\
\hline Area irrigated (\% area equipped for irrigation) & & & 81.9 \\
\hline Employment in agriculture (\%) & 13.5 & 5.2 & 2.9 \\
\hline Employment in agriculture, female (\%) & 15.9 & 4.7 & 1.9 \\
\hline Fertilizers, Nitrogen (kg of nutrients per ha) & & 85.6 & 116.9 \\
\hline Fertilizers, Phosphate (kg of nutrients per ha) & & 58.5 & 59.3 \\
\hline Fertilizers, Potash (kg nutrients per ha) & & 14.1 & 34.5 \\
\hline Energy consump, power irrigation (mln kWh) & 67 & 91 & 91 \\
\hline Agr value added per worker (constant US\$) & 8337 & 12294 & 13906 \\
\hline \multicolumn{4}{|l|}{ Hunger dimensions } \\
\hline \multicolumn{4}{|l|}{ Dietary energy supply (kcal/pc/day) } \\
\hline Average dietary energy supply adequacy (\%) & 114 & 107 & 104 \\
\hline Dietary en supp, cereals/roots/tubers (\%) & 30 & 28 & 28 \\
\hline Prevalence of undernourishment (\%) & $<5.0$ & $<5.0$ & $<5.0$ \\
\hline GDP per capita (US\$, PPP) & 22199 & 27893 & 27394 \\
\hline Domestic food price volatility (index) & & 17.1 & 12.7 \\
\hline Cereal import dependency ratio (\%) & 73.1 & 86.4 & 89.4 \\
\hline \multicolumn{4}{|l|}{ Underweight, children under-5 (\%) } \\
\hline Improved water source (\% pop) & 100 & 100 & 100 \\
\hline \multicolumn{4}{|l|}{ Food supply } \\
\hline Food production value, (2004-2006 mln I\$) & 396 & 437 & 328 \\
\hline Agriculture, value added (\% GDP) & 7 & 4 & 2 \\
\hline Food exports (mln US\$) & 199 & 91 & 233 \\
\hline Food imports (mln US\$) & 193 & 255 & 773 \\
\hline \multicolumn{4}{|l|}{ Production indices (2004-06=100) } \\
\hline Net food & 98 & 108 & 81 \\
\hline Net crops & 136 & 111 & 68 \\
\hline Cereals & 142 & 54 & 101 \\
\hline Vegetable oils & 55 & 100 & 42 \\
\hline Roots and tubers & 137 & 85 & 81 \\
\hline Fruit and vegetables & 145 & 118 & 66 \\
\hline \multicolumn{4}{|l|}{ Sugar } \\
\hline Livestock & 70 & 104 & 90 \\
\hline Milk & 73 & 98 & 105 \\
\hline Meat & 67 & 106 & 84 \\
\hline Fish & 5 & 119 & 11 \\
\hline \multicolumn{4}{|l|}{ Net trade (mln US\$) } \\
\hline Cereals & -81 & -108 & -240 \\
\hline Fruit and vegetables & 150 & 28 & -17 \\
\hline Meat & -15 & -11 & -86 \\
\hline Dairy products & -10 & -8 & 7 \\
\hline Fish & -19 & -24 & -70 \\
\hline \multicolumn{4}{|l|}{ Environment } \\
\hline Forest area (\%) & 17 & 19 & 19 \\
\hline Renewable water res withdrawn (\% of total) & & & 86 \\
\hline Terrestrial protect areas (\% total land area) & 14 & 39 & 41 \\
\hline Organic area (\% total agricultural area) & & & 3 \\
\hline Water withdrawal by agriculture (\% of total) & & & 86 \\
\hline Biofuel production (thousand kt of oil eq.) & & 0 & 162 \\
\hline Wood pellet prod. (1 000 tonnes) & & & 0 \\
\hline Net GHG emissions from AFOLU ( $\mathrm{CO}_{2}$ eq, $\left.\mathrm{Mt}\right)$ & 0 & 0 & 0 \\
\hline
\end{tabular}




\begin{tabular}{|c|c|c|c|}
\hline & 1990 & 2000 & 2014 \\
\hline \multicolumn{4}{|l|}{ The setting } \\
\hline Population, total (mln) & 10.3 & 10.2 & 10.7 \\
\hline Population, rural (mln) & 2.6 & 2.7 & 2.9 \\
\hline Govt expenditure on ag (\% total outlays) & & 2.9 & 3.9 \\
\hline Area harvested (mln ha) & & 6 & 8 \\
\hline Cropping intensity ratio & & 1.5 & \\
\hline Water resources (1 $000 \mathrm{~m}^{3} /$ person/year) & 1 & 1 & 1 \\
\hline Area equipped for irrigation (1 $000 \mathrm{ha})$ & & & 32 \\
\hline Area irrigated (\% area equipped for irrigation) & & & 51.7 \\
\hline Employment in agriculture (\%) & 7.7 & 5.1 & 3.1 \\
\hline Employment in agriculture, female (\%) & 6.3 & 3.7 & 1.9 \\
\hline Fertilizers, Nitrogen (kg of nutrients per ha) & & 67.8 & 74.8 \\
\hline Fertilizers, Phosphate (kg of nutrients per ha) & & 14.2 & 14 \\
\hline Fertilizers, Potash (kg nutrients per ha) & & 9.6 & 17.1 \\
\hline Energy consump, power irrigation (mln kWh) & 0 & & 31 \\
\hline Agr value added per worker (constant US\$) & 4724 & 5709 & 7841 \\
\hline \multicolumn{4}{|l|}{ Hunger dimensions } \\
\hline \multicolumn{4}{|l|}{ Dietary energy supply (kcal/pc/day) } \\
\hline Average dietary energy supply adequacy (\%) & 118 & 122 & 130 \\
\hline Dietary en supp, cereals/roots/tubers (\%) & 34 & 31 & 31 \\
\hline Prevalence of undernourishment (\%) & $<5.0$ & $<5.0$ & $<5.0$ \\
\hline GDP per capita (US\$, PPP) & 19839 & 21003 & 28124 \\
\hline Domestic food price volatility (index) & & 6.6 & 10.7 \\
\hline Cereal import dependency ratio (\%) & -0.7 & -12.7 & -44 \\
\hline Underweight, children under-5 (\%) & 0.9 & 2.1 & \\
\hline Improved water source (\% pop) & 99.8 & 99.8 & 99.8 \\
\hline \multicolumn{4}{|l|}{ Food supply } \\
\hline Food production value, (2004-2006 mln I\$) & 4817 & 3748 & 3476 \\
\hline Agriculture, value added (\% GDP) & 5 & 3 & 3 \\
\hline Food exports (mln US\$) & 738 & 821 & 5473 \\
\hline Food imports (mln US\$) & 621 & 1040 & 6240 \\
\hline \multicolumn{4}{|l|}{ Production indices (2004-06=100) } \\
\hline Net food & 128 & 100 & 92 \\
\hline Net crops & 109 & 102 & 102 \\
\hline Cereals & 85 & 85 & 101 \\
\hline Vegetable oils & 39 & 88 & 140 \\
\hline Roots and tubers & 285 & 172 & 61 \\
\hline Fruit and vegetables & 199 & 185 & 78 \\
\hline Sugar & 127 & 83 & 110 \\
\hline Livestock & 132 & 102 & 85 \\
\hline Milk & 126 & 102 & 103 \\
\hline Meat & 139 & 101 & 74 \\
\hline Fish & 0 & 98 & 94 \\
\hline \multicolumn{4}{|l|}{ Net trade (mln US\$) } \\
\hline Cereals & 5 & 77 & 531 \\
\hline Fruit and vegetables & -133 & -283 & -1028 \\
\hline Meat & 45 & -18 & -800 \\
\hline Dairy products & 184 & 108 & 214 \\
\hline Fish & -31 & -48 & -126 \\
\hline \multicolumn{4}{|l|}{ Environment } \\
\hline Forest area (\%) & 34 & 34 & 34 \\
\hline Renewable water res withdrawn (\% of total) & & & 2 \\
\hline Terrestrial protect areas (\% total land area) & 15 & 16 & 22 \\
\hline Organic area (\% total agricultural area) & & & 11 \\
\hline Water withdrawal by agriculture (\% of total) & & & 2 \\
\hline Biofuel production (thousand kt of oil eq.) & 162 & 1817 & 5382 \\
\hline Wood pellet prod. (1 000 tonnes) & & & 165 \\
\hline Net GHG emissions from AFOLU ( $\mathrm{CO}_{2}$ eq, $\mathrm{Mt}$ ) & 9 & -6 & -6 \\
\hline
\end{tabular}




\begin{tabular}{|c|c|c|c|}
\hline & 1990 & 2000 & 2014 \\
\hline \multicolumn{4}{|l|}{ The setting } \\
\hline Population, total (mln) & 12.1 & 16.1 & 20.8 \\
\hline Population, rural (mln) & 7.3 & 9.1 & 9.7 \\
\hline \multicolumn{4}{|l|}{ Govt expenditure on ag (\% total outlays) } \\
\hline Area harvested (mln ha) & 5 & 7 & 8 \\
\hline Cropping intensity ratio & 0.2 & 0.3 & \\
\hline Water resources (1 $000 \mathrm{~m}^{3} /$ person/year) & 7 & 5 & 4 \\
\hline Area equipped for irrigation (1 $000 \mathrm{ha})$ & & & 73 \\
\hline Area irrigated (\% area equipped for irrigation) & & 92 & \\
\hline \multicolumn{4}{|l|}{ Employment in agriculture (\%) } \\
\hline \multicolumn{4}{|l|}{ Employment in agriculture, female (\%) } \\
\hline Fertilizers, Nitrogen (kg of nutrients per ha) & & 7.1 & 11.6 \\
\hline Fertilizers, Phosphate (kg of nutrients per ha) & & 11.7 & 5.3 \\
\hline Fertilizers, Potash (kg nutrients per ha) & & 10.5 & 8.6 \\
\hline Energy consump, power irrigation (mln kWh) & 0 & 87 & 87 \\
\hline Agr value added per worker (constant US\$) & & & 1350 \\
\hline \multicolumn{4}{|l|}{ Hunger dimensions } \\
\hline Dietary energy supply (kcal/pc/day) & 2548 & 2627 & 2802 \\
\hline Average dietary energy supply adequacy (\%) & 121 & 124 & 131 \\
\hline Dietary en supp, cereals/roots/tubers (\%) & 66 & 65 & 67 \\
\hline Prevalence of undernourishment (\%) & 11.2 & 14.9 & 13.4 \\
\hline GDP per capita (US\$, PPP) & 3234 & 3049 & 3108 \\
\hline Domestic food price volatility (index) & & 20.3 & 8.8 \\
\hline Cereal import dependency ratio (\%) & 37.6 & 44.6 & 52.4 \\
\hline Underweight, children under-5 (\%) & & 18.2 & 15.7 \\
\hline Improved water source (\% pop) & 76 & 77.5 & 80.2 \\
\hline \multicolumn{4}{|l|}{ Food supply } \\
\hline Food production value, (2004-2006 mln I\$) & 3166 & 4537 & 6009 \\
\hline Agriculture, value added (\% GDP) & 32 & 25 & 22 \\
\hline Food exports (mln US\$) & 1064 & 1342 & 4193 \\
\hline Food imports (mln US\$) & 379 & 308 & 1883 \\
\hline \multicolumn{4}{|l|}{ Production indices (2004-06=100) } \\
\hline Net food & 66 & 95 & 126 \\
\hline Net crops & 68 & 101 & 124 \\
\hline Cereals & 88 & 90 & 224 \\
\hline Vegetable oils & 92 & 99 & 152 \\
\hline Roots and tubers & 62 & 87 & 110 \\
\hline Fruit and vegetables & 77 & 111 & 105 \\
\hline Sugar & 94 & 115 & 135 \\
\hline Livestock & 81 & 90 & 131 \\
\hline Milk & 61 & 90 & 109 \\
\hline Meat & 84 & 89 & 131 \\
\hline Fish & 185 & 158 & 159 \\
\hline \multicolumn{4}{|l|}{ Net trade (mln US\$) } \\
\hline Cereals & -169 & -143 & -1355 \\
\hline Fruit and vegetables & 64 & 168 & 457 \\
\hline Meat & -41 & -12 & -76 \\
\hline Dairy products & -47 & -25 & -89 \\
\hline Fish & 9 & -3 & -242 \\
\hline \multicolumn{4}{|l|}{ Environment } \\
\hline Forest area (\%) & 32 & 32 & 33 \\
\hline Renewable water res withdrawn (\% of total) & & & 38 \\
\hline Terrestrial protect areas (\% total land area) & 23 & 23 & 23 \\
\hline Organic area (\% total agricultural area) & & & 0 \\
\hline Water withdrawal by agriculture (\% of total) & & & 38 \\
\hline Biofuel production (thousand kt of oil eq.) & 4 & 5 & 5 \\
\hline \multicolumn{4}{|l|}{ Wood pellet prod. (1 000 tonnes) } \\
\hline Net GHG emissions from AFOLU ( $\mathrm{CO}_{2}$ eq, $\left.\mathrm{Mt}\right)$ & 6 & 0 & 10 \\
\hline
\end{tabular}




\begin{tabular}{|c|c|c|c|}
\hline & 1990 & 2000 & 2014 \\
\hline \multicolumn{4}{|l|}{ The setting } \\
\hline Population, total (mln) & 34.9 & 46.9 & 69.4 \\
\hline Population, rural (mln) & 25.2 & 33.2 & 44.4 \\
\hline \multicolumn{4}{|l|}{ Govt expenditure on ag (\% total outlays) } \\
\hline Area harvested (mln ha) & 20 & 17 & 18 \\
\hline Cropping intensity ratio & 0.8 & 0.7 & \\
\hline Water resources (1 $000 \mathrm{~m}^{3} /$ person/year) & 37 & 27 & 19 \\
\hline Area equipped for irrigation (1 $000 \mathrm{ha})$ & & & 11 \\
\hline Area irrigated (\% area equipped for irrigation) & & 76.2 & \\
\hline \multicolumn{4}{|l|}{ Employment in agriculture (\%) } \\
\hline \multicolumn{4}{|l|}{ Employment in agriculture, female (\%) } \\
\hline Fertilizers, Nitrogen (kg of nutrients per ha) & & 0.2 & 0.3 \\
\hline Fertilizers, Phosphate (kg of nutrients per ha) & & 0.1 & 0.3 \\
\hline Fertilizers, Potash (kg nutrients per ha) & & 0.1 & 0.3 \\
\hline Energy consump, power irrigation (mln kWh) & 0 & 0 & 0 \\
\hline Agr value added per worker (constant US\$) & 265 & 226 & 227 \\
\hline \multicolumn{4}{|l|}{ Hunger dimensions } \\
\hline \multicolumn{4}{|l|}{ Dietary energy supply (kcal/pc/day) } \\
\hline \multicolumn{4}{|l|}{ Average dietary energy supply adequacy (\%) } \\
\hline \multicolumn{4}{|l|}{ Dietary en supp, cereals/roots/tubers (\%) } \\
\hline \multicolumn{4}{|l|}{ Prevalence of undernourishment (\%) } \\
\hline GDP per capita (US\$, PPP) & 1375 & 575 & 783 \\
\hline \multicolumn{4}{|l|}{ Domestic food price volatility (index) } \\
\hline Cereal import dependency ratio (\%) & 18.4 & 20.4 & 33.7 \\
\hline Underweight, children under-5 (\%) & & 33.6 & 23.4 \\
\hline Improved water source (\% pop) & 43.2 & 44 & 46.5 \\
\hline \multicolumn{4}{|l|}{ Food supply } \\
\hline Food production value, (2004-2006 mln I\$) & 4247 & 3686 & 4236 \\
\hline Agriculture, value added (\% GDP) & 31 & 32 & 21 \\
\hline Food exports (mln US\$) & 8 & 4 & 32 \\
\hline Food imports (mln US\$) & 221 & 193 & 950 \\
\hline \multicolumn{4}{|l|}{ Production indices (2004-06=100) } \\
\hline Net food & 117 & 102 & 117 \\
\hline Net crops & 122 & 102 & 116 \\
\hline Cereals & 103 & 103 & 117 \\
\hline Vegetable oils & 117 & 97 & 146 \\
\hline Roots and tubers & 121 & 105 & 112 \\
\hline Fruit and vegetables & 126 & 97 & 116 \\
\hline Sugar & 111 & 110 & 131 \\
\hline Livestock & 99 & 101 & 121 \\
\hline Milk & 139 & 90 & 170 \\
\hline Meat & 99 & 101 & 121 \\
\hline Fish & 69 & 104 & 97 \\
\hline \multicolumn{4}{|l|}{ Net trade (mln US\$) } \\
\hline Cereals & -121 & -74 & -418 \\
\hline Fruit and vegetables & -11 & -11 & -63 \\
\hline Meat & -49 & -50 & -173 \\
\hline Dairy products & -16 & -15 & -49 \\
\hline Fish & -40 & -26 & -175 \\
\hline \multicolumn{4}{|l|}{ Environment } \\
\hline Forest area (\%) & 71 & 69 & 68 \\
\hline Renewable water res withdrawn (\% of total) & & & 10 \\
\hline Terrestrial protect areas (\% total land area) & 10 & 10 & 12 \\
\hline Organic area (\% total agricultural area) & & & 0 \\
\hline Water withdrawal by agriculture (\% of total) & & & 10 \\
\hline Biofuel production (thousand kt of oil eq.) & 2 & 2 & \\
\hline \multicolumn{4}{|l|}{ Wood pellet prod. (1 000 tonnes) } \\
\hline Net GHG emissions from AFOLU ( $\mathrm{CO}_{2}$ eq, $\left.\mathrm{Mt}\right)$ & 187 & 184 & 182 \\
\hline
\end{tabular}




\begin{tabular}{|c|c|c|c|}
\hline & 1990 & 2000 & 2014 \\
\hline \multicolumn{4}{|l|}{ The setting } \\
\hline Population, total (mln) & 5.1 & 5.3 & 5.6 \\
\hline Population, rural (mln) & 0.8 & 0.8 & 0.7 \\
\hline \multicolumn{4}{|l|}{ Govt expenditure on ag (\% total outlays) } \\
\hline Area harvested (mln ha) & 10 & 9 & 9 \\
\hline Cropping intensity ratio & 3.4 & 3.6 & \\
\hline Water resources (1 $000 \mathrm{~m}^{3} /$ person/year) & 1 & 1 & 1 \\
\hline Area equipped for irrigation (1 $000 \mathrm{ha})$ & & & 435 \\
\hline Area irrigated (\% area equipped for irrigation) & & & 58.4 \\
\hline Employment in agriculture (\%) & 5.5 & 3.3 & 2.6 \\
\hline Employment in agriculture, female (\%) & 2.8 & 1.6 & 1.1 \\
\hline Fertilizers, Nitrogen (kg of nutrients per ha) & & 96.3 & 72.9 \\
\hline Fertilizers, Phosphate (kg of nutrients per ha) & & 7 & 13.8 \\
\hline Fertilizers, Potash (kg nutrients per ha) & & 33.1 & 25.7 \\
\hline \multicolumn{4}{|l|}{ Energy consump, power irrigation (mln kWh) } \\
\hline Agr value added per worker (constant US\$) & 12426 & 29317 & 41870 \\
\hline \multicolumn{4}{|l|}{ Hunger dimensions } \\
\hline \multicolumn{4}{|l|}{ Dietary energy supply (kcal/pc/day) } \\
\hline Average dietary energy supply adequacy (\%) & 130 & 131 & 132 \\
\hline Dietary en supp, cereals/roots/tubers (\%) & 28 & 29 & 29 \\
\hline Prevalence of undernourishment (\%) & $<5.0$ & $<5.0$ & $<5.0$ \\
\hline GDP per capita (US\$, PPP) & 33256 & 41693 & 42483 \\
\hline Domestic food price volatility (index) & & 5.6 & 6 \\
\hline Cereal import dependency ratio (\%) & -26.9 & -15.9 & -13.7 \\
\hline \multicolumn{4}{|l|}{ Underweight, children under-5 (\%) } \\
\hline Improved water source (\% pop) & 100 & 100 & 100 \\
\hline \multicolumn{4}{|l|}{ Food supply } \\
\hline Food production value, (2004-2006 mln I\$) & 5522 & 5738 & 5980 \\
\hline Agriculture, value added (\% GDP) & 4 & 2 & 1 \\
\hline Food exports (mln US\$) & 6719 & 6794 & 13771 \\
\hline Food imports (mln US\$) & 1540 & 2485 & 6842 \\
\hline \multicolumn{4}{|l|}{ Production indices $(2004-06=100)$} \\
\hline Net food & 95 & 98 & 103 \\
\hline Net crops & 116 & 103 & 104 \\
\hline Cereals & 103 & 104 & 99 \\
\hline Vegetable oils & 191 & 72 & 166 \\
\hline Roots and tubers & 97 & 109 & 105 \\
\hline Fruit and vegetables & 112 & 86 & 108 \\
\hline Sugar & 134 & 127 & 87 \\
\hline Livestock & 82 & 95 & 99 \\
\hline Milk & 103 & 103 & 111 \\
\hline Meat & 73 & 92 & 94 \\
\hline Fish & 152 & 158 & 70 \\
\hline \multicolumn{4}{|l|}{ Net trade (mln US\$) } \\
\hline Cereals & 686 & 448 & 477 \\
\hline Fruit and vegetables & -223 & -454 & -1084 \\
\hline Meat & 3082 & 2809 & 3840 \\
\hline Dairy products & 1125 & 1142 & 1910 \\
\hline Fish & 1049 & 949 & 1029 \\
\hline \multicolumn{4}{|l|}{ Environment } \\
\hline Forest area (\%) & 10 & 11 & 13 \\
\hline Renewable water res withdrawn (\% of total) & & & 36 \\
\hline Terrestrial protect areas (\% total land area) & 12 & 18 & 18 \\
\hline Organic area (\% total agricultural area) & & & 7 \\
\hline Water withdrawal by agriculture (\% of total) & & & 36 \\
\hline Biofuel production (thousand kt of oil eq.) & 12 & 28 & 2102 \\
\hline Wood pellet prod. (1 000 tonnes) & & & 92 \\
\hline Net GHG emissions from AFOLU ( $\mathrm{CO}_{2}$ eq, $\mathrm{Mt}$ ) & 13 & 11 & 11 \\
\hline
\end{tabular}




\begin{tabular}{|c|c|c|c|}
\hline & 1990 & 2000 & 2014 \\
\hline \multicolumn{4}{|l|}{ The setting } \\
\hline Population, total (mln) & 0.6 & 0.7 & 0.9 \\
\hline Population, rural (mln) & 0.1 & 0.2 & 0.2 \\
\hline \multicolumn{4}{|l|}{ Govt expenditure on ag (\% total outlays) } \\
\hline Area harvested (mln ha) & 0 & 0 & 0 \\
\hline Cropping intensity ratio & 0 & 0 & \\
\hline Water resources (1 $000 \mathrm{~m}^{3} /$ person/year) & 1 & 0 & 0 \\
\hline Area equipped for irrigation (1 $000 \mathrm{ha}$ ) & & & 1 \\
\hline Area irrigated (\% area equipped for irrigation) & & 38.3 & \\
\hline \multicolumn{4}{|l|}{ Employment in agriculture (\%) } \\
\hline \multicolumn{4}{|l|}{ Employment in agriculture, female (\%) } \\
\hline \multicolumn{4}{|l|}{ Fertilizers, Nitrogen (kg of nutrients per ha) } \\
\hline \multicolumn{4}{|l|}{ Fertilizers, Phosphate (kg of nutrients per ha) } \\
\hline \multicolumn{4}{|l|}{ Fertilizers, Potash (kg nutrients per ha) } \\
\hline \multicolumn{4}{|l|}{ Energy consump, power irrigation (mln kWh) } \\
\hline Agr value added per worker (constant US\$) & 108 & 81 & 89 \\
\hline \multicolumn{4}{|l|}{ Hunger dimensions } \\
\hline Dietary energy supply (kcal/pc/day) & 1559 & 1959 & 2662 \\
\hline Average dietary energy supply adequacy (\%) & 71 & 86 & 114 \\
\hline Dietary en supp, cereals/roots/tubers (\%) & 56 & 58 & 56 \\
\hline Prevalence of undernourishment (\%) & 75.9 & 52.4 & 17.3 \\
\hline GDP per capita (US\$, PPP) & 3050 & 2078 & 2902 \\
\hline \multicolumn{4}{|l|}{ Domestic food price volatility (index) } \\
\hline Cereal import dependency ratio (\%) & 100 & 100 & 100 \\
\hline Underweight, children under-5 (\%) & & 25.4 & 29.8 \\
\hline Improved water source (\% pop) & 76.6 & 82.3 & 92.1 \\
\hline \multicolumn{4}{|l|}{ Food supply } \\
\hline Food production value, (2004-2006 mln I\$) & 51 & 46 & 73 \\
\hline Agriculture, value added (\% GDP) & 3 & 4 & 4 \\
\hline Food exports (mln US\$) & 7 & 3 & 66 \\
\hline Food imports (mln US\$) & 50 & 77 & 760 \\
\hline \multicolumn{4}{|l|}{ Production indices $(2004-06=100)$} \\
\hline Net food & 94 & 85 & 134 \\
\hline Net crops & 82 & 103 & 137 \\
\hline Cereals & 100 & 110 & 161 \\
\hline \multicolumn{4}{|l|}{ Vegetable oils } \\
\hline \multicolumn{4}{|l|}{ Roots and tubers } \\
\hline Fruit and vegetables & 78 & 98 & 134 \\
\hline Sugar & 73 & 100 & 100 \\
\hline Livestock & 96 & 82 & 134 \\
\hline Milk & 78 & 92 & 107 \\
\hline Meat & 98 & 81 & 137 \\
\hline \multicolumn{4}{|l|}{ Fish } \\
\hline \multicolumn{4}{|l|}{ Net trade (mln US\$) } \\
\hline Cereals & -20 & -35 & -265 \\
\hline Fruit and vegetables & -8 & -4 & -26 \\
\hline Meat & -2 & -1 & -19 \\
\hline Dairy products & -6 & -16 & -17 \\
\hline Fish & 0 & -2 & -3 \\
\hline \multicolumn{4}{|l|}{ Environment } \\
\hline Forest area (\%) & 0 & 0 & 0 \\
\hline Renewable water res withdrawn (\% of total) & & 16 & 16 \\
\hline Terrestrial protect areas (\% total land area) & 0 & 0 & 0 \\
\hline \multicolumn{4}{|l|}{ Organic area (\% total agricultural area) } \\
\hline Water withdrawal by agriculture (\% of total) & & 16 & 16 \\
\hline Biofuel production (thousand kt of oil eq.) & & & \\
\hline Wood pellet prod. (1 000 tonnes) & & & \\
\hline Net GHG emissions from AFOLU ( $\mathrm{CO}_{2}$ eq, $\left.\mathrm{Mt}\right)$ & 0 & 1 & 1 \\
\hline
\end{tabular}




\begin{tabular}{|c|c|c|c|}
\hline & 1990 & 2000 & 2014 \\
\hline \multicolumn{4}{|l|}{ The setting } \\
\hline Population, total (mln) & 7.2 & 8.7 & 10.5 \\
\hline Population, rural (mln) & 3.2 & 3.3 & 3 \\
\hline \multicolumn{4}{|l|}{ Govt expenditure on ag (\% total outlays) } \\
\hline Area harvested (mln ha) & 7 & 5 & 5 \\
\hline Cropping intensity ratio & 2.6 & 1.8 & \\
\hline Water resources (1 $000 \mathrm{~m}^{3} /$ person/year) & 3 & 2 & 2 \\
\hline Area equipped for irrigation (1 $000 \mathrm{ha})$ & & & 307 \\
\hline \multicolumn{4}{|l|}{ Area irrigated (\% area equipped for irrigation) } \\
\hline Employment in agriculture (\%) & 16.9 & 15.9 & 14.5 \\
\hline Employment in agriculture, female (\%) & 2.8 & 2.5 & 2.5 \\
\hline Fertilizers, Nitrogen (kg of nutrients per ha) & & 49.6 & 63.1 \\
\hline Fertilizers, Phosphate (kg of nutrients per ha) & & 0 & 18.8 \\
\hline Fertilizers, Potash (kg nutrients per ha) & & 2.6 & 11.7 \\
\hline Energy consump, power irrigation (mln kWh) & 0 & 0 & 0 \\
\hline Agr value added per worker (constant US\$) & 2460 & 3559 & 8181 \\
\hline \multicolumn{4}{|l|}{ Hunger dimensions } \\
\hline Dietary energy supply (kcal/pc/day) & 2205 & 2211 & 2609 \\
\hline Average dietary energy supply adequacy (\%) & 97 & 96 & 111 \\
\hline Dietary en supp, cereals/roots/tubers (\%) & 34 & 32 & 32 \\
\hline Prevalence of undernourishment (\%) & 30.7 & 30.7 & 12.5 \\
\hline GDP per capita (US\$, PPP) & 5284 & 7963 & 11796 \\
\hline Domestic food price volatility (index) & & 8 & 5.2 \\
\hline Cereal import dependency ratio (\%) & 71.8 & 75.8 & 73.9 \\
\hline Underweight, children under-5 (\%) & 8.4 & 3.5 & 4 \\
\hline Improved water source (\% pop) & 88.8 & 85.8 & 80.9 \\
\hline \multicolumn{4}{|l|}{ Food supply } \\
\hline Food production value, (2004-2006 mln I\$) & 1480 & 1792 & 2891 \\
\hline Agriculture, value added (\% GDP) & 15 & 7 & 6 \\
\hline Food exports (mln US\$) & 301 & 243 & 793 \\
\hline Food imports (mln US\$) & 230 & 379 & 1457 \\
\hline \multicolumn{4}{|l|}{ Production indices (2004-06=100) } \\
\hline Net food & 69 & 84 & 136 \\
\hline Net crops & 82 & 88 & 148 \\
\hline Cereals & 69 & 90 & 128 \\
\hline Vegetable oils & 67 & 91 & 174 \\
\hline Roots and tubers & 80 & 103 & 118 \\
\hline Fruit and vegetables & 61 & 84 & 174 \\
\hline Sugar & 128 & 89 & 94 \\
\hline Livestock & 58 & 80 & 114 \\
\hline Milk & 77 & 94 & 148 \\
\hline Meat & 56 & 79 & 107 \\
\hline \multicolumn{4}{|l|}{ Fish } \\
\hline \multicolumn{4}{|l|}{ Net trade (mln US\$) } \\
\hline Cereals & -113 & -143 & -695 \\
\hline Fruit and vegetables & 29 & 65 & 220 \\
\hline Meat & 22 & -10 & -46 \\
\hline Dairy products & -32 & -42 & -77 \\
\hline \multicolumn{4}{|l|}{ Fish } \\
\hline \multicolumn{4}{|l|}{ Environment } \\
\hline Forest area $(\%)$ & 41 & 41 & 41 \\
\hline Renewable water res withdrawn (\% of total) & & & 80 \\
\hline Terrestrial protect areas (\% total land area) & 20 & 22 & 19 \\
\hline Organic area (\% total agricultural area) & & & 8 \\
\hline Water withdrawal by agriculture (\% of total) & & & 80 \\
\hline Biofuel production (thousand kt of oil eq.) & 15 & 20 & 22 \\
\hline \multicolumn{4}{|l|}{ Wood pellet prod. (1 000 tonnes) } \\
\hline Net GHG emissions from AFOLU ( $\mathrm{CO}_{2}$ eq, $\mathrm{Mt}$ ) & 9 & 5 & 8 \\
\hline
\end{tabular}




\begin{tabular}{|c|c|c|c|}
\hline & 1990 & 2000 & 2014 \\
\hline \multicolumn{4}{|l|}{ The setting } \\
\hline Population, total (mln) & 10.1 & 12.5 & 16 \\
\hline Population, rural (mln) & 4.5 & 5 & 4.9 \\
\hline Govt expenditure on ag (\% total outlays) & & & 68.6 \\
\hline Area harvested (mln ha) & 6 & 8 & 7 \\
\hline Cropping intensity ratio & 0.7 & 1 & \\
\hline Water resources (1 $000 \mathrm{~m}^{3} /$ person/year) & 45 & 36 & 29 \\
\hline Area equipped for irrigation (1 $000 \mathrm{ha}$ ) & & & 1500 \\
\hline Area irrigated (\% area equipped for irrigation) & & & 62.8 \\
\hline Employment in agriculture (\%) & 7.5 & 29.3 & 27.8 \\
\hline Employment in agriculture, female (\%) & 2.5 & 21 & 21.2 \\
\hline Fertilizers, Nitrogen (kg of nutrients per ha) & & 92.3 & 145.5 \\
\hline Fertilizers, Phosphate (kg of nutrients per ha) & & 26.1 & 28.6 \\
\hline Fertilizers, Potash (kg nutrients per ha) & & 38.5 & 73.1 \\
\hline Energy consump, power irrigation (mln kWh) & 0 & 411 & 411 \\
\hline Agr value added per worker (constant US\$) & 1946 & 2606 & 4322 \\
\hline \multicolumn{4}{|l|}{ Hunger dimensions } \\
\hline Dietary energy supply (kcal/pc/day) & 2328 & 2275 & 2498 \\
\hline Average dietary energy supply adequacy (\%) & 108 & 104 & 112 \\
\hline Dietary en supp, cereals/roots/tubers (\%) & 40 & 39 & 34 \\
\hline Prevalence of undernourishment (\%) & 17 & 17.8 & 11.1 \\
\hline GDP per capita (US\$, PPP) & 7542 & 7444 & 10541 \\
\hline Domestic food price volatility (index) & & 22.2 & 5.7 \\
\hline Cereal import dependency ratio (\%) & 17.3 & 30.6 & 36.4 \\
\hline Underweight, children under-5 (\%) & & 12.5 & 6.4 \\
\hline Improved water source (\% pop) & 73.9 & 79.6 & 86.4 \\
\hline \multicolumn{4}{|l|}{ Food supply } \\
\hline Food production value, (2004-2006 mln I\$) & 3423 & 5605 & 6972 \\
\hline Agriculture, value added (\% GDP) & 21 & 16 & 9 \\
\hline Food exports (mln US\$) & 622 & 1090 & 3422 \\
\hline Food imports (mln US\$) & 142 & 249 & 1262 \\
\hline \multicolumn{4}{|l|}{ Production indices $(2004-06=100)$} \\
\hline Net food & 57 & 93 & 115 \\
\hline Net crops & 66 & 96 & 104 \\
\hline Cereals & 55 & 79 & 116 \\
\hline Vegetable oils & 59 & 77 & 119 \\
\hline Roots and tubers & 108 & 65 & 89 \\
\hline Fruit and vegetables & 59 & 101 & 101 \\
\hline Sugar & 86 & 81 & 108 \\
\hline Livestock & 51 & 91 & 131 \\
\hline Milk & 57 & 99 & 129 \\
\hline Meat & 42 & 80 & 130 \\
\hline Fish & 64 & 116 & 150 \\
\hline \multicolumn{4}{|l|}{ Net trade (mln US\$) } \\
\hline Cereals & -97 & -93 & -487 \\
\hline Fruit and vegetables & 474 & 890 & 2303 \\
\hline Meat & 0 & 3 & -30 \\
\hline Dairy products & -1 & 0 & 28 \\
\hline Fish & 466 & 585 & 2687 \\
\hline \multicolumn{4}{|l|}{ Environment } \\
\hline Forest area (\%) & 50 & 48 & 38 \\
\hline Renewable water res withdrawn (\% of total) & & & 81 \\
\hline Terrestrial protect areas (\% total land area) & 22 & 24 & 24 \\
\hline Organic area (\% total agricultural area) & & & 1 \\
\hline Water withdrawal by agriculture (\% of total) & & & 81 \\
\hline Biofuel production (thousand kt of oil eq.) & 8 & 13 & 13 \\
\hline \multicolumn{4}{|l|}{ Wood pellet prod. (1 000 tonnes) } \\
\hline Net GHG emissions from AFOLU ( $\mathrm{CO}_{2}$ eq, $\mathrm{Mt}$ ) & 90 & 93 & 97 \\
\hline
\end{tabular}




\begin{tabular}{|c|c|c|c|}
\hline & 1990 & 2000 & 2014 \\
\hline \multicolumn{4}{|l|}{ The setting } \\
\hline Population, total (mln) & 56.3 & 66.1 & 83.4 \\
\hline Population, rural (mln) & 31.8 & 37.8 & 46.7 \\
\hline Govt expenditure on ag (\% total outlays) & & 5.1 & 1.9 \\
\hline Area harvested (mln ha) & 13 & 20 & 24 \\
\hline Cropping intensity ratio & 4.9 & 6.1 & \\
\hline Water resources (1 $000 \mathrm{~m}^{3} /$ person/year) & 1 & 1 & 1 \\
\hline Area equipped for irrigation (1 $000 \mathrm{ha}$ ) & & & 3650 \\
\hline Area irrigated (\% area equipped for irrigation) & & 100 & \\
\hline Employment in agriculture (\%) & 39 & 29.6 & 29.2 \\
\hline Employment in agriculture, female (\%) & 52 & 39.4 & 43.3 \\
\hline Fertilizers, Nitrogen (kg of nutrients per ha) & & 550 & 430.7 \\
\hline Fertilizers, Phosphate (kg of nutrients per ha) & & 61.4 & 133.2 \\
\hline Fertilizers, Potash (kg nutrients per ha) & & 16.3 & 11.5 \\
\hline Energy consump, power irrigation (mln kWh) & 165 & 482 & 948 \\
\hline Agr value added per worker (constant US\$) & 1210 & 1677 & 2562 \\
\hline \multicolumn{4}{|l|}{ Hunger dimensions } \\
\hline Dietary energy supply (kcal/pc/day) & 3211 & 3378 & 3547 \\
\hline Average dietary energy supply adequacy (\%) & 143 & 148 & 152 \\
\hline Dietary en supp, cereals/roots/tubers (\%) & 68 & 66 & 65 \\
\hline Prevalence of undernourishment (\%) & $<5.0$ & $<5.0$ & $<5.0$ \\
\hline GDP per capita (US\$, PPP) & 6024 & 7812 & 10734 \\
\hline Domestic food price volatility (index) & & 3.7 & 9.8 \\
\hline Cereal import dependency ratio (\%) & 36 & 33.8 & 44.2 \\
\hline Underweight, children under-5 (\%) & 8.2 & 4.3 & 6.8 \\
\hline Improved water source (\% pop) & 92.9 & 96.1 & 99.3 \\
\hline \multicolumn{4}{|l|}{ Food supply } \\
\hline Food production value, (2004-2006 mln I\$) & 9850 & 15585 & 21720 \\
\hline Agriculture, value added (\% GDP) & 19 & 17 & 14 \\
\hline Food exports (mln US\$) & 201 & 309 & 3523 \\
\hline Food imports (mln US\$) & 2501 & 2737 & 13416 \\
\hline \multicolumn{4}{|l|}{ Production indices $(2004-06=100)$} \\
\hline Net food & 54 & 85 & 119 \\
\hline Net crops & 56 & 87 & 114 \\
\hline Cereals & 57 & 92 & 101 \\
\hline Vegetable oils & 40 & 84 & 108 \\
\hline Roots and tubers & 60 & 63 & 168 \\
\hline Fruit and vegetables & 52 & 84 & 119 \\
\hline Sugar & 57 & 93 & 141 \\
\hline Livestock & 51 & 82 & 131 \\
\hline Milk & 48 & 79 & 121 \\
\hline Meat & 51 & 83 & 136 \\
\hline Fish & 34 & 80 & 160 \\
\hline \multicolumn{4}{|l|}{ Net trade (mln US\$) } \\
\hline Cereals & -1302 & -1175 & -5791 \\
\hline Fruit and vegetables & 55 & -80 & 762 \\
\hline Meat & -192 & -303 & -1154 \\
\hline Dairy products & -179 & -174 & -400 \\
\hline Fish & -68 & -170 & -764 \\
\hline \multicolumn{4}{|l|}{ Environment } \\
\hline Forest area (\%) & 0 & 0 & 0 \\
\hline Renewable water res withdrawn (\% of total) & & 86 & 86 \\
\hline Terrestrial protect areas (\% total land area) & 0 & 4 & 11 \\
\hline Organic area (\% total agricultural area) & & & 2 \\
\hline Water withdrawal by agriculture (\% of total) & & 86 & 86 \\
\hline Biofuel production (thousand kt of oil eq.) & 44 & 57 & 58 \\
\hline Wood pellet prod. (1 000 tonnes) & & & 20 \\
\hline Net GHG emissions from AFOLU ( $\mathrm{CO}_{2}$ eq, $\mathrm{Mt}$ ) & 18 & 24 & 29 \\
\hline
\end{tabular}




\begin{tabular}{|c|c|c|c|}
\hline & 1990 & 2000 & 2014 \\
\hline \multicolumn{4}{|l|}{ The setting } \\
\hline Population, total (mln) & 5.3 & 6 & 6.4 \\
\hline Population, rural (mln) & 2.7 & 2.4 & 2.2 \\
\hline Govt expenditure on ag (\% total outlays) & & 1 & 1.8 \\
\hline Area harvested (mln ha) & 3 & 5 & 7 \\
\hline Cropping intensity ratio & 2.1 & 3.4 & \\
\hline Water resources (1 $000 \mathrm{~m}^{3} /$ person/year) & 5 & 4 & 4 \\
\hline Area equipped for irrigation (1 $000 \mathrm{ha}$ ) & & & 45 \\
\hline Area irrigated (\% area equipped for irrigation) & & & 74.8 \\
\hline Employment in agriculture (\%) & 7.4 & 21.6 & 21 \\
\hline Employment in agriculture, female (\%) & 2.7 & 3.8 & 5 \\
\hline Fertilizers, Nitrogen (kg of nutrients per ha) & & 53.6 & 104.5 \\
\hline Fertilizers, Phosphate (kg of nutrients per ha) & & 15.4 & 39.8 \\
\hline Fertilizers, Potash (kg nutrients per ha) & & 9.2 & 28.2 \\
\hline Energy consump, power irrigation (mln kWh) & 11 & 11 & 11 \\
\hline Agr value added per worker (constant US\$) & 2133 & 2382 & 3489 \\
\hline \multicolumn{4}{|l|}{ Hunger dimensions } \\
\hline Dietary energy supply (kcal/pc/day) & 2391 & 2527 & 2568 \\
\hline Average dietary energy supply adequacy (\%) & 111 & 116 & 113 \\
\hline Dietary en supp, cereals/roots/tubers (\%) & 58 & 52 & 49 \\
\hline Prevalence of undernourishment (\%) & 15.4 & 12.5 & 12.6 \\
\hline GDP per capita (US\$, PPP) & 4454 & 6266 & 7515 \\
\hline Domestic food price volatility (index) & & 14.3 & 3 \\
\hline Cereal import dependency ratio (\%) & 27.8 & 41.5 & 41.8 \\
\hline Underweight, children under-5 (\%) & 7.2 & 6.1 & 6.6 \\
\hline Improved water source (\% pop) & 74.8 & 83.6 & 90.1 \\
\hline \multicolumn{4}{|l|}{ Food supply } \\
\hline Food production value, (2004-2006 mln I\$) & 635 & 804 & 950 \\
\hline Agriculture, value added (\% GDP) & 17 & 10 & 11 \\
\hline Food exports (mln US\$) & 60 & 244 & 679 \\
\hline Food imports (mln US\$) & 127 & 584 & 1297 \\
\hline \multicolumn{4}{|l|}{ Production indices $(2004-06=100)$} \\
\hline Net food & 75 & 95 & 113 \\
\hline Net crops & 94 & 102 & 113 \\
\hline Cereals & 97 & 90 & 118 \\
\hline Vegetable oils & 180 & 129 & 123 \\
\hline Roots and tubers & 21 & 155 & 141 \\
\hline Fruit and vegetables & 86 & 83 & 105 \\
\hline Sugar & 61 & 106 & 147 \\
\hline Livestock & 62 & 90 & 107 \\
\hline Milk & 64 & 90 & 113 \\
\hline Meat & 58 & 91 & 104 \\
\hline Fish & 20 & 22 & 127 \\
\hline \multicolumn{4}{|l|}{ Net trade (mln US\$) } \\
\hline Cereals & -33 & -118 & -208 \\
\hline Fruit and vegetables & -14 & -84 & -107 \\
\hline Meat & 0 & -38 & -115 \\
\hline Dairy products & -20 & -75 & -135 \\
\hline Fish & 9 & 18 & 63 \\
\hline \multicolumn{4}{|l|}{ Environment } \\
\hline Forest area (\%) & 18 & 16 & 13 \\
\hline Renewable water res withdrawn (\% of total) & & & 68 \\
\hline Terrestrial protect areas (\% total land area) & 0 & 0 & 8 \\
\hline Organic area (\% total agricultural area) & & & 0 \\
\hline Water withdrawal by agriculture (\% of total) & & & 68 \\
\hline Biofuel production (thousand kt of oil eq.) & 6 & 9 & 22 \\
\hline \multicolumn{4}{|l|}{ Wood pellet prod. (1 000 tonnes) } \\
\hline Net GHG emissions from AFOLU ( $\mathrm{CO}_{2}$ eq, $\mathrm{Mt}$ ) & 4 & 4 & 4 \\
\hline
\end{tabular}




\section{Eritrea}

\begin{tabular}{|c|c|c|c|}
\hline & 1990 & 2000 & 2014 \\
\hline \multicolumn{4}{|l|}{ The setting } \\
\hline Population, total (mln) & 3.3 & 3.9 & 6.5 \\
\hline Population, rural (mln) & 2.8 & 3.2 & 5.1 \\
\hline \multicolumn{4}{|l|}{ Govt expenditure on ag (\% total outlays) } \\
\hline Area harvested (mln ha) & & 0 & 0 \\
\hline Cropping intensity ratio & & 0 & \\
\hline Water resources (1 $000 \mathrm{~m}^{3} /$ person/year) & 2 & 2 & 1 \\
\hline Area equipped for irrigation (1 $000 \mathrm{ha}$ ) & & & 21 \\
\hline Area irrigated (\% area equipped for irrigation) & 62.5 & 62.5 & \\
\hline \multicolumn{4}{|l|}{ Employment in agriculture (\%) } \\
\hline \multicolumn{4}{|l|}{ Employment in agriculture, female (\%) } \\
\hline Fertilizers, Nitrogen (kg of nutrients per ha) & & 1.1 & 1.2 \\
\hline Fertilizers, Phosphate (kg of nutrients per ha) & & 0.2 & 0.1 \\
\hline Fertilizers, Potash (kg nutrients per ha) & & 0.2 & 0.1 \\
\hline Energy consump, power irrigation (mln kWh) & 0 & 0 & 0 \\
\hline Agr value added per worker (constant US\$) & 162 & 132 & 106 \\
\hline \multicolumn{4}{|l|}{ Hunger dimensions } \\
\hline \multicolumn{4}{|l|}{ Dietary energy supply (kcal/pc/day) } \\
\hline \multicolumn{4}{|l|}{ Average dietary energy supply adequacy (\%) } \\
\hline \multicolumn{4}{|l|}{ Dietary en supp, cereals/roots/tubers (\%) } \\
\hline \multicolumn{4}{|l|}{ Prevalence of undernourishment (\%) } \\
\hline GDP per capita (US\$, PPP) & 1175 & 1446 & 1157 \\
\hline \multicolumn{4}{|l|}{ Domestic food price volatility (index) } \\
\hline Cereal import dependency ratio (\%) & 52.2 & 51.3 & 50.7 \\
\hline Underweight, children under-5 (\%) & 36.9 & 34.5 & 38.8 \\
\hline Improved water source (\% pop) & 42.6 & 53.7 & 60.2 \\
\hline \multicolumn{4}{|l|}{ Food supply } \\
\hline Food production value, $(2004-2006 \mathrm{mln} I \$)$ & 137 & 174 & 243 \\
\hline Agriculture, value added (\% GDP) & 22 & 13 & 15 \\
\hline Food exports (mln US\$) & 0 & 1 & 0 \\
\hline Food imports (mln US\$) & 30 & 69 & 97 \\
\hline \multicolumn{4}{|l|}{ Production indices $(2004-06=100)$} \\
\hline Net food & 63 & 79 & 111 \\
\hline Net crops & 61 & 69 & 94 \\
\hline Cereals & 30 & 43 & 103 \\
\hline Vegetable oils & 78 & 55 & 35 \\
\hline Roots and tubers & 113 & 123 & 60 \\
\hline Fruit and vegetables & 75 & 84 & 142 \\
\hline \multicolumn{4}{|l|}{ Sugar } \\
\hline Livestock & 64 & 87 & 123 \\
\hline Milk & 48 & 68 & 114 \\
\hline Meat & 69 & 97 & 129 \\
\hline Fish & 0 & 187 & 59 \\
\hline \multicolumn{4}{|l|}{ Net trade (mln US\$) } \\
\hline Cereals & & -33 & -51 \\
\hline Fruit and vegetables & & -14 & -4 \\
\hline \multicolumn{4}{|l|}{ Meat } \\
\hline Dairy products & & -5 & 0 \\
\hline Fish & & 2 & 0 \\
\hline \multicolumn{4}{|l|}{ Environment } \\
\hline Forest area (\%) & 16 & 16 & 15 \\
\hline Renewable water res withdrawn (\% of total) & & & 94 \\
\hline Terrestrial protect areas (\% total land area) & 5 & 5 & 5 \\
\hline \multicolumn{4}{|l|}{ Organic area (\% total agricultural area) } \\
\hline Water withdrawal by agriculture (\% of total) & & & 94 \\
\hline Biofuel production (thousand kt of oil eq.) & & 1 & 1 \\
\hline \multicolumn{4}{|l|}{ Wood pellet prod. (1 000 tonnes) } \\
\hline Net GHG emissions from AFOLU ( $\mathrm{CO}_{2}$ eq, $\mathrm{Mt}$ ) & 3 & 5 & 5 \\
\hline
\end{tabular}




\begin{tabular}{|c|c|c|c|}
\hline & 1990 & 2000 & 2014 \\
\hline \multicolumn{4}{|l|}{ The setting } \\
\hline Population, total (mln) & 1.5 & 1.4 & 1.3 \\
\hline Population, rural (mln) & 0.4 & 0.4 & 0.4 \\
\hline \multicolumn{4}{|l|}{ Govt expenditure on ag (\% total outlays) } \\
\hline Area harvested (mln ha) & & 1 & 1 \\
\hline Cropping intensity ratio & & 0.7 & \\
\hline Water resources (1 $000 \mathrm{~m}^{3} /$ person/year) & 9 & 9 & 10 \\
\hline Area equipped for irrigation (1 $000 \mathrm{ha}$ ) & & & 4 \\
\hline Area irrigated (\% area equipped for irrigation) & & & 71.2 \\
\hline Employment in agriculture (\%) & 21 & 7.1 & 4.7 \\
\hline Employment in agriculture, female (\%) & 15.2 & 4.4 & 2.6 \\
\hline Fertilizers, Nitrogen (kg of nutrients per ha) & & 42.7 & 53.1 \\
\hline Fertilizers, Phosphate (kg of nutrients per ha) & & 11.3 & 10.9 \\
\hline Fertilizers, Potash (kg nutrients per ha) & & 17.6 & 17 \\
\hline Energy consump, power irrigation (mln kWh) & 0 & 7 & 7 \\
\hline Agr value added per worker (constant US\$) & & 6329 & 13786 \\
\hline \multicolumn{4}{|l|}{ Hunger dimensions } \\
\hline \multicolumn{4}{|l|}{ Dietary energy supply (kcal/pc/day) } \\
\hline Average dietary energy supply adequacy (\%) & 106 & 122 & 131 \\
\hline Dietary en supp, cereals/roots/tubers (\%) & 41 & 36 & 37 \\
\hline Prevalence of undernourishment (\%) & $<5.0$ & $<5.0$ & $<5.0$ \\
\hline GDP per capita (US\$, PPP) & & 14875 & 25254 \\
\hline Domestic food price volatility (index) & & 3.4 & 7.4 \\
\hline Cereal import dependency ratio (\%) & 17.6 & 25.6 & -19.8 \\
\hline \multicolumn{4}{|l|}{ Underweight, children under-5 (\%) } \\
\hline Improved water source (\% pop) & 99.2 & 99.1 & 99.1 \\
\hline \multicolumn{4}{|l|}{ Food supply } \\
\hline Food production value, (2004-2006 mln I\$) & 559 & 391 & 589 \\
\hline Agriculture, value added (\% GDP) & & 5 & 4 \\
\hline Food exports (mln US\$) & 119 & 199 & 977 \\
\hline Food imports (mln US\$) & 91 & 340 & 959 \\
\hline \multicolumn{4}{|l|}{ Production indices $(2004-06=100)$} \\
\hline Net food & 127 & 89 & 134 \\
\hline Net crops & 136 & 126 & 148 \\
\hline Cereals & 115 & 102 & 155 \\
\hline Vegetable oils & 3 & 49 & 221 \\
\hline Roots and tubers & 283 & 268 & 77 \\
\hline Fruit and vegetables & 189 & 137 & 104 \\
\hline \multicolumn{4}{|l|}{ Sugar } \\
\hline Livestock & 131 & 89 & 120 \\
\hline Milk & 120 & 94 & 115 \\
\hline Meat & 146 & 78 & 132 \\
\hline Fish & 377 & 124 & 77 \\
\hline \multicolumn{4}{|l|}{ Net trade (mln US\$) } \\
\hline Cereals & -8 & -37 & 72 \\
\hline Fruit and vegetables & -2 & -54 & -170 \\
\hline Meat & 9 & -24 & -45 \\
\hline Dairy products & 49 & 28 & 149 \\
\hline Fish & 47 & 47 & 81 \\
\hline \multicolumn{4}{|l|}{ Environment } \\
\hline Forest area (\%) & 50 & 53 & 52 \\
\hline Renewable water res withdrawn (\% of total) & & & 0 \\
\hline Terrestrial protect areas (\% total land area) & 13 & 17 & 21 \\
\hline Organic area (\% total agricultural area) & & & 15 \\
\hline Water withdrawal by agriculture (\% of total) & & & 0 \\
\hline Biofuel production (thousand kt of oil eq.) & & 9 & 8 \\
\hline Wood pellet prod. (1 000 tonnes) & & & 720 \\
\hline Net GHG emissions from AFOLU ( $\mathrm{CO}_{2}$ eq, $\mathrm{Mt}$ ) & 10 & 10 & 12 \\
\hline
\end{tabular}




\begin{tabular}{|c|c|c|c|}
\hline & 1990 & 2000 & 2014 \\
\hline \multicolumn{4}{|l|}{ The setting } \\
\hline Population, total (mln) & 53.4 & 66 & 96.5 \\
\hline Population, rural (mln) & 46.2 & 56.3 & 79.3 \\
\hline Govt expenditure on ag (\% total outlays) & & 5 & 17.5 \\
\hline Area harvested (mln ha) & & 8 & 23 \\
\hline Cropping intensity ratio & & 0.3 & \\
\hline Water resources (1 $000 \mathrm{~m}^{3} /$ person/year) & 2 & 2 & 1 \\
\hline Area equipped for irrigation (1 $000 \mathrm{ha}$ ) & & & 290 \\
\hline \multicolumn{4}{|l|}{ Area irrigated (\% area equipped for irrigation) } \\
\hline Employment in agriculture (\%) & & 89.3 & 79.3 \\
\hline Employment in agriculture, female (\%) & & & 74.8 \\
\hline Fertilizers, Nitrogen (kg of nutrients per ha) & & 3.1 & 11.7 \\
\hline Fertilizers, Phosphate (kg of nutrients per ha) & & 2.6 & 12 \\
\hline Fertilizers, Potash (kg nutrients per ha) & & 0 & 0 \\
\hline Energy consump, power irrigation (mln kWh) & 0 & 15 & 15 \\
\hline Agr value added per worker (constant US\$) & 180 & 166 & 278 \\
\hline \multicolumn{4}{|l|}{ Hunger dimensions } \\
\hline Dietary energy supply (kcal/pc/day) & 1545 & 1782 & 2164 \\
\hline Average dietary energy supply adequacy (\%) & 72 & 83 & 98 \\
\hline Dietary en supp, cereals/roots/tubers (\%) & 83 & 82 & 76 \\
\hline Prevalence of undernourishment (\%) & 72.5 & 57.9 & 33.1 \\
\hline GDP per capita (US\$, PPP) & 652 & 623 & 1336 \\
\hline Domestic food price volatility (index) & & 8.8 & 9 \\
\hline Cereal import dependency ratio (\%) & 17.3 & 10.8 & 10.7 \\
\hline Underweight, children under-5 (\%) & 43.3 & 42 & 25.2 \\
\hline Improved water source (\% pop) & 13.2 & 29 & 51.5 \\
\hline \multicolumn{4}{|l|}{ Food supply } \\
\hline Food production value, (2004-2006 mln I\$) & 3599 & 4856 & 10294 \\
\hline Agriculture, value added (\% GDP) & 52 & 48 & 42 \\
\hline Food exports (mln US\$) & 10 & 66 & 1264 \\
\hline Food imports (mln US\$) & 120 & 296 & 1393 \\
\hline \multicolumn{4}{|l|}{ Production indices $(2004-06=100)$} \\
\hline Net food & 52 & 70 & 147 \\
\hline Net crops & 52 & 75 & 157 \\
\hline Cereals & 45 & 67 & 196 \\
\hline Vegetable oils & 31 & 33 & 161 \\
\hline Roots and tubers & 62 & 82 & 146 \\
\hline Fruit and vegetables & 43 & 67 & 142 \\
\hline Sugar & 65 & 83 & 105 \\
\hline Livestock & 53 & 64 & 130 \\
\hline Milk & 32 & 41 & 170 \\
\hline Meat & 63 & 74 & 112 \\
\hline Fish & 51 & 160 & 392 \\
\hline \multicolumn{4}{|l|}{ Net trade (mln US\$) } \\
\hline Cereals & -82 & -227 & -700 \\
\hline Fruit and vegetables & -3 & 0 & 435 \\
\hline Meat & 0 & 2 & 74 \\
\hline Dairy products & -3 & -3 & -10 \\
\hline Fish & 0 & 0 & -2 \\
\hline \multicolumn{4}{|l|}{ Environment } \\
\hline Forest area (\%) & 15 & 14 & 12 \\
\hline Renewable water res withdrawn (\% of total) & & 94 & 94 \\
\hline Terrestrial protect areas (\% total land area) & 18 & 18 & 18 \\
\hline Organic area (\% total agricultural area) & & & 0 \\
\hline Water withdrawal by agriculture (\% of total) & & 94 & 94 \\
\hline Biofuel production (thousand kt of oil eq.) & & 71 & 137 \\
\hline \multicolumn{4}{|l|}{ Wood pellet prod. (1 000 tonnes) } \\
\hline Net GHG emissions from AFOLU ( $\mathrm{CO}_{2}$ eq, $\mathrm{Mt}$ ) & 62 & 82 & 115 \\
\hline
\end{tabular}




\begin{tabular}{|c|c|c|c|}
\hline & 1990 & 2000 & 2014 \\
\hline \multicolumn{4}{|l|}{ The setting } \\
\hline Population, total (mln) & 0.7 & 0.8 & 0.9 \\
\hline Population, rural (mln) & 0.4 & 0.4 & 0.4 \\
\hline Govt expenditure on ag (\% total outlays) & & 0.8 & 1.2 \\
\hline Area harvested (mln ha) & 4 & 4 & 2 \\
\hline Cropping intensity ratio & 9.8 & 8.4 & \\
\hline Water resources (1 $000 \mathrm{~m}^{3} /$ person/year) & 39 & 35 & 32 \\
\hline Area equipped for irrigation (1 $000 \mathrm{ha}$ ) & & & 4 \\
\hline \multicolumn{4}{|l|}{ Area irrigated (\% area equipped for irrigation) } \\
\hline \multicolumn{4}{|l|}{ Employment in agriculture (\%) } \\
\hline \multicolumn{4}{|l|}{ Employment in agriculture, female (\%) } \\
\hline Fertilizers, Nitrogen (kg of nutrients per ha) & & 18.7 & 24.4 \\
\hline Fertilizers, Phosphate (kg of nutrients per ha) & & 0.3 & 0.5 \\
\hline Fertilizers, Potash (kg nutrients per ha) & & 0.2 & 7.9 \\
\hline \multicolumn{4}{|l|}{ Energy consump, power irrigation (mln kWh) } \\
\hline Agr value added per worker (constant US\$) & 2902 & 2873 & 2934 \\
\hline \multicolumn{4}{|l|}{ Hunger dimensions } \\
\hline Dietary energy supply (kcal/pc/day) & 2797 & 2859 & 2930 \\
\hline Average dietary energy supply adequacy (\%) & 122 & 124 & 124 \\
\hline Dietary en supp, cereals/roots/tubers (\%) & 47 & 48 & 48 \\
\hline Prevalence of undernourishment (\%) & 5.7 & $<5.0$ & $<5.0$ \\
\hline GDP per capita (US\$, PPP) & 5694 & 6444 & 7502 \\
\hline Domestic food price volatility (index) & & 13.6 & 8.3 \\
\hline Cereal import dependency ratio (\%) & 88.6 & 93.6 & 93.1 \\
\hline Underweight, children under-5 (\%) & 6.9 & 6.9 & \\
\hline Improved water source (\% pop) & 85.3 & 91.2 & 96.3 \\
\hline \multicolumn{4}{|l|}{ Food supply } \\
\hline Food production value, (2004-2006 mln I\$) & 247 & 231 & 196 \\
\hline Agriculture, value added (\% GDP) & 20 & 17 & 12 \\
\hline Food exports (mln US\$) & 177 & 142 & 201 \\
\hline Food imports (mln US\$) & 78 & 96 & 287 \\
\hline \multicolumn{4}{|l|}{ Production indices $(2004-06=100)$} \\
\hline Net food & 108 & 101 & 86 \\
\hline Net crops & 116 & 105 & 76 \\
\hline Cereals & 188 & 94 & 38 \\
\hline Vegetable oils & 141 & 96 & 127 \\
\hline Roots and tubers & 30 & 56 & 126 \\
\hline Fruit and vegetables & 50 & 95 & 83 \\
\hline Sugar & 134 & 120 & 53 \\
\hline Livestock & 92 & 92 & 110 \\
\hline Milk & 106 & 102 & 112 \\
\hline Meat & 88 & 89 & 104 \\
\hline Fish & 68 & 90 & 91 \\
\hline \multicolumn{4}{|l|}{ Net trade (mln US\$) } \\
\hline Cereals & -22 & -26 & -42 \\
\hline Fruit and vegetables & -10 & -8 & -25 \\
\hline Meat & -11 & -16 & -29 \\
\hline Dairy products & -8 & -9 & -37 \\
\hline Fish & 17 & 26 & 173 \\
\hline \multicolumn{4}{|l|}{ Environment } \\
\hline Forest area (\%) & 52 & 54 & 56 \\
\hline Renewable water res withdrawn (\% of total) & & 59 & 59 \\
\hline Terrestrial protect areas (\% total land area) & 1 & 2 & 4 \\
\hline Organic area (\% total agricultural area) & & & 1 \\
\hline Water withdrawal by agriculture (\% of total) & & 59 & 59 \\
\hline Biofuel production (thousand kt of oil eq.) & 8 & 9 & 4 \\
\hline \multicolumn{4}{|l|}{ Wood pellet prod. (1 000 tonnes) } \\
\hline Net GHG emissions from AFOLU ( $\mathrm{CO}_{2}$ eq, $\left.\mathrm{Mt}\right)$ & 1 & 1 & 0 \\
\hline
\end{tabular}




\section{Finland}

\begin{tabular}{|c|c|c|c|}
\hline & 1990 & 2000 & 2014 \\
\hline \multicolumn{4}{|l|}{ The setting } \\
\hline Population, total (mln) & 5 & 5.2 & 5.4 \\
\hline Population, rural (mln) & 1 & 0.9 & 0.9 \\
\hline \multicolumn{4}{|l|}{ Govt expenditure on ag (\% total outlays) } \\
\hline Area harvested (mln ha) & 4 & 4 & 4 \\
\hline Cropping intensity ratio & 1.8 & 1.8 & \\
\hline Water resources (1 $000 \mathrm{~m}^{3} /$ person/year) & 22 & 21 & 20 \\
\hline Area equipped for irrigation ( $1000 \mathrm{ha}$ ) & & & 69 \\
\hline Area irrigated (\% area equipped for irrigation) & & & 21.9 \\
\hline Employment in agriculture (\%) & 8.8 & 6 & 4.1 \\
\hline Employment in agriculture, female (\%) & 6.7 & 3.9 & 2.3 \\
\hline Fertilizers, Nitrogen (kg of nutrients per ha) & & 74 & 85.5 \\
\hline Fertilizers, Phosphate (kg of nutrients per ha) & & 10 & 32.3 \\
\hline Fertilizers, Potash (kg nutrients per ha) & & 34.3 & 70.8 \\
\hline Energy consump, power irrigation (mln kWh) & 0 & & 132 \\
\hline Agr value added per worker (constant US\$) & 22008 & 33255 & 71465 \\
\hline \multicolumn{4}{|l|}{ Hunger dimensions } \\
\hline \multicolumn{4}{|l|}{ Dietary energy supply (kcal/pc/day) } \\
\hline Average dietary energy supply adequacy (\%) & 121 & 124 & 131 \\
\hline Dietary en supp, cereals/roots/tubers (\%) & 28 & 32 & 32 \\
\hline Prevalence of undernourishment (\%) & $<5.0$ & $<5.0$ & $<5.0$ \\
\hline GDP per capita (US\$, PPP) & 28598 & 34517 & 38821 \\
\hline Domestic food price volatility (index) & & 4.5 & 6.2 \\
\hline Cereal import dependency ratio (\%) & -35.6 & -8.3 & -21.3 \\
\hline \multicolumn{4}{|l|}{ Underweight, children under-5 (\%) } \\
\hline Improved water source (\% pop) & 100 & 100 & 100 \\
\hline \multicolumn{4}{|l|}{ Food supply } \\
\hline Food production value, (2004-2006 mln I\$) & 2116 & 1924 & 1876 \\
\hline Agriculture, value added (\% GDP) & 6 & 3 & 3 \\
\hline Food exports (mln US\$) & 547 & 613 & 1485 \\
\hline Food imports (mln US\$) & 851 & 1156 & 3483 \\
\hline \multicolumn{4}{|l|}{ Production indices $(2004-06=100)$} \\
\hline Net food & 109 & 99 & 96 \\
\hline Net crops & 111 & 105 & 100 \\
\hline Cereals & 111 & 106 & 107 \\
\hline Vegetable oils & 107 & 65 & 73 \\
\hline Roots and tubers & 133 & 118 & 92 \\
\hline Fruit and vegetables & 87 & 104 & 116 \\
\hline Sugar & 106 & 98 & 45 \\
\hline Livestock & 107 & 95 & 98 \\
\hline Milk & 116 & 101 & 96 \\
\hline Meat & 97 & 89 & 99 \\
\hline Fish & 100 & 113 & 119 \\
\hline \multicolumn{4}{|l|}{ Net trade (mln US\$) } \\
\hline Cereals & 64 & -96 & -129 \\
\hline Fruit and vegetables & -441 & -372 & -974 \\
\hline Meat & 54 & -22 & -276 \\
\hline Dairy products & 175 & 168 & 178 \\
\hline Fish & -117 & -99 & -387 \\
\hline \multicolumn{4}{|l|}{ Environment } \\
\hline Forest area (\%) & 72 & 74 & 73 \\
\hline Renewable water res withdrawn (\% of total) & & & 3 \\
\hline Terrestrial protect areas (\% total land area) & 4 & 13 & 15 \\
\hline Organic area (\% total agricultural area) & & & 9 \\
\hline Water withdrawal by agriculture (\% of total) & & & 3 \\
\hline Biofuel production (thousand kt of oil eq.) & 16 & 72 & 7773 \\
\hline Wood pellet prod. (1 000 tonnes) & & & 324 \\
\hline Net GHG emissions from AFOLU ( $\mathrm{CO}_{2}$ eq, $\left.\mathrm{Mt}\right)$ & -10 & -19 & 12 \\
\hline
\end{tabular}




\begin{tabular}{|c|c|c|c|}
\hline & 1990 & 2000 & 2014 \\
\hline \multicolumn{4}{|l|}{ The setting } \\
\hline Population, total (mln) & 56.8 & 59.2 & 64.6 \\
\hline Population, rural (mln) & 14.7 & 13.7 & 8.2 \\
\hline \multicolumn{4}{|l|}{ Govt expenditure on ag (\% total outlays) } \\
\hline Area harvested (mln ha) & 55 & 66 & 68 \\
\hline Cropping intensity ratio & 1.8 & 2.2 & \\
\hline Water resources (1 $000 \mathrm{~m}^{3} /$ person/year) & 4 & 4 & 3 \\
\hline Area equipped for irrigation (1 $000 \mathrm{ha})$ & & & 2600 \\
\hline Area irrigated (\% area equipped for irrigation) & & & 57.2 \\
\hline Employment in agriculture (\%) & 5.4 & 4.1 & 2.9 \\
\hline Employment in agriculture, female (\%) & 4.5 & 2.9 & 1.8 \\
\hline Fertilizers, Nitrogen (kg of nutrients per ha) & & 129.4 & 104.7 \\
\hline Fertilizers, Phosphate (kg of nutrients per ha) & & 40.6 & 13.8 \\
\hline Fertilizers, Potash (kg nutrients per ha) & & 53.3 & 18.4 \\
\hline Energy consump, power irrigation (mln kWh) & 98 & 4829 & 4875 \\
\hline Agr value added per worker (constant US\$) & 23375 & 42551 & 84574 \\
\hline \multicolumn{4}{|l|}{ Hunger dimensions } \\
\hline \multicolumn{4}{|l|}{ Dietary energy supply (kcal/pc/day) } \\
\hline Average dietary energy supply adequacy (\%) & 142 & 145 & 141 \\
\hline Dietary en supp, cereals/roots/tubers (\%) & 27 & 28 & 29 \\
\hline Prevalence of undernourishment (\%) & $<5.0$ & $<5.0$ & $<5.0$ \\
\hline GDP per capita (US\$, PPP) & 29476 & 34774 & 37217 \\
\hline Domestic food price volatility (index) & & 4.6 & 4.8 \\
\hline Cereal import dependency ratio (\%) & -125.7 & -100.2 & -90.4 \\
\hline \multicolumn{4}{|l|}{ Underweight, children under-5 (\%) } \\
\hline Improved water source (\% pop) & 100 & 100 & 100 \\
\hline \multicolumn{4}{|l|}{ Food supply } \\
\hline Food production value, (2004-2006 mln I\$) & 38720 & 40321 & 38188 \\
\hline Agriculture, value added (\% GDP) & & 2 & 2 \\
\hline Food exports (mln US\$) & 23576 & 21950 & 46001 \\
\hline Food imports (mln US\$) & 15148 & 15627 & 37016 \\
\hline \multicolumn{4}{|l|}{ Production indices $(2004-06=100)$} \\
\hline Net food & 100 & 104 & 99 \\
\hline Net crops & 97 & 103 & 94 \\
\hline Cereals & 84 & 101 & 104 \\
\hline Vegetable oils & 80 & 94 & 104 \\
\hline Roots and tubers & 69 & 95 & 103 \\
\hline Fruit and vegetables & 114 & 109 & 80 \\
\hline Sugar & 104 & 102 & 110 \\
\hline Livestock & 100 & 105 & 100 \\
\hline Milk & 106 & 102 & 97 \\
\hline Meat & 97 & 107 & 102 \\
\hline Fish & 106 & 108 & 84 \\
\hline \multicolumn{4}{|l|}{ Net trade (mln US\$) } \\
\hline Cereals & 6079 & 3909 & 9659 \\
\hline Fruit and vegetables & -2297 & -2102 & -5751 \\
\hline Meat & -877 & 532 & -1270 \\
\hline Dairy products & 2367 & 1740 & 4015 \\
\hline Fish & -1878 & -1888 & -4281 \\
\hline \multicolumn{4}{|l|}{ Environment } \\
\hline Forest area (\%) & 27 & 28 & 29 \\
\hline Renewable water res withdrawn (\% of total) & & & 12 \\
\hline Terrestrial protect areas (\% total land area) & 11 & 17 & 25 \\
\hline Organic area (\% total agricultural area) & & & 4 \\
\hline Water withdrawal by agriculture (\% of total) & & & 12 \\
\hline Biofuel production (thousand kt of oil eq.) & 211 & 8109 & 53997 \\
\hline Wood pellet prod. (1 000 tonnes) & & & 1200 \\
\hline Net GHG emissions from AFOLU ( $\mathrm{CO}_{2}$ eq, $\mathrm{Mt}$ ) & 77 & 52 & 44 \\
\hline
\end{tabular}




\begin{tabular}{|c|c|c|c|}
\hline & 1990 & 2000 & 2014 \\
\hline \multicolumn{4}{|l|}{ The setting } \\
\hline Population, total (mln) & 0.9 & 1.2 & 1.7 \\
\hline Population, rural (mln) & 0.3 & 0.2 & 0.2 \\
\hline \multicolumn{4}{|l|}{ Govt expenditure on ag (\% total outlays) } \\
\hline Area harvested (mln ha) & 1 & 1 & 1 \\
\hline Cropping intensity ratio & 0.1 & 0.1 & \\
\hline Water resources (1 $000 \mathrm{~m}^{3} /$ person/year) & 175 & 135 & 99 \\
\hline Area equipped for irrigation (1 $000 \mathrm{ha})$ & & & 4 \\
\hline \multicolumn{4}{|l|}{ Area irrigated (\% area equipped for irrigation) } \\
\hline Employment in agriculture (\%) & 41.6 & 41.6 & 24.2 \\
\hline Employment in agriculture, female (\%) & 60.8 & 60.8 & 33.7 \\
\hline Fertilizers, Nitrogen (kg of nutrients per ha) & & 0.6 & 6.8 \\
\hline Fertilizers, Phosphate (kg of nutrients per ha) & & 0.4 & 4.8 \\
\hline Fertilizers, Potash (kg nutrients per ha) & & 2.6 & 5.7 \\
\hline \multicolumn{4}{|l|}{ Energy consump, power irrigation (mln kWh) } \\
\hline Agr value added per worker (constant US\$) & 1106 & 1383 & 2741 \\
\hline \multicolumn{4}{|l|}{ Hunger dimensions } \\
\hline Dietary energy supply (kcal/pc/day) & 2498 & 2709 & 2807 \\
\hline Average dietary energy supply adequacy (\%) & 114 & 122 & 125 \\
\hline Dietary en supp, cereals/roots/tubers (\%) & 46 & 49 & 51 \\
\hline Prevalence of undernourishment (\%) & 10.1 & $<5.0$ & $<5.0$ \\
\hline GDP per capita (US\$, PPP) & 19147 & 17438 & 18646 \\
\hline Domestic food price volatility (index) & & 5.9 & 21 \\
\hline Cereal import dependency ratio (\%) & 78.2 & 85.2 & 81.9 \\
\hline Underweight, children under-5 (\%) & & 8.8 & 6.5 \\
\hline Improved water source (\% pop) & & 83.8 & 92.2 \\
\hline \multicolumn{4}{|l|}{ Food supply } \\
\hline Food production value, (2004-2006 mln I\$) & 188 & 213 & 260 \\
\hline Agriculture, value added (\% GDP) & 7 & 6 & 4 \\
\hline Food exports (mln US\$) & 3 & 2 & 0 \\
\hline Food imports (mln US\$) & 88 & 141 & 489 \\
\hline \multicolumn{4}{|l|}{ Production indices $(2004-06=100)$} \\
\hline Net food & 87 & 99 & 121 \\
\hline Net crops & 76 & 98 & 123 \\
\hline Cereals & 70 & 80 & 138 \\
\hline Vegetable oils & 72 & 99 & 86 \\
\hline Roots and tubers & 78 & 98 & 127 \\
\hline Fruit and vegetables & 81 & 98 & 109 \\
\hline Sugar & 107 & 115 & 137 \\
\hline Livestock & 87 & 102 & 120 \\
\hline Milk & 43 & 83 & 108 \\
\hline Meat & 88 & 102 & 121 \\
\hline Fish & 46 & 110 & 76 \\
\hline \multicolumn{4}{|l|}{ Net trade (mln US\$) } \\
\hline Cereals & -33 & -43 & -137 \\
\hline Fruit and vegetables & -13 & -12 & -36 \\
\hline Meat & & -46 & -170 \\
\hline Dairy products & -17 & -17 & -44 \\
\hline Fish & -8 & 3 & -41 \\
\hline \multicolumn{4}{|l|}{ Environment } \\
\hline Forest area (\%) & 85 & 85 & 85 \\
\hline Renewable water res withdrawn (\% of total) & & & 29 \\
\hline Terrestrial protect areas (\% total land area) & 5 & 6 & 20 \\
\hline \multicolumn{4}{|l|}{ Organic area (\% total agricultural area) } \\
\hline Water withdrawal by agriculture (\% of total) & & & 29 \\
\hline Biofuel production (thousand kt of oil eq.) & 1 & 0 & 1 \\
\hline \multicolumn{4}{|l|}{ Wood pellet prod. (1 000 tonnes) } \\
\hline Net GHG emissions from AFOLU ( $\mathrm{CO}_{2}$ eq, $\left.\mathrm{Mt}\right)$ & 1 & 1 & 1 \\
\hline
\end{tabular}




\begin{tabular}{|c|c|c|c|}
\hline & 1990 & 2000 & 2014 \\
\hline \multicolumn{4}{|l|}{ The setting } \\
\hline Population, total (mln) & 0.9 & 1.2 & 1.9 \\
\hline Population, rural (mln) & 0.6 & 0.6 & 0.8 \\
\hline \multicolumn{4}{|l|}{ Govt expenditure on ag (\% total outlays) } \\
\hline Area harvested (mln ha) & 0 & 0 & 0 \\
\hline Cropping intensity ratio & 0.2 & 0.3 & \\
\hline Water resources (1 $000 \mathrm{~m}^{3} /$ person/year) & 9 & 7 & 4 \\
\hline Area equipped for irrigation (1 $000 \mathrm{ha}$ ) & & & 5 \\
\hline Area irrigated (\% area equipped for irrigation) & 64.7 & & \\
\hline Employment in agriculture (\%) & 64.7 & 64.7 & \\
\hline Employment in agriculture, female (\%) & 76.5 & 76.5 & \\
\hline Fertilizers, Nitrogen (kg of nutrients per ha) & & 5.6 & 2.6 \\
\hline Fertilizers, Phosphate (kg of nutrients per ha) & & 2.1 & 2.2 \\
\hline Fertilizers, Potash (kg nutrients per ha) & & 1.3 & 1.7 \\
\hline Energy consump, power irrigation (mln kWh) & 0 & 0 & 0 \\
\hline Agr value added per worker (constant US\$) & 307 & 331 & 267 \\
\hline \multicolumn{4}{|l|}{ Hunger dimensions } \\
\hline Dietary energy supply (kcal/pc/day) & 2447 & 2536 & 2882 \\
\hline Average dietary energy supply adequacy (\%) & 112 & 116 & 131 \\
\hline Dietary en supp, cereals/roots/tubers (\%) & 57 & 55 & 63 \\
\hline Prevalence of undernourishment (\%) & 14.6 & 14.1 & 5.4 \\
\hline GDP per capita (US\$, PPP) & 1517 & 1564 & 1608 \\
\hline Domestic food price volatility (index) & & 3.7 & 2.7 \\
\hline Cereal import dependency ratio (\%) & 51.5 & 39.5 & 43.6 \\
\hline Underweight, children under-5 (\%) & & 15.4 & 17.4 \\
\hline Improved water source (\% pop) & 75.8 & 82.8 & 90.1 \\
\hline \multicolumn{4}{|l|}{ Food supply } \\
\hline Food production value, (2004-2006 mln I\$) & 72 & 118 & 116 \\
\hline \multicolumn{4}{|l|}{ Agriculture, value added (\% GDP) } \\
\hline Food exports (mln US\$) & 11 & 13 & 23 \\
\hline Food imports (mln US\$) & 45 & 60 & 98 \\
\hline \multicolumn{4}{|l|}{ Production indices (2004-06=100) } \\
\hline Net food & 60 & 99 & 97 \\
\hline Net crops & 57 & 101 & 97 \\
\hline Cereals & 44 & 85 & 114 \\
\hline Vegetable oils & 63 & 115 & 77 \\
\hline Roots and tubers & 66 & 79 & 121 \\
\hline Fruit and vegetables & 63 & 82 & 124 \\
\hline \multicolumn{4}{|l|}{ Sugar } \\
\hline Livestock & 76 & 85 & 99 \\
\hline Milk & 69 & 89 & 110 \\
\hline Meat & 77 & 84 & 97 \\
\hline Fish & 62 & 83 & 125 \\
\hline \multicolumn{4}{|l|}{ Net trade (mln US\$) } \\
\hline Cereals & & -24 & -58 \\
\hline Fruit and vegetables & -6 & -5 & -4 \\
\hline Meat & -2 & -1 & -2 \\
\hline Dairy products & & -3 & 0 \\
\hline Fish & 4 & 3 & 1 \\
\hline \multicolumn{4}{|l|}{ Environment } \\
\hline Forest area (\%) & 44 & 46 & 48 \\
\hline Renewable water res withdrawn (\% of total) & & 43 & 43 \\
\hline Terrestrial protect areas (\% total land area) & 2 & 4 & 5 \\
\hline Organic area (\% total agricultural area) & & & 0 \\
\hline Water withdrawal by agriculture (\% of total) & & 43 & 43 \\
\hline \multicolumn{4}{|l|}{ Biofuel production (thousand kt of oil eq.) } \\
\hline \multicolumn{4}{|l|}{ Wood pellet prod. (1 000 tonnes) } \\
\hline Net GHG emissions from AFOLU ( $\mathrm{CO}_{2}$ eq, $\mathrm{Mt}$ ) & 1 & 1 & 1 \\
\hline
\end{tabular}




\begin{tabular}{|c|c|c|c|}
\hline & 1990 & 2000 & 2014 \\
\hline \multicolumn{4}{|l|}{ The setting } \\
\hline Population, total (mln) & 5.3 & 4.7 & 4.3 \\
\hline Population, rural (mln) & 2.4 & 2.2 & 2 \\
\hline Govt expenditure on ag (\% total outlays) & & 2 & 0.5 \\
\hline Area harvested (mln ha) & & 0 & 0 \\
\hline Cropping intensity ratio & & 0.2 & \\
\hline Water resources (1 $000 \mathrm{~m}^{3} /$ person/year) & 12 & 13 & 15 \\
\hline Area equipped for irrigation (1 $000 \mathrm{ha}$ ) & & & 433 \\
\hline Area irrigated (\% area equipped for irrigation) & & 63 & \\
\hline Employment in agriculture (\%) & & 52.1 & 53.4 \\
\hline Employment in agriculture, female (\%) & & 57.5 & 56.6 \\
\hline Fertilizers, Nitrogen (kg of nutrients per ha) & & 13.9 & 33.6 \\
\hline Fertilizers, Phosphate (kg of nutrients per ha) & & 0.5 & 9.4 \\
\hline Fertilizers, Potash (kg nutrients per ha) & & 0 & 2 \\
\hline Energy consump, power irrigation (mln kWh) & 0 & 171 & 68 \\
\hline Agr value added per worker (constant US\$) & 2680 & 1652 & 2997 \\
\hline \multicolumn{4}{|l|}{ Hunger dimensions } \\
\hline Dietary energy supply (kcal/pc/day) & 1852 & 2573 & 2852 \\
\hline Average dietary energy supply adequacy (\%) & 76 & 104 & 115 \\
\hline Dietary en supp, cereals/roots/tubers (\%) & 64 & 61 & 58 \\
\hline Prevalence of undernourishment (\%) & 67.1 & 14.8 & 8 \\
\hline GDP per capita (US\$, PPP) & 8018 & 3269 & 6930 \\
\hline \multicolumn{4}{|l|}{ Domestic food price volatility (index) } \\
\hline Cereal import dependency ratio (\%) & 57.9 & 58.4 & 68.6 \\
\hline Underweight, children under-5 (\%) & & 2.7 & 1.1 \\
\hline Improved water source (\% pop) & 85 & 89.2 & 98.7 \\
\hline \multicolumn{4}{|l|}{ Food supply } \\
\hline Food production value, (2004-2006 mln I\$) & 893 & 866 & 775 \\
\hline Agriculture, value added (\% GDP) & 32 & 22 & 9 \\
\hline Food exports (mln US\$) & 21 & 48 & 260 \\
\hline Food imports (mln US\$) & 200 & 214 & 961 \\
\hline \multicolumn{4}{|l|}{ Production indices $(2004-06=100)$} \\
\hline Net food & 100 & 97 & 87 \\
\hline Net crops & 135 & 100 & 100 \\
\hline Cereals & 71 & 71 & 90 \\
\hline Vegetable oils & 13 & 10 & 33 \\
\hline Roots and tubers & 97 & 81 & 106 \\
\hline Fruit and vegetables & 146 & 107 & 94 \\
\hline \multicolumn{4}{|l|}{ Sugar } \\
\hline Livestock & 74 & 95 & 74 \\
\hline Milk & 53 & 84 & 85 \\
\hline Meat & 97 & 108 & 56 \\
\hline Fish & 855 & 15 & 103 \\
\hline \multicolumn{4}{|l|}{ Net trade (mln US\$) } \\
\hline Cereals & -137 & -145 & -272 \\
\hline Fruit and vegetables & 6 & 19 & 13 \\
\hline Meat & -22 & -12 & -140 \\
\hline Dairy products & -3 & 8 & -36 \\
\hline Fish & 0 & -1 & -39 \\
\hline \multicolumn{4}{|l|}{ Environment } \\
\hline Forest area (\%) & 40 & 40 & 39 \\
\hline Renewable water res withdrawn (\% of total) & & & 58 \\
\hline Terrestrial protect areas (\% total land area) & 3 & 4 & 4 \\
\hline Organic area (\% total agricultural area) & & & 0 \\
\hline Water withdrawal by agriculture (\% of total) & & & 58 \\
\hline Biofuel production (thousand kt of oil eq.) & & 0 & 0 \\
\hline \multicolumn{4}{|l|}{ Wood pellet prod. (1 000 tonnes) } \\
\hline Net GHG emissions from AFOLU ( $\mathrm{CO}_{2}$ eq, $\left.\mathrm{Mt}\right)$ & -1 & -1 & -1 \\
\hline
\end{tabular}




\begin{tabular}{|c|c|c|c|}
\hline & 1990 & 2000 & 2014 \\
\hline \multicolumn{4}{|l|}{ The setting } \\
\hline Population, total (mln) & 80.5 & 83.5 & 82.7 \\
\hline Population, rural (mln) & 21.6 & 22.5 & 21.2 \\
\hline \multicolumn{4}{|l|}{ Govt expenditure on ag (\% total outlays) } \\
\hline Area harvested (mln ha) & 38 & 45 & 48 \\
\hline Cropping intensity ratio & 2.1 & 2.7 & \\
\hline Water resources (1 $000 \mathrm{~m}^{3} /$ person/year) & 2 & 2 & 2 \\
\hline Area equipped for irrigation (1 $000 \mathrm{ha})$ & & & 650 \\
\hline Area irrigated (\% area equipped for irrigation) & & & 45.5 \\
\hline Employment in agriculture (\%) & 3.5 & 2.6 & 1.5 \\
\hline Employment in agriculture, female (\%) & 3.4 & 2.1 & 1.1 \\
\hline Fertilizers, Nitrogen (kg of nutrients per ha) & & 154.5 & 139.3 \\
\hline Fertilizers, Phosphate (kg of nutrients per ha) & & 24 & 24 \\
\hline Fertilizers, Potash (kg nutrients per ha) & & 41.1 & 35.6 \\
\hline Energy consump, power irrigation (mln kWh) & 2 & 4 & 974 \\
\hline Agr value added per worker (constant US\$) & 19540 & 21233 & 39490 \\
\hline \multicolumn{4}{|l|}{ Hunger dimensions } \\
\hline \multicolumn{4}{|l|}{ Dietary energy supply (kcal/pc/day) } \\
\hline Average dietary energy supply adequacy (\%) & 129 & 131 & 139 \\
\hline Dietary en supp, cereals/roots/tubers (\%) & 26 & 28 & 28 \\
\hline Prevalence of undernourishment (\%) & $<5.0$ & $<5.0$ & $<5.0$ \\
\hline GDP per capita (US\$, PPP) & 31476 & 36953 & 42884 \\
\hline Domestic food price volatility (index) & & 5.6 & 5.6 \\
\hline Cereal import dependency ratio (\%) & -19.1 & -24 & -9.2 \\
\hline Underweight, children under-5 (\%) & & & 1.1 \\
\hline Improved water source (\% pop) & 100 & 100 & 100 \\
\hline \multicolumn{4}{|l|}{ Food supply } \\
\hline Food production value, (2004-2006 mln I\$) & 34013 & 32193 & 33635 \\
\hline Agriculture, value added (\% GDP) & 1 & 1 & 1 \\
\hline Food exports (mln US\$) & 14211 & 16288 & 54646 \\
\hline Food imports (mln US\$) & 26636 & 23074 & 63113 \\
\hline \multicolumn{4}{|l|}{ Production indices (2004-06=100) } \\
\hline Net food & 109 & 103 & 107 \\
\hline Net crops & 93 & 107 & 98 \\
\hline Cereals & 76 & 95 & 102 \\
\hline Vegetable oils & 41 & 70 & 110 \\
\hline Roots and tubers & 125 & 119 & 83 \\
\hline Fruit and vegetables & 129 & 148 & 94 \\
\hline Sugar & 125 & 114 & 94 \\
\hline Livestock & 115 & 99 & 110 \\
\hline Milk & 111 & 100 & 110 \\
\hline Meat & 118 & 96 & 111 \\
\hline Fish & 121 & 83 & 79 \\
\hline \multicolumn{4}{|l|}{ Net trade (mln US\$) } \\
\hline Cereals & -21 & 1832 & 3535 \\
\hline Fruit and vegetables & -9355 & -7134 & -13940 \\
\hline Meat & -1613 & -1497 & 3092 \\
\hline Dairy products & 664 & 1494 & 3109 \\
\hline Fish & -1234 & -1160 & -2549 \\
\hline \multicolumn{4}{|l|}{ Environment } \\
\hline Forest area (\%) & 31 & 32 & 32 \\
\hline Renewable water res withdrawn (\% of total) & & & 0 \\
\hline Terrestrial protect areas (\% total land area) & 35 & 42 & 48 \\
\hline Organic area (\% total agricultural area) & & & 6 \\
\hline Water withdrawal by agriculture (\% of total) & & & 0 \\
\hline Biofuel production (thousand kt of oil eq.) & 24 & 30 & 77610 \\
\hline Wood pellet prod. (1 000 tonnes) & & & 2078 \\
\hline Net GHG emissions from AFOLU ( $\mathrm{CO}_{2}$ eq, $\mathrm{Mt}$ ) & 28 & -3 & -19 \\
\hline
\end{tabular}




\begin{tabular}{|c|c|c|c|}
\hline & 1990 & 2000 & 2014 \\
\hline \multicolumn{4}{|l|}{ The setting } \\
\hline Population, total (mln) & 14.6 & 18.8 & 26.4 \\
\hline Population, rural (mln) & 9.3 & 10.6 & 12.2 \\
\hline Govt expenditure on ag (\% total outlays) & & & 1.5 \\
\hline Area harvested (mln ha) & 4 & 13 & 23 \\
\hline Cropping intensity ratio & 0.3 & 0.9 & \\
\hline Water resources (1 $000 \mathrm{~m}^{3} /$ person/year) & 4 & 3 & 2 \\
\hline Area equipped for irrigation (1 $000 \mathrm{ha}$ ) & & & 34 \\
\hline Area irrigated (\% area equipped for irrigation) & & 90.3 & \\
\hline Employment in agriculture (\%) & 62 & 55 & 41.5 \\
\hline Employment in agriculture, female (\%) & 58.7 & 50.3 & 37.7 \\
\hline Fertilizers, Nitrogen (kg of nutrients per ha) & & 2 & 9 \\
\hline Fertilizers, Phosphate (kg of nutrients per ha) & & 0.7 & 15.1 \\
\hline Fertilizers, Potash (kg nutrients per ha) & & 4.1 & 10.8 \\
\hline Energy consump, power irrigation (mln kWh) & 0 & 15 & 15 \\
\hline Agr value added per worker (constant US\$) & & & 752 \\
\hline \multicolumn{4}{|l|}{ Hunger dimensions } \\
\hline Dietary energy supply (kcal/pc/day) & 2238 & 2504 & 3295 \\
\hline Average dietary energy supply adequacy (\%) & 103 & 113 & 147 \\
\hline Dietary en supp, cereals/roots/tubers (\%) & 70 & 69 & 66 \\
\hline Prevalence of undernourishment (\%) & 33.9 & 17.5 & $<5.0$ \\
\hline GDP per capita (US\$, PPP) & 1892 & 2240 & 3864 \\
\hline Domestic food price volatility (index) & & 9.8 & 18.3 \\
\hline Cereal import dependency ratio (\%) & 24.1 & 23.4 & 26.1 \\
\hline Underweight, children under-5 (\%) & 25.1 & 18.8 & 13.4 \\
\hline Improved water source (\% pop) & 54.4 & 70.8 & 87.2 \\
\hline \multicolumn{4}{|l|}{ Food supply } \\
\hline Food production value, $(2004-2006 \mathrm{mln}$ I\$) & 1941 & 4121 & 7581 \\
\hline Agriculture, value added (\% GDP) & 45 & 39 & 21 \\
\hline Food exports (mln US\$) & 403 & 507 & 2514 \\
\hline Food imports (mln US\$) & 156 & 274 & 1396 \\
\hline \multicolumn{4}{|l|}{ Production indices $(2004-06=100)$} \\
\hline Net food & 37 & 78 & 144 \\
\hline Net crops & 34 & 77 & 144 \\
\hline Cereals & 42 & 91 & 151 \\
\hline Vegetable oils & 51 & 71 & 98 \\
\hline Roots and tubers & 28 & 86 & 156 \\
\hline Fruit and vegetables & 41 & 79 & 152 \\
\hline Sugar & 79 & 100 & 107 \\
\hline Livestock & 78 & 92 & 136 \\
\hline Milk & 58 & 94 & 114 \\
\hline Meat & 82 & 93 & 135 \\
\hline Fish & 100 & 115 & 83 \\
\hline \multicolumn{4}{|l|}{ Net trade (mln US\$) } \\
\hline Cereals & -75 & -120 & -528 \\
\hline Fruit and vegetables & 3 & 21 & 96 \\
\hline Meat & & -18 & -253 \\
\hline Dairy products & -9 & -24 & -76 \\
\hline Fish & 10 & -5 & -214 \\
\hline \multicolumn{4}{|l|}{ Environment } \\
\hline Forest area (\%) & 33 & 27 & 21 \\
\hline Renewable water res withdrawn (\% of total) & & 66 & 66 \\
\hline Terrestrial protect areas (\% total land area) & 15 & 15 & 15 \\
\hline Organic area (\% total agricultural area) & & & 0 \\
\hline Water withdrawal by agriculture (\% of total) & & 66 & 66 \\
\hline Biofuel production (thousand kt of oil eq.) & 0 & 0 & \\
\hline \multicolumn{4}{|l|}{ Wood pellet prod. (1 000 tonnes) } \\
\hline Net GHG emissions from AFOLU ( $\mathrm{CO}_{2}$ eq, $\mathrm{Mt}$ ) & 43 & 46 & 40 \\
\hline
\end{tabular}




\begin{tabular}{|c|c|c|c|}
\hline & 1990 & 2000 & 2014 \\
\hline \multicolumn{4}{|l|}{ The setting } \\
\hline Population, total (mln) & 10.2 & 11 & 11.1 \\
\hline Population, rural (mln) & 4.2 & 4.4 & 4.2 \\
\hline \multicolumn{4}{|l|}{ Govt expenditure on ag (\% total outlays) } \\
\hline Area harvested (mln ha) & 4 & 5 & 5 \\
\hline Cropping intensity ratio & 0.5 & 0.6 & \\
\hline Water resources (1 $000 \mathrm{~m}^{3} /$ person/year) & 7 & 6 & 6 \\
\hline Area equipped for irrigation (1 $000 \mathrm{ha})$ & & & 1555 \\
\hline Area irrigated (\% area equipped for irrigation) & & & 82.3 \\
\hline Employment in agriculture (\%) & 23.9 & 17.4 & 13 \\
\hline Employment in agriculture, female (\%) & 30.3 & 19.6 & 12.9 \\
\hline Fertilizers, Nitrogen (kg of nutrients per ha) & & 105 & 55.1 \\
\hline Fertilizers, Phosphate (kg of nutrients per ha) & & 40.2 & 35.6 \\
\hline Fertilizers, Potash (kg nutrients per ha) & & 16.9 & 10 \\
\hline \multicolumn{4}{|l|}{ Energy consump, power irrigation (mln kWh) } \\
\hline Agr value added per worker (constant US\$) & & 12580 & 13670 \\
\hline \multicolumn{4}{|l|}{ Hunger dimensions } \\
\hline \multicolumn{4}{|l|}{ Dietary energy supply (kcal/pc/day) } \\
\hline Average dietary energy supply adequacy (\%) & 142 & 143 & 135 \\
\hline Dietary en supp, cereals/roots/tubers (\%) & 33 & 32 & 30 \\
\hline Prevalence of undernourishment (\%) & $<5.0$ & $<5.0$ & $<5.0$ \\
\hline GDP per capita (US\$, PPP) & 21085 & 25030 & 24305 \\
\hline Domestic food price volatility (index) & & 10.2 & 11.2 \\
\hline Cereal import dependency ratio (\%) & -17.6 & 17.2 & 16.7 \\
\hline \multicolumn{4}{|l|}{ Underweight, children under-5 (\%) } \\
\hline Improved water source (\% pop) & 96.2 & 98.9 & 99.8 \\
\hline \multicolumn{4}{|l|}{ Food supply } \\
\hline Food production value, (2004-2006 mln I\$) & 6393 & 7850 & 6481 \\
\hline Agriculture, value added (\% GDP) & & 6 & 4 \\
\hline Food exports (mln US\$) & 1823 & 1647 & 4293 \\
\hline Food imports (mln US\$) & 2278 & 2268 & 5644 \\
\hline \multicolumn{4}{|l|}{ Production indices $(2004-06=100)$} \\
\hline Net food & 87 & 106 & 88 \\
\hline Net crops & 80 & 110 & 84 \\
\hline Cereals & 88 & 100 & 92 \\
\hline Vegetable oils & 44 & 105 & 87 \\
\hline Roots and tubers & 104 & 114 & 89 \\
\hline Fruit and vegetables & 102 & 112 & 89 \\
\hline Sugar & 122 & 134 & 15 \\
\hline Livestock & 100 & 93 & 91 \\
\hline Milk & 88 & 98 & 90 \\
\hline Meat & 109 & 88 & 91 \\
\hline Fish & 71 & 97 & 104 \\
\hline \multicolumn{4}{|l|}{ Net trade (mln US\$) } \\
\hline Cereals & -74 & -211 & -388 \\
\hline Fruit and vegetables & 938 & 733 & 1480 \\
\hline Meat & -837 & -654 & -1399 \\
\hline Dairy products & -433 & -333 & -471 \\
\hline Fish & -107 & -48 & 152 \\
\hline \multicolumn{4}{|l|}{ Environment } \\
\hline Forest area (\%) & 26 & 28 & 31 \\
\hline Renewable water res withdrawn (\% of total) & & & 89 \\
\hline Terrestrial protect areas (\% total land area) & 9 & 22 & 35 \\
\hline Organic area (\% total agricultural area) & & & 6 \\
\hline Water withdrawal by agriculture (\% of total) & & & 89 \\
\hline Biofuel production (thousand kt of oil eq.) & & 0 & 3361 \\
\hline Wood pellet prod. (1 000 tonnes) & & & 0 \\
\hline Net GHG emissions from AFOLU ( $\mathrm{CO}_{2}$ eq, $\left.\mathrm{Mt}\right)$ & 11 & 8 & 6 \\
\hline
\end{tabular}




\begin{tabular}{|c|c|c|c|}
\hline & 1990 & 2000 & 2014 \\
\hline \multicolumn{4}{|l|}{ The setting } \\
\hline Population, total (mln) & 8.9 & 11.2 & 15.9 \\
\hline Population, rural (mln) & 5.2 & 6.1 & 7.8 \\
\hline Govt expenditure on ag (\% total outlays) & & 3.3 & 2.1 \\
\hline Area harvested (mln ha) & 10 & 17 & 26 \\
\hline Cropping intensity ratio & 2.2 & 3.7 & \\
\hline Water resources (1 $000 \mathrm{~m}^{3} /$ person/year) & 14 & 11 & 8 \\
\hline Area equipped for irrigation (1 $000 \mathrm{ha}$ ) & & & 338 \\
\hline Area irrigated (\% area equipped for irrigation) & & 100 & \\
\hline Employment in agriculture (\%) & 12.9 & 39.8 & 32.3 \\
\hline Employment in agriculture, female (\%) & 3.2 & 18.8 & 12.6 \\
\hline Fertilizers, Nitrogen (kg of nutrients per ha) & & 60.9 & 116.1 \\
\hline Fertilizers, Phosphate (kg of nutrients per ha) & & 24.1 & 32.8 \\
\hline Fertilizers, Potash (kg nutrients per ha) & & 6.6 & 9.7 \\
\hline Energy consump, power irrigation (mln kWh) & 0 & 0 & 246 \\
\hline Agr value added per worker (constant US\$) & 1477 & 1945 & 2009 \\
\hline \multicolumn{4}{|l|}{ Hunger dimensions } \\
\hline Dietary energy supply (kcal/pc/day) & 2326 & 2174 & 2435 \\
\hline Average dietary energy supply adequacy (\%) & 114 & 106 & 116 \\
\hline Dietary en supp, cereals/roots/tubers (\%) & 59 & 53 & 47 \\
\hline Prevalence of undernourishment (\%) & 15.3 & 22.1 & 15.6 \\
\hline GDP per capita (US\$, PPP) & 5315 & 6313 & 7062 \\
\hline Domestic food price volatility (index) & & 8 & 5.5 \\
\hline Cereal import dependency ratio (\%) & 24.4 & 40 & 44.3 \\
\hline Underweight, children under-5 (\%) & & 19.6 & 13 \\
\hline Improved water source (\% pop) & 81.4 & 87.4 & 93.8 \\
\hline \multicolumn{4}{|l|}{ Food supply } \\
\hline Food production value, (2004-2006 mln I\$) & 1615 & 2327 & 4330 \\
\hline Agriculture, value added (\% GDP) & & 14 & 11 \\
\hline Food exports (mln US\$) & 388 & 833 & 3101 \\
\hline Food imports (mln US\$) & 145 & 485 & 1749 \\
\hline \multicolumn{4}{|l|}{ Production indices $(2004-06=100)$} \\
\hline Net food & 56 & 81 & 151 \\
\hline Net crops & 60 & 88 & 154 \\
\hline Cereals & 121 & 98 & 150 \\
\hline Vegetable oils & 26 & 62 & 327 \\
\hline Roots and tubers & 40 & 52 & 123 \\
\hline Fruit and vegetables & 46 & 84 & 169 \\
\hline Sugar & 52 & 89 & 142 \\
\hline Livestock & 60 & 73 & 121 \\
\hline Milk & 61 & 65 & 120 \\
\hline Meat & 67 & 86 & 120 \\
\hline Fish & 31 & 171 & 153 \\
\hline \multicolumn{4}{|l|}{ Net trade (mln US\$) } \\
\hline Cereals & -46 & -74 & -589 \\
\hline Fruit and vegetables & 102 & 288 & 1003 \\
\hline Meat & 26 & -27 & -88 \\
\hline Dairy products & -20 & -61 & -162 \\
\hline Fish & 10 & 27 & 20 \\
\hline \multicolumn{4}{|l|}{ Environment } \\
\hline Forest area (\%) & 44 & 39 & 33 \\
\hline Renewable water res withdrawn (\% of total) & & & 57 \\
\hline Terrestrial protect areas (\% total land area) & 26 & 30 & 31 \\
\hline Organic area (\% total agricultural area) & & & 0 \\
\hline Water withdrawal by agriculture (\% of total) & & & 57 \\
\hline Biofuel production (thousand kt of oil eq.) & 24 & 42 & 109 \\
\hline \multicolumn{4}{|l|}{ Wood pellet prod. (1 000 tonnes) } \\
\hline Net GHG emissions from AFOLU ( $\mathrm{CO}_{2}$ eq, $\mathrm{Mt}$ ) & 22 & 23 & 24 \\
\hline
\end{tabular}




\begin{tabular}{|c|c|c|c|}
\hline & 1990 & 2000 & 2014 \\
\hline \multicolumn{4}{|l|}{ The setting } \\
\hline Population, total (mln) & 6 & 8.7 & 12 \\
\hline Population, rural (mln) & 4.3 & 6 & 7.6 \\
\hline \multicolumn{4}{|l|}{ Govt expenditure on ag (\% total outlays) } \\
\hline Area harvested (mln ha) & 1 & 2 & 3 \\
\hline Cropping intensity ratio & 0.1 & 0.1 & \\
\hline Water resources (1 $000 \mathrm{~m}^{3} /$ person/year) & 38 & 26 & 19 \\
\hline Area equipped for irrigation (1 $000 \mathrm{ha})$ & & & 95 \\
\hline Area irrigated (\% area equipped for irrigation) & & 100 & \\
\hline Employment in agriculture (\%) & & 76 & \\
\hline Employment in agriculture, female (\%) & & 78.8 & \\
\hline Fertilizers, Nitrogen (kg of nutrients per ha) & & 0.7 & 2.1 \\
\hline Fertilizers, Phosphate (kg of nutrients per ha) & & 0.1 & 0.6 \\
\hline Fertilizers, Potash (kg nutrients per ha) & & 0 & 0.7 \\
\hline Energy consump, power irrigation (mln kWh) & 0 & 4 & 1 \\
\hline Agr value added per worker (constant US\$) & 150 & 165 & 215 \\
\hline \multicolumn{4}{|l|}{ Hunger dimensions } \\
\hline Dietary energy supply (kcal/pc/day) & 2431 & 2346 & 2604 \\
\hline Average dietary energy supply adequacy (\%) & 113 & 108 & 117 \\
\hline Dietary en supp, cereals/roots/tubers (\%) & 62 & 62 & 62 \\
\hline Prevalence of undernourishment (\%) & 22.7 & 27.2 & 16.8 \\
\hline GDP per capita (US\$, PPP) & 1128 & 1139 & 1213 \\
\hline Domestic food price volatility (index) & & 13.2 & 7.3 \\
\hline Cereal import dependency ratio (\%) & 27.4 & 20.4 & 13.8 \\
\hline Underweight, children under-5 (\%) & & 29.1 & 16.3 \\
\hline Improved water source (\% pop) & 52.4 & 62.7 & 74.8 \\
\hline \multicolumn{4}{|l|}{ Food supply } \\
\hline Food production value, $(2004-2006 \mathrm{mln}$ I\$) & 956 & 1346 & 1988 \\
\hline Agriculture, value added (\% GDP) & 24 & 22 & 20 \\
\hline Food exports (mln US\$) & 18 & 17 & 58 \\
\hline Food imports (mln US\$) & 104 & 123 & 490 \\
\hline \multicolumn{4}{|l|}{ Production indices $(2004-06=100)$} \\
\hline Net food & 59 & 84 & 124 \\
\hline Net crops & 60 & 85 & 121 \\
\hline Cereals & 49 & 82 & 156 \\
\hline Vegetable oils & 51 & 85 & 97 \\
\hline Roots and tubers & 70 & 96 & 117 \\
\hline Fruit and vegetables & 72 & 84 & 102 \\
\hline Sugar & 80 & 96 & 107 \\
\hline Livestock & 43 & 78 & 132 \\
\hline Milk & 47 & 78 & 131 \\
\hline Meat & 43 & 79 & 132 \\
\hline Fish & 42 & 88 & 122 \\
\hline \multicolumn{4}{|l|}{ Net trade (mln US\$) } \\
\hline Cereals & -51 & -56 & -224 \\
\hline Fruit and vegetables & -3 & -7 & -19 \\
\hline Meat & & -3 & -22 \\
\hline Dairy products & & -8 & -41 \\
\hline Fish & -6 & 4 & 4 \\
\hline \multicolumn{4}{|l|}{ Environment } \\
\hline Forest area (\%) & 30 & 28 & 26 \\
\hline Renewable water res withdrawn (\% of total) & & 53 & 53 \\
\hline Terrestrial protect areas (\% total land area) & 7 & 7 & 28 \\
\hline \multicolumn{4}{|l|}{ Organic area (\% total agricultural area) } \\
\hline Water withdrawal by agriculture (\% of total) & & 53 & 53 \\
\hline Biofuel production (thousand kt of oil eq.) & 1 & 1 & 1 \\
\hline \multicolumn{4}{|l|}{ Wood pellet prod. (1 000 tonnes) } \\
\hline Net GHG emissions from AFOLU ( $\mathrm{CO}_{2}$ eq, $\mathrm{Mt}$ ) & 19 & 21 & 25 \\
\hline
\end{tabular}




\begin{tabular}{|c|c|c|c|}
\hline & 1990 & 2000 & 2014 \\
\hline \multicolumn{4}{|l|}{ The setting } \\
\hline Population, total (mln) & 1 & 1.3 & 1.7 \\
\hline Population, rural (mln) & 0.7 & 0.8 & 0.9 \\
\hline \multicolumn{4}{|l|}{ Govt expenditure on ag (\% total outlays) } \\
\hline Area harvested (mln ha) & 0 & 0 & 0 \\
\hline Cropping intensity ratio & 0.2 & 0.2 & \\
\hline Water resources (1 $000 \mathrm{~m}^{3} /$ person/year) & 31 & 25 & 18 \\
\hline Area equipped for irrigation (1 $000 \mathrm{ha})$ & & & 25 \\
\hline Area irrigated (\% area equipped for irrigation) & & 100 & \\
\hline \multicolumn{4}{|l|}{ Employment in agriculture (\%) } \\
\hline \multicolumn{4}{|l|}{ Employment in agriculture, female (\%) } \\
\hline \multicolumn{4}{|l|}{ Fertilizers, Nitrogen (kg of nutrients per ha) } \\
\hline \multicolumn{4}{|l|}{ Fertilizers, Phosphate (kg of nutrients per ha) } \\
\hline \multicolumn{4}{|l|}{ Fertilizers, Potash (kg nutrients per ha) } \\
\hline \multicolumn{4}{|l|}{ Energy consump, power irrigation (mln kWh) } \\
\hline Agr value added per worker (constant US\$) & & 567 & 655 \\
\hline \multicolumn{4}{|l|}{ Hunger dimensions } \\
\hline Dietary energy supply (kcal/pc/day) & 2340 & 2222 & 2371 \\
\hline Average dietary energy supply adequacy (\%) & 108 & 102 & 107 \\
\hline Dietary en supp, cereals/roots/tubers (\%) & 69 & 70 & 64 \\
\hline Prevalence of undernourishment (\%) & 21.4 & 28.4 & 22 \\
\hline GDP per capita (US\$, PPP) & 1564 & 1330 & 1362 \\
\hline \multicolumn{4}{|l|}{ Domestic food price volatility (index) } \\
\hline Cereal import dependency ratio (\%) & 34.9 & 37.5 & 31.4 \\
\hline Underweight, children under-5 (\%) & & 21.9 & 18.1 \\
\hline Improved water source (\% pop) & 35.8 & 51.9 & 73.6 \\
\hline \multicolumn{4}{|l|}{ Food supply } \\
\hline Food production value, $(2004-2006 \mathrm{mln}$ I\$) & 147 & 206 & 333 \\
\hline Agriculture, value added (\% GDP) & 61 & 43 & 44 \\
\hline Food exports (mln US\$) & 13 & 50 & 113 \\
\hline Food imports (mln US\$) & 19 & 35 & 71 \\
\hline \multicolumn{4}{|l|}{ Production indices $(2004-06=100)$} \\
\hline Net food & 62 & 87 & 141 \\
\hline Net crops & 60 & 86 & 143 \\
\hline Cereals & 93 & 93 & 150 \\
\hline Vegetable oils & 81 & 94 & 155 \\
\hline Roots and tubers & 65 & 89 & 119 \\
\hline Fruit and vegetables & 72 & 94 & 125 \\
\hline Sugar & 100 & 100 & 115 \\
\hline Livestock & 71 & 90 & 129 \\
\hline Milk & 82 & 93 & 125 \\
\hline Meat & 69 & 89 & 131 \\
\hline \multicolumn{4}{|l|}{ Fish } \\
\hline \multicolumn{4}{|l|}{ Net trade (mln US\$) } \\
\hline Cereals & -12 & -24 & -37 \\
\hline Fruit and vegetables & 11 & 48 & 108 \\
\hline Meat & -1 & -2 & -2 \\
\hline Dairy products & -1 & -1 & -4 \\
\hline Fish & 3 & 3 & 2 \\
\hline \multicolumn{4}{|l|}{ Environment } \\
\hline Forest area (\%) & 79 & 75 & 71 \\
\hline Renewable water res withdrawn (\% of total) & & 82 & 82 \\
\hline Terrestrial protect areas (\% total land area) & 8 & 16 & 16 \\
\hline Organic area (\% total agricultural area) & & & 0 \\
\hline Water withdrawal by agriculture (\% of total) & & 82 & 82 \\
\hline \multicolumn{4}{|l|}{ Biofuel production (thousand kt of oil eq.) } \\
\hline \multicolumn{4}{|l|}{ Wood pellet prod. (1 000 tonnes) } \\
\hline Net GHG emissions from AFOLU ( $\mathrm{CO}_{2}$ eq, $\mathrm{Mt}$ ) & 3 & 3 & 4 \\
\hline
\end{tabular}




\begin{tabular}{|c|c|c|c|}
\hline & 1990 & 2000 & 2014 \\
\hline \multicolumn{4}{|l|}{ The setting } \\
\hline Population, total (mln) & 0.7 & 0.7 & 0.8 \\
\hline Population, rural (mln) & 0.5 & 0.5 & 0.6 \\
\hline \multicolumn{4}{|l|}{ Govt expenditure on ag (\% total outlays) } \\
\hline Area harvested (mln ha) & 3 & 3 & 2 \\
\hline Cropping intensity ratio & 1.6 & 1.6 & \\
\hline Water resources (1 $000 \mathrm{~m}^{3} /$ person/year) & 374 & 364 & 339 \\
\hline Area equipped for irrigation (1 $000 \mathrm{ha}$ ) & & & 143 \\
\hline Area irrigated (\% area equipped for irrigation) & & & 89.2 \\
\hline Employment in agriculture (\%) & & 21.4 & \\
\hline Employment in agriculture, female (\%) & & 7.1 & \\
\hline Fertilizers, Nitrogen (kg of nutrients per ha) & & 21.4 & 20.3 \\
\hline Fertilizers, Phosphate (kg of nutrients per ha) & & 1.6 & 3.8 \\
\hline Fertilizers, Potash (kg nutrients per ha) & & 0.1 & 1.4 \\
\hline Energy consump, power irrigation (mln kWh) & 0 & 0 & 0 \\
\hline Agr value added per worker (constant US\$) & 2525 & 4504 & 5035 \\
\hline \multicolumn{4}{|l|}{ Hunger dimensions } \\
\hline Dietary energy supply (kcal/pc/day) & 2470 & 2722 & 2727 \\
\hline Average dietary energy supply adequacy (\%) & 107 & 118 & 117 \\
\hline Dietary en supp, cereals/roots/tubers (\%) & 55 & 49 & 50 \\
\hline Prevalence of undernourishment (\%) & 20.5 & 10.4 & 11.2 \\
\hline GDP per capita (US\$, PPP) & 3231 & 5071 & 6336 \\
\hline \multicolumn{4}{|l|}{ Domestic food price volatility (index) } \\
\hline Cereal import dependency ratio (\%) & -65.9 & -74.1 & -21.2 \\
\hline Underweight, children under-5 (\%) & 16.1 & 11.9 & 11.1 \\
\hline Improved water source (\% pop) & 77.1 & 86.2 & 97.6 \\
\hline \multicolumn{4}{|l|}{ Food supply } \\
\hline Food production value, (2004-2006 mln I\$) & 156 & 308 & 410 \\
\hline Agriculture, value added (\% GDP) & 38 & 31 & 18 \\
\hline Food exports (mln US\$) & 97 & 171 & 387 \\
\hline Food imports (mln US\$) & 32 & 65 & 202 \\
\hline \multicolumn{4}{|l|}{ Production indices $(2004-06=100)$} \\
\hline Net food & 49 & 97 & 130 \\
\hline Net crops & 59 & 101 & 129 \\
\hline Cereals & 34 & 98 & 179 \\
\hline Vegetable oils & 53 & 111 & 90 \\
\hline Roots and tubers & 60 & 149 & 75 \\
\hline Fruit and vegetables & 85 & 191 & 137 \\
\hline Sugar & 83 & 84 & 76 \\
\hline Livestock & 37 & 68 & 126 \\
\hline Milk & 52 & 85 & 125 \\
\hline Meat & 30 & 61 & 126 \\
\hline Fish & 67 & 90 & 90 \\
\hline \multicolumn{4}{|l|}{ Net trade (mln US\$) } \\
\hline Cereals & 2 & 23 & 125 \\
\hline Fruit and vegetables & -3 & -6 & -17 \\
\hline Meat & -1 & -8 & -8 \\
\hline Dairy products & -3 & -14 & -41 \\
\hline Fish & 27 & 49 & 74 \\
\hline \multicolumn{4}{|l|}{ Environment } \\
\hline Forest area (\%) & 77 & 77 & 77 \\
\hline Renewable water res withdrawn (\% of total) & & & 94 \\
\hline Terrestrial protect areas (\% total land area) & 0 & 2 & 5 \\
\hline Organic area (\% total agricultural area) & & & 0 \\
\hline Water withdrawal by agriculture (\% of total) & & & 94 \\
\hline Biofuel production (thousand kt of oil eq.) & 3 & 7 & 7 \\
\hline \multicolumn{4}{|l|}{ Wood pellet prod. (1 000 tonnes) } \\
\hline Net GHG emissions from AFOLU ( $\mathrm{CO}_{2}$ eq, $\left.\mathrm{Mt}\right)$ & 5 & 5 & 6 \\
\hline
\end{tabular}




\section{Haiti}

\begin{tabular}{|c|c|c|c|}
\hline & 1990 & 2000 & 2014 \\
\hline \multicolumn{4}{|l|}{ The setting } \\
\hline Population, total (mln) & 7.1 & 8.6 & 10.5 \\
\hline Population, rural (mln) & 5.1 & 5.5 & 4.5 \\
\hline \multicolumn{4}{|l|}{ Govt expenditure on ag (\% total outlays) } \\
\hline Area harvested (mln ha) & 2 & 1 & 2 \\
\hline Cropping intensity ratio & 0.9 & 0.6 & \\
\hline Water resources (1 $000 \mathrm{~m}^{3} /$ person/year) & 2 & 2 & 1 \\
\hline Area equipped for irrigation (1 $000 \mathrm{ha})$ & & & 97 \\
\hline Area irrigated (\% area equipped for irrigation) & & & 82 \\
\hline Employment in agriculture (\%) & 65.6 & 50.5 & \\
\hline Employment in agriculture, female (\%) & 49.6 & 37.3 & \\
\hline \multicolumn{4}{|l|}{ Fertilizers, Nitrogen (kg of nutrients per ha) } \\
\hline \multicolumn{4}{|l|}{ Fertilizers, Phosphate (kg of nutrients per ha) } \\
\hline \multicolumn{4}{|l|}{ Fertilizers, Potash (kg nutrients per ha) } \\
\hline Energy consump, power irrigation (mln kWh) & 0 & 0 & 0 \\
\hline \multicolumn{4}{|l|}{ Agr value added per worker (constant US\$) } \\
\hline \multicolumn{4}{|l|}{ Hunger dimensions } \\
\hline Dietary energy supply (kcal/pc/day) & 1714 & 1937 & 2014 \\
\hline Average dietary energy supply adequacy (\%) & 78 & 87 & 87 \\
\hline Dietary en supp, cereals/roots/tubers (\%) & 58 & 53 & 53 \\
\hline Prevalence of undernourishment (\%) & 62.6 & 55.2 & 52.3 \\
\hline GDP per capita (US\$, PPP) & & 1734 & 1648 \\
\hline Domestic food price volatility (index) & & 9.2 & 3.4 \\
\hline Cereal import dependency ratio (\%) & 48.1 & 58 & 51.8 \\
\hline Underweight, children under-5 (\%) & 23.7 & 13.9 & 11.6 \\
\hline Improved water source (\% pop) & 60.8 & 60.9 & 62.4 \\
\hline \multicolumn{4}{|l|}{ Food supply } \\
\hline Food production value, (2004-2006 mln I\$) & 852 & 887 & 1092 \\
\hline \multicolumn{4}{|l|}{ Agriculture, value added (\% GDP) } \\
\hline Food exports (mln US\$) & 12 & 16 & 18 \\
\hline Food imports (mln US\$) & 187 & 301 & 911 \\
\hline \multicolumn{4}{|l|}{ Production indices $(2004-06=100)$} \\
\hline Net food & 94 & 98 & 121 \\
\hline Net crops & 105 & 99 & 122 \\
\hline Cereals & 95 & 110 & 153 \\
\hline Vegetable oils & 134 & 92 & 98 \\
\hline Roots and tubers & 87 & 90 & 181 \\
\hline Fruit and vegetables & 107 & 106 & 90 \\
\hline Sugar & 142 & 76 & 113 \\
\hline Livestock & 60 & 93 & 107 \\
\hline Milk & 80 & 93 & 132 \\
\hline Meat & 56 & 92 & 104 \\
\hline Fish & 47 & 67 & 157 \\
\hline \multicolumn{4}{|l|}{ Net trade (mln US\$) } \\
\hline Cereals & -94 & -123 & \\
\hline Fruit and vegetables & -2 & -9 & -35 \\
\hline Meat & -4 & -19 & -103 \\
\hline Dairy products & & -27 & -76 \\
\hline Fish & -3 & -2 & -24 \\
\hline \multicolumn{4}{|l|}{ Environment } \\
\hline Forest area (\%) & 4 & 4 & 4 \\
\hline Renewable water res withdrawn (\% of total) & & & 83 \\
\hline Terrestrial protect areas (\% total land area) & 0 & 0 & 0 \\
\hline Organic area (\% total agricultural area) & & & 0 \\
\hline Water withdrawal by agriculture (\% of total) & & & 83 \\
\hline Biofuel production (thousand kt of oil eq.) & 4 & 3 & 0 \\
\hline \multicolumn{4}{|l|}{ Wood pellet prod. (1 000 tonnes) } \\
\hline Net GHG emissions from AFOLU ( $\mathrm{CO}_{2}$ eq, $\left.\mathrm{Mt}\right)$ & 3 & 4 & 4 \\
\hline
\end{tabular}




\begin{tabular}{|c|c|c|c|}
\hline & 1990 & 2000 & 2014 \\
\hline \multicolumn{4}{|l|}{ The setting } \\
\hline Population, total (mln) & 4.9 & 6.2 & 8.3 \\
\hline Population, rural (mln) & 2.9 & 3.4 & 3.8 \\
\hline \multicolumn{4}{|l|}{ Govt expenditure on ag (\% total outlays) } \\
\hline Area harvested (mln ha) & 3 & 4 & 6 \\
\hline Cropping intensity ratio & 0.9 & 1.4 & \\
\hline Water resources (1 $000 \mathrm{~m}^{3} /$ person/year) & 19 & 15 & 11 \\
\hline Area equipped for irrigation (1 $000 \mathrm{ha})$ & & & 90 \\
\hline Area irrigated (\% area equipped for irrigation) & & & 92.9 \\
\hline Employment in agriculture (\%) & 50.1 & 37.4 & 35.3 \\
\hline Employment in agriculture, female (\%) & 5.8 & 8.7 & 9.4 \\
\hline Fertilizers, Nitrogen (kg of nutrients per ha) & & 48.4 & 72.3 \\
\hline Fertilizers, Phosphate (kg of nutrients per ha) & & 5.9 & 5 \\
\hline Fertilizers, Potash (kg nutrients per ha) & & 0.6 & 5.9 \\
\hline Energy consump, power irrigation (mln kWh) & 0 & 0 & 0 \\
\hline Agr value added per worker (constant US\$) & 1222 & 1434 & 2647 \\
\hline \multicolumn{4}{|l|}{ Hunger dimensions } \\
\hline Dietary energy supply (kcal/pc/day) & 2335 & 2406 & 2723 \\
\hline Average dietary energy supply adequacy (\%) & 112 & 114 & 122 \\
\hline Dietary en supp, cereals/roots/tubers (\%) & 50 & 48 & 47 \\
\hline Prevalence of undernourishment (\%) & 21.9 & 19 & 12.3 \\
\hline GDP per capita (US\$, PPP) & 3205 & 3483 & 4445 \\
\hline Domestic food price volatility (index) & & 21.6 & 4.8 \\
\hline Cereal import dependency ratio (\%) & 25.2 & 44.9 & 56.5 \\
\hline Underweight, children under-5 (\%) & 15.8 & 12.5 & 7.1 \\
\hline Improved water source (\% pop) & 72.8 & 80.8 & 89.6 \\
\hline \multicolumn{4}{|l|}{ Food supply } \\
\hline Food production value, (2004-2006 mln I\$) & 984 & 1054 & 1755 \\
\hline Agriculture, value added (\% GDP) & 22 & 16 & 14 \\
\hline Food exports (mln US\$) & 456 & 226 & 923 \\
\hline Food imports (mln US\$) & 83 & 323 & 1083 \\
\hline \multicolumn{4}{|l|}{ Production indices $(2004-06=100)$} \\
\hline Net food & 66 & 71 & 119 \\
\hline Net crops & 72 & 69 & 126 \\
\hline Cereals & 125 & 106 & 126 \\
\hline Vegetable oils & 35 & 43 & 189 \\
\hline Roots and tubers & 60 & 76 & 126 \\
\hline Fruit and vegetables & 81 & 53 & 114 \\
\hline Sugar & 52 & 72 & 110 \\
\hline Livestock & 52 & 78 & 110 \\
\hline Milk & 51 & 83 & 101 \\
\hline Meat & 51 & 73 & 114 \\
\hline Fish & 31 & 44 & 126 \\
\hline \multicolumn{4}{|l|}{ Net trade (mln US\$) } \\
\hline Cereals & -32 & -113 & -411 \\
\hline Fruit and vegetables & 399 & 136 & 263 \\
\hline Meat & 26 & -21 & -38 \\
\hline Dairy products & -10 & -37 & -41 \\
\hline Fish & 29 & 173 & 241 \\
\hline \multicolumn{4}{|l|}{ Environment } \\
\hline Forest area (\%) & 73 & 57 & 44 \\
\hline Renewable water res withdrawn (\% of total) & & 73 & 73 \\
\hline Terrestrial protect areas (\% total land area) & 16 & 21 & 21 \\
\hline Organic area (\% total agricultural area) & & & 1 \\
\hline Water withdrawal by agriculture (\% of total) & & 73 & 73 \\
\hline Biofuel production (thousand kt of oil eq.) & 5 & 8 & 23 \\
\hline Wood pellet prod. (1 000 tonnes) & & & 4 \\
\hline Net GHG emissions from AFOLU ( $\mathrm{CO}_{2}$ eq, $\mathrm{Mt}$ ) & 45 & 46 & 34 \\
\hline
\end{tabular}




\begin{tabular}{|c|c|c|c|}
\hline & 1990 & 2000 & 2014 \\
\hline \multicolumn{4}{|l|}{ The setting } \\
\hline Population, total (mln) & 10.4 & 10.2 & 9.9 \\
\hline Population, rural (mln) & 3.5 & 3.6 & 2.9 \\
\hline \multicolumn{4}{|l|}{ Govt expenditure on ag (\% total outlays) } \\
\hline Area harvested (mln ha) & 13 & 10 & 14 \\
\hline Cropping intensity ratio & 1.9 & 1.7 & \\
\hline Water resources (1 $000 \mathrm{~m}^{3} /$ person/year) & 10 & 10 & 10 \\
\hline Area equipped for irrigation (1 $000 \mathrm{ha}$ ) & & & 172 \\
\hline Area irrigated (\% area equipped for irrigation) & & & 62.2 \\
\hline Employment in agriculture (\%) & 18.2 & 6.5 & 5.2 \\
\hline Employment in agriculture, female (\%) & 15 & 3.6 & 2.9 \\
\hline Fertilizers, Nitrogen (kg of nutrients per ha) & & 62.9 & 68.8 \\
\hline Fertilizers, Phosphate (kg of nutrients per ha) & & 14.6 & 14.1 \\
\hline Fertilizers, Potash (kg nutrients per ha) & & 18.1 & 14.2 \\
\hline Energy consump, power irrigation (mln kWh) & 5 & 7 & 235 \\
\hline Agr value added per worker (constant US\$) & & 6603 & 11505 \\
\hline \multicolumn{4}{|l|}{ Hunger dimensions } \\
\hline \multicolumn{4}{|l|}{ Dietary energy supply (kcal/pc/day) } \\
\hline Average dietary energy supply adequacy (\%) & 125 & 122 & 111 \\
\hline Dietary en supp, cereals/roots/tubers (\%) & 28 & 29 & 30 \\
\hline Prevalence of undernourishment (\%) & $<5.0$ & $<5.0$ & $<5.0$ \\
\hline GDP per capita (US\$, PPP) & 14408 & 17707 & 22707 \\
\hline Domestic food price volatility (index) & & 6.9 & 5.8 \\
\hline Cereal import dependency ratio (\%) & -20.1 & -28.4 & -81.1 \\
\hline \multicolumn{4}{|l|}{ Underweight, children under-5 (\%) } \\
\hline Improved water source (\% pop) & 95.7 & 99 & 100 \\
\hline \multicolumn{4}{|l|}{ Food supply } \\
\hline Food production value, (2004-2006 mln I\$) & 7118 & 5334 & 5189 \\
\hline Agriculture, value added (\% GDP) & & 6 & 4 \\
\hline Food exports (mln US\$) & 1991 & 1820 & 7988 \\
\hline Food imports (mln US\$) & 312 & 522 & 3590 \\
\hline \multicolumn{4}{|l|}{ Production indices $(2004-06=100)$} \\
\hline Net food & 122 & 91 & 89 \\
\hline Net crops & 101 & 76 & 87 \\
\hline Cereals & 79 & 63 & 86 \\
\hline Vegetable oils & 57 & 46 & 134 \\
\hline Roots and tubers & 170 & 130 & 66 \\
\hline Fruit and vegetables & 135 & 104 & 84 \\
\hline Sugar & 150 & 62 & 30 \\
\hline Livestock & 169 & 121 & 88 \\
\hline Milk & 150 & 114 & 93 \\
\hline Meat & 181 & 128 & 86 \\
\hline Fish & 160 & 94 & 101 \\
\hline \multicolumn{4}{|l|}{ Net trade (mln US\$) } \\
\hline Cereals & 106 & 220 & 1593 \\
\hline Fruit and vegetables & 374 & 216 & 288 \\
\hline Meat & 819 & 525 & 760 \\
\hline Dairy products & 85 & 37 & -39 \\
\hline Fish & -37 & -34 & -60 \\
\hline \multicolumn{4}{|l|}{ Environment } \\
\hline Forest area (\%) & 20 & 21 & 23 \\
\hline Renewable water res withdrawn (\% of total) & & & 6 \\
\hline Terrestrial protect areas (\% total land area) & 6 & 7 & 23 \\
\hline Organic area (\% total agricultural area) & & & 2 \\
\hline Water withdrawal by agriculture (\% of total) & & & 6 \\
\hline Biofuel production (thousand kt of oil eq.) & & 0 & 3843 \\
\hline Wood pellet prod. (1 000 tonnes) & & & 4 \\
\hline Net GHG emissions from AFOLU ( $\mathrm{CO}_{2}$ eq, $\left.\mathrm{Mt}\right)$ & 16 & 11 & 10 \\
\hline
\end{tabular}




\begin{tabular}{|c|c|c|c|}
\hline & 1990 & 2000 & 2014 \\
\hline \multicolumn{4}{|l|}{ The setting } \\
\hline Population, total (mln) & 0.3 & 0.3 & 0.3 \\
\hline Population, rural (mln) & 0 & 0 & 0 \\
\hline \multicolumn{4}{|l|}{ Govt expenditure on ag (\% total outlays) } \\
\hline Area harvested (mln ha) & 1 & 3 & 0 \\
\hline Cropping intensity ratio & 0.6 & 1.5 & \\
\hline Water resources (1 $000 \mathrm{~m}^{3} /$ person/year) & 667 & 605 & 515 \\
\hline \multicolumn{4}{|l|}{ Area equipped for irrigation (1 $000 \mathrm{ha})$} \\
\hline \multicolumn{4}{|l|}{ Area irrigated (\% area equipped for irrigation) } \\
\hline Employment in agriculture (\%) & 9.2 & 8.3 & 5.5 \\
\hline Employment in agriculture, female (\%) & 3.3 & 4.4 & 2.1 \\
\hline Fertilizers, Nitrogen (kg of nutrients per ha) & & 80.2 & 77.8 \\
\hline Fertilizers, Phosphate (kg of nutrients per ha) & & 26.3 & 58 \\
\hline Fertilizers, Potash (kg nutrients per ha) & & 16.4 & 40.7 \\
\hline \multicolumn{4}{|l|}{ Energy consump, power irrigation (mln kWh) } \\
\hline Agr value added per worker (constant US\$) & & 60555 & 74231 \\
\hline \multicolumn{4}{|l|}{ Hunger dimensions } \\
\hline \multicolumn{4}{|l|}{ Dietary energy supply (kcal/pc/day) } \\
\hline Average dietary energy supply adequacy (\%) & 124 & 125 & 132 \\
\hline Dietary en supp, cereals/roots/tubers (\%) & 26 & 24 & 23 \\
\hline Prevalence of undernourishment (\%) & $<5.0$ & $<5.0$ & $<5.0$ \\
\hline GDP per capita (US\$, PPP) & 28658 & 33590 & 40789 \\
\hline Domestic food price volatility (index) & & 6.1 & 5.4 \\
\hline \multicolumn{4}{|l|}{ Cereal import dependency ratio (\%) } \\
\hline \multicolumn{4}{|l|}{ Underweight, children under-5 (\%) } \\
\hline Improved water source (\% pop) & 100 & 100 & 100 \\
\hline \multicolumn{4}{|l|}{ Food supply } \\
\hline Food production value, (2004-2006 mln I\$) & 83 & 91 & 110 \\
\hline Agriculture, value added (\% GDP) & & 8 & 7 \\
\hline Food exports (mln US\$) & 13 & 11 & 50 \\
\hline Food imports (mln US\$) & 95 & 122 & 292 \\
\hline \multicolumn{4}{|l|}{ Production indices $(2004-06=100)$} \\
\hline Net food & 86 & 95 & 114 \\
\hline Net crops & 41 & 77 & 79 \\
\hline \multicolumn{4}{|l|}{ Cereals } \\
\hline \multicolumn{4}{|l|}{ Vegetable oils } \\
\hline Roots and tubers & 55 & 72 & 44 \\
\hline Fruit and vegetables & 24 & 84 & 120 \\
\hline \multicolumn{4}{|l|}{ Sugar } \\
\hline Livestock & 89 & 96 & 115 \\
\hline Milk & 100 & 92 & 109 \\
\hline Meat & 80 & 98 & 120 \\
\hline Fish & 95 & 125 & 87 \\
\hline \multicolumn{4}{|l|}{ Net trade (mln US\$) } \\
\hline Cereals & -25 & -36 & -80 \\
\hline Fruit and vegetables & -33 & -38 & -96 \\
\hline Meat & 10 & 4 & 20 \\
\hline Dairy products & 0 & -1 & 5 \\
\hline Fish & 1223 & 1162 & 2093 \\
\hline \multicolumn{4}{|l|}{ Environment } \\
\hline Forest area (\%) & 0 & 0 & 0 \\
\hline Renewable water res withdrawn (\% of total) & & & 42 \\
\hline Terrestrial protect areas (\% total land area) & 10 & 10 & 20 \\
\hline Organic area (\% total agricultural area) & & & 1 \\
\hline Water withdrawal by agriculture (\% of total) & & & 42 \\
\hline Biofuel production (thousand kt of oil eq.) & & & 0 \\
\hline Wood pellet prod. (1 000 tonnes) & & & 0 \\
\hline Net GHG emissions from AFOLU ( $\left.\mathrm{CO}_{2} \mathrm{eq}, \mathrm{Mt}\right)$ & 1 & 0 & 0 \\
\hline
\end{tabular}




\begin{tabular}{|c|c|c|c|}
\hline & 1990 & 2000 & 2014 \\
\hline \multicolumn{4}{|l|}{ The setting } \\
\hline Population, total (mln) & 868.9 & 1042.3 & 1267.4 \\
\hline Population, rural (mln) & 646.9 & 753.9 & 857.1 \\
\hline Govt expenditure on ag (\% total outlays) & & 4.1 & 6.5 \\
\hline Area harvested (mln ha) & 226 & 299 & 341 \\
\hline Cropping intensity ratio & 1.2 & 1.7 & \\
\hline Water resources (1 $000 \mathrm{~m}^{3} /$ person/year) & 2 & 2 & 2 \\
\hline Area equipped for irrigation (1 $000 \mathrm{ha})$ & & & 66700 \\
\hline Area irrigated (\% area equipped for irrigation) & & & 93.9 \\
\hline Employment in agriculture (\%) & & 59.9 & 47.2 \\
\hline Employment in agriculture, female (\%) & & 74.8 & 59.8 \\
\hline Fertilizers, Nitrogen (kg of nutrients per ha) & & 69.3 & 107.9 \\
\hline Fertilizers, Phosphate (kg of nutrients per ha) & & 25.9 & 43.1 \\
\hline Fertilizers, Potash (kg nutrients per ha) & & 10 & 12.7 \\
\hline Energy consump, power irrigation (mln kWh) & 2 & 1859 & 4881 \\
\hline Agr value added per worker (constant US\$) & 459 & 528 & 689 \\
\hline \multicolumn{4}{|l|}{ Hunger dimensions } \\
\hline Dietary energy supply (kcal/pc/day) & 2298 & 2370 & 2462 \\
\hline Average dietary energy supply adequacy (\%) & 106 & 107 & 108 \\
\hline Dietary en supp, cereals/roots/tubers (\%) & 66 & 63 & 59 \\
\hline Prevalence of undernourishment (\%) & 22.4 & 17 & 15.3 \\
\hline GDP per capita (US\$, PPP) & 1777 & 2548 & 5244 \\
\hline Domestic food price volatility (index) & & 3.9 & 8.4 \\
\hline Cereal import dependency ratio (\%) & -0.2 & -1.4 & -3.1 \\
\hline Underweight, children under-5 (\%) & 50.7 & 44.4 & 43.5 \\
\hline Improved water source (\% pop) & 70.3 & 80.6 & 92.6 \\
\hline \multicolumn{4}{|l|}{ Food supply } \\
\hline Food production value, (2004-2006 mln I\$) & 119804 & 156265 & 236540 \\
\hline Agriculture, value added (\% GDP) & 29 & 23 & 17 \\
\hline Food exports (mln US\$) & 1013 & 2826 & 20835 \\
\hline Food imports (mln US\$) & 758 & 2062 & 16523 \\
\hline \multicolumn{4}{|l|}{ Production indices $(2004-06=100)$} \\
\hline Net food & 70 & 92 & 139 \\
\hline Net crops & 73 & 92 & 142 \\
\hline Cereals & 82 & 98 & 123 \\
\hline Vegetable oils & 78 & 81 & 134 \\
\hline Roots and tubers & 55 & 88 & 152 \\
\hline Fruit and vegetables & 61 & 89 & 163 \\
\hline Sugar & 90 & 119 & 136 \\
\hline Livestock & 61 & 85 & 135 \\
\hline Milk & 56 & 83 & 141 \\
\hline Meat & 77 & 90 & 117 \\
\hline Fish & 57 & 85 & 139 \\
\hline \multicolumn{4}{|l|}{ Net trade (mln US\$) } \\
\hline Cereals & 191 & 803 & 9154 \\
\hline Fruit and vegetables & -34 & 434 & -1551 \\
\hline Meat & 79 & 325 & 3145 \\
\hline Dairy products & -1 & 12 & 56 \\
\hline Fish & 467 & 1402 & 3326 \\
\hline \multicolumn{4}{|l|}{ Environment } \\
\hline Forest area (\%) & 22 & 22 & 23 \\
\hline Renewable water res withdrawn (\% of total) & & & 90 \\
\hline Terrestrial protect areas (\% total land area) & 5 & 5 & 5 \\
\hline Organic area (\% total agricultural area) & & & 0 \\
\hline Water withdrawal by agriculture (\% of total) & & & 90 \\
\hline Biofuel production (thousand kt of oil eq.) & 304 & 510 & 2505 \\
\hline \multicolumn{4}{|l|}{ Wood pellet prod. (1 000 tonnes) } \\
\hline Net GHG emissions from AFOLU ( $\mathrm{CO}_{2}$ eq, $\mathrm{Mt}$ ) & 476 & 505 & 532 \\
\hline
\end{tabular}




\begin{tabular}{|c|c|c|c|}
\hline & 1990 & 2000 & 2014 \\
\hline \multicolumn{4}{|l|}{ The setting } \\
\hline Population, total (mln) & 178.6 & 208.9 & 252.8 \\
\hline Population, rural (mln) & 124 & 121.2 & 118.8 \\
\hline Govt expenditure on ag (\% total outlays) & & 3 & 0.9 \\
\hline Area harvested (mln ha) & 52 & 62 & 120 \\
\hline Cropping intensity ratio & 1.2 & 1.3 & \\
\hline Water resources (1 $000 \mathrm{~m}^{3} /$ person/year) & 11 & 10 & 8 \\
\hline Area equipped for irrigation (1 $000 \mathrm{ha}$ ) & & & 6722 \\
\hline \multicolumn{4}{|l|}{ Area irrigated (\% area equipped for irrigation) } \\
\hline Employment in agriculture (\%) & 55.9 & 45.3 & 35.1 \\
\hline Employment in agriculture, female (\%) & 56.3 & 46.7 & 34.5 \\
\hline Fertilizers, Nitrogen (kg of nutrients per ha) & & 94.6 & 125.4 \\
\hline Fertilizers, Phosphate (kg of nutrients per ha) & & 23.5 & 29 \\
\hline Fertilizers, Potash (kg nutrients per ha) & & 13 & 40.4 \\
\hline Energy consump, power irrigation (mln kWh) & 0 & 0 & 0 \\
\hline Agr value added per worker (constant US\$) & 613 & 662 & 1079 \\
\hline \multicolumn{4}{|l|}{ Hunger dimensions } \\
\hline Dietary energy supply (kcal/pc/day) & 2440 & 2442 & 2771 \\
\hline Average dietary energy supply adequacy (\%) & 110 & 108 & 122 \\
\hline Dietary en supp, cereals/roots/tubers (\%) & 72 & 72 & 70 \\
\hline Prevalence of undernourishment (\%) & 18.1 & 17.2 & 7.6 \\
\hline GDP per capita (US\$, PPP) & 4295 & 5552 & 9254 \\
\hline Domestic food price volatility (index) & & 12.5 & 10.7 \\
\hline Cereal import dependency ratio (\%) & 8.4 & 13.4 & 12.7 \\
\hline Underweight, children under-5 (\%) & 29.8 & 24.8 & 19.9 \\
\hline Improved water source (\% pop) & 69.7 & 77.7 & 84.9 \\
\hline \multicolumn{4}{|l|}{ Food supply } \\
\hline Food production value, $(2004-2006 \mathrm{mln}$ I\$) & 27270 & 34289 & 60205 \\
\hline Agriculture, value added (\% GDP) & 19 & 16 & 14 \\
\hline Food exports (mln US\$) & 922 & 2984 & 24711 \\
\hline Food imports (mln US\$) & 828 & 2583 & 11598 \\
\hline \multicolumn{4}{|l|}{ Production indices $(2004-06=100)$} \\
\hline Net food & 62 & 78 & 137 \\
\hline Net crops & 62 & 79 & 137 \\
\hline Cereals & 81 & 94 & 134 \\
\hline Vegetable oils & 23 & 55 & 194 \\
\hline Roots and tubers & 81 & 84 & 121 \\
\hline Fruit and vegetables & 42 & 65 & 116 \\
\hline Sugar & 99 & 84 & 119 \\
\hline Livestock & 63 & 74 & 140 \\
\hline Milk & 68 & 89 & 156 \\
\hline Meat & 67 & 74 & 139 \\
\hline Fish & 51 & 83 & 169 \\
\hline \multicolumn{4}{|l|}{ Net trade (mln US\$) } \\
\hline Cereals & -289 & -1026 & -3708 \\
\hline Fruit and vegetables & 188 & 54 & -732 \\
\hline Meat & 3 & -62 & -158 \\
\hline Dairy products & -55 & -180 & -1002 \\
\hline Fish & 936 & 1489 & 3228 \\
\hline \multicolumn{4}{|l|}{ Environment } \\
\hline Forest area (\%) & 65 & 55 & 51 \\
\hline Renewable water res withdrawn (\% of total) & & 82 & 82 \\
\hline Terrestrial protect areas (\% total land area) & 10 & 14 & 15 \\
\hline Organic area (\% total agricultural area) & & & 0 \\
\hline Water withdrawal by agriculture (\% of total) & & 82 & 82 \\
\hline Biofuel production (thousand kt of oil eq.) & 59 & 42 & 510 \\
\hline Wood pellet prod. (1 000 tonnes) & & & 80 \\
\hline Net GHG emissions from AFOLU ( $\mathrm{CO}_{2}$ eq, $\mathrm{Mt}$ ) & 760 & 951 & 1383 \\
\hline
\end{tabular}


Iran (Islamic Republic of)

\begin{tabular}{|c|c|c|c|}
\hline & 1990 & 2000 & 2014 \\
\hline \multicolumn{4}{|l|}{ The setting } \\
\hline Population, total (mln) & 56.4 & 65.9 & 78.5 \\
\hline Population, rural (mln) & 24.6 & 23.7 & 23.9 \\
\hline Govt expenditure on ag (\% total outlays) & & 3 & 1.4 \\
\hline Area harvested (mln ha) & 14 & 13 & 22 \\
\hline Cropping intensity ratio & 0.2 & 0.2 & \\
\hline Water resources (1 $000 \mathrm{~m}^{3} /$ person/year) & 2 & 2 & 2 \\
\hline Area equipped for irrigation (1 $000 \mathrm{ha})$ & & & 9553 \\
\hline Area irrigated (\% area equipped for irrigation) & & & 77.4 \\
\hline Employment in agriculture (\%) & & 23 & 21.2 \\
\hline Employment in agriculture, female (\%) & & 16.7 & 30.6 \\
\hline Fertilizers, Nitrogen (kg of nutrients per ha) & & 52.5 & 17.6 \\
\hline Fertilizers, Phosphate (kg of nutrients per ha) & & 21.6 & 7.5 \\
\hline Fertilizers, Potash (kg nutrients per ha) & & 6.3 & 1.3 \\
\hline Energy consump, power irrigation (mln kWh) & 2 & 219 & 1688 \\
\hline Agr value added per worker (constant US\$) & 2122 & 2558 & 3313 \\
\hline \multicolumn{4}{|l|}{ Hunger dimensions } \\
\hline Dietary energy supply (kcal/pc/day) & 3063 & 3045 & 3195 \\
\hline Average dietary energy supply adequacy (\%) & 139 & 130 & 133 \\
\hline Dietary en supp, cereals/roots/tubers (\%) & 62 & 60 & 53 \\
\hline Prevalence of undernourishment (\%) & $<5.0$ & 5.2 & 5.1 \\
\hline GDP per capita (US\$, PPP) & 8679 & 10694 & 15090 \\
\hline Domestic food price volatility (index) & & 15.9 & 13 \\
\hline Cereal import dependency ratio (\%) & 22 & 40.8 & 28.7 \\
\hline Underweight, children under-5 (\%) & & 9.5 & \\
\hline Improved water source (\% pop) & 92.2 & 94.1 & 95.9 \\
\hline \multicolumn{4}{|l|}{ Food supply } \\
\hline Food production value, (2004-2006 mln I\$) & 12210 & 17582 & 25588 \\
\hline Agriculture, value added (\% GDP) & 19 & 14 & 10 \\
\hline Food exports (mln US\$) & 345 & 904 & 3970 \\
\hline Food imports (mln US\$) & 2211 & 2484 & 9668 \\
\hline \multicolumn{4}{|l|}{ Production indices (2004-06=100) } \\
\hline Net food & 54 & 78 & 113 \\
\hline Net crops & 55 & 76 & 117 \\
\hline Cereals & 60 & 59 & 103 \\
\hline Vegetable oils & 45 & 56 & 105 \\
\hline Roots and tubers & 55 & 80 & 124 \\
\hline Fruit and vegetables & 52 & 83 & 112 \\
\hline Sugar & 51 & 63 & 92 \\
\hline Livestock & 53 & 81 & 105 \\
\hline Milk & 56 & 83 & 105 \\
\hline Meat & 53 & 79 & 108 \\
\hline Fish & 51 & 81 & 169 \\
\hline \multicolumn{4}{|l|}{ Net trade (mln US\$) } \\
\hline Cereals & -981 & -1465 & -4387 \\
\hline Fruit and vegetables & 262 & 452 & 1305 \\
\hline Meat & -290 & -33 & -506 \\
\hline Dairy products & -161 & -49 & 188 \\
\hline Fish & 37 & 14 & 178 \\
\hline \multicolumn{4}{|l|}{ Environment } \\
\hline Forest area (\%) & 7 & 7 & 7 \\
\hline Renewable water res withdrawn (\% of total) & & & 92 \\
\hline Terrestrial protect areas (\% total land area) & 6 & 6 & 7 \\
\hline Organic area (\% total agricultural area) & & & 0 \\
\hline Water withdrawal by agriculture (\% of total) & & & 92 \\
\hline Biofuel production (thousand kt of oil eq.) & 4 & 13 & 1 \\
\hline \multicolumn{4}{|l|}{ Wood pellet prod. (1 000 tonnes) } \\
\hline Net GHG emissions from AFOLU ( $\mathrm{CO}_{2}$ eq, $\mathrm{Mt}$ ) & 37 & 43 & 38 \\
\hline
\end{tabular}




\begin{tabular}{|c|c|c|c|}
\hline & 1990 & 2000 & 2014 \\
\hline \multicolumn{4}{|l|}{ The setting } \\
\hline Population, total (mln) & 17.5 & 23.8 & 34.8 \\
\hline Population, rural (mln) & 5.3 & 7.7 & 11.7 \\
\hline Govt expenditure on ag (\% total outlays) & & & 0.4 \\
\hline Area harvested (mln ha) & 3 & 3 & 5 \\
\hline Cropping intensity ratio & 0.4 & 0.4 & \\
\hline Water resources (1 $000 \mathrm{~m}^{3} /$ person/year) & 5 & 4 & 3 \\
\hline Area equipped for irrigation (1 $000 \mathrm{ha})$ & & & 3525 \\
\hline Area irrigated (\% area equipped for irrigation) & 54.9 & & \\
\hline Employment in agriculture (\%) & & & 23.4 \\
\hline Employment in agriculture, female (\%) & & & 50.7 \\
\hline Fertilizers, Nitrogen (kg of nutrients per ha) & & 0 & 41.3 \\
\hline Fertilizers, Phosphate (kg of nutrients per ha) & & 0 & 14.4 \\
\hline Fertilizers, Potash (kg nutrients per ha) & & 0 & 0.9 \\
\hline Energy consump, power irrigation (mln kWh) & 0 & 19 & 19 \\
\hline Agr value added per worker (constant US\$) & 3190 & 4968 & 7046 \\
\hline \multicolumn{4}{|l|}{ Hunger dimensions } \\
\hline Dietary energy supply (kcal/pc/day) & 2246 & 2208 & 2549 \\
\hline Average dietary energy supply adequacy (\%) & 107 & 104 & 118 \\
\hline Dietary en supp, cereals/roots/tubers (\%) & 62 & 61 & 62 \\
\hline Prevalence of undernourishment (\%) & 15.3 & 24.6 & 23.2 \\
\hline GDP per capita (US\$, PPP) & 11212 & 11764 & 14472 \\
\hline Domestic food price volatility (index) & & & 16.4 \\
\hline Cereal import dependency ratio (\%) & 35 & 73.3 & 56.8 \\
\hline Underweight, children under-5 (\%) & 10.4 & 12.9 & 8.5 \\
\hline Improved water source (\% pop) & 78.3 & 80.1 & 85.4 \\
\hline \multicolumn{4}{|l|}{ Food supply } \\
\hline Food production value, (2004-2006 mln I\$) & 2725 & 2324 & 2917 \\
\hline \multicolumn{4}{|l|}{ Agriculture, value added (\% GDP) } \\
\hline Food exports (mln US\$) & 40 & 6 & 57 \\
\hline Food imports (mln US\$) & 1511 & 1831 & 6333 \\
\hline \multicolumn{4}{|l|}{ Production indices (2004-06=100) } \\
\hline Net food & 118 & 100 & 126 \\
\hline Net crops & 101 & 91 & 132 \\
\hline Cereals & 88 & 15 & 205 \\
\hline Vegetable oils & 95 & 61 & 60 \\
\hline Roots and tubers & 26 & 71 & 76 \\
\hline Fruit and vegetables & 111 & 117 & 116 \\
\hline Sugar & 3204 & 895 & 356 \\
\hline Livestock & 169 & 124 & 127 \\
\hline Milk & 158 & 193 & 97 \\
\hline Meat & 176 & 108 & 143 \\
\hline Fish & 50 & 47 & 147 \\
\hline \multicolumn{4}{|l|}{ Net trade (mln US\$) } \\
\hline Cereals & -692 & -1244 & -2538 \\
\hline Fruit and vegetables & -52 & -27 & -498 \\
\hline Meat & -149 & 0 & -838 \\
\hline Dairy products & -152 & -135 & -195 \\
\hline Fish & & 0 & -42 \\
\hline \multicolumn{4}{|l|}{ Environment } \\
\hline Forest area (\%) & 2 & 2 & 2 \\
\hline Renewable water res withdrawn (\% of total) & & 79 & 79 \\
\hline Terrestrial protect areas (\% total land area) & 0 & 0 & 0 \\
\hline \multicolumn{4}{|l|}{ Organic area (\% total agricultural area) } \\
\hline Water withdrawal by agriculture (\% of total) & & 79 & 79 \\
\hline Biofuel production (thousand kt of oil eq.) & 0 & 0 & \\
\hline \multicolumn{4}{|l|}{ Wood pellet prod. (1 000 tonnes) } \\
\hline Net GHG emissions from AFOLU ( $\mathrm{CO}_{2}$ eq, $\left.\mathrm{Mt}\right)$ & 6 & 6 & 7 \\
\hline
\end{tabular}




\begin{tabular}{|c|c|c|c|}
\hline & 1990 & 2000 & 2014 \\
\hline \multicolumn{4}{|l|}{ The setting } \\
\hline Population, total (mln) & 3.5 & 3.8 & 4.7 \\
\hline Population, rural (mln) & 1.5 & 1.6 & 1.7 \\
\hline \multicolumn{4}{|l|}{ Govt expenditure on ag (\% total outlays) } \\
\hline Area harvested (mln ha) & 2 & 3 & 2 \\
\hline Cropping intensity ratio & 0.3 & 0.7 & \\
\hline Water resources (1 $000 \mathrm{~m}^{3} /$ person/year) & 15 & 14 & 11 \\
\hline \multicolumn{4}{|l|}{ Area equipped for irrigation (1 $000 \mathrm{ha})$} \\
\hline Area irrigated (\% area equipped for irrigation) & & 100 & \\
\hline Employment in agriculture (\%) & 12.7 & 6.5 & 4.7 \\
\hline Employment in agriculture, female (\%) & 3.6 & 1.8 & 1.2 \\
\hline Fertilizers, Nitrogen (kg of nutrients per ha) & & 331 & 239.3 \\
\hline Fertilizers, Phosphate (kg of nutrients per ha) & & 82.9 & 65.9 \\
\hline Fertilizers, Potash (kg nutrients per ha) & & 119.9 & 81.5 \\
\hline \multicolumn{4}{|l|}{ Energy consump, power irrigation (mln kWh) } \\
\hline Agr value added per worker (constant US\$) & & 15132 & 7881 \\
\hline \multicolumn{4}{|l|}{ Hunger dimensions } \\
\hline \multicolumn{4}{|l|}{ Dietary energy supply (kcal/pc/day) } \\
\hline Average dietary energy supply adequacy (\%) & 145 & 149 & 146 \\
\hline Dietary en supp, cereals/roots/tubers (\%) & 34 & 31 & 34 \\
\hline Prevalence of undernourishment (\%) & $<5.0$ & $<5.0$ & $<5.0$ \\
\hline GDP per capita (US\$, PPP) & 22468 & 41198 & 44647 \\
\hline Domestic food price volatility (index) & & 3.8 & 3.3 \\
\hline Cereal import dependency ratio (\%) & 6.5 & 21.5 & 32 \\
\hline \multicolumn{4}{|l|}{ Underweight, children under-5 (\%) } \\
\hline Improved water source (\% pop) & 99.8 & 99.8 & 99.9 \\
\hline \multicolumn{4}{|l|}{ Food supply } \\
\hline Food production value, (2004-2006 mln I\$) & 4231 & 4614 & 4317 \\
\hline Agriculture, value added (\% GDP) & & 3 & 2 \\
\hline Food exports (mln US\$) & 4410 & 5651 & 9303 \\
\hline Food imports (mln US\$) & 1401 & 2199 & 5985 \\
\hline \multicolumn{4}{|l|}{ Production indices $(2004-06=100)$} \\
\hline Net food & 97 & 106 & 99 \\
\hline Net crops & 91 & 100 & 94 \\
\hline Cereals & 87 & 98 & 105 \\
\hline Vegetable oils & 155 & 66 & 379 \\
\hline Roots and tubers & 127 & 101 & 86 \\
\hline Fruit and vegetables & 72 & 88 & 98 \\
\hline Sugar & 91 & 112 & 86 \\
\hline Livestock & 96 & 106 & 100 \\
\hline Milk & 101 & 96 & 104 \\
\hline Meat & 93 & 112 & 96 \\
\hline Fish & 78 & 105 & 90 \\
\hline \multicolumn{4}{|l|}{ Net trade (mln US\$) } \\
\hline Cereals & 104 & 622 & 591 \\
\hline Fruit and vegetables & -243 & -306 & -995 \\
\hline Meat & 1221 & 1361 & 2787 \\
\hline Dairy products & 765 & 804 & 1374 \\
\hline Fish & 169 & 196 & 389 \\
\hline \multicolumn{4}{|l|}{ Environment } \\
\hline Forest area (\%) & 7 & 9 & 11 \\
\hline Renewable water res withdrawn (\% of total) & & & 0 \\
\hline Terrestrial protect areas (\% total land area) & 1 & 8 & 14 \\
\hline Organic area (\% total agricultural area) & & & 1 \\
\hline Water withdrawal by agriculture (\% of total) & & & 0 \\
\hline Biofuel production (thousand kt of oil eq.) & 0 & 5 & 1871 \\
\hline Wood pellet prod. (1 000 tonnes) & & & 32 \\
\hline Net GHG emissions from AFOLU ( $\mathrm{CO}_{2}$ eq, $\mathrm{Mt}$ ) & 21 & 21 & 19 \\
\hline
\end{tabular}




\begin{tabular}{|c|c|c|c|}
\hline & 1990 & 2000 & 2014 \\
\hline \multicolumn{4}{|l|}{ The setting } \\
\hline Population, total (mln) & 4.5 & 6 & 7.8 \\
\hline Population, rural (mln) & 0.4 & 0.5 & 0.6 \\
\hline \multicolumn{4}{|l|}{ Govt expenditure on ag (\% total outlays) } \\
\hline Area harvested (mln ha) & 2 & 2 & 1 \\
\hline Cropping intensity ratio & 3.5 & 3 & \\
\hline Water resources (1 $000 \mathrm{~m}^{3} /$ person/year) & 0 & 0 & 0 \\
\hline Area equipped for irrigation (1 $000 \mathrm{ha})$ & & & 225 \\
\hline Area irrigated (\% area equipped for irrigation) & & & 80.7 \\
\hline Employment in agriculture (\%) & 4.1 & 2.2 & 1.7 \\
\hline Employment in agriculture, female (\%) & 2.3 & 0.9 & 0.7 \\
\hline Fertilizers, Nitrogen (kg of nutrients per ha) & & 150.3 & 216.4 \\
\hline Fertilizers, Phosphate (kg of nutrients per ha) & & 35.6 & 40.4 \\
\hline Fertilizers, Potash (kg nutrients per ha) & & 97 & 108.2 \\
\hline Energy consump, power irrigation (mln kWh) & 306 & 555 & 552 \\
\hline \multicolumn{4}{|l|}{ Agr value added per worker (constant US\$) } \\
\hline \multicolumn{4}{|l|}{ Hunger dimensions } \\
\hline \multicolumn{4}{|l|}{ Dietary energy supply (kcal/pc/day) } \\
\hline Average dietary energy supply adequacy (\%) & 148 & 154 & 159 \\
\hline Dietary en supp, cereals/roots/tubers (\%) & 37 & 33 & 35 \\
\hline Prevalence of undernourishment (\%) & $<5.0$ & $<5.0$ & $<5.0$ \\
\hline GDP per capita (US\$, PPP) & 17152 & 25739 & 30927 \\
\hline Domestic food price volatility (index) & & 10.5 & 5.9 \\
\hline Cereal import dependency ratio (\%) & 91.1 & 94.8 & 93.3 \\
\hline \multicolumn{4}{|l|}{ Underweight, children under-5 (\%) } \\
\hline Improved water source (\% pop) & 100 & 100 & 100 \\
\hline \multicolumn{4}{|l|}{ Food supply } \\
\hline Food production value, (2004-2006 mln I\$) & 1859 & 2250 & 2836 \\
\hline \multicolumn{4}{|l|}{ Agriculture, value added (\% GDP) } \\
\hline Food exports (mln US\$) & 976 & 754 & 1875 \\
\hline Food imports (mln US\$) & 932 & 1467 & 3810 \\
\hline \multicolumn{4}{|l|}{ Production indices (2004-06=100) } \\
\hline Net food & 72 & 87 & 110 \\
\hline Net crops & 96 & 87 & 106 \\
\hline Cereals & 149 & 64 & 114 \\
\hline Vegetable oils & 98 & 104 & 105 \\
\hline Roots and tubers & 36 & 66 & 101 \\
\hline Fruit and vegetables & 97 & 90 & 106 \\
\hline \multicolumn{4}{|l|}{ Sugar } \\
\hline Livestock & 57 & 87 & 114 \\
\hline Milk & 82 & 101 & 119 \\
\hline Meat & 42 & 81 & 110 \\
\hline Fish & 90 & 99 & 96 \\
\hline \multicolumn{4}{|l|}{ Net trade (mln US\$) } \\
\hline Cereals & -322 & -465 & -1247 \\
\hline Fruit and vegetables & 638 & 286 & 861 \\
\hline Meat & -32 & -110 & -391 \\
\hline Dairy products & -3 & -22 & -39 \\
\hline Fish & -76 & -121 & -359 \\
\hline \multicolumn{4}{|l|}{ Environment } \\
\hline Forest area (\%) & 6 & 7 & 7 \\
\hline Renewable water res withdrawn (\% of total) & & & 58 \\
\hline Terrestrial protect areas (\% total land area) & 16 & 17 & 17 \\
\hline \multicolumn{4}{|l|}{ Organic area (\% total agricultural area) } \\
\hline Water withdrawal by agriculture (\% of total) & & & 58 \\
\hline Biofuel production (thousand kt of oil eq.) & & & 0 \\
\hline \multicolumn{4}{|l|}{ Wood pellet prod. (1 000 tonnes) } \\
\hline Net GHG emissions from AFOLU ( $\mathrm{CO}_{2}$ eq, $\mathrm{Mt}$ ) & 1 & 1 & 1 \\
\hline
\end{tabular}




\begin{tabular}{|c|c|c|c|}
\hline & 1990 & 2000 & 2014 \\
\hline \multicolumn{4}{|l|}{ The setting } \\
\hline Population, total (mln) & 56.8 & 57 & 61.1 \\
\hline Population, rural (mln) & 18.9 & 18.7 & 19 \\
\hline \multicolumn{4}{|l|}{ Govt expenditure on ag (\% total outlays) } \\
\hline Area harvested (mln ha) & 17 & 21 & 16 \\
\hline Cropping intensity ratio & 1 & 1.3 & \\
\hline Water resources (1 $000 \mathrm{~m}^{3} /$ person/year) & 3 & 3 & 3 \\
\hline Area equipped for irrigation (1 $000 \mathrm{ha})$ & & & 3950 \\
\hline Area irrigated (\% area equipped for irrigation) & & & 67.5 \\
\hline Employment in agriculture (\%) & 8.8 & 5.2 & 3.7 \\
\hline Employment in agriculture, female (\%) & 9.1 & 4.4 & 2.6 \\
\hline Fertilizers, Nitrogen (kg of nutrients per ha) & & 106.4 & 96.3 \\
\hline Fertilizers, Phosphate (kg of nutrients per ha) & & 39.7 & 29.2 \\
\hline Fertilizers, Potash (kg nutrients per ha) & & 31.6 & 25.4 \\
\hline Energy consump, power irrigation (mln kWh) & 1039 & 1149 & 2993 \\
\hline Agr value added per worker (constant US\$) & 15178 & 31601 & 52411 \\
\hline \multicolumn{4}{|l|}{ Hunger dimensions } \\
\hline \multicolumn{4}{|l|}{ Dietary energy supply (kcal/pc/day) } \\
\hline Average dietary energy supply adequacy (\%) & 138 & 145 & 140 \\
\hline Dietary en supp, cereals/roots/tubers (\%) & 34 & 34 & 34 \\
\hline Prevalence of undernourishment (\%) & $<5.0$ & $<5.0$ & $<5.0$ \\
\hline GDP per capita (US\$, PPP) & 30746 & 36073 & 33924 \\
\hline Domestic food price volatility (index) & & 4.5 & 5 \\
\hline Cereal import dependency ratio (\%) & 10.7 & 17.6 & 26.6 \\
\hline \multicolumn{4}{|l|}{ Underweight, children under-5 (\%) } \\
\hline Improved water source (\% pop) & 100 & 100 & 100 \\
\hline \multicolumn{4}{|l|}{ Food supply } \\
\hline Food production value, (2004-2006 mln I\$) & 27827 & 31115 & 29303 \\
\hline Agriculture, value added (\% GDP) & 3 & 3 & 2 \\
\hline Food exports (mln US\$) & 8203 & 10851 & 27468 \\
\hline Food imports (mln US\$) & 15686 & 13790 & 31717 \\
\hline \multicolumn{4}{|l|}{ Production indices $(2004-06=100)$} \\
\hline Net food & 90 & 101 & 95 \\
\hline Net crops & 85 & 98 & 89 \\
\hline Cereals & 80 & 95 & 86 \\
\hline Vegetable oils & 40 & 80 & 78 \\
\hline Roots and tubers & 130 & 117 & 72 \\
\hline Fruit and vegetables & 93 & 102 & 96 \\
\hline Sugar & 129 & 135 & 24 \\
\hline Livestock & 98 & 105 & 93 \\
\hline Milk & 101 & 113 & 93 \\
\hline Meat & 97 & 101 & 92 \\
\hline Fish & 115 & 113 & 74 \\
\hline \multicolumn{4}{|l|}{ Net trade (mln US\$) } \\
\hline Cereals & -554 & 537 & 403 \\
\hline Fruit and vegetables & 1851 & 1473 & 3252 \\
\hline Meat & -3504 & -2053 & -3134 \\
\hline Dairy products & -2089 & -1468 & -1447 \\
\hline Fish & -2220 & -2163 & -4830 \\
\hline \multicolumn{4}{|l|}{ Environment } \\
\hline Forest area (\%) & 26 & 28 & 32 \\
\hline Renewable water res withdrawn (\% of total) & & 44 & 44 \\
\hline Terrestrial protect areas (\% total land area) & 6 & 17 & 22 \\
\hline Organic area (\% total agricultural area) & & & 9 \\
\hline Water withdrawal by agriculture (\% of total) & & 44 & 44 \\
\hline Biofuel production (thousand kt of oil eq.) & 0 & 5 & 21619 \\
\hline Wood pellet prod. (1 000 tonnes) & & & 450 \\
\hline Net GHG emissions from AFOLU ( $\mathrm{CO}_{2}$ eq, $\left.\mathrm{Mt}\right)$ & 23 & 1 & -1 \\
\hline
\end{tabular}




\begin{tabular}{|c|c|c|c|}
\hline & 1990 & 2000 & 2014 \\
\hline \multicolumn{4}{|l|}{ The setting } \\
\hline Population, total (mln) & 2.4 & 2.6 & 2.8 \\
\hline Population, rural (mln) & 1.2 & 1.2 & 1.3 \\
\hline Govt expenditure on ag (\% total outlays) & & 1.6 & 1.6 \\
\hline Area harvested (mln ha) & 2 & 2 & 1 \\
\hline Cropping intensity ratio & 5.2 & 4.2 & \\
\hline Water resources (1 $000 \mathrm{~m}^{3} /$ person/year) & 4 & 4 & 3 \\
\hline Area equipped for irrigation (1 $000 \mathrm{ha}$ ) & & & 25 \\
\hline Area irrigated (\% area equipped for irrigation) & & 100 & \\
\hline Employment in agriculture (\%) & 24.4 & 20.8 & 18.1 \\
\hline Employment in agriculture, female (\%) & 12 & 9.2 & 7.9 \\
\hline Fertilizers, Nitrogen (kg of nutrients per ha) & & 72.2 & 50.5 \\
\hline Fertilizers, Phosphate (kg of nutrients per ha) & & 36.4 & 30.8 \\
\hline Fertilizers, Potash (kg nutrients per ha) & & 3.5 & 3.2 \\
\hline Energy consump, power irrigation (mln kWh) & 0 & 13 & 13 \\
\hline Agr value added per worker (constant US\$) & 2631 & 2695 & 3725 \\
\hline \multicolumn{4}{|l|}{ Hunger dimensions } \\
\hline Dietary energy supply (kcal/pc/day) & 2683 & 2751 & 2793 \\
\hline Average dietary energy supply adequacy (\%) & 115 & 117 & 116 \\
\hline Dietary en supp, cereals/roots/tubers (\%) & 41 & 38 & 40 \\
\hline Prevalence of undernourishment (\%) & 9.6 & 7.8 & 8.7 \\
\hline GDP per capita (US\$, PPP) & 7391 & 8139 & 8608 \\
\hline Domestic food price volatility (index) & & 5.1 & 7 \\
\hline Cereal import dependency ratio (\%) & 99.1 & 99.6 & 99.5 \\
\hline Underweight, children under-5 (\%) & 8.9 & 3.8 & 3.2 \\
\hline Improved water source (\% pop) & 93.4 & 93.4 & 93.1 \\
\hline \multicolumn{4}{|l|}{ Food supply } \\
\hline Food production value, $(2004-2006 \mathrm{mln}$ I\$) & 481 & 526 & 563 \\
\hline Agriculture, value added (\% GDP) & 8 & 7 & 7 \\
\hline Food exports (mln US\$) & 174 & 166 & 234 \\
\hline Food imports (mln US\$) & 216 & 320 & 824 \\
\hline \multicolumn{4}{|l|}{ Production indices $(2004-06=100)$} \\
\hline Net food & 91 & 99 & 106 \\
\hline Net crops & 98 & 104 & 108 \\
\hline Cereals & 140 & 98 & 166 \\
\hline Vegetable oils & 31 & 63 & 109 \\
\hline Roots and tubers & 130 & 118 & 124 \\
\hline Fruit and vegetables & 90 & 109 & 107 \\
\hline Sugar & 146 & 119 & 82 \\
\hline Livestock & 80 & 91 & 103 \\
\hline Milk & 106 & 101 & 110 \\
\hline Meat & 70 & 88 & 100 \\
\hline Fish & 82 & 48 & 124 \\
\hline \multicolumn{4}{|l|}{ Net trade (mln US\$) } \\
\hline Cereals & -70 & -109 & -269 \\
\hline Fruit and vegetables & 54 & 17 & -35 \\
\hline Meat & -31 & -43 & -99 \\
\hline Dairy products & -28 & -22 & -38 \\
\hline Fish & -28 & -42 & -99 \\
\hline \multicolumn{4}{|l|}{ Environment } \\
\hline Forest area (\%) & 32 & 31 & 31 \\
\hline Renewable water res withdrawn (\% of total) & & & 55 \\
\hline Terrestrial protect areas (\% total land area) & 10 & 16 & 16 \\
\hline Organic area (\% total agricultural area) & & & 0 \\
\hline Water withdrawal by agriculture (\% of total) & & & 55 \\
\hline Biofuel production (thousand kt of oil eq.) & 5 & 5 & 2 \\
\hline \multicolumn{4}{|l|}{ Wood pellet prod. (1 000 tonnes) } \\
\hline Net GHG emissions from AFOLU ( $\mathrm{CO}_{2}$ eq, $\mathrm{Mt}$ ) & 2 & 2 & 1 \\
\hline
\end{tabular}




\begin{tabular}{|c|c|c|c|}
\hline & 1990 & 2000 & 2014 \\
\hline \multicolumn{4}{|l|}{ The setting } \\
\hline Population, total (mln) & 122.2 & 125.7 & 127 \\
\hline Population, rural (mln) & 27.7 & 26.8 & 8.8 \\
\hline \multicolumn{4}{|l|}{ Govt expenditure on ag (\% total outlays) } \\
\hline Area harvested (mln ha) & 14 & 13 & 12 \\
\hline Cropping intensity ratio & 2.5 & 2.4 & \\
\hline Water resources (1 $000 \mathrm{~m}^{3} /$ person/year) & 4 & 3 & 3 \\
\hline Area equipped for irrigation (1 $000 \mathrm{ha})$ & & & 2469 \\
\hline Area irrigated (\% area equipped for irrigation) & & & 92.9 \\
\hline Employment in agriculture (\%) & 7.2 & 5.1 & 3.7 \\
\hline Employment in agriculture, female (\%) & 8.5 & 5.5 & 3.7 \\
\hline Fertilizers, Nitrogen (kg of nutrients per ha) & & 123.7 & 101.9 \\
\hline Fertilizers, Phosphate (kg of nutrients per ha) & & 137.7 & 96.1 \\
\hline Fertilizers, Potash (kg nutrients per ha) & & 73.8 & 61.2 \\
\hline Energy consump, power irrigation (mln kWh) & 256 & 647 & 1063 \\
\hline Agr value added per worker (constant US\$) & 15305 & 25258 & 50720 \\
\hline \multicolumn{4}{|l|}{ Hunger dimensions } \\
\hline \multicolumn{4}{|l|}{ Dietary energy supply (kcal/pc/day) } \\
\hline Average dietary energy supply adequacy (\%) & 120 & 119 & 112 \\
\hline Dietary en supp, cereals/roots/tubers (\%) & 42 & 41 & 41 \\
\hline Prevalence of undernourishment (\%) & $<5.0$ & $<5.0$ & $<5.0$ \\
\hline GDP per capita (US\$, PPP) & 29548 & 32193 & 35614 \\
\hline Domestic food price volatility (index) & & 5.3 & 5.6 \\
\hline Cereal import dependency ratio (\%) & 78 & 79.6 & 79.7 \\
\hline \multicolumn{4}{|l|}{ Underweight, children under-5 (\%) } \\
\hline Improved water source (\% pop) & 100 & 100 & 100 \\
\hline \multicolumn{4}{|l|}{ Food supply } \\
\hline Food production value, (2004-2006 mln I\$) & 20634 & 18741 & 17730 \\
\hline Agriculture, value added (\% GDP) & 2 & 2 & 1 \\
\hline Food exports (mln US\$) & 642 & 893 & 1750 \\
\hline Food imports (mln US\$) & 17884 & 24832 & 45107 \\
\hline \multicolumn{4}{|l|}{ Production indices $(2004-06=100)$} \\
\hline Net food & 114 & 104 & 98 \\
\hline Net crops & 123 & 107 & 94 \\
\hline Cereals & 119 & 107 & 97 \\
\hline Vegetable oils & 135 & 118 & 93 \\
\hline Roots and tubers & 130 & 106 & 94 \\
\hline Fruit and vegetables & 125 & 108 & 93 \\
\hline Sugar & 106 & 91 & 83 \\
\hline Livestock & 107 & 101 & 101 \\
\hline Milk & 99 & 103 & 91 \\
\hline Meat & 114 & 99 & 106 \\
\hline Fish & 204 & 115 & 84 \\
\hline \multicolumn{4}{|l|}{ Net trade (mln US\$) } \\
\hline Cereals & -4434 & -4352 & -10064 \\
\hline Fruit and vegetables & -3581 & -6149 & -9682 \\
\hline Meat & -5026 & -8531 & -13206 \\
\hline Dairy products & -470 & -692 & -1450 \\
\hline Fish & -9861 & -14711 & -16174 \\
\hline \multicolumn{4}{|l|}{ Environment } \\
\hline Forest area (\%) & 68 & 68 & 69 \\
\hline Renewable water res withdrawn (\% of total) & & 63 & 63 \\
\hline Terrestrial protect areas (\% total land area) & 13 & 16 & 17 \\
\hline Organic area (\% total agricultural area) & & & 0 \\
\hline Water withdrawal by agriculture (\% of total) & & 63 & 63 \\
\hline Biofuel production (thousand kt of oil eq.) & 6 & 9 & 8 \\
\hline Wood pellet prod. (1 000 tonnes) & & & 90 \\
\hline Net GHG emissions from AFOLU ( $\mathrm{CO}_{2}$ eq, $\left.\mathrm{Mt}\right)$ & -44 & -48 & -113 \\
\hline
\end{tabular}




\begin{tabular}{|c|c|c|c|}
\hline & 1990 & 2000 & 2014 \\
\hline \multicolumn{4}{|l|}{ The setting } \\
\hline Population, total (mln) & 3.4 & 4.8 & 7.5 \\
\hline Population, rural (mln) & 0.9 & 1 & 1.2 \\
\hline Govt expenditure on ag (\% total outlays) & & 1.1 & 0.7 \\
\hline Area harvested (mln ha) & 1 & 1 & 0 \\
\hline Cropping intensity ratio & 0.8 & 0.9 & \\
\hline Water resources (1 $000 \mathrm{~m}^{3} /$ person/year) & 0 & 0 & 0 \\
\hline Area equipped for irrigation (1 $000 \mathrm{ha}$ ) & & & 96 \\
\hline Area irrigated (\% area equipped for irrigation) & & & 91.3 \\
\hline Employment in agriculture (\%) & & 4.9 & 2 \\
\hline Employment in agriculture, female (\%) & & 3.6 & 0.9 \\
\hline Fertilizers, Nitrogen (kg of nutrients per ha) & & 318.2 & 144.3 \\
\hline Fertilizers, Phosphate (kg of nutrients per ha) & & 598 & 121.8 \\
\hline Fertilizers, Potash (kg nutrients per ha) & & 0 & 994.4 \\
\hline Energy consump, power irrigation (mln kWh) & 29 & 106 & 157 \\
\hline Agr value added per worker (constant US\$) & 2540 & 1858 & 4848 \\
\hline \multicolumn{4}{|l|}{ Hunger dimensions } \\
\hline Dietary energy supply (kcal/pc/day) & 2697 & 2742 & 3132 \\
\hline Average dietary energy supply adequacy (\%) & 122 & 123 & 137 \\
\hline Dietary en supp, cereals/roots/tubers (\%) & 48 & 50 & 48 \\
\hline Prevalence of undernourishment (\%) & 6.7 & 7 & $<5.0$ \\
\hline GDP per capita (US\$, PPP) & 7058 & 7695 & 11405 \\
\hline Domestic food price volatility (index) & & 8.9 & 6.1 \\
\hline Cereal import dependency ratio (\%) & 92.2 & 97.4 & 96.2 \\
\hline Underweight, children under-5 (\%) & 4.8 & 3.6 & 3 \\
\hline Improved water source (\% pop) & 96.7 & 96.7 & 96.1 \\
\hline \multicolumn{4}{|l|}{ Food supply } \\
\hline Food production value, $(2004-2006 \mathrm{mln}$ I\$) & 557 & 777 & 1315 \\
\hline Agriculture, value added (\% GDP) & 8 & 2 & 4 \\
\hline Food exports (mln US\$) & 98 & 164 & 1217 \\
\hline Food imports (mln US\$) & 635 & 676 & 3016 \\
\hline \multicolumn{4}{|l|}{ Production indices $(2004-06=100)$} \\
\hline Net food & 58 & 81 & 137 \\
\hline Net crops & 67 & 78 & 131 \\
\hline Cereals & 198 & 75 & 150 \\
\hline Vegetable oils & 45 & 96 & 91 \\
\hline Roots and tubers & 52 & 57 & 63 \\
\hline Fruit and vegetables & 70 & 74 & 144 \\
\hline \multicolumn{4}{|l|}{ Sugar } \\
\hline Livestock & 44 & 87 & 147 \\
\hline Milk & 34 & 69 & 105 \\
\hline Meat & 42 & 91 & 175 \\
\hline Fish & 40 & 108 & 118 \\
\hline \multicolumn{4}{|l|}{ Net trade (mln US\$) } \\
\hline Cereals & -253 & -245 & -961 \\
\hline Fruit and vegetables & 12 & 15 & 307 \\
\hline Meat & -58 & -48 & -279 \\
\hline Dairy products & -62 & -60 & -203 \\
\hline Fish & -10 & -20 & -105 \\
\hline \multicolumn{4}{|l|}{ Environment } \\
\hline Forest area (\%) & 1 & 1 & 1 \\
\hline Renewable water res withdrawn (\% of total) & & & 65 \\
\hline Terrestrial protect areas (\% total land area) & 1 & 2 & 2 \\
\hline Organic area (\% total agricultural area) & & & 0 \\
\hline Water withdrawal by agriculture (\% of total) & & & 65 \\
\hline Biofuel production (thousand kt of oil eq.) & & 0 & 0 \\
\hline \multicolumn{4}{|l|}{ Wood pellet prod. (1 000 tonnes) } \\
\hline Net GHG emissions from AFOLU ( $\mathrm{CO}_{2}$ eq, $\mathrm{Mt}$ ) & 1 & 1 & 1 \\
\hline
\end{tabular}




\begin{tabular}{|c|c|c|c|}
\hline & 1990 & 2000 & 2014 \\
\hline \multicolumn{4}{|l|}{ The setting } \\
\hline Population, total (mln) & 15.9 & 14.6 & 16.6 \\
\hline Population, rural (mln) & 7 & 6.5 & 7.8 \\
\hline \multicolumn{4}{|l|}{ Govt expenditure on ag (\% total outlays) } \\
\hline Area harvested (mln ha) & & 12 & 18 \\
\hline Cropping intensity ratio & & 0.1 & \\
\hline Water resources (1 $000 \mathrm{~m}^{3} /$ person/year) & 7 & 7 & 7 \\
\hline Area equipped for irrigation (1 $000 \mathrm{ha}$ ) & & & 2066 \\
\hline Area irrigated (\% area equipped for irrigation) & & & 61.2 \\
\hline Employment in agriculture (\%) & & 35.3 & 25.5 \\
\hline Employment in agriculture, female (\%) & & 34 & 29.2 \\
\hline Fertilizers, Nitrogen (kg of nutrients per ha) & & 0.9 & 1 \\
\hline Fertilizers, Phosphate (kg of nutrients per ha) & & 0.6 & 0.7 \\
\hline Fertilizers, Potash (kg nutrients per ha) & & 0 & 0 \\
\hline Energy consump, power irrigation (mln kWh) & 1325 & 1325 & 98 \\
\hline Agr value added per worker (constant US\$) & 3162 & 2084 & 3973 \\
\hline \multicolumn{4}{|l|}{ Hunger dimensions } \\
\hline Dietary energy supply (kcal/pc/day) & 2873 & 2813 & 3154 \\
\hline Average dietary energy supply adequacy (\%) & 126 & 121 & 136 \\
\hline Dietary en supp, cereals/roots/tubers (\%) & 60 & 57 & 39 \\
\hline Prevalence of undernourishment (\%) & $<5.0$ & $<5.0$ & $<5.0$ \\
\hline GDP per capita (US\$, PPP) & 12728 & 9706 & 22470 \\
\hline \multicolumn{4}{|l|}{ Domestic food price volatility (index) } \\
\hline Cereal import dependency ratio (\%) & -17.1 & -48.3 & -50.6 \\
\hline Underweight, children under-5 (\%) & & 3.8 & 3.7 \\
\hline Improved water source (\% pop) & 94.1 & 93.8 & 93.1 \\
\hline \multicolumn{4}{|l|}{ Food supply } \\
\hline Food production value, $(2004-2006 \mathrm{mln}$ I\$) & 7543 & 4689 & 7421 \\
\hline Agriculture, value added (\% GDP) & 18 & 9 & 5 \\
\hline Food exports (mln US\$) & 564 & 572 & 2634 \\
\hline Food imports (mln US\$) & 301 & 346 & 3307 \\
\hline \multicolumn{4}{|l|}{ Production indices $(2004-06=100)$} \\
\hline Net food & 128 & 79 & 125 \\
\hline Net crops & 102 & 79 & 146 \\
\hline Cereals & 145 & 86 & 134 \\
\hline Vegetable oils & 41 & 45 & 281 \\
\hline Roots and tubers & 84 & 67 & 144 \\
\hline Fruit and vegetables & 39 & 75 & 161 \\
\hline Sugar & 241 & 78 & 19 \\
\hline Livestock & 155 & 81 & 111 \\
\hline Milk & 117 & 79 & 104 \\
\hline Meat & 182 & 84 & 113 \\
\hline Fish & 257 & 111 & 105 \\
\hline \multicolumn{4}{|l|}{ Net trade (mln US\$) } \\
\hline Cereals & 514 & 511 & 2019 \\
\hline Fruit and vegetables & -70 & -9 & -840 \\
\hline Meat & 12 & -25 & -424 \\
\hline Dairy products & -14 & -38 & -365 \\
\hline Fish & & -7 & -20 \\
\hline \multicolumn{4}{|l|}{ Environment } \\
\hline Forest area (\%) & 1 & 1 & 1 \\
\hline Renewable water res withdrawn (\% of total) & & & 66 \\
\hline Terrestrial protect areas (\% total land area) & 2 & 3 & 3 \\
\hline Organic area (\% total agricultural area) & & & 0 \\
\hline Water withdrawal by agriculture (\% of total) & & & 66 \\
\hline \multicolumn{4}{|l|}{ Biofuel production (thousand kt of oil eq.) } \\
\hline Wood pellet prod. (1 000 tonnes) & & & 0 \\
\hline Net GHG emissions from AFOLU ( $\mathrm{CO}_{2}$ eq, $\mathrm{Mt}$ ) & 33 & 15 & 19 \\
\hline
\end{tabular}




\begin{tabular}{|c|c|c|c|}
\hline & 1990 & 2000 & 2014 \\
\hline \multicolumn{4}{|l|}{ The setting } \\
\hline Population, total (mln) & 23.4 & 31.3 & 45.5 \\
\hline Population, rural (mln) & 19.5 & 25.1 & 34.1 \\
\hline Govt expenditure on ag (\% total outlays) & & 4.6 & 5.1 \\
\hline Area harvested (mln ha) & 5 & 4 & 6 \\
\hline Cropping intensity ratio & 0.2 & 0.1 & \\
\hline Water resources (1 $000 \mathrm{~m}^{3} /$ person/year) & 1 & 1 & 1 \\
\hline Area equipped for irrigation (1 $000 \mathrm{ha}$ ) & & & 103 \\
\hline Area irrigated (\% area equipped for irrigation) & & 94.2 & \\
\hline Employment in agriculture (\%) & & & 61.1 \\
\hline Employment in agriculture, female (\%) & & & 68 \\
\hline Fertilizers, Nitrogen (kg of nutrients per ha) & & 13.2 & 20.3 \\
\hline Fertilizers, Phosphate (kg of nutrients per ha) & & 17.6 & 18.3 \\
\hline Fertilizers, Potash (kg nutrients per ha) & & 2.3 & 5.7 \\
\hline Energy consump, power irrigation (mln kWh) & 53 & 53 & 154 \\
\hline Agr value added per worker (constant US\$) & 415 & 352 & 396 \\
\hline \multicolumn{4}{|l|}{ Hunger dimensions } \\
\hline Dietary energy supply (kcal/pc/day) & 1958 & 2064 & 2210 \\
\hline Average dietary energy supply adequacy (\%) & 93 & 96 & 102 \\
\hline Dietary en supp, cereals/roots/tubers (\%) & 55 & 56 & 56 \\
\hline Prevalence of undernourishment (\%) & 37.5 & 32.3 & 21.5 \\
\hline GDP per capita (US\$, PPP) & 2376 & 2143 & 2705 \\
\hline Domestic food price volatility (index) & & 9.8 & 6 \\
\hline Cereal import dependency ratio (\%) & 13.7 & 25.4 & 36.4 \\
\hline Underweight, children under-5 (\%) & 20.1 & 17.5 & 16.4 \\
\hline Improved water source (\% pop) & 42.7 & 51.8 & 61.7 \\
\hline \multicolumn{4}{|l|}{ Food supply } \\
\hline Food production value, (2004-2006 mln I\$) & 3564 & 4002 & 6840 \\
\hline Agriculture, value added (\% GDP) & 30 & 32 & 30 \\
\hline Food exports (mln US\$) & 146 & 227 & 382 \\
\hline Food imports (mln US\$) & 194 & 447 & 1216 \\
\hline \multicolumn{4}{|l|}{ Production indices $(2004-06=100)$} \\
\hline Net food & 64 & 72 & 123 \\
\hline Net crops & 68 & 77 & 127 \\
\hline Cereals & 78 & 72 & 123 \\
\hline Vegetable oils & 69 & 101 & 111 \\
\hline Roots and tubers & 46 & 42 & 127 \\
\hline Fruit and vegetables & 46 & 83 & 130 \\
\hline Sugar & 99 & 82 & 123 \\
\hline Livestock & 64 & 69 & 118 \\
\hline Milk & 67 & 66 & 122 \\
\hline Meat & 59 & 69 & 115 \\
\hline Fish & 139 & 148 & 128 \\
\hline \multicolumn{4}{|l|}{ Net trade (mln US\$) } \\
\hline Cereals & -51 & -246 & -606 \\
\hline Fruit and vegetables & 102 & 149 & 102 \\
\hline Meat & 5 & 1 & 7 \\
\hline Dairy products & 1 & -4 & -25 \\
\hline Fish & 26 & 34 & 51 \\
\hline \multicolumn{4}{|l|}{ Environment } \\
\hline Forest area (\%) & 7 & 6 & 6 \\
\hline Renewable water res withdrawn (\% of total) & & 79 & 79 \\
\hline Terrestrial protect areas (\% total land area) & 12 & 12 & 12 \\
\hline Organic area (\% total agricultural area) & & & 0 \\
\hline Water withdrawal by agriculture (\% of total) & & 79 & 79 \\
\hline Biofuel production (thousand kt of oil eq.) & 43 & 49 & 54 \\
\hline \multicolumn{4}{|l|}{ Wood pellet prod. (1 000 tonnes) } \\
\hline Net GHG emissions from AFOLU ( $\mathrm{CO}_{2}$ eq, $\mathrm{Mt}$ ) & 33 & 30 & 51 \\
\hline
\end{tabular}




\begin{tabular}{|c|c|c|c|}
\hline & 1990 & 2000 & 2014 \\
\hline \multicolumn{4}{|l|}{ The setting } \\
\hline Population, total (mln) & 2.1 & 1.9 & 3.5 \\
\hline Population, rural (mln) & 0 & 0 & 0.1 \\
\hline Govt expenditure on ag (\% total outlays) & & 0 & 0 \\
\hline Area harvested (mln ha) & 1 & 1 & 0 \\
\hline Cropping intensity ratio & 9.6 & 4.7 & \\
\hline Water resources (1 $000 \mathrm{~m}^{3} /$ person/year) & 0 & 0 & 0 \\
\hline Area equipped for irrigation (1 $000 \mathrm{ha}$ ) & & & 10 \\
\hline Area irrigated (\% area equipped for irrigation) & & & 100 \\
\hline Employment in agriculture (\%) & 1.3 & 0 & 2.7 \\
\hline Employment in agriculture, female (\%) & 0.1 & & 0 \\
\hline Fertilizers, Nitrogen (kg of nutrients per ha) & & 0 & 0 \\
\hline Fertilizers, Phosphate (kg of nutrients per ha) & & 0 & 42.6 \\
\hline Fertilizers, Potash (kg nutrients per ha) & & 0 & 294.5 \\
\hline Energy consump, power irrigation (mln kWh) & 0 & 4 & 4 \\
\hline \multicolumn{4}{|l|}{ Agr value added per worker (constant US\$) } \\
\hline \multicolumn{4}{|l|}{ Hunger dimensions } \\
\hline Dietary energy supply (kcal/pc/day) & 2529 & 3449 & 3304 \\
\hline Average dietary energy supply adequacy (\%) & 106 & 142 & 135 \\
\hline Dietary en supp, cereals/roots/tubers (\%) & 42 & 40 & 43 \\
\hline Prevalence of undernourishment (\%) & 31.5 & $<5.0$ & $<5.0$ \\
\hline GDP per capita (US\$, PPP) & & 74824 & 82358 \\
\hline Domestic food price volatility (index) & & 6 & 3.7 \\
\hline Cereal import dependency ratio (\%) & 99.5 & 99.7 & 97.8 \\
\hline Underweight, children under-5 (\%) & & 2.3 & 2.2 \\
\hline Improved water source (\% pop) & 99 & 99 & 99 \\
\hline \multicolumn{4}{|l|}{ Food supply } \\
\hline Food production value, (2004-2006 mln I\$) & 90 & 143 & 303 \\
\hline Agriculture, value added (\% GDP) & & & 0 \\
\hline Food exports (mln US\$) & 32 & 43 & 131 \\
\hline Food imports (mln US\$) & 492 & 1088 & 2236 \\
\hline \multicolumn{4}{|l|}{ Production indices $(2004-06=100)$} \\
\hline Net food & 50 & 80 & 170 \\
\hline Net crops & 40 & 74 & 163 \\
\hline Cereals & 52 & 78 & 749 \\
\hline Vegetable oils & 29 & 12 & 155 \\
\hline Roots and tubers & 8 & 86 & 226 \\
\hline Fruit and vegetables & 40 & 73 & 156 \\
\hline \multicolumn{4}{|l|}{ Sugar } \\
\hline Livestock & 58 & 84 & 177 \\
\hline Milk & 56 & 76 & 142 \\
\hline Meat & 65 & 84 & 183 \\
\hline Fish & 79 & 131 & 88 \\
\hline \multicolumn{4}{|l|}{ Net trade (mln US\$) } \\
\hline Cereals & -53 & -218 & -585 \\
\hline Fruit and vegetables & -144 & -280 & -306 \\
\hline Meat & -34 & -119 & -430 \\
\hline Dairy products & -56 & -158 & -222 \\
\hline Fish & 1 & -20 & -112 \\
\hline \multicolumn{4}{|l|}{ Environment } \\
\hline Forest area (\%) & 0 & 0 & 0 \\
\hline Renewable water res withdrawn (\% of total) & & 54 & 54 \\
\hline Terrestrial protect areas (\% total land area) & 2 & 2 & 18 \\
\hline \multicolumn{4}{|l|}{ Organic area (\% total agricultural area) } \\
\hline Water withdrawal by agriculture (\% of total) & & 54 & 54 \\
\hline \multicolumn{4}{|l|}{ Biofuel production (thousand kt of oil eq.) } \\
\hline \multicolumn{4}{|l|}{ Wood pellet prod. (1 000 tonnes) } \\
\hline Net GHG emissions from AFOLU ( $\mathrm{CO}_{2}$ eq, $\mathrm{Mt}$ ) & 0 & 0 & 0 \\
\hline
\end{tabular}




\begin{tabular}{|c|c|c|c|}
\hline & 1990 & 2000 & 2014 \\
\hline \multicolumn{4}{|l|}{ The setting } \\
\hline Population, total (mln) & 4.5 & 5 & 5.6 \\
\hline Population, rural (mln) & 2.8 & 3.2 & 3.6 \\
\hline Govt expenditure on ag (\% total outlays) & & 4.5 & 1.5 \\
\hline Area harvested (mln ha) & & 2 & 2 \\
\hline Cropping intensity ratio & & 0.1 & \\
\hline Water resources (1 $000 \mathrm{~m}^{3} /$ person/year) & 5 & 5 & 4 \\
\hline Area equipped for irrigation (1 $000 \mathrm{ha}$ ) & & & 1023 \\
\hline Area irrigated (\% area equipped for irrigation) & & & 99.8 \\
\hline Employment in agriculture (\%) & 32.7 & 53.1 & 34 \\
\hline Employment in agriculture, female (\%) & & 54.8 & 35.4 \\
\hline Fertilizers, Nitrogen (kg of nutrients per ha) & & 23.4 & 19.8 \\
\hline Fertilizers, Phosphate (kg of nutrients per ha) & & 1.3 & 1.2 \\
\hline Fertilizers, Potash (kg nutrients per ha) & & 0 & 0.6 \\
\hline Energy consump, power irrigation (mln kWh) & 0 & 89 & 1 \\
\hline Agr value added per worker (constant US\$) & 835 & 1133 & 1418 \\
\hline \multicolumn{4}{|l|}{ Hunger dimensions } \\
\hline Dietary energy supply (kcal/pc/day) & 2495 & 2453 & 2855 \\
\hline Average dietary energy supply adequacy (\%) & 111 & 106 & 122 \\
\hline Dietary en supp, cereals/roots/tubers (\%) & 58 & 59 & 54 \\
\hline Prevalence of undernourishment (\%) & 15.9 & 15.2 & 6 \\
\hline GDP per capita (US\$, PPP) & 3475 & 2074 & 3110 \\
\hline \multicolumn{4}{|l|}{ Domestic food price volatility (index) } \\
\hline Cereal import dependency ratio (\%) & 32.6 & 8.2 & 23.4 \\
\hline Underweight, children under-5 (\%) & & 8.2 & 2.8 \\
\hline Improved water source (\% pop) & 72.9 & 78.7 & 87.6 \\
\hline \multicolumn{4}{|l|}{ Food supply } \\
\hline Food production value, (2004-2006 mln I\$) & 1084 & 1377 & 1619 \\
\hline Agriculture, value added (\% GDP) & 34 & 37 & 17 \\
\hline Food exports (mln US\$) & 5 & 18 & 203 \\
\hline Food imports (mln US\$) & 134 & 56 & 614 \\
\hline \multicolumn{4}{|l|}{ Production indices $(2004-06=100)$} \\
\hline Net food & 75 & 95 & 112 \\
\hline Net crops & 55 & 94 & 112 \\
\hline Cereals & 91 & 97 & 106 \\
\hline Vegetable oils & 16 & 64 & 63 \\
\hline Roots and tubers & 27 & 83 & 109 \\
\hline Fruit and vegetables & 34 & 100 & 132 \\
\hline Sugar & 57 & 117 & 51 \\
\hline Livestock & 106 & 99 & 113 \\
\hline Milk & 79 & 93 & 118 \\
\hline Meat & 119 & 105 & 107 \\
\hline Fish & 3021 & 254 & 755 \\
\hline \multicolumn{4}{|l|}{ Net trade (mln US\$) } \\
\hline Cereals & & -35 & -185 \\
\hline Fruit and vegetables & 1 & 7 & 106 \\
\hline Meat & & -2 & -73 \\
\hline Dairy products & & 1 & 9 \\
\hline Fish & & -2 & -15 \\
\hline \multicolumn{4}{|l|}{ Environment } \\
\hline Forest area (\%) & 4 & 4 & 5 \\
\hline Renewable water res withdrawn (\% of total) & & & 93 \\
\hline Terrestrial protect areas (\% total land area) & 6 & 7 & 6 \\
\hline Organic area (\% total agricultural area) & & & 0 \\
\hline Water withdrawal by agriculture (\% of total) & & & 93 \\
\hline \multicolumn{4}{|l|}{ Biofuel production (thousand kt of oil eq.) } \\
\hline \multicolumn{4}{|l|}{ Wood pellet prod. (1 000 tonnes) } \\
\hline Net GHG emissions from AFOLU ( $\mathrm{CO}_{2}$ eq, $\mathrm{Mt}$ ) & 2 & 1 & -11 \\
\hline
\end{tabular}




\begin{tabular}{|c|c|c|c|}
\hline & 1990 & 2000 & 2014 \\
\hline \multicolumn{4}{|l|}{ The setting } \\
\hline Population, total (mln) & 4.2 & 5.4 & 6.9 \\
\hline Population, rural (mln) & 3.6 & 4.2 & 4.3 \\
\hline \multicolumn{4}{|l|}{ Govt expenditure on ag (\% total outlays) } \\
\hline Area harvested (mln ha) & 2 & 2 & 4 \\
\hline Cropping intensity ratio & 0.9 & 1.3 & \\
\hline Water resources (1 $000 \mathrm{~m}^{3} /$ person/year) & 79 & 62 & 49 \\
\hline Area equipped for irrigation (1 $000 \mathrm{ha})$ & & & 310 \\
\hline Area irrigated (\% area equipped for irrigation) & & & 87.3 \\
\hline Employment in agriculture (\%) & & 85.4 & \\
\hline Employment in agriculture, female (\%) & & 89.3 & \\
\hline \multicolumn{4}{|l|}{ Fertilizers, Nitrogen (kg of nutrients per ha) } \\
\hline \multicolumn{4}{|l|}{ Fertilizers, Phosphate (kg of nutrients per ha) } \\
\hline \multicolumn{4}{|l|}{ Fertilizers, Potash (kg nutrients per ha) } \\
\hline Energy consump, power irrigation (mln kWh) & 0 & 0 & 0 \\
\hline Agr value added per worker (constant US\$) & 345 & 432 & 522 \\
\hline \multicolumn{4}{|l|}{ Hunger dimensions } \\
\hline Dietary energy supply (kcal/pc/day) & 1967 & 2083 & 2399 \\
\hline Average dietary energy supply adequacy (\%) & 90 & 94 & 104 \\
\hline Dietary en supp, cereals/roots/tubers (\%) & 83 & 78 & 72 \\
\hline Prevalence of undernourishment (\%) & 44.1 & 39.2 & 18.9 \\
\hline GDP per capita (US\$, PPP) & 1622 & 2327 & 4667 \\
\hline Domestic food price volatility (index) & & 12.2 & 3.6 \\
\hline Cereal import dependency ratio (\%) & 1.1 & 1.8 & -5.1 \\
\hline Underweight, children under-5 (\%) & 39.8 & 36.4 & 26.5 \\
\hline Improved water source (\% pop) & & 45.5 & 71.5 \\
\hline \multicolumn{4}{|l|}{ Food supply } \\
\hline Food production value, (2004-2006 mln I\$) & 579 & 968 & 1807 \\
\hline Agriculture, value added (\% GDP) & 61 & 45 & 28 \\
\hline Food exports (mln US\$) & 23 & 14 & 39 \\
\hline Food imports (mln US\$) & 11 & 32 & 199 \\
\hline \multicolumn{4}{|l|}{ Production indices $(2004-06=100)$} \\
\hline Net food & 49 & 82 & 153 \\
\hline Net crops & 51 & 84 & 163 \\
\hline Cereals & 55 & 82 & 146 \\
\hline Vegetable oils & 39 & 68 & 198 \\
\hline Roots and tubers & 110 & 87 & 524 \\
\hline Fruit and vegetables & 21 & 76 & 153 \\
\hline Sugar & 45 & 140 & 555 \\
\hline Livestock & 50 & 83 & 126 \\
\hline Milk & 73 & 93 & 112 \\
\hline Meat & 50 & 83 & 126 \\
\hline Fish & 31 & 80 & 159 \\
\hline \multicolumn{4}{|l|}{ Net trade (mln US\$) } \\
\hline Cereals & -2 & -14 & -21 \\
\hline Fruit and vegetables & 0 & 1 & -2 \\
\hline Meat & -1 & 0 & 0 \\
\hline \multicolumn{4}{|l|}{ Dairy products } \\
\hline Fish & 0 & -2 & -8 \\
\hline \multicolumn{4}{|l|}{ Environment } \\
\hline Forest area (\%) & 75 & 72 & 68 \\
\hline Renewable water res withdrawn (\% of total) & & & 91 \\
\hline Terrestrial protect areas (\% total land area) & 1 & 17 & 17 \\
\hline Organic area (\% total agricultural area) & & & 0 \\
\hline Water withdrawal by agriculture (\% of total) & & & 91 \\
\hline \multicolumn{4}{|l|}{ Biofuel production (thousand kt of oil eq.) } \\
\hline \multicolumn{4}{|l|}{ Wood pellet prod. (1 000 tonnes) } \\
\hline Net GHG emissions from AFOLU ( $\mathrm{CO}_{2}$ eq, $\mathrm{Mt}$ ) & 29 & 27 & 33 \\
\hline
\end{tabular}




\begin{tabular}{|c|c|c|c|}
\hline & 1990 & 2000 & 2014 \\
\hline \multicolumn{4}{|l|}{ The setting } \\
\hline Population, total (mln) & 2.6 & 2.4 & 2 \\
\hline Population, rural (mln) & 0.8 & 0.8 & 0.7 \\
\hline Govt expenditure on ag (\% total outlays) & & 9.9 & 12.5 \\
\hline Area harvested (mln ha) & & 1 & 2 \\
\hline Cropping intensity ratio & & 0.6 & \\
\hline Water resources (1 $000 \mathrm{~m}^{3} /$ person/year) & 14 & 15 & 17 \\
\hline Area equipped for irrigation (1 $000 \mathrm{ha}$ ) & & & 1 \\
\hline Area irrigated (\% area equipped for irrigation) & & & 74.7 \\
\hline Employment in agriculture (\%) & & 14.5 & 8.4 \\
\hline Employment in agriculture, female (\%) & & 12.2 & 4.9 \\
\hline Fertilizers, Nitrogen (kg of nutrients per ha) & & 25.2 & 55.9 \\
\hline Fertilizers, Phosphate (kg of nutrients per ha) & & 11.7 & 16.8 \\
\hline Fertilizers, Potash (kg nutrients per ha) & & 12.6 & 17.5 \\
\hline Energy consump, power irrigation (mln kWh) & 0 & 0 & 0 \\
\hline Agr value added per worker (constant US\$) & & 3838 & 7081 \\
\hline \multicolumn{4}{|l|}{ Hunger dimensions } \\
\hline \multicolumn{4}{|l|}{ Dietary energy supply (kcal/pc/day) } \\
\hline Average dietary energy supply adequacy (\%) & 133 & 115 & 134 \\
\hline Dietary en supp, cereals/roots/tubers (\%) & 47 & 41 & 33 \\
\hline Prevalence of undernourishment (\%) & $<5.0$ & $<5.0$ & $<5.0$ \\
\hline GDP per capita (US\$, PPP) & 13310 & 11256 & 21833 \\
\hline Domestic food price volatility (index) & & 6.9 & 7.9 \\
\hline Cereal import dependency ratio (\%) & 2.4 & 4.7 & -72.2 \\
\hline \multicolumn{4}{|l|}{ Underweight, children under-5 (\%) } \\
\hline Improved water source (\% pop) & 98.5 & 98.4 & 98.4 \\
\hline \multicolumn{4}{|l|}{ Food supply } \\
\hline Food production value, (2004-2006 mln I\$) & 1116 & 570 & 797 \\
\hline Agriculture, value added (\% GDP) & & 5 & 3 \\
\hline Food exports (mln US\$) & 89 & 88 & 1548 \\
\hline Food imports (mln US\$) & 38 & 398 & 1474 \\
\hline \multicolumn{4}{|l|}{ Production indices $(2004-06=100)$} \\
\hline Net food & 169 & 86 & 121 \\
\hline Net crops & 126 & 82 & 125 \\
\hline Cereals & 96 & 76 & 183 \\
\hline Vegetable oils & 4 & 9 & 239 \\
\hline Roots and tubers & 203 & 128 & 41 \\
\hline Fruit and vegetables & 195 & 82 & 62 \\
\hline Sugar & 60 & 82 & 2 \\
\hline Livestock & 196 & 94 & 117 \\
\hline Milk & 144 & 103 & 114 \\
\hline Meat & 318 & 84 & 123 \\
\hline Fish & 330 & 98 & 84 \\
\hline \multicolumn{4}{|l|}{ Net trade (mln US\$) } \\
\hline Cereals & 1 & -45 & 369 \\
\hline Fruit and vegetables & -4 & -122 & -178 \\
\hline Meat & 21 & -34 & -96 \\
\hline Dairy products & 29 & 13 & 102 \\
\hline Fish & 27 & 13 & 53 \\
\hline \multicolumn{4}{|l|}{ Environment } \\
\hline Forest area (\%) & 51 & 52 & 54 \\
\hline Renewable water res withdrawn (\% of total) & & 13 & 13 \\
\hline Terrestrial protect areas (\% total land area) & 7 & 16 & 19 \\
\hline Organic area (\% total agricultural area) & & & 11 \\
\hline Water withdrawal by agriculture (\% of total) & & 13 & 13 \\
\hline Biofuel production (thousand kt of oil eq.) & 1 & 8 & 1195 \\
\hline Wood pellet prod. (1 000 tonnes) & & & 1280 \\
\hline Net GHG emissions from AFOLU ( $\mathrm{CO}_{2}$ eq, $\mathrm{Mt}$ ) & -5 & -7 & -12 \\
\hline
\end{tabular}




\begin{tabular}{|c|c|c|c|}
\hline & 1990 & 2000 & 2014 \\
\hline \multicolumn{4}{|l|}{ The setting } \\
\hline Population, total (mln) & 2.7 & 3.2 & 5 \\
\hline Population, rural (mln) & 0.5 & 0.5 & 0.6 \\
\hline \multicolumn{4}{|l|}{ Govt expenditure on ag (\% total outlays) } \\
\hline Area harvested (mln ha) & 1 & 1 & 0 \\
\hline Cropping intensity ratio & 2 & 1.5 & \\
\hline Water resources (1 $000 \mathrm{~m}^{3} /$ person/year) & 2 & 1 & 1 \\
\hline Area equipped for irrigation (1 $000 \mathrm{ha})$ & & & 104 \\
\hline Area irrigated (\% area equipped for irrigation) & & 86.5 & \\
\hline Employment in agriculture (\%) & & & 6.3 \\
\hline Employment in agriculture, female (\%) & & & 5.7 \\
\hline Fertilizers, Nitrogen (kg of nutrients per ha) & & 123.5 & 74.1 \\
\hline Fertilizers, Phosphate (kg of nutrients per ha) & & 0 & 182.4 \\
\hline Fertilizers, Potash (kg nutrients per ha) & & 8.9 & 26.4 \\
\hline Energy consump, power irrigation (mln kWh) & 82 & 91 & 91 \\
\hline Agr value added per worker (constant US\$) & & 15404 & 35125 \\
\hline \multicolumn{4}{|l|}{ Hunger dimensions } \\
\hline Dietary energy supply (kcal/pc/day) & 3264 & 3342 & 3213 \\
\hline Average dietary energy supply adequacy (\%) & 141 & 142 & 130 \\
\hline Dietary en supp, cereals/roots/tubers (\%) & 37 & 37 & 39 \\
\hline Prevalence of undernourishment (\%) & $<5.0$ & $<5.0$ & $<5.0$ \\
\hline GDP per capita (US\$, PPP) & 7734 & 12335 & 16623 \\
\hline \multicolumn{4}{|l|}{ Domestic food price volatility (index) } \\
\hline Cereal import dependency ratio (\%) & 89.1 & 87.5 & 88.3 \\
\hline Underweight, children under-5 (\%) & & 3.5 & \\
\hline Improved water source (\% pop) & 100 & 100 & 100 \\
\hline \multicolumn{4}{|l|}{ Food supply } \\
\hline Food production value, (2004-2006 mln I\$) & 1016 & 1198 & 1163 \\
\hline Agriculture, value added (\% GDP) & & 7 & 6 \\
\hline Food exports (mln US\$) & 90 & 96 & 478 \\
\hline Food imports (mln US\$) & 552 & 855 & 2348 \\
\hline \multicolumn{4}{|l|}{ Production indices $(2004-06=100)$} \\
\hline Net food & 84 & 99 & 96 \\
\hline Net crops & 97 & 104 & 99 \\
\hline Cereals & 42 & 70 & 101 \\
\hline Vegetable oils & 47 & 134 & 72 \\
\hline Roots and tubers & 51 & 59 & 88 \\
\hline Fruit and vegetables & 124 & 106 & 107 \\
\hline Sugar & 143 & 521 & 2 \\
\hline Livestock & 54 & 87 & 93 \\
\hline Milk & 55 & 88 & 161 \\
\hline Meat & 49 & 84 & 75 \\
\hline Fish & 32 & 88 & 110 \\
\hline \multicolumn{4}{|l|}{ Net trade (mln US\$) } \\
\hline Cereals & -100 & -170 & -521 \\
\hline Fruit and vegetables & -77 & -97 & -92 \\
\hline Meat & -35 & -58 & -184 \\
\hline Dairy products & -68 & -148 & -287 \\
\hline Fish & -10 & -44 & -132 \\
\hline \multicolumn{4}{|l|}{ Environment } \\
\hline Forest area (\%) & 13 & 13 & 13 \\
\hline Renewable water res withdrawn (\% of total) & & & 60 \\
\hline Terrestrial protect areas (\% total land area) & 0 & 1 & 1 \\
\hline Organic area (\% total agricultural area) & & & 0 \\
\hline Water withdrawal by agriculture (\% of total) & & & 60 \\
\hline \multicolumn{4}{|l|}{ Biofuel production (thousand kt of oil eq.) } \\
\hline \multicolumn{4}{|l|}{ Wood pellet prod. (1 000 tonnes) } \\
\hline Net GHG emissions from AFOLU ( $\mathrm{CO}_{2}$ eq, $\left.\mathrm{Mt}\right)$ & 0 & 1 & 1 \\
\hline
\end{tabular}




\begin{tabular}{|c|c|c|c|}
\hline & 1990 & 2000 & 2014 \\
\hline \multicolumn{4}{|l|}{ The setting } \\
\hline Population, total (mln) & 1.6 & 1.9 & 2.1 \\
\hline Population, rural (mln) & 1.4 & 1.5 & 1.5 \\
\hline Govt expenditure on ag (\% total outlays) & & & 2.4 \\
\hline Area harvested (mln ha) & 0 & 0 & 0 \\
\hline Cropping intensity ratio & 0.1 & 0.1 & \\
\hline Water resources (1 $000 \mathrm{~m}^{3} /$ person/year) & 2 & 2 & 1 \\
\hline Area equipped for irrigation (1 $000 \mathrm{ha}$ ) & & & 3 \\
\hline Area irrigated (\% area equipped for irrigation) & & 2.5 & \\
\hline Employment in agriculture (\%) & & 72.3 & \\
\hline Employment in agriculture, female (\%) & & 64.9 & \\
\hline \multicolumn{4}{|l|}{ Fertilizers, Nitrogen (kg of nutrients per ha) } \\
\hline \multicolumn{4}{|l|}{ Fertilizers, Phosphate (kg of nutrients per ha) } \\
\hline \multicolumn{4}{|l|}{ Fertilizers, Potash (kg nutrients per ha) } \\
\hline \multicolumn{4}{|l|}{ Energy consump, power irrigation (mln kWh) } \\
\hline Agr value added per worker (constant US\$) & 427 & 393 & 364 \\
\hline \multicolumn{4}{|l|}{ Hunger dimensions } \\
\hline Dietary energy supply (kcal/pc/day) & 2388 & 2468 & 2593 \\
\hline Average dietary energy supply adequacy (\%) & 112 & 115 & 116 \\
\hline Dietary en supp, cereals/roots/tubers (\%) & 80 & 80 & 80 \\
\hline Prevalence of undernourishment (\%) & 15.3 & 13 & 11.2 \\
\hline GDP per capita (US\$, PPP) & 1307 & 1637 & 2494 \\
\hline Domestic food price volatility (index) & & 12.2 & 6.4 \\
\hline Cereal import dependency ratio (\%) & 70.1 & 54.1 & 78.2 \\
\hline Underweight, children under-5 (\%) & 18.9 & 15 & 13.5 \\
\hline Improved water source (\% pop) & 77.5 & 79.1 & 81.3 \\
\hline \multicolumn{4}{|l|}{ Food supply } \\
\hline Food production value, (2004-2006 mln I\$) & 118 & 122 & 132 \\
\hline Agriculture, value added (\% GDP) & 25 & 12 & 6 \\
\hline Food exports (mln US\$) & 6 & 2 & 0 \\
\hline Food imports (mln US\$) & 112 & 111 & 189 \\
\hline \multicolumn{4}{|l|}{ Production indices (2004-06=100) } \\
\hline Net food & 100 & 103 & 112 \\
\hline Net crops & 128 & 113 & 113 \\
\hline Cereals & 217 & 133 & 93 \\
\hline \multicolumn{4}{|l|}{ Vegetable oils } \\
\hline Roots and tubers & 46 & 88 & 126 \\
\hline Fruit and vegetables & 93 & 76 & 95 \\
\hline \multicolumn{4}{|l|}{ Sugar } \\
\hline Livestock & 85 & 95 & 111 \\
\hline Milk & 83 & 110 & 138 \\
\hline Meat & 85 & 96 & 107 \\
\hline Fish & 64 & 86 & 1179 \\
\hline \multicolumn{4}{|l|}{ Net trade (mln US\$) } \\
\hline Cereals & -36 & -36 & -96 \\
\hline Fruit and vegetables & -24 & -27 & -33 \\
\hline Meat & -9 & -11 & -20 \\
\hline \multicolumn{4}{|l|}{ Dairy products } \\
\hline Fish & & -9 & -3 \\
\hline \multicolumn{4}{|l|}{ Environment } \\
\hline Forest area (\%) & 1 & 1 & 1 \\
\hline Renewable water res withdrawn (\% of total) & & 9 & 9 \\
\hline Terrestrial protect areas (\% total land area) & 0 & 0 & 1 \\
\hline Organic area (\% total agricultural area) & & & 0 \\
\hline Water withdrawal by agriculture (\% of total) & & 9 & 9 \\
\hline \multicolumn{4}{|l|}{ Biofuel production (thousand kt of oil eq.) } \\
\hline \multicolumn{4}{|l|}{ Wood pellet prod. (1 000 tonnes) } \\
\hline Net GHG emissions from AFOLU ( $\mathrm{CO}_{2}$ eq, $\mathrm{Mt}$ ) & 1 & 2 & 1 \\
\hline
\end{tabular}




\section{Liberia}

\begin{tabular}{|c|c|c|c|}
\hline & 1990 & 2000 & 2014 \\
\hline \multicolumn{4}{|l|}{ The setting } \\
\hline Population, total (mln) & 2.1 & 2.9 & 4.4 \\
\hline Population, rural (mln) & 0.9 & 1.6 & 2.2 \\
\hline Govt expenditure on ag (\% total outlays) & & & 2.4 \\
\hline Area harvested (mln ha) & 0 & 1 & 1 \\
\hline Cropping intensity ratio & 0.2 & 0.2 & \\
\hline Water resources (1 $000 \mathrm{~m}^{3} /$ person/year) & 110 & 80 & 54 \\
\hline Area equipped for irrigation (1000 ha) & & & 3 \\
\hline \multicolumn{4}{|l|}{ Area irrigated (\% area equipped for irrigation) } \\
\hline Employment in agriculture (\%) & & & 48.9 \\
\hline Employment in agriculture, female (\%) & & & 48.3 \\
\hline \multicolumn{4}{|l|}{ Fertilizers, Nitrogen (kg of nutrients per ha) } \\
\hline \multicolumn{4}{|l|}{ Fertilizers, Phosphate (kg of nutrients per ha) } \\
\hline \multicolumn{4}{|l|}{ Fertilizers, Potash (kg nutrients per ha) } \\
\hline \multicolumn{4}{|l|}{ Energy consump, power irrigation (mln kWh) } \\
\hline Agr value added per worker (constant US\$) & & 574 & 790 \\
\hline \multicolumn{4}{|l|}{ Hunger dimensions } \\
\hline Dietary energy supply (kcal/pc/day) & 2161 & 2084 & 2336 \\
\hline Average dietary energy supply adequacy (\%) & 101 & 98 & 108 \\
\hline Dietary en supp, cereals/roots/tubers (\%) & 61 & 65 & 66 \\
\hline Prevalence of undernourishment (\%) & 30.7 & 36.5 & 32.5 \\
\hline GDP per capita (US\$, PPP) & 502 & 473 & 850 \\
\hline \multicolumn{4}{|l|}{ Domestic food price volatility (index) } \\
\hline Cereal import dependency ratio (\%) & 75.3 & 64.3 & 61.1 \\
\hline Underweight, children under-5 (\%) & & 22.8 & 15.3 \\
\hline Improved water source (\% pop) & & 61.2 & 74.6 \\
\hline \multicolumn{4}{|l|}{ Food supply } \\
\hline Food production value, (2004-2006 mln I\$) & 207 & 270 & 323 \\
\hline \multicolumn{4}{|l|}{ Agriculture, value added (\% GDP) } \\
\hline Food exports (mln US\$) & 5 & 5 & 31 \\
\hline Food imports (mln US\$) & 47 & 84 & 308 \\
\hline \multicolumn{4}{|l|}{ Production indices $(2004-06=100)$} \\
\hline Net food & 77 & 100 & 120 \\
\hline Net crops & 63 & 100 & 96 \\
\hline Cereals & 128 & 130 & 166 \\
\hline Vegetable oils & 56 & 100 & 105 \\
\hline Roots and tubers & 75 & 91 & 101 \\
\hline Fruit and vegetables & 59 & 95 & 104 \\
\hline Sugar & 82 & 98 & 104 \\
\hline Livestock & 79 & 101 & 140 \\
\hline Milk & 80 & 92 & 91 \\
\hline Meat & 78 & 102 & 144 \\
\hline Fish & 56 & 99 & 82 \\
\hline \multicolumn{4}{|l|}{ Net trade (mln US\$) } \\
\hline Cereals & -27 & -52 & -167 \\
\hline Fruit and vegetables & -4 & -5 & -11 \\
\hline Meat & -3 & -6 & -33 \\
\hline Dairy products & -2 & -3 & -8 \\
\hline Fish & -6 & -3 & -10 \\
\hline \multicolumn{4}{|l|}{ Environment } \\
\hline Forest area (\%) & 51 & 48 & 44 \\
\hline Renewable water res withdrawn (\% of total) & & 9 & 9 \\
\hline Terrestrial protect areas (\% total land area) & 2 & 2 & 3 \\
\hline \multicolumn{4}{|l|}{ Organic area (\% total agricultural area) } \\
\hline Water withdrawal by agriculture (\% of total) & & 9 & 9 \\
\hline Biofuel production (thousand kt of oil eq.) & 0 & 0 & \\
\hline \multicolumn{4}{|l|}{ Wood pellet prod. (1 000 tonnes) } \\
\hline Net GHG emissions from AFOLU ( $\mathrm{CO}_{2}$ eq, $\mathrm{Mt}$ ) & 15 & 15 & 16 \\
\hline
\end{tabular}




\begin{tabular}{|c|c|c|c|}
\hline & 1990 & 2000 & 2014 \\
\hline \multicolumn{4}{|l|}{ The setting } \\
\hline Population, total (mln) & 4.3 & 5.2 & 6.3 \\
\hline Population, rural (mln) & 1 & 1.2 & 1.4 \\
\hline \multicolumn{4}{|l|}{ Govt expenditure on ag (\% total outlays) } \\
\hline Area harvested (mln ha) & 1 & 1 & 0 \\
\hline Cropping intensity ratio & 0 & 0.1 & \\
\hline Water resources (1 $000 \mathrm{~m}^{3} /$ person/year) & 0 & 0 & 0 \\
\hline Area equipped for irrigation (1 $000 \mathrm{ha}$ ) & & & 470 \\
\hline Area irrigated (\% area equipped for irrigation) & & 67.2 & \\
\hline \multicolumn{4}{|l|}{ Employment in agriculture (\%) } \\
\hline \multicolumn{4}{|l|}{ Employment in agriculture, female (\%) } \\
\hline Fertilizers, Nitrogen (kg of nutrients per ha) & & 17.8 & 26.3 \\
\hline Fertilizers, Phosphate (kg of nutrients per ha) & & 11.9 & 4.3 \\
\hline Fertilizers, Potash (kg nutrients per ha) & & 3.3 & 0.3 \\
\hline \multicolumn{4}{|l|}{ Energy consump, power irrigation (mln kWh) } \\
\hline Agr value added per worker (constant US\$) & & & 13815 \\
\hline \multicolumn{4}{|l|}{ Hunger dimensions } \\
\hline \multicolumn{4}{|l|}{ Dietary energy supply (kcal/pc/day) } \\
\hline \multicolumn{4}{|l|}{ Average dietary energy supply adequacy (\%) } \\
\hline \multicolumn{4}{|l|}{ Dietary en supp, cereals/roots/tubers (\%) } \\
\hline \multicolumn{4}{|l|}{ Prevalence of undernourishment (\%) } \\
\hline GDP per capita (US\$, PPP) & & 23085 & 20371 \\
\hline \multicolumn{4}{|l|}{ Domestic food price volatility (index) } \\
\hline Cereal import dependency ratio (\%) & 91.9 & 91.3 & 92 \\
\hline Underweight, children under-5 (\%) & & 4.3 & 5.6 \\
\hline Improved water source (\% pop) & 54.4 & 54.4 & \\
\hline \multicolumn{4}{|l|}{ Food supply } \\
\hline Food production value, (2004-2006 mln I\$) & 757 & 943 & 1103 \\
\hline Agriculture, value added (\% GDP) & & 4 & 2 \\
\hline Food exports (mln US\$) & 38 & 50 & 2 \\
\hline Food imports (mln US\$) & 1000 & 991 & 3287 \\
\hline \multicolumn{4}{|l|}{ Production indices $(2004-06=100)$} \\
\hline Net food & 76 & 94 & 110 \\
\hline Net crops & 78 & 97 & 110 \\
\hline Cereals & 126 & 102 & 152 \\
\hline Vegetable oils & 40 & 91 & 76 \\
\hline Roots and tubers & 54 & 77 & 120 \\
\hline Fruit and vegetables & 82 & 100 & 119 \\
\hline \multicolumn{4}{|l|}{ Sugar } \\
\hline Livestock & 72 & 91 & 113 \\
\hline Milk & 67 & 90 & 97 \\
\hline Meat & 76 & 90 & 122 \\
\hline Fish & 66 & 133 & 96 \\
\hline \multicolumn{4}{|l|}{ Net trade (mln US\$) } \\
\hline Cereals & -458 & -561 & -1233 \\
\hline Fruit and vegetables & -76 & -101 & -355 \\
\hline Meat & -12 & -9 & -287 \\
\hline Dairy products & -108 & -66 & -425 \\
\hline Fish & -15 & 3 & -246 \\
\hline \multicolumn{4}{|l|}{ Environment } \\
\hline Forest area (\%) & 0 & 0 & 0 \\
\hline Renewable water res withdrawn (\% of total) & & 83 & 83 \\
\hline Terrestrial protect areas (\% total land area) & 0 & 0 & 0 \\
\hline \multicolumn{4}{|l|}{ Organic area (\% total agricultural area) } \\
\hline Water withdrawal by agriculture (\% of total) & & 83 & 83 \\
\hline \multicolumn{4}{|l|}{ Biofuel production (thousand kt of oil eq.) } \\
\hline Wood pellet prod. (1 000 tonnes) & & & \\
\hline Net GHG emissions from AFOLU ( $\mathrm{CO}_{2}$ eq, $\left.\mathrm{Mt}\right)$ & 2 & 2 & 3 \\
\hline
\end{tabular}




\begin{tabular}{|c|c|c|c|}
\hline & 1990 & 2000 & 2014 \\
\hline \multicolumn{4}{|l|}{ The setting } \\
\hline Population, total (mln) & 3.7 & 3.5 & 3 \\
\hline Population, rural (mln) & 1.2 & 1.2 & 1 \\
\hline \multicolumn{4}{|l|}{ Govt expenditure on ag (\% total outlays) } \\
\hline Area harvested (mln ha) & & 3 & 4 \\
\hline Cropping intensity ratio & & 0.8 & \\
\hline Water resources (1 $000 \mathrm{~m}^{3} /$ person/year) & 7 & 7 & 8 \\
\hline Area equipped for irrigation (1 $000 \mathrm{ha}$ ) & & & 4 \\
\hline Area irrigated (\% area equipped for irrigation) & & & 22.4 \\
\hline Employment in agriculture (\%) & & 18.7 & 8.9 \\
\hline Employment in agriculture, female (\%) & & 15.1 & 6.4 \\
\hline Fertilizers, Nitrogen (kg of nutrients per ha) & & 25.4 & 29.2 \\
\hline Fertilizers, Phosphate (kg of nutrients per ha) & & 44.2 & 42.5 \\
\hline Fertilizers, Potash (kg nutrients per ha) & & 77.8 & 27.3 \\
\hline Energy consump, power irrigation (mln kWh) & 0 & 18 & 18 \\
\hline Agr value added per worker (constant US\$) & & & 12444 \\
\hline \multicolumn{4}{|l|}{ Hunger dimensions } \\
\hline \multicolumn{4}{|l|}{ Dietary energy supply (kcal/pc/day) } \\
\hline Average dietary energy supply adequacy (\%) & 118 & 129 & 143 \\
\hline Dietary en supp, cereals/roots/tubers (\%) & 53 & 49 & 39 \\
\hline Prevalence of undernourishment (\%) & $<5.0$ & $<5.0$ & $<5.0$ \\
\hline GDP per capita (US\$, PPP) & 15611 & 11887 & 24470 \\
\hline Domestic food price volatility (index) & & 6 & 5.5 \\
\hline Cereal import dependency ratio (\%) & 6.8 & -7.9 & -74.7 \\
\hline \multicolumn{4}{|l|}{ Underweight, children under-5 (\%) } \\
\hline Improved water source (\% pop) & 87.1 & 91 & 95.9 \\
\hline \multicolumn{4}{|l|}{ Food supply } \\
\hline Food production value, (2004-2006 mln I\$) & 1839 & 1403 & 1799 \\
\hline Agriculture, value added (\% GDP) & & & 3 \\
\hline Food exports (mln US\$) & 193 & 308 & 3785 \\
\hline Food imports (mln US\$) & 151 & 314 & 2621 \\
\hline \multicolumn{4}{|l|}{ Production indices (2004-06=100) } \\
\hline Net food & 120 & 91 & 117 \\
\hline Net crops & 118 & 114 & 156 \\
\hline Cereals & 98 & 101 & 193 \\
\hline Vegetable oils & 1 & 44 & 294 \\
\hline Roots and tubers & 255 & 225 & 58 \\
\hline Fruit and vegetables & 129 & 101 & 93 \\
\hline Sugar & 106 & 109 & 120 \\
\hline Livestock & 124 & 88 & 97 \\
\hline Milk & 111 & 92 & 92 \\
\hline Meat & 149 & 85 & 102 \\
\hline Fish & 220 & 52 & 62 \\
\hline \multicolumn{4}{|l|}{ Net trade (mln US\$) } \\
\hline Cereals & -22 & -23 & 620 \\
\hline Fruit and vegetables & 6 & -56 & -120 \\
\hline Meat & 25 & 5 & 48 \\
\hline Dairy products & 45 & 107 & 436 \\
\hline Fish & -1 & -22 & 23 \\
\hline \multicolumn{4}{|l|}{ Environment } \\
\hline Forest area (\%) & 31 & 32 & 35 \\
\hline Renewable water res withdrawn (\% of total) & & & 3 \\
\hline Terrestrial protect areas (\% total land area) & 2 & 12 & 17 \\
\hline Organic area (\% total agricultural area) & & & 6 \\
\hline Water withdrawal by agriculture (\% of total) & & & 3 \\
\hline Biofuel production (thousand kt of oil eq.) & 7 & 10 & 2431 \\
\hline Wood pellet prod. (1 000 tonnes) & & & 250 \\
\hline Net GHG emissions from AFOLU ( $\mathrm{CO}_{2}$ eq, $\mathrm{Mt}$ ) & 8 & 7 & 9 \\
\hline
\end{tabular}




\begin{tabular}{|c|c|c|c|}
\hline & 1990 & 2000 & 2014 \\
\hline \multicolumn{4}{|l|}{ The setting } \\
\hline Population, total (mln) & & 0.4 & 0.5 \\
\hline Population, rural (mln) & & 0.1 & 0.1 \\
\hline \multicolumn{4}{|l|}{ Govt expenditure on ag (\% total outlays) } \\
\hline Area harvested (mln ha) & & 1 & 0 \\
\hline Cropping intensity ratio & & 5.3 & \\
\hline Water resources (1 $000 \mathrm{~m}^{3} /$ person/year) & 9 & 8 & 7 \\
\hline \multicolumn{4}{|l|}{ Area equipped for irrigation (1 $000 \mathrm{ha})$} \\
\hline Area irrigated (\% area equipped for irrigation) & & 100 & \\
\hline Employment in agriculture (\%) & 3.3 & 2.4 & 1.3 \\
\hline Employment in agriculture, female (\%) & 2.9 & 1.5 & 0.9 \\
\hline Fertilizers, Nitrogen (kg of nutrients per ha) & & 208.1 & 359.1 \\
\hline Fertilizers, Phosphate (kg of nutrients per ha) & & 28.9 & 12.7 \\
\hline Fertilizers, Potash (kg nutrients per ha) & & 30.4 & 32.2 \\
\hline \multicolumn{4}{|l|}{ Energy consump, power irrigation (mln kWh) } \\
\hline Agr value added per worker (constant US\$) & & 61218 & 36314 \\
\hline \multicolumn{4}{|l|}{ Hunger dimensions } \\
\hline \multicolumn{4}{|l|}{ Dietary energy supply (kcal/pc/day) } \\
\hline Average dietary energy supply adequacy (\%) & & 140 & 140 \\
\hline Dietary en supp, cereals/roots/tubers (\%) & & 25 & 30 \\
\hline Prevalence of undernourishment (\%) & $<5.0$ & $<5.0$ & $<5.0$ \\
\hline GDP per capita (US\$, PPP) & 56922 & 81425 & 88850 \\
\hline Domestic food price volatility (index) & & 6.8 & 8.9 \\
\hline Cereal import dependency ratio (\%) & & -4.3 & 14.6 \\
\hline \multicolumn{4}{|l|}{ Underweight, children under-5 (\%) } \\
\hline Improved water source (\% pop) & 100 & 100 & 100 \\
\hline \multicolumn{4}{|l|}{ Food supply } \\
\hline Food production value, (2004-2006 mln I\$) & & 204 & 166 \\
\hline Agriculture, value added (\% GDP) & & 1 & 0 \\
\hline Food exports (mln US\$) & & 250 & 918 \\
\hline Food imports (mln US\$) & & 500 & 1534 \\
\hline \multicolumn{4}{|l|}{ Production indices (2004-06=100) } \\
\hline Net food & & 110 & 90 \\
\hline Net crops & & 96 & 86 \\
\hline Cereals & & 91 & 104 \\
\hline Vegetable oils & & 53 & 96 \\
\hline Roots and tubers & & 124 & 87 \\
\hline Fruit and vegetables & & 113 & 59 \\
\hline \multicolumn{4}{|l|}{ Sugar } \\
\hline Livestock & & 113 & 96 \\
\hline Milk & & 98 & 111 \\
\hline Meat & & 132 & 76 \\
\hline \multicolumn{4}{|l|}{ Fish } \\
\hline \multicolumn{4}{|l|}{ Net trade (mln US\$) } \\
\hline Cereals & & -41 & -96 \\
\hline Fruit and vegetables & & -90 & -201 \\
\hline Meat & & -72 & -177 \\
\hline Dairy products & & 8 & -12 \\
\hline Fish & & -43 & -94 \\
\hline \multicolumn{4}{|l|}{ Environment } \\
\hline Forest area (\%) & & 34 & 33 \\
\hline Renewable water res withdrawn (\% of total) & & 0 & 0 \\
\hline Terrestrial protect areas (\% total land area) & 15 & 33 & 40 \\
\hline Organic area (\% total agricultural area) & & & 3 \\
\hline Water withdrawal by agriculture (\% of total) & & 0 & 0 \\
\hline Biofuel production (thousand kt of oil eq.) & 0 & 0 & 12 \\
\hline Wood pellet prod. (1 000 tonnes) & & & 45 \\
\hline Net GHG emissions from AFOLU ( $\mathrm{CO}_{2}$ eq, $\left.\mathrm{Mt}\right)$ & & 0 & 1 \\
\hline
\end{tabular}




\begin{tabular}{|c|c|c|c|}
\hline & 1990 & 2000 & 2014 \\
\hline \multicolumn{4}{|l|}{ The setting } \\
\hline Population, total (mln) & 2 & 2.1 & 2.1 \\
\hline Population, rural (mln) & 0.8 & 0.8 & 0.9 \\
\hline \multicolumn{4}{|l|}{ Govt expenditure on ag (\% total outlays) } \\
\hline Area harvested (mln ha) & & 1 & 1 \\
\hline Cropping intensity ratio & & 0.5 & \\
\hline Water resources (1 $000 \mathrm{~m}^{3} /$ person/year) & 3 & 3 & 3 \\
\hline Area equipped for irrigation (1 $000 \mathrm{ha}$ ) & & & 128 \\
\hline \multicolumn{4}{|l|}{ Area irrigated (\% area equipped for irrigation) } \\
\hline Employment in agriculture (\%) & & 22 & 17.3 \\
\hline Employment in agriculture, female (\%) & & 22.2 & 17.6 \\
\hline Fertilizers, Nitrogen (kg of nutrients per ha) & & 25.5 & 39.4 \\
\hline Fertilizers, Phosphate (kg of nutrients per ha) & & 0 & 9.5 \\
\hline Fertilizers, Potash (kg nutrients per ha) & & 7.3 & 8.3 \\
\hline \multicolumn{4}{|l|}{ Energy consump, power irrigation (mln kWh) } \\
\hline Agr value added per worker (constant US\$) & 3249 & 5271 & 11460 \\
\hline \multicolumn{4}{|l|}{ Hunger dimensions } \\
\hline \multicolumn{4}{|l|}{ Dietary energy supply (kcal/pc/day) } \\
\hline Average dietary energy supply adequacy (\%) & 100 & 110 & 118 \\
\hline Dietary en supp, cereals/roots/tubers (\%) & 48 & 40 & 37 \\
\hline Prevalence of undernourishment (\%) & $<5.0$ & $<5.0$ & $<5.0$ \\
\hline GDP per capita (US\$, PPP) & 9803 & 8757 & 11609 \\
\hline Domestic food price volatility (index) & & 10.2 & 7.9 \\
\hline Cereal import dependency ratio (\%) & 22.4 & 23.1 & 25.4 \\
\hline Underweight, children under-5 (\%) & & 1.9 & 1.3 \\
\hline Improved water source (\% pop) & 99.3 & 99.3 & 99.4 \\
\hline \multicolumn{4}{|l|}{ Food supply } \\
\hline Food production value, (2004-2006 mln I\$) & 506 & 638 & 764 \\
\hline Agriculture, value added (\% GDP) & 9 & 12 & 10 \\
\hline Food exports (mln US\$) & 113 & 64 & 367 \\
\hline Food imports (mln US\$) & 164 & 182 & 666 \\
\hline \multicolumn{4}{|l|}{ Production indices (2004-06=100) } \\
\hline Net food & 78 & 98 & 118 \\
\hline Net crops & 72 & 98 & 115 \\
\hline Cereals & 73 & 88 & 89 \\
\hline Vegetable oils & 72 & 118 & 101 \\
\hline Roots and tubers & 50 & 84 & 100 \\
\hline Fruit and vegetables & 70 & 101 & 123 \\
\hline Sugar & 122 & 125 & 21 \\
\hline Livestock & 98 & 94 & 116 \\
\hline Milk & 68 & 92 & 149 \\
\hline Meat & 135 & 86 & 82 \\
\hline Fish & 0 & 142 & 166 \\
\hline \multicolumn{4}{|l|}{ Net trade (mln US\$) } \\
\hline Cereals & -15 & -37 & -38 \\
\hline Fruit and vegetables & 27 & 13 & 108 \\
\hline Meat & -30 & -39 & -132 \\
\hline Dairy products & -15 & -11 & -42 \\
\hline Fish & -6 & -7 & -23 \\
\hline \multicolumn{4}{|l|}{ Environment } \\
\hline Forest area (\%) & 36 & 38 & 40 \\
\hline Renewable water res withdrawn (\% of total) & & & 12 \\
\hline Terrestrial protect areas (\% total land area) & 5 & 6 & 7 \\
\hline Organic area (\% total agricultural area) & & & 0 \\
\hline Water withdrawal by agriculture (\% of total) & & & 12 \\
\hline Biofuel production (thousand kt of oil eq.) & & & 54 \\
\hline Wood pellet prod. (1 000 tonnes) & & & 0 \\
\hline Net GHG emissions from AFOLU ( $\mathrm{CO}_{2}$ eq, $\mathrm{Mt}$ ) & 1 & 1 & 1 \\
\hline
\end{tabular}




\begin{tabular}{|c|c|c|c|}
\hline & 1990 & 2000 & 2014 \\
\hline \multicolumn{4}{|l|}{ The setting } \\
\hline Population, total (mln) & 11.5 & 15.7 & 23.6 \\
\hline Population, rural (mln) & 8.8 & 11.5 & 15.4 \\
\hline \multicolumn{4}{|l|}{ Govt expenditure on ag (\% total outlays) } \\
\hline Area harvested (mln ha) & 3 & 3 & 4 \\
\hline Cropping intensity ratio & 0.1 & 0.1 & \\
\hline Water resources (1 $000 \mathrm{~m}^{3} /$ person/year) & 29 & 21 & 15 \\
\hline Area equipped for irrigation (1 $000 \mathrm{ha}$ ) & & & 1086 \\
\hline Area irrigated (\% area equipped for irrigation) & & 50.6 & \\
\hline Employment in agriculture (\%) & & 78 & 80.4 \\
\hline Employment in agriculture, female (\%) & & 79.3 & 81.1 \\
\hline Fertilizers, Nitrogen (kg of nutrients per ha) & & 1.4 & 0.7 \\
\hline Fertilizers, Phosphate (kg of nutrients per ha) & & 0.3 & 0.7 \\
\hline Fertilizers, Potash (kg nutrients per ha) & & 0.5 & 0.6 \\
\hline Energy consump, power irrigation (mln kWh) & 0 & 6 & 6 \\
\hline Agr value added per worker (constant US\$) & 245 & 225 & 175 \\
\hline \multicolumn{4}{|l|}{ Hunger dimensions } \\
\hline Dietary energy supply (kcal/pc/day) & 2075 & 2002 & 2059 \\
\hline Average dietary energy supply adequacy (\%) & 100 & 96 & 97 \\
\hline Dietary en supp, cereals/roots/tubers (\%) & 74 & 77 & 79 \\
\hline Prevalence of undernourishment (\%) & 29.7 & 34.8 & 32.8 \\
\hline GDP per capita (US\$, PPP) & 1661 & 1446 & 1369 \\
\hline Domestic food price volatility (index) & & 7.6 & 3.5 \\
\hline Cereal import dependency ratio (\%) & 5.9 & 12.2 & 8.7 \\
\hline Underweight, children under-5 (\%) & 40.9 & 35.5 & \\
\hline Improved water source (\% pop) & 28.6 & 38.1 & 49.6 \\
\hline \multicolumn{4}{|l|}{ Food supply } \\
\hline Food production value, (2004-2006 mln I\$) & 2232 & 2428 & 3312 \\
\hline Agriculture, value added (\% GDP) & 29 & 29 & 26 \\
\hline Food exports (mln US\$) & 125 & 95 & 274 \\
\hline Food imports (mln US\$) & 66 & 100 & 340 \\
\hline \multicolumn{4}{|l|}{ Production indices $(2004-06=100)$} \\
\hline Net food & 81 & 88 & 121 \\
\hline Net crops & 79 & 83 & 117 \\
\hline Cereals & 71 & 73 & 109 \\
\hline Vegetable oils & 94 & 101 & 95 \\
\hline Roots and tubers & 82 & 91 & 118 \\
\hline Fruit and vegetables & 82 & 89 & 122 \\
\hline Sugar & 82 & 89 & 132 \\
\hline Livestock & 99 & 102 & 125 \\
\hline Milk & 94 & 106 & 113 \\
\hline Meat & 103 & 101 & 130 \\
\hline Fish & 72 & 89 & 79 \\
\hline \multicolumn{4}{|l|}{ Net trade (mln US\$) } \\
\hline Cereals & -39 & -62 & -178 \\
\hline Fruit and vegetables & 13 & 19 & 42 \\
\hline Meat & 0 & 0 & -1 \\
\hline Dairy products & -7 & -6 & -13 \\
\hline Fish & 45 & 31 & 103 \\
\hline \multicolumn{4}{|l|}{ Environment } \\
\hline Forest area (\%) & 24 & 23 & 21 \\
\hline Renewable water res withdrawn (\% of total) & & 98 & 98 \\
\hline Terrestrial protect areas (\% total land area) & 2 & 3 & 5 \\
\hline Organic area (\% total agricultural area) & & & 0 \\
\hline Water withdrawal by agriculture (\% of total) & & 98 & 98 \\
\hline Biofuel production (thousand kt of oil eq.) & 3 & 2 & 2 \\
\hline \multicolumn{4}{|l|}{ Wood pellet prod. (1 000 tonnes) } \\
\hline Net GHG emissions from AFOLU ( $\mathrm{CO}_{2}$ eq, $\mathrm{Mt}$ ) & 59 & 56 & 53 \\
\hline
\end{tabular}




\begin{tabular}{|c|c|c|c|}
\hline & 1990 & 2000 & 2014 \\
\hline \multicolumn{4}{|l|}{ The setting } \\
\hline Population, total (mln) & 9.4 & 11.3 & 16.8 \\
\hline Population, rural (mln) & 8.4 & 9.7 & 14.1 \\
\hline \multicolumn{4}{|l|}{ Govt expenditure on ag (\% total outlays) } \\
\hline Area harvested (mln ha) & 2 & 5 & 9 \\
\hline Cropping intensity ratio & 0.4 & 1 & \\
\hline Water resources (1 $000 \mathrm{~m}^{3} /$ person/year) & 2 & 2 & 1 \\
\hline Area equipped for irrigation (1 $000 \mathrm{ha})$ & & & 74 \\
\hline Area irrigated (\% area equipped for irrigation) & 96 & & \\
\hline \multicolumn{4}{|l|}{ Employment in agriculture (\%) } \\
\hline \multicolumn{4}{|l|}{ Employment in agriculture, female (\%) } \\
\hline Fertilizers, Nitrogen (kg of nutrients per ha) & & 20.3 & 30.2 \\
\hline Fertilizers, Phosphate (kg of nutrients per ha) & & 6.9 & 6 \\
\hline Fertilizers, Potash (kg nutrients per ha) & & 3.9 & 3.6 \\
\hline Energy consump, power irrigation (mln kWh) & 1 & 30 & 117 \\
\hline Agr value added per worker (constant US\$) & 120 & 213 & 253 \\
\hline \multicolumn{4}{|l|}{ Hunger dimensions } \\
\hline Dietary energy supply (kcal/pc/day) & 1860 & 2207 & 2355 \\
\hline Average dietary energy supply adequacy (\%) & 89 & 105 & 111 \\
\hline Dietary en supp, cereals/roots/tubers (\%) & 73 & 75 & 71 \\
\hline Prevalence of undernourishment (\%) & 46.9 & 28.6 & 20.8 \\
\hline GDP per capita (US\$, PPP) & 540 & 632 & 755 \\
\hline Domestic food price volatility (index) & & 18.1 & 23.6 \\
\hline Cereal import dependency ratio (\%) & 28.2 & 3.6 & 1.6 \\
\hline Underweight, children under-5 (\%) & 24.4 & 21.5 & 16.7 \\
\hline Improved water source (\% pop) & 42.1 & 62.5 & 85 \\
\hline \multicolumn{4}{|l|}{ Food supply } \\
\hline Food production value, $(2004-2006 \mathrm{mln}$ I\$) & 739 & 1580 & 3105 \\
\hline Agriculture, value added (\% GDP) & 45 & 40 & 33 \\
\hline Food exports (mln US\$) & 35 & 29 & 207 \\
\hline Food imports (mln US\$) & 68 & 29 & 194 \\
\hline \multicolumn{4}{|l|}{ Production indices $(2004-06=100)$} \\
\hline Net food & 47 & 100 & 196 \\
\hline Net crops & 51 & 99 & 182 \\
\hline Cereals & 72 & 136 & 203 \\
\hline Vegetable oils & 23 & 67 & 245 \\
\hline Roots and tubers & 12 & 105 & 214 \\
\hline Fruit and vegetables & 57 & 80 & 121 \\
\hline Sugar & 77 & 91 & 125 \\
\hline Livestock & 68 & 93 & 253 \\
\hline Milk & 107 & 102 & 318 \\
\hline Meat & 64 & 92 & 266 \\
\hline Fish & 116 & 79 & 181 \\
\hline \multicolumn{4}{|l|}{ Net trade (mln US\$) } \\
\hline Cereals & -42 & -11 & -94 \\
\hline Fruit and vegetables & 2 & 1 & 44 \\
\hline Meat & 0 & 0 & -1 \\
\hline Dairy products & -5 & -3 & -8 \\
\hline Fish & -1 & 0 & -1 \\
\hline \multicolumn{4}{|l|}{ Environment } \\
\hline Forest area (\%) & 41 & 38 & 34 \\
\hline Renewable water res withdrawn (\% of total) & & & 86 \\
\hline Terrestrial protect areas (\% total land area) & 15 & 16 & 18 \\
\hline Organic area (\% total agricultural area) & & & 0 \\
\hline Water withdrawal by agriculture (\% of total) & & & 86 \\
\hline Biofuel production (thousand kt of oil eq.) & 5 & 5 & 7 \\
\hline \multicolumn{4}{|l|}{ Wood pellet prod. (1 000 tonnes) } \\
\hline Net GHG emissions from AFOLU ( $\mathrm{CO}_{2}$ eq, $\mathrm{Mt}$ ) & 9 & 8 & 11 \\
\hline
\end{tabular}




\begin{tabular}{|c|c|c|c|}
\hline & 1990 & 2000 & 2014 \\
\hline \multicolumn{4}{|l|}{ The setting } \\
\hline Population, total (mln) & 18.2 & 23.4 & 30.2 \\
\hline Population, rural (mln) & 9.1 & 8.9 & 7.6 \\
\hline Govt expenditure on ag (\% total outlays) & & 3 & 2.6 \\
\hline Area harvested (mln ha) & 31 & 57 & 100 \\
\hline Cropping intensity ratio & 4.5 & 8.1 & \\
\hline Water resources (1 $000 \mathrm{~m}^{3} /$ person/year) & 32 & 25 & 20 \\
\hline Area equipped for irrigation (1 $000 \mathrm{ha})$ & & & 380 \\
\hline Area irrigated (\% area equipped for irrigation) & & 100 & \\
\hline Employment in agriculture (\%) & 26 & 18.4 & 12.6 \\
\hline Employment in agriculture, female (\%) & 25.3 & 14 & 8.2 \\
\hline Fertilizers, Nitrogen (kg of nutrients per ha) & & 470.8 & 578.1 \\
\hline Fertilizers, Phosphate (kg of nutrients per ha) & & 26.1 & 231.7 \\
\hline Fertilizers, Potash (kg nutrients per ha) & & 807.2 & 760.8 \\
\hline Energy consump, power irrigation (mln kWh) & 0 & 0 & 17 \\
\hline Agr value added per worker (constant US\$) & 4812 & 5485 & 10127 \\
\hline \multicolumn{4}{|l|}{ Hunger dimensions } \\
\hline Dietary energy supply (kcal/pc/day) & 2801 & 2858 & 3018 \\
\hline Average dietary energy supply adequacy (\%) & 125 & 125 & 128 \\
\hline Dietary en supp, cereals/roots/tubers (\%) & 44 & 45 & 45 \\
\hline Prevalence of undernourishment (\%) & $<5.0$ & $<5.0$ & $<5.0$ \\
\hline GDP per capita (US\$, PPP) & 10159 & 15695 & 22589 \\
\hline Domestic food price volatility (index) & & 3.8 & 4.3 \\
\hline Cereal import dependency ratio (\%) & 70.2 & 75.5 & 76 \\
\hline Underweight, children under-5 (\%) & 22.1 & 16.7 & 12.9 \\
\hline Improved water source (\% pop) & 88.2 & 96.4 & 99.6 \\
\hline \multicolumn{4}{|l|}{ Food supply } \\
\hline Food production value, (2004-2006 mln I\$) & 5983 & 8710 & 14311 \\
\hline Agriculture, value added (\% GDP) & 15 & 9 & 9 \\
\hline Food exports (mln US\$) & 2893 & 4189 & 21754 \\
\hline Food imports (mln US\$) & 1630 & 2730 & 12373 \\
\hline \multicolumn{4}{|l|}{ Production indices $(2004-06=100)$} \\
\hline Net food & 54 & 78 & 128 \\
\hline Net crops & 60 & 78 & 118 \\
\hline Cereals & 83 & 95 & 117 \\
\hline Vegetable oils & 41 & 73 & 128 \\
\hline Roots and tubers & 409 & 150 & 118 \\
\hline Fruit and vegetables & 84 & 94 & 113 \\
\hline Sugar & 269 & 228 & 44 \\
\hline Livestock & 59 & 78 & 139 \\
\hline Milk & 79 & 75 & 174 \\
\hline Meat & 57 & 76 & 136 \\
\hline Fish & 69 & 99 & 121 \\
\hline \multicolumn{4}{|l|}{ Net trade (mln US\$) } \\
\hline Cereals & -469 & -611 & -1571 \\
\hline Fruit and vegetables & -77 & -211 & -995 \\
\hline Meat & -76 & -187 & -614 \\
\hline Dairy products & -161 & -246 & -534 \\
\hline Fish & 83 & 52 & -223 \\
\hline \multicolumn{4}{|l|}{ Environment } \\
\hline Forest area $(\%)$ & 68 & 66 & 62 \\
\hline Renewable water res withdrawn (\% of total) & & & 22 \\
\hline Terrestrial protect areas (\% total land area) & 17 & 18 & 18 \\
\hline Organic area (\% total agricultural area) & & & 0 \\
\hline Water withdrawal by agriculture (\% of total) & & & 22 \\
\hline Biofuel production (thousand kt of oil eq.) & 3 & 3 & 230 \\
\hline Wood pellet prod. (1 000 tonnes) & & & 180 \\
\hline Net GHG emissions from AFOLU ( $\mathrm{CO}_{2}$ eq, $\mathrm{Mt}$ ) & -232 & -209 & 160 \\
\hline
\end{tabular}




\begin{tabular}{|c|c|c|c|}
\hline & 1990 & 2000 & 2014 \\
\hline \multicolumn{4}{|l|}{ The setting } \\
\hline Population, total (mln) & 0.2 & 0.3 & 0.4 \\
\hline Population, rural (mln) & 0.2 & 0.2 & 0.2 \\
\hline Govt expenditure on ag (\% total outlays) & & 0.7 & 1.3 \\
\hline Area harvested (mln ha) & 0 & 0 & 0 \\
\hline Cropping intensity ratio & 18.8 & 34.8 & \\
\hline Water resources (1 $000 \mathrm{~m}^{3} /$ person/year) & 0 & 0 & 0 \\
\hline \multicolumn{4}{|l|}{ Area equipped for irrigation (1 $000 \mathrm{ha})$} \\
\hline \multicolumn{4}{|l|}{ Area irrigated (\% area equipped for irrigation) } \\
\hline Employment in agriculture (\%) & 25.2 & 13.7 & 11.5 \\
\hline Employment in agriculture, female (\%) & 13.5 & 5.4 & 7.1 \\
\hline Fertilizers, Nitrogen (kg of nutrients per ha) & & 4 & 50 \\
\hline Fertilizers, Phosphate (kg of nutrients per ha) & & 2 & 43.7 \\
\hline Fertilizers, Potash (kg nutrients per ha) & & 2 & 44.3 \\
\hline \multicolumn{4}{|l|}{ Energy consump, power irrigation ( $\mathrm{mln} \mathrm{kWh}$ ) } \\
\hline Agr value added per worker (constant US\$) & & 3268 & 3304 \\
\hline \multicolumn{4}{|l|}{ Hunger dimensions } \\
\hline Dietary energy supply (kcal/pc/day) & 2433 & 2465 & 2879 \\
\hline Average dietary energy supply adequacy (\%) & 119 & 116 & 128 \\
\hline Dietary en supp, cereals/roots/tubers (\%) & 45 & 45 & 41 \\
\hline Prevalence of undernourishment (\%) & 11.1 & 11.8 & 5.9 \\
\hline GDP per capita (US\$, PPP) & & 7695 & 11283 \\
\hline Domestic food price volatility (index) & & 57.6 & 14.2 \\
\hline Cereal import dependency ratio (\%) & 100 & 100 & 100 \\
\hline Underweight, children under-5 (\%) & & 25.7 & 17.8 \\
\hline Improved water source (\% pop) & 93.2 & 95.2 & 98.6 \\
\hline \multicolumn{4}{|l|}{ Food supply } \\
\hline Food production value, $(2004-2006 \mathrm{mln} I \$)$ & 9 & 10 & 7 \\
\hline Agriculture, value added (\% GDP) & & 9 & 4 \\
\hline Food exports (mln US\$) & 0 & 0 & 0 \\
\hline Food imports (mln US\$) & 21 & 62 & 256 \\
\hline \multicolumn{4}{|l|}{ Production indices $(2004-06=100)$} \\
\hline Net food & 76 & 89 & 64 \\
\hline Net crops & 74 & 87 & 59 \\
\hline Cereals & 6 & 89 & 146 \\
\hline Vegetable oils & 68 & 84 & 3 \\
\hline Roots and tubers & 240 & 127 & 70 \\
\hline Fruit and vegetables & 62 & 77 & 36 \\
\hline \multicolumn{4}{|l|}{ Sugar } \\
\hline Livestock & 103 & 116 & 108 \\
\hline \multicolumn{4}{|l|}{ Milk } \\
\hline Meat & 103 & 116 & 108 \\
\hline \multicolumn{4}{|l|}{ Fish } \\
\hline \multicolumn{4}{|l|}{ Net trade (mln US\$) } \\
\hline Cereals & & -14 & -43 \\
\hline \multicolumn{4}{|l|}{ Fruit and vegetables } \\
\hline Meat & & -9 & -40 \\
\hline Dairy products & & -11 & -32 \\
\hline Fish & 38 & 41 & 135 \\
\hline \multicolumn{4}{|l|}{ Environment } \\
\hline Forest area (\%) & 3 & 3 & 3 \\
\hline Renewable water res withdrawn (\% of total) & & & 0 \\
\hline \multicolumn{4}{|l|}{ Terrestrial protect areas (\% total land area) } \\
\hline \multicolumn{4}{|l|}{ Organic area (\% total agricultural area) } \\
\hline Water withdrawal by agriculture (\% of total) & & & 0 \\
\hline \multicolumn{4}{|l|}{ Biofuel production (thousand kt of oil eq.) } \\
\hline \multicolumn{4}{|l|}{ Wood pellet prod. (1 000 tonnes) } \\
\hline Net GHG emissions from AFOLU ( $\mathrm{CO}_{2}$ eq, $\mathrm{Mt}$ ) & 0 & 0 & 0 \\
\hline
\end{tabular}




\begin{tabular}{|c|c|c|c|}
\hline & 1990 & 2000 & 2014 \\
\hline \multicolumn{4}{|l|}{ The setting } \\
\hline Population, total (mln) & 8 & 10.3 & 15.8 \\
\hline Population, rural (mln) & 6.1 & 7.4 & 10 \\
\hline \multicolumn{4}{|l|}{ Govt expenditure on ag (\% total outlays) } \\
\hline Area harvested (mln ha) & 2 & 2 & 6 \\
\hline Cropping intensity ratio & 0.1 & 0.1 & \\
\hline Water resources (1 $000 \mathrm{~m}^{3} /$ person/year) & 15 & 12 & 8 \\
\hline Area equipped for irrigation (1 $000 \mathrm{ha})$ & & & 378 \\
\hline Area irrigated (\% area equipped for irrigation) & & 74.6 & \\
\hline Employment in agriculture (\%) & & & 66 \\
\hline Employment in agriculture, female (\%) & & & 63.9 \\
\hline Fertilizers, Nitrogen (kg of nutrients per ha) & & 0 & 18.5 \\
\hline Fertilizers, Phosphate (kg of nutrients per ha) & & 0 & 4.6 \\
\hline Fertilizers, Potash (kg nutrients per ha) & & 0 & 2.9 \\
\hline Energy consump, power irrigation (mln kWh) & 0 & 0 & 0 \\
\hline Agr value added per worker (constant US\$) & 598 & 581 & 842 \\
\hline \multicolumn{4}{|l|}{ Hunger dimensions } \\
\hline Dietary energy supply (kcal/pc/day) & 2344 & 2469 & 2829 \\
\hline Average dietary energy supply adequacy (\%) & 112 & 118 & 136 \\
\hline Dietary en supp, cereals/roots/tubers (\%) & 69 & 68 & 68 \\
\hline Prevalence of undernourishment (\%) & 17.7 & 13.9 & $<5.0$ \\
\hline GDP per capita (US\$, PPP) & 1100 & 1269 & 1589 \\
\hline Domestic food price volatility (index) & & 10.2 & 9.4 \\
\hline Cereal import dependency ratio (\%) & 3 & 6.7 & 4.7 \\
\hline Underweight, children under-5 (\%) & & 30.1 & 27.9 \\
\hline Improved water source (\% pop) & 28.1 & 45.5 & 67.2 \\
\hline \multicolumn{4}{|l|}{ Food supply } \\
\hline Food production value, $(2004-2006 \mathrm{mln}$ I\$) & 1245 & 1579 & 3288 \\
\hline Agriculture, value added (\% GDP) & 46 & 42 & 42 \\
\hline Food exports (mln US\$) & 92 & 124 & 150 \\
\hline Food imports (mln US\$) & 88 & 82 & 382 \\
\hline \multicolumn{4}{|l|}{ Production indices $(2004-06=100)$} \\
\hline Net food & 57 & 72 & 151 \\
\hline Net crops & 54 & 69 & 139 \\
\hline Cereals & 50 & 72 & 183 \\
\hline Vegetable oils & 68 & 68 & 119 \\
\hline Roots and tubers & 21 & 47 & 143 \\
\hline Fruit and vegetables & 55 & 82 & 117 \\
\hline Sugar & 92 & 93 & 107 \\
\hline Livestock & 63 & 72 & 147 \\
\hline Milk & 42 & 48 & 137 \\
\hline Meat & 73 & 84 & 151 \\
\hline Fish & 70 & 109 & 101 \\
\hline \multicolumn{4}{|l|}{ Net trade (mln US\$) } \\
\hline Cereals & -14 & -24 & -191 \\
\hline Fruit and vegetables & -11 & -3 & -1 \\
\hline Meat & -2 & 0 & -1 \\
\hline Dairy products & -22 & -16 & -29 \\
\hline Fish & -3 & -1 & -11 \\
\hline \multicolumn{4}{|l|}{ Environment } \\
\hline Forest area (\%) & 12 & 11 & 10 \\
\hline Renewable water res withdrawn (\% of total) & & & 98 \\
\hline Terrestrial protect areas (\% total land area) & 3 & 3 & 6 \\
\hline Organic area (\% total agricultural area) & & & 0 \\
\hline Water withdrawal by agriculture (\% of total) & & & 98 \\
\hline Biofuel production (thousand kt of oil eq.) & 1 & 1 & 1 \\
\hline \multicolumn{4}{|l|}{ Wood pellet prod. (1 000 tonnes) } \\
\hline Net GHG emissions from AFOLU ( $\mathrm{CO}_{2}$ eq, $\mathrm{Mt}$ ) & 23 & 26 & 34 \\
\hline
\end{tabular}




\begin{tabular}{|c|c|c|c|}
\hline & 1990 & 2000 & 2014 \\
\hline \multicolumn{4}{|l|}{ The setting } \\
\hline Population, total (mln) & 0.4 & 0.4 & 0.4 \\
\hline Population, rural (mln) & 0 & 0 & 0 \\
\hline \multicolumn{4}{|l|}{ Govt expenditure on ag (\% total outlays) } \\
\hline Area harvested (mln ha) & 0 & 0 & 0 \\
\hline Cropping intensity ratio & 26.7 & 46.1 & \\
\hline Water resources (1 $000 \mathrm{~m}^{3} /$ person/year) & 0 & 0 & 0 \\
\hline Area equipped for irrigation (1 $000 \mathrm{ha}$ ) & & & 4 \\
\hline Area irrigated (\% area equipped for irrigation) & & & 87.8 \\
\hline Employment in agriculture (\%) & & 1.7 & 1 \\
\hline Employment in agriculture, female (\%) & & 0.7 & 0.5 \\
\hline Fertilizers, Nitrogen (kg of nutrients per ha) & & 56 & 218.8 \\
\hline Fertilizers, Phosphate (kg of nutrients per ha) & & 18.1 & 0 \\
\hline Fertilizers, Potash (kg nutrients per ha) & & 20.1 & 0.3 \\
\hline Energy consump, power irrigation (mln kWh) & 1 & 1 & 1 \\
\hline Agr value added per worker (constant US\$) & 35909 & 39112 & 56327 \\
\hline \multicolumn{4}{|l|}{ Hunger dimensions } \\
\hline \multicolumn{4}{|l|}{ Dietary energy supply (kcal/pc/day) } \\
\hline Average dietary energy supply adequacy (\%) & 127 & 133 & 135 \\
\hline Dietary en supp, cereals/roots/tubers (\%) & 36 & 37 & 35 \\
\hline Prevalence of undernourishment (\%) & $<5.0$ & $<5.0$ & $<5.0$ \\
\hline GDP per capita (US\$, PPP) & 16596 & 25841 & 28822 \\
\hline Domestic food price volatility (index) & & 15 & 8.6 \\
\hline Cereal import dependency ratio (\%) & 95.4 & 94.6 & 89.3 \\
\hline \multicolumn{4}{|l|}{ Underweight, children under-5 (\%) } \\
\hline Improved water source (\% pop) & 99.8 & 100 & 100 \\
\hline \multicolumn{4}{|l|}{ Food supply } \\
\hline Food production value, (2004-2006 mln I\$) & 62 & 84 & 72 \\
\hline Agriculture, value added (\% GDP) & 3 & 2 & 2 \\
\hline Food exports (mln US\$) & 18 & 33 & 121 \\
\hline Food imports (mln US\$) & 146 & 203 & 470 \\
\hline \multicolumn{4}{|l|}{ Production indices $(2004-06=100)$} \\
\hline Net food & 79 & 107 & 92 \\
\hline Net crops & 78 & 102 & 99 \\
\hline Cereals & 64 & 90 & 134 \\
\hline Vegetable oils & 400 & 75 & 125 \\
\hline Roots and tubers & 81 & 170 & 61 \\
\hline Fruit and vegetables & 78 & 95 & 100 \\
\hline \multicolumn{4}{|l|}{ Sugar } \\
\hline Livestock & 81 & 110 & 86 \\
\hline Milk & 60 & 117 & 99 \\
\hline Meat & 87 & 112 & 82 \\
\hline Fish & 32 & 114 & 255 \\
\hline \multicolumn{4}{|l|}{ Net trade (mln US\$) } \\
\hline Cereals & -32 & -51 & -78 \\
\hline Fruit and vegetables & -20 & -33 & -78 \\
\hline Meat & -25 & -31 & -96 \\
\hline Dairy products & -23 & -25 & -53 \\
\hline Fish & -12 & -9 & 15 \\
\hline \multicolumn{4}{|l|}{ Environment } \\
\hline Forest area (\%) & 1 & 1 & 1 \\
\hline Renewable water res withdrawn (\% of total) & & 35 & 35 \\
\hline Terrestrial protect areas (\% total land area) & 0 & 15 & 22 \\
\hline Organic area (\% total agricultural area) & & & 0 \\
\hline Water withdrawal by agriculture (\% of total) & & 35 & 35 \\
\hline \multicolumn{4}{|l|}{ Biofuel production (thousand kt of oil eq.) } \\
\hline Wood pellet prod. (1 000 tonnes) & & & 0 \\
\hline Net GHG emissions from AFOLU ( $\mathrm{CO}_{2}$ eq, $\mathrm{Mt}$ ) & 0 & 0 & 0 \\
\hline
\end{tabular}




\begin{tabular}{|c|c|c|c|}
\hline & 1990 & 2000 & 2014 \\
\hline \multicolumn{4}{|l|}{ The setting } \\
\hline Population, total (mln) & 2 & 2.7 & 4 \\
\hline Population, rural (mln) & 1.2 & 1.6 & 2.3 \\
\hline \multicolumn{4}{|l|}{ Govt expenditure on ag (\% total outlays) } \\
\hline Area harvested (mln ha) & 0 & 0 & 0 \\
\hline Cropping intensity ratio & 0 & 0 & \\
\hline Water resources (1 $000 \mathrm{~m}^{3} /$ person/year) & 6 & 4 & 3 \\
\hline Area equipped for irrigation (1 $000 \mathrm{ha})$ & & & 45 \\
\hline \multicolumn{4}{|l|}{ Area irrigated (\% area equipped for irrigation) } \\
\hline \multicolumn{4}{|l|}{ Employment in agriculture (\%) } \\
\hline \multicolumn{4}{|l|}{ Employment in agriculture, female (\%) } \\
\hline \multicolumn{4}{|l|}{ Fertilizers, Nitrogen (kg of nutrients per ha) } \\
\hline \multicolumn{4}{|l|}{ Fertilizers, Phosphate (kg of nutrients per ha) } \\
\hline \multicolumn{4}{|l|}{ Fertilizers, Potash (kg nutrients per ha) } \\
\hline \multicolumn{4}{|l|}{ Energy consump, power irrigation (mln kWh) } \\
\hline Agr value added per worker (constant US\$) & 1122 & 1010 & 1102 \\
\hline \multicolumn{4}{|l|}{ Hunger dimensions } \\
\hline Dietary energy supply (kcal/pc/day) & 2532 & 2626 & 2945 \\
\hline Average dietary energy supply adequacy (\%) & 117 & 120 & 133 \\
\hline Dietary en supp, cereals/roots/tubers (\%) & 57 & 51 & 51 \\
\hline Prevalence of undernourishment (\%) & 14.1 & 11.5 & 5.8 \\
\hline GDP per capita (US\$, PPP) & 2459 & 2436 & 2945 \\
\hline Domestic food price volatility (index) & & 6.4 & 3.1 \\
\hline Cereal import dependency ratio (\%) & 62.3 & 68.2 & 74 \\
\hline Underweight, children under-5 (\%) & 43.3 & 30.4 & 19.5 \\
\hline Improved water source (\% pop) & 30.3 & 40.4 & 49.6 \\
\hline \multicolumn{4}{|l|}{ Food supply } \\
\hline Food production value, $(2004-2006 \mathrm{mln}$ I\$) & 335 & 387 & 521 \\
\hline Agriculture, value added (\% GDP) & 30 & 36 & 23 \\
\hline Food exports (mln US\$) & 43 & 13 & 19 \\
\hline Food imports (mln US\$) & 105 & 135 & 409 \\
\hline \multicolumn{4}{|l|}{ Production indices (2004-06=100) } \\
\hline Net food & 77 & 89 & 120 \\
\hline Net crops & 65 & 107 & 166 \\
\hline Cereals & 68 & 114 & 215 \\
\hline Vegetable oils & 148 & 163 & 103 \\
\hline Roots and tubers & 80 & 90 & 103 \\
\hline Fruit and vegetables & 53 & 100 & 89 \\
\hline \multicolumn{4}{|l|}{ Sugar } \\
\hline Livestock & 79 & 86 & 112 \\
\hline Milk & 73 & 89 & 110 \\
\hline Meat & 82 & 85 & 114 \\
\hline \multicolumn{4}{|l|}{ Fish } \\
\hline \multicolumn{4}{|l|}{ Net trade (mln US\$) } \\
\hline Cereals & -40 & -53 & -219 \\
\hline Fruit and vegetables & -6 & -8 & -29 \\
\hline Meat & 0 & -3 & -12 \\
\hline Dairy products & -20 & -11 & -66 \\
\hline Fish & 120 & 87 & 340 \\
\hline \multicolumn{4}{|l|}{ Environment } \\
\hline Forest area (\%) & 0 & 0 & 0 \\
\hline Renewable water res withdrawn (\% of total) & & & 91 \\
\hline Terrestrial protect areas (\% total land area) & 1 & 1 & 1 \\
\hline \multicolumn{4}{|l|}{ Organic area (\% total agricultural area) } \\
\hline Water withdrawal by agriculture (\% of total) & & & 91 \\
\hline \multicolumn{4}{|l|}{ Biofuel production (thousand kt of oil eq.) } \\
\hline Wood pellet prod. (1 000 tonnes) & & & \\
\hline Net GHG emissions from AFOLU ( $\mathrm{CO}_{2}$ eq, $\mathrm{Mt}$ ) & 6 & 8 & 8 \\
\hline
\end{tabular}




\begin{tabular}{|c|c|c|c|}
\hline & 1990 & 2000 & 2014 \\
\hline \multicolumn{4}{|l|}{ The setting } \\
\hline Population, total (mln) & 1.1 & 1.2 & 1.2 \\
\hline Population, rural (mln) & 0.6 & 0.7 & 0.7 \\
\hline \multicolumn{4}{|l|}{ Govt expenditure on ag (\% total outlays) } \\
\hline Area harvested (mln ha) & 6 & 5 & 4 \\
\hline Cropping intensity ratio & 50 & 50.6 & \\
\hline Water resources (1 $000 \mathrm{~m}^{3} /$ person/year) & 3 & 2 & 2 \\
\hline Area equipped for irrigation (1 $000 \mathrm{ha}$ ) & & & 19 \\
\hline Area irrigated (\% area equipped for irrigation) & & 98 & \\
\hline Employment in agriculture (\%) & 16.7 & 12.1 & 7.8 \\
\hline Employment in agriculture, female (\%) & 14.3 & 10.6 & 6.5 \\
\hline Fertilizers, Nitrogen (kg of nutrients per ha) & & 122.1 & 120 \\
\hline Fertilizers, Phosphate (kg of nutrients per ha) & & 46.5 & 29.7 \\
\hline Fertilizers, Potash (kg nutrients per ha) & & 130.9 & 74.5 \\
\hline Energy consump, power irrigation (mln kWh) & 0 & 39 & 45 \\
\hline Agr value added per worker (constant US\$) & 4407 & 5667 & 9121 \\
\hline \multicolumn{4}{|l|}{ Hunger dimensions } \\
\hline Dietary energy supply (kcal/pc/day) & 2813 & 2907 & 3121 \\
\hline Average dietary energy supply adequacy (\%) & 120 & 123 & 128 \\
\hline Dietary en supp, cereals/roots/tubers (\%) & 47 & 47 & 49 \\
\hline Prevalence of undernourishment (\%) & 7.9 & 7.1 & $<5.0$ \\
\hline GDP per capita (US\$, PPP) & 7570 & 11353 & 17146 \\
\hline Domestic food price volatility (index) & & 7.4 & 11.7 \\
\hline Cereal import dependency ratio (\%) & 99.3 & 99.9 & 100 \\
\hline Underweight, children under-5 (\%) & & 13 & \\
\hline Improved water source (\% pop) & 99.2 & 99.2 & 99.8 \\
\hline \multicolumn{4}{|l|}{ Food supply } \\
\hline Food production value, (2004-2006 mln I\$) & 230 & 243 & 241 \\
\hline Agriculture, value added (\% GDP) & 13 & 7 & 3 \\
\hline Food exports (mln US\$) & 365 & 233 & 320 \\
\hline Food imports (mln US\$) & 162 & 229 & 698 \\
\hline \multicolumn{4}{|l|}{ Production indices (2004-06=100) } \\
\hline Net food & 90 & 95 & 94 \\
\hline Net crops & 107 & 102 & 82 \\
\hline Cereals & 537 & 146 & 430 \\
\hline Vegetable oils & 354 & 101 & 104 \\
\hline Roots and tubers & 148 & 114 & 144 \\
\hline Fruit and vegetables & 57 & 105 & 110 \\
\hline Sugar & 111 & 102 & 76 \\
\hline Livestock & 46 & 73 & 130 \\
\hline Milk & 275 & 118 & 125 \\
\hline Meat & 43 & 67 & 139 \\
\hline Fish & 143 & 98 & 79 \\
\hline \multicolumn{4}{|l|}{ Net trade (mln US\$) } \\
\hline Cereals & -53 & -60 & -174 \\
\hline Fruit and vegetables & -17 & -34 & -82 \\
\hline Meat & -21 & -25 & -72 \\
\hline Dairy products & -34 & -40 & -116 \\
\hline Fish & -7 & -5 & 129 \\
\hline \multicolumn{4}{|l|}{ Environment } \\
\hline Forest area (\%) & 19 & 19 & 17 \\
\hline Renewable water res withdrawn (\% of total) & & 68 & 68 \\
\hline Terrestrial protect areas (\% total land area) & 2 & 4 & 4 \\
\hline Organic area (\% total agricultural area) & & & 0 \\
\hline Water withdrawal by agriculture (\% of total) & & 68 & 68 \\
\hline Biofuel production (thousand kt of oil eq.) & 12 & 12 & 11 \\
\hline Wood pellet prod. (1 000 tonnes) & & & 0 \\
\hline Net GHG emissions from AFOLU ( $\mathrm{CO}_{2}$ eq, $\mathrm{Mt}$ ) & 0 & 0 & 0 \\
\hline
\end{tabular}




\begin{tabular}{|c|c|c|c|}
\hline & 1990 & 2000 & 2014 \\
\hline \multicolumn{4}{|l|}{ The setting } \\
\hline Population, total (mln) & 86.1 & 103.9 & 123.8 \\
\hline Population, rural (mln) & 24.6 & 26.3 & 26 \\
\hline Govt expenditure on ag (\% total outlays) & & 3.4 & 3 \\
\hline Area harvested (mln ha) & 40 & 44 & 61 \\
\hline Cropping intensity ratio & 0.4 & 0.4 & \\
\hline Water resources (1 $000 \mathrm{~m}^{3} /$ person/year) & 5 & 4 & 4 \\
\hline Area equipped for irrigation (1 $000 \mathrm{ha}$ ) & & & 6500 \\
\hline Area irrigated (\% area equipped for irrigation) & & & 86.1 \\
\hline Employment in agriculture (\%) & 22.6 & 18 & 13.4 \\
\hline Employment in agriculture, female (\%) & 3.4 & 7.2 & 3.6 \\
\hline Fertilizers, Nitrogen (kg of nutrients per ha) & & 38.6 & 58.6 \\
\hline Fertilizers, Phosphate (kg of nutrients per ha) & & 20.5 & 4.6 \\
\hline Fertilizers, Potash (kg nutrients per ha) & & 7.7 & 8.8 \\
\hline Energy consump, power irrigation (mln kWh) & 28 & 985 & 985 \\
\hline Agr value added per worker (constant US\$) & 2712 & 3111 & 4416 \\
\hline \multicolumn{4}{|l|}{ Hunger dimensions } \\
\hline Dietary energy supply (kcal/pc/day) & 3004 & 3035 & 3081 \\
\hline Average dietary energy supply adequacy (\%) & 132 & 132 & 130 \\
\hline Dietary en supp, cereals/roots/tubers (\%) & 47 & 47 & 44 \\
\hline Prevalence of undernourishment (\%) & 6.7 & $<5.0$ & $<5.0$ \\
\hline GDP per capita (US\$, PPP) & 12479 & 14704 & 16291 \\
\hline Domestic food price volatility (index) & & 4.4 & 4.7 \\
\hline Cereal import dependency ratio (\%) & 23.2 & 33.3 & 30.7 \\
\hline Underweight, children under-5 (\%) & & 6 & 2.8 \\
\hline Improved water source (\% pop) & 82.3 & 88.6 & 94.9 \\
\hline \multicolumn{4}{|l|}{ Food supply } \\
\hline Food production value, $(2004-2006 \mathrm{mln}$ I\$) & 20133 & 27000 & 35142 \\
\hline Agriculture, value added (\% GDP) & 8 & 4 & 3 \\
\hline Food exports (mln US\$) & 2075 & 4980 & 16230 \\
\hline Food imports (mln US\$) & 4166 & 7599 & 21503 \\
\hline \multicolumn{4}{|l|}{ Production indices $(2004-06=100)$} \\
\hline Net food & 66 & 88 & 115 \\
\hline Net crops & 74 & 89 & 117 \\
\hline Cereals & 83 & 90 & 107 \\
\hline Vegetable oils & 121 & 92 & 129 \\
\hline Roots and tubers & 77 & 100 & 106 \\
\hline Fruit and vegetables & 60 & 87 & 122 \\
\hline Sugar & 79 & 88 & 122 \\
\hline Livestock & 60 & 87 & 113 \\
\hline Milk & 62 & 93 & 110 \\
\hline Meat & 60 & 85 & 113 \\
\hline Fish & 96 & 95 & 124 \\
\hline \multicolumn{4}{|l|}{ Net trade (mln US\$) } \\
\hline Cereals & -1064 & -1570 & -4463 \\
\hline Fruit and vegetables & 1051 & 2357 & 6934 \\
\hline Meat & -373 & -1423 & -2427 \\
\hline Dairy products & -714 & -526 & -1434 \\
\hline Fish & 297 & 564 & 416 \\
\hline \multicolumn{4}{|l|}{ Environment } \\
\hline Forest area (\%) & 36 & 34 & 33 \\
\hline Renewable water res withdrawn (\% of total) & & & 77 \\
\hline Terrestrial protect areas (\% total land area) & 2 & 8 & 13 \\
\hline Organic area (\% total agricultural area) & & & 0 \\
\hline Water withdrawal by agriculture (\% of total) & & & 77 \\
\hline Biofuel production (thousand kt of oil eq.) & 161 & 204 & 219 \\
\hline Wood pellet prod. (1 000 tonnes) & & & 4 \\
\hline Net GHG emissions from AFOLU ( $\mathrm{CO}_{2}$ eq, $\mathrm{Mt}$ ) & 123 & 113 & 108 \\
\hline
\end{tabular}




\begin{tabular}{|c|c|c|c|}
\hline & 1990 & 2000 & 2014 \\
\hline \multicolumn{4}{|l|}{ The setting } \\
\hline Population, total (mln) & 2.2 & 2.4 & 2.9 \\
\hline Population, rural (mln) & 0.9 & 1 & 0.8 \\
\hline Govt expenditure on ag (\% total outlays) & & 2.1 & 5.9 \\
\hline Area harvested (mln ha) & 1 & 0 & 0 \\
\hline Cropping intensity ratio & 0 & 0 & \\
\hline Water resources (1 $000 \mathrm{~m}^{3} /$ person/year) & 16 & 15 & 12 \\
\hline Area equipped for irrigation (1 $000 \mathrm{ha}$ ) & & & 84 \\
\hline Area irrigated (\% area equipped for irrigation) & 74.6 & 74.6 & \\
\hline Employment in agriculture (\%) & 39.5 & 48.6 & 32.6 \\
\hline Employment in agriculture, female (\%) & 36.9 & 46.5 & 32.2 \\
\hline Fertilizers, Nitrogen (kg of nutrients per ha) & & 5.7 & 25.1 \\
\hline Fertilizers, Phosphate (kg of nutrients per ha) & & 0 & 0 \\
\hline Fertilizers, Potash (kg nutrients per ha) & & 0 & 0 \\
\hline Energy consump, power irrigation (mln kWh) & 94 & 94 & 94 \\
\hline Agr value added per worker (constant US\$) & 2426 & 2161 & 4301 \\
\hline \multicolumn{4}{|l|}{ Hunger dimensions } \\
\hline Dietary energy supply (kcal/pc/day) & 1852 & 2177 & 2539 \\
\hline Average dietary energy supply adequacy (\%) & 83 & 94 & 108 \\
\hline Dietary en supp, cereals/roots/tubers (\%) & 44 & 46 & 47 \\
\hline Prevalence of undernourishment (\%) & 46.1 & 38.2 & 21.5 \\
\hline GDP per capita (US\$, PPP) & 4303 & 3910 & 9132 \\
\hline Domestic food price volatility (index) & & 47.6 & 16.7 \\
\hline Cereal import dependency ratio (\%) & 15.8 & 55.3 & 35.1 \\
\hline Underweight, children under-5 (\%) & 10.8 & 11.6 & 1.6 \\
\hline Improved water source (\% pop) & 62.3 & 68.4 & 84.6 \\
\hline \multicolumn{4}{|l|}{ Food supply } \\
\hline Food production value, (2004-2006 mln I\$) & 829 & 891 & 891 \\
\hline Agriculture, value added (\% GDP) & 13 & 31 & 16 \\
\hline Food exports (mln US\$) & 91 & 20 & 42 \\
\hline Food imports (mln US\$) & 56 & 76 & 388 \\
\hline \multicolumn{4}{|l|}{ Production indices $(2004-06=100)$} \\
\hline Net food & 135 & 145 & 145 \\
\hline Net crops & 226 & 73 & 247 \\
\hline Cereals & 613 & 127 & 389 \\
\hline Vegetable oils & & & 109 \\
\hline Roots and tubers & 125 & 60 & 223 \\
\hline Fruit and vegetables & 65 & 66 & 177 \\
\hline \multicolumn{4}{|l|}{ Sugar } \\
\hline Livestock & 133 & 150 & 136 \\
\hline Milk & 77 & 113 & 134 \\
\hline Meat & 147 & 161 & 139 \\
\hline \multicolumn{4}{|l|}{ Fish } \\
\hline \multicolumn{4}{|l|}{ Net trade (mln US\$) } \\
\hline Cereals & -10 & -44 & -87 \\
\hline Fruit and vegetables & & -8 & -51 \\
\hline Meat & 40 & 16 & -3 \\
\hline Dairy products & -6 & -4 & -9 \\
\hline Fish & -2 & 0 & -4 \\
\hline \multicolumn{4}{|l|}{ Environment } \\
\hline Forest area (\%) & 8 & 8 & 7 \\
\hline Renewable water res withdrawn (\% of total) & & & 44 \\
\hline Terrestrial protect areas (\% total land area) & 4 & 13 & 14 \\
\hline \multicolumn{4}{|l|}{ Organic area (\% total agricultural area) } \\
\hline Water withdrawal by agriculture (\% of total) & & & 44 \\
\hline \multicolumn{4}{|l|}{ Biofuel production (thousand kt of oil eq.) } \\
\hline \multicolumn{4}{|l|}{ Wood pellet prod. (1 000 tonnes) } \\
\hline Net GHG emissions from AFOLU ( $\mathrm{CO}_{2}$ eq, $\left.\mathrm{Mt}\right)$ & 42 & 46 & 44 \\
\hline
\end{tabular}




\begin{tabular}{|c|c|c|c|}
\hline & 1990 & 2000 & 2014 \\
\hline \multicolumn{4}{|l|}{ The setting } \\
\hline Population, total (mln) & & & 0.6 \\
\hline Population, rural (mln) & & & 0.2 \\
\hline \multicolumn{4}{|l|}{ Govt expenditure on ag (\% total outlays) } \\
\hline Area harvested (mln ha) & & & 0 \\
\hline \multicolumn{4}{|l|}{ Cropping intensity ratio } \\
\hline \multicolumn{4}{|l|}{ Water resources (1 $000 \mathrm{~m}^{3} /$ person/year) } \\
\hline Area equipped for irrigation (1 000 ha) & & & 2 \\
\hline \multicolumn{4}{|l|}{ Area irrigated (\% area equipped for irrigation) } \\
\hline Employment in agriculture (\%) & & 9 & 5.7 \\
\hline Employment in agriculture, female (\%) & & 6.9 & 4.8 \\
\hline Fertilizers, Nitrogen (kg of nutrients per ha) & & & 7.3 \\
\hline Fertilizers, Phosphate (kg of nutrients per ha) & & & 2.8 \\
\hline Fertilizers, Potash (kg nutrients per ha) & & & 2.3 \\
\hline Energy consump, power irrigation (mln kWh) & 0 & & 5 \\
\hline Agr value added per worker (constant US\$) & & & 5620 \\
\hline \multicolumn{4}{|l|}{ Hunger dimensions } \\
\hline \multicolumn{4}{|l|}{ Dietary energy supply (kcal/pc/day) } \\
\hline Average dietary energy supply adequacy (\%) & & & 147 \\
\hline Dietary en supp, cereals/roots/tubers (\%) & & & 35 \\
\hline Prevalence of undernourishment (\%) & $<5.0$ & $<5.0$ & $<5.0$ \\
\hline GDP per capita (US\$, PPP) & & 9703 & 14152 \\
\hline Domestic food price volatility (index) & & & 9.1 \\
\hline Cereal import dependency ratio (\%) & & & 89.1 \\
\hline Underweight, children under-5 (\%) & & & 1 \\
\hline Improved water source (\% pop) & 97.4 & 97.8 & 98 \\
\hline \multicolumn{4}{|l|}{ Food supply } \\
\hline Food production value, (2004-2006 mln I\$) & & & 167 \\
\hline Agriculture, value added (\% GDP) & & 12 & 10 \\
\hline Food exports (mln US\$) & & & 38 \\
\hline Food imports (mln US\$) & & & 422 \\
\hline \multicolumn{4}{|l|}{ Production indices $(2004-06=100)$} \\
\hline Net food & & & 95 \\
\hline Net crops & & & 102 \\
\hline Cereals & & & 96 \\
\hline Vegetable oils & & & 154 \\
\hline Roots and tubers & & & 85 \\
\hline Fruit and vegetables & & & 106 \\
\hline \multicolumn{4}{|l|}{ Sugar } \\
\hline Livestock & & & 88 \\
\hline Milk & & & 100 \\
\hline Meat & & & 40 \\
\hline Fish & 0 & 0 & 382 \\
\hline \multicolumn{4}{|l|}{ Net trade (mln US\$) } \\
\hline Cereals & & & -70 \\
\hline Fruit and vegetables & & & -39 \\
\hline Meat & & & -100 \\
\hline Dairy products & & & -52 \\
\hline Fish & & & -14 \\
\hline \multicolumn{4}{|l|}{ Environment } \\
\hline Forest area (\%) & & & 40 \\
\hline Renewable water res withdrawn (\% of total) & & & 1 \\
\hline Terrestrial protect areas (\% total land area) & 14 & 14 & 15 \\
\hline Organic area (\% total agricultural area) & & & 1 \\
\hline Water withdrawal by agriculture (\% of total) & & & 1 \\
\hline \multicolumn{4}{|l|}{ Biofuel production (thousand kt of oil eq.) } \\
\hline Wood pellet prod. (1 000 tonnes) & & & 3 \\
\hline Net GHG emissions from AFOLU $\left(\mathrm{CO}_{2} \mathrm{eq}, \mathrm{Mt}\right)$ & & & 0 \\
\hline
\end{tabular}




\begin{tabular}{|c|c|c|c|}
\hline & 1990 & 2000 & 2014 \\
\hline \multicolumn{4}{|l|}{ The setting } \\
\hline Population, total (mln) & 24.7 & 28.7 & 33.5 \\
\hline Population, rural (mln) & 12.7 & 13.4 & 14 \\
\hline \multicolumn{4}{|l|}{ Govt expenditure on ag (\% total outlays) } \\
\hline Area harvested (mln ha) & 6 & 5 & 10 \\
\hline Cropping intensity ratio & 0.2 & 0.2 & \\
\hline Water resources (1 $000 \mathrm{~m}^{3} /$ person/year) & 1 & 1 & 1 \\
\hline Area equipped for irrigation (1 $000 \mathrm{ha})$ & & & 1458 \\
\hline \multicolumn{4}{|l|}{ Area irrigated (\% area equipped for irrigation) } \\
\hline Employment in agriculture (\%) & 3.9 & 5.1 & 39.2 \\
\hline Employment in agriculture, female (\%) & 3.1 & 5.2 & 59.2 \\
\hline Fertilizers, Nitrogen (kg of nutrients per ha) & & 22.9 & 21.1 \\
\hline Fertilizers, Phosphate (kg of nutrients per ha) & & 24.2 & 3.2 \\
\hline Fertilizers, Potash (kg nutrients per ha) & & 8.2 & 3.9 \\
\hline Energy consump, power irrigation (mln kWh) & 258 & 258 & 998 \\
\hline Agr value added per worker (constant US\$) & 2019 & 1565 & 4507 \\
\hline \multicolumn{4}{|l|}{ Hunger dimensions } \\
\hline Dietary energy supply (kcal/pc/day) & 2927 & 3086 & 3343 \\
\hline Average dietary energy supply adequacy (\%) & 131 & 134 & 142 \\
\hline Dietary en supp, cereals/roots/tubers (\%) & 63 & 64 & 61 \\
\hline Prevalence of undernourishment (\%) & 7.5 & 6.5 & $<5.0$ \\
\hline GDP per capita (US\$, PPP) & 3901 & 4430 & 6967 \\
\hline Domestic food price volatility (index) & & 11.1 & 4.9 \\
\hline Cereal import dependency ratio (\%) & 35.4 & 58.2 & 36.4 \\
\hline Underweight, children under-5 (\%) & 8.1 & 9.9 & 3.1 \\
\hline Improved water source (\% pop) & 72.8 & 78.1 & 83.6 \\
\hline \multicolumn{4}{|l|}{ Food supply } \\
\hline Food production value, $(2004-2006 \mathrm{mln}$ I\$) & 4748 & 4954 & 9139 \\
\hline Agriculture, value added (\% GDP) & 19 & 15 & 16 \\
\hline Food exports (mln US\$) & 572 & 601 & 1870 \\
\hline Food imports (mln US\$) & 514 & 1284 & 4322 \\
\hline \multicolumn{4}{|l|}{ Production indices $(2004-06=100)$} \\
\hline Net food & 69 & 72 & 134 \\
\hline Net crops & 69 & 65 & 129 \\
\hline Cereals & 82 & 23 & 136 \\
\hline Vegetable oils & 76 & 65 & 179 \\
\hline Roots and tubers & 56 & 70 & 131 \\
\hline Fruit and vegetables & 57 & 75 & 119 \\
\hline Sugar & 102 & 108 & 71 \\
\hline Livestock & 71 & 88 & 147 \\
\hline Milk & 64 & 83 & 160 \\
\hline Meat & 72 & 88 & 150 \\
\hline Fish & 60 & 96 & 133 \\
\hline \multicolumn{4}{|l|}{ Net trade (mln US\$) } \\
\hline Cereals & -219 & -727 & -2228 \\
\hline Fruit and vegetables & 476 & 463 & 1245 \\
\hline Meat & -8 & -3 & -27 \\
\hline Dairy products & -67 & -44 & -148 \\
\hline Fish & 520 & 941 & 1582 \\
\hline \multicolumn{4}{|l|}{ Environment } \\
\hline Forest area (\%) & 11 & 11 & 12 \\
\hline Renewable water res withdrawn (\% of total) & & & 88 \\
\hline Terrestrial protect areas (\% total land area) & 0 & 1 & 22 \\
\hline Organic area (\% total agricultural area) & & & 0 \\
\hline Water withdrawal by agriculture (\% of total) & & & 88 \\
\hline Biofuel production (thousand kt of oil eq.) & 3 & 4 & 2 \\
\hline \multicolumn{4}{|l|}{ Wood pellet prod. (1 000 tonnes) } \\
\hline Net GHG emissions from AFOLU ( $\mathrm{CO}_{2}$ eq, $\mathrm{Mt}$ ) & 4 & 3 & 14 \\
\hline
\end{tabular}




\begin{tabular}{|c|c|c|c|}
\hline & 1990 & 2000 & 2014 \\
\hline \multicolumn{4}{|l|}{ The setting } \\
\hline Population, total (mln) & 13.6 & 18.3 & 26.5 \\
\hline Population, rural (mln) & 10.7 & 13 & 18 \\
\hline \multicolumn{4}{|l|}{ Govt expenditure on ag (\% total outlays) } \\
\hline Area harvested (mln ha) & 5 & 6 & 11 \\
\hline Cropping intensity ratio & 0.1 & 0.1 & \\
\hline Water resources (1 $000 \mathrm{~m}^{3} /$ person/year) & 16 & 12 & 8 \\
\hline Area equipped for irrigation (1 $000 \mathrm{ha})$ & & & 118 \\
\hline Area irrigated (\% area equipped for irrigation) & & 33.9 & \\
\hline Employment in agriculture (\%) & & 80.5 & \\
\hline Employment in agriculture, female (\%) & & 89.9 & \\
\hline Fertilizers, Nitrogen (kg of nutrients per ha) & & 0.4 & 4.6 \\
\hline Fertilizers, Phosphate (kg of nutrients per ha) & & 0.3 & 0.6 \\
\hline Fertilizers, Potash (kg nutrients per ha) & & 0 & 0.8 \\
\hline \multicolumn{4}{|l|}{ Energy consump, power irrigation (mln kWh) } \\
\hline Agr value added per worker (constant US\$) & 167 & 158 & 283 \\
\hline \multicolumn{4}{|l|}{ Hunger dimensions } \\
\hline Dietary energy supply (kcal/pc/day) & 1691 & 1991 & 2306 \\
\hline Average dietary energy supply adequacy (\%) & 80 & 94 & 109 \\
\hline Dietary en supp, cereals/roots/tubers (\%) & 76 & 77 & 73 \\
\hline Prevalence of undernourishment (\%) & 59.4 & 42 & 26.2 \\
\hline GDP per capita (US\$, PPP) & 462 & 582 & 1070 \\
\hline Domestic food price volatility (index) & & 5.3 & 6.7 \\
\hline Cereal import dependency ratio (\%) & 57.3 & 23.1 & 27.3 \\
\hline Underweight, children under-5 (\%) & & 21.2 & 15.6 \\
\hline Improved water source (\% pop) & 33.6 & 41.1 & 49.2 \\
\hline \multicolumn{4}{|l|}{ Food supply } \\
\hline Food production value, (2004-2006 mln I\$) & 1093 & 1535 & 2828 \\
\hline Agriculture, value added (\% GDP) & 37 & 24 & 29 \\
\hline Food exports (mln US\$) & 31 & 47 & 297 \\
\hline Food imports (mln US\$) & 191 & 194 & 632 \\
\hline \multicolumn{4}{|l|}{ Production indices $(2004-06=100)$} \\
\hline Net food & 62 & 88 & 162 \\
\hline Net crops & 60 & 77 & 164 \\
\hline Cereals & 55 & 119 & 173 \\
\hline Vegetable oils & 72 & 90 & 188 \\
\hline Roots and tubers & 76 & 93 & 176 \\
\hline Fruit and vegetables & 85 & 61 & 192 \\
\hline Sugar & 16 & 19 & 184 \\
\hline Livestock & 45 & 107 & 129 \\
\hline Milk & 94 & 92 & 103 \\
\hline Meat & 41 & 111 & 122 \\
\hline Fish & 36 & 41 & 222 \\
\hline \multicolumn{4}{|l|}{ Net trade (mln US\$) } \\
\hline Cereals & -98 & -74 & -370 \\
\hline Fruit and vegetables & 2 & 11 & 36 \\
\hline Meat & -6 & -8 & -29 \\
\hline Dairy products & -7 & -13 & -44 \\
\hline Fish & 41 & 90 & -23 \\
\hline \multicolumn{4}{|l|}{ Environment } \\
\hline Forest area (\%) & 55 & 52 & 49 \\
\hline Renewable water res withdrawn (\% of total) & & 78 & 78 \\
\hline Terrestrial protect areas (\% total land area) & 15 & 15 & 18 \\
\hline Organic area (\% total agricultural area) & & & 0 \\
\hline Water withdrawal by agriculture (\% of total) & & 78 & 78 \\
\hline Biofuel production (thousand kt of oil eq.) & 1 & 1 & 7 \\
\hline \multicolumn{4}{|l|}{ Wood pellet prod. (1 000 tonnes) } \\
\hline Net GHG emissions from AFOLU ( $\mathrm{CO}_{2}$ eq, $\left.\mathrm{Mt}\right)$ & 51 & 49 & 49 \\
\hline
\end{tabular}




\begin{tabular}{|c|c|c|c|}
\hline & 1990 & 2000 & 2014 \\
\hline \multicolumn{4}{|l|}{ The setting } \\
\hline Population, total (mln) & 42.1 & 48.5 & 53.7 \\
\hline Population, rural (mln) & 31.8 & 35.3 & 35.3 \\
\hline Govt expenditure on ag (\% total outlays) & & 10.3 & 6.3 \\
\hline Area harvested (mln ha) & 14 & 22 & 30 \\
\hline Cropping intensity ratio & 1.4 & 2 & \\
\hline Water resources (1 $000 \mathrm{~m}^{3} /$ person/year) & 28 & 24 & 22 \\
\hline Area equipped for irrigation (1 $000 \mathrm{ha})$ & & & 2295 \\
\hline \multicolumn{4}{|l|}{ Area irrigated (\% area equipped for irrigation) } \\
\hline Employment in agriculture (\%) & 69.7 & 62.7 & \\
\hline \multicolumn{4}{|l|}{ Employment in agriculture, female (\%) } \\
\hline Fertilizers, Nitrogen (kg of nutrients per ha) & & 7 & 12.2 \\
\hline Fertilizers, Phosphate (kg of nutrients per ha) & & 2.4 & 2 \\
\hline Fertilizers, Potash (kg nutrients per ha) & & 0.9 & 1.5 \\
\hline Energy consump, power irrigation (mln kWh) & 0 & 0 & 0 \\
\hline \multicolumn{4}{|l|}{ Agr value added per worker (constant US\$) } \\
\hline \multicolumn{4}{|l|}{ Hunger dimensions } \\
\hline Dietary energy supply (kcal/pc/day) & 1715 & 1902 & 2598 \\
\hline Average dietary energy supply adequacy (\%) & 78 & 84 & 112 \\
\hline Dietary en supp, cereals/roots/tubers (\%) & 69 & 68 & 52 \\
\hline Prevalence of undernourishment (\%) & 62.5 & 52.4 & 14.9 \\
\hline \multicolumn{4}{|l|}{ GDP per capita (US\$, PPP) } \\
\hline Domestic food price volatility (index) & & 5.2 & 8.1 \\
\hline Cereal import dependency ratio (\%) & -5.1 & -3.1 & -2.9 \\
\hline Underweight, children under-5 (\%) & 28.8 & 30.1 & 22.6 \\
\hline Improved water source (\% pop) & 55.6 & 66.9 & 85.7 \\
\hline \multicolumn{4}{|l|}{ Food supply } \\
\hline Food production value, (2004-2006 mln I\$) & 5462 & 9244 & 16517 \\
\hline Agriculture, value added (\% GDP) & 57 & 57 & \\
\hline Food exports (mln US\$) & 184 & 354 & 1584 \\
\hline Food imports (mln US\$) & 75 & 227 & 898 \\
\hline \multicolumn{4}{|l|}{ Production indices $(2004-06=100)$} \\
\hline Net food & 43 & 73 & 130 \\
\hline Net crops & 43 & 71 & 121 \\
\hline Cereals & 49 & 76 & 105 \\
\hline Vegetable oils & 36 & 54 & 141 \\
\hline Roots and tubers & 30 & 53 & 179 \\
\hline Fruit and vegetables & 48 & 80 & 120 \\
\hline Sugar & 27 & 76 & 130 \\
\hline Livestock & 25 & 50 & 192 \\
\hline Milk & 53 & 62 & 172 \\
\hline Meat & 21 & 47 & 193 \\
\hline Fish & 33 & 53 & 208 \\
\hline \multicolumn{4}{|l|}{ Net trade (mln US\$) } \\
\hline Cereals & 62 & 11 & -24 \\
\hline Fruit and vegetables & 88 & 252 & 1345 \\
\hline Meat & 0 & 0 & -16 \\
\hline \multicolumn{4}{|l|}{ Dairy products } \\
\hline Fish & 13 & 183 & 638 \\
\hline \multicolumn{4}{|l|}{ Environment } \\
\hline Forest area (\%) & 60 & 53 & 48 \\
\hline Renewable water res withdrawn (\% of total) & & 89 & 89 \\
\hline Terrestrial protect areas (\% total land area) & 3 & 4 & 7 \\
\hline Organic area (\% total agricultural area) & & & 0 \\
\hline Water withdrawal by agriculture (\% of total) & & 89 & 89 \\
\hline Biofuel production (thousand kt of oil eq.) & 3 & 26 & 27 \\
\hline \multicolumn{4}{|l|}{ Wood pellet prod. (1 000 tonnes) } \\
\hline Net GHG emissions from AFOLU ( $\mathrm{CO}_{2}$ eq, $\left.\mathrm{Mt}\right)$ & 156 & 158 & 153 \\
\hline
\end{tabular}




\begin{tabular}{|c|c|c|c|}
\hline & 1990 & 2000 & 2014 \\
\hline \multicolumn{4}{|l|}{ The setting } \\
\hline Population, total (mln) & 1.4 & 1.9 & 2.3 \\
\hline Population, rural (mln) & 1 & 1.3 & 1.4 \\
\hline Govt expenditure on ag (\% total outlays) & & 5 & 6.5 \\
\hline Area harvested (mln ha) & 0 & 0 & 0 \\
\hline Cropping intensity ratio & 0 & 0 & \\
\hline Water resources (1 $000 \mathrm{~m}^{3} /$ person/year) & 28 & 21 & 17 \\
\hline Area equipped for irrigation (1 $000 \mathrm{ha})$ & & & 8 \\
\hline Area irrigated (\% area equipped for irrigation) & 100 & & \\
\hline Employment in agriculture (\%) & 48.2 & 31.1 & 27.4 \\
\hline Employment in agriculture, female (\%) & 52 & 29.1 & 26.6 \\
\hline Fertilizers, Nitrogen (kg of nutrients per ha) & & 1 & 5 \\
\hline Fertilizers, Phosphate (kg of nutrients per ha) & & 0.3 & 0.5 \\
\hline Fertilizers, Potash (kg nutrients per ha) & & 0.2 & 0.6 \\
\hline Energy consump, power irrigation (mln kWh) & 8 & 8 & 11 \\
\hline Agr value added per worker (constant US\$) & 1845 & 2651 & 2080 \\
\hline \multicolumn{4}{|l|}{ Hunger dimensions } \\
\hline Dietary energy supply (kcal/pc/day) & 2010 & 2229 & 2043 \\
\hline Average dietary energy supply adequacy (\%) & 91 & 99 & 88 \\
\hline Dietary en supp, cereals/roots/tubers (\%) & 63 & 59 & 55 \\
\hline Prevalence of undernourishment (\%) & 38.3 & 30.4 & 42.3 \\
\hline GDP per capita (US\$, PPP) & 5716 & 6110 & 9276 \\
\hline Domestic food price volatility (index) & & 7.3 & 7.2 \\
\hline Cereal import dependency ratio (\%) & 69.9 & 69.1 & 55.9 \\
\hline Underweight, children under-5 (\%) & 21.5 & 20.3 & 13.2 \\
\hline Improved water source (\% pop) & 67.2 & 79.1 & 91.7 \\
\hline \multicolumn{4}{|l|}{ Food supply } \\
\hline Food production value, (2004-2006 mln I\$) & 364 & 375 & 406 \\
\hline Agriculture, value added (\% GDP) & 10 & 12 & 6 \\
\hline Food exports (mln US\$) & 141 & 67 & 362 \\
\hline Food imports (mln US\$) & 107 & 181 & 599 \\
\hline \multicolumn{4}{|l|}{ Production indices (2004-06=100) } \\
\hline Net food & 81 & 84 & 91 \\
\hline Net crops & 55 & 77 & 107 \\
\hline Cereals & 66 & 83 & 54 \\
\hline Vegetable oils & 168 & 177 & 38 \\
\hline Roots and tubers & 67 & 81 & 114 \\
\hline Fruit and vegetables & 22 & 50 & 153 \\
\hline \multicolumn{4}{|l|}{ Sugar } \\
\hline Livestock & 89 & 87 & 84 \\
\hline Milk & 68 & 79 & 107 \\
\hline Meat & 93 & 89 & 81 \\
\hline Fish & 48 & 108 & 89 \\
\hline \multicolumn{4}{|l|}{ Net trade (mln US\$) } \\
\hline Cereals & & -36 & -122 \\
\hline Fruit and vegetables & & -26 & -76 \\
\hline Meat & 53 & 10 & 127 \\
\hline Dairy products & & -11 & -43 \\
\hline Fish & 58 & 259 & 717 \\
\hline \multicolumn{4}{|l|}{ Environment } \\
\hline Forest area (\%) & 11 & 10 & 9 \\
\hline Renewable water res withdrawn (\% of total) & & 70 & 70 \\
\hline Terrestrial protect areas (\% total land area) & 12 & 20 & 43 \\
\hline Organic area (\% total agricultural area) & & & 0 \\
\hline Water withdrawal by agriculture (\% of total) & & 70 & 70 \\
\hline \multicolumn{4}{|l|}{ Biofuel production (thousand kt of oil eq.) } \\
\hline \multicolumn{4}{|l|}{ Wood pellet prod. (1 000 tonnes) } \\
\hline Net GHG emissions from AFOLU ( $\mathrm{CO}_{2}$ eq, $\mathrm{Mt}$ ) & 15 & 16 & 19 \\
\hline
\end{tabular}




\begin{tabular}{|c|c|c|c|}
\hline & 1990 & 2000 & 2014 \\
\hline \multicolumn{4}{|l|}{ The setting } \\
\hline Population, total (mln) & 18.1 & 23.2 & 28.1 \\
\hline Population, rural (mln) & 16.5 & 20.1 & 23.1 \\
\hline Govt expenditure on ag (\% total outlays) & & 4.9 & 8.5 \\
\hline Area harvested (mln ha) & 6 & 7 & 9 \\
\hline Cropping intensity ratio & 1.4 & 1.7 & \\
\hline Water resources (1 $000 \mathrm{~m}^{3} /$ person/year) & 12 & 9 & 8 \\
\hline Area equipped for irrigation (1000 ha) & & & 1332 \\
\hline \multicolumn{4}{|l|}{ Area irrigated (\% area equipped for irrigation) } \\
\hline Employment in agriculture (\%) & 81.2 & 65.7 & \\
\hline Employment in agriculture, female (\%) & 90.5 & 72.8 & \\
\hline Fertilizers, Nitrogen (kg of nutrients per ha) & & 2.4 & 23.4 \\
\hline Fertilizers, Phosphate (kg of nutrients per ha) & & 2.3 & 4.3 \\
\hline Fertilizers, Potash (kg nutrients per ha) & & 0.4 & 0.7 \\
\hline Energy consump, power irrigation (mln kWh) & 0 & 0 & 0 \\
\hline Agr value added per worker (constant US\$) & 269 & 263 & 271 \\
\hline \multicolumn{4}{|l|}{ Hunger dimensions } \\
\hline Dietary energy supply (kcal/pc/day) & 2222 & 2280 & 2670 \\
\hline Average dietary energy supply adequacy (\%) & 106 & 108 & 122 \\
\hline Dietary en supp, cereals/roots/tubers (\%) & 76 & 74 & 70 \\
\hline Prevalence of undernourishment (\%) & 24.2 & 22.2 & 7.7 \\
\hline GDP per capita (US\$, PPP) & 1240 & 1577 & 2173 \\
\hline Domestic food price volatility (index) & & 10 & 10.2 \\
\hline Cereal import dependency ratio (\%) & 1.2 & 1.7 & 3.9 \\
\hline Underweight, children under-5 (\%) & & 43 & 29.1 \\
\hline Improved water source (\% pop) & 66.5 & 77.1 & 88.1 \\
\hline \multicolumn{4}{|l|}{ Food supply } \\
\hline Food production value, (2004-2006 mln I\$) & 2783 & 3714 & 5553 \\
\hline Agriculture, value added (\% GDP) & 52 & 41 & 34 \\
\hline Food exports (mln US\$) & 39 & 28 & 170 \\
\hline Food imports (mln US\$) & 69 & 168 & 829 \\
\hline \multicolumn{4}{|l|}{ Production indices $(2004-06=100)$} \\
\hline Net food & 65 & 87 & 130 \\
\hline Net crops & 63 & 85 & 130 \\
\hline Cereals & 77 & 94 & 109 \\
\hline Vegetable oils & 74 & 88 & 115 \\
\hline Roots and tubers & 41 & 67 & 146 \\
\hline Fruit and vegetables & 46 & 77 & 168 \\
\hline Sugar & 41 & 88 & 124 \\
\hline Livestock & 71 & 89 & 136 \\
\hline Milk & 68 & 86 & 131 \\
\hline Meat & 73 & 92 & 138 \\
\hline Fish & 34 & 74 & 135 \\
\hline \multicolumn{4}{|l|}{ Net trade (mln US\$) } \\
\hline Cereals & -5 & -49 & -200 \\
\hline Fruit and vegetables & 16 & -18 & -91 \\
\hline Meat & 0 & 0 & 7 \\
\hline Dairy products & -5 & -2 & -11 \\
\hline Fish & 0 & 0 & -7 \\
\hline \multicolumn{4}{|l|}{ Environment } \\
\hline Forest area (\%) & 34 & 27 & 25 \\
\hline Renewable water res withdrawn (\% of total) & & & 98 \\
\hline Terrestrial protect areas (\% total land area) & 8 & 17 & 16 \\
\hline Organic area (\% total agricultural area) & & & 0 \\
\hline Water withdrawal by agriculture (\% of total) & & & 98 \\
\hline Biofuel production (thousand kt of oil eq.) & 11 & 17 & 21 \\
\hline \multicolumn{4}{|l|}{ Wood pellet prod. (1 000 tonnes) } \\
\hline Net GHG emissions from AFOLU ( $\mathrm{CO}_{2}$ eq, $\mathrm{Mt}$ ) & 49 & 57 & 28 \\
\hline
\end{tabular}




\begin{tabular}{|c|c|c|c|}
\hline & 1990 & 2000 & 2014 \\
\hline \multicolumn{4}{|l|}{ The setting } \\
\hline Population, total (mln) & 14.9 & 15.9 & 16.8 \\
\hline Population, rural (mln) & 4.7 & 3.7 & 2.6 \\
\hline \multicolumn{4}{|l|}{ Govt expenditure on ag (\% total outlays) } \\
\hline Area harvested (mln ha) & 15 & 26 & 7 \\
\hline Cropping intensity ratio & 7.3 & 13.5 & \\
\hline Water resources (1 $000 \mathrm{~m}^{3} /$ person/year) & 6 & 6 & 5 \\
\hline Area equipped for irrigation (1 $000 \mathrm{ha}$ ) & & & 486 \\
\hline Area irrigated (\% area equipped for irrigation) & & & 44.2 \\
\hline Employment in agriculture (\%) & 4.5 & 3 & 2.5 \\
\hline Employment in agriculture, female (\%) & 3.4 & 2.2 & 1.5 \\
\hline Fertilizers, Nitrogen (kg of nutrients per ha) & & 320.7 & 274.5 \\
\hline Fertilizers, Phosphate (kg of nutrients per ha) & & 57.9 & 14.8 \\
\hline Fertilizers, Potash (kg nutrients per ha) & & 59.7 & 20.8 \\
\hline Energy consump, power irrigation (mln kWh) & 6 & 6 & 6 \\
\hline Agr value added per worker (constant US\$) & 31040 & 43101 & 70859 \\
\hline \multicolumn{4}{|l|}{ Hunger dimensions } \\
\hline \multicolumn{4}{|l|}{ Dietary energy supply (kcal/pc/day) } \\
\hline Average dietary energy supply adequacy (\%) & 128 & 127 & 123 \\
\hline Dietary en supp, cereals/roots/tubers (\%) & 22 & 21 & 27 \\
\hline Prevalence of undernourishment (\%) & $<5.0$ & $<5.0$ & $<5.0$ \\
\hline GDP per capita (US\$, PPP) & 32534 & 41771 & 45021 \\
\hline Domestic food price volatility (index) & & 4.8 & 5.6 \\
\hline Cereal import dependency ratio (\%) & 68.8 & 74.1 & 84.3 \\
\hline \multicolumn{4}{|l|}{ Underweight, children under-5 (\%) } \\
\hline Improved water source (\% pop) & 100 & 100 & 100 \\
\hline \multicolumn{4}{|l|}{ Food supply } \\
\hline Food production value, (2004-2006 mln I\$) & 12169 & 12319 & 13343 \\
\hline Agriculture, value added (\% GDP) & 4 & 2 & 2 \\
\hline Food exports (mln US\$) & 21160 & 17073 & 56768 \\
\hline Food imports (mln US\$) & 12317 & 11252 & 41002 \\
\hline \multicolumn{4}{|l|}{ Production indices $(2004-06=100)$} \\
\hline Net food & 103 & 105 & 113 \\
\hline Net crops & 90 & 104 & 109 \\
\hline Cereals & 82 & 100 & 104 \\
\hline Vegetable oils & 255 & 65 & 93 \\
\hline Roots and tubers & 101 & 119 & 100 \\
\hline Fruit and vegetables & 78 & 94 & 117 \\
\hline Sugar & 147 & 116 & 97 \\
\hline Livestock & 106 & 107 & 115 \\
\hline Milk & 102 & 102 & 114 \\
\hline Meat & 109 & 111 & 116 \\
\hline Fish & 87 & 98 & 66 \\
\hline \multicolumn{4}{|l|}{ Net trade (mln US\$) } \\
\hline Cereals & -328 & 1 & -853 \\
\hline Fruit and vegetables & 1421 & 1538 & 5085 \\
\hline Meat & 4286 & 2525 & 4732 \\
\hline Dairy products & 1972 & 1307 & 3839 \\
\hline Fish & 543 & 183 & 270 \\
\hline \multicolumn{4}{|l|}{ Environment } \\
\hline Forest area (\%) & 10 & 11 & 11 \\
\hline Renewable water res withdrawn (\% of total) & & & 1 \\
\hline Terrestrial protect areas (\% total land area) & 12 & 19 & 20 \\
\hline Organic area (\% total agricultural area) & & & 3 \\
\hline Water withdrawal by agriculture (\% of total) & & & 1 \\
\hline Biofuel production (thousand kt of oil eq.) & 6 & 12 & 10350 \\
\hline Wood pellet prod. (1 000 tonnes) & & & 300 \\
\hline Net GHG emissions from AFOLU ( $\mathrm{CO}_{2}$ eq, $\mathrm{Mt}$ ) & 24 & 21 & 20 \\
\hline
\end{tabular}




\begin{tabular}{|c|c|c|c|}
\hline & 1990 & 2000 & 2014 \\
\hline \multicolumn{4}{|l|}{ The setting } \\
\hline Population, total (mln) & 3.4 & 3.9 & 4.6 \\
\hline Population, rural (mln) & 0.5 & 0.6 & 0.6 \\
\hline \multicolumn{4}{|l|}{ Govt expenditure on ag (\% total outlays) } \\
\hline Area harvested (mln ha) & 4 & 2 & 1 \\
\hline Cropping intensity ratio & 0.2 & 0.1 & \\
\hline Water resources (1 $000 \mathrm{~m}^{3} /$ person/year) & 96 & 85 & 73 \\
\hline Area equipped for irrigation (1 $000 \mathrm{ha}$ ) & & & 722 \\
\hline Area irrigated (\% area equipped for irrigation) & & & 82.2 \\
\hline Employment in agriculture (\%) & 10.6 & 8.7 & 6.6 \\
\hline Employment in agriculture, female (\%) & 7.8 & 6 & 4.4 \\
\hline Fertilizers, Nitrogen (kg of nutrients per ha) & & 835.9 & 519.9 \\
\hline Fertilizers, Phosphate (kg of nutrients per ha) & & 1063.7 & 892.6 \\
\hline Fertilizers, Potash (kg nutrients per ha) & & 379.6 & 73.3 \\
\hline Energy consump, power irrigation (mln kWh) & 2 & 2 & 1028 \\
\hline Agr value added per worker (constant US\$) & 21800 & 26763 & 28677 \\
\hline \multicolumn{4}{|l|}{ Hunger dimensions } \\
\hline \multicolumn{4}{|l|}{ Dietary energy supply (kcal/pc/day) } \\
\hline Average dietary energy supply adequacy (\%) & 129 & 127 & 128 \\
\hline Dietary en supp, cereals/roots/tubers (\%) & 29 & 30 & 28 \\
\hline Prevalence of undernourishment (\%) & $<5.0$ & $<5.0$ & $<5.0$ \\
\hline GDP per capita (US\$, PPP) & 23424 & 27422 & 33020 \\
\hline \multicolumn{4}{|l|}{ Domestic food price volatility (index) } \\
\hline Cereal import dependency ratio (\%) & 20.6 & 28.8 & 28.3 \\
\hline \multicolumn{4}{|l|}{ Underweight, children under-5 (\%) } \\
\hline Improved water source (\% pop) & 100 & 100 & 100 \\
\hline \multicolumn{4}{|l|}{ Food supply } \\
\hline Food production value, (2004-2006 mln I\$) & 6171 & 8174 & 10334 \\
\hline Agriculture, value added (\% GDP) & 6 & 8 & 7 \\
\hline Food exports (mln US\$) & 3420 & 4914 & 17306 \\
\hline Food imports (mln US\$) & 479 & 774 & 2780 \\
\hline \multicolumn{4}{|l|}{ Production indices (2004-06=100) } \\
\hline Net food & 65 & 87 & 110 \\
\hline Net crops & 75 & 98 & 118 \\
\hline Cereals & 98 & 102 & 132 \\
\hline Vegetable oils & 45 & 90 & 93 \\
\hline Roots and tubers & 52 & 100 & 112 \\
\hline Fruit and vegetables & 73 & 97 & 117 \\
\hline \multicolumn{4}{|l|}{ Sugar } \\
\hline Livestock & 68 & 87 & 107 \\
\hline Milk & 50 & 82 & 126 \\
\hline Meat & 80 & 89 & 85 \\
\hline Fish & 61 & 102 & 86 \\
\hline \multicolumn{4}{|l|}{ Net trade (mln US\$) } \\
\hline Cereals & -68 & -71 & 62 \\
\hline Fruit and vegetables & 480 & 533 & 1252 \\
\hline Meat & 1356 & 1663 & 4183 \\
\hline Dairy products & 1206 & 2109 & 9117 \\
\hline Fish & 403 & 612 & 1090 \\
\hline \multicolumn{4}{|l|}{ Environment } \\
\hline Forest area (\%) & 29 & 31 & 31 \\
\hline Renewable water res withdrawn (\% of total) & & 74 & 74 \\
\hline Terrestrial protect areas (\% total land area) & 26 & 27 & 27 \\
\hline Organic area (\% total agricultural area) & & & 1 \\
\hline Water withdrawal by agriculture (\% of total) & & 74 & 74 \\
\hline Biofuel production (thousand kt of oil eq.) & 25 & 40 & 70 \\
\hline Wood pellet prod. (1 000 tonnes) & & & 30 \\
\hline Net GHG emissions from AFOLU ( $\mathrm{CO}_{2}$ eq, $\mathrm{Mt}$ ) & 50 & 20 & 21 \\
\hline
\end{tabular}




\begin{tabular}{|c|c|c|c|}
\hline & 1990 & 2000 & 2014 \\
\hline \multicolumn{4}{|l|}{ The setting } \\
\hline Population, total (mln) & 4.1 & 5.1 & 6.2 \\
\hline Population, rural (mln) & 2 & 2.3 & 2.6 \\
\hline \multicolumn{4}{|l|}{ Govt expenditure on ag (\% total outlays) } \\
\hline Area harvested (mln ha) & 2 & 4 & 7 \\
\hline Cropping intensity ratio & 0.6 & 0.7 & \\
\hline Water resources (1 $000 \mathrm{~m}^{3} /$ person/year) & 40 & 32 & 27 \\
\hline Area equipped for irrigation (1 $000 \mathrm{ha}$ ) & & & 199 \\
\hline Area irrigated (\% area equipped for irrigation) & & & 72.4 \\
\hline Employment in agriculture (\%) & 39.3 & 43.5 & 32.2 \\
\hline Employment in agriculture, female (\%) & & 10.4 & 15.2 \\
\hline Fertilizers, Nitrogen (kg of nutrients per ha) & & 17.9 & 36.8 \\
\hline Fertilizers, Phosphate (kg of nutrients per ha) & & 9.8 & 7.3 \\
\hline Fertilizers, Potash (kg nutrients per ha) & & 4.2 & 9.6 \\
\hline Energy consump, power irrigation (mln kWh) & 0 & 0 & 0 \\
\hline Agr value added per worker (constant US\$) & & 2265 & 3973 \\
\hline \multicolumn{4}{|l|}{ Hunger dimensions } \\
\hline Dietary energy supply (kcal/pc/day) & 1855 & 2173 & 2628 \\
\hline Average dietary energy supply adequacy (\%) & 88 & 100 & 117 \\
\hline Dietary en supp, cereals/roots/tubers (\%) & 48 & 51 & 51 \\
\hline Prevalence of undernourishment (\%) & 51.5 & 34.8 & 17.1 \\
\hline GDP per capita (US\$, PPP) & 3082 & 3489 & 4494 \\
\hline Domestic food price volatility (index) & & 7.7 & 6.4 \\
\hline Cereal import dependency ratio (\%) & 24.6 & 29.2 & 31.5 \\
\hline Underweight, children under-5 (\%) & 9.6 & 7.8 & 5.7 \\
\hline Improved water source (\% pop) & 73.9 & 80 & 85 \\
\hline \multicolumn{4}{|l|}{ Food supply } \\
\hline Food production value, (2004-2006 mln I\$) & 569 & 915 & 1579 \\
\hline Agriculture, value added (\% GDP) & & 20 & 21 \\
\hline Food exports (mln US\$) & 126 & 211 & 1238 \\
\hline Food imports (mln US\$) & 93 & 225 & 716 \\
\hline \multicolumn{4}{|l|}{ Production indices $(2004-06=100)$} \\
\hline Net food & 51 & 82 & 141 \\
\hline Net crops & 60 & 88 & 141 \\
\hline Cereals & 52 & 90 & 117 \\
\hline Vegetable oils & 32 & 67 & 126 \\
\hline Roots and tubers & 54 & 60 & 355 \\
\hline Fruit and vegetables & 133 & 92 & 133 \\
\hline Sugar & 58 & 86 & 171 \\
\hline Livestock & 45 & 79 & 136 \\
\hline Milk & 25 & 90 & 123 \\
\hline Meat & 52 & 72 & 143 \\
\hline Fish & 9 & 77 & 183 \\
\hline \multicolumn{4}{|l|}{ Net trade (mln US\$) } \\
\hline Cereals & -40 & -72 & -231 \\
\hline Fruit and vegetables & 19 & -8 & 54 \\
\hline Meat & 50 & 54 & 434 \\
\hline Dairy products & -8 & 2 & 139 \\
\hline Fish & 10 & 121 & 193 \\
\hline \multicolumn{4}{|l|}{ Environment } \\
\hline Forest area (\%) & 38 & 32 & 25 \\
\hline Renewable water res withdrawn (\% of total) & & & 77 \\
\hline Terrestrial protect areas (\% total land area) & 15 & 30 & 31 \\
\hline Organic area (\% total agricultural area) & & & 1 \\
\hline Water withdrawal by agriculture (\% of total) & & & 77 \\
\hline Biofuel production (thousand kt of oil eq.) & 6 & 10 & 13 \\
\hline \multicolumn{4}{|l|}{ Wood pellet prod. (1 000 tonnes) } \\
\hline Net GHG emissions from AFOLU ( $\mathrm{CO}_{2}$ eq, $\left.\mathrm{Mt}\right)$ & 35 & 35 & 37 \\
\hline
\end{tabular}




\begin{tabular}{|c|c|c|c|}
\hline & 1990 & 2000 & 2014 \\
\hline \multicolumn{4}{|l|}{ The setting } \\
\hline Population, total (mln) & 7.8 & 11 & 18.5 \\
\hline Population, rural (mln) & 6.6 & 9.2 & 15.1 \\
\hline \multicolumn{4}{|l|}{ Govt expenditure on ag (\% total outlays) } \\
\hline Area harvested (mln ha) & 7 & 7 & 10 \\
\hline Cropping intensity ratio & 0.2 & 0.2 & \\
\hline Water resources (1 $000 \mathrm{~m}^{3} /$ person/year) & 4 & 3 & 2 \\
\hline Area equipped for irrigation (1 $000 \mathrm{ha})$ & & & 100 \\
\hline Area irrigated (\% area equipped for irrigation) & & & 88 \\
\hline Employment in agriculture (\%) & & & 56.9 \\
\hline Employment in agriculture, female (\%) & & & 37.8 \\
\hline Fertilizers, Nitrogen (kg of nutrients per ha) & & 0.2 & 0.8 \\
\hline Fertilizers, Phosphate (kg of nutrients per ha) & & 0.1 & 0.2 \\
\hline Fertilizers, Potash (kg nutrients per ha) & & 0 & 0.3 \\
\hline \multicolumn{4}{|l|}{ Energy consump, power irrigation (mln kWh) } \\
\hline Agr value added per worker (constant US\$) & & & 233 \\
\hline \multicolumn{4}{|l|}{ Hunger dimensions } \\
\hline Dietary energy supply (kcal/pc/day) & 2026 & 2297 & 2583 \\
\hline Average dietary energy supply adequacy (\%) & 97 & 110 & 124 \\
\hline Dietary en supp, cereals/roots/tubers (\%) & 76 & 69 & 61 \\
\hline Prevalence of undernourishment (\%) & 32.4 & 22.8 & 9.7 \\
\hline GDP per capita (US\$, PPP) & 924 & 778 & 887 \\
\hline Domestic food price volatility (index) & & 16.5 & 9.4 \\
\hline Cereal import dependency ratio (\%) & 4.4 & 8.6 & 7.3 \\
\hline Underweight, children under-5 (\%) & 41 & 43.6 & 37.9 \\
\hline Improved water source (\% pop) & 34.3 & 42.2 & 52.3 \\
\hline \multicolumn{4}{|l|}{ Food supply } \\
\hline Food production value, (2004-2006 mln I\$) & 1004 & 1508 & 2859 \\
\hline Agriculture, value added (\% GDP) & 35 & 38 & 37 \\
\hline Food exports (mln US\$) & 50 & 76 & 191 \\
\hline Food imports (mln US\$) & 97 & 93 & 460 \\
\hline \multicolumn{4}{|l|}{ Production indices (2004-06=100) } \\
\hline Net food & 47 & 71 & 134 \\
\hline Net crops & 47 & 64 & 160 \\
\hline Cereals & 62 & 61 & 123 \\
\hline Vegetable oils & 5 & 60 & 206 \\
\hline Roots and tubers & 88 & 109 & 185 \\
\hline Fruit and vegetables & 34 & 74 & 166 \\
\hline Sugar & 43 & 88 & 84 \\
\hline Livestock & 46 & 80 & 103 \\
\hline Milk & 53 & 81 & 129 \\
\hline Meat & 44 & 79 & 95 \\
\hline Fish & 8 & 37 & 103 \\
\hline \multicolumn{4}{|l|}{ Net trade (mln US\$) } \\
\hline Cereals & -39 & -39 & -236 \\
\hline Fruit and vegetables & -6 & 18 & 60 \\
\hline Meat & -1 & 0 & -1 \\
\hline Dairy products & -9 & -9 & -38 \\
\hline Fish & -1 & 1 & -1 \\
\hline \multicolumn{4}{|l|}{ Environment } \\
\hline Forest area (\%) & 2 & 1 & 1 \\
\hline Renewable water res withdrawn (\% of total) & & & 67 \\
\hline Terrestrial protect areas (\% total land area) & 7 & 7 & 17 \\
\hline Organic area (\% total agricultural area) & & & 0 \\
\hline Water withdrawal by agriculture (\% of total) & & & 67 \\
\hline Biofuel production (thousand kt of oil eq.) & & & 0 \\
\hline \multicolumn{4}{|l|}{ Wood pellet prod. (1 000 tonnes) } \\
\hline Net GHG emissions from AFOLU ( $\mathrm{CO}_{2}$ eq, $\left.\mathrm{Mt}\right)$ & 17 & 21 & 22 \\
\hline
\end{tabular}




\begin{tabular}{|c|c|c|c|}
\hline & 1990 & 2000 & 2014 \\
\hline \multicolumn{4}{|l|}{ The setting } \\
\hline Population, total (mln) & 95.6 & 122.9 & 178.5 \\
\hline Population, rural (mln) & 61.9 & 70.8 & 86.6 \\
\hline Govt expenditure on ag (\% total outlays) & & 0.8 & 0.9 \\
\hline Area harvested (mln ha) & 34 & 65 & 100 \\
\hline Cropping intensity ratio & 0.5 & 0.9 & \\
\hline Water resources (1 $000 \mathrm{~m}^{3} /$ person/year) & 3 & 2 & 2 \\
\hline Area equipped for irrigation (1 $000 \mathrm{ha}$ ) & & & 293 \\
\hline \multicolumn{4}{|l|}{ Area irrigated (\% area equipped for irrigation) } \\
\hline \multicolumn{4}{|l|}{ Employment in agriculture (\%) } \\
\hline \multicolumn{4}{|l|}{ Employment in agriculture, female (\%) } \\
\hline Fertilizers, Nitrogen (kg of nutrients per ha) & & 4.8 & 3.3 \\
\hline Fertilizers, Phosphate (kg of nutrients per ha) & & 0.6 & 0.8 \\
\hline Fertilizers, Potash (kg nutrients per ha) & & 0.7 & 0.7 \\
\hline Energy consump, power irrigation (mln kWh) & 0 & & 0 \\
\hline Agr value added per worker (constant US\$) & 1044 & 1489 & 4760 \\
\hline \multicolumn{4}{|l|}{ Hunger dimensions } \\
\hline Dietary energy supply (kcal/pc/day) & 2487 & 2619 & 2656 \\
\hline Average dietary energy supply adequacy (\%) & 116 & 121 & 124 \\
\hline Dietary en supp, cereals/roots/tubers (\%) & 68 & 65 & 66 \\
\hline Prevalence of undernourishment (\%) & 16.1 & 9.2 & 6.7 \\
\hline GDP per capita (US\$, PPP) & 3030 & 2836 & 5423 \\
\hline Domestic food price volatility (index) & & 11.5 & 4 \\
\hline Cereal import dependency ratio (\%) & 6.4 & 13.6 & 21.7 \\
\hline Underweight, children under-5 (\%) & 35.1 & 27.2 & 31 \\
\hline Improved water source (\% pop) & 45.6 & 54.8 & 64 \\
\hline \multicolumn{4}{|l|}{ Food supply } \\
\hline Food production value, (2004-2006 mln I\$) & 15138 & 25335 & 36075 \\
\hline Agriculture, value added (\% GDP) & 32 & 26 & 20 \\
\hline Food exports (mln US\$) & 158 & 262 & 1219 \\
\hline Food imports (mln US\$) & 480 & 1017 & 6402 \\
\hline \multicolumn{4}{|l|}{ Production indices $(2004-06=100)$} \\
\hline Net food & 48 & 81 & 115 \\
\hline Net crops & 47 & 79 & 109 \\
\hline Cereals & 66 & 82 & 102 \\
\hline Vegetable oils & 46 & 76 & 87 \\
\hline Roots and tubers & 39 & 76 & 118 \\
\hline Fruit and vegetables & 54 & 85 & 103 \\
\hline Sugar & 100 & 76 & 158 \\
\hline Livestock & 60 & 86 & 124 \\
\hline Milk & 80 & 88 & 129 \\
\hline Meat & 58 & 87 & 122 \\
\hline Fish & 55 & 81 & 174 \\
\hline \multicolumn{4}{|l|}{ Net trade (mln US\$) } \\
\hline Cereals & -119 & -493 & -3211 \\
\hline Fruit and vegetables & -2 & -17 & 159 \\
\hline Meat & 0 & -1 & -11 \\
\hline Dairy products & -73 & -134 & -519 \\
\hline Fish & -166 & -169 & -1142 \\
\hline \multicolumn{4}{|l|}{ Environment } \\
\hline Forest area (\%) & 19 & 14 & 9 \\
\hline Renewable water res withdrawn (\% of total) & & & 54 \\
\hline Terrestrial protect areas (\% total land area) & 12 & 13 & 14 \\
\hline Organic area (\% total agricultural area) & & & 0 \\
\hline Water withdrawal by agriculture (\% of total) & & & 54 \\
\hline Biofuel production (thousand kt of oil eq.) & 1 & 1 & 1 \\
\hline \multicolumn{4}{|l|}{ Wood pellet prod. (1 000 tonnes) } \\
\hline Net GHG emissions from AFOLU ( $\mathrm{CO}_{2}$ eq, $\left.\mathrm{Mt}\right)$ & 221 & 230 & 237 \\
\hline
\end{tabular}




\begin{tabular}{|c|c|c|c|}
\hline & 1990 & 2000 & 2014 \\
\hline \multicolumn{4}{|l|}{ The setting } \\
\hline Population, total (mln) & 20.2 & 22.8 & 25 \\
\hline Population, rural (mln) & 8.4 & 9.3 & 9.8 \\
\hline \multicolumn{4}{|l|}{ Govt expenditure on ag (\% total outlays) } \\
\hline Area harvested (mln ha) & 6 & 4 & 5 \\
\hline Cropping intensity ratio & 2.5 & 1.5 & \\
\hline Water resources (1 $000 \mathrm{~m}^{3} /$ person/year) & 4 & 3 & 3 \\
\hline Area equipped for irrigation (1 $000 \mathrm{ha}$ ) & & & 1460 \\
\hline \multicolumn{4}{|l|}{ Area irrigated (\% area equipped for irrigation) } \\
\hline \multicolumn{4}{|l|}{ Employment in agriculture (\%) } \\
\hline \multicolumn{4}{|l|}{ Employment in agriculture, female (\%) } \\
\hline \multicolumn{4}{|l|}{ Fertilizers, Nitrogen (kg of nutrients per ha) } \\
\hline \multicolumn{4}{|l|}{ Fertilizers, Phosphate (kg of nutrients per ha) } \\
\hline \multicolumn{4}{|l|}{ Fertilizers, Potash (kg nutrients per ha) } \\
\hline Energy consump, power irrigation (mln kWh) & 0 & 0 & 0 \\
\hline \multicolumn{4}{|l|}{ Agr value added per worker (constant US\$) } \\
\hline \multicolumn{4}{|l|}{ Hunger dimensions } \\
\hline Dietary energy supply (kcal/pc/day) & 2248 & 2118 & 2103 \\
\hline Average dietary energy supply adequacy (\%) & 96 & 90 & 88 \\
\hline Dietary en supp, cereals/roots/tubers (\%) & 66 & 68 & 69 \\
\hline Prevalence of undernourishment (\%) & 26.7 & 37.9 & 41.8 \\
\hline \multicolumn{4}{|l|}{ GDP per capita (US\$, PPP) } \\
\hline \multicolumn{4}{|l|}{ Domestic food price volatility (index) } \\
\hline Cereal import dependency ratio (\%) & 13.5 & 39.1 & 12.2 \\
\hline Underweight, children under-5 (\%) & & 24.7 & 15.2 \\
\hline Improved water source (\% pop) & 100 & 99.7 & 98.1 \\
\hline \multicolumn{4}{|l|}{ Food supply } \\
\hline Food production value, (2004-2006 mln I\$) & 3232 & 2961 & 3651 \\
\hline \multicolumn{4}{|l|}{ Agriculture, value added (\% GDP) } \\
\hline Food exports (mln US\$) & 31 & 21 & 18 \\
\hline Food imports (mln US\$) & 174 & 428 & 510 \\
\hline \multicolumn{4}{|l|}{ Production indices (2004-06=100) } \\
\hline Net food & 91 & 83 & 102 \\
\hline Net crops & 99 & 85 & 103 \\
\hline Cereals & 114 & 65 & 115 \\
\hline Vegetable oils & 129 & 101 & 99 \\
\hline Roots and tubers & 42 & 91 & 91 \\
\hline Fruit and vegetables & 101 & 94 & 97 \\
\hline \multicolumn{4}{|l|}{ Sugar } \\
\hline Livestock & 98 & 78 & 103 \\
\hline Milk & 80 & 81 & 89 \\
\hline Meat & 91 & 76 & 105 \\
\hline Fish & 198 & 104 & 104 \\
\hline \multicolumn{4}{|l|}{ Net trade (mln US\$) } \\
\hline Cereals & -85 & -339 & -346 \\
\hline Fruit and vegetables & 20 & 8 & 6 \\
\hline Meat & -8 & -3 & -3 \\
\hline \multicolumn{4}{|l|}{ Dairy products } \\
\hline Fish & 65 & 74 & 14 \\
\hline Environment & & & \\
\hline Forest area (\%) & 68 & 58 & 45 \\
\hline Renewable water res withdrawn (\% of total) & & & 76 \\
\hline Terrestrial protect areas (\% total land area) & 2 & 2 & 2 \\
\hline Organic area (\% total agricultural area) & & & \\
\hline Water withdrawal by agriculture (\% of total) & & & 76 \\
\hline Biofuel production (thousand kt of oil eq.) & & & \\
\hline Wood pellet prod. (1 000 tonnes) & & & \\
\hline Net GHG emissions from AFOLU ( $\mathrm{CO}_{2}$ eq, $\mathrm{Mt}$ ) & 21 & 18 & 19 \\
\hline
\end{tabular}




\begin{tabular}{|c|c|c|c|}
\hline & 1990 & 2000 & 2014 \\
\hline \multicolumn{4}{|l|}{ The setting } \\
\hline Population, total (mln) & 4.2 & 4.5 & 5.1 \\
\hline Population, rural (mln) & 1.2 & 1.1 & 1 \\
\hline \multicolumn{4}{|l|}{ Govt expenditure on ag (\% total outlays) } \\
\hline Area harvested (mln ha) & 2 & 3 & 1 \\
\hline Cropping intensity ratio & 2.5 & 3.1 & \\
\hline Water resources (1 $000 \mathrm{~m}^{3} /$ person/year) & 93 & 87 & 78 \\
\hline Area equipped for irrigation (1 $000 \mathrm{ha})$ & & & 90 \\
\hline Area irrigated (\% area equipped for irrigation) & & & 47.8 \\
\hline Employment in agriculture (\%) & 6.4 & 4.3 & 2.2 \\
\hline Employment in agriculture, female (\%) & 3.9 & 2.3 & 0.8 \\
\hline Fertilizers, Nitrogen (kg of nutrients per ha) & & 117.8 & 116.1 \\
\hline Fertilizers, Phosphate (kg of nutrients per ha) & & 33.1 & 23.6 \\
\hline Fertilizers, Potash (kg nutrients per ha) & & 62.6 & 48 \\
\hline \multicolumn{4}{|l|}{ Energy consump, power irrigation (mln kWh) } \\
\hline Agr value added per worker (constant US\$) & 20498 & 33262 & 76958 \\
\hline \multicolumn{4}{|l|}{ Hunger dimensions } \\
\hline \multicolumn{4}{|l|}{ Dietary energy supply (kcal/pc/day) } \\
\hline Average dietary energy supply adequacy (\%) & 128 & 135 & 138 \\
\hline Dietary en supp, cereals/roots/tubers (\%) & 32 & 32 & 31 \\
\hline Prevalence of undernourishment (\%) & $<5.0$ & $<5.0$ & $<5.0$ \\
\hline GDP per capita (US\$, PPP) & 42752 & 58093 & 62411 \\
\hline Domestic food price volatility (index) & & 7 & 11.3 \\
\hline Cereal import dependency ratio (\%) & 30 & 30.1 & 40.2 \\
\hline \multicolumn{4}{|l|}{ Underweight, children under-5 (\%) } \\
\hline Improved water source (\% pop) & 100 & 100 & 100 \\
\hline \multicolumn{4}{|l|}{ Food supply } \\
\hline Food production value, (2004-2006 mln I\$) & 1397 & 1278 & 1351 \\
\hline Agriculture, value added (\% GDP) & 3 & 2 & 2 \\
\hline Food exports (mln US\$) & 191 & 192 & 449 \\
\hline Food imports (mln US\$) & 963 & 1160 & 4706 \\
\hline \multicolumn{4}{|l|}{ Production indices $(2004-06=100)$} \\
\hline Net food & 108 & 99 & 104 \\
\hline Net crops & 136 & 92 & 77 \\
\hline Cereals & 114 & 99 & 67 \\
\hline Vegetable oils & 85 & 81 & 100 \\
\hline Roots and tubers & 134 & 85 & 88 \\
\hline Fruit and vegetables & 190 & 85 & 89 \\
\hline \multicolumn{4}{|l|}{ Sugar } \\
\hline Livestock & 101 & 101 & 109 \\
\hline Milk & 126 & 110 & 101 \\
\hline Meat & 80 & 94 & 115 \\
\hline Fish & 57 & 104 & 109 \\
\hline \multicolumn{4}{|l|}{ Net trade (mln US\$) } \\
\hline Cereals & -149 & -185 & -818 \\
\hline Fruit and vegetables & -358 & -427 & -1400 \\
\hline Meat & 0 & -19 & -198 \\
\hline Dairy products & 79 & 56 & -25 \\
\hline Fish & 1822 & 2935 & 7547 \\
\hline \multicolumn{4}{|l|}{ Environment } \\
\hline Forest area (\%) & 25 & 25 & 28 \\
\hline Renewable water res withdrawn (\% of total) & & & 29 \\
\hline Terrestrial protect areas (\% total land area) & 7 & 9 & 16 \\
\hline Organic area (\% total agricultural area) & & & 6 \\
\hline Water withdrawal by agriculture (\% of total) & & & 29 \\
\hline Biofuel production (thousand kt of oil eq.) & 1 & 1 & 1 \\
\hline Wood pellet prod. (1 000 tonnes) & & & 56 \\
\hline Net GHG emissions from AFOLU ( $\mathrm{CO}_{2}$ eq, $\left.\mathrm{Mt}\right)$ & -6 & -9 & -20 \\
\hline
\end{tabular}




\begin{tabular}{|c|c|c|c|}
\hline & 1990 & 2000 & 2014 \\
\hline \multicolumn{4}{|l|}{ The setting } \\
\hline Population, total (mln) & 111.1 & 143.8 & 185.1 \\
\hline Population, rural (mln) & 77.1 & 96.2 & 116.3 \\
\hline Govt expenditure on ag (\% total outlays) & & 0.8 & 1.2 \\
\hline Area harvested (mln ha) & 35 & 46 & 64 \\
\hline Cropping intensity ratio & 1.4 & 1.7 & \\
\hline Water resources (1 $000 \mathrm{~m}^{3} /$ person/year) & 2 & 2 & 1 \\
\hline Area equipped for irrigation (1 $000 \mathrm{ha}$ ) & & & 20200 \\
\hline \multicolumn{4}{|l|}{ Area irrigated (\% area equipped for irrigation) } \\
\hline Employment in agriculture (\%) & 51.1 & 48.4 & 43.7 \\
\hline Employment in agriculture, female (\%) & 72.2 & 72.9 & 75.7 \\
\hline Fertilizers, Nitrogen (kg of nutrients per ha) & & 113.8 & 134.4 \\
\hline Fertilizers, Phosphate (kg of nutrients per ha) & & 31 & 31.6 \\
\hline Fertilizers, Potash (kg nutrients per ha) & & 1.1 & 0.9 \\
\hline \multicolumn{4}{|l|}{ Energy consump, power irrigation (mln kWh) } \\
\hline Agr value added per worker (constant US\$) & 857 & 1064 & 1083 \\
\hline \multicolumn{4}{|l|}{ Hunger dimensions } \\
\hline Dietary energy supply (kcal/pc/day) & 2358 & 2377 & 2444 \\
\hline Average dietary energy supply adequacy (\%) & 111 & 109 & 108 \\
\hline Dietary en supp, cereals/roots/tubers (\%) & 55 & 52 & 50 \\
\hline Prevalence of undernourishment (\%) & 24 & 22.4 & 22 \\
\hline GDP per capita (US\$, PPP) & 2961 & 3366 & 4454 \\
\hline Domestic food price volatility (index) & & 8.5 & 13.2 \\
\hline Cereal import dependency ratio (\%) & 5 & -3.5 & -12.2 \\
\hline Underweight, children under-5 (\%) & 39 & 31.3 & 31.6 \\
\hline Improved water source (\% pop) & 85.3 & 88.3 & 91.4 \\
\hline \multicolumn{4}{|l|}{ Food supply } \\
\hline Food production value, (2004-2006 mln I\$) & 17572 & 25714 & 27300 \\
\hline Agriculture, value added (\% GDP) & 26 & 26 & 25 \\
\hline Food exports (mln US\$) & 399 & 777 & 3621 \\
\hline Food imports (mln US\$) & 1014 & 1249 & 4018 \\
\hline \multicolumn{4}{|l|}{ Production indices $(2004-06=100)$} \\
\hline Net food & 60 & 88 & 94 \\
\hline Net crops & 68 & 90 & 107 \\
\hline Cereals & 64 & 94 & 108 \\
\hline Vegetable oils & 61 & 77 & 93 \\
\hline Roots and tubers & 49 & 100 & 187 \\
\hline Fruit and vegetables & 61 & 87 & 104 \\
\hline Sugar & 74 & 96 & 131 \\
\hline Livestock & 56 & 86 & 81 \\
\hline Milk & 50 & 86 & 51 \\
\hline Meat & 68 & 85 & 145 \\
\hline Fish & 85 & 112 & 111 \\
\hline \multicolumn{4}{|l|}{ Net trade (mln US\$) } \\
\hline Cereals & -176 & 385 & 2125 \\
\hline Fruit and vegetables & 4 & -86 & -163 \\
\hline Meat & 0 & 6 & 204 \\
\hline Dairy products & -24 & -16 & -45 \\
\hline Fish & 95 & 150 & 277 \\
\hline \multicolumn{4}{|l|}{ Environment } \\
\hline Forest area (\%) & 3 & 3 & 2 \\
\hline Renewable water res withdrawn (\% of total) & & & 94 \\
\hline Terrestrial protect areas (\% total land area) & 10 & 10 & 11 \\
\hline Organic area (\% total agricultural area) & & & 0 \\
\hline Water withdrawal by agriculture (\% of total) & & & 94 \\
\hline Biofuel production (thousand kt of oil eq.) & 49 & 180 & 255 \\
\hline \multicolumn{4}{|l|}{ Wood pellet prod. (1 000 tonnes) } \\
\hline Net GHG emissions from AFOLU ( $\mathrm{CO}_{2}$ eq, $\mathrm{Mt}$ ) & 103 & 121 & 161 \\
\hline
\end{tabular}




\begin{tabular}{|c|c|c|c|}
\hline & 1990 & 2000 & 2014 \\
\hline \multicolumn{4}{|l|}{ The setting } \\
\hline Population, total (mln) & 2.5 & 3.1 & 3.9 \\
\hline Population, rural (mln) & 1.1 & 1 & 0.9 \\
\hline Govt expenditure on ag (\% total outlays) & & 3.5 & 1.8 \\
\hline Area harvested (mln ha) & 1 & 2 & 2 \\
\hline Cropping intensity ratio & 0.6 & 0.8 & \\
\hline Water resources (1 $000 \mathrm{~m}^{3} /$ person/year) & 56 & 46 & 36 \\
\hline Area equipped for irrigation (1 $000 \mathrm{ha}$ ) & & & 32 \\
\hline Area irrigated (\% area equipped for irrigation) & & & 100 \\
\hline Employment in agriculture (\%) & 23.1 & 17 & 16.7 \\
\hline Employment in agriculture, female (\%) & 3 & 1.7 & 8.5 \\
\hline Fertilizers, Nitrogen (kg of nutrients per ha) & & 32.2 & 32.1 \\
\hline Fertilizers, Phosphate (kg of nutrients per ha) & & 10.9 & 19.9 \\
\hline Fertilizers, Potash (kg nutrients per ha) & & 8.1 & 12.5 \\
\hline Energy consump, power irrigation (mln kWh) & 0 & 19 & 18 \\
\hline Agr value added per worker (constant US\$) & 2344 & 3122 & 4187 \\
\hline \multicolumn{4}{|l|}{ Hunger dimensions } \\
\hline Dietary energy supply (kcal/pc/day) & 2329 & 2203 & 2745 \\
\hline Average dietary energy supply adequacy (\%) & 104 & 98 & 120 \\
\hline Dietary en supp, cereals/roots/tubers (\%) & 44 & 40 & 45 \\
\hline Prevalence of undernourishment (\%) & 23.9 & 27.4 & 10 \\
\hline GDP per capita (US\$, PPP) & 7463 & 9954 & 18793 \\
\hline Domestic food price volatility (index) & & 3.6 & 2.1 \\
\hline Cereal import dependency ratio (\%) & 47.9 & 63.9 & 69.4 \\
\hline Underweight, children under-5 (\%) & & 5.1 & 3.9 \\
\hline Improved water source (\% pop) & 83.9 & 90.2 & 94.3 \\
\hline \multicolumn{4}{|l|}{ Food supply } \\
\hline Food production value, $(2004-2006 \mathrm{mln}$ I\$) & 754 & 788 & 953 \\
\hline Agriculture, value added (\% GDP) & 10 & 7 & 3 \\
\hline Food exports (mln US\$) & 292 & 249 & 287 \\
\hline Food imports (mln US\$) & 144 & 308 & 1031 \\
\hline \multicolumn{4}{|l|}{ Production indices $(2004-06=100)$} \\
\hline Net food & 92 & 96 & 117 \\
\hline Net crops & 123 & 98 & 98 \\
\hline Cereals & 99 & 89 & 125 \\
\hline Vegetable oils & 5 & 87 & 82 \\
\hline Roots and tubers & 59 & 76 & 87 \\
\hline Fruit and vegetables & 153 & 103 & 81 \\
\hline Sugar & 73 & 101 & 139 \\
\hline Livestock & 65 & 95 & 135 \\
\hline Milk & 69 & 95 & 115 \\
\hline Meat & 66 & 98 & 141 \\
\hline Fish & 52 & 90 & 82 \\
\hline \multicolumn{4}{|l|}{ Net trade (mln US\$) } \\
\hline Cereals & -41 & -102 & -376 \\
\hline Fruit and vegetables & 193 & 113 & -21 \\
\hline Meat & -3 & -15 & -49 \\
\hline Dairy products & -5 & -9 & -60 \\
\hline Fish & 57 & 241 & 68 \\
\hline \multicolumn{4}{|l|}{ Environment } \\
\hline Forest area (\%) & 51 & 45 & 43 \\
\hline Renewable water res withdrawn (\% of total) & & & 43 \\
\hline Terrestrial protect areas (\% total land area) & 18 & 19 & 21 \\
\hline Organic area (\% total agricultural area) & & & 0 \\
\hline Water withdrawal by agriculture (\% of total) & & & 43 \\
\hline Biofuel production (thousand kt of oil eq.) & 1 & 4 & 7 \\
\hline \multicolumn{4}{|l|}{ Wood pellet prod. (1 000 tonnes) } \\
\hline Net GHG emissions from AFOLU ( $\mathrm{CO}_{2}$ eq, $\mathrm{Mt}$ ) & 23 & 22 & 10 \\
\hline
\end{tabular}




\begin{tabular}{|c|c|c|c|}
\hline & 1990 & 2000 & 2014 \\
\hline \multicolumn{4}{|l|}{ The setting } \\
\hline Population, total (mln) & 4.2 & 5.3 & 6.9 \\
\hline Population, rural (mln) & 2.2 & 2.4 & 2.5 \\
\hline Govt expenditure on ag (\% total outlays) & & & 3.4 \\
\hline Area harvested (mln ha) & 4 & 3 & 9 \\
\hline Cropping intensity ratio & 0.2 & 0.1 & \\
\hline Water resources (1 $000 \mathrm{~m}^{3} /$ person/year) & 91 & 72 & 57 \\
\hline Area equipped for irrigation (1 $000 \mathrm{ha}$ ) & & & 136 \\
\hline Area irrigated (\% area equipped for irrigation) & & & 100 \\
\hline Employment in agriculture (\%) & 2.1 & 31.9 & 27.2 \\
\hline Employment in agriculture, female (\%) & 0.8 & 20.3 & 23 \\
\hline Fertilizers, Nitrogen (kg of nutrients per ha) & & 12.7 & 21.8 \\
\hline Fertilizers, Phosphate (kg of nutrients per ha) & & 28.8 & 34.3 \\
\hline Fertilizers, Potash (kg nutrients per ha) & & 24.7 & 27.3 \\
\hline \multicolumn{4}{|l|}{ Energy consump, power irrigation (mln kWh) } \\
\hline Agr value added per worker (constant US\$) & 1714 & 1694 & 3173 \\
\hline \multicolumn{4}{|l|}{ Hunger dimensions } \\
\hline Dietary energy supply (kcal/pc/day) & 2460 & 2623 & 2645 \\
\hline Average dietary energy supply adequacy (\%) & 110 & 115 & 113 \\
\hline Dietary en supp, cereals/roots/tubers (\%) & 42 & 42 & 44 \\
\hline Prevalence of undernourishment (\%) & 19.2 & 13.3 & 11.1 \\
\hline GDP per capita (US\$, PPP) & 5985 & 6031 & 7833 \\
\hline Domestic food price volatility (index) & & 8.1 & 11.2 \\
\hline Cereal import dependency ratio (\%) & 4.2 & -22.9 & -144.3 \\
\hline Underweight, children under-5 (\%) & 2.8 & & 3.4 \\
\hline Improved water source (\% pop) & 53.1 & 73.5 & 93.8 \\
\hline \multicolumn{4}{|l|}{ Food supply } \\
\hline Food production value, (2004-2006 mln I\$) & 1978 & 2497 & 5562 \\
\hline Agriculture, value added (\% GDP) & 18 & 16 & 21 \\
\hline Food exports (mln US\$) & 410 & 459 & 3786 \\
\hline Food imports (mln US\$) & 31 & 150 & 471 \\
\hline \multicolumn{4}{|l|}{ Production indices $(2004-06=100)$} \\
\hline Net food & 63 & 79 & 176 \\
\hline Net crops & 71 & 69 & 169 \\
\hline Cereals & 37 & 47 & 298 \\
\hline Vegetable oils & 54 & 77 & 215 \\
\hline Roots and tubers & 71 & 54 & 55 \\
\hline Fruit and vegetables & 92 & 86 & 92 \\
\hline Sugar & 69 & 63 & 156 \\
\hline Livestock & 69 & 91 & 134 \\
\hline Milk & 60 & 88 & 138 \\
\hline Meat & 74 & 94 & 134 \\
\hline Fish & 52 & 117 & 95 \\
\hline \multicolumn{4}{|l|}{ Net trade (mln US\$) } \\
\hline Cereals & 11 & -20 & 891 \\
\hline Fruit and vegetables & 7 & -23 & -42 \\
\hline Meat & 134 & 70 & 781 \\
\hline Dairy products & -2 & -11 & -20 \\
\hline Fish & -1 & -2 & -7 \\
\hline \multicolumn{4}{|l|}{ Environment } \\
\hline Forest area (\%) & 53 & 49 & 43 \\
\hline Renewable water res withdrawn (\% of total) & & & 79 \\
\hline Terrestrial protect areas (\% total land area) & 3 & 5 & 6 \\
\hline Organic area (\% total agricultural area) & & & 0 \\
\hline Water withdrawal by agriculture (\% of total) & & & 79 \\
\hline Biofuel production (thousand kt of oil eq.) & 3 & 2 & 4 \\
\hline \multicolumn{4}{|l|}{ Wood pellet prod. (1 000 tonnes) } \\
\hline Net GHG emissions from AFOLU ( $\mathrm{CO}_{2}$ eq, $\left.\mathrm{Mt}\right)$ & 87 & 89 & 100 \\
\hline
\end{tabular}




\begin{tabular}{|c|c|c|c|}
\hline & 1990 & 2000 & 2014 \\
\hline \multicolumn{4}{|l|}{ The setting } \\
\hline Population, total (mln) & 21.8 & 26 & 30.8 \\
\hline Population, rural (mln) & 6.8 & 7 & 6.7 \\
\hline \multicolumn{4}{|l|}{ Govt expenditure on ag (\% total outlays) } \\
\hline Area harvested (mln ha) & 7 & 8 & 11 \\
\hline Cropping intensity ratio & 0.3 & 0.3 & \\
\hline Water resources (1 $000 \mathrm{~m}^{3} /$ person/year) & 87 & 73 & 62 \\
\hline Area equipped for irrigation (1 $000 \mathrm{ha})$ & & & 2580 \\
\hline Area irrigated (\% area equipped for irrigation) & & & 70.1 \\
\hline Employment in agriculture (\%) & 1.2 & 0.6 & 25.8 \\
\hline Employment in agriculture, female (\%) & 0.6 & 0.2 & 22.5 \\
\hline Fertilizers, Nitrogen (kg of nutrients per ha) & & 53.2 & 67.5 \\
\hline Fertilizers, Phosphate (kg of nutrients per ha) & & 16.3 & 25.1 \\
\hline Fertilizers, Potash (kg nutrients per ha) & & 12.7 & 11.7 \\
\hline Energy consump, power irrigation (mln kWh) & 0 & 70 & 70 \\
\hline Agr value added per worker (constant US\$) & 1025 & 1408 & 1949 \\
\hline \multicolumn{4}{|l|}{ Hunger dimensions } \\
\hline Dietary energy supply (kcal/pc/day) & 2168 & 2321 & 2733 \\
\hline Average dietary energy supply adequacy (\%) & 100 & 106 & 120 \\
\hline Dietary en supp, cereals/roots/tubers (\%) & 60 & 59 & 57 \\
\hline Prevalence of undernourishment (\%) & 28.6 & 21.6 & 8.1 \\
\hline GDP per capita (US\$, PPP) & 5280 & 6485 & 11396 \\
\hline Domestic food price volatility (index) & & 4 & 3.4 \\
\hline Cereal import dependency ratio (\%) & 60.6 & 45.4 & 48.4 \\
\hline Underweight, children under-5 (\%) & 8.8 & 5.2 & 3.5 \\
\hline Improved water source (\% pop) & 74.4 & 80.6 & 86.8 \\
\hline \multicolumn{4}{|l|}{ Food supply } \\
\hline Food production value, (2004-2006 mln I\$) & 3085 & 5319 & 9145 \\
\hline Agriculture, value added (\% GDP) & 10 & 9 & 7 \\
\hline Food exports (mln US\$) & 121 & 326 & 2738 \\
\hline Food imports (mln US\$) & 595 & 698 & 3238 \\
\hline \multicolumn{4}{|l|}{ Production indices $(2004-06=100)$} \\
\hline Net food & 50 & 85 & 147 \\
\hline Net crops & 46 & 86 & 140 \\
\hline Cereals & 45 & 89 & 137 \\
\hline Vegetable oils & 70 & 78 & 284 \\
\hline Roots and tubers & 43 & 103 & 138 \\
\hline Fruit and vegetables & 43 & 83 & 151 \\
\hline Sugar & 93 & 105 & 153 \\
\hline Livestock & 55 & 84 & 153 \\
\hline Milk & 58 & 79 & 133 \\
\hline Meat & 55 & 85 & 156 \\
\hline Fish & 79 & 123 & 69 \\
\hline \multicolumn{4}{|l|}{ Net trade (mln US\$) } \\
\hline Cereals & -381 & -321 & -1332 \\
\hline Fruit and vegetables & 40 & 196 & 1765 \\
\hline Meat & -28 & -26 & -93 \\
\hline Dairy products & -42 & -65 & -127 \\
\hline Fish & 398 & 1113 & 3177 \\
\hline \multicolumn{4}{|l|}{ Environment } \\
\hline Forest area (\%) & 55 & 54 & 53 \\
\hline Renewable water res withdrawn (\% of total) & & & 89 \\
\hline Terrestrial protect areas (\% total land area) & 5 & 8 & 19 \\
\hline Organic area (\% total agricultural area) & & & 1 \\
\hline Water withdrawal by agriculture (\% of total) & & & 89 \\
\hline Biofuel production (thousand kt of oil eq.) & 8 & 10 & 259 \\
\hline \multicolumn{4}{|l|}{ Wood pellet prod. (1 000 tonnes) } \\
\hline Net GHG emissions from AFOLU ( $\mathrm{CO}_{2}$ eq, $\mathrm{Mt}$ ) & 84 & 67 & 97 \\
\hline
\end{tabular}




\begin{tabular}{|c|c|c|c|}
\hline & 1990 & 2000 & 2014 \\
\hline \multicolumn{4}{|l|}{ The setting } \\
\hline Population, total (mln) & 61.9 & 77.7 & 100.1 \\
\hline Population, rural (mln) & 31.8 & 40.4 & 50.5 \\
\hline Govt expenditure on ag (\% total outlays) & & 3.9 & 3.7 \\
\hline Area harvested (mln ha) & 25 & 24 & 32 \\
\hline Cropping intensity ratio & 2.3 & 2.2 & \\
\hline Water resources (1 $000 \mathrm{~m}^{3} /$ person/year) & 8 & 6 & 5 \\
\hline Area equipped for irrigation (1 $000 \mathrm{ha})$ & & & 1627 \\
\hline Area irrigated (\% area equipped for irrigation) & 95 & 95 & \\
\hline Employment in agriculture (\%) & 45.2 & 37.1 & 32.2 \\
\hline Employment in agriculture, female (\%) & 31.3 & 23.9 & 20.1 \\
\hline Fertilizers, Nitrogen (kg of nutrients per ha) & & 115.8 & 80.7 \\
\hline Fertilizers, Phosphate (kg of nutrients per ha) & & 42.2 & 18.2 \\
\hline Fertilizers, Potash (kg nutrients per ha) & & 15.7 & 14.7 \\
\hline Energy consump, power irrigation (mln kWh) & 0 & 0 & 37 \\
\hline Agr value added per worker (constant US\$) & 826 & 882 & 1152 \\
\hline \multicolumn{4}{|l|}{ Hunger dimensions } \\
\hline Dietary energy supply (kcal/pc/day) & 2256 & 2375 & 2604 \\
\hline Average dietary energy supply adequacy (\%) & 106 & 110 & 118 \\
\hline Dietary en supp, cereals/roots/tubers (\%) & 57 & 57 & 59 \\
\hline Prevalence of undernourishment (\%) & 26.4 & 21.3 & 13.9 \\
\hline GDP per capita (US\$, PPP) & 4010 & 4243 & 6326 \\
\hline Domestic food price volatility (index) & & 3.3 & 2.6 \\
\hline Cereal import dependency ratio (\%) & 16.8 & 23.5 & 21.9 \\
\hline Underweight, children under-5 (\%) & 29.9 & 20.7 & 20.2 \\
\hline Improved water source (\% pop) & 83.6 & 87.6 & 91.8 \\
\hline \multicolumn{4}{|l|}{ Food supply } \\
\hline Food production value, (2004-2006 mln I\$) & 11600 & 14232 & 20587 \\
\hline Agriculture, value added (\% GDP) & 22 & 14 & 11 \\
\hline Food exports (mln US\$) & 996 & 1317 & 3280 \\
\hline Food imports (mln US\$) & 993 & 1991 & 4745 \\
\hline \multicolumn{4}{|l|}{ Production indices (2004-06=100) } \\
\hline Net food & 68 & 84 & 122 \\
\hline Net crops & 76 & 85 & 118 \\
\hline Cereals & 70 & 83 & 127 \\
\hline Vegetable oils & 81 & 88 & 112 \\
\hline Roots and tubers & 107 & 101 & 128 \\
\hline Fruit and vegetables & 75 & 85 & 118 \\
\hline Sugar & 79 & 75 & 99 \\
\hline Livestock & 48 & 84 & 127 \\
\hline Milk & 209 & 84 & 160 \\
\hline Meat & 46 & 84 & 128 \\
\hline Fish & 78 & 81 & 111 \\
\hline \multicolumn{4}{|l|}{ Net trade (mln US\$) } \\
\hline Cereals & -535 & -690 & -1724 \\
\hline Fruit and vegetables & 365 & 497 & 1229 \\
\hline Meat & -23 & -160 & -443 \\
\hline Dairy products & -266 & -389 & -638 \\
\hline Fish & 311 & 298 & 566 \\
\hline \multicolumn{4}{|l|}{ Environment } \\
\hline Forest area (\%) & 22 & 24 & 26 \\
\hline Renewable water res withdrawn (\% of total) & & & 82 \\
\hline Terrestrial protect areas (\% total land area) & 9 & 11 & 11 \\
\hline Organic area (\% total agricultural area) & & & 1 \\
\hline Water withdrawal by agriculture (\% of total) & & & 82 \\
\hline Biofuel production (thousand kt of oil eq.) & 55 & 52 & 2975 \\
\hline \multicolumn{4}{|l|}{ Wood pellet prod. (1 000 tonnes) } \\
\hline Net GHG emissions from AFOLU ( $\mathrm{CO}_{2}$ eq, $\left.\mathrm{Mt}\right)$ & 51 & 42 & 50 \\
\hline
\end{tabular}




\section{Poland}

\begin{tabular}{|c|c|c|c|}
\hline & 1990 & 2000 & 2014 \\
\hline \multicolumn{4}{|l|}{ The setting } \\
\hline Population, total (mln) & 38.1 & 38.4 & 38.2 \\
\hline Population, rural (mln) & 14.8 & 14.7 & 15 \\
\hline \multicolumn{4}{|l|}{ Govt expenditure on ag (\% total outlays) } \\
\hline Area harvested (mln ha) & 36 & 24 & 28 \\
\hline Cropping intensity ratio & 1.9 & 1.3 & \\
\hline Water resources (1 $000 \mathrm{~m}^{3} /$ person/year) & 2 & 2 & 2 \\
\hline Area equipped for irrigation (1 $000 \mathrm{ha})$ & & & 97 \\
\hline Area irrigated (\% area equipped for irrigation) & & & 62.3 \\
\hline Employment in agriculture (\%) & 25.2 & 18.8 & 12.6 \\
\hline Employment in agriculture, female (\%) & & 18.3 & 11.7 \\
\hline Fertilizers, Nitrogen (kg of nutrients per ha) & & 71.1 & 136.5 \\
\hline Fertilizers, Phosphate (kg of nutrients per ha) & & 25.6 & 36 \\
\hline Fertilizers, Potash (kg nutrients per ha) & & 32.2 & 40.8 \\
\hline Energy consump, power irrigation (mln kWh) & 3 & 8 & 25 \\
\hline Agr value added per worker (constant US\$) & & 1970 & 3320 \\
\hline \multicolumn{4}{|l|}{ Hunger dimensions } \\
\hline \multicolumn{4}{|l|}{ Dietary energy supply (kcal/pc/day) } \\
\hline Average dietary energy supply adequacy (\%) & 136 & 136 & 139 \\
\hline Dietary en supp, cereals/roots/tubers (\%) & 43 & 42 & 41 \\
\hline Prevalence of undernourishment (\%) & $<5.0$ & $<5.0$ & $<5.0$ \\
\hline GDP per capita (US\$, PPP) & 10080 & 14553 & 22835 \\
\hline Domestic food price volatility (index) & & 5.4 & 7 \\
\hline Cereal import dependency ratio (\%) & 5.1 & 5.3 & -2.5 \\
\hline \multicolumn{4}{|l|}{ Underweight, children under-5 (\%) } \\
\hline \multicolumn{4}{|l|}{ Improved water source (\% pop) } \\
\hline \multicolumn{4}{|l|}{ Food supply } \\
\hline Food production value, (2004-2006 mln I\$) & 18397 & 15289 & 17813 \\
\hline Agriculture, value added (\% GDP) & & 3 & 3 \\
\hline Food exports (mln US\$) & 1388 & 1930 & 17021 \\
\hline Food imports (mln US\$) & 536 & 1743 & 10716 \\
\hline \multicolumn{4}{|l|}{ Production indices $(2004-06=100)$} \\
\hline Net food & 111 & 92 & 107 \\
\hline Net crops & 133 & 108 & 106 \\
\hline Cereals & 104 & 84 & 111 \\
\hline Vegetable oils & 78 & 60 & 170 \\
\hline Roots and tubers & 330 & 222 & 56 \\
\hline Fruit and vegetables & 71 & 84 & 118 \\
\hline Sugar & 139 & 109 & 88 \\
\hline Livestock & 116 & 94 & 108 \\
\hline Milk & 133 & 100 & 107 \\
\hline Meat & 106 & 91 & 109 \\
\hline Fish & 237 & 126 & 124 \\
\hline \multicolumn{4}{|l|}{ Net trade (mln US\$) } \\
\hline Cereals & -211 & -267 & 845 \\
\hline Fruit and vegetables & 273 & 61 & 1228 \\
\hline Meat & 288 & 228 & 2657 \\
\hline Dairy products & 88 & 138 & 1186 \\
\hline Fish & 142 & -55 & -53 \\
\hline \multicolumn{4}{|l|}{ Environment } \\
\hline Forest area (\%) & 29 & 30 & 31 \\
\hline Renewable water res withdrawn (\% of total) & & & 10 \\
\hline Terrestrial protect areas (\% total land area) & 16 & 23 & 34 \\
\hline Organic area (\% total agricultural area) & & & 5 \\
\hline Water withdrawal by agriculture (\% of total) & & & 10 \\
\hline Biofuel production (thousand kt of oil eq.) & 0 & 1 & 7194 \\
\hline Wood pellet prod. (1 000 tonnes) & & & 620 \\
\hline Net GHG emissions from AFOLU ( $\mathrm{CO}_{2}$ eq, $\mathrm{Mt}$ ) & 20 & 3 & -12 \\
\hline
\end{tabular}




\section{Portugal}

\begin{tabular}{|c|c|c|c|}
\hline & 1990 & 2000 & 2014 \\
\hline \multicolumn{4}{|l|}{ The setting } \\
\hline Population, total (mln) & 9.9 & 10.3 & 10.6 \\
\hline Population, rural (mln) & 5.2 & 4.7 & 4 \\
\hline \multicolumn{4}{|l|}{ Govt expenditure on ag (\% total outlays) } \\
\hline Area harvested (mln ha) & 2 & 2 & 1 \\
\hline Cropping intensity ratio & 0.6 & 0.6 & \\
\hline Water resources (1 $000 \mathrm{~m}^{3} /$ person/year) & 8 & 8 & 7 \\
\hline Area equipped for irrigation (1 $000 \mathrm{ha})$ & & & 540 \\
\hline Area irrigated (\% area equipped for irrigation) & & & 72.2 \\
\hline Employment in agriculture (\%) & 17.9 & 12.5 & 10.5 \\
\hline Employment in agriculture, female (\%) & 21.1 & 14.2 & 8.7 \\
\hline Fertilizers, Nitrogen (kg of nutrients per ha) & & 71.9 & 93.4 \\
\hline Fertilizers, Phosphate (kg of nutrients per ha) & & 58.5 & 34.2 \\
\hline Fertilizers, Potash (kg nutrients per ha) & & 46.6 & 28.5 \\
\hline Energy consump, power irrigation (mln kWh) & 45 & 125 & 125 \\
\hline Agr value added per worker (constant US\$) & & 6739 & 9588 \\
\hline \multicolumn{4}{|l|}{ Hunger dimensions } \\
\hline \multicolumn{4}{|l|}{ Dietary energy supply (kcal/pc/day) } \\
\hline Average dietary energy supply adequacy (\%) & 138 & 141 & 133 \\
\hline Dietary en supp, cereals/roots/tubers (\%) & 36 & 32 & 32 \\
\hline Prevalence of undernourishment (\%) & $<5.0$ & $<5.0$ & $<5.0$ \\
\hline GDP per capita (US\$, PPP) & 20282 & 26147 & 25933 \\
\hline Domestic food price volatility (index) & & 8.2 & 9 \\
\hline Cereal import dependency ratio (\%) & 60.1 & 66.8 & 77.1 \\
\hline \multicolumn{4}{|l|}{ Underweight, children under-5 (\%) } \\
\hline Improved water source (\% pop) & 96.1 & 97.9 & 99.8 \\
\hline \multicolumn{4}{|l|}{ Food supply } \\
\hline Food production value, (2004-2006 mln I\$) & 4016 & 4046 & 4240 \\
\hline Agriculture, value added (\% GDP) & & 4 & 2 \\
\hline Food exports (mln US\$) & 371 & 694 & 3367 \\
\hline Food imports (mln US\$) & 1691 & 2625 & 7452 \\
\hline \multicolumn{4}{|l|}{ Production indices $(2004-06=100)$} \\
\hline Net food & 99 & 99 & 104 \\
\hline Net crops & 111 & 101 & 105 \\
\hline Cereals & 124 & 141 & 112 \\
\hline Vegetable oils & 76 & 91 & 116 \\
\hline Roots and tubers & 197 & 114 & 74 \\
\hline Fruit and vegetables & 111 & 95 & 109 \\
\hline Sugar & 3 & 89 & 3 \\
\hline Livestock & 85 & 101 & 103 \\
\hline Milk & 80 & 103 & 93 \\
\hline Meat & 89 & 102 & 108 \\
\hline Fish & 143 & 86 & 88 \\
\hline \multicolumn{4}{|l|}{ Net trade (mln US\$) } \\
\hline Cereals & -333 & -517 & -1293 \\
\hline Fruit and vegetables & -202 & -327 & -151 \\
\hline Meat & -259 & -444 & -838 \\
\hline Dairy products & 20 & -60 & -249 \\
\hline Fish & -326 & -579 & -858 \\
\hline \multicolumn{4}{|l|}{ Environment } \\
\hline Forest area (\%) & 36 & 37 & 38 \\
\hline Renewable water res withdrawn (\% of total) & & 73 & 73 \\
\hline Terrestrial protect areas (\% total land area) & 7 & 22 & 22 \\
\hline Organic area (\% total agricultural area) & & & 6 \\
\hline Water withdrawal by agriculture (\% of total) & & 73 & 73 \\
\hline Biofuel production (thousand kt of oil eq.) & 34 & 29 & 8629 \\
\hline Wood pellet prod. (1 000 tonnes) & & & 800 \\
\hline Net GHG emissions from AFOLU ( $\mathrm{CO}_{2}$ eq, $\left.\mathrm{Mt}\right)$ & 9 & 8 & 6 \\
\hline
\end{tabular}




\begin{tabular}{|c|c|c|c|}
\hline & 1990 & 2000 & 2014 \\
\hline \multicolumn{4}{|l|}{ The setting } \\
\hline Population, total (mln) & 43 & 46 & 49.5 \\
\hline Population, rural (mln) & 11.2 & 9.4 & 7.9 \\
\hline Govt expenditure on ag (\% total outlays) & & 5.9 & 2.9 \\
\hline Area harvested (mln ha) & 10 & 12 & 6 \\
\hline Cropping intensity ratio & 4.6 & 6.2 & \\
\hline Water resources (1 $000 \mathrm{~m}^{3} /$ person/year) & 2 & 2 & 1 \\
\hline Area equipped for irrigation (1 $000 \mathrm{ha})$ & & & 778 \\
\hline Area irrigated (\% area equipped for irrigation) & & & 100 \\
\hline Employment in agriculture (\%) & 17.9 & 10.6 & 6.6 \\
\hline Employment in agriculture, female (\%) & 20.3 & 12.2 & 6.9 \\
\hline Fertilizers, Nitrogen (kg of nutrients per ha) & & 202.4 & 198.9 \\
\hline Fertilizers, Phosphate (kg of nutrients per ha) & & 132.3 & 140.2 \\
\hline Fertilizers, Potash (kg nutrients per ha) & & 134.1 & 141.9 \\
\hline Energy consump, power irrigation (mln kWh) & 0 & 0 & 0 \\
\hline Agr value added per worker (constant US\$) & 5936 & 11116 & 28795 \\
\hline \multicolumn{4}{|l|}{ Hunger dimensions } \\
\hline Dietary energy supply (kcal/pc/day) & 2981 & 3087 & 3443 \\
\hline Average dietary energy supply adequacy (\%) & 124 & 128 & 140 \\
\hline Dietary en supp, cereals/roots/tubers (\%) & 53 & 49 & 44 \\
\hline Prevalence of undernourishment (\%) & $<5.0$ & $<5.0$ & $<5.0$ \\
\hline GDP per capita (US\$, PPP) & 12087 & 20757 & 32708 \\
\hline Domestic food price volatility (index) & & 9 & 9.1 \\
\hline Cereal import dependency ratio (\%) & 68.9 & 70.8 & 74.2 \\
\hline Underweight, children under-5 (\%) & & 0.9 & 0.6 \\
\hline Improved water source (\% pop) & 90.1 & 93.4 & 97.8 \\
\hline \multicolumn{4}{|l|}{ Food supply } \\
\hline Food production value, (2004-2006 mln I\$) & 7778 & 10168 & 10238 \\
\hline Agriculture, value added (\% GDP) & 8 & 4 & 2 \\
\hline Food exports (mln US\$) & 639 & 980 & 2705 \\
\hline Food imports (mln US\$) & 2882 & 4993 & 16704 \\
\hline \multicolumn{4}{|l|}{ Production indices (2004-06=100) } \\
\hline Net food & 79 & 103 & 104 \\
\hline Net crops & 92 & 105 & 94 \\
\hline Cereals & 120 & 110 & 86 \\
\hline Vegetable oils & 170 & 111 & 96 \\
\hline Roots and tubers & 65 & 100 & 102 \\
\hline Fruit and vegetables & 75 & 102 & 96 \\
\hline \multicolumn{4}{|l|}{ Sugar } \\
\hline Livestock & 62 & 101 & 119 \\
\hline Milk & 79 & 101 & 94 \\
\hline Meat & 58 & 104 & 126 \\
\hline Fish & 135 & 100 & 95 \\
\hline \multicolumn{4}{|l|}{ Net trade (mln US\$) } \\
\hline Cereals & -1303 & -1513 & -4752 \\
\hline Fruit and vegetables & 26 & -283 & -2343 \\
\hline Meat & -296 & -1078 & -2944 \\
\hline Dairy products & -14 & -133 & -595 \\
\hline Fish & 998 & 2 & -1723 \\
\hline \multicolumn{4}{|l|}{ Environment } \\
\hline Forest area $(\%)$ & 66 & 65 & 64 \\
\hline Renewable water res withdrawn (\% of total) & & 62 & 62 \\
\hline Terrestrial protect areas (\% total land area) & 4 & 4 & 6 \\
\hline Organic area (\% total agricultural area) & & & 1 \\
\hline Water withdrawal by agriculture (\% of total) & & 62 & 62 \\
\hline Biofuel production (thousand kt of oil eq.) & 1 & 7 & 8904 \\
\hline Wood pellet prod. (1 000 tonnes) & & & 15 \\
\hline Net GHG emissions from AFOLU ( $\mathrm{CO}_{2}$ eq, $\mathrm{Mt}$ ) & -13 & -13 & -18 \\
\hline
\end{tabular}




\begin{tabular}{|c|c|c|c|}
\hline & 1990 & 2000 & 2014 \\
\hline \multicolumn{4}{|l|}{ The setting } \\
\hline Population, total (mln) & 4.4 & 4.1 & 3.5 \\
\hline Population, rural (mln) & 2.3 & 2.3 & 1.7 \\
\hline Govt expenditure on ag (\% total outlays) & & 2.2 & 4.1 \\
\hline Area harvested (mln ha) & & 2 & 3 \\
\hline Cropping intensity ratio & & 0.7 & \\
\hline Water resources (1 $000 \mathrm{~m}^{3} /$ person/year) & 3 & 3 & 3 \\
\hline Area equipped for irrigation (1 $000 \mathrm{ha})$ & & & 228 \\
\hline Area irrigated (\% area equipped for irrigation) & 72.1 & & \\
\hline Employment in agriculture (\%) & 33.8 & 50.9 & 26.4 \\
\hline Employment in agriculture, female (\%) & & 49.8 & 23.2 \\
\hline Fertilizers, Nitrogen (kg of nutrients per ha) & & 7.2 & 14.8 \\
\hline Fertilizers, Phosphate (kg of nutrients per ha) & & 0.2 & 3.1 \\
\hline Fertilizers, Potash (kg nutrients per ha) & & 0.1 & 1.2 \\
\hline Energy consump, power irrigation (mln kWh) & 14 & 14 & 309 \\
\hline Agr value added per worker (constant US\$) & 935 & 845 & 2638 \\
\hline \multicolumn{4}{|l|}{ Hunger dimensions } \\
\hline \multicolumn{4}{|l|}{ Dietary energy supply (kcal/pc/day) } \\
\hline Average dietary energy supply adequacy (\%) & 104 & 103 & 116 \\
\hline Dietary en supp, cereals/roots/tubers (\%) & 53 & 61 & 46 \\
\hline Prevalence of undernourishment (\%) & $<5.0$ & $<5.0$ & $<5.0$ \\
\hline GDP per capita (US\$, PPP) & 6419 & 2321 & 4521 \\
\hline Domestic food price volatility (index) & & 11.2 & 5.7 \\
\hline Cereal import dependency ratio (\%) & 12.9 & -7.3 & -12.5 \\
\hline Underweight, children under-5 (\%) & & & 2.2 \\
\hline Improved water source (\% pop) & 92.7 & 93.4 & 96.5 \\
\hline \multicolumn{4}{|l|}{ Food supply } \\
\hline Food production value, (2004-2006 mln I\$) & 2362 & 1220 & 1262 \\
\hline Agriculture, value added (\% GDP) & 36 & 29 & 15 \\
\hline Food exports (mln US\$) & 154 & 123 & 604 \\
\hline Food imports (mln US\$) & 60 & 37 & 464 \\
\hline \multicolumn{4}{|l|}{ Production indices $(2004-06=100)$} \\
\hline Net food & 164 & 85 & 88 \\
\hline Net crops & 170 & 89 & 102 \\
\hline Cereals & 123 & 70 & 101 \\
\hline Vegetable oils & 52 & 72 & 151 \\
\hline Roots and tubers & 204 & 92 & 67 \\
\hline Fruit and vegetables & 207 & 102 & 102 \\
\hline Sugar & 200 & 92 & 98 \\
\hline Livestock & 174 & 94 & 96 \\
\hline Milk & 152 & 90 & 83 \\
\hline Meat & 232 & 103 & 115 \\
\hline Fish & 150 & 27 & 152 \\
\hline \multicolumn{4}{|l|}{ Net trade (mln US\$) } \\
\hline Cereals & -52 & 6 & -42 \\
\hline Fruit and vegetables & 92 & 43 & 149 \\
\hline Meat & 32 & 10 & -23 \\
\hline Dairy products & 7 & 5 & -28 \\
\hline Fish & & -4 & -53 \\
\hline \multicolumn{4}{|l|}{ Environment } \\
\hline Forest area $(\%)$ & 10 & 10 & 12 \\
\hline Renewable water res withdrawn (\% of total) & & & 4 \\
\hline Terrestrial protect areas (\% total land area) & 1 & 2 & 4 \\
\hline Organic area (\% total agricultural area) & & & 1 \\
\hline Water withdrawal by agriculture (\% of total) & & & 4 \\
\hline Biofuel production (thousand kt of oil eq.) & & 0 & 0 \\
\hline Wood pellet prod. (1 000 tonnes) & & & 0 \\
\hline Net GHG emissions from AFOLU ( $\mathrm{CO}_{2}$ eq, $\mathrm{Mt}$ ) & 2 & 1 & 1 \\
\hline
\end{tabular}




\begin{tabular}{|c|c|c|c|}
\hline & 1990 & 2000 & 2014 \\
\hline \multicolumn{4}{|l|}{ The setting } \\
\hline Population, total (mln) & 23.4 & 22.4 & 21.6 \\
\hline Population, rural (mln) & 10.9 & 10.5 & 10.2 \\
\hline \multicolumn{4}{|l|}{ Govt expenditure on ag (\% total outlays) } \\
\hline Area harvested (mln ha) & 17 & 10 & 21 \\
\hline Cropping intensity ratio & 1.2 & 0.7 & \\
\hline Water resources (1 $000 \mathrm{~m}^{3} /$ person/year) & 9 & 9 & 10 \\
\hline Area equipped for irrigation (1 $000 \mathrm{ha})$ & & & 3149 \\
\hline Area irrigated (\% area equipped for irrigation) & & & 28.2 \\
\hline Employment in agriculture (\%) & 29.1 & 42.8 & 29 \\
\hline Employment in agriculture, female (\%) & 34.3 & 45.5 & 30.1 \\
\hline Fertilizers, Nitrogen (kg of nutrients per ha) & & 26.9 & 33 \\
\hline Fertilizers, Phosphate (kg of nutrients per ha) & & 10.1 & 12.8 \\
\hline Fertilizers, Potash (kg nutrients per ha) & & 1.6 & 4 \\
\hline Energy consump, power irrigation (mln kWh) & 5025 & 5025 & 872 \\
\hline Agr value added per worker (constant US\$) & 3825 & 3839 & 9117 \\
\hline \multicolumn{4}{|l|}{ Hunger dimensions } \\
\hline \multicolumn{4}{|l|}{ Dietary energy supply (kcal/pc/day) } \\
\hline Average dietary energy supply adequacy (\%) & 120 & 127 & 136 \\
\hline Dietary en supp, cereals/roots/tubers (\%) & 49 & 49 & 45 \\
\hline Prevalence of undernourishment (\%) & $<5.0$ & $<5.0$ & $<5.0$ \\
\hline GDP per capita (US\$, PPP) & 11449 & 9970 & 18184 \\
\hline Domestic food price volatility (index) & & 12.6 & 4.3 \\
\hline Cereal import dependency ratio (\%) & 9.7 & -0.2 & -22.6 \\
\hline Underweight, children under-5 (\%) & 5 & 3.7 & \\
\hline Improved water source (\% pop) & 75.3 & 84.2 & 87.7 \\
\hline \multicolumn{4}{|l|}{ Food supply } \\
\hline Food production value, (2004-2006 mln I\$) & 8050 & 7090 & 8556 \\
\hline Agriculture, value added (\% GDP) & 24 & 12 & 5 \\
\hline Food exports (mln US\$) & 58 & 276 & 4012 \\
\hline Food imports (mln US\$) & 875 & 599 & 4203 \\
\hline \multicolumn{4}{|l|}{ Production indices $(2004-06=100)$} \\
\hline Net food & 93 & 81 & 98 \\
\hline Net crops & 82 & 71 & 103 \\
\hline Cereals & 85 & 51 & 106 \\
\hline Vegetable oils & 38 & 47 & 168 \\
\hline Roots and tubers & 71 & 83 & 73 \\
\hline Fruit and vegetables & 85 & 95 & 93 \\
\hline Sugar & 385 & 78 & 121 \\
\hline Livestock & 114 & 89 & 86 \\
\hline Milk & 65 & 78 & 86 \\
\hline Meat & 166 & 103 & 86 \\
\hline Fish & 927 & 124 & 111 \\
\hline \multicolumn{4}{|l|}{ Net trade (mln US\$) } \\
\hline Cereals & -141 & -75 & 944 \\
\hline Fruit and vegetables & -50 & -75 & -478 \\
\hline Meat & -214 & -72 & -226 \\
\hline Dairy products & -45 & -16 & -203 \\
\hline Fish & -37 & -27 & -184 \\
\hline \multicolumn{4}{|l|}{ Environment } \\
\hline Forest area (\%) & 28 & 28 & 29 \\
\hline Renewable water res withdrawn (\% of total) & & & 17 \\
\hline Terrestrial protect areas (\% total land area) & 18 & 17 & 19 \\
\hline Organic area (\% total agricultural area) & & & 2 \\
\hline Water withdrawal by agriculture (\% of total) & & & 17 \\
\hline Biofuel production (thousand kt of oil eq.) & & 16 & 330 \\
\hline Wood pellet prod. (1 000 tonnes) & & & 810 \\
\hline Net GHG emissions from AFOLU ( $\mathrm{CO}_{2}$ eq, $\mathrm{Mt}$ ) & 31 & 17 & 4 \\
\hline
\end{tabular}




\section{Russian Federation}

\begin{tabular}{|c|c|c|c|}
\hline & 1990 & 2000 & 2014 \\
\hline \multicolumn{4}{|l|}{ The setting } \\
\hline Population, total (mln) & 148.8 & 146.8 & 142.5 \\
\hline Population, rural (mln) & 39.6 & 39.1 & 36.6 \\
\hline \multicolumn{4}{|l|}{ Govt expenditure on ag (\% total outlays) } \\
\hline Area harvested (mln ha) & & 64 & 90 \\
\hline Cropping intensity ratio & & 0.3 & \\
\hline Water resources (1 $000 \mathrm{~m}^{3} /$ person/year) & 30 & 31 & 32 \\
\hline Area equipped for irrigation (1 $000 \mathrm{ha}$ ) & & & 4300 \\
\hline Area irrigated (\% area equipped for irrigation) & & & 39.5 \\
\hline Employment in agriculture (\%) & 13.9 & 14.5 & 9.7 \\
\hline Employment in agriculture, female (\%) & & 11.7 & 6.7 \\
\hline Fertilizers, Nitrogen (kg of nutrients per ha) & & 6.9 & 9.9 \\
\hline Fertilizers, Phosphate (kg of nutrients per ha) & & 2.5 & 3.5 \\
\hline Fertilizers, Potash (kg nutrients per ha) & & 1.6 & 2.3 \\
\hline \multicolumn{4}{|l|}{ Energy consump, power irrigation (mln kWh) } \\
\hline Agr value added per worker (constant US\$) & 3635 & 3743 & 5973 \\
\hline \multicolumn{4}{|l|}{ Hunger dimensions } \\
\hline \multicolumn{4}{|l|}{ Dietary energy supply (kcal/pc/day) } \\
\hline Average dietary energy supply adequacy (\%) & 120 & 116 & 136 \\
\hline Dietary en supp, cereals/roots/tubers (\%) & 49 & 47 & 41 \\
\hline Prevalence of undernourishment (\%) & $<5.0$ & $<5.0$ & $<5.0$ \\
\hline GDP per capita (US\$, PPP) & 19349 & 13173 & 23564 \\
\hline Domestic food price volatility (index) & & 6.3 & 5.2 \\
\hline Cereal import dependency ratio (\%) & 14.8 & 5 & -27.5 \\
\hline \multicolumn{4}{|l|}{ Underweight, children under-5 (\%) } \\
\hline Improved water source (\% pop) & 93.2 & 95.1 & 97 \\
\hline \multicolumn{4}{|l|}{ Food supply } \\
\hline Food production value, (2004-2006 mln I\$) & 45882 & 33710 & 46439 \\
\hline Agriculture, value added (\% GDP) & 17 & 6 & 4 \\
\hline Food exports (mln US\$) & 137 & 797 & 11606 \\
\hline Food imports (mln US\$) & 8892 & 4942 & 28907 \\
\hline \multicolumn{4}{|l|}{ Production indices (2004-06=100) } \\
\hline Net food & 121 & 89 & 122 \\
\hline Net crops & 95 & 79 & 116 \\
\hline Cereals & 116 & 80 & 122 \\
\hline Vegetable oils & 46 & 64 & 195 \\
\hline Roots and tubers & 96 & 86 & 82 \\
\hline Fruit and vegetables & 70 & 82 & 97 \\
\hline Sugar & 103 & 57 & 160 \\
\hline Livestock & 151 & 97 & 122 \\
\hline Milk & 147 & 102 & 97 \\
\hline Meat & 161 & 93 & 150 \\
\hline Fish & 234 & 124 & 138 \\
\hline \multicolumn{4}{|l|}{ Net trade (mln US\$) } \\
\hline Cereals & -2068 & -600 & 5160 \\
\hline Fruit and vegetables & -1613 & -1145 & -9689 \\
\hline Meat & -1554 & -1025 & -7087 \\
\hline Dairy products & -552 & -153 & -2805 \\
\hline Fish & 1452 & 1333 & 411 \\
\hline \multicolumn{4}{|l|}{ Environment } \\
\hline Forest area (\%) & 49 & 49 & 49 \\
\hline Renewable water res withdrawn (\% of total) & & 20 & 20 \\
\hline Terrestrial protect areas (\% total land area) & 5 & 11 & 11 \\
\hline Organic area (\% total agricultural area) & & & 0 \\
\hline Water withdrawal by agriculture (\% of total) & & 20 & 20 \\
\hline \multicolumn{4}{|l|}{ Biofuel production (thousand kt of oil eq.) } \\
\hline Wood pellet prod. (1 000 tonnes) & & & 892 \\
\hline Net GHG emissions from AFOLU ( $\mathrm{CO}_{2}$ eq, $\mathrm{Mt}$ ) & 404 & 310 & 25 \\
\hline
\end{tabular}




\begin{tabular}{|c|c|c|c|}
\hline & 1990 & 2000 & 2014 \\
\hline \multicolumn{4}{|l|}{ The setting } \\
\hline Population, total (mln) & 7.2 & 8.4 & 12.1 \\
\hline Population, rural (mln) & 6.8 & 7.2 & 9.7 \\
\hline \multicolumn{4}{|l|}{ Govt expenditure on ag (\% total outlays) } \\
\hline Area harvested (mln ha) & 3 & 3 & 6 \\
\hline Cropping intensity ratio & 1.5 & 1.7 & \\
\hline Water resources (1 $000 \mathrm{~m}^{3} /$ person/year) & 2 & 2 & 1 \\
\hline Area equipped for irrigation (1 $000 \mathrm{ha})$ & & & 10 \\
\hline Area irrigated (\% area equipped for irrigation) & & & 82.5 \\
\hline Employment in agriculture (\%) & 90.1 & & 78.8 \\
\hline Employment in agriculture, female (\%) & 95.9 & & \\
\hline Fertilizers, Nitrogen (kg of nutrients per ha) & & 0.8 & 1.9 \\
\hline Fertilizers, Phosphate (kg of nutrients per ha) & & 0.7 & 2.1 \\
\hline Fertilizers, Potash (kg nutrients per ha) & & 0.7 & 0 \\
\hline \multicolumn{4}{|l|}{ Energy consump, power irrigation (mln kWh) } \\
\hline Agr value added per worker (constant US\$) & 198 & 228 & 312 \\
\hline \multicolumn{4}{|l|}{ Hunger dimensions } \\
\hline Dietary energy supply (kcal/pc/day) & 1822 & 1781 & 2232 \\
\hline Average dietary energy supply adequacy (\%) & 86 & 86 & 104 \\
\hline Dietary en supp, cereals/roots/tubers (\%) & 50 & 53 & 50 \\
\hline Prevalence of undernourishment (\%) & 56.1 & 60.6 & 32.7 \\
\hline GDP per capita (US\$, PPP) & 856 & 764 & 1426 \\
\hline Domestic food price volatility (index) & & 15.3 & 10.5 \\
\hline Cereal import dependency ratio (\%) & 32.8 & 19.6 & 23.7 \\
\hline Underweight, children under-5 (\%) & 24.3 & 20.3 & 11.7 \\
\hline Improved water source (\% pop) & 60.3 & 66.2 & 70.7 \\
\hline \multicolumn{4}{|l|}{ Food supply } \\
\hline Food production value, (2004-2006 mln I\$) & 1035 & 1175 & 2472 \\
\hline Agriculture, value added (\% GDP) & 33 & 37 & 33 \\
\hline Food exports (mln US\$) & 0 & 0 & 49 \\
\hline Food imports (mln US\$) & 42 & 50 & 224 \\
\hline \multicolumn{4}{|l|}{ Production indices $(2004-06=100)$} \\
\hline Net food & 72 & 82 & 173 \\
\hline Net crops & 76 & 84 & 175 \\
\hline Cereals & 65 & 60 & 254 \\
\hline Vegetable oils & 83 & 64 & 127 \\
\hline Roots and tubers & 40 & 91 & 216 \\
\hline Fruit and vegetables & 92 & 80 & 148 \\
\hline Sugar & 61 & 68 & 195 \\
\hline Livestock & 59 & 72 & 138 \\
\hline Milk & 62 & 78 & 141 \\
\hline Meat & 58 & 69 & 138 \\
\hline Fish & 33 & 92 & 325 \\
\hline \multicolumn{4}{|l|}{ Net trade (mln US\$) } \\
\hline Cereals & -17 & -21 & -55 \\
\hline Fruit and vegetables & -3 & -2 & -10 \\
\hline Meat & & 0 & 0 \\
\hline Dairy products & -5 & -1 & -1 \\
\hline Fish & 0 & 0 & -7 \\
\hline \multicolumn{4}{|l|}{ Environment } \\
\hline Forest area (\%) & 13 & 14 & 18 \\
\hline Renewable water res withdrawn (\% of total) & & 68 & 68 \\
\hline Terrestrial protect areas (\% total land area) & 10 & 10 & 11 \\
\hline Organic area (\% total agricultural area) & & & 0 \\
\hline Water withdrawal by agriculture (\% of total) & & 68 & 68 \\
\hline Biofuel production (thousand kt of oil eq.) & 0 & 0 & 0 \\
\hline \multicolumn{4}{|l|}{ Wood pellet prod. (1 000 tonnes) } \\
\hline Net GHG emissions from AFOLU ( $\mathrm{CO}_{2}$ eq, $\mathrm{Mt}$ ) & 12 & 11 & 2 \\
\hline
\end{tabular}




\begin{tabular}{|c|c|c|c|}
\hline & 1990 & 2000 & 2014 \\
\hline \multicolumn{4}{|l|}{ The setting } \\
\hline Population, total (mln) & 0.2 & 0.2 & 0.2 \\
\hline Population, rural (mln) & 0.1 & 0.1 & 0.2 \\
\hline Govt expenditure on ag (\% total outlays) & & 3.9 & 1.8 \\
\hline Area harvested (mln ha) & 0 & 0 & 0 \\
\hline Cropping intensity ratio & 7.4 & 6.9 & \\
\hline \multicolumn{4}{|l|}{ Water resources (1 $000 \mathrm{~m}^{3} /$ person/year) } \\
\hline \multicolumn{4}{|l|}{ Area equipped for irrigation (1 $000 \mathrm{ha})$} \\
\hline \multicolumn{4}{|l|}{ Area irrigated (\% area equipped for irrigation) } \\
\hline Employment in agriculture (\%) & & 39.9 & \\
\hline Employment in agriculture, female (\%) & & 15.8 & \\
\hline Fertilizers, Nitrogen (kg of nutrients per ha) & & 0.5 & 0.2 \\
\hline Fertilizers, Phosphate (kg of nutrients per ha) & & 0.9 & 0.4 \\
\hline Fertilizers, Potash (kg nutrients per ha) & & 0.4 & 0.2 \\
\hline \multicolumn{4}{|l|}{ Energy consump, power irrigation (mln kWh) } \\
\hline Agr value added per worker (constant US\$) & & 2956 & 3019 \\
\hline \multicolumn{4}{|l|}{ Hunger dimensions } \\
\hline Dietary energy supply (kcal/pc/day) & 2394 & 2761 & 2908 \\
\hline Average dietary energy supply adequacy (\%) & 106 & 123 & 128 \\
\hline Dietary en supp, cereals/roots/tubers (\%) & 30 & 31 & 29 \\
\hline Prevalence of undernourishment (\%) & 13.9 & 6.6 & $<5.0$ \\
\hline GDP per capita (US\$, PPP) & 3657 & 4336 & 5584 \\
\hline \multicolumn{4}{|l|}{ Domestic food price volatility (index) } \\
\hline \multicolumn{4}{|l|}{ Cereal import dependency ratio (\%) } \\
\hline Underweight, children under-5 (\%) & & 1.7 & \\
\hline Improved water source (\% pop) & 88.9 & 93.3 & 98.5 \\
\hline \multicolumn{4}{|l|}{ Food supply } \\
\hline Food production value, (2004-2006 mln I\$) & 44 & 44 & 53 \\
\hline \multicolumn{4}{|l|}{ Agriculture, value added (\% GDP) } \\
\hline Food exports (mln US\$) & 7 & 3 & 6 \\
\hline Food imports (mln US\$) & 16 & 21 & 80 \\
\hline \multicolumn{4}{|l|}{ Production indices $(2004-06=100)$} \\
\hline Net food & 91 & 92 & 110 \\
\hline Net crops & 88 & 91 & 110 \\
\hline \multicolumn{4}{|l|}{ Cereals } \\
\hline Vegetable oils & 91 & 93 & 125 \\
\hline Roots and tubers & 119 & 86 & 104 \\
\hline Fruit and vegetables & 76 & 92 & 97 \\
\hline Sugar & 117 & 100 & 100 \\
\hline Livestock & 102 & 97 & 111 \\
\hline Milk & 74 & 92 & 91 \\
\hline Meat & 102 & 95 & 114 \\
\hline Fish & 14 & 81 & 106 \\
\hline \multicolumn{4}{|l|}{ Net trade (mln US\$) } \\
\hline Cereals & -5 & -3 & -21 \\
\hline Fruit and vegetables & 3 & 1 & -3 \\
\hline Meat & -5 & -9 & -29 \\
\hline Dairy products & -1 & -2 & -9 \\
\hline Fish & -3 & -1 & 1 \\
\hline \multicolumn{4}{|l|}{ Environment } \\
\hline Forest area (\%) & 46 & 60 & 60 \\
\hline \multicolumn{4}{|l|}{ Renewable water res withdrawn (\% of total) } \\
\hline Terrestrial protect areas (\% total land area) & 2 & 6 & 7 \\
\hline Organic area (\% total agricultural area) & & & 96 \\
\hline \multicolumn{4}{|l|}{ Water withdrawal by agriculture (\% of total) } \\
\hline Biofuel production (thousand kt of oil eq.) & 0 & 0 & 0 \\
\hline \multicolumn{4}{|l|}{ Wood pellet prod. (1 000 tonnes) } \\
\hline Net GHG emissions from AFOLU ( $\mathrm{CO}_{2}$ eq, $\left.\mathrm{Mt}\right)$ & 0 & -1 & 0 \\
\hline
\end{tabular}




\begin{tabular}{|c|c|c|c|}
\hline & 1990 & 2000 & 2014 \\
\hline \multicolumn{4}{|l|}{ The setting } \\
\hline Population, total (mln) & 0.1 & 0.1 & 0.2 \\
\hline Population, rural (mln) & 0.1 & 0.1 & 0.1 \\
\hline \multicolumn{4}{|l|}{ Govt expenditure on ag (\% total outlays) } \\
\hline Area harvested (mln ha) & 0 & 0 & 0 \\
\hline Cropping intensity ratio & 3 & 2 & \\
\hline Water resources (1 $000 \mathrm{~m}^{3} /$ person/year) & 19 & 16 & 11 \\
\hline Area equipped for irrigation (1 $000 \mathrm{ha})$ & & & 10 \\
\hline \multicolumn{4}{|l|}{ Area irrigated (\% area equipped for irrigation) } \\
\hline Employment in agriculture (\%) & 39.9 & 27.9 & \\
\hline Employment in agriculture, female (\%) & 35 & 22.8 & \\
\hline \multicolumn{4}{|l|}{ Fertilizers, Nitrogen (kg of nutrients per ha) } \\
\hline \multicolumn{4}{|l|}{ Fertilizers, Phosphate (kg of nutrients per ha) } \\
\hline \multicolumn{4}{|l|}{ Fertilizers, Potash (kg nutrients per ha) } \\
\hline \multicolumn{4}{|l|}{ Energy consump, power irrigation (mln kWh) } \\
\hline Agr value added per worker (constant US\$) & & & 725 \\
\hline \multicolumn{4}{|l|}{ Hunger dimensions } \\
\hline Dietary energy supply (kcal/pc/day) & 2202 & 2348 & 2655 \\
\hline Average dietary energy supply adequacy (\%) & 102 & 107 & 120 \\
\hline Dietary en supp, cereals/roots/tubers (\%) & 53 & 50 & 47 \\
\hline Prevalence of undernourishment (\%) & 24 & 21.5 & 6.4 \\
\hline GDP per capita (US\$, PPP) & & 2157 & 2876 \\
\hline Domestic food price volatility (index) & & 4.5 & 50.5 \\
\hline \multicolumn{4}{|l|}{ Cereal import dependency ratio (\%) } \\
\hline Underweight, children under-5 (\%) & & 10.1 & 14.4 \\
\hline Improved water source (\% pop) & & 78.2 & 97 \\
\hline \multicolumn{4}{|l|}{ Food supply } \\
\hline Food production value, (2004-2006 mln I\$) & 12 & 25 & 29 \\
\hline Agriculture, value added (\% GDP) & & 12 & 20 \\
\hline Food exports (mln US\$) & 3 & 4 & 5 \\
\hline Food imports (mln US\$) & 7 & 6 & 32 \\
\hline \multicolumn{4}{|l|}{ Production indices $(2004-06=100)$} \\
\hline Net food & 47 & 96 & 113 \\
\hline Net crops & 48 & 97 & 111 \\
\hline Cereals & 97 & 80 & 27 \\
\hline Vegetable oils & 36 & 78 & 142 \\
\hline Roots and tubers & 20 & 97 & 36 \\
\hline Fruit and vegetables & 29 & 88 & 128 \\
\hline \multicolumn{4}{|l|}{ Sugar } \\
\hline Livestock & 45 & 85 & 135 \\
\hline Milk & 83 & 97 & 121 \\
\hline Meat & 47 & 86 & 136 \\
\hline \multicolumn{4}{|l|}{ Fish } \\
\hline \multicolumn{4}{|l|}{ Net trade (mln US\$) } \\
\hline Cereals & & & -14 \\
\hline Fruit and vegetables & -1 & -1 & -3 \\
\hline \multicolumn{4}{|l|}{ Meat } \\
\hline Dairy products & & & -3 \\
\hline Fish & -1 & 0 & 0 \\
\hline \multicolumn{4}{|l|}{ Environment } \\
\hline Forest area (\%) & 28 & 28 & 28 \\
\hline \multicolumn{4}{|l|}{ Renewable water res withdrawn (\% of total) } \\
\hline \multicolumn{4}{|l|}{ Terrestrial protect areas (\% total land area) } \\
\hline Organic area (\% total agricultural area) & & & 8 \\
\hline Water withdrawal by agriculture (\% of total) & & & \\
\hline Biofuel production (thousand kt of oil eq.) & & & \\
\hline Wood pellet prod. (1 000 tonnes) & & & \\
\hline Net GHG emissions from AFOLU ( $\mathrm{CO}_{2}$ eq, $\mathrm{Mt}$ ) & 0 & 0 & 0 \\
\hline
\end{tabular}




\begin{tabular}{|c|c|c|c|}
\hline & 1990 & 2000 & 2014 \\
\hline \multicolumn{4}{|l|}{ The setting } \\
\hline Population, total (mln) & 16.2 & 20.1 & 29.4 \\
\hline Population, rural (mln) & 3.8 & 4.1 & 5 \\
\hline \multicolumn{4}{|l|}{ Govt expenditure on ag (\% total outlays) } \\
\hline Area harvested (mln ha) & 4 & 2 & 1 \\
\hline Cropping intensity ratio & 0 & 0 & \\
\hline Water resources (1 $000 \mathrm{~m}^{3} /$ person/year) & 0 & 0 & 0 \\
\hline Area equipped for irrigation (1 $000 \mathrm{ha}$ ) & & & 1620 \\
\hline Area irrigated (\% area equipped for irrigation) & 100 & & \\
\hline Employment in agriculture (\%) & 7.7 & 6.1 & 4.7 \\
\hline Employment in agriculture, female (\%) & 0.4 & 2.3 & 0.2 \\
\hline Fertilizers, Nitrogen (kg of nutrients per ha) & & 69.5 & 138.7 \\
\hline Fertilizers, Phosphate (kg of nutrients per ha) & & 45.7 & 216.8 \\
\hline Fertilizers, Potash (kg nutrients per ha) & & 3.9 & 1.1 \\
\hline Energy consump, power irrigation (mln kWh) & 2558 & 2558 & 2204 \\
\hline Agr value added per worker (constant US\$) & 8298 & 14933 & 27684 \\
\hline \multicolumn{4}{|l|}{ Hunger dimensions } \\
\hline Dietary energy supply (kcal/pc/day) & 2744 & 3089 & 3266 \\
\hline Average dietary energy supply adequacy (\%) & 123 & 137 & 137 \\
\hline Dietary en supp, cereals/roots/tubers (\%) & 52 & 51 & 48 \\
\hline Prevalence of undernourishment (\%) & $<5.0$ & $<5.0$ & $<5.0$ \\
\hline GDP per capita (US\$, PPP) & 35239 & 37066 & 52068 \\
\hline Domestic food price volatility (index) & & 5.6 & 3.8 \\
\hline Cereal import dependency ratio (\%) & 47.5 & 73.2 & 88.1 \\
\hline Underweight, children under-5 (\%) & & 13.5 & 5.3 \\
\hline Improved water source (\% pop) & 92 & 95 & 97 \\
\hline \multicolumn{4}{|l|}{ Food supply } \\
\hline Food production value, (2004-2006 mln I\$) & 2305 & 2651 & 3526 \\
\hline Agriculture, value added (\% GDP) & 6 & 5 & 2 \\
\hline Food exports (mln US\$) & 311 & 377 & 3308 \\
\hline Food imports (mln US\$) & 2964 & 4547 & 18512 \\
\hline \multicolumn{4}{|l|}{ Production indices $(2004-06=100)$} \\
\hline Net food & 71 & 81 & 108 \\
\hline Net crops & 85 & 75 & 89 \\
\hline Cereals & 131 & 69 & 31 \\
\hline Vegetable oils & 49 & 84 & 77 \\
\hline Roots and tubers & 14 & 91 & 108 \\
\hline Fruit and vegetables & 71 & 76 & 110 \\
\hline \multicolumn{4}{|l|}{ Sugar } \\
\hline Livestock & 57 & 89 & 129 \\
\hline Milk & 38 & 76 & 183 \\
\hline Meat & 63 & 95 & 107 \\
\hline Fish & 57 & 74 & 131 \\
\hline \multicolumn{4}{|l|}{ Net trade (mln US\$) } \\
\hline Cereals & -277 & -1539 & -5783 \\
\hline Fruit and vegetables & -511 & -585 & -1844 \\
\hline Meat & -403 & -563 & -2435 \\
\hline Dairy products & -436 & -464 & -891 \\
\hline Fish & -55 & -100 & -571 \\
\hline \multicolumn{4}{|l|}{ Environment } \\
\hline Forest area (\%) & 0 & 0 & 0 \\
\hline Renewable water res withdrawn (\% of total) & & & 88 \\
\hline Terrestrial protect areas (\% total land area) & 8 & 31 & 31 \\
\hline Organic area (\% total agricultural area) & & & 0 \\
\hline Water withdrawal by agriculture (\% of total) & & & 88 \\
\hline \multicolumn{4}{|l|}{ Biofuel production (thousand kt of oil eq.) } \\
\hline \multicolumn{4}{|l|}{ Wood pellet prod. (1 000 tonnes) } \\
\hline Net GHG emissions from AFOLU ( $\mathrm{CO}_{2}$ eq, $\mathrm{Mt}$ ) & 5 & 5 & 8 \\
\hline
\end{tabular}




\begin{tabular}{|c|c|c|c|}
\hline & 1990 & 2000 & 2014 \\
\hline \multicolumn{4}{|l|}{ The setting } \\
\hline Population, total (mln) & 7.5 & 9.9 & 14.5 \\
\hline Population, rural (mln) & 4.6 & 5.9 & 8.2 \\
\hline \multicolumn{4}{|l|}{ Govt expenditure on ag (\% total outlays) } \\
\hline Area harvested (mln ha) & 1 & 1 & 1 \\
\hline Cropping intensity ratio & 0.1 & 0.1 & \\
\hline Water resources (1 $000 \mathrm{~m}^{3} /$ person/year) & 5 & 4 & 3 \\
\hline Area equipped for irrigation (1 $000 \mathrm{ha}$ ) & & & 120 \\
\hline Area irrigated (\% area equipped for irrigation) & & 96.6 & \\
\hline Employment in agriculture (\%) & & 45.6 & 33.7 \\
\hline Employment in agriculture, female (\%) & & 43.7 & 33 \\
\hline Fertilizers, Nitrogen (kg of nutrients per ha) & & 4.4 & 6.2 \\
\hline Fertilizers, Phosphate (kg of nutrients per ha) & & 3.5 & 0.5 \\
\hline Fertilizers, Potash (kg nutrients per ha) & & 2.8 & 1.3 \\
\hline Energy consump, power irrigation (mln kWh) & 1 & 1 & 1 \\
\hline Agr value added per worker (constant US\$) & 400 & 412 & 354 \\
\hline \multicolumn{4}{|l|}{ Hunger dimensions } \\
\hline Dietary energy supply (kcal/pc/day) & 2161 & 2164 & 2208 \\
\hline Average dietary energy supply adequacy (\%) & 99 & 98 & 99 \\
\hline Dietary en supp, cereals/roots/tubers (\%) & 60 & 61 & 60 \\
\hline Prevalence of undernourishment (\%) & 27.1 & 29.4 & 23 \\
\hline GDP per capita (US\$, PPP) & 1856 & 1916 & 2170 \\
\hline Domestic food price volatility (index) & & 14.9 & 8.7 \\
\hline Cereal import dependency ratio (\%) & 39.5 & 48.3 & 46.9 \\
\hline Underweight, children under-5 (\%) & 21.9 & 20.3 & 16.8 \\
\hline Improved water source (\% pop) & 59.9 & 66.4 & 74.1 \\
\hline \multicolumn{4}{|l|}{ Food supply } \\
\hline Food production value, (2004-2006 mln I\$) & 823 & 1139 & 1369 \\
\hline Agriculture, value added (\% GDP) & 20 & 19 & 17 \\
\hline Food exports (mln US\$) & 155 & 115 & 318 \\
\hline Food imports (mln US\$) & 377 & 351 & 1533 \\
\hline \multicolumn{4}{|l|}{ Production indices $(2004-06=100)$} \\
\hline Net food & 77 & 107 & 128 \\
\hline Net crops & 78 & 111 & 125 \\
\hline Cereals & 85 & 92 & 125 \\
\hline Vegetable oils & 101 & 155 & 117 \\
\hline Roots and tubers & 30 & 58 & 67 \\
\hline Fruit and vegetables & 47 & 80 & 162 \\
\hline Sugar & 85 & 103 & 107 \\
\hline Livestock & 75 & 92 & 133 \\
\hline Milk & 77 & 102 & 191 \\
\hline Meat & 77 & 95 & 123 \\
\hline Fish & 79 & 109 & 117 \\
\hline \multicolumn{4}{|l|}{ Net trade (mln US\$) } \\
\hline Cereals & -180 & -170 & -726 \\
\hline Fruit and vegetables & -28 & -16 & -42 \\
\hline Meat & -6 & -5 & -14 \\
\hline Dairy products & -42 & -31 & -71 \\
\hline Fish & 169 & 259 & 251 \\
\hline \multicolumn{4}{|l|}{ Environment } \\
\hline Forest area (\%) & 49 & 46 & 44 \\
\hline Renewable water res withdrawn (\% of total) & & 93 & 93 \\
\hline Terrestrial protect areas (\% total land area) & 25 & 25 & 25 \\
\hline Organic area (\% total agricultural area) & & & 0 \\
\hline Water withdrawal by agriculture (\% of total) & & 93 & 93 \\
\hline Biofuel production (thousand kt of oil eq.) & 2 & 5 & 5 \\
\hline \multicolumn{4}{|l|}{ Wood pellet prod. (1 000 tonnes) } \\
\hline Net GHG emissions from AFOLU ( $\mathrm{CO}_{2}$ eq, $\left.\mathrm{Mt}\right)$ & 18 & 19 & 17 \\
\hline
\end{tabular}




\section{Serbia}

\begin{tabular}{|c|c|c|c|}
\hline & 1990 & 2000 & 2014 \\
\hline \multicolumn{4}{|l|}{ The setting } \\
\hline Population, total (mln) & & & 9.5 \\
\hline Population, rural (mln) & & & 4 \\
\hline Govt expenditure on ag (\% total outlays) & & & 2.5 \\
\hline Area harvested (mln ha) & & & 9 \\
\hline \multicolumn{4}{|l|}{ Cropping intensity ratio } \\
\hline Water resources (1 $000 \mathrm{~m}^{3} /$ person/year) & & & 17 \\
\hline Area equipped for irrigation (1 $000 \mathrm{ha}$ ) & & & 95 \\
\hline Area irrigated (\% area equipped for irrigation) & & & 37.2 \\
\hline Employment in agriculture (\%) & & & 21 \\
\hline Employment in agriculture, female (\%) & & & 18.9 \\
\hline Fertilizers, Nitrogen (kg of nutrients per ha) & & & 107.9 \\
\hline Fertilizers, Phosphate (kg of nutrients per ha) & & & 39.2 \\
\hline Fertilizers, Potash (kg nutrients per ha) & & & 28 \\
\hline \multicolumn{4}{|l|}{ Energy consump, power irrigation (mln kWh) } \\
\hline Agr value added per worker (constant US\$) & & & 5299 \\
\hline \multicolumn{4}{|l|}{ Hunger dimensions } \\
\hline \multicolumn{4}{|l|}{ Dietary energy supply (kcal/pc/day) } \\
\hline Average dietary energy supply adequacy (\%) & & & 108 \\
\hline Dietary en supp, cereals/roots/tubers (\%) & & & 40 \\
\hline Prevalence of undernourishment (\%) & $<5.0$ & $<5.0$ & $<5.0$ \\
\hline GDP per capita (US\$, PPP) & & & 12892 \\
\hline Domestic food price volatility (index) & & 23.7 & 8.5 \\
\hline Cereal import dependency ratio (\%) & & & -31.8 \\
\hline Underweight, children under-5 (\%) & & & 1.8 \\
\hline Improved water source (\% pop) & & & 99.2 \\
\hline \multicolumn{4}{|l|}{ Food supply } \\
\hline Food production value, $(2004-2006 \mathrm{mln}$ I\$) & & & 4062 \\
\hline Agriculture, value added (\% GDP) & & 20 & 10 \\
\hline Food exports (mln US\$) & & & 2207 \\
\hline Food imports (mln US\$) & & & 932 \\
\hline \multicolumn{4}{|l|}{ Production indices $(2004-06=100)$} \\
\hline Net food & & & 108 \\
\hline Net crops & & & 105 \\
\hline Cereals & & & 110 \\
\hline Vegetable oils & & & 122 \\
\hline Roots and tubers & & & 70 \\
\hline Fruit and vegetables & & & 107 \\
\hline Sugar & & & 94 \\
\hline Livestock & & & 101 \\
\hline Milk & & & 93 \\
\hline Meat & & & 104 \\
\hline Fish & 0 & 0 & 441 \\
\hline \multicolumn{4}{|l|}{ Net trade (mln US\$) } \\
\hline Cereals & & & 737 \\
\hline Fruit and vegetables & & & 231 \\
\hline Meat & & & -15 \\
\hline Dairy products & & & 21 \\
\hline Fish & & & -94 \\
\hline \multicolumn{4}{|l|}{ Environment } \\
\hline Forest area (\%) & & & 32 \\
\hline Renewable water res withdrawn (\% of total) & & & 2 \\
\hline Terrestrial protect areas (\% total land area) & & & 6 \\
\hline Organic area (\% total agricultural area) & & & 0 \\
\hline Water withdrawal by agriculture (\% of total) & & & 2 \\
\hline Biofuel production (thousand kt of oil eq.) & & & 0 \\
\hline Wood pellet prod. (1 000 tonnes) & & & 212 \\
\hline Net GHG emissions from AFOLU ( $\mathrm{CO}_{2}$ eq, $\left.\mathrm{Mt}\right)$ & & & -69 \\
\hline
\end{tabular}




\begin{tabular}{|c|c|c|c|}
\hline & 1990 & 2000 & 2014 \\
\hline \multicolumn{4}{|l|}{ The setting } \\
\hline Population, total (mln) & 4 & 4.1 & 6.2 \\
\hline Population, rural (mln) & 2.7 & 2.7 & 3.7 \\
\hline Govt expenditure on ag (\% total outlays) & & 2 & 1.1 \\
\hline Area harvested (mln ha) & 1 & 1 & 4 \\
\hline Cropping intensity ratio & 0.2 & 0.3 & \\
\hline Water resources (1 $000 \mathrm{~m}^{3} /$ person/year) & 40 & 39 & 26 \\
\hline Area equipped for irrigation (1 $000 \mathrm{ha})$ & & & 30 \\
\hline \multicolumn{4}{|l|}{ Area irrigated (\% area equipped for irrigation) } \\
\hline Employment in agriculture (\%) & & 67.3 & \\
\hline Employment in agriculture, female (\%) & & 69.5 & \\
\hline \multicolumn{4}{|l|}{ Fertilizers, Nitrogen (kg of nutrients per ha) } \\
\hline \multicolumn{4}{|l|}{ Fertilizers, Phosphate (kg of nutrients per ha) } \\
\hline \multicolumn{4}{|l|}{ Fertilizers, Potash (kg nutrients per ha) } \\
\hline Energy consump, power irrigation (mln kWh) & 0 & 0 & 0 \\
\hline Agr value added per worker (constant US\$) & 733 & 748 & 927 \\
\hline \multicolumn{4}{|l|}{ Hunger dimensions } \\
\hline Dietary energy supply (kcal/pc/day) & 2057 & 2018 & 2408 \\
\hline Average dietary energy supply adequacy (\%) & 96 & 94 & 111 \\
\hline Dietary en supp, cereals/roots/tubers (\%) & 61 & 64 & 63 \\
\hline Prevalence of undernourishment (\%) & 40.7 & 38 & 22.7 \\
\hline GDP per capita (US\$, PPP) & 1333 & 1006 & 1495 \\
\hline Domestic food price volatility (index) & & 13.7 & 3.3 \\
\hline Cereal import dependency ratio (\%) & 35 & 50.3 & 19.7 \\
\hline Underweight, children under-5 (\%) & 25.4 & 24.7 & 18.1 \\
\hline Improved water source (\% pop) & 36.7 & 47.3 & 60.1 \\
\hline \multicolumn{4}{|l|}{ Food supply } \\
\hline Food production value, $(2004-2006 \mathrm{mln}$ I\$) & 392 & 297 & 1172 \\
\hline Agriculture, value added (\% GDP) & 47 & 58 & 62 \\
\hline Food exports (mln US\$) & 7 & 2 & 30 \\
\hline Food imports (mln US\$) & 96 & 104 & 278 \\
\hline \multicolumn{4}{|l|}{ Production indices $(2004-06=100)$} \\
\hline Net food & 58 & 44 & 172 \\
\hline Net crops & 58 & 42 & 170 \\
\hline Cereals & 65 & 24 & 164 \\
\hline Vegetable oils & 84 & 61 & 121 \\
\hline Roots and tubers & 14 & 22 & 256 \\
\hline Fruit and vegetables & 73 & 77 & 128 \\
\hline Sugar & 100 & 116 & 110 \\
\hline Livestock & 90 & 77 & 198 \\
\hline Milk & 124 & 72 & 214 \\
\hline Meat & 78 & 73 & 191 \\
\hline Fish & 40 & 52 & 140 \\
\hline \multicolumn{4}{|l|}{ Net trade (mln US\$) } \\
\hline Cereals & -55 & -63 & -140 \\
\hline Fruit and vegetables & -3 & -6 & -16 \\
\hline Meat & -2 & -1 & -32 \\
\hline \multicolumn{4}{|l|}{ Dairy products } \\
\hline Fish & 14 & 10 & 9 \\
\hline \multicolumn{4}{|l|}{ Environment } \\
\hline Forest area (\%) & 43 & 40 & 37 \\
\hline Renewable water res withdrawn (\% of total) & & & 22 \\
\hline Terrestrial protect areas (\% total land area) & 4 & 7 & 10 \\
\hline Organic area (\% total agricultural area) & & & 2 \\
\hline Water withdrawal by agriculture (\% of total) & & & 22 \\
\hline Biofuel production (thousand kt of oil eq.) & 0 & 0 & 0 \\
\hline \multicolumn{4}{|l|}{ Wood pellet prod. (1 000 tonnes) } \\
\hline Net GHG emissions from AFOLU ( $\mathrm{CO}_{2}$ eq, $\mathrm{Mt}$ ) & 7 & 7 & 9 \\
\hline
\end{tabular}




\begin{tabular}{|c|c|c|c|}
\hline & 1990 & 2000 & 2014 \\
\hline \multicolumn{4}{|l|}{ The setting } \\
\hline Population, total (mln) & 5.3 & 5.4 & 5.5 \\
\hline Population, rural (mln) & 2.3 & 2.4 & 2.5 \\
\hline \multicolumn{4}{|l|}{ Govt expenditure on ag (\% total outlays) } \\
\hline Area harvested (mln ha) & & 2 & 3 \\
\hline Cropping intensity ratio & & 0.9 & \\
\hline Water resources (1 $000 \mathrm{~m}^{3} /$ person/year) & 9 & 9 & 9 \\
\hline Area equipped for irrigation (1 $000 \mathrm{ha}$ ) & & & 87 \\
\hline Area irrigated (\% area equipped for irrigation) & & & 22.7 \\
\hline Employment in agriculture (\%) & & 6.7 & 3.2 \\
\hline Employment in agriculture, female (\%) & & 4 & 1.7 \\
\hline Fertilizers, Nitrogen (kg of nutrients per ha) & & 56.6 & 77 \\
\hline Fertilizers, Phosphate (kg of nutrients per ha) & & 11.5 & 15 \\
\hline Fertilizers, Potash (kg nutrients per ha) & & 10.9 & 10.5 \\
\hline \multicolumn{4}{|l|}{ Energy consump, power irrigation (mln kWh) } \\
\hline Agr value added per worker (constant US\$) & & 6219 & 18288 \\
\hline \multicolumn{4}{|l|}{ Hunger dimensions } \\
\hline \multicolumn{4}{|l|}{ Dietary energy supply (kcal/pc/day) } \\
\hline Average dietary energy supply adequacy (\%) & 119 & 111 & 114 \\
\hline Dietary en supp, cereals/roots/tubers (\%) & 39 & 38 & 35 \\
\hline Prevalence of undernourishment (\%) & $<5.0$ & $<5.0$ & $<5.0$ \\
\hline GDP per capita (US\$, PPP) & 11536 & 15242 & 25759 \\
\hline Domestic food price volatility (index) & & 7.4 & 9.2 \\
\hline Cereal import dependency ratio (\%) & -3.3 & -7.4 & -27.5 \\
\hline \multicolumn{4}{|l|}{ Underweight, children under-5 (\%) } \\
\hline Improved water source (\% pop) & 99.8 & 99.8 & 100 \\
\hline \multicolumn{4}{|l|}{ Food supply } \\
\hline Food production value, (2004-2006 mln I\$) & 1850 & 1431 & 1386 \\
\hline Agriculture, value added (\% GDP) & & 4 & 4 \\
\hline Food exports (mln US\$) & 274 & 285 & 3875 \\
\hline Food imports (mln US\$) & 351 & 469 & 3864 \\
\hline \multicolumn{4}{|l|}{ Production indices $(2004-06=100)$} \\
\hline Net food & 116 & 90 & 87 \\
\hline Net crops & 112 & 81 & 97 \\
\hline Cereals & 91 & 64 & 101 \\
\hline Vegetable oils & 29 & 55 & 124 \\
\hline Roots and tubers & 288 & 135 & 46 \\
\hline Fruit and vegetables & 171 & 139 & 90 \\
\hline Sugar & 72 & 61 & 73 \\
\hline Livestock & 139 & 103 & 79 \\
\hline Milk & 114 & 99 & 88 \\
\hline Meat & 164 & 110 & 67 \\
\hline Fish & 0 & 80 & 110 \\
\hline \multicolumn{4}{|l|}{ Net trade (mln US\$) } \\
\hline Cereals & -21 & -22 & 194 \\
\hline Fruit and vegetables & -16 & -103 & -488 \\
\hline Meat & -6 & -40 & -381 \\
\hline Dairy products & 0 & 20 & -25 \\
\hline Fish & -23 & -28 & -78 \\
\hline \multicolumn{4}{|l|}{ Environment } \\
\hline Forest area (\%) & 40 & 40 & 40 \\
\hline Renewable water res withdrawn (\% of total) & & & 3 \\
\hline Terrestrial protect areas (\% total land area) & 19 & 21 & 36 \\
\hline Organic area (\% total agricultural area) & & & 9 \\
\hline Water withdrawal by agriculture (\% of total) & & & 3 \\
\hline Biofuel production (thousand kt of oil eq.) & & 0 & 3056 \\
\hline Wood pellet prod. (1 000 tonnes) & & & 100 \\
\hline Net GHG emissions from AFOLU ( $\mathrm{CO}_{2}$ eq, $\mathrm{Mt}$ ) & 4 & -7 & -4 \\
\hline
\end{tabular}




\begin{tabular}{|c|c|c|c|}
\hline & 1990 & 2000 & 2014 \\
\hline \multicolumn{4}{|l|}{ The setting } \\
\hline Population, total (mln) & 2 & 2 & 2.1 \\
\hline Population, rural (mln) & 1 & 1 & 1 \\
\hline Govt expenditure on ag (\% total outlays) & & 3.2 & 3.3 \\
\hline Area harvested (mln ha) & & 0 & 0 \\
\hline Cropping intensity ratio & & 1 & \\
\hline Water resources (1 $000 \mathrm{~m}^{3} /$ person/year) & 16 & 16 & 15 \\
\hline Area equipped for irrigation (1 $000 \mathrm{ha})$ & & & 6 \\
\hline \multicolumn{4}{|l|}{ Area irrigated (\% area equipped for irrigation) } \\
\hline Employment in agriculture (\%) & 10.7 & 9.5 & 8.3 \\
\hline Employment in agriculture, female (\%) & 10.1 & 9.7 & 7.8 \\
\hline Fertilizers, Nitrogen (kg of nutrients per ha) & & 196.7 & 151.4 \\
\hline Fertilizers, Phosphate (kg of nutrients per ha) & & 86.9 & 51.1 \\
\hline Fertilizers, Potash (kg nutrients per ha) & & 116.7 & 62.5 \\
\hline Energy consump, power irrigation (mln kWh) & 0 & & 15 \\
\hline Agr value added per worker (constant US\$) & & 42840 & 163437 \\
\hline \multicolumn{4}{|l|}{ Hunger dimensions } \\
\hline \multicolumn{4}{|l|}{ Dietary energy supply (kcal/pc/day) } \\
\hline Average dietary energy supply adequacy (\%) & 110 & 121 & 125 \\
\hline Dietary en supp, cereals/roots/tubers (\%) & 40 & 39 & 38 \\
\hline Prevalence of undernourishment (\%) & $<5.0$ & $<5.0$ & $<5.0$ \\
\hline GDP per capita (US\$, PPP) & & 22488 & 27368 \\
\hline Domestic food price volatility (index) & & 4.2 & 9.4 \\
\hline Cereal import dependency ratio (\%) & 56.3 & 50.3 & 36.9 \\
\hline \multicolumn{4}{|l|}{ Underweight, children under-5 (\%) } \\
\hline Improved water source (\% pop) & 99.6 & 99.6 & 99.6 \\
\hline \multicolumn{4}{|l|}{ Food supply } \\
\hline Food production value, $(2004-2006 \mathrm{mln}$ I\$) & 661 & 722 & 608 \\
\hline Agriculture, value added (\% GDP) & & 3 & 2 \\
\hline Food exports (mln US\$) & 210 & 218 & 1207 \\
\hline Food imports (mln US\$) & 418 & 481 & 1987 \\
\hline \multicolumn{4}{|l|}{ Production indices $(2004-06=100)$} \\
\hline Net food & 90 & 99 & 83 \\
\hline Net crops & 81 & 102 & 74 \\
\hline Cereals & 76 & 90 & 83 \\
\hline Vegetable oils & 53 & 26 & 196 \\
\hline Roots and tubers & 105 & 132 & 58 \\
\hline Fruit and vegetables & 78 & 104 & 74 \\
\hline Sugar & 54 & 142 & 107 \\
\hline Livestock & 93 & 93 & 85 \\
\hline Milk & 84 & 99 & 91 \\
\hline Meat & 98 & 86 & 79 \\
\hline Fish & 0 & 119 & 64 \\
\hline \multicolumn{4}{|l|}{ Net trade (mln US\$) } \\
\hline Cereals & -92 & -56 & -167 \\
\hline Fruit and vegetables & -53 & -117 & -246 \\
\hline Meat & 16 & -35 & -122 \\
\hline Dairy products & 12 & 19 & 1 \\
\hline Fish & -14 & -20 & -61 \\
\hline \multicolumn{4}{|l|}{ Environment } \\
\hline Forest area (\%) & 60 & 61 & 62 \\
\hline Renewable water res withdrawn (\% of total) & & & 0 \\
\hline Terrestrial protect areas (\% total land area) & 8 & 9 & 55 \\
\hline Organic area (\% total agricultural area) & & & 7 \\
\hline Water withdrawal by agriculture (\% of total) & & & 0 \\
\hline Biofuel production (thousand kt of oil eq.) & & 0 & 514 \\
\hline Wood pellet prod. (1 000 tonnes) & & & 100 \\
\hline Net GHG emissions from AFOLU ( $\mathrm{CO}_{2}$ eq, $\mathrm{Mt}$ ) & -7 & -8 & -13 \\
\hline
\end{tabular}




\begin{tabular}{|c|c|c|c|}
\hline & 1990 & 2000 & 2014 \\
\hline \multicolumn{4}{|l|}{ The setting } \\
\hline Population, total (mln) & 0.3 & 0.4 & 0.6 \\
\hline Population, rural (mln) & 0.3 & 0.3 & 0.4 \\
\hline \multicolumn{4}{|l|}{ Govt expenditure on ag (\% total outlays) } \\
\hline Area harvested (mln ha) & 0 & 0 & 0 \\
\hline Cropping intensity ratio & 3.2 & 3.2 & \\
\hline Water resources (1 $000 \mathrm{~m}^{3} /$ person/year) & 143 & 108 & 80 \\
\hline \multicolumn{4}{|l|}{ Area equipped for irrigation (1 $000 \mathrm{ha})$} \\
\hline \multicolumn{4}{|l|}{ Area irrigated (\% area equipped for irrigation) } \\
\hline \multicolumn{4}{|l|}{ Employment in agriculture (\%) } \\
\hline \multicolumn{4}{|l|}{ Employment in agriculture, female (\%) } \\
\hline \multicolumn{4}{|l|}{ Fertilizers, Nitrogen (kg of nutrients per ha) } \\
\hline \multicolumn{4}{|l|}{ Fertilizers, Phosphate (kg of nutrients per ha) } \\
\hline \multicolumn{4}{|l|}{ Fertilizers, Potash (kg nutrients per ha) } \\
\hline \multicolumn{4}{|l|}{ Energy consump, power irrigation (mln kWh) } \\
\hline Agr value added per worker (constant US\$) & 853 & 811 & 1084 \\
\hline \multicolumn{4}{|l|}{ Hunger dimensions } \\
\hline Dietary energy supply (kcal/pc/day) & 2210 & 2326 & 2471 \\
\hline Average dietary energy supply adequacy (\%) & 105 & 109 & 115 \\
\hline Dietary en supp, cereals/roots/tubers (\%) & 67 & 70 & 67 \\
\hline Prevalence of undernourishment (\%) & 23.1 & 15 & 10.9 \\
\hline GDP per capita (US\$, PPP) & 1776 & 1715 & 2003 \\
\hline \multicolumn{4}{|l|}{ Domestic food price volatility (index) } \\
\hline Cereal import dependency ratio (\%) & 95.3 & 94.8 & 97 \\
\hline Underweight, children under-5 (\%) & & & 11.5 \\
\hline Improved water source (\% pop) & & 79.7 & 80.5 \\
\hline \multicolumn{4}{|l|}{ Food supply } \\
\hline Food production value, (2004-2006 mln I\$) & 64 & 84 & 121 \\
\hline Agriculture, value added (\% GDP) & 29 & 35 & 36 \\
\hline Food exports (mln US\$) & 18 & 40 & 83 \\
\hline Food imports (mln US\$) & 12 & 14 & 88 \\
\hline \multicolumn{4}{|l|}{ Production indices $(2004-06=100)$} \\
\hline Net food & 62 & 82 & 118 \\
\hline Net crops & 61 & 81 & 119 \\
\hline Cereals & 32 & 159 & 146 \\
\hline Vegetable oils & 61 & 93 & 112 \\
\hline Roots and tubers & 67 & 84 & 120 \\
\hline Fruit and vegetables & 66 & 76 & 115 \\
\hline \multicolumn{4}{|l|}{ Sugar } \\
\hline Livestock & 78 & 90 & 106 \\
\hline Milk & 96 & 104 & 103 \\
\hline Meat & 78 & 90 & 104 \\
\hline Fish & 118 & 56 & 105 \\
\hline \multicolumn{4}{|l|}{ Net trade (mln US\$) } \\
\hline Cereals & -5 & -11 & -51 \\
\hline Fruit and vegetables & -2 & 0 & -3 \\
\hline Meat & & & -11 \\
\hline Dairy products & & & -3 \\
\hline Fish & 22 & 12 & 34 \\
\hline \multicolumn{4}{|l|}{ Environment } \\
\hline Forest area (\%) & 83 & 81 & 79 \\
\hline \multicolumn{4}{|l|}{ Renewable water res withdrawn (\% of total) } \\
\hline Terrestrial protect areas (\% total land area) & 0 & 1 & 2 \\
\hline Organic area (\% total agricultural area) & & & 1 \\
\hline Water withdrawal by agriculture (\% of total) & & & \\
\hline Biofuel production (thousand kt of oil eq.) & 2 & 2 & 2 \\
\hline Wood pellet prod. (1 000 tonnes) & & & \\
\hline Net GHG emissions from AFOLU ( $\mathrm{CO}_{2}$ eq, $\mathrm{Mt}$ ) & 2 & 2 & 2 \\
\hline
\end{tabular}




\section{Somalia}

\begin{tabular}{|c|c|c|c|}
\hline & 1990 & 2000 & 2014 \\
\hline \multicolumn{4}{|l|}{ The setting } \\
\hline Population, total (mln) & 6.3 & 7.4 & 10.8 \\
\hline Population, rural (mln) & 4.4 & 4.9 & 6.6 \\
\hline \multicolumn{4}{|l|}{ Govt expenditure on ag (\% total outlays) } \\
\hline Area harvested (mln ha) & 1 & 1 & 0 \\
\hline Cropping intensity ratio & 0 & 0 & \\
\hline Water resources (1 $000 \mathrm{~m}^{3} /$ person/year) & 2 & 2 & 1 \\
\hline Area equipped for irrigation (1 $000 \mathrm{ha})$ & & & 200 \\
\hline Area irrigated (\% area equipped for irrigation) & & 32.5 & \\
\hline \multicolumn{4}{|l|}{ Employment in agriculture (\%) } \\
\hline \multicolumn{4}{|l|}{ Employment in agriculture, female (\%) } \\
\hline \multicolumn{4}{|l|}{ Fertilizers, Nitrogen (kg of nutrients per ha) } \\
\hline \multicolumn{4}{|l|}{ Fertilizers, Phosphate (kg of nutrients per ha) } \\
\hline \multicolumn{4}{|l|}{ Fertilizers, Potash (kg nutrients per ha) } \\
\hline \multicolumn{4}{|l|}{ Energy consump, power irrigation (mln kWh) } \\
\hline \multicolumn{4}{|l|}{ Agr value added per worker (constant US\$) } \\
\hline \multicolumn{4}{|l|}{ Hunger dimensions } \\
\hline \multicolumn{4}{|l|}{ Dietary energy supply (kcal/pc/day) } \\
\hline \multicolumn{4}{|l|}{ Average dietary energy supply adequacy (\%) } \\
\hline \multicolumn{4}{|l|}{ Dietary en supp, cereals/roots/tubers (\%) } \\
\hline \multicolumn{4}{|l|}{ Prevalence of undernourishment (\%) } \\
\hline \multicolumn{4}{|l|}{ GDP per capita (US\$, PPP) } \\
\hline \multicolumn{4}{|l|}{ Domestic food price volatility (index) } \\
\hline Cereal import dependency ratio (\%) & 51.2 & 37.5 & 69.7 \\
\hline Underweight, children under-5 (\%) & & 22.8 & 32.8 \\
\hline Improved water source (\% pop) & 21 & 23.5 & 31.7 \\
\hline \multicolumn{4}{|l|}{ Food supply } \\
\hline Food production value, $(2004-2006 \mathrm{mln}$ I\$) & 1460 & 1437 & 1881 \\
\hline Agriculture, value added (\% GDP) & 65 & & \\
\hline Food exports (mln US\$) & 70 & 108 & 314 \\
\hline Food imports (mln US\$) & 77 & 132 & 853 \\
\hline \multicolumn{4}{|l|}{ Production indices $(2004-06=100)$} \\
\hline Net food & 91 & 90 & 117 \\
\hline Net crops & 122 & 87 & 124 \\
\hline Cereals & 175 & 113 & 114 \\
\hline Vegetable oils & 91 & 49 & 186 \\
\hline Roots and tubers & 53 & 80 & 103 \\
\hline Fruit and vegetables & 121 & 88 & 109 \\
\hline Sugar & 131 & 120 & 120 \\
\hline Livestock & 86 & 90 & 116 \\
\hline Milk & 98 & 93 & 110 \\
\hline Meat & 72 & 86 & 124 \\
\hline Fish & & & \\
\hline Net trade (mln US\$) & & & \\
\hline Cereals & -60 & -80 & -230 \\
\hline Fruit and vegetables & 24 & 4 & -239 \\
\hline Meat & 0 & 0 & 0 \\
\hline Dairy products & -3 & -1 & -37 \\
\hline Fish & 15 & 2 & -5 \\
\hline Environment & & & \\
\hline Forest area (\%) & 13 & 12 & 11 \\
\hline Renewable water res withdrawn (\% of total) & & 100 & 100 \\
\hline Terrestrial protect areas (\% total land area) & 1 & 1 & 1 \\
\hline Organic area (\% total agricultural area) & & & 0 \\
\hline Water withdrawal by agriculture (\% of total) & & 100 & 100 \\
\hline Biofuel production (thousand kt of oil eq.) & 1 & 0 & 1 \\
\hline Wood pellet prod. (1 000 tonnes) & & & \\
\hline Net GHG emissions from AFOLU ( $\mathrm{CO}_{2}$ eq, $\left.\mathrm{Mt}\right)$ & 37 & 37 & 36 \\
\hline
\end{tabular}




\begin{tabular}{|c|c|c|c|}
\hline & 1990 & 2000 & 2014 \\
\hline \multicolumn{4}{|l|}{ The setting } \\
\hline Population, total (mln) & 36.8 & 44.8 & 53.1 \\
\hline Population, rural (mln) & 17.6 & 19.3 & 19.5 \\
\hline \multicolumn{4}{|l|}{ Govt expenditure on ag (\% total outlays) } \\
\hline Area harvested (mln ha) & 18 & 24 & 18 \\
\hline Cropping intensity ratio & 0.2 & 0.2 & \\
\hline Water resources (1 $000 \mathrm{~m}^{3} /$ person/year) & 1 & 1 & 1 \\
\hline Area equipped for irrigation (1 $000 \mathrm{ha}$ ) & & & 1670 \\
\hline Area irrigated (\% area equipped for irrigation) & & & 95.9 \\
\hline Employment in agriculture (\%) & & 15.6 & 4.6 \\
\hline Employment in agriculture, female (\%) & & 16.1 & 3.9 \\
\hline Fertilizers, Nitrogen (kg of nutrients per ha) & & 30.9 & 35.8 \\
\hline Fertilizers, Phosphate (kg of nutrients per ha) & & 14.8 & 15.8 \\
\hline Fertilizers, Potash (kg nutrients per ha) & & 9.4 & 10.3 \\
\hline Energy consump, power irrigation (mln kWh) & 246 & 2406 & 2406 \\
\hline Agr value added per worker (constant US\$) & 3308 & 3866 & 7238 \\
\hline \multicolumn{4}{|l|}{ Hunger dimensions } \\
\hline Dietary energy supply (kcal/pc/day) & 2821 & 2878 & 3136 \\
\hline Average dietary energy supply adequacy (\%) & 121 & 121 & 131 \\
\hline Dietary en supp, cereals/roots/tubers (\%) & 57 & 57 & 53 \\
\hline Prevalence of undernourishment (\%) & 5 & $<5.0$ & $<5.0$ \\
\hline GDP per capita (US\$, PPP) & 10364 & 9927 & 12454 \\
\hline Domestic food price volatility (index) & & 7.1 & 6.2 \\
\hline Cereal import dependency ratio (\%) & 10 & 4.8 & 2.8 \\
\hline Underweight, children under-5 (\%) & & 10.1 & 8.7 \\
\hline Improved water source (\% pop) & 81.3 & 86.8 & 95.1 \\
\hline \multicolumn{4}{|l|}{ Food supply } \\
\hline Food production value, (2004-2006 mln I\$) & 8301 & 10029 & 12753 \\
\hline Agriculture, value added (\% GDP) & 5 & 3 & 2 \\
\hline Food exports (mln US\$) & 1286 & 1501 & 4764 \\
\hline Food imports (mln US\$) & 593 & 876 & 4679 \\
\hline \multicolumn{4}{|l|}{ Production indices $(2004-06=100)$} \\
\hline Net food & 80 & 96 & 122 \\
\hline Net crops & 84 & 103 & 115 \\
\hline Cereals & 97 & 123 & 125 \\
\hline Vegetable oils & 102 & 92 & 120 \\
\hline Roots and tubers & 68 & 95 & 124 \\
\hline Fruit and vegetables & 72 & 89 & 113 \\
\hline Sugar & 89 & 118 & 89 \\
\hline Livestock & 80 & 90 & 131 \\
\hline Milk & 87 & 89 & 119 \\
\hline Meat & 77 & 89 & 134 \\
\hline Fish & 69 & 83 & 54 \\
\hline \multicolumn{4}{|l|}{ Net trade (mln US\$) } \\
\hline Cereals & 60 & -181 & -855 \\
\hline Fruit and vegetables & 585 & 739 & 2404 \\
\hline Meat & -52 & -48 & -533 \\
\hline Dairy products & 22 & -9 & -77 \\
\hline Fish & -13 & 211 & 255 \\
\hline \multicolumn{4}{|l|}{ Environment } \\
\hline Forest area (\%) & 8 & 8 & 8 \\
\hline Renewable water res withdrawn (\% of total) & & 63 & 63 \\
\hline Terrestrial protect areas (\% total land area) & 7 & 7 & 6 \\
\hline Organic area (\% total agricultural area) & & & 0 \\
\hline Water withdrawal by agriculture (\% of total) & & 63 & 63 \\
\hline Biofuel production (thousand kt of oil eq.) & 69 & 138 & 135 \\
\hline Wood pellet prod. (1 000 tonnes) & & & 75 \\
\hline Net GHG emissions from AFOLU ( $\mathrm{CO}_{2}$ eq, $\left.\mathrm{Mt}\right)$ & 35 & 32 & 32 \\
\hline
\end{tabular}




\section{South Sudan}

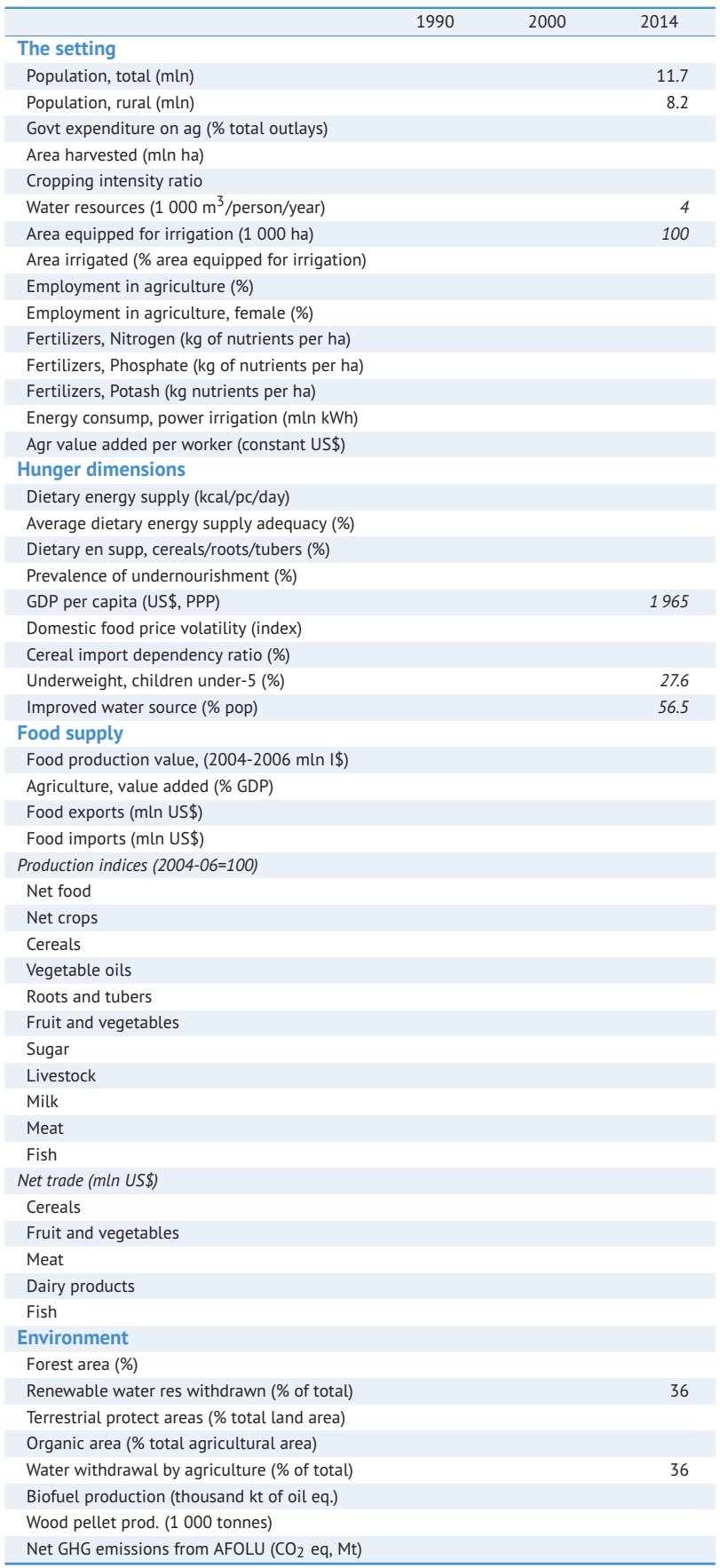




\begin{tabular}{|c|c|c|c|}
\hline & 1990 & 2000 & 2014 \\
\hline \multicolumn{4}{|l|}{ The setting } \\
\hline Population, total (mln) & 38.9 & 40.3 & 47.1 \\
\hline Population, rural (mln) & 9.6 & 9.6 & 10.4 \\
\hline \multicolumn{4}{|l|}{ Govt expenditure on ag (\% total outlays) } \\
\hline Area harvested (mln ha) & 19 & 25 & 25 \\
\hline Cropping intensity ratio & 0.6 & 0.8 & \\
\hline Water resources (1 $000 \mathrm{~m}^{3} /$ person/year) & 3 & 3 & 2 \\
\hline Area equipped for irrigation (1 $000 \mathrm{ha})$ & & & 3800 \\
\hline Area irrigated (\% area equipped for irrigation) & & & 89.1 \\
\hline Employment in agriculture (\%) & 11.8 & 6.7 & 4.4 \\
\hline Employment in agriculture, female (\%) & 10.2 & 4.8 & 2.5 \\
\hline Fertilizers, Nitrogen (kg of nutrients per ha) & & 92.1 & 68 \\
\hline Fertilizers, Phosphate (kg of nutrients per ha) & & 47.2 & 30.4 \\
\hline Fertilizers, Potash (kg nutrients per ha) & & 36 & 25.9 \\
\hline Energy consump, power irrigation (mln kWh) & 217 & 309 & 4708 \\
\hline Agr value added per worker (constant US\$) & & 26526 & 43431 \\
\hline \multicolumn{4}{|l|}{ Hunger dimensions } \\
\hline \multicolumn{4}{|l|}{ Dietary energy supply (kcal/pc/day) } \\
\hline Average dietary energy supply adequacy (\%) & 132 & 133 & 125 \\
\hline Dietary en supp, cereals/roots/tubers (\%) & 28 & 26 & 25 \\
\hline Prevalence of undernourishment (\%) & $<5.0$ & $<5.0$ & $<5.0$ \\
\hline GDP per capita (US\$, PPP) & 24126 & 30647 & 31683 \\
\hline Domestic food price volatility (index) & & 4.6 & 8.4 \\
\hline Cereal import dependency ratio (\%) & 13.3 & 21.3 & 34.2 \\
\hline \multicolumn{4}{|l|}{ Underweight, children under-5 (\%) } \\
\hline Improved water source (\% pop) & 100 & 100 & 100 \\
\hline \multicolumn{4}{|l|}{ Food supply } \\
\hline Food production value, (2004-2006 mln I\$) & 24546 & 29439 & 32045 \\
\hline Agriculture, value added (\% GDP) & & 4 & 2 \\
\hline Food exports (mln US\$) & 6513 & 11438 & 33621 \\
\hline Food imports (mln US\$) & 4942 & 6846 & 22087 \\
\hline \multicolumn{4}{|l|}{ Production indices $(2004-06=100)$} \\
\hline Net food & 83 & 100 & 109 \\
\hline Net crops & 89 & 103 & 115 \\
\hline Cereals & 95 & 129 & 134 \\
\hline Vegetable oils & 79 & 104 & 160 \\
\hline Roots and tubers & 198 & 116 & 84 \\
\hline Fruit and vegetables & 86 & 96 & 102 \\
\hline Sugar & 110 & 118 & 36 \\
\hline Livestock & 72 & 95 & 100 \\
\hline Milk & 92 & 96 & 106 \\
\hline Meat & 66 & 96 & 100 \\
\hline Fish & 115 & 119 & 110 \\
\hline \multicolumn{4}{|l|}{ Net trade (mln US\$) } \\
\hline Cereals & -319 & -591 & -3199 \\
\hline Fruit and vegetables & 3142 & 5004 & 12711 \\
\hline Meat & -429 & 516 & 3573 \\
\hline Dairy products & -259 & -404 & -1089 \\
\hline Fish & -1543 & -1754 & -2467 \\
\hline \multicolumn{4}{|l|}{ Environment } \\
\hline Forest area $(\%)$ & 28 & 34 & 37 \\
\hline Renewable water res withdrawn (\% of total) & & & 60 \\
\hline Terrestrial protect areas (\% total land area) & 13 & 27 & 29 \\
\hline Organic area (\% total agricultural area) & & & 6 \\
\hline Water withdrawal by agriculture (\% of total) & & & 60 \\
\hline Biofuel production (thousand kt of oil eq.) & 37 & 2183 & 22769 \\
\hline Wood pellet prod. (1 000 tonnes) & & & 350 \\
\hline Net GHG emissions from AFOLU ( $\mathrm{CO}_{2}$ eq, $\mathrm{Mt}$ ) & 24 & -2 & 18 \\
\hline
\end{tabular}




\begin{tabular}{|c|c|c|c|}
\hline & 1990 & 2000 & 2014 \\
\hline \multicolumn{4}{|l|}{ The setting } \\
\hline Population, total (mln) & 17.3 & 18.8 & 21.4 \\
\hline Population, rural (mln) & 14.3 & 15.9 & 18.2 \\
\hline Govt expenditure on ag (\% total outlays) & & 4.1 & 5.7 \\
\hline Area harvested (mln ha) & 3 & 3 & 5 \\
\hline Cropping intensity ratio & 1.1 & 1.2 & \\
\hline Water resources (1 $000 \mathrm{~m}^{3} /$ person/year) & 3 & 3 & 2 \\
\hline Area equipped for irrigation (1 $000 \mathrm{ha}$ ) & & & 570 \\
\hline Area irrigated (\% area equipped for irrigation) & & & 81.1 \\
\hline Employment in agriculture (\%) & 47.8 & 34 & 39.4 \\
\hline Employment in agriculture, female (\%) & 42.6 & 39.2 & 34.7 \\
\hline Fertilizers, Nitrogen (kg of nutrients per ha) & & 158.6 & 130.9 \\
\hline Fertilizers, Phosphate (kg of nutrients per ha) & & 39.4 & 50 \\
\hline Fertilizers, Potash (kg nutrients per ha) & & 61.2 & 18.6 \\
\hline Energy consump, power irrigation (mln kWh) & 0 & 0 & 0 \\
\hline Agr value added per worker (constant US\$) & 650 & 778 & 1046 \\
\hline \multicolumn{4}{|l|}{ Hunger dimensions } \\
\hline Dietary energy supply (kcal/pc/day) & 2180 & 2352 & 2585 \\
\hline Average dietary energy supply adequacy (\%) & 97 & 103 & 114 \\
\hline Dietary en supp, cereals/roots/tubers (\%) & 59 & 56 & 58 \\
\hline Prevalence of undernourishment (\%) & 31.9 & 29.9 & 22.9 \\
\hline GDP per capita (US\$, PPP) & 3340 & 4946 & 9426 \\
\hline Domestic food price volatility (index) & & 5.9 & 8.3 \\
\hline Cereal import dependency ratio (\%) & 37.5 & 36 & 25.4 \\
\hline Underweight, children under-5 (\%) & 33.8 & 22.8 & 26.3 \\
\hline Improved water source (\% pop) & 67.6 & 79.3 & 93.8 \\
\hline \multicolumn{4}{|l|}{ Food supply } \\
\hline Food production value, $(2004-2006 \mathrm{mln}$ I\$) & 1657 & 1843 & 2737 \\
\hline Agriculture, value added (\% GDP) & 26 & 20 & 10 \\
\hline Food exports (mln US\$) & 119 & 192 & 626 \\
\hline Food imports (mln US\$) & 436 & 633 & 1624 \\
\hline \multicolumn{4}{|l|}{ Production indices $(2004-06=100)$} \\
\hline Net food & 86 & 95 & 142 \\
\hline Net crops & 88 & 96 & 136 \\
\hline Cereals & 82 & 93 & 154 \\
\hline Vegetable oils & 99 & 118 & 146 \\
\hline Roots and tubers & 150 & 97 & 124 \\
\hline Fruit and vegetables & 95 & 98 & 126 \\
\hline Sugar & 79 & 103 & 92 \\
\hline Livestock & 75 & 88 & 137 \\
\hline Milk & 154 & 94 & 195 \\
\hline Meat & 53 & 83 & 107 \\
\hline Fish & 66 & 107 & 188 \\
\hline \multicolumn{4}{|l|}{ Net trade (mln US\$) } \\
\hline Cereals & -175 & -158 & -347 \\
\hline Fruit and vegetables & 23 & -23 & -85 \\
\hline Meat & 0 & -2 & 0 \\
\hline Dairy products & -56 & -116 & -305 \\
\hline Fish & -23 & 62 & 55 \\
\hline \multicolumn{4}{|l|}{ Environment } \\
\hline Forest area (\%) & 37 & 33 & 29 \\
\hline Renewable water res withdrawn (\% of total) & & & 87 \\
\hline Terrestrial protect areas (\% total land area) & 20 & 22 & 22 \\
\hline Organic area (\% total agricultural area) & & & 1 \\
\hline Water withdrawal by agriculture (\% of total) & & & 87 \\
\hline Biofuel production (thousand kt of oil eq.) & 1 & 2 & 1 \\
\hline \multicolumn{4}{|l|}{ Wood pellet prod. (1 000 tonnes) } \\
\hline Net GHG emissions from AFOLU ( $\mathrm{CO}_{2}$ eq, $\mathrm{Mt}$ ) & 15 & 14 & 13 \\
\hline
\end{tabular}




\section{Sudan}

\begin{tabular}{|c|c|c|c|}
\hline & 1990 & 2000 & 2014 \\
\hline \multicolumn{4}{|l|}{ The setting } \\
\hline Population, total (mln) & & & 38.8 \\
\hline Population, rural (mln) & & & 27.1 \\
\hline \multicolumn{4}{|l|}{ Govt expenditure on ag (\% total outlays) } \\
\hline \multicolumn{4}{|l|}{ Area harvested (mln ha) } \\
\hline \multicolumn{4}{|l|}{ Cropping intensity ratio } \\
\hline Water resources (1 $000 \mathrm{~m}^{3} /$ person/year) & & & 1 \\
\hline Area equipped for irrigation (1 $000 \mathrm{ha}$ ) & & & 1890 \\
\hline \multicolumn{4}{|l|}{ Area irrigated (\% area equipped for irrigation) } \\
\hline \multicolumn{4}{|l|}{ Employment in agriculture (\%) } \\
\hline \multicolumn{4}{|l|}{ Employment in agriculture, female (\%) } \\
\hline Fertilizers, Nitrogen (kg of nutrients per ha) & & & 7.2 \\
\hline Fertilizers, Phosphate (kg of nutrients per ha) & & & 3.3 \\
\hline Fertilizers, Potash (kg nutrients per ha) & & & 0.1 \\
\hline \multicolumn{4}{|l|}{ Energy consump, power irrigation (mln kWh) } \\
\hline Agr value added per worker (constant US\$) & 820 & 1217 & 1390 \\
\hline \multicolumn{4}{|l|}{ Hunger dimensions } \\
\hline \multicolumn{4}{|l|}{ Dietary energy supply (kcal/pc/day) } \\
\hline \multicolumn{4}{|l|}{ Average dietary energy supply adequacy (\%) } \\
\hline \multicolumn{4}{|l|}{ Dietary en supp, cereals/roots/tubers (\%) } \\
\hline \multicolumn{4}{|l|}{ Prevalence of undernourishment (\%) } \\
\hline GDP per capita (US\$, PPP) & 1860 & 2396 & 3265 \\
\hline \multicolumn{4}{|l|}{ Domestic food price volatility (index) } \\
\hline Cereal import dependency ratio (\%) & & & 25 \\
\hline Underweight, children under-5 (\%) & 31.8 & 38.4 & 27 \\
\hline Improved water source (\% pop) & 67.5 & 62 & 55.5 \\
\hline \multicolumn{4}{|l|}{ Food supply } \\
\hline \multicolumn{4}{|l|}{ Food production value, (2004-2006 mln I\$) } \\
\hline Agriculture, value added (\% GDP) & 41 & 42 & 29 \\
\hline \multicolumn{4}{|l|}{ Food exports (mln US\$) } \\
\hline \multicolumn{4}{|l|}{ Food imports (mln US\$) } \\
\hline Production indices $(2004-06=100)$ & & & \\
\hline Net food & & & \\
\hline Net crops & & & \\
\hline Cereals & & & \\
\hline Vegetable oils & & & \\
\hline Roots and tubers & & & \\
\hline Fruit and vegetables & & & \\
\hline Sugar & & & \\
\hline Livestock & & & \\
\hline Milk & & & \\
\hline Meat & & & \\
\hline Fish & & & \\
\hline Net trade (mln US\$) & & & \\
\hline Cereals & & & \\
\hline Fruit and vegetables & & & \\
\hline Meat & & & \\
\hline Dairy products & & & \\
\hline Fish & & & -5 \\
\hline Environment & & & \\
\hline Forest area (\%) & & & \\
\hline Renewable water res withdrawn (\% of total) & & & 96 \\
\hline Terrestrial protect areas (\% total land area) & 4 & 4 & 7 \\
\hline Organic area (\% total agricultural area) & & & \\
\hline Water withdrawal by agriculture (\% of total) & & & 96 \\
\hline Biofuel production (thousand kt of oil eq.) & & & \\
\hline Wood pellet prod. (1 000 tonnes) & & & \\
\hline Net GHG emissions from AFOLU ( $\mathrm{CO}_{2}$ eq, $\left.\mathrm{Mt}\right)$ & & & \\
\hline
\end{tabular}




\section{Suriname}

\begin{tabular}{|c|c|c|c|}
\hline & 1990 & 2000 & 2014 \\
\hline \multicolumn{4}{|l|}{ The setting } \\
\hline Population, total (mln) & 0.4 & 0.5 & 0.5 \\
\hline Population, rural (mln) & 0.2 & 0.2 & 0.2 \\
\hline \multicolumn{4}{|l|}{ Govt expenditure on ag (\% total outlays) } \\
\hline Area harvested (mln ha) & 0 & 0 & 0 \\
\hline Cropping intensity ratio & 3.9 & 4.5 & \\
\hline Water resources (1 $000 \mathrm{~m}^{3} /$ person/year) & 243 & 212 & 184 \\
\hline Area equipped for irrigation (1 $000 \mathrm{ha})$ & & & 57 \\
\hline Area irrigated (\% area equipped for irrigation) & & & 100 \\
\hline Employment in agriculture (\%) & 3.7 & 6.1 & \\
\hline Employment in agriculture, female (\%) & 2.2 & 1.8 & \\
\hline Fertilizers, Nitrogen (kg of nutrients per ha) & & 101.7 & 42.2 \\
\hline Fertilizers, Phosphate (kg of nutrients per ha) & & 4.8 & 1 \\
\hline Fertilizers, Potash (kg nutrients per ha) & & 2.5 & 19 \\
\hline Energy consump, power irrigation (mln kWh) & 0 & 2 & 2 \\
\hline Agr value added per worker (constant US\$) & 2939 & 2769 & 3802 \\
\hline \multicolumn{4}{|l|}{ Hunger dimensions } \\
\hline Dietary energy supply (kcal/pc/day) & 2521 & 2514 & 2773 \\
\hline Average dietary energy supply adequacy (\%) & 108 & 107 & 116 \\
\hline Dietary en supp, cereals/roots/tubers (\%) & 50 & 45 & 43 \\
\hline Prevalence of undernourishment (\%) & 14.8 & 14.1 & 8.3 \\
\hline GDP per capita (US\$, PPP) & 10625 & 9908 & 15556 \\
\hline Domestic food price volatility (index) & & 18.6 & 9.7 \\
\hline Cereal import dependency ratio (\%) & -14.6 & 4.5 & 6.3 \\
\hline Underweight, children under-5 (\%) & & 11.4 & 5.8 \\
\hline Improved water source (\% pop) & & 88.9 & 95.2 \\
\hline \multicolumn{4}{|l|}{ Food supply } \\
\hline Food production value, (2004-2006 mln I\$) & 107 & 86 & 136 \\
\hline Agriculture, value added (\% GDP) & 9 & 11 & 7 \\
\hline Food exports (mln US\$) & 38 & 54 & 95 \\
\hline Food imports (mln US\$) & 46 & 74 & 207 \\
\hline \multicolumn{4}{|l|}{ Production indices $(2004-06=100)$} \\
\hline Net food & 122 & 97 & 154 \\
\hline Net crops & 109 & 97 & 155 \\
\hline Cereals & 115 & 95 & 153 \\
\hline Vegetable oils & 406 & 108 & 126 \\
\hline Roots and tubers & 57 & 76 & 193 \\
\hline Fruit and vegetables & 97 & 95 & 159 \\
\hline Sugar & 75 & 138 & 139 \\
\hline Livestock & 163 & 89 & 115 \\
\hline Milk & 212 & 103 & 72 \\
\hline Meat & 160 & 82 & 122 \\
\hline Fish & 24 & 78 & 126 \\
\hline \multicolumn{4}{|l|}{ Net trade (mln US\$) } \\
\hline Cereals & 15 & 8 & -5 \\
\hline Fruit and vegetables & 6 & 14 & 31 \\
\hline Meat & -1 & -28 & -38 \\
\hline Dairy products & & -9 & -15 \\
\hline Fish & 2 & 35 & 67 \\
\hline \multicolumn{4}{|l|}{ Environment } \\
\hline Forest area (\%) & 95 & 95 & 95 \\
\hline Renewable water res withdrawn (\% of total) & & & 70 \\
\hline Terrestrial protect areas (\% total land area) & 3 & 15 & 15 \\
\hline \multicolumn{4}{|l|}{ Organic area (\% total agricultural area) } \\
\hline Water withdrawal by agriculture (\% of total) & & & 70 \\
\hline Biofuel production (thousand kt of oil eq.) & 0 & 0 & 0 \\
\hline \multicolumn{4}{|l|}{ Wood pellet prod. (1 000 tonnes) } \\
\hline Net GHG emissions from AFOLU ( $\mathrm{CO}_{2}$ eq, $\mathrm{Mt}$ ) & 3 & 3 & 6 \\
\hline
\end{tabular}




\begin{tabular}{|c|c|c|c|}
\hline & 1990 & 2000 & 2014 \\
\hline \multicolumn{4}{|l|}{ The setting } \\
\hline Population, total (mln) & 0.9 & 1.1 & 1.3 \\
\hline Population, rural (mln) & 0.7 & 0.8 & 1 \\
\hline Govt expenditure on ag (\% total outlays) & & 5.1 & 4.9 \\
\hline Area harvested (mln ha) & 4 & 4 & 5 \\
\hline Cropping intensity ratio & 3.1 & 3.2 & \\
\hline Water resources (1 $000 \mathrm{~m}^{3} /$ person/year) & 5 & 4 & 4 \\
\hline Area equipped for irrigation (1 $000 \mathrm{ha}$ ) & & & 50 \\
\hline Area irrigated (\% area equipped for irrigation) & & 89.9 & \\
\hline \multicolumn{4}{|l|}{ Employment in agriculture (\%) } \\
\hline \multicolumn{4}{|l|}{ Employment in agriculture, female (\%) } \\
\hline \multicolumn{4}{|l|}{ Fertilizers, Nitrogen (kg of nutrients per ha) } \\
\hline \multicolumn{4}{|l|}{ Fertilizers, Phosphate (kg of nutrients per ha) } \\
\hline \multicolumn{4}{|l|}{ Fertilizers, Potash (kg nutrients per ha) } \\
\hline Energy consump, power irrigation (mln kWh) & 0 & 58 & 58 \\
\hline Agr value added per worker (constant US\$) & 1173 & 1168 & 1373 \\
\hline \multicolumn{4}{|l|}{ Hunger dimensions } \\
\hline Dietary energy supply (kcal/pc/day) & 2308 & 2312 & 2239 \\
\hline Average dietary energy supply adequacy (\%) & 109 & 105 & 98 \\
\hline Dietary en supp, cereals/roots/tubers (\%) & 57 & 54 & 59 \\
\hline Prevalence of undernourishment (\%) & 16.8 & 21.7 & 26.5 \\
\hline GDP per capita (US\$, PPP) & 5372 & 5829 & 6471 \\
\hline \multicolumn{4}{|l|}{ Domestic food price volatility (index) } \\
\hline Cereal import dependency ratio (\%) & 55.2 & 54.9 & 72.9 \\
\hline Underweight, children under-5 (\%) & & 9.1 & 5.8 \\
\hline Improved water source (\% pop) & 38.9 & 51.9 & 74.1 \\
\hline \multicolumn{4}{|l|}{ Food supply } \\
\hline Food production value, $(2004-2006 \mathrm{mln} I \$)$ & 250 & 238 & 308 \\
\hline Agriculture, value added (\% GDP) & 10 & 12 & 7 \\
\hline Food exports (mln US\$) & 319 & 284 & 234 \\
\hline Food imports (mln US\$) & 79 & 151 & 152 \\
\hline \multicolumn{4}{|l|}{ Production indices $(2004-06=100)$} \\
\hline Net food & 91 & 87 & 112 \\
\hline Net crops & 99 & 89 & 111 \\
\hline Cereals & 162 & 163 & 118 \\
\hline Vegetable oils & 307 & 136 & 59 \\
\hline Roots and tubers & 79 & 95 & 127 \\
\hline Fruit and vegetables & 131 & 93 & 117 \\
\hline Sugar & 77 & 78 & 109 \\
\hline Livestock & 87 & 88 & 117 \\
\hline Milk & 109 & 97 & 110 \\
\hline Meat & 83 & 86 & 119 \\
\hline Fish & 183 & 198 & 167 \\
\hline \multicolumn{4}{|l|}{ Net trade (mln US\$) } \\
\hline Cereals & -22 & -32 & -96 \\
\hline Fruit and vegetables & 18 & 10 & 11 \\
\hline Meat & -5 & 5 & -2 \\
\hline Dairy products & -6 & -13 & -3 \\
\hline Fish & & -6 & -4 \\
\hline \multicolumn{4}{|l|}{ Environment } \\
\hline Forest area (\%) & 27 & 30 & 33 \\
\hline Renewable water res withdrawn (\% of total) & & 96 & 96 \\
\hline Terrestrial protect areas (\% total land area) & 3 & 3 & 3 \\
\hline Organic area (\% total agricultural area) & & & 0 \\
\hline Water withdrawal by agriculture (\% of total) & & 96 & 96 \\
\hline Biofuel production (thousand kt of oil eq.) & 9 & 9 & 11 \\
\hline \multicolumn{4}{|l|}{ Wood pellet prod. (1 000 tonnes) } \\
\hline Net GHG emissions from AFOLU ( $\mathrm{CO}_{2}$ eq, $\mathrm{Mt}$ ) & 2 & 1 & 1 \\
\hline
\end{tabular}




\begin{tabular}{|c|c|c|c|}
\hline & 1990 & 2000 & 2014 \\
\hline \multicolumn{4}{|l|}{ The setting } \\
\hline Population, total (mln) & 8.6 & 8.9 & 9.6 \\
\hline Population, rural (mln) & 1.4 & 1.4 & 1.4 \\
\hline \multicolumn{4}{|l|}{ Govt expenditure on ag (\% total outlays) } \\
\hline Area harvested (mln ha) & 6 & 6 & 5 \\
\hline Cropping intensity ratio & 1.9 & 1.8 & \\
\hline Water resources (1 $000 \mathrm{~m}^{3} /$ person/year) & 20 & 20 & 18 \\
\hline Area equipped for irrigation (1 $000 \mathrm{ha}$ ) & & & 164 \\
\hline Area irrigated (\% area equipped for irrigation) & & & 33.9 \\
\hline Employment in agriculture (\%) & 3.4 & 2.4 & 2 \\
\hline Employment in agriculture, female (\%) & 1.8 & 1.2 & 1 \\
\hline Fertilizers, Nitrogen (kg of nutrients per ha) & & 67.6 & 57 \\
\hline Fertilizers, Phosphate (kg of nutrients per ha) & & 14.3 & 4 \\
\hline Fertilizers, Potash (kg nutrients per ha) & & 16.4 & 8.3 \\
\hline \multicolumn{4}{|l|}{ Energy consump, power irrigation (mln kWh) } \\
\hline Agr value added per worker (constant US\$) & 18605 & 23810 & 42496 \\
\hline \multicolumn{4}{|l|}{ Hunger dimensions } \\
\hline \multicolumn{4}{|l|}{ Dietary energy supply (kcal/pc/day) } \\
\hline Average dietary energy supply adequacy (\%) & 123 & 122 & 126 \\
\hline Dietary en supp, cereals/roots/tubers (\%) & 27 & 28 & 28 \\
\hline Prevalence of undernourishment (\%) & $<5.0$ & $<5.0$ & $<5.0$ \\
\hline GDP per capita (US\$, PPP) & 30901 & 36816 & 43540 \\
\hline Domestic food price volatility (index) & & 4.3 & 6.7 \\
\hline Cereal import dependency ratio (\%) & -10.7 & -31.2 & -15 \\
\hline \multicolumn{4}{|l|}{ Underweight, children under-5 (\%) } \\
\hline Improved water source (\% pop) & 100 & 100 & 100 \\
\hline \multicolumn{4}{|l|}{ Food supply } \\
\hline Food production value, $(2004-2006 \mathrm{mln} 1 \$)$ & 3108 & 2871 & 2630 \\
\hline Agriculture, value added (\% GDP) & 4 & 2 & 1 \\
\hline Food exports (mln US\$) & 900 & 1168 & 3841 \\
\hline Food imports (mln US\$) & 2026 & 2507 & 8537 \\
\hline \multicolumn{4}{|l|}{ Production indices $(2004-06=100)$} \\
\hline Net food & 110 & 102 & 93 \\
\hline Net crops & 130 & 106 & 102 \\
\hline Cereals & 126 & 113 & 99 \\
\hline Vegetable oils & 186 & 57 & 154 \\
\hline Roots and tubers & 131 & 106 & 89 \\
\hline Fruit and vegetables & 130 & 90 & 110 \\
\hline Sugar & 121 & 114 & 102 \\
\hline Livestock & 103 & 103 & 93 \\
\hline Milk & 109 & 104 & 90 \\
\hline Meat & 97 & 102 & 92 \\
\hline Fish & 96 & 126 & 70 \\
\hline \multicolumn{4}{|l|}{ Net trade (mln US\$) } \\
\hline Cereals & 87 & 108 & 227 \\
\hline Fruit and vegetables & -947 & -900 & -2003 \\
\hline Meat & -76 & -270 & -1413 \\
\hline Dairy products & 21 & -10 & -581 \\
\hline Fish & -276 & -240 & -752 \\
\hline \multicolumn{4}{|l|}{ Environment } \\
\hline Forest area (\%) & 66 & 67 & 69 \\
\hline Renewable water res withdrawn (\% of total) & & & 4 \\
\hline Terrestrial protect areas (\% total land area) & 6 & 13 & 15 \\
\hline Organic area (\% total agricultural area) & & & 16 \\
\hline Water withdrawal by agriculture (\% of total) & & & 4 \\
\hline Biofuel production (thousand kt of oil eq.) & 216 & 143 & 5563 \\
\hline Wood pellet prod. (1 000 tonnes) & & & 1577 \\
\hline Net GHG emissions from AFOLU ( $\mathrm{CO}_{2}$ eq, $\left.\mathrm{Mt}\right)$ & 30 & 10 & -16 \\
\hline
\end{tabular}




\begin{tabular}{|c|c|c|c|}
\hline & 1990 & 2000 & 2014 \\
\hline \multicolumn{4}{|l|}{ The setting } \\
\hline Population, total (mln) & 6.7 & 7.2 & 8.2 \\
\hline Population, rural (mln) & 1.8 & 1.9 & 2.1 \\
\hline \multicolumn{4}{|l|}{ Govt expenditure on ag (\% total outlays) } \\
\hline Area harvested (mln ha) & 1 & 1 & 1 \\
\hline Cropping intensity ratio & 0.8 & 0.9 & \\
\hline Water resources (1 $000 \mathrm{~m}^{3} /$ person/year) & 8 & 7 & 7 \\
\hline Area equipped for irrigation (1 $000 \mathrm{ha}$ ) & & & 63 \\
\hline Area irrigated (\% area equipped for irrigation) & & & 59.3 \\
\hline Employment in agriculture (\%) & 4.2 & 4.5 & 3.5 \\
\hline Employment in agriculture, female (\%) & 3.5 & 3.7 & 2.9 \\
\hline Fertilizers, Nitrogen (kg of nutrients per ha) & & 106.1 & 120.5 \\
\hline Fertilizers, Phosphate (kg of nutrients per ha) & & 38.7 & 40 \\
\hline Fertilizers, Potash (kg nutrients per ha) & & 58.5 & 48.6 \\
\hline \multicolumn{4}{|l|}{ Energy consump, power irrigation (mln kWh) } \\
\hline Agr value added per worker (constant US\$) & 19890 & 23390 & 27288 \\
\hline \multicolumn{4}{|l|}{ Hunger dimensions } \\
\hline \multicolumn{4}{|l|}{ Dietary energy supply (kcal/pc/day) } \\
\hline Average dietary energy supply adequacy (\%) & 132 & 133 & 137 \\
\hline Dietary en supp, cereals/roots/tubers (\%) & 25 & 26 & 24 \\
\hline Prevalence of undernourishment (\%) & $<5.0$ & $<5.0$ & $<5.0$ \\
\hline GDP per capita (US\$, PPP) & 46600 & 49083 & 54993 \\
\hline Domestic food price volatility (index) & & 6.2 & 6.6 \\
\hline Cereal import dependency ratio (\%) & 33.5 & 36.9 & 51.5 \\
\hline \multicolumn{4}{|l|}{ Underweight, children under-5 (\%) } \\
\hline Improved water source (\% pop) & 100 & 100 & 100 \\
\hline \multicolumn{4}{|l|}{ Food supply } \\
\hline Food production value, (2004-2006 mln I\$) & 2794 & 2680 & 2675 \\
\hline Agriculture, value added (\% GDP) & 2 & 1 & 1 \\
\hline Food exports (mln US\$) & 1232 & 1358 & 3613 \\
\hline Food imports (mln US\$) & 2678 & 2704 & 6756 \\
\hline \multicolumn{4}{|l|}{ Production indices $(2004-06=100)$} \\
\hline Net food & 106 & 101 & 101 \\
\hline Net crops & 119 & 119 & 89 \\
\hline Cereals & 118 & 114 & 80 \\
\hline Vegetable oils & 59 & 70 & 111 \\
\hline Roots and tubers & 150 & 129 & 71 \\
\hline Fruit and vegetables & 126 & 124 & 94 \\
\hline Sugar & 64 & 103 & 101 \\
\hline Livestock & 104 & 97 & 104 \\
\hline Milk & 98 & 99 & 102 \\
\hline Meat & 113 & 93 & 107 \\
\hline Fish & 146 & 96 & 114 \\
\hline \multicolumn{4}{|l|}{ Net trade (mln US\$) } \\
\hline Cereals & -171 & -88 & -384 \\
\hline Fruit and vegetables & -1106 & -971 & -2035 \\
\hline Meat & -372 & -392 & -794 \\
\hline Dairy products & 232 & 130 & 266 \\
\hline Fish & -379 & -351 & -710 \\
\hline \multicolumn{4}{|l|}{ Environment } \\
\hline Forest area (\%) & 29 & 30 & 32 \\
\hline Renewable water res withdrawn (\% of total) & & 2 & 2 \\
\hline Terrestrial protect areas (\% total land area) & 15 & 22 & 26 \\
\hline Organic area (\% total agricultural area) & & & 8 \\
\hline Water withdrawal by agriculture (\% of total) & & 2 & 2 \\
\hline Biofuel production (thousand kt of oil eq.) & 1 & 4 & 196 \\
\hline Wood pellet prod. (1 000 tonnes) & & & 168 \\
\hline Net GHG emissions from AFOLU ( $\mathrm{CO}_{2}$ eq, $\mathrm{Mt}$ ) & 5 & 2 & 2 \\
\hline
\end{tabular}




\begin{tabular}{|c|c|c|c|}
\hline & 1990 & 2000 & 2014 \\
\hline \multicolumn{4}{|l|}{ The setting } \\
\hline Population, total (mln) & 12.5 & 16.4 & 22 \\
\hline Population, rural (mln) & 6.4 & 7.9 & 9.4 \\
\hline Govt expenditure on ag (\% total outlays) & & & 4.4 \\
\hline Area harvested (mln ha) & 4 & 4 & 4 \\
\hline Cropping intensity ratio & 0.3 & 0.3 & \\
\hline Water resources (1 $000 \mathrm{~m}^{3} /$ person/year) & 1 & 1 & 1 \\
\hline Area equipped for irrigation (1 $000 \mathrm{ha})$ & & & 1428 \\
\hline Area irrigated (\% area equipped for irrigation) & & 95.5 & \\
\hline Employment in agriculture (\%) & 31.1 & 32.9 & 14.3 \\
\hline Employment in agriculture, female (\%) & 52.7 & 61.7 & 22.2 \\
\hline Fertilizers, Nitrogen (kg of nutrients per ha) & & 52.2 & 23.6 \\
\hline Fertilizers, Phosphate (kg of nutrients per ha) & & 22 & 6.2 \\
\hline Fertilizers, Potash (kg nutrients per ha) & & 1.7 & 0.1 \\
\hline Energy consump, power irrigation (mln kWh) & 77 & 77 & 719 \\
\hline Agr value added per worker (constant US\$) & & & 4736 \\
\hline \multicolumn{4}{|l|}{ Hunger dimensions } \\
\hline \multicolumn{4}{|l|}{ Dietary energy supply (kcal/pc/day) } \\
\hline \multicolumn{4}{|l|}{ Average dietary energy supply adequacy (\%) } \\
\hline \multicolumn{4}{|l|}{ Dietary en supp, cereals/roots/tubers (\%) } \\
\hline \multicolumn{4}{|l|}{ Prevalence of undernourishment (\%) } \\
\hline \multicolumn{4}{|l|}{ GDP per capita (US\$, PPP) } \\
\hline \multicolumn{4}{|l|}{ Domestic food price volatility (index) } \\
\hline Cereal import dependency ratio (\%) & 13.1 & 19.8 & 43.2 \\
\hline Underweight, children under-5 (\%) & 11.5 & 6 & 10.1 \\
\hline Improved water source (\% pop) & 85.7 & 87.5 & 90.1 \\
\hline \multicolumn{4}{|l|}{ Food supply } \\
\hline Food production value, (2004-2006 mln I\$) & 3151 & 4824 & 5121 \\
\hline Agriculture, value added (\% GDP) & 30 & 24 & 18 \\
\hline Food exports (mln US\$) & 527 & 423 & 571 \\
\hline Food imports (mln US\$) & 649 & 650 & 1878 \\
\hline \multicolumn{4}{|l|}{ Production indices $(2004-06=100)$} \\
\hline Net food & 51 & 78 & 82 \\
\hline Net crops & 52 & 81 & 76 \\
\hline Cereals & 49 & 59 & 71 \\
\hline Vegetable oils & 51 & 96 & 78 \\
\hline Roots and tubers & 67 & 84 & 76 \\
\hline Fruit and vegetables & 71 & 89 & 87 \\
\hline Sugar & 34 & 94 & 25 \\
\hline Livestock & 53 & 75 & 84 \\
\hline Milk & 58 & 71 & 100 \\
\hline Meat & 49 & 79 & 78 \\
\hline Fish & 34 & 78 & 51 \\
\hline \multicolumn{4}{|l|}{ Net trade (mln US\$) } \\
\hline Cereals & -345 & -237 & -612 \\
\hline Fruit and vegetables & 197 & 197 & -91 \\
\hline Meat & -2 & 0 & -18 \\
\hline Dairy products & -4 & -46 & -78 \\
\hline Fish & -1 & -41 & -74 \\
\hline \multicolumn{4}{|l|}{ Environment } \\
\hline Forest area (\%) & 2 & 2 & 3 \\
\hline Renewable water res withdrawn (\% of total) & & & 88 \\
\hline Terrestrial protect areas (\% total land area) & 0 & 0 & 1 \\
\hline Organic area (\% total agricultural area) & & & 0 \\
\hline Water withdrawal by agriculture (\% of total) & & & 88 \\
\hline \multicolumn{4}{|l|}{ Biofuel production (thousand kt of oil eq.) } \\
\hline \multicolumn{4}{|l|}{ Wood pellet prod. (1 000 tonnes) } \\
\hline Net GHG emissions from AFOLU ( $\mathrm{CO}_{2}$ eq, $\left.\mathrm{Mt}\right)$ & 6 & 5 & 6 \\
\hline
\end{tabular}




\begin{tabular}{|c|c|c|c|}
\hline & 1990 & 2000 & 2014 \\
\hline \multicolumn{4}{|l|}{ The setting } \\
\hline Population, total (mln) & 5.6 & 6.2 & 8.4 \\
\hline Population, rural (mln) & 3.9 & 4.5 & 6.2 \\
\hline Govt expenditure on ag (\% total outlays) & & 2.9 & 2 \\
\hline Area harvested (mln ha) & & 1 & 1 \\
\hline Cropping intensity ratio & & 0.1 & \\
\hline Water resources (1 $000 \mathrm{~m}^{3} /$ person/year) & 4 & 4 & 3 \\
\hline Area equipped for irrigation (1 $000 \mathrm{ha})$ & & & 742 \\
\hline Area irrigated (\% area equipped for irrigation) & & & 90.9 \\
\hline \multicolumn{4}{|l|}{ Employment in agriculture (\%) } \\
\hline \multicolumn{4}{|l|}{ Employment in agriculture, female (\%) } \\
\hline Fertilizers, Nitrogen (kg of nutrients per ha) & & 0 & 58.7 \\
\hline Fertilizers, Phosphate (kg of nutrients per ha) & & 0 & 0 \\
\hline Fertilizers, Potash (kg nutrients per ha) & & 0 & 0 \\
\hline Energy consump, power irrigation (mln kWh) & 0 & 0 & 0 \\
\hline Agr value added per worker (constant US\$) & 539 & 518 & 1167 \\
\hline \multicolumn{4}{|l|}{ Hunger dimensions } \\
\hline Dietary energy supply (kcal/pc/day) & 2048 & 1953 & 2140 \\
\hline Average dietary energy supply adequacy (\%) & 97 & 90 & 96 \\
\hline Dietary en supp, cereals/roots/tubers (\%) & 64 & 72 & 62 \\
\hline Prevalence of undernourishment (\%) & 28.1 & 38.8 & 34 \\
\hline GDP per capita (US\$, PPP) & 3635 & 1186 & 2432 \\
\hline \multicolumn{4}{|l|}{ Domestic food price volatility (index) } \\
\hline Cereal import dependency ratio (\%) & 85.4 & 45.4 & 43.7 \\
\hline Underweight, children under-5 (\%) & & & 13.3 \\
\hline Improved water source (\% pop) & 58.2 & 59.6 & 71.7 \\
\hline \multicolumn{4}{|l|}{ Food supply } \\
\hline Food production value, (2004-2006 mln I\$) & 672 & 597 & 1292 \\
\hline Agriculture, value added (\% GDP) & 33 & 27 & 27 \\
\hline Food exports (mln US\$) & 12 & 25 & 41 \\
\hline Food imports (mln US\$) & 238 & 123 & 572 \\
\hline \multicolumn{4}{|l|}{ Production indices (2004-06=100) } \\
\hline Net food & 81 & 72 & 156 \\
\hline Net crops & 80 & 67 & 151 \\
\hline Cereals & 29 & 66 & 135 \\
\hline Vegetable oils & 112 & 68 & 93 \\
\hline Roots and tubers & 26 & 54 & 204 \\
\hline Fruit and vegetables & 78 & 72 & 202 \\
\hline \multicolumn{4}{|l|}{ Sugar } \\
\hline Livestock & 87 & 56 & 164 \\
\hline Milk & 91 & 59 & 159 \\
\hline Meat & 81 & 53 & 161 \\
\hline Fish & 1811 & 118 & 737 \\
\hline \multicolumn{4}{|l|}{ Net trade (mln US\$) } \\
\hline Cereals & & -33 & -316 \\
\hline Fruit and vegetables & 7 & 25 & 26 \\
\hline Meat & & -14 & -26 \\
\hline Dairy products & & & -2 \\
\hline Fish & & 0 & -3 \\
\hline \multicolumn{4}{|l|}{ Environment } \\
\hline Forest area (\%) & 3 & 3 & 3 \\
\hline Renewable water res withdrawn (\% of total) & & & 91 \\
\hline Terrestrial protect areas (\% total land area) & 2 & 4 & 5 \\
\hline Organic area (\% total agricultural area) & & & 0 \\
\hline Water withdrawal by agriculture (\% of total) & & & 91 \\
\hline \multicolumn{4}{|l|}{ Biofuel production (thousand kt of oil eq.) } \\
\hline \multicolumn{4}{|l|}{ Wood pellet prod. (1 000 tonnes) } \\
\hline Net GHG emissions from AFOLU ( $\mathrm{CO}_{2}$ eq, $\mathrm{Mt}$ ) & 3 & 3 & 5 \\
\hline
\end{tabular}




\begin{tabular}{|c|c|c|c|}
\hline & 1990 & 2000 & 2014 \\
\hline \multicolumn{4}{|l|}{ The setting } \\
\hline Population, total (mln) & 25.5 & 34 & 50.8 \\
\hline Population, rural (mln) & 20.7 & 26.4 & 36.5 \\
\hline Govt expenditure on ag (\% total outlays) & & 3.5 & 1.6 \\
\hline Area harvested (mln ha) & 9 & 6 & 10 \\
\hline Cropping intensity ratio & 0.3 & 0.2 & \\
\hline Water resources (1 $000 \mathrm{~m}^{3} /$ person/year) & 4 & 3 & 2 \\
\hline Area equipped for irrigation (1 $000 \mathrm{ha}$ ) & & & 184 \\
\hline \multicolumn{4}{|l|}{ Area irrigated (\% area equipped for irrigation) } \\
\hline Employment in agriculture (\%) & 84.2 & 82.1 & 76.5 \\
\hline Employment in agriculture, female (\%) & 90.4 & 84 & 80 \\
\hline Fertilizers, Nitrogen (kg of nutrients per ha) & & 3.1 & 3.4 \\
\hline Fertilizers, Phosphate (kg of nutrients per ha) & & 0.7 & 0.5 \\
\hline Fertilizers, Potash (kg nutrients per ha) & & 0.6 & 0.5 \\
\hline \multicolumn{4}{|l|}{ Energy consump, power irrigation (mln kWh) } \\
\hline Agr value added per worker (constant US\$) & 257 & 275 & 356 \\
\hline \multicolumn{4}{|l|}{ Hunger dimensions } \\
\hline Dietary energy supply (kcal/pc/day) & 2109 & 2032 & 2203 \\
\hline Average dietary energy supply adequacy (\%) & 101 & 96 & 105 \\
\hline Dietary en supp, cereals/roots/tubers (\%) & 68 & 65 & 57 \\
\hline Prevalence of undernourishment (\%) & 27.1 & 36.8 & 32.1 \\
\hline GDP per capita (US\$, PPP) & 1460 & 1477 & 2365 \\
\hline Domestic food price volatility (index) & & 7.1 & 4.8 \\
\hline Cereal import dependency ratio (\%) & 7.1 & 9.6 & 13.2 \\
\hline Underweight, children under-5 (\%) & 25.1 & 25.3 & 13.6 \\
\hline Improved water source (\% pop) & 55 & 54.3 & 53.2 \\
\hline \multicolumn{4}{|l|}{ Food supply } \\
\hline Food production value, (2004-2006 mln I\$) & 3648 & 4101 & 8650 \\
\hline Agriculture, value added (\% GDP) & 46 & 33 & 31 \\
\hline Food exports (mln US\$) & 67 & 184 & 726 \\
\hline Food imports (mln US\$) & 66 & 308 & 1115 \\
\hline \multicolumn{4}{|l|}{ Production indices $(2004-06=100)$} \\
\hline Net food & 65 & 73 & 154 \\
\hline Net crops & 62 & 66 & 157 \\
\hline Cereals & 66 & 63 & 156 \\
\hline Vegetable oils & 32 & 43 & 336 \\
\hline Roots and tubers & 117 & 86 & 133 \\
\hline Fruit and vegetables & 44 & 54 & 133 \\
\hline Sugar & 58 & 60 & 130 \\
\hline Livestock & 71 & 89 & 143 \\
\hline Milk & 42 & 56 & 141 \\
\hline Meat & 87 & 107 & 150 \\
\hline Fish & 108 & 89 & 109 \\
\hline \multicolumn{4}{|l|}{ Net trade (mln US\$) } \\
\hline Cereals & -16 & -112 & -370 \\
\hline Fruit and vegetables & 27 & 107 & 313 \\
\hline Meat & 7 & 0 & -7 \\
\hline Dairy products & -5 & -5 & -14 \\
\hline Fish & 6 & 94 & 101 \\
\hline \multicolumn{4}{|l|}{ Environment } \\
\hline Forest area (\%) & 47 & 42 & 37 \\
\hline Renewable water res withdrawn (\% of total) & & 89 & 89 \\
\hline Terrestrial protect areas (\% total land area) & 27 & 28 & 32 \\
\hline Organic area (\% total agricultural area) & & & 0 \\
\hline Water withdrawal by agriculture (\% of total) & & 89 & 89 \\
\hline Biofuel production (thousand kt of oil eq.) & 3 & 3 & 7 \\
\hline \multicolumn{4}{|l|}{ Wood pellet prod. (1 000 tonnes) } \\
\hline Net GHG emissions from AFOLU ( $\mathrm{CO}_{2}$ eq, $\mathrm{Mt}$ ) & 135 & 134 & 147 \\
\hline
\end{tabular}




\begin{tabular}{|c|c|c|c|}
\hline & 1990 & 2000 & 2014 \\
\hline \multicolumn{4}{|l|}{ The setting } \\
\hline Population, total (mln) & 56.6 & 62.3 & 67.2 \\
\hline Population, rural (mln) & 39.9 & 42.9 & 43.5 \\
\hline \multicolumn{4}{|l|}{ Govt expenditure on ag (\% total outlays) } \\
\hline Area harvested (mln ha) & 34 & 54 & 100 \\
\hline Cropping intensity ratio & 1.6 & 2.7 & \\
\hline Water resources (1 $000 \mathrm{~m}^{3} /$ person/year) & 8 & 7 & 7 \\
\hline Area equipped for irrigation (1 $000 \mathrm{ha}$ ) & & & 6415 \\
\hline Area irrigated (\% area equipped for irrigation) & & & 78.9 \\
\hline Employment in agriculture (\%) & 64 & 48.8 & 39.6 \\
\hline Employment in agriculture, female (\%) & 65 & 47.5 & 37.8 \\
\hline Fertilizers, Nitrogen (kg of nutrients per ha) & & 82.5 & 96.5 \\
\hline Fertilizers, Phosphate (kg of nutrients per ha) & & 37 & 30.2 \\
\hline Fertilizers, Potash (kg nutrients per ha) & & 29.7 & 26.5 \\
\hline \multicolumn{4}{|l|}{ Energy consump, power irrigation (mln kWh) } \\
\hline Agr value added per worker (constant US\$) & 645 & 803 & 1195 \\
\hline \multicolumn{4}{|l|}{ Hunger dimensions } \\
\hline Dietary energy supply (kcal/pc/day) & 2300 & 2580 & 2821 \\
\hline Average dietary energy supply adequacy (\%) & 97 & 107 & 115 \\
\hline Dietary en supp, cereals/roots/tubers (\%) & 54 & 52 & 50 \\
\hline Prevalence of undernourishment (\%) & 32.6 & 19 & 7.9 \\
\hline GDP per capita (US\$, PPP) & 6369 & 8939 & 13932 \\
\hline Domestic food price volatility (index) & & 3.7 & 2.8 \\
\hline Cereal import dependency ratio (\%) & -35.3 & -39 & -41.6 \\
\hline Underweight, children under-5 (\%) & 16.3 & 15.4 & 9.2 \\
\hline Improved water source (\% pop) & 86.4 & 91.7 & 95.8 \\
\hline \multicolumn{4}{|l|}{ Food supply } \\
\hline Food production value, (2004-2006 mln I\$) & 15396 & 20766 & 28642 \\
\hline Agriculture, value added (\% GDP) & 12 & 9 & 12 \\
\hline Food exports (mln US\$) & 3257 & 4860 & 18764 \\
\hline Food imports (mln US\$) & 443 & 1170 & 6443 \\
\hline \multicolumn{4}{|l|}{ Production indices (2004-06=100) } \\
\hline Net food & 69 & 93 & 128 \\
\hline Net crops & 62 & 91 & 129 \\
\hline Cereals & 60 & 89 & 123 \\
\hline Vegetable oils & 38 & 70 & 197 \\
\hline Roots and tubers & 100 & 94 & 148 \\
\hline Fruit and vegetables & 56 & 100 & 111 \\
\hline Sugar & 62 & 100 & 185 \\
\hline Livestock & 81 & 96 & 130 \\
\hline Milk & 15 & 62 & 130 \\
\hline Meat & 82 & 96 & 129 \\
\hline Fish & 68 & 91 & 71 \\
\hline \multicolumn{4}{|l|}{ Net trade (mln US\$) } \\
\hline Cereals & 1199 & 1549 & 3892 \\
\hline Fruit and vegetables & 1454 & 1086 & 3039 \\
\hline Meat & 310 & 776 & 2522 \\
\hline Dairy products & -152 & -214 & -448 \\
\hline Fish & 1471 & 3586 & 5022 \\
\hline \multicolumn{4}{|l|}{ Environment } \\
\hline Forest area (\%) & 38 & 37 & 37 \\
\hline Renewable water res withdrawn (\% of total) & & & 90 \\
\hline Terrestrial protect areas (\% total land area) & 12 & 17 & 19 \\
\hline Organic area (\% total agricultural area) & & & 0 \\
\hline Water withdrawal by agriculture (\% of total) & & & 90 \\
\hline Biofuel production (thousand kt of oil eq.) & 115 & 194 & 13727 \\
\hline Wood pellet prod. (1 000 tonnes) & & & 20 \\
\hline Net GHG emissions from AFOLU ( $\mathrm{CO}_{2}$ eq, $\mathrm{Mt}$ ) & 76 & 67 & 70 \\
\hline
\end{tabular}




\begin{tabular}{|c|c|c|c|}
\hline & 1990 & 2000 & 2014 \\
\hline \multicolumn{4}{|l|}{ The setting } \\
\hline Population, total (mln) & 0.8 & 0.9 & 1.2 \\
\hline Population, rural (mln) & 0.6 & 0.6 & 0.8 \\
\hline \multicolumn{4}{|l|}{ Govt expenditure on ag (\% total outlays) } \\
\hline Area harvested (mln ha) & 0 & 0 & 0 \\
\hline Cropping intensity ratio & 1 & 0.7 & \\
\hline Water resources (1 $000 \mathrm{~m}^{3} /$ person/year) & 11 & 10 & 7 \\
\hline Area equipped for irrigation (1 $000 \mathrm{ha}$ ) & & & 35 \\
\hline Area irrigated (\% area equipped for irrigation) & & 83.4 & \\
\hline Employment in agriculture (\%) & & & 50.6 \\
\hline Employment in agriculture, female (\%) & & & 50.2 \\
\hline \multicolumn{4}{|l|}{ Fertilizers, Nitrogen (kg of nutrients per ha) } \\
\hline \multicolumn{4}{|l|}{ Fertilizers, Phosphate (kg of nutrients per ha) } \\
\hline \multicolumn{4}{|l|}{ Fertilizers, Potash (kg nutrients per ha) } \\
\hline \multicolumn{4}{|l|}{ Energy consump, power irrigation (mln kWh) } \\
\hline Agr value added per worker (constant US\$) & & 509 & 363 \\
\hline \multicolumn{4}{|l|}{ Hunger dimensions } \\
\hline Dietary energy supply (kcal/pc/day) & 2008 & 1875 & 2179 \\
\hline Average dietary energy supply adequacy (\%) & 96 & 94 & 106 \\
\hline Dietary en supp, cereals/roots/tubers (\%) & 74 & 74 & 70 \\
\hline Prevalence of undernourishment (\%) & 38.5 & 43.9 & 27.9 \\
\hline GDP per capita (US\$, PPP) & & 1322 & 2040 \\
\hline \multicolumn{4}{|l|}{ Domestic food price volatility (index) } \\
\hline Cereal import dependency ratio (\%) & 16.9 & 30.3 & 10.6 \\
\hline Underweight, children under-5 (\%) & & 41.5 & 45.3 \\
\hline Improved water source (\% pop) & & 54.3 & 70.5 \\
\hline \multicolumn{4}{|l|}{ Food supply } \\
\hline Food production value, (2004-2006 mln I\$) & 95 & 90 & 122 \\
\hline Agriculture, value added (\% GDP) & & 28 & 18 \\
\hline Food exports (mln US\$) & 0 & 0 & 0 \\
\hline Food imports (mln US\$) & 21 & 35 & 73 \\
\hline \multicolumn{4}{|l|}{ Production indices (2004-06=100) } \\
\hline Net food & 94 & 89 & 121 \\
\hline Net crops & 80 & 89 & 114 \\
\hline Cereals & 91 & 97 & 140 \\
\hline Vegetable oils & 43 & 84 & 96 \\
\hline Roots and tubers & 89 & 94 & 60 \\
\hline Fruit and vegetables & 91 & 67 & 167 \\
\hline \multicolumn{4}{|l|}{ Sugar } \\
\hline Livestock & 104 & 91 & 112 \\
\hline Milk & 76 & 76 & 114 \\
\hline Meat & 105 & 90 & 112 \\
\hline Fish & 0 & 99 & 89 \\
\hline \multicolumn{4}{|l|}{ Net trade (mln US\$) } \\
\hline \multicolumn{4}{|l|}{ Cereals } \\
\hline Fruit and vegetables & -6 & -9 & -6 \\
\hline \multicolumn{4}{|l|}{ Meat } \\
\hline \multicolumn{4}{|l|}{ Dairy products } \\
\hline Fish & & & -2 \\
\hline \multicolumn{4}{|l|}{ Environment } \\
\hline Forest area (\%) & 65 & 57 & 48 \\
\hline Renewable water res withdrawn (\% of total) & & & 91 \\
\hline Terrestrial protect areas (\% total land area) & & 3 & 9 \\
\hline Organic area (\% total agricultural area) & & & 6 \\
\hline Water withdrawal by agriculture (\% of total) & & & 91 \\
\hline \multicolumn{4}{|l|}{ Biofuel production (thousand kt of oil eq.) } \\
\hline \multicolumn{4}{|l|}{ Wood pellet prod. (1 000 tonnes) } \\
\hline Net GHG emissions from AFOLU ( $\mathrm{CO}_{2}$ eq, $\mathrm{Mt}$ ) & 4 & 4 & 6 \\
\hline
\end{tabular}




\begin{tabular}{|c|c|c|c|}
\hline & 1990 & 2000 & 2014 \\
\hline \multicolumn{4}{|l|}{ The setting } \\
\hline Population, total (mln) & 3.8 & 4.9 & 7 \\
\hline Population, rural (mln) & 2.7 & 3.3 & 4.2 \\
\hline \multicolumn{4}{|l|}{ Govt expenditure on ag (\% total outlays) } \\
\hline Area harvested (mln ha) & 1 & 1 & 2 \\
\hline Cropping intensity ratio & 0.3 & 0.4 & \\
\hline Water resources (1 $000 \mathrm{~m}^{3} /$ person/year) & 4 & 3 & 2 \\
\hline Area equipped for irrigation (1 $000 \mathrm{ha})$ & & & 7 \\
\hline Area irrigated (\% area equipped for irrigation) & & 85.6 & \\
\hline Employment in agriculture (\%) & & & 54.1 \\
\hline Employment in agriculture, female (\%) & & & 48.2 \\
\hline Fertilizers, Nitrogen (kg of nutrients per ha) & & 2.6 & 2.6 \\
\hline Fertilizers, Phosphate (kg of nutrients per ha) & & 2.4 & 2.1 \\
\hline Fertilizers, Potash (kg nutrients per ha) & & 2.4 & 0.4 \\
\hline Energy consump, power irrigation (mln kWh) & 1 & 1 & 1 \\
\hline Agr value added per worker (constant US\$) & 582 & 636 & 681 \\
\hline \multicolumn{4}{|l|}{ Hunger dimensions } \\
\hline Dietary energy supply (kcal/pc/day) & 1967 & 2158 & 2600 \\
\hline Average dietary energy supply adequacy (\%) & 93 & 101 & 120 \\
\hline Dietary en supp, cereals/roots/tubers (\%) & 75 & 76 & 72 \\
\hline Prevalence of undernourishment (\%) & 40.6 & 29.2 & 12.9 \\
\hline GDP per capita (US\$, PPP) & 1350 & 1305 & 1346 \\
\hline Domestic food price volatility (index) & & 26.1 & 15.5 \\
\hline Cereal import dependency ratio (\%) & 10.9 & 12.4 & 14 \\
\hline Underweight, children under-5 (\%) & & 23.2 & 16.5 \\
\hline Improved water source (\% pop) & 48.4 & 53.4 & 60 \\
\hline \multicolumn{4}{|l|}{ Food supply } \\
\hline Food production value, (2004-2006 mln I\$) & 390 & 513 & 763 \\
\hline Agriculture, value added (\% GDP) & 34 & 35 & 42 \\
\hline Food exports (mln US\$) & 41 & 19 & 147 \\
\hline Food imports (mln US\$) & 79 & 36 & 185 \\
\hline \multicolumn{4}{|l|}{ Production indices (2004-06=100) } \\
\hline Net food & 64 & 84 & 125 \\
\hline Net crops & 68 & 91 & 117 \\
\hline Cereals & 57 & 88 & 151 \\
\hline Vegetable oils & 87 & 86 & 120 \\
\hline Roots and tubers & 71 & 95 & 115 \\
\hline Fruit and vegetables & 97 & 98 & 101 \\
\hline \multicolumn{4}{|l|}{ Sugar } \\
\hline Livestock & 66 & 82 & 144 \\
\hline Milk & 58 & 93 & 105 \\
\hline Meat & 65 & 82 & 146 \\
\hline Fish & 59 & 83 & 74 \\
\hline \multicolumn{4}{|l|}{ Net trade (mln US\$) } \\
\hline Cereals & -17 & -11 & -47 \\
\hline Fruit and vegetables & -8 & -1 & -7 \\
\hline Meat & -2 & -3 & -12 \\
\hline Dairy products & -6 & -1 & -6 \\
\hline Fish & -14 & -9 & -32 \\
\hline \multicolumn{4}{|l|}{ Environment } \\
\hline Forest area (\%) & 13 & 9 & 5 \\
\hline Renewable water res withdrawn (\% of total) & & 45 & 45 \\
\hline Terrestrial protect areas (\% total land area) & 11 & 12 & 25 \\
\hline Organic area (\% total agricultural area) & & & 0 \\
\hline Water withdrawal by agriculture (\% of total) & & 45 & 45 \\
\hline Biofuel production (thousand kt of oil eq.) & 1 & 1 & 1 \\
\hline \multicolumn{4}{|l|}{ Wood pellet prod. (1 000 tonnes) } \\
\hline Net GHG emissions from AFOLU ( $\mathrm{CO}_{2}$ eq, $\left.\mathrm{Mt}\right)$ & 11 & 10 & 2 \\
\hline
\end{tabular}




\begin{tabular}{|c|c|c|c|}
\hline & 1990 & 2000 & 2014 \\
\hline \multicolumn{4}{|l|}{ The setting } \\
\hline Population, total (mln) & 1.2 & 1.3 & 1.3 \\
\hline Population, rural (mln) & 1.1 & 1.1 & 1.1 \\
\hline \multicolumn{4}{|l|}{ Govt expenditure on ag (\% total outlays) } \\
\hline Area harvested (mln ha) & 1 & 1 & 0 \\
\hline Cropping intensity ratio & 19.2 & 20.5 & \\
\hline Water resources (1 $000 \mathrm{~m}^{3} /$ person/year) & 3 & 3 & 3 \\
\hline Area equipped for irrigation (1 000 ha) & & & 7 \\
\hline Area irrigated (\% area equipped for irrigation) & & 85 & \\
\hline Employment in agriculture (\%) & 12.3 & 7.2 & 3.8 \\
\hline Employment in agriculture, female (\%) & 6.3 & 2.9 & 1.8 \\
\hline Fertilizers, Nitrogen (kg of nutrients per ha) & & 106.3 & 506.8 \\
\hline Fertilizers, Phosphate (kg of nutrients per ha) & & 8.1 & 48.3 \\
\hline Fertilizers, Potash (kg nutrients per ha) & & 36.3 & 64.3 \\
\hline Energy consump, power irrigation (mln kWh) & 2 & 2 & 2 \\
\hline Agr value added per worker (constant US\$) & 2048 & 2484 & 1888 \\
\hline \multicolumn{4}{|l|}{ Hunger dimensions } \\
\hline Dietary energy supply (kcal/pc/day) & 2554 & 2761 & 2965 \\
\hline Average dietary energy supply adequacy (\%) & 109 & 114 & 122 \\
\hline Dietary en supp, cereals/roots/tubers (\%) & 39 & 37 & 37 \\
\hline Prevalence of undernourishment (\%) & 14.7 & 13 & 8 \\
\hline GDP per capita (US\$, PPP) & 13464 & 17721 & 29469 \\
\hline Domestic food price volatility (index) & & 12.8 & 16.5 \\
\hline Cereal import dependency ratio (\%) & 92.6 & 98.2 & 98 \\
\hline Underweight, children under-5 (\%) & & 4.4 & \\
\hline Improved water source (\% pop) & 90.3 & 92.2 & 93.6 \\
\hline \multicolumn{4}{|l|}{ Food supply } \\
\hline Food production value, (2004-2006 mln I\$) & 131 & 146 & 143 \\
\hline Agriculture, value added (\% GDP) & 3 & 1 & 1 \\
\hline Food exports (mln US\$) & 74 & 134 & 114 \\
\hline Food imports (mln US\$) & 206 & 259 & 756 \\
\hline \multicolumn{4}{|l|}{ Production indices (2004-06=100) } \\
\hline Net food & 88 & 98 & 96 \\
\hline Net crops & 141 & 132 & 60 \\
\hline Cereals & 472 & 208 & 141 \\
\hline Vegetable oils & 222 & 128 & 92 \\
\hline Roots and tubers & 56 & 57 & 118 \\
\hline Fruit and vegetables & 65 & 80 & 88 \\
\hline Sugar & 244 & 227 & 134 \\
\hline Livestock & 55 & 75 & 121 \\
\hline Milk & 151 & 142 & 69 \\
\hline Meat & 51 & 73 & 123 \\
\hline Fish & 83 & 95 & 88 \\
\hline \multicolumn{4}{|l|}{ Net trade (mln US\$) } \\
\hline Cereals & -43 & -20 & -145 \\
\hline Fruit and vegetables & -34 & -28 & -106 \\
\hline Meat & -18 & -22 & -99 \\
\hline Dairy products & -37 & -37 & -93 \\
\hline Fish & -2 & 4 & -30 \\
\hline \multicolumn{4}{|l|}{ Environment } \\
\hline Forest area $(\%)$ & 47 & 46 & 44 \\
\hline Renewable water res withdrawn (\% of total) & & 9 & 9 \\
\hline Terrestrial protect areas (\% total land area) & 30 & 32 & 33 \\
\hline \multicolumn{4}{|l|}{ Organic area (\% total agricultural area) } \\
\hline Water withdrawal by agriculture (\% of total) & & 9 & 9 \\
\hline Biofuel production (thousand kt of oil eq.) & 3 & 4 & 0 \\
\hline \multicolumn{4}{|l|}{ Wood pellet prod. (1 000 tonnes) } \\
\hline Net GHG emissions from AFOLU ( $\mathrm{CO}_{2}$ eq, $\left.\mathrm{Mt}\right)$ & 1 & 1 & 1 \\
\hline
\end{tabular}




\section{Tunisia}

\begin{tabular}{|c|c|c|c|}
\hline & 1990 & 2000 & 2014 \\
\hline \multicolumn{4}{|l|}{ The setting } \\
\hline Population, total (mln) & 8.1 & 9.6 & 11.1 \\
\hline Population, rural (mln) & 3.4 & 3.5 & 3.7 \\
\hline Govt expenditure on ag (\% total outlays) & & 8.9 & 5.4 \\
\hline Area harvested (mln ha) & 2 & 2 & 2 \\
\hline Cropping intensity ratio & 0.2 & 0.2 & \\
\hline Water resources (1 $000 \mathrm{~m}^{3} /$ person/year) & 1 & 0 & 0 \\
\hline Area equipped for irrigation (1 $000 \mathrm{ha}$ ) & & & 459 \\
\hline Area irrigated (\% area equipped for irrigation) & & & 89 \\
\hline Employment in agriculture (\%) & 25.8 & & 16.2 \\
\hline Employment in agriculture, female (\%) & 22.7 & & \\
\hline Fertilizers, Nitrogen (kg of nutrients per ha) & & 18.9 & 30.7 \\
\hline Fertilizers, Phosphate (kg of nutrients per ha) & & 14.1 & 22.6 \\
\hline Fertilizers, Potash (kg nutrients per ha) & & 3.4 & 2.3 \\
\hline Energy consump, power irrigation (mln kWh) & 0 & 147 & 366 \\
\hline Agr value added per worker (constant US\$) & 3019 & 3601 & 4424 \\
\hline \multicolumn{4}{|l|}{ Hunger dimensions } \\
\hline Dietary energy supply (kcal/pc/day) & 3141 & 3296 & 3465 \\
\hline Average dietary energy supply adequacy (\%) & 140 & 142 & 146 \\
\hline Dietary en supp, cereals/roots/tubers (\%) & 55 & 53 & 53 \\
\hline Prevalence of undernourishment (\%) & $<5.0$ & $<5.0$ & $<5.0$ \\
\hline GDP per capita (US\$, PPP) & 5502 & 7464 & 10768 \\
\hline Domestic food price volatility (index) & & 4.2 & 4.7 \\
\hline Cereal import dependency ratio (\%) & 41.5 & 61 & 55.3 \\
\hline Underweight, children under-5 (\%) & & 3.5 & 2.3 \\
\hline Improved water source (\% pop) & 81.5 & 89.4 & 96.8 \\
\hline \multicolumn{4}{|l|}{ Food supply } \\
\hline Food production value, (2004-2006 mln I\$) & 2287 & 2781 & 3916 \\
\hline Agriculture, value added (\% GDP) & 18 & 11 & 9 \\
\hline Food exports (mln US\$) & 240 & 373 & 1309 \\
\hline Food imports (mln US\$) & 480 & 535 & 2144 \\
\hline \multicolumn{4}{|l|}{ Production indices $(2004-06=100)$} \\
\hline Net food & 69 & 84 & 119 \\
\hline Net crops & 75 & 78 & 117 \\
\hline Cereals & 79 & 54 & 65 \\
\hline Vegetable oils & 85 & 57 & 113 \\
\hline Roots and tubers & 62 & 83 & 111 \\
\hline Fruit and vegetables & 61 & 91 & 132 \\
\hline \multicolumn{4}{|l|}{ Sugar } \\
\hline Livestock & 58 & 101 & 120 \\
\hline Milk & 46 & 98 & 126 \\
\hline Meat & 64 & 104 & 117 \\
\hline Fish & 79 & 86 & 109 \\
\hline \multicolumn{4}{|l|}{ Net trade (mln US\$) } \\
\hline Cereals & -202 & -232 & -813 \\
\hline Fruit and vegetables & 60 & 39 & 299 \\
\hline Meat & -27 & -7 & -41 \\
\hline Dairy products & -42 & -12 & -7 \\
\hline Fish & 105 & 75 & 89 \\
\hline \multicolumn{4}{|l|}{ Environment } \\
\hline Forest area (\%) & 4 & 5 & 7 \\
\hline Renewable water res withdrawn (\% of total) & & 82 & 82 \\
\hline Terrestrial protect areas (\% total land area) & 1 & 1 & 5 \\
\hline Organic area (\% total agricultural area) & & & 1 \\
\hline Water withdrawal by agriculture (\% of total) & & 82 & 82 \\
\hline Biofuel production (thousand kt of oil eq.) & & 6 & 6 \\
\hline \multicolumn{4}{|l|}{ Wood pellet prod. (1 000 tonnes) } \\
\hline Net GHG emissions from AFOLU ( $\mathrm{CO}_{2}$ eq, $\mathrm{Mt}$ ) & 3 & 4 & 4 \\
\hline
\end{tabular}




\begin{tabular}{|c|c|c|c|}
\hline & 1990 & 2000 & 2014 \\
\hline \multicolumn{4}{|l|}{ The setting } \\
\hline Population, total (mln) & 54 & 63.2 & 75.8 \\
\hline Population, rural (mln) & 22 & 22.3 & 19.5 \\
\hline \multicolumn{4}{|l|}{ Govt expenditure on ag (\% total outlays) } \\
\hline Area harvested (mln ha) & 30 & 32 & 37 \\
\hline Cropping intensity ratio & 0.8 & 0.8 & \\
\hline Water resources (1 $000 \mathrm{~m}^{3} /$ person/year) & 4 & 3 & 3 \\
\hline Area equipped for irrigation (1 $000 \mathrm{ha})$ & & & 5215 \\
\hline Area irrigated (\% area equipped for irrigation) & & & 98.9 \\
\hline Employment in agriculture (\%) & 46.9 & 36 & 23.6 \\
\hline Employment in agriculture, female (\%) & 75.8 & 60.5 & 37.2 \\
\hline Fertilizers, Nitrogen (kg of nutrients per ha) & & 57.4 & 71.6 \\
\hline Fertilizers, Phosphate (kg of nutrients per ha) & & 23.4 & 29.8 \\
\hline Fertilizers, Potash (kg nutrients per ha) & & 3.6 & 4.7 \\
\hline Energy consump, power irrigation (mln kWh) & 0 & 637 & 959 \\
\hline Agr value added per worker (constant US\$) & 3499 & 4573 & 6863 \\
\hline \multicolumn{4}{|l|}{ Hunger dimensions } \\
\hline Dietary energy supply (kcal/pc/day) & 3723 & 3634 & 3703 \\
\hline Average dietary energy supply adequacy (\%) & 163 & 157 & 156 \\
\hline Dietary en supp, cereals/roots/tubers (\%) & 54 & 54 & 47 \\
\hline Prevalence of undernourishment (\%) & $<5.0$ & $<5.0$ & $<5.0$ \\
\hline GDP per capita (US\$, PPP) & 10670 & 13025 & 18567 \\
\hline Domestic food price volatility (index) & & 19.8 & 12.9 \\
\hline Cereal import dependency ratio (\%) & -7.4 & -0.2 & 0.8 \\
\hline Underweight, children under-5 (\%) & 8.7 & 7 & 1.9 \\
\hline Improved water source (\% pop) & 85.4 & 92.8 & 99.7 \\
\hline \multicolumn{4}{|l|}{ Food supply } \\
\hline Food production value, (2004-2006 mln I\$) & 22979 & 27212 & 37484 \\
\hline Agriculture, value added (\% GDP) & 18 & 11 & 8 \\
\hline Food exports (mln US\$) & 2309 & 2865 & 12641 \\
\hline Food imports (mln US\$) & 1371 & 1366 & 7787 \\
\hline \multicolumn{4}{|l|}{ Production indices $(2004-06=100)$} \\
\hline Net food & 80 & 94 & 130 \\
\hline Net crops & 79 & 96 & 115 \\
\hline Cereals & 85 & 91 & 109 \\
\hline Vegetable oils & 78 & 103 & 122 \\
\hline Roots and tubers & 98 & 121 & 90 \\
\hline Fruit and vegetables & 72 & 93 & 121 \\
\hline Sugar & 97 & 131 & 115 \\
\hline Livestock & 83 & 92 & 163 \\
\hline Milk & 87 & 87 & 162 \\
\hline Meat & 85 & 93 & 174 \\
\hline Fish & 62 & 94 & 98 \\
\hline \multicolumn{4}{|l|}{ Net trade (mln US\$) } \\
\hline Cereals & -497 & -17 & 519 \\
\hline Fruit and vegetables & 1682 & 1625 & 5738 \\
\hline Meat & 7 & 11 & 469 \\
\hline Dairy products & -9 & -17 & 87 \\
\hline Fish & 42 & 39 & 137 \\
\hline \multicolumn{4}{|l|}{ Environment } \\
\hline Forest area (\%) & 13 & 13 & 15 \\
\hline Renewable water res withdrawn (\% of total) & & 74 & 74 \\
\hline Terrestrial protect areas (\% total land area) & 2 & 2 & 2 \\
\hline Organic area (\% total agricultural area) & & & 2 \\
\hline Water withdrawal by agriculture (\% of total) & & 74 & 74 \\
\hline Biofuel production (thousand kt of oil eq.) & 15 & 58 & 236 \\
\hline \multicolumn{4}{|l|}{ Wood pellet prod. (1 000 tonnes) } \\
\hline Net GHG emissions from AFOLU ( $\mathrm{CO}_{2}$ eq, $\left.\mathrm{Mt}\right)$ & 40 & 20 & 14 \\
\hline
\end{tabular}




\section{Turkmenistan}

\begin{tabular}{|c|c|c|c|}
\hline & 1990 & 2000 & 2014 \\
\hline \multicolumn{4}{|l|}{ The setting } \\
\hline Population, total (mln) & 4 & 4.5 & 5.3 \\
\hline Population, rural (mln) & 2.2 & 2.4 & 2.7 \\
\hline \multicolumn{4}{|l|}{ Govt expenditure on ag (\% total outlays) } \\
\hline Area harvested (mln ha) & & 2 & 5 \\
\hline Cropping intensity ratio & & 0 & \\
\hline Water resources (1 $000 \mathrm{~m}^{3} /$ person/year) & 6 & 6 & 5 \\
\hline Area equipped for irrigation (1 $000 \mathrm{ha})$ & & & 1995 \\
\hline Area irrigated (\% area equipped for irrigation) & & & 100 \\
\hline \multicolumn{4}{|l|}{ Employment in agriculture (\%) } \\
\hline \multicolumn{4}{|l|}{ Employment in agriculture, female (\%) } \\
\hline \multicolumn{4}{|l|}{ Fertilizers, Nitrogen (kg of nutrients per ha) } \\
\hline \multicolumn{4}{|l|}{ Fertilizers, Phosphate (kg of nutrients per ha) } \\
\hline \multicolumn{4}{|l|}{ Fertilizers, Potash (kg nutrients per ha) } \\
\hline Energy consump, power irrigation (mln kWh) & 0 & 1 & 0 \\
\hline Agr value added per worker (constant US\$) & 1737 & 1354 & 2796 \\
\hline \multicolumn{4}{|l|}{ Hunger dimensions } \\
\hline Dietary energy supply (kcal/pc/day) & 2562 & 2614 & 3010 \\
\hline Average dietary energy supply adequacy (\%) & 116 & 114 & 128 \\
\hline Dietary en supp, cereals/roots/tubers (\%) & 59 & 63 & 59 \\
\hline Prevalence of undernourishment (\%) & 8.6 & 9 & $<5.0$ \\
\hline GDP per capita (US\$, PPP) & 8353 & 5351 & 13555 \\
\hline \multicolumn{4}{|l|}{ Domestic food price volatility (index) } \\
\hline \multicolumn{4}{|l|}{ Cereal import dependency ratio (\%) } \\
\hline Underweight, children under-5 (\%) & & 10.5 & \\
\hline Improved water source (\% pop) & & 83.3 & 71.1 \\
\hline \multicolumn{4}{|l|}{ Food supply } \\
\hline Food production value, (2004-2006 mln I\$) & 905 & 1253 & 2365 \\
\hline Agriculture, value added (\% GDP) & 32 & 24 & 15 \\
\hline Food exports (mln US\$) & 2 & 6 & 9 \\
\hline Food imports (mln US\$) & 231 & 82 & 373 \\
\hline \multicolumn{4}{|l|}{ Production indices $(2004-06=100)$} \\
\hline Net food & 48 & 66 & 124 \\
\hline Net crops & 75 & 71 & 77 \\
\hline Cereals & 31 & 54 & 52 \\
\hline Vegetable oils & 125 & 117 & 63 \\
\hline Roots and tubers & 17 & 49 & 156 \\
\hline Fruit and vegetables & 46 & 64 & 115 \\
\hline Sugar & & 99 & 103 \\
\hline Livestock & 46 & 64 & 136 \\
\hline Milk & 41 & 57 & 126 \\
\hline Meat & 49 & 70 & 150 \\
\hline Fish & 299 & 82 & 100 \\
\hline \multicolumn{4}{|l|}{ Net trade (mln US\$) } \\
\hline \multicolumn{4}{|l|}{ Cereals } \\
\hline Fruit and vegetables & -15 & -5 & -13 \\
\hline Meat & & -25 & \\
\hline Dairy products & & 0 & \\
\hline Fish & & 0 & -7 \\
\hline \multicolumn{4}{|l|}{ Environment } \\
\hline Forest area (\%) & 9 & 9 & 9 \\
\hline Renewable water res withdrawn (\% of total) & & & 94 \\
\hline Terrestrial protect areas (\% total land area) & 3 & 3 & 3 \\
\hline \multicolumn{4}{|l|}{ Organic area (\% total agricultural area) } \\
\hline Water withdrawal by agriculture (\% of total) & & & 94 \\
\hline \multicolumn{4}{|l|}{ Biofuel production (thousand kt of oil eq.) } \\
\hline Wood pellet prod. (1 000 tonnes) & & & 0 \\
\hline Net GHG emissions from AFOLU ( $\mathrm{CO}_{2}$ eq, $\mathrm{Mt}$ ) & 4 & 5 & 8 \\
\hline
\end{tabular}




\begin{tabular}{|c|c|c|c|}
\hline & 1990 & 2000 & 2014 \\
\hline \multicolumn{4}{|l|}{ The setting } \\
\hline Population, total (mln) & 17.5 & 24.3 & 38.8 \\
\hline Population, rural (mln) & 15.6 & 21.3 & 32.3 \\
\hline Govt expenditure on ag (\% total outlays) & & 4.2 & 3.6 \\
\hline Area harvested (mln ha) & 8 & 10 & 9 \\
\hline Cropping intensity ratio & 0.7 & 0.8 & \\
\hline Water resources (1 $000 \mathrm{~m}^{3} /$ person/year) & 3 & 2 & 2 \\
\hline Area equipped for irrigation (1 $000 \mathrm{ha})$ & & & 14 \\
\hline Area irrigated (\% area equipped for irrigation) & & & 95 \\
\hline Employment in agriculture (\%) & & 68.7 & 65.6 \\
\hline Employment in agriculture, female (\%) & & 75.7 & \\
\hline Fertilizers, Nitrogen (kg of nutrients per ha) & & 0.7 & 1 \\
\hline Fertilizers, Phosphate (kg of nutrients per ha) & & 0.5 & 0.4 \\
\hline Fertilizers, Potash (kg nutrients per ha) & & 0.3 & 0.4 \\
\hline Energy consump, power irrigation (mln kWh) & 0 & 1 & 5 \\
\hline Agr value added per worker (constant US\$) & 205 & 220 & 215 \\
\hline \multicolumn{4}{|l|}{ Hunger dimensions } \\
\hline Dietary energy supply (kcal/pc/day) & 2231 & 2265 & 2273 \\
\hline Average dietary energy supply adequacy (\%) & 107 & 109 & 108 \\
\hline Dietary en supp, cereals/roots/tubers (\%) & 44 & 45 & 45 \\
\hline Prevalence of undernourishment (\%) & 25 & 28.4 & 25.3 \\
\hline GDP per capita (US\$, PPP) & 767 & 1042 & 1621 \\
\hline Domestic food price volatility (index) & & 19 & 21.8 \\
\hline Cereal import dependency ratio (\%) & -1.1 & 5.6 & 9.1 \\
\hline Underweight, children under-5 (\%) & & 19 & 14.1 \\
\hline Improved water source (\% pop) & 41.6 & 56.5 & 74.8 \\
\hline \multicolumn{4}{|l|}{ Food supply } \\
\hline Food production value, (2004-2006 mln I\$) & 3220 & 4101 & 5360 \\
\hline Agriculture, value added (\% GDP) & 57 & 29 & 28 \\
\hline Food exports (mln US\$) & 14 & 20 & 446 \\
\hline Food imports (mln US\$) & 20 & 111 & 793 \\
\hline \multicolumn{4}{|l|}{ Production indices (2004-06=100) } \\
\hline Net food & 68 & 86 & 113 \\
\hline Net crops & 72 & 93 & 108 \\
\hline Cereals & 65 & 85 & 136 \\
\hline Vegetable oils & 45 & 62 & 126 \\
\hline Roots and tubers & 61 & 91 & 102 \\
\hline Fruit and vegetables & 84 & 100 & 100 \\
\hline Sugar & 26 & 62 & 141 \\
\hline Livestock & 53 & 67 & 127 \\
\hline Milk & 42 & 50 & 118 \\
\hline Meat & 57 & 73 & 130 \\
\hline Fish & 61 & 55 & 129 \\
\hline \multicolumn{4}{|l|}{ Net trade (mln US\$) } \\
\hline Cereals & -1 & -57 & -185 \\
\hline Fruit and vegetables & 5 & 5 & 13 \\
\hline Meat & -1 & 0 & -1 \\
\hline Dairy products & -5 & -1 & 16 \\
\hline Fish & 1 & 29 & 61 \\
\hline \multicolumn{4}{|l|}{ Environment } \\
\hline Forest area (\%) & 24 & 19 & 14 \\
\hline Renewable water res withdrawn (\% of total) & & & 41 \\
\hline Terrestrial protect areas (\% total land area) & 8 & 9 & 11 \\
\hline Organic area (\% total agricultural area) & & & 2 \\
\hline Water withdrawal by agriculture (\% of total) & & & 41 \\
\hline Biofuel production (thousand kt of oil eq.) & 1 & 4 & 11 \\
\hline \multicolumn{4}{|l|}{ Wood pellet prod. (1 000 tonnes) } \\
\hline Net GHG emissions from AFOLU ( $\mathrm{CO}_{2}$ eq, $\left.\mathrm{Mt}\right)$ & 29 & 31 & 42 \\
\hline
\end{tabular}




\begin{tabular}{|c|c|c|c|}
\hline & 1990 & 2000 & 2014 \\
\hline \multicolumn{4}{|l|}{ The setting } \\
\hline Population, total (mln) & 51.6 & 49.1 & 44.9 \\
\hline Population, rural (mln) & 17.1 & 16.1 & 13.7 \\
\hline Govt expenditure on ag (\% total outlays) & & 4.9 & 2.2 \\
\hline Area harvested (mln ha) & & 24 & 63 \\
\hline Cropping intensity ratio & & 0.6 & \\
\hline Water resources (1 $000 \mathrm{~m}^{3} /$ person/year) & 3 & 3 & 3 \\
\hline Area equipped for irrigation (1 $000 \mathrm{ha}$ ) & & & 2167 \\
\hline Area irrigated (\% area equipped for irrigation) & & 33.6 & \\
\hline Employment in agriculture (\%) & 19.8 & 23.4 & 17.2 \\
\hline \multicolumn{4}{|l|}{ Employment in agriculture, female (\%) } \\
\hline Fertilizers, Nitrogen (kg of nutrients per ha) & & 11.1 & 28.5 \\
\hline Fertilizers, Phosphate (kg of nutrients per ha) & & 2.4 & 6.8 \\
\hline Fertilizers, Potash (kg nutrients per ha) & & 2.3 & 6 \\
\hline Energy consump, power irrigation (mln kWh) & 4012 & 4012 & 4012 \\
\hline Agr value added per worker (constant US\$) & 1923 & 1966 & 5495 \\
\hline \multicolumn{4}{|l|}{ Hunger dimensions } \\
\hline \multicolumn{4}{|l|}{ Dietary energy supply (kcal/pc/day) } \\
\hline Average dietary energy supply adequacy (\%) & 130 & 116 & 126 \\
\hline Dietary en supp, cereals/roots/tubers (\%) & 50 & 50 & 42 \\
\hline Prevalence of undernourishment (\%) & $<5.0$ & $<5.0$ & $<5.0$ \\
\hline GDP per capita (US\$, PPP) & 10507 & 4817 & 8508 \\
\hline Domestic food price volatility (index) & & 8.7 & 3.9 \\
\hline Cereal import dependency ratio (\%) & 2.2 & -15.2 & -60.3 \\
\hline Underweight, children under-5 (\%) & & 4.1 & \\
\hline Improved water source (\% pop) & & 96.9 & 98 \\
\hline \multicolumn{4}{|l|}{ Food supply } \\
\hline Food production value, (2004-2006 mln I\$) & 22215 & 15084 & 23686 \\
\hline Agriculture, value added (\% GDP) & 26 & 17 & 12 \\
\hline Food exports (mln US\$) & 589 & 1475 & 16140 \\
\hline Food imports (mln US\$) & 643 & 666 & 4577 \\
\hline \multicolumn{4}{|l|}{ Production indices $(2004-06=100)$} \\
\hline Net food & 130 & 88 & 138 \\
\hline Net crops & 112 & 77 & 162 \\
\hline Cereals & 116 & 62 & 178 \\
\hline Vegetable oils & 43 & 72 & 288 \\
\hline Roots and tubers & 108 & 99 & 116 \\
\hline Fruit and vegetables & 114 & 88 & 140 \\
\hline Sugar & 186 & 73 & 59 \\
\hline Livestock & 152 & 97 & 105 \\
\hline Milk & 135 & 93 & 85 \\
\hline Meat & 189 & 110 & 122 \\
\hline Fish & 362 & 162 & 87 \\
\hline \multicolumn{4}{|l|}{ Net trade (mln US\$) } \\
\hline Cereals & -294 & -93 & 7003 \\
\hline Fruit and vegetables & 33 & -16 & -882 \\
\hline Meat & 42 & 218 & -361 \\
\hline Dairy products & 23 & 143 & 318 \\
\hline Fish & -6 & -69 & -735 \\
\hline \multicolumn{4}{|l|}{ Environment } \\
\hline Forest area (\%) & 16 & 16 & 17 \\
\hline Renewable water res withdrawn (\% of total) & & & 6 \\
\hline Terrestrial protect areas (\% total land area) & 2 & 4 & 4 \\
\hline Organic area (\% total agricultural area) & & & 1 \\
\hline Water withdrawal by agriculture (\% of total) & & & 6 \\
\hline \multicolumn{4}{|l|}{ Biofuel production (thousand kt of oil eq.) } \\
\hline Wood pellet prod. (1 000 tonnes) & & & 706 \\
\hline Net GHG emissions from AFOLU ( $\mathrm{CO}_{2}$ eq, $\mathrm{Mt}$ ) & 23 & -11 & 5 \\
\hline
\end{tabular}




\section{United Arab Emirates}

\begin{tabular}{|c|c|c|c|}
\hline & 1990 & 2000 & 2014 \\
\hline \multicolumn{4}{|l|}{ The setting } \\
\hline Population, total (mln) & 1.8 & 3 & 9.4 \\
\hline Population, rural (mln) & 0.4 & 0.6 & 1.4 \\
\hline \multicolumn{4}{|l|}{ Govt expenditure on ag (\% total outlays) } \\
\hline Area harvested ( $\mathrm{mln}$ ha) & 1 & 3 & 1 \\
\hline Cropping intensity ratio & 3.1 & 5.2 & \\
\hline Water resources (1 $000 \mathrm{~m}^{3} /$ person/year) & 0 & 0 & 0 \\
\hline Area equipped for irrigation (1 $000 \mathrm{ha})$ & & & 92 \\
\hline Area irrigated (\% area equipped for irrigation) & & & 82.6 \\
\hline Employment in agriculture (\%) & & 7.9 & 3.8 \\
\hline Employment in agriculture, female (\%) & & 0.1 & 0.1 \\
\hline Fertilizers, Nitrogen (kg of nutrients per ha) & & 767.4 & 516.2 \\
\hline Fertilizers, Phosphate (kg of nutrients per ha) & & 32.2 & 0 \\
\hline Fertilizers, Potash (kg nutrients per ha) & & 139.8 & 89.4 \\
\hline Energy consump, power irrigation (mln kWh) & 100 & 100 & 481 \\
\hline Agr value added per worker (constant US\$) & & 30097 & 12079 \\
\hline \multicolumn{4}{|l|}{ Hunger dimensions } \\
\hline Dietary energy supply (kcal/pc/day) & 3250 & 3330 & 3328 \\
\hline Average dietary energy supply adequacy (\%) & 135 & 135 & 129 \\
\hline Dietary en supp, cereals/roots/tubers (\%) & 32 & 42 & 42 \\
\hline Prevalence of undernourishment (\%) & $<5.0$ & $<5.0$ & $<5.0$ \\
\hline GDP per capita (US\$, PPP) & 115748 & 108909 & 57045 \\
\hline \multicolumn{4}{|l|}{ Domestic food price volatility (index) } \\
\hline Cereal import dependency ratio (\%) & 99.7 & 100 & 94.7 \\
\hline \multicolumn{4}{|l|}{ Underweight, children under-5 (\%) } \\
\hline Improved water source (\% pop) & 99.7 & 99.7 & 99.6 \\
\hline \multicolumn{4}{|l|}{ Food supply } \\
\hline Food production value, $(2004-2006 \mathrm{mln}$ I\$) & 197 & 1119 & 439 \\
\hline Agriculture, value added (\% GDP) & & 2 & 1 \\
\hline Food exports (mln US\$) & 501 & 584 & 2579 \\
\hline Food imports (mln US\$) & 1429 & 2040 & 10821 \\
\hline \multicolumn{4}{|l|}{ Production indices $(2004-06=100)$} \\
\hline Net food & 31 & 174 & 68 \\
\hline Net crops & 30 & 201 & 43 \\
\hline Cereals & 12330 & 1560 & 579943 \\
\hline \multicolumn{4}{|l|}{ Vegetable oils } \\
\hline Roots and tubers & 56 & 220 & 141 \\
\hline Fruit and vegetables & 29 & 201 & 38 \\
\hline \multicolumn{4}{|l|}{ Sugar } \\
\hline Livestock & 43 & 80 & 153 \\
\hline Milk & 41 & 79 & 147 \\
\hline Meat & 42 & 79 & 153 \\
\hline Fish & 102 & 113 & 78 \\
\hline \multicolumn{4}{|l|}{ Net trade (mln US\$) } \\
\hline Cereals & -120 & -397 & -1790 \\
\hline Fruit and vegetables & -266 & -357 & -1907 \\
\hline Meat & -169 & -140 & -1217 \\
\hline Dairy products & -135 & -163 & -617 \\
\hline Fish & 8 & -19 & -396 \\
\hline \multicolumn{4}{|l|}{ Environment } \\
\hline Forest area (\%) & 3 & 4 & 4 \\
\hline Renewable water res withdrawn (\% of total) & & & 83 \\
\hline Terrestrial protect areas (\% total land area) & 13 & 13 & 18 \\
\hline Organic area (\% total agricultural area) & & & 1 \\
\hline Water withdrawal by agriculture (\% of total) & & & 83 \\
\hline \multicolumn{4}{|l|}{ Biofuel production (thousand kt of oil eq.) } \\
\hline \multicolumn{4}{|l|}{ Wood pellet prod. (1 000 tonnes) } \\
\hline Net GHG emissions from AFOLU ( $\mathrm{CO}_{2}$ eq, $\mathrm{Mt}$ ) & 0 & 0 & 1 \\
\hline
\end{tabular}




\begin{tabular}{|c|c|c|c|}
\hline & 1990 & 2000 & 2014 \\
\hline \multicolumn{4}{|l|}{ The setting } \\
\hline Population, total (mln) & 57.4 & 59.2 & 63.7 \\
\hline Population, rural (mln) & 12.6 & 12.7 & 12.8 \\
\hline \multicolumn{4}{|l|}{ Govt expenditure on ag (\% total outlays) } \\
\hline Area harvested (mln ha) & 23 & 24 & 20 \\
\hline Cropping intensity ratio & 1.2 & 1.4 & \\
\hline Water resources (1 $000 \mathrm{~m}^{3} /$ person/year) & 3 & 2 & 2 \\
\hline Area equipped for irrigation (1 $000 \mathrm{ha}$ ) & & & 95 \\
\hline Area irrigated (\% area equipped for irrigation) & & & 60.6 \\
\hline Employment in agriculture (\%) & 2 & 1.5 & 1.2 \\
\hline Employment in agriculture, female (\%) & 1.1 & 0.8 & 0.6 \\
\hline Fertilizers, Nitrogen (kg of nutrients per ha) & & 198.8 & 160.2 \\
\hline Fertilizers, Phosphate (kg of nutrients per ha) & & 49.1 & 31.2 \\
\hline Fertilizers, Potash (kg nutrients per ha) & & 66.3 & 43 \\
\hline Energy consump, power irrigation (mln kWh) & 9 & 11 & 214 \\
\hline Agr value added per worker (constant US\$) & 20139 & 24176 & 29212 \\
\hline \multicolumn{4}{|l|}{ Hunger dimensions } \\
\hline \multicolumn{4}{|l|}{ Dietary energy supply (kcal/pc/day) } \\
\hline Average dietary energy supply adequacy (\%) & 131 & 136 & 137 \\
\hline Dietary en supp, cereals/roots/tubers (\%) & 28 & 31 & 32 \\
\hline Prevalence of undernourishment (\%) & $<5.0$ & $<5.0$ & $<5.0$ \\
\hline GDP per capita (US\$, PPP) & 26424 & 32543 & 36932 \\
\hline Domestic food price volatility (index) & & 7.4 & 5 \\
\hline Cereal import dependency ratio (\%) & -12.7 & -6 & -3.2 \\
\hline \multicolumn{4}{|l|}{ Underweight, children under-5 (\%) } \\
\hline Improved water source (\% pop) & 100 & 100 & 100 \\
\hline \multicolumn{4}{|l|}{ Food supply } \\
\hline Food production value, (2004-2006 mln I\$) & 17024 & 16492 & 15878 \\
\hline Agriculture, value added (\% GDP) & & 1 & 1 \\
\hline Food exports (mln US\$) & 6169 & 6927 & 13824 \\
\hline Food imports (mln US\$) & 15794 & 17484 & 42602 \\
\hline \multicolumn{4}{|l|}{ Production indices $(2004-06=100)$} \\
\hline Net food & 107 & 104 & 100 \\
\hline Net crops & 105 & 105 & 93 \\
\hline Cereals & 103 & 112 & 91 \\
\hline Vegetable oils & 71 & 64 & 117 \\
\hline Roots and tubers & 105 & 109 & 92 \\
\hline Fruit and vegetables & 137 & 111 & 102 \\
\hline Sugar & 94 & 108 & 96 \\
\hline Livestock & 107 & 102 & 103 \\
\hline Milk & 106 & 100 & 96 \\
\hline Meat & 109 & 104 & 107 \\
\hline Fish & 97 & 109 & 99 \\
\hline \multicolumn{4}{|l|}{ Net trade (mln US\$) } \\
\hline Cereals & 447 & 140 & -2032 \\
\hline Fruit and vegetables & -4775 & -5249 & -11552 \\
\hline Meat & -2282 & -2655 & -6490 \\
\hline Dairy products & -705 & -719 & -2053 \\
\hline Fish & -949 & -925 & -1703 \\
\hline \multicolumn{4}{|l|}{ Environment } \\
\hline Forest area $(\%)$ & 11 & 12 & 12 \\
\hline Renewable water res withdrawn (\% of total) & & & 10 \\
\hline Terrestrial protect areas (\% total land area) & 23 & 25 & 28 \\
\hline Organic area (\% total agricultural area) & & & 3 \\
\hline Water withdrawal by agriculture (\% of total) & & & 10 \\
\hline Biofuel production (thousand kt of oil eq.) & 2 & 52 & 4306 \\
\hline Wood pellet prod. (1 000 tonnes) & & & 335 \\
\hline Net GHG emissions from AFOLU ( $\mathrm{CO}_{2}$ eq, $\left.\mathrm{Mt}\right)$ & 64 & 55 & 41 \\
\hline
\end{tabular}




\begin{tabular}{|c|c|c|c|}
\hline & 1990 & 2000 & 2014 \\
\hline \multicolumn{4}{|l|}{ The setting } \\
\hline Population, total (mln) & 254.5 & 284.6 & 322.6 \\
\hline Population, rural (mln) & 62.9 & 59.5 & 54.4 \\
\hline Govt expenditure on ag (\% total outlays) & & 1 & 0.6 \\
\hline Area harvested (mln ha) & 312 & 343 & 437 \\
\hline Cropping intensity ratio & 0.7 & 0.8 & \\
\hline Water resources (1 $000 \mathrm{~m}^{3} /$ person/year) & 12 & 11 & 10 \\
\hline Area equipped for irrigation ( $1000 \mathrm{ha}$ ) & & & 26400 \\
\hline Area irrigated (\% area equipped for irrigation) & & & 86 \\
\hline Employment in agriculture (\%) & 2.9 & 2.6 & 1.6 \\
\hline Employment in agriculture, female (\%) & 1.3 & 1.4 & 0.8 \\
\hline Fertilizers, Nitrogen (kg of nutrients per ha) & & 67.2 & 78 \\
\hline Fertilizers, Phosphate (kg of nutrients per ha) & & 24.4 & 25.7 \\
\hline Fertilizers, Potash (kg nutrients per ha) & & 28 & 27.5 \\
\hline Energy consump, power irrigation (mln kWh) & & & 26904 \\
\hline Agr value added per worker (constant US\$) & & 38473 & 69457 \\
\hline \multicolumn{4}{|l|}{ Hunger dimensions } \\
\hline \multicolumn{4}{|l|}{ Dietary energy supply (kcal/pc/day) } \\
\hline Average dietary energy supply adequacy (\%) & 143 & 146 & 147 \\
\hline Dietary en supp, cereals/roots/tubers (\%) & 26 & 25 & 25 \\
\hline Prevalence of undernourishment (\%) & $<5.0$ & $<5.0$ & $<5.0$ \\
\hline GDP per capita (US\$, PPP) & 37026 & 45986 & 51340 \\
\hline Domestic food price volatility (index) & & 0 & 0 \\
\hline Cereal import dependency ratio (\%) & -33.7 & -33 & -24 \\
\hline Underweight, children under-5 (\%) & 0.9 & 1.3 & 0.5 \\
\hline Improved water source (\% pop) & 98.4 & 98.8 & 99.2 \\
\hline \multicolumn{4}{|l|}{ Food supply } \\
\hline Food production value, $(2004-2006 \mathrm{mln}$ I\$) & 148524 & 182863 & 215750 \\
\hline Agriculture, value added (\% GDP) & & 1 & 1 \\
\hline Food exports (mln US\$) & 28597 & 39317 & 111636 \\
\hline Food imports (mln US\$) & 16696 & 26914 & 63704 \\
\hline \multicolumn{4}{|l|}{ Production indices $(2004-06=100)$} \\
\hline Net food & 78 & 96 & 113 \\
\hline Net crops & 79 & 95 & 109 \\
\hline Cereals & 86 & 94 & 119 \\
\hline Vegetable oils & 63 & 88 & 102 \\
\hline Roots and tubers & 90 & 117 & 100 \\
\hline Fruit and vegetables & 82 & 106 & 98 \\
\hline Sugar & 85 & 115 & 98 \\
\hline Livestock & 79 & 97 & 108 \\
\hline Milk & 84 & 95 & 114 \\
\hline Meat & 77 & 99 & 106 \\
\hline Fish & 108 & 95 & 104 \\
\hline \multicolumn{4}{|l|}{ Net trade (mln US\$) } \\
\hline Cereals & 11772 & 8515 & 16260 \\
\hline Fruit and vegetables & -1316 & -2679 & -2493 \\
\hline Meat & 26 & 3536 & 11327 \\
\hline Dairy products & -266 & -423 & 2126 \\
\hline Fish & -2553 & -7395 & -11803 \\
\hline \multicolumn{4}{|l|}{ Environment } \\
\hline Forest area (\%) & 32 & 33 & 33 \\
\hline Renewable water res withdrawn (\% of total) & & & 40 \\
\hline Terrestrial protect areas (\% total land area) & 14 & 14 & 14 \\
\hline Organic area (\% total agricultural area) & & & 0 \\
\hline Water withdrawal by agriculture (\% of total) & & & 40 \\
\hline Biofuel production (thousand kt of oil eq.) & 659 & 1583 & 29835 \\
\hline Wood pellet prod. (1 000 tonnes) & & & 6900 \\
\hline Net GHG emissions from AFOLU ( $\mathrm{CO}_{2}$ eq, $\mathrm{Mt}$ ) & 146 & 47 & -58 \\
\hline
\end{tabular}




\begin{tabular}{|c|c|c|c|}
\hline & 1990 & 2000 & 2014 \\
\hline \multicolumn{4}{|l|}{ The setting } \\
\hline Population, total (mln) & 3.1 & 3.3 & 3.4 \\
\hline Population, rural (mln) & 0.3 & 0.3 & 0.2 \\
\hline Govt expenditure on ag (\% total outlays) & & 1.2 & 1.4 \\
\hline Area harvested (mln ha) & 1 & 2 & 4 \\
\hline Cropping intensity ratio & 0.1 & 0.1 & \\
\hline Water resources (1 $000 \mathrm{~m}^{3} /$ person/year) & 55 & 52 & 51 \\
\hline Area equipped for irrigation (1 $000 \mathrm{ha})$ & & & 238 \\
\hline Area irrigated (\% area equipped for irrigation) & & & 100 \\
\hline Employment in agriculture (\%) & 0 & 4.1 & 10.9 \\
\hline Employment in agriculture, female (\%) & 0 & 1.1 & 4.8 \\
\hline Fertilizers, Nitrogen (kg of nutrients per ha) & & 50.7 & 83.3 \\
\hline Fertilizers, Phosphate (kg of nutrients per ha) & & 64.1 & 90.9 \\
\hline Fertilizers, Potash (kg nutrients per ha) & & 3.1 & 18.4 \\
\hline Energy consump, power irrigation (mln kWh) & 0 & 0 & 82 \\
\hline Agr value added per worker (constant US\$) & 5475 & 6967 & 10297 \\
\hline \multicolumn{4}{|l|}{ Hunger dimensions } \\
\hline Dietary energy supply (kcal/pc/day) & 2781 & 2855 & 2900 \\
\hline Average dietary energy supply adequacy (\%) & 117 & 120 & 120 \\
\hline Dietary en supp, cereals/roots/tubers (\%) & 37 & 39 & 45 \\
\hline Prevalence of undernourishment (\%) & 5.8 & $<5.0$ & $<5.0$ \\
\hline GDP per capita (US\$, PPP) & 9617 & 12584 & 18966 \\
\hline Domestic food price volatility (index) & & 5.1 & 6.4 \\
\hline Cereal import dependency ratio (\%) & -35.5 & -116.2 & -123.7 \\
\hline Underweight, children under-5 (\%) & & 5.7 & 4.5 \\
\hline Improved water source (\% pop) & 95.5 & 97.1 & 99.5 \\
\hline \multicolumn{4}{|l|}{ Food supply } \\
\hline Food production value, (2004-2006 mln I\$) & 1947 & 2700 & 4211 \\
\hline Agriculture, value added (\% GDP) & 9 & 7 & 9 \\
\hline Food exports (mln US\$) & 614 & 863 & 5368 \\
\hline Food imports (mln US\$) & 46 & 270 & 701 \\
\hline \multicolumn{4}{|l|}{ Production indices (2004-06=100) } \\
\hline Net food & 60 & 83 & 130 \\
\hline Net crops & 49 & 70 & 197 \\
\hline Cereals & 39 & 82 & 149 \\
\hline Vegetable oils & 14 & 12 & 384 \\
\hline Roots and tubers & 98 & 76 & 83 \\
\hline Fruit and vegetables & 76 & 91 & 102 \\
\hline Sugar & 609 & 95 & 239 \\
\hline Livestock & 70 & 90 & 104 \\
\hline Milk & 61 & 90 & 134 \\
\hline Meat & 67 & 88 & 95 \\
\hline Fish & 71 & 89 & 47 \\
\hline \multicolumn{4}{|l|}{ Net trade (mln US\$) } \\
\hline Cereals & 156 & 156 & 1154 \\
\hline Fruit and vegetables & 15 & -20 & -35 \\
\hline Meat & 309 & 429 & 1555 \\
\hline Dairy products & 60 & 123 & 751 \\
\hline Fish & 68 & 98 & 134 \\
\hline \multicolumn{4}{|l|}{ Environment } \\
\hline Forest area (\%) & 5 & 8 & 10 \\
\hline Renewable water res withdrawn (\% of total) & & 87 & 87 \\
\hline Terrestrial protect areas (\% total land area) & 2 & 2 & 3 \\
\hline Organic area (\% total agricultural area) & & & 6 \\
\hline Water withdrawal by agriculture (\% of total) & & 87 & 87 \\
\hline Biofuel production (thousand kt of oil eq.) & 2 & 1 & 302 \\
\hline Wood pellet prod. (1 000 tonnes) & & & 3 \\
\hline Net GHG emissions from AFOLU ( $\mathrm{CO}_{2}$ eq, $\left.\mathrm{Mt}\right)$ & 21 & 1 & 4 \\
\hline
\end{tabular}




\begin{tabular}{|c|c|c|c|}
\hline & 1990 & 2000 & 2014 \\
\hline \multicolumn{4}{|l|}{ The setting } \\
\hline Population, total (mln) & 22 & 24.8 & 29.3 \\
\hline Population, rural (mln) & 13.4 & 15.5 & 18.7 \\
\hline \multicolumn{4}{|l|}{ Govt expenditure on ag (\% total outlays) } \\
\hline Area harvested (mln ha) & & 4 & 7 \\
\hline Cropping intensity ratio & & 0.1 & \\
\hline Water resources (1 $000 \mathrm{~m}^{3} /$ person/year) & 2 & 2 & 2 \\
\hline Area equipped for irrigation (1 $000 \mathrm{ha})$ & & & 4215 \\
\hline Area irrigated (\% area equipped for irrigation) & & & 88.1 \\
\hline Employment in agriculture (\%) & & 38.5 & \\
\hline \multicolumn{4}{|l|}{ Employment in agriculture, female (\%) } \\
\hline Fertilizers, Nitrogen (kg of nutrients per ha) & & 0 & 167.9 \\
\hline Fertilizers, Phosphate (kg of nutrients per ha) & & 0 & 27.6 \\
\hline Fertilizers, Potash (kg nutrients per ha) & & 0 & 8 \\
\hline Energy consump, power irrigation (mln kWh) & 0 & 11 & 11 \\
\hline Agr value added per worker (constant US\$) & 913 & 989 & 2352 \\
\hline \multicolumn{4}{|l|}{ Hunger dimensions } \\
\hline Dietary energy supply (kcal/pc/day) & 2704 & 2349 & 2851 \\
\hline Average dietary energy supply adequacy (\%) & 125 & 105 & 122 \\
\hline Dietary en supp, cereals/roots/tubers (\%) & 61 & 59 & 58 \\
\hline Prevalence of undernourishment (\%) & $<5.0$ & 11.5 & $<5.0$ \\
\hline GDP per capita (US\$, PPP) & 3043 & 2481 & 5002 \\
\hline \multicolumn{4}{|l|}{ Domestic food price volatility (index) } \\
\hline Cereal import dependency ratio (\%) & 63.2 & 15.7 & 18.2 \\
\hline Underweight, children under-5 (\%) & & 7.1 & 4.4 \\
\hline Improved water source (\% pop) & 90 & 88.7 & 87.3 \\
\hline \multicolumn{4}{|l|}{ Food supply } \\
\hline Food production value, (2004-2006 mln I\$) & 4803 & 5281 & 10915 \\
\hline Agriculture, value added (\% GDP) & 33 & 34 & 19 \\
\hline Food exports (mln US\$) & 75 & 144 & 470 \\
\hline Food imports (mln US\$) & 653 & 362 & 1133 \\
\hline \multicolumn{4}{|l|}{ Production indices $(2004-06=100)$} \\
\hline Net food & 72 & 79 & 163 \\
\hline Net crops & 81 & 76 & 144 \\
\hline Cereals & 35 & 61 & 123 \\
\hline Vegetable oils & 113 & 83 & 90 \\
\hline Roots and tubers & 47 & 76 & 238 \\
\hline Fruit and vegetables & 69 & 76 & 216 \\
\hline \multicolumn{4}{|l|}{ Sugar } \\
\hline Livestock & 81 & 79 & 166 \\
\hline Milk & 80 & 79 & 173 \\
\hline Meat & 79 & 79 & 157 \\
\hline Fish & 547 & 183 & 223 \\
\hline \multicolumn{4}{|l|}{ Net trade (mln US\$) } \\
\hline Cereals & & -135 & -483 \\
\hline Fruit and vegetables & 38 & 96 & 412 \\
\hline Meat & & -21 & -31 \\
\hline Dairy products & & -19 & -26 \\
\hline Fish & & -1 & -3 \\
\hline \multicolumn{4}{|l|}{ Environment } \\
\hline Forest area (\%) & 7 & 8 & 8 \\
\hline Renewable water res withdrawn (\% of total) & & & 90 \\
\hline Terrestrial protect areas (\% total land area) & 2 & 2 & 3 \\
\hline Organic area (\% total agricultural area) & & & 0 \\
\hline Water withdrawal by agriculture (\% of total) & & & 90 \\
\hline \multicolumn{4}{|l|}{ Biofuel production (thousand kt of oil eq.) } \\
\hline Wood pellet prod. (1 000 tonnes) & & & 0 \\
\hline Net GHG emissions from AFOLU ( $\mathrm{CO}_{2}$ eq, $\mathrm{Mt}$ ) & 12 & 14 & 25 \\
\hline
\end{tabular}




\begin{tabular}{|c|c|c|c|}
\hline & 1990 & 2000 & 2014 \\
\hline \multicolumn{4}{|l|}{ The setting } \\
\hline Population, total (mln) & 0.1 & 0.2 & 0.3 \\
\hline Population, rural (mln) & 0.1 & 0.1 & 0.2 \\
\hline Govt expenditure on ag (\% total outlays) & & 20 & 15 \\
\hline Area harvested (mln ha) & 0 & 0 & 0 \\
\hline Cropping intensity ratio & 2.3 & 1.4 & \\
\hline \multicolumn{4}{|l|}{ Water resources (1 $000 \mathrm{~m}^{3} /$ person/year) } \\
\hline \multicolumn{4}{|l|}{ Area equipped for irrigation (1 $000 \mathrm{ha})$} \\
\hline \multicolumn{4}{|l|}{ Area irrigated (\% area equipped for irrigation) } \\
\hline Employment in agriculture (\%) & & & 60.5 \\
\hline Employment in agriculture, female (\%) & & & 62.3 \\
\hline \multicolumn{4}{|l|}{ Fertilizers, Nitrogen (kg of nutrients per ha) } \\
\hline \multicolumn{4}{|l|}{ Fertilizers, Phosphate (kg of nutrients per ha) } \\
\hline \multicolumn{4}{|l|}{ Fertilizers, Potash (kg nutrients per ha) } \\
\hline \multicolumn{4}{|l|}{ Energy consump, power irrigation (mln kWh) } \\
\hline Agr value added per worker (constant US\$) & 2223 & 2374 & 2839 \\
\hline \multicolumn{4}{|l|}{ Hunger dimensions } \\
\hline Dietary energy supply (kcal/pc/day) & 2533 & 2696 & 2868 \\
\hline Average dietary energy supply adequacy (\%) & 120 & 126 & 131 \\
\hline Dietary en supp, cereals/roots/tubers (\%) & 44 & 48 & 47 \\
\hline Prevalence of undernourishment (\%) & 11.9 & 8.1 & 6.4 \\
\hline GDP per capita (US\$, PPP) & 2550 & 2823 & 2895 \\
\hline \multicolumn{4}{|l|}{ Domestic food price volatility (index) } \\
\hline Cereal import dependency ratio (\%) & 92.1 & 94.5 & 95.8 \\
\hline Underweight, children under-5 (\%) & & 10.6 & 11.7 \\
\hline Improved water source (\% pop) & 61.9 & 76 & 90.7 \\
\hline \multicolumn{4}{|l|}{ Food supply } \\
\hline Food production value, (2004-2006 mln I\$) & 68 & 60 & 82 \\
\hline Agriculture, value added (\% GDP) & 21 & 26 & 28 \\
\hline Food exports (mln US\$) & 11 & 13 & 31 \\
\hline Food imports (mln US\$) & 10 & 13 & 54 \\
\hline \multicolumn{4}{|l|}{ Production indices $(2004-06=100)$} \\
\hline Net food & 114 & 101 & 138 \\
\hline Net crops & 122 & 98 & 146 \\
\hline Cereals & 79 & 104 & 110 \\
\hline Vegetable oils & 137 & 98 & 161 \\
\hline Roots and tubers & 89 & 89 & 118 \\
\hline Fruit and vegetables & 79 & 95 & 106 \\
\hline \multicolumn{4}{|l|}{ Sugar } \\
\hline Livestock & 87 & 110 & 112 \\
\hline Milk & 79 & 108 & 115 \\
\hline Meat & 88 & 110 & 111 \\
\hline Fish & 19 & 32 & 26 \\
\hline \multicolumn{4}{|l|}{ Net trade (mln US\$) } \\
\hline Cereals & -4 & -6 & -26 \\
\hline Fruit and vegetables & -1 & -2 & 3 \\
\hline Meat & 2 & 1 & -7 \\
\hline Dairy products & & -1 & -4 \\
\hline Fish & 46 & 57 & 35 \\
\hline \multicolumn{4}{|l|}{ Environment } \\
\hline Forest area (\%) & 36 & 36 & 36 \\
\hline \multicolumn{4}{|l|}{ Renewable water res withdrawn (\% of total) } \\
\hline Terrestrial protect areas (\% total land area) & 4 & 4 & 4 \\
\hline Organic area (\% total agricultural area) & & & 2 \\
\hline \multicolumn{4}{|l|}{ Water withdrawal by agriculture (\% of total) } \\
\hline \multicolumn{4}{|l|}{ Biofuel production (thousand kt of oil eq.) } \\
\hline Wood pellet prod. (1 000 tonnes) & & & \\
\hline Net GHG emissions from AFOLU ( $\mathrm{CO}_{2}$ eq, $\left.\mathrm{Mt}\right)$ & 0 & 0 & 0 \\
\hline
\end{tabular}




\begin{tabular}{|c|c|c|c|}
\hline & 1990 & 2000 & 2014 \\
\hline \multicolumn{4}{|l|}{ The setting } \\
\hline Population, total (mln) & 19.7 & 24.4 & 30.9 \\
\hline Population, rural (mln) & 3.1 & 2.5 & 1.8 \\
\hline \multicolumn{4}{|l|}{ Govt expenditure on ag (\% total outlays) } \\
\hline Area harvested (mln ha) & 7 & 9 & 7 \\
\hline Cropping intensity ratio & 0.3 & 0.4 & \\
\hline Water resources (1 $000 \mathrm{~m}^{3} /$ person/year) & 67 & 54 & 44 \\
\hline Area equipped for irrigation (1 $000 \mathrm{ha})$ & & & 1055 \\
\hline Area irrigated (\% area equipped for irrigation) & & & 92.8 \\
\hline Employment in agriculture (\%) & 13.4 & 10.6 & 7.7 \\
\hline Employment in agriculture, female (\%) & 2.2 & 1.6 & 1.8 \\
\hline Fertilizers, Nitrogen (kg of nutrients per ha) & & 125.8 & 104.9 \\
\hline Fertilizers, Phosphate (kg of nutrients per ha) & & 17.3 & 25.6 \\
\hline Fertilizers, Potash (kg nutrients per ha) & & 25.8 & 37 \\
\hline Energy consump, power irrigation (mln kWh) & 203 & 203 & 694 \\
\hline Agr value added per worker (constant US\$) & 4543 & 5789 & 8533 \\
\hline \multicolumn{4}{|l|}{ Hunger dimensions } \\
\hline Dietary energy supply (kcal/pc/day) & 2456 & 2459 & 2992 \\
\hline Average dietary energy supply adequacy (\%) & 109 & 107 & 128 \\
\hline Dietary en supp, cereals/roots/tubers (\%) & 39 & 39 & 41 \\
\hline Prevalence of undernourishment (\%) & 13.2 & 16.6 & $<5.0$ \\
\hline GDP per capita (US\$, PPP) & 14539 & 14461 & 17615 \\
\hline Domestic food price volatility (index) & & 6.6 & 12.8 \\
\hline Cereal import dependency ratio (\%) & 54.9 & 51.3 & 56.6 \\
\hline Underweight, children under-5 (\%) & 6.7 & 3.9 & 2.9 \\
\hline Improved water source (\% pop) & 89.9 & 92.1 & 92.9 \\
\hline \multicolumn{4}{|l|}{ Food supply } \\
\hline Food production value, (2004-2006 mln I\$) & 4038 & 5354 & 7244 \\
\hline Agriculture, value added (\% GDP) & 5 & 4 & 5 \\
\hline Food exports (mln US\$) & 168 & 196 & 15 \\
\hline Food imports (mln US\$) & 564 & 1300 & 7416 \\
\hline \multicolumn{4}{|l|}{ Production indices $(2004-06=100)$} \\
\hline Net food & 73 & 97 & 131 \\
\hline Net crops & 76 & 99 & 127 \\
\hline Cereals & 50 & 76 & 97 \\
\hline Vegetable oils & 86 & 104 & 71 \\
\hline Roots and tubers & 57 & 95 & 149 \\
\hline Fruit and vegetables & 85 & 116 & 158 \\
\hline Sugar & 69 & 92 & 77 \\
\hline Livestock & 72 & 97 & 140 \\
\hline Milk & 112 & 106 & 197 \\
\hline Meat & 66 & 95 & 130 \\
\hline Fish & 74 & 82 & 50 \\
\hline \multicolumn{4}{|l|}{ Net trade (mln US\$) } \\
\hline Cereals & -290 & -399 & -1846 \\
\hline Fruit and vegetables & -5 & -204 & -550 \\
\hline Meat & 19 & -12 & -1248 \\
\hline Dairy products & -42 & -176 & -1232 \\
\hline Fish & 93 & 96 & -188 \\
\hline \multicolumn{4}{|l|}{ Environment } \\
\hline Forest area (\%) & 59 & 56 & 52 \\
\hline Renewable water res withdrawn (\% of total) & & & 74 \\
\hline Terrestrial protect areas (\% total land area) & 40 & 53 & 53 \\
\hline Organic area (\% total agricultural area) & & & 0 \\
\hline Water withdrawal by agriculture (\% of total) & & & 74 \\
\hline Biofuel production (thousand kt of oil eq.) & 14 & 16 & 28 \\
\hline \multicolumn{4}{|l|}{ Wood pellet prod. (1 000 tonnes) } \\
\hline Net GHG emissions from AFOLU ( $\mathrm{CO}_{2}$ eq, $\mathrm{Mt}$ ) & 139 & 141 & 144 \\
\hline
\end{tabular}




\begin{tabular}{|c|c|c|c|}
\hline & 1990 & 2000 & 2014 \\
\hline \multicolumn{4}{|l|}{ The setting } \\
\hline Population, total (mln) & 68.9 & 80.9 & 92.5 \\
\hline Population, rural (mln) & 55 & 61.2 & 62 \\
\hline Govt expenditure on ag (\% total outlays) & & & 2.5 \\
\hline Area harvested (mln ha) & 20 & 35 & 49 \\
\hline Cropping intensity ratio & 3 & 3.9 & \\
\hline Water resources (1 $000 \mathrm{~m}^{3} /$ person/year) & 13 & 11 & 10 \\
\hline Area equipped for irrigation (1 $000 \mathrm{ha}$ ) & & & 4600 \\
\hline Area irrigated (\% area equipped for irrigation) & & & 100 \\
\hline Employment in agriculture (\%) & & 65.3 & 47.4 \\
\hline Employment in agriculture, female (\%) & & 66.3 & 49.5 \\
\hline Fertilizers, Nitrogen (kg of nutrients per ha) & & 187.1 & 143.3 \\
\hline Fertilizers, Phosphate (kg of nutrients per ha) & & 89.8 & 75.2 \\
\hline Fertilizers, Potash (kg nutrients per ha) & & 65.3 & 78.5 \\
\hline Energy consump, power irrigation (mln kWh) & 0 & 0 & 3 \\
\hline Agr value added per worker (constant US\$) & 266 & 353 & 489 \\
\hline \multicolumn{4}{|l|}{ Hunger dimensions } \\
\hline Dietary energy supply (kcal/pc/day) & 2002 & 2246 & 2796 \\
\hline Average dietary energy supply adequacy (\%) & 93 & 100 & 121 \\
\hline Dietary en supp, cereals/roots/tubers (\%) & 77 & 71 & 60 \\
\hline Prevalence of undernourishment (\%) & 41.3 & 28.1 & 11.8 \\
\hline GDP per capita (US\$, PPP) & 1501 & 2650 & 5125 \\
\hline \multicolumn{4}{|l|}{ Domestic food price volatility (index) } \\
\hline Cereal import dependency ratio (\%) & -10.6 & -15.3 & -11 \\
\hline Underweight, children under-5 (\%) & 36.9 & 26.7 & 12 \\
\hline Improved water source (\% pop) & 61.6 & 77.4 & 95 \\
\hline \multicolumn{4}{|l|}{ Food supply } \\
\hline Food production value, (2004-2006 mln I\$) & 10134 & 15838 & 27498 \\
\hline Agriculture, value added (\% GDP) & 39 & 23 & 18 \\
\hline Food exports (mln US\$) & 492 & 1406 & 7147 \\
\hline Food imports (mln US\$) & 90 & 625 & 7062 \\
\hline \multicolumn{4}{|l|}{ Production indices $(2004-06=100)$} \\
\hline Net food & 49 & 77 & 134 \\
\hline Net crops & 46 & 80 & 133 \\
\hline Cereals & 52 & 89 & 123 \\
\hline Vegetable oils & 52 & 74 & 105 \\
\hline Roots and tubers & 50 & 43 & 134 \\
\hline Fruit and vegetables & 51 & 80 & 150 \\
\hline Sugar & 34 & 95 & 128 \\
\hline Livestock & 39 & 68 & 147 \\
\hline Milk & 29 & 46 & 214 \\
\hline Meat & 38 & 68 & 145 \\
\hline Fish & 27 & 62 & 176 \\
\hline \multicolumn{4}{|l|}{ Net trade (mln US\$) } \\
\hline Cereals & 262 & 519 & 2081 \\
\hline Fruit and vegetables & 49 & 230 & 1519 \\
\hline Meat & 28 & 119 & -1474 \\
\hline Dairy products & -8 & -141 & -581 \\
\hline Fish & 176 & 1447 & 5462 \\
\hline \multicolumn{4}{|l|}{ Environment } \\
\hline Forest area (\%) & 29 & 38 & 45 \\
\hline Renewable water res withdrawn (\% of total) & & & 95 \\
\hline Terrestrial protect areas (\% total land area) & 5 & 6 & 6 \\
\hline Organic area (\% total agricultural area) & & & 0 \\
\hline Water withdrawal by agriculture (\% of total) & & & 95 \\
\hline Biofuel production (thousand kt of oil eq.) & 12 & 29 & 23 \\
\hline Wood pellet prod. (1 000 tonnes) & & & 800 \\
\hline Net GHG emissions from AFOLU ( $\mathrm{CO}_{2}$ eq, $\mathrm{Mt}$ ) & 64 & 15 & 46 \\
\hline
\end{tabular}




\begin{tabular}{|c|c|c|c|}
\hline & 1990 & 2000 & 2014 \\
\hline \multicolumn{4}{|l|}{ The setting } \\
\hline Population, total (mln) & 2.1 & 3.2 & 4.4 \\
\hline Population, rural (mln) & 0.7 & 0.9 & 1.1 \\
\hline \multicolumn{4}{|l|}{ Govt expenditure on ag (\% total outlays) } \\
\hline Area harvested (mln ha) & 0 & 1 & 0 \\
\hline Cropping intensity ratio & 0 & 2.1 & \\
\hline Water resources (1 $000 \mathrm{~m}^{3} /$ person/year) & 0 & 0 & 0 \\
\hline Area equipped for irrigation (1 $000 \mathrm{ha})$ & & & 24 \\
\hline \multicolumn{4}{|l|}{ Area irrigated (\% area equipped for irrigation) } \\
\hline Employment in agriculture (\%) & & 13.7 & 11.5 \\
\hline Employment in agriculture, female (\%) & & 34.6 & 23.7 \\
\hline \multicolumn{4}{|l|}{ Fertilizers, Nitrogen (kg of nutrients per ha) } \\
\hline \multicolumn{4}{|l|}{ Fertilizers, Phosphate (kg of nutrients per ha) } \\
\hline \multicolumn{4}{|l|}{ Fertilizers, Potash (kg nutrients per ha) } \\
\hline \multicolumn{4}{|l|}{ Energy consump, power irrigation (mln kWh) } \\
\hline Agr value added per worker (constant US\$) & & 2778 & 2279 \\
\hline \multicolumn{4}{|l|}{ Hunger dimensions } \\
\hline \multicolumn{4}{|l|}{ Dietary energy supply (kcal/pc/day) } \\
\hline \multicolumn{4}{|l|}{ Average dietary energy supply adequacy (\%) } \\
\hline \multicolumn{4}{|l|}{ Dietary en supp, cereals/roots/tubers (\%) } \\
\hline \multicolumn{4}{|l|}{ Prevalence of undernourishment (\%) } \\
\hline GDP per capita (US\$, PPP) & & 4206 & 4484 \\
\hline \multicolumn{4}{|l|}{ Domestic food price volatility (index) } \\
\hline Cereal import dependency ratio (\%) & 0 & 93.5 & 95.5 \\
\hline Underweight, children under-5 (\%) & & 3.6 & 1.4 \\
\hline Improved water source (\% pop) & 96.6 & 92 & 81.8 \\
\hline \multicolumn{4}{|l|}{ Food supply } \\
\hline Food production value, (2004-2006 mln I\$) & & 520 & 551 \\
\hline Agriculture, value added (\% GDP) & & 11 & 5 \\
\hline Food exports (mln US\$) & 0 & 73 & 91 \\
\hline Food imports (mln US\$) & 23 & 410 & 461 \\
\hline \multicolumn{4}{|l|}{ Production indices (2004-06=100) } \\
\hline Net food & & 88 & 93 \\
\hline Net crops & & 86 & 94 \\
\hline Cereals & & 107 & 41 \\
\hline Vegetable oils & & 42 & 85 \\
\hline Roots and tubers & & 88 & 88 \\
\hline Fruit and vegetables & & 99 & 92 \\
\hline \multicolumn{4}{|l|}{ Sugar } \\
\hline Livestock & & 91 & 91 \\
\hline Milk & & 71 & 102 \\
\hline Meat & & 100 & 95 \\
\hline Fish & 0 & 111 & 93 \\
\hline \multicolumn{4}{|l|}{ Net trade (mln US\$) } \\
\hline Cereals & & -131 & -119 \\
\hline Fruit and vegetables & -1 & -48 & -74 \\
\hline Meat & & -12 & -4 \\
\hline Dairy products & & -15 & -25 \\
\hline Fish & & & -10 \\
\hline \multicolumn{4}{|l|}{ Environment } \\
\hline Forest area (\%) & 2 & 2 & 2 \\
\hline Renewable water res withdrawn (\% of total) & & & 45 \\
\hline Terrestrial protect areas (\% total land area) & 1 & 1 & 1 \\
\hline Organic area (\% total agricultural area) & & & 2 \\
\hline Water withdrawal by agriculture (\% of total) & & & 45 \\
\hline Biofuel production (thousand kt of oil eq.) & & & \\
\hline Wood pellet prod. (1 000 tonnes) & & & \\
\hline Net GHG emissions from AFOLU ( $\mathrm{CO}_{2}$ eq, $\mathrm{Mt}$ ) & 0 & 0 & 0 \\
\hline
\end{tabular}




\begin{tabular}{|c|c|c|c|}
\hline & 1990 & 2000 & 2014 \\
\hline \multicolumn{4}{|l|}{ The setting } \\
\hline Population, total (mln) & 11.8 & 17.5 & 25 \\
\hline Population, rural (mln) & 9.3 & 12.9 & 16.5 \\
\hline Govt expenditure on ag (\% total outlays) & & 0.9 & 1.1 \\
\hline Area harvested (mln ha) & 1 & 1 & 1 \\
\hline Cropping intensity ratio & 0 & 0 & \\
\hline Water resources (1 $000 \mathrm{~m}^{3} /$ person/year) & 0 & 0 & 0 \\
\hline Area equipped for irrigation (1 $000 \mathrm{ha})$ & & & 680 \\
\hline \multicolumn{4}{|l|}{ Area irrigated (\% area equipped for irrigation) } \\
\hline Employment in agriculture (\%) & 52.6 & 54.1 & 24.7 \\
\hline Employment in agriculture, female (\%) & 83.1 & 87.8 & 28 \\
\hline Fertilizers, Nitrogen (kg of nutrients per ha) & & 3.9 & 9.3 \\
\hline Fertilizers, Phosphate (kg of nutrients per ha) & & 0 & 0 \\
\hline Fertilizers, Potash (kg nutrients per ha) & & 0.6 & 0.5 \\
\hline Energy consump, power irrigation (mln kWh) & 0 & 2 & 2 \\
\hline Agr value added per worker (constant US\$) & & & 824 \\
\hline \multicolumn{4}{|l|}{ Hunger dimensions } \\
\hline Dietary energy supply (kcal/pc/day) & 2083 & 2081 & 2212 \\
\hline Average dietary energy supply adequacy (\%) & 104 & 101 & 102 \\
\hline Dietary en supp, cereals/roots/tubers (\%) & 68 & 67 & 64 \\
\hline Prevalence of undernourishment (\%) & 27.9 & 29.6 & 25.9 \\
\hline GDP per capita (US\$, PPP) & 3441 & 4018 & 3832 \\
\hline Domestic food price volatility (index) & & & 11 \\
\hline Cereal import dependency ratio (\%) & 69.9 & 78.6 & 81.2 \\
\hline Underweight, children under-5 (\%) & 29.6 & 43.1 & 35.5 \\
\hline Improved water source (\% pop) & 66.3 & 59.9 & 54.9 \\
\hline \multicolumn{4}{|l|}{ Food supply } \\
\hline Food production value, $(2004-2006 \mathrm{mln}$ I\$) & 726 & 1058 & 1774 \\
\hline Agriculture, value added (\% GDP) & 24 & 14 & 10 \\
\hline Food exports (mln US\$) & 30 & 32 & 180 \\
\hline Food imports (mln US\$) & 613 & 719 & 3682 \\
\hline \multicolumn{4}{|l|}{ Production indices $(2004-06=100)$} \\
\hline Net food & 57 & 83 & 138 \\
\hline Net crops & 61 & 84 & 122 \\
\hline Cereals & 124 & 109 & 141 \\
\hline Vegetable oils & 45 & 94 & 121 \\
\hline Roots and tubers & 75 & 99 & 133 \\
\hline Fruit and vegetables & 50 & 79 & 117 \\
\hline \multicolumn{4}{|l|}{ Sugar } \\
\hline Livestock & 50 & 80 & 159 \\
\hline Milk & 68 & 81 & 126 \\
\hline Meat & 48 & 83 & 170 \\
\hline Fish & 32 & 47 & 87 \\
\hline \multicolumn{4}{|l|}{ Net trade (mln US\$) } \\
\hline Cereals & -251 & -302 & -1917 \\
\hline Fruit and vegetables & -16 & -13 & -146 \\
\hline Meat & -27 & -62 & -217 \\
\hline Dairy products & -62 & -78 & -285 \\
\hline Fish & 14 & 16 & 193 \\
\hline \multicolumn{4}{|l|}{ Environment } \\
\hline Forest area (\%) & 1 & 1 & 1 \\
\hline Renewable water res withdrawn (\% of total) & & & 91 \\
\hline Terrestrial protect areas (\% total land area) & & 1 & 1 \\
\hline \multicolumn{4}{|l|}{ Organic area (\% total agricultural area) } \\
\hline Water withdrawal by agriculture (\% of total) & & & 91 \\
\hline \multicolumn{4}{|l|}{ Biofuel production (thousand kt of oil eq.) } \\
\hline \multicolumn{4}{|l|}{ Wood pellet prod. (1 000 tonnes) } \\
\hline Net GHG emissions from AFOLU ( $\mathrm{CO}_{2}$ eq, $\mathrm{Mt}$ ) & 4 & 5 & 7 \\
\hline
\end{tabular}




\begin{tabular}{|c|c|c|c|}
\hline & 1990 & 2000 & 2014 \\
\hline \multicolumn{4}{|l|}{ The setting } \\
\hline Population, total (mln) & 7.8 & 10.1 & 15 \\
\hline Population, rural (mln) & 4.8 & 6.6 & 8.9 \\
\hline Govt expenditure on ag (\% total outlays) & & & 9.7 \\
\hline Area harvested (mln ha) & 1 & 2 & 4 \\
\hline Cropping intensity ratio & 0.1 & 0.1 & \\
\hline Water resources (1 $000 \mathrm{~m}^{3} /$ person/year) & 13 & 10 & 7 \\
\hline Area equipped for irrigation (1 $000 \mathrm{ha}$ ) & & & 156 \\
\hline Area irrigated (\% area equipped for irrigation) & & 100 & \\
\hline Employment in agriculture (\%) & 49.8 & 71.6 & 72.2 \\
\hline Employment in agriculture, female (\%) & 56 & 79.6 & 78.9 \\
\hline Fertilizers, Nitrogen (kg of nutrients per ha) & & 14.9 & 10.6 \\
\hline Fertilizers, Phosphate (kg of nutrients per ha) & & 5.1 & 4.5 \\
\hline Fertilizers, Potash (kg nutrients per ha) & & 6.2 & 3.1 \\
\hline Energy consump, power irrigation (mln kWh) & 43 & 43 & 56 \\
\hline Agr value added per worker (constant US\$) & 458 & 486 & 353 \\
\hline \multicolumn{4}{|l|}{ Hunger dimensions } \\
\hline Dietary energy supply (kcal/pc/day) & 1996 & 1893 & 1942 \\
\hline Average dietary energy supply adequacy (\%) & 96 & 91 & 92 \\
\hline Dietary en supp, cereals/roots/tubers (\%) & 78 & 77 & 72 \\
\hline Prevalence of undernourishment (\%) & 35.2 & 42.9 & 48.4 \\
\hline GDP per capita (US\$, PPP) & 2407 & 2202 & 3800 \\
\hline Domestic food price volatility (index) & & 9.2 & 3.2 \\
\hline Cereal import dependency ratio (\%) & 24.3 & 7.1 & -8.2 \\
\hline Underweight, children under-5 (\%) & 21.2 & 23.3 & 14.9 \\
\hline Improved water source (\% pop) & 49.1 & 53.1 & 63.3 \\
\hline \multicolumn{4}{|l|}{ Food supply } \\
\hline Food production value, (2004-2006 mln I\$) & 709 & 812 & 1772 \\
\hline Agriculture, value added (\% GDP) & 21 & 18 & 10 \\
\hline Food exports (mln US\$) & 11 & 42 & 756 \\
\hline Food imports (mln US\$) & 49 & 62 & 378 \\
\hline \multicolumn{4}{|l|}{ Production indices $(2004-06=100)$} \\
\hline Net food & 74 & 84 & 184 \\
\hline Net crops & 61 & 72 & 158 \\
\hline Cereals & 89 & 90 & 215 \\
\hline Vegetable oils & 41 & 68 & 196 \\
\hline Roots and tubers & 62 & 78 & 113 \\
\hline Fruit and vegetables & 92 & 89 & 121 \\
\hline Sugar & 48 & 68 & 169 \\
\hline Livestock & 78 & 91 & 216 \\
\hline Milk & 95 & 91 & 108 \\
\hline Meat & 80 & 91 & 234 \\
\hline Fish & 95 & 102 & 153 \\
\hline \multicolumn{4}{|l|}{ Net trade (mln US\$) } \\
\hline Cereals & -25 & -16 & 443 \\
\hline Fruit and vegetables & 3 & -1 & -27 \\
\hline Meat & 0 & -1 & -13 \\
\hline Dairy products & -2 & -3 & -22 \\
\hline Fish & 0 & -1 & -32 \\
\hline \multicolumn{4}{|l|}{ Environment } \\
\hline Forest area (\%) & 71 & 69 & 66 \\
\hline Renewable water res withdrawn (\% of total) & & 73 & 73 \\
\hline Terrestrial protect areas (\% total land area) & 36 & 36 & 38 \\
\hline Organic area (\% total agricultural area) & & & 0 \\
\hline Water withdrawal by agriculture (\% of total) & & 73 & 73 \\
\hline Biofuel production (thousand kt of oil eq.) & 3 & 5 & 10 \\
\hline \multicolumn{4}{|l|}{ Wood pellet prod. (1 000 tonnes) } \\
\hline Net GHG emissions from AFOLU ( $\mathrm{CO}_{2}$ eq, $\mathrm{Mt}$ ) & 94 & 92 & 94 \\
\hline
\end{tabular}




\begin{tabular}{|c|c|c|c|}
\hline & 1990 & 2000 & 2014 \\
\hline \multicolumn{4}{|l|}{ The setting } \\
\hline Population, total (mln) & 10.5 & 12.5 & 14.6 \\
\hline Population, rural (mln) & 7.4 & 8.3 & 8.7 \\
\hline Govt expenditure on ag (\% total outlays) & & & 16 \\
\hline Area harvested (mln ha) & 3 & 4 & 4 \\
\hline Cropping intensity ratio & 0.2 & 0.3 & \\
\hline Water resources (1 $000 \mathrm{~m}^{3} /$ person/year) & 2 & 2 & 1 \\
\hline Area equipped for irrigation (1 $000 \mathrm{ha}$ ) & & & 174 \\
\hline Area irrigated (\% area equipped for irrigation) & & 71.4 & \\
\hline Employment in agriculture (\%) & & 60 & \\
\hline Employment in agriculture, female (\%) & & 69.5 & \\
\hline Fertilizers, Nitrogen (kg of nutrients per ha) & & 21.2 & 16.6 \\
\hline Fertilizers, Phosphate (kg of nutrients per ha) & & 12.2 & 10.3 \\
\hline Fertilizers, Potash (kg nutrients per ha) & & 6.6 & 2.2 \\
\hline Energy consump, power irrigation (mln kWh) & 197 & 305 & 305 \\
\hline Agr value added per worker (constant US\$) & 399 & 474 & 279 \\
\hline \multicolumn{4}{|l|}{ Hunger dimensions } \\
\hline Dietary energy supply (kcal/pc/day) & 1943 & 2001 & 2176 \\
\hline Average dietary energy supply adequacy (\%) & 89 & 89 & 96 \\
\hline Dietary en supp, cereals/roots/tubers (\%) & 65 & 58 & 60 \\
\hline Prevalence of undernourishment (\%) & 45.1 & 43.7 & 34 \\
\hline GDP per capita (US\$, PPP) & 2532 & 2521 & 1773 \\
\hline \multicolumn{4}{|l|}{ Domestic food price volatility (index) } \\
\hline Cereal import dependency ratio (\%) & 8.3 & 1.8 & 43.9 \\
\hline Underweight, children under-5 (\%) & & 11.5 & 11.2 \\
\hline Improved water source (\% pop) & 79.2 & 79.5 & 79.9 \\
\hline \multicolumn{4}{|l|}{ Food supply } \\
\hline Food production value, (2004-2006 mln I\$) & 1199 & 1422 & 1261 \\
\hline Agriculture, value added (\% GDP) & 16 & 18 & 14 \\
\hline Food exports (mln US\$) & 225 & 243 & 147 \\
\hline Food imports (mln US\$) & 40 & 87 & 1121 \\
\hline \multicolumn{4}{|l|}{ Production indices (2004-06=100) } \\
\hline Net food & 93 & 110 & 98 \\
\hline Net crops & 117 & 157 & 108 \\
\hline Cereals & 146 & 142 & 55 \\
\hline Vegetable oils & 156 & 203 & 128 \\
\hline Roots and tubers & 53 & 83 & 118 \\
\hline Fruit and vegetables & 73 & 85 & 110 \\
\hline Sugar & 82 & 112 & 103 \\
\hline Livestock & 76 & 97 & 105 \\
\hline Milk & 119 & 121 & 104 \\
\hline Meat & 66 & 91 & 105 \\
\hline Fish & 196 & 116 & 157 \\
\hline \multicolumn{4}{|l|}{ Net trade (mln US\$) } \\
\hline Cereals & 83 & -7 & -583 \\
\hline Fruit and vegetables & 10 & 31 & -62 \\
\hline Meat & 7 & 22 & -25 \\
\hline Dairy products & 5 & 6 & -40 \\
\hline Fish & -1 & -4 & -21 \\
\hline \multicolumn{4}{|l|}{ Environment } \\
\hline Forest area (\%) & 57 & 49 & 39 \\
\hline Renewable water res withdrawn (\% of total) & & 79 & 79 \\
\hline Terrestrial protect areas (\% total land area) & 18 & 18 & 27 \\
\hline Organic area (\% total agricultural area) & & & 0 \\
\hline Water withdrawal by agriculture (\% of total) & & 79 & 79 \\
\hline Biofuel production (thousand kt of oil eq.) & 12 & 14 & 8 \\
\hline \multicolumn{4}{|l|}{ Wood pellet prod. (1 000 tonnes) } \\
\hline Net GHG emissions from AFOLU ( $\mathrm{CO}_{2}$ eq, $\mathrm{Mt}$ ) & 50 & 49 & 48 \\
\hline
\end{tabular}





\section{Definitions}

\section{Agricultural area}

The sum arable land, permanent crops, and permanent pastures.

Source: FAO, Statistics Division

Owner: FAO

\section{Agricultural production indices}

The FAO indices of agricultural production show the relative level of the aggregate volume of agricultural production for each year in comparison with the base period 2004-2006. They are based on the sum of price-weighted quantities of different agricultural commodities produced after deductions of quantities used as seed and feed weighted in a similar manner. All the indices are calculated by the Laspeyres formula. Production quantities - except for fish of each commodity - except for fish - are weighted by 2004-2006 average international commodity prices and summed for each year To obtain the index, the aggregate for a given year is divided by the average aggregate for the base period 2004-2006. The fish index is only normalized to the base period 2004-06.Since the FAO indices are based on the concept of agriculture as a single enterprise, amounts of seed and feed are subtracted from the production data to avoid double counting them, once in the production data and once with the crops or livestock produced from them. Deductions for seed (in the case of eggs, for hatching) and for livestock and poultry feed apply to both domestically produced and imported commodities. They cover only primary agricultural products destined to animal feed (e.g. maize, potatoes, milk, etc.). Processed and semi-processed feed items such as bran, oilcakes, meals and molasses have been completely excluded from the calculations at all stages. When calculating indices of agricultural, food and nonfood production, all intermediate primary inputs of agricultural origin are deducted. However, for indices of any other commodity group, only inputs originating from within the same group are deducted; thus, only seed is removed from the group "crops" and from all crop subgroups, such as cereals, oil crops, etc.; and both feed and seed originating from within the livestock sector (e.g. milk feed, hatching eggs) are removed from the group "livestock products". For the main two livestock subgroups, namely, meat and milk only feed originating from the respective subgroup is removed. International commodity prices are used in order to avoid the use of exchange rates for obtaining continental and world aggregates and to improve international comparative analysis of productivity at the national level. These "international prices", expressed in "international dollars," are derived using a Geary-Khamis formula for the agricultural sector. This method assigns a single "price" to each commodity. The commodities covered in the computation of indices of agricultural production are all crops and livestock products originating in each country. The category of food production includes commodities that are considered edible and that contain nutrients, although it does not include fish. Accordingly, coffee and tea are excluded along with inedible commodities because, although edible, they have practically no nutritive value. Indices for meat production are computed based on data for production from indigenous animals, which takes account of the meat equivalent of exported live animals but excludes the meat equivalent of imported live animals. For index purposes, annual changes in livestock and poultry numbers or in their average live weight are not taken into account.

Source: FAO, Statistics Division and Fisheries
Division

Owner: FAO

Agriculture orientation index (AOI)

Measures the ratio of the agriculture share of government expenditures, over the agriculture contribution to the economy. An AOl greater than 1 gives more prominence to agriculture than its contribution to the economy, as measured by GDP, while those with an $\mathrm{AOI}$ less than 1 give more prominence to non-agricultural sectors.

Source: FAO, Statistics Division

Owner: FAO

\section{Agriculture value added per worker}

Agriculture comprises value added from forestry, hunting, and fishing as well as cultivation of crops and livestock production. Data are in constant 2000 U.S. dollars. Agricultural productivity is measured by value added per unit of input. Agricultural value added includes that from forestry and fishing. Data on employment are drawn from labor force surveys, household surveys, official estimates, censuses and administrative records of social insurance schemes, and establishment surveys when no other information is available. The concept of employment generally refers to people above a certain age who worked, or who held a job, during a reference period. Employment data include both full-time and part-time workers.

Source: World Bank

Owner: IMF and FAO

\section{Aid flows (Official Development Assistance,} ODA)

Consists of disbursements of loans made on concessional terms (net of repayments of principal) and grants by official agencies of the members of the Development Assistance Committee (DAC), by multilateral institutions, and by non-DAC countries to promote economic development and welfare in countries and territories in the DAC list of ODA recipients. It includes loans with a grant element of at least 25 percent (calculated at a rate of discount of 10 percent)

Source: World Bank

Owner: Development Assistance Committee of the Organisation for Economic Co-operation and Development, Geographical Distribution of Financial Flows to Developing Countries, Development Co-operation Report, and International Development Statistics database.

Anemia among children under 5 years of age, prevalence (\%)

The percentage of children under age 5 whose hemoglobin level is less than 110 grams per liter at sea level.

Source: World Bank

Owner: WHO. Global anemia prevalence and trends 1995-2011. Geneva: World Health Organization; forthcoming. Stevens $G A$, Finucane $M M$, De-Regil LM, et al. Global, regional, and national trends in hemoglobin concentration and prevalence of total and severe anemia in children and pregnant and non-pregnant women for 1995-2011: a systematic analysis of population-representative data. The Lancet Global Health 2013; 1(1): e16-e25.

\section{Aquaculture fish production}

Defined as the farming of aquatic organisms. Farmingimplies some form of intervention in the rearing process to enhance production, such as regular stocking, feeding, protection 
from predators, etc. Farming also implies individual or corporate ownership of the stock being cultivated. For statistical purposes, aquatic organisms which are harvested by an individual or corporate body which has owned them throughout their rearing period contribute to aquaculture, while aquatic organisms which are exploitable by the public as a common property resource, with or without appropriate licenses, are the harvest of fisheries. In the case of capture-based aquaculture, only the incremental growth (or weight gain) in captivity, could and should be reported as the production from aquaculture. Data included here covers an aquaculture production of fish, molluscs, crustaceans and miscellaneous aquatic animals but excluding production for marine mammals, crocodiles, corals, pearls, sponges and aquatic plants. Fish production is the sum of aquaculture and capture fish production.

Source: Fisheries and Aquaculture Department (Fishery and Aquaculture statistics)

Owner: FAO

\section{Arable land}

The land under temporary agricultural crops (multiple-cropped areas are counted only once), temporary meadows for mowing or pasture, land under market and kitchen gardens and land temporarily fallow (less than five years). The abandoned land resulting from shifting cultivation is not included in this category. Data for arable land are not meant to indicate the amount of land that is potentially cultivable.

Source: FAO, Statistics Division

Owner: FAO

\section{Area equipped for irrigation}

Area equipped to provide water (via irrigation) to crops. It includes areas equipped for full/partial control irrigation, equipped lowland areas, and areas equipped for spate irrigation.

Source: Land and Water Division (AQUASTAT) Owner: FAO

\section{Area harvested}

Refer to the area from which a crop is gathered. Area harvested, therefore, excludes the area from which, although sown or planted, there was no harvest due to damage, failure, etc It is usually net of temporary crops and sometimes gross of permanent crops. Net area differs from gross area insofar as the latter includes uncultivated patches, footpaths, ditches, headlands, shoulders, shelterbelts, etc. If the crop under consideration is harvested more than once during the year as a consequence of successive cropping (i.e. the same crop is sown or planted more than once in the same field during the year), the area is counted as many times as harvested

Source: FAO, Statistics Division (FAOSTAT)

Owner: FAO

\section{Area irrigated (\%)}

Percent of area equipped for irrigation that is actually irrigated in any given year, expressed in percentage. Irrigated land that is cultivated more than once a year is counted only once. Source: Land and Water Division (AQUASTAT) Owner: FAO

\section{Average dietary energy supply adequacy (\%)}

Expresses the Dietary Energy Supply (DES) as a percentage of the Average Dietary Energy Requirement (ADER). Each country's or region's average supply of calories for food consumption is normalized by the average dietary energy requirement estimated for its population to provide an index of adequacy of the food supply in terms of calories.

Source: FAO, Statistics Division

Owner: FAO

\section{Average protein supply of animal origin} (g/capita/day)

Includes the following groups: meat; offals; animal fats and products; milk and products; eggs, fish, seafood and products; and aquatic products, other.

Source: FAO, Statistics Division

Owner: FAO

\section{Bioenergy consumption in agriculture}

\section{total Bioenergy consumption}

total Energy consumption for power irrigation,

\section{Biofuel production (thousand kt of oil eq.)}

Fuel that is produced through biological processes, including agriculture. They can be derived directly from plants, or indirectly from agricultural, commercial, domestic, and/or industrial wastes.

Source: United Nations Statistics Division

Owner: United Nations Statistics Division

\section{Capture fish production}

Defined as the hunting, collecting and gathering activities directed at removing or collecting live wild aquatic organisms. The capture production statistics here indicates the nominal catches of aquatic organisms, killed, caught, trapped or collected for all commercial, industrial, recreational and subsistence purposes in live weight equivalent. Data included here covers capture production of fish, molluscs, crustaceans and miscellaneous aquatic animals but excluding production for marine mammals, crocodiles, corals, pearls, sponges and aquatic plants. Fish production is the sum of aquaculture and capture fish production.

Source: Fisheries and Aquaculture Department (Fishery and Aquaculture statistics)

Owner: FAO

\section{Cattle}

Common ox (Bos taurus); zebu, humped ox (Bos indicus); Asiatic ox (subgenus Bibos); Tibetan yak (Poephagus grunniens).

Source: FAO, Statistics Division

Owner: FAO

\section{Cereal import dependency ratio}

Tells how much of the available domestic food supply of cereals has been imported and how much comes from the country's own production. It is computed as (cereal imports - cereal exports)/(cereal production + cereal imports - cereal exports) ${ }^{*} 100$. Given this formula the indicator assumes only values <= 100. Negative values indicate that the country is a net exporter of cereals.

Source: FAO, Statistics Division Owner: FAO

\section{Cereals}

Include wheat, rice paddy, barley, maize, popcorn, rye, oats, millets, sorghum, 
buckwheat, quinoa, fonio, triticale, canary seed, mixed grain and cereals nes.

Source: FAO, Statistics Division

Owner: FAO

\section{Children under 5 years of age affected by wasting (\%)}

The proportion of children under five whose weight for height is more than two standard deviations below the median for the international reference population ages 0-59.

Source: World Bank

Owner: World Health Organization, Global Database on Child Growth and Malnutrition

\section{Children under 5 years of age who are stunted} (\%)

Hheight-for-age less than -2 standard deviations of the WHO Child Growth Standards median, among children aged $0-5$ years.

Source: World Bank

Owner: World Health Organization, Global Database on Child Growth and Malnutrition

\section{Credit to agriculture}

This dataset is built by compiling official country data published on-line by national central banks in their monetary and financial statistics publications, either through annual or quarterly reports.

Source: FAO, Statistics Division

Owner: FAO

\section{Crop production}

Refer to the actual harvested production from the field or orchard and gardens, excluding harvesting and threshing losses and that part of crop not harvested for any reason. Production therefore includes the quantities of the commodity sold in the market (marketed production) and the quantities consumed or used by the producers (auto-consumption). When the production data available refers to a production period falling into two successive calendar years and it is not possible to allocate the relative production to each of them, it is usual to refer production data to that year into which the bulk of the production falls. Crop production data are recorded in tonnes (t). In many countries, crop production data are obtained as a function of the estimated yield and the total area. If such a compilation method of production statistics is enforced by the country, it must be ensured that the total area does not refer to sown or planted area, which would give then the biological production, but to the actually harvested area during the year.

Source: FAO, Statistics Division

Owner: FAO

\section{Cropland}

Includes arable land and permanent crops.

Source: FAO, Statistics Division

Owner: FAO

\section{Cropping Intensity Ratio}

The ratio of area harvested to total arable land. The cropping intensity may exceed 100 percent where more than one crop cycle is permitted each year on the same area.

Source: FAO, Statistics Division (FAOSTAT)

Owner: FAO

\section{Dietary energy supply (kcal/pc/day)}

The food available for human consumption, expressed in kilocalories per person per day. At the country level, it is calculated as the food remaining for human use after taking out all non-food utilization, including exports, industrial use, animal feed, seed, wastage and changes in stocks.

Source: FAO, Statistics Division

Owner: FAO

\section{Dietary energy supply, cereals, roots and tubers}

(\%)

Expresses the energy supply (in kcal/caput/day) provided by cereals, roots and tubers as a percentage of the total Dietary Energy Supply (DES) (in kcal/caput/day) calculated from the corresponding countries in the FAOSTAT food balance sheets.

Source: FAO, Statistics Division

Owner: FAO

\section{Domestic food price level index}

An indicator of the relative price of food in a country. The indicator is calculated from the 2011 International Comparison Program (ICP) data from the World Bank as well as general and food consumer price indices from the International Labour Organization made consistent and available on FAOSTAT. Specifically, the ratio of food and non-alcoholic beverages expenditure to actual individual consumption is calculated in purchasing power parity terms relative to the United States. To control for inflation, this ratio is forecasted and backcasted using the ratio of a country's Food Consumer Price Index (FPI) and General Consumer Price Index (CPI) using a 2011 base year, relative to the United States. The indicator is computed for countries for which International Comparison Program data as well as general and food consumer price indices are available.

Source: FAO, Statistics Division

Owner: ILO and World Bank

\section{Domestic food price volatility}

Measures the variability in the relative price of food in a country. The indicator is calculated from the monthly domestic food price level index using monthly consumer and general food price indices and purchasing power parity data from the International Comparison Program conducted by the World Bank (see the Relative Price of Food Indicator for more information). Month-to-month growth rates are calculated, and the standard deviation of these growth rates are calculated over the previous 8 months (8-months rolling standard deviation). The average of these standard deviations is then computed to obtain an annual volatility indicator.

Source: FAO, Statistics Division

Owner: FAO, ILO and World Bank

\section{Employment in agriculture (\%)}

People who work for a public or private employer and receive remuneration in wages, salary, commission, tips, piece rates, or pay in kind. Agriculture corresponds to division 1 (ISIC revision 2) or tabulation categories $A$ and $B$ (ISIC revision 3) and includes hunting, forestry, and fishing.

Source: World Bank

Owner: ILO

\section{Fertilizers (kg/ha)}

Collected through individual countries in product weight through the new FAO Questionnaire on Agricultural Resources - 
Fertilizers and then converted to nutrients and validated for consistency regarding summary totals of production, import, export, non-fertilizer use and consumption for the three types of fertilizers: nitrogen $(\mathrm{N})$, phosphate (P205), potash (K2O) and including complex fertilizers (NP, PK, NK and NPK).Fertilizers may be organic, inorganic or mineral. Organic fertilizers play an important role in crop production and are derived from animals, plants and compost. Mineral fertilizers are available to farmers in solid or liquid form, and are delivered to the farm either in bulk, in bags or in pressurized containers. All fertilizers contain at least one of the major plant nutrients: nitrogen $(\mathrm{N})$, phosphorus $(\mathrm{P})$ or potassium $(\mathrm{K})$.

Source: FAO, Statistics Division

Owner: FAO

\section{Food imports and exports (mln US\$)}

Food comprises the commodities in SITC sections 0 (food and live animals), 1 (beverages and tobacco), and 4 (animal and vegetable oils and fats) and SITC division 22 (oil seeds, oil nuts, and oil kernels)

Source: FAO, Statistics Division and Fisheries Division

Owner: FAO

\section{Food production, average value}

In International Dollars (I\$) divided by the total population. It provides a cross country comparable measure of the relative economic size of the food production.

Source: FAO, Statistics Division

Owner: FAO

\section{Forestry production}

The solid volume or weight of all production of the products specified below. It includes: the production of products that may immediately be consumed in the production of another product (e.g. wood pulp, which may immediately be converted into paper as part of a continuous process). It excludes: the production of veneer sheets that are used for plywood production within the same country. It is reported in cubic metres of solid volume in the case of roundwood, sawnwood and wood based panels and metric tons in the case of charcoal, pulp and paper products.

Source: FAO, Statistics Division

Owner: Forestry Division, FAO

\section{GDP per capita (US\$, PPP)}

Gross domestic product converted to international dollars using purchasing power parity rates. An international dollar has the same purchasing power over GDP as the U.S. dollar has in the United States. GDP at purchaser's prices is the sum of gross value added by all resident producers in the economy plus any product taxes and minus any subsidies not included in the value of the products. It is calculated without making deductions for depreciation of fabricated assets or for depletion and degradation of natural resources. Data are in constant 2011 international dollars.

Source: FAO, Statistics Division

Owner: World Bank

\section{Government expenditure on agriculture (\%}

\section{total outlays)}

Refers to Core Areas of Government Functions Relevant to the Agriculture Sector based on the Classification of Functions of Government (COFOG) as outlined in the IMFs Government Finance Statistics Manual, 2001 (GFSM 2001).
Statistics on expenditures in agriculture, forestry and fisheries and on environmental protection can be used to study the effectiveness of government programs that support an enabling environment for essential public goods with high economic and social returns.

Source: FAO, Statistics Division (FAOSTAT)

Owner: FAO and IMF

\section{Greenhouse gas emissions from agriculture,} forestry and other land use (AFOLU)

Contains all the emissions produced in the different agricultural emissions sub-domains, providing a picture of the contribution to the total amount of GHG emissions from agriculture. GHG Emissions from agriculture consist of non- $\mathrm{CO} 2$ gases, namely methane (CH4) and nitrous oxide (N2O), produced by crop and livestock production and management activities. Land use includes $\mathrm{CO} 2$ emissions by sources and removals by sinks from forest land, cropland and grassland, as well as emissions of non-CO2 from fires of biomass and organic soils.

Source: FAO, Statistics Division

Owner: FAO

\section{Import value index $(2004-2006=100)$}

Value indices represent the change in the currentvalues of Import c.i.f. (cost, insurance and freight) all expressed in US dollars. For countries which report import values on an f.o.b. (free on board) basis, these are adjusted to approximate c.i.f. values (by a standard factor of 112 percent)

Source: FAO, Statistics Division

Owner: FAO

\section{Improved water source (\%)}

Refers to the percentage of the population with reasonable access to an adequate amount of water from an improved source, such as a household connection, public standpipe, borehole, protected well or spring, and rainwater collection. Unimproved sources include vendors, tanker trucks, and unprotected wells and springs. Reasonable access is defined as the availability of at least 20 liters a person a day from a source within one kilometer of the dwelling.

Source: World Bank

Owner: World Health Organization and United Nations Children's Fund, Joint Measurement Programme (JMP)

\section{Labour force participation rate}

The proportion of the population ages 15-64 that is economically active: all people who supply labour for the production of goods and services during a specified period.

Source: World Bank

Owner: KILM database, ILO

\section{Life expectancy at birth (years)}

Indicates the number of years a newborn infant would live if prevailing patterns of mortality at the time of its birth were to stay the same throughout its life.

Source: World Bank

Owner: UNPD World Population Prospects 2010

\section{Livestock primary production}

Include products from live and slaughtered animals. Products from slaughtered animals include meat, offals, raw fats, fresh hides and skins. Products from live animals include 
milk, eggs, honey, beeswax and fibres of animal origin. All data shown relate to total meat production from both commercial and farm slaughter. Data are given in terms of dressed carcass weight, i.e. excluding offals and slaughter fats. Production of beef and buffalo meat includes veal; mutton and goat meat includes meat from lambs and kids, respectively; pig meat includes bacon and ham in fresh equivalent. Poultry meat includes meat from all domestic birds and refers, wherever possible, to ready-to-cook weight. Cow milk production relates to total production of whole fresh milk, excluding the milk sucked by young animals but including amounts fed to livestock. The concept of production of buffalo, sheep and goat milk is the same as for cow milk; however, the cover- age is probably less adequate. Egg production covers all domestic birds which have contributed to egg production during the year, wherever they lay and the corresponding total production, including eggs intended to be used for hatching but excluding waste on farms.

Source: FAO, Statistics Division

Owner: FAO

Whole fresh milk production from Buffalos, Camels, Cows, Goats and Sheep.

Source: FAO, Statistics Division

Owner: FAO

\section{Net trade}

The sum of exports and imports of goods and services.Forest area (\%): The land spanning more than 0.5 hectares with trees higher than 5 meters and a canopy cover of more than 10 percent, or trees able to reach these thresholds in situ. It does not include land that is predominantly under agricultural or urban land use. Forest is determined both by the presence of trees and the absence of other predominant land uses. The trees should be able to reach a minimum height of 5 metres $(m)$ in situ. Areas under reforestation that have not yet reached but are expected to reach a canopy cover of 10 percent and a tree height of $5 \mathrm{~m}$ are included, as are temporarily unstocked areas, resulting from human intervention or natural causes, which are expected to regenerate. Includes: areas with bamboo and palms provided that height and canopy cover criteria are met; forest roads, firebreaks and other small open areas; forest in national parks, nature reserves and other protected areas such as those of specific scientific, historical, cultural or spiritual interest; windbreaks, shelterbelts and corridors of trees with an area of more than 0.5 ha and width of more than 20 $\mathrm{m}$; plantations primarily used for forestry or protective purposes, such as: rubber-wood plantations and cork, oak stands. Excludes: tree stands in agricultural production systems, for example in fruit plantations and agroforestry systems. The term also excludes trees in urban parks and gardens.

Source: FAO, Statistics Division

Owner: FAO

\section{Organic area (\%)}

Sum of areas under "Agricultural area certified organic" and "Agricultural area in conversion to organic". Agricultural area certified organic is the land area exclusively dedicated to organic agriculture and managed by applying organic agriculture methods. It refers to the land area fully converted to organic agriculture. It is the portion of land area (including arable lands, pastures or wild areas) managed (cultivated) or wild harvested in accordance with specific organic standards or technical regulations and that has been inspected and approved by a certification body. Agricultural area in conversion to organic is the land area which is going through the organic conversion process, usually two years period of conversion to organic land.

Source: FAO, Statistics Division

Owner: FAO

\section{Other naturally regenerated forest}

Naturally regenerated forest is forest predominantly composed of trees established through natural regeneration. Other naturally regenerated forest is forest where there are clearly visible indications of human activities. Source: Global Forest Resources Assessment, $\mathrm{FAO}$

Owner: FAO

Per capita food production variability (constant 2004-2006 thousand int\$ per capita)

Corresponds to the variability of the "food net per capita production value in constant 2004-2006 international dollars" as disseminated in FAOSTAT.

Source: FAO, Statistics Division

Owner: FAO

\section{Per capita food supply variability} (kcal/capita/day)

Corresponds to the variability of the "food supply in $\mathrm{kcal} / \mathrm{caput} / \mathrm{day}$ " as disseminated in FAOSTAT.

Source: FAO, Statistics Division

Owner: FAO

\section{Pesticides}

Refer to insecticides, fungicides, herbicides, disinfectants and any substance or mixture of substances intended for preventing, destroying or controlling any pest, including vectors of human or animal disease, unwanted species of plants or animals causing harm during or otherwise interfering with the production, processing, storage, transport or marketing of food, agricultural commodities, wood and wood products or animal feedstuffs, or substances which may be administered to animals for the control of insects, arachnids or other pests in or on their bodies. The term includes substances intended for use as a plant growth regulator, defoliant, desiccant or agent for thinning fruit or preventing the premature fall of fruit, and substances applied to crops either before or after harvest to protect the commodity from deterioration during storage and transport. Pesticides use data refers to quantities of pesticides applied to crops and seeds in the agriculture sector. Figures are expressed in metric tons of active ingredients. However, due to some country reporting practices, the data may be reported by: use in formulated product; sales; distribution or imports for use in the agricultural sector.

Source: FAO, Statistics Division

Owner: FAO

\section{Planted forest}

Planted forest is forest predominantly composed of trees establishedthrough planting and/or deliberate seeding.

Source: Global Forest Resources Assessment, FAO

Owner: FAO

Political stability and absence of violence/terrorism (index)

Measures perceptions of the likelihood that the government will be destabilized or overthrown 
by unconstitutional or violent means, including politically-motivated violence and terrorism.

Source: World Bank

Owner: Brookings Institution and World Bank

\section{Population with access to sanitation facilities}

(\%)

Refers to the percentage of the population with at least adequate access to excreta disposal facilities that can effectively prevent human, animal, and insect contact with excreta. Improved facilities range from simple but protected pit latrines to flush toilets with a sewerage connection. To be effective, facilities must be correctly constructed and properly maintained.

Source: World Bank

Owner: World Health Organization and United Nations Children's Fund, Joint Measurement Programme (JMP)

\section{Population, rural}

De facto population living in areas classified as rural (that is, it is the difference between the total population of a country and its urban population). Data refer to 1 July of the year indicated and are presented in thousands.

Source: FAO, Statistics Division

Owner: United Nations Population Division, World Urbanization Prospects.

\section{Population, total}

De facto population in a country, area or region as of 1 July of the year indicated.

Source: FAO, Statistics Division

Owner: World Population Prospects: The 2012 Revision from the UN Population Division

\section{Prevalence of undernourishment}

Expresses the probability that a randomly selected individual from the population consumes an amount of calories that is insufficient to cover her/his energy requirement for an active and healthy life. The indicator is computed by comparing a probability distribution of habitual daily dietary energy consumption with a threshold level called the minimum dietary energy Requirement. Both are based on the notion of an average individual in the reference population.

Source: FAO, Statistics Division

Owner: FAO

\section{Primary forest}

Naturally regenerated forest of native species, where there are no clearly visible indications of human activities and the ecological processes are not significantly disturbed.

Source: Global Forest Resources Assessment, $\mathrm{FAO}$

Owner: FAO

\section{Renewable water resources withdrawn (\% of total)}

The total volume of groundwater and surface water withdrawn from their sources for human use (in the agricultural, municipal and industrial sectors), expressed as a percentage of the total actual renewable water resources. Source: Land and Water Division (AQUASTAT) Owner: FAO

\section{Rice}

Oryza spp., mainly oryza sativa. Rice grain after threshing and winnowing. Also known as rice in the husk and rough rice. Used mainly for human food.

Source: FAO, Statistics Division

Owner: FAO

\section{Road density (per 100 sq km of land area)}

Includes motorways, highways, and main or national roads, secondary or regional roads, and all other roads in a country. A motorway is a road designed and built for motor traffic that separates the traffic flowing in opposite directions.

Source: World Bank

Owner: International Road Federation, World Road Statistics and electronic files

\section{Terrestrial protected areas (\% total land area)}

Totally or partially protected areas of at least 1,000 hectares that are designated by national authorities as scientific reserves with limited public access, national parks, natural monuments, nature reserves or wildlife sanctuaries, protected landscapes, and areas managed mainly for sustainable use. Marine areas, unclassified areas, littoral (intertidal) areas, and sites protected under local or provincial law are excluded.

Source: World Bank

Owner: United Nations Environmental Program and the World Conservation Monitoring Centre, as compiled by the World Resources Institute

\section{The depth of the food deficit (\%)}

Indicates how many calories would be needed to lift the undernourished from their status, everything else being constant. The average intensity of food deprivation of the undernourished, estimated as the difference between the average dietary energy requirement and the average dietary energy consumption of the undernourished population (food-deprived), is multiplied by the number of undernourished to provide an estimate of the total food deficit in the country, which is then normalized by the total population.

Source: FAO, Statistics Division

Owner: FAO

\section{Total land area}

The total area of the country excluding area under inland water bodies.

Source: FAO, Statistics Division

Owner: FAO

\section{Underweight, children under-5 (\%)}

Percentage of underweight (weight-for-age less than -2 standard deviations of the WHO Child Growth Standards median) among children aged 0-5 years.

Source: World Bank

Owner: World Health Organization, Global Database on Child Growth and Malnutrition. Country-level data are unadjusted data from national surveys, and thus may not be comparable across countries.

\section{Value added from agriculture (\% GDP)}

Agriculture corresponds to ISIC divisions 1-5 and includes forestry, hunting, and fishing, as well as cultivation of crops and livestock production. Value added is the net output of a sector after adding up all outputs and subtracting intermediate inputs. It is calculated without making deductions for depreciation of fabricated assets or depletion and degradation of natural resources. The origin of value added 
is determined by the International Standard Industrial Classification (ISIC), revision 3.

Source: World Development Indicators, World Bank

Owner: IMF

\section{Value of food (excluding fish) imports over total} merchandise exports

Provides a measure of vulnerability and captures the adequacy of foreign exchange reserves to pay for food imports, which has implications for national food security depending on production and trade patterns.

Source: FAO, Statistics Division

Owner: FAO

\section{Water resources per capita (m3/person/year)}

Total annual internal renewable water resources per inhabitant.

Source: Land and Water Division (AQUASTAT)

Owner: FAO

\section{Water withdrawal, agriculture (\% of total)}

Annual quantity of water withdrawn for irrigation, livestock and aquaculture purposes. It includes renewable freshwater resources as well as over-abstraction of renewable groundwater or withdrawal of fossil groundwater, use of agricultural drainage water, (treated) wastewater and desalinated water.

Source: Land and Water Division (AQUASTAT)

Owner: FAO

\section{Water withdrawal, industrial (\% of total water} withdrawal)

Industrial water withdrawal as percentage of total water withdrawal.

Source: Land and Water Division (AOUASTAT)

Owner: FAO

\section{Wheat}

Triticum spp.: common (T. aestivum) durum (T. durum) spelt (T. spelta). Common and durum wheat are the main types. Among common wheat, the main varieties are spring and winter, hard and soft, and red and white. At the national level, different varieties should be reported separately, reflecting their different uses. Used mainly for human food.

Source: FAO, Statistics Division

Owner: FAO

\section{Wood pellet production (thousand tonnes)}

The most common type of pellet fuel and are generally made from compacted sawdust and related industrial wastes from the milling of lumber, manufacture of wood products and furniture, and construction. Other industrial waste sources include Empty Fruit Bunches [EFB], palm kernel shells, coconut shells, and tree tops and branches discarded during logging operations.

Source: FAO, Statistics Division

Owner: Forestry Division, FAO 


\section{Notes}

The country classification adopted in this publication is based on the United Nations M49 classification (http://unstats.un.org/unsd/methods/m49/m49.htm). The country names have been abbreviated for the purpose of this publication. The official FAO names can be found at http://termportal.fao.org/faonocs/ appl/.

Following the creation of the Republic of South Sudan in July 2011, the M49 classification considered the Sudan as part of the Northern Africa region, and South Sudan as part of Eastern Africa. In this report, data for the Sudan are therefore included in the Northern Africa region.

The asterisk in charts and maps indicates the most recent year available in the specified time interval. In the country profiles, when the country data have not been reported for the reference year, data in italics indicate that the value for the most recent year available is shown.

In the tables, a blank means not applicable or, for an aggregate, not analytically meaningful. A 0 or 0.0 means zero or a number that is small enough to round to zero at the displayed number of decimal places.

The $\sim$ in the maps refers to the range specified in the class intervals.

In addition:

- $<5.0$ proportion less than 5 percent

- $<0.1$ less than 100000 people

- ns not statistically significant

Two types of aggregations are used in the book: sum and weighted mean. Two restrictions are imposed when computing the aggregation: i) the sufficiency condition - the aggregation is computed only when sufficient countries have reported data, and the current threshold is set at 50 percent of the variable and the weighting variable, if present; and ii) the comparability condition - as aggregations are usually computed over time, this condition is designed to ensure that the number of countries is comparable over several years; under the current restriction the number of countries may not vary by more than 15 over time.

This publication was carried out under the direction of Pietro Gennari (Chief Statistician and Director, ESS). It was prepared by the Statistics Division (Amy Heyman and Markus Kainu). A special thanks the team leaders for their input and to Nicola Selleri (ESS) for the cover design. 



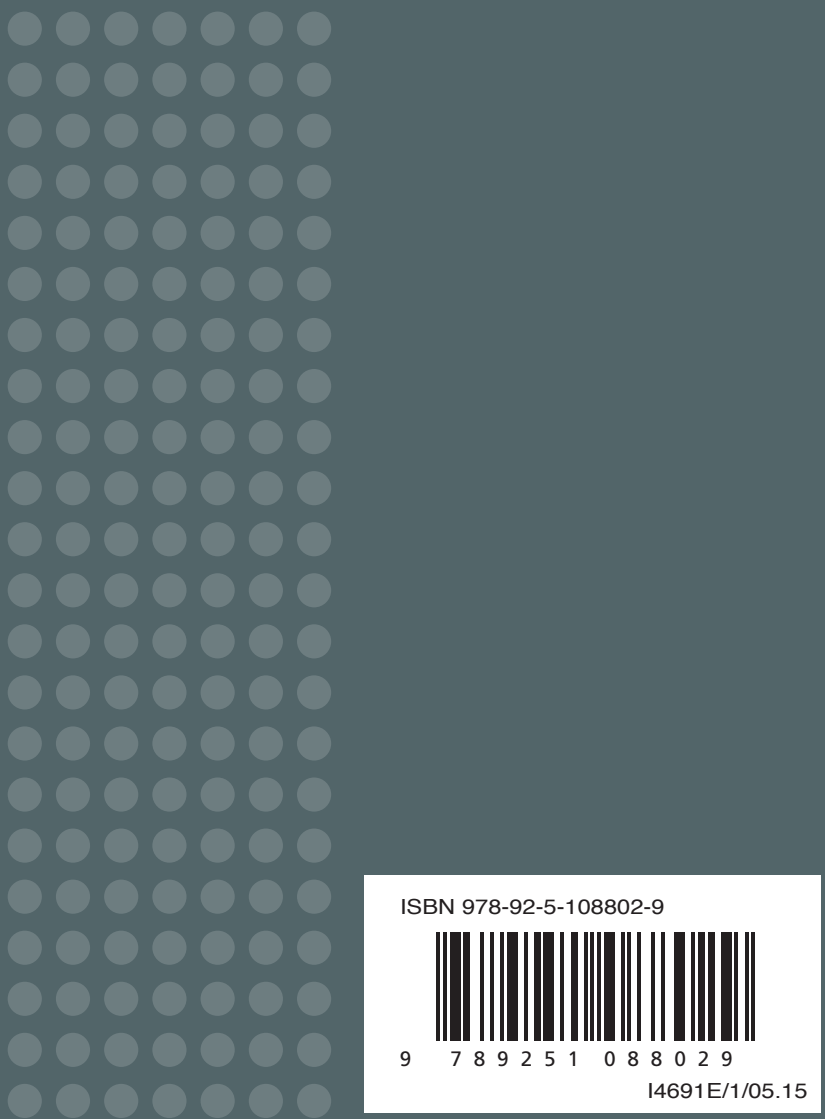

